\title{
Compresibilidad y cambios térmicos en el comportamiento ondulatorio de la fluidización rápida
}

\author{
Tesis que presenta: \\ José Rosario Guadalupe Sánchez López \\ Para obtener el grado de \\ Doctor en Ciencias en Ingeniería Química
}

Asesor:

Dr. Alberto Soria López

Posgrado en Ingeniería Química del Departamento de

Ingeniería de Procesos e Hidráulica de la

División de Ciencias Básicas e Ingeniería

Universidad Autónoma Metropolitana-Iztapalapa

miércoles 25 de julio de 2012 
COMPRESIBILIDAD Y CAMBIOS TERMICOS EN EL

COMPORTAMIENTO ONDULATORIO

DE LA FLUIDIZACION RAPIDA
En México, D.F., se presentaron a las 11:00 horas del día 25 del mes de julio del año 2012 en la Unidad Iztapalapa de la Universidad Autónoma Metropolitana, los suscritos miembros del jurado:

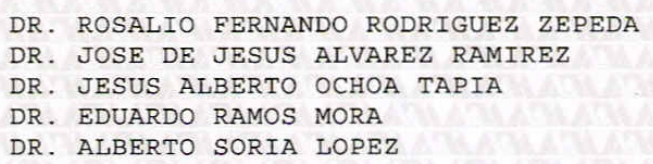

Bajo la Presidencia del primero y con carácter de Secretario el último, se reunieron a la presentación de la Disertación pública cuya denominación aparece al margen, para la obtención del grado de:

DOCTOR EN CIENCIAS (INGENIERIA QUIMICA)

DE: JOSE ROSARIO GUADALUPE SANCHEZ LOPEZ

y de acuerdo con el artículo 78 fracción IV del Reglamento de Estudios Superiores de la Universidad Autónoma Metropolitana, los miembros del jurado resolvieron:

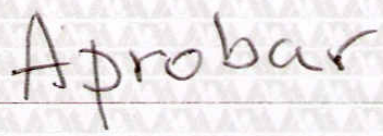

Acto continuo, el presidente del jurado comunicó al interesado el resultado de la evaluación $y$, en caso aprobatorio, le fue tomada la protesta.
LIC. JULIO CA RR LARA ISASSI DIRECTOR DE SISTEMAS ES\&OLARES

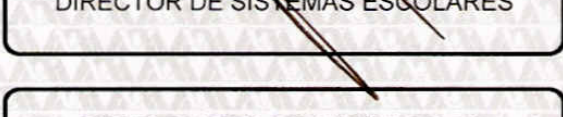

DIRECTOR DE LA DIVISIÓN DE CBI
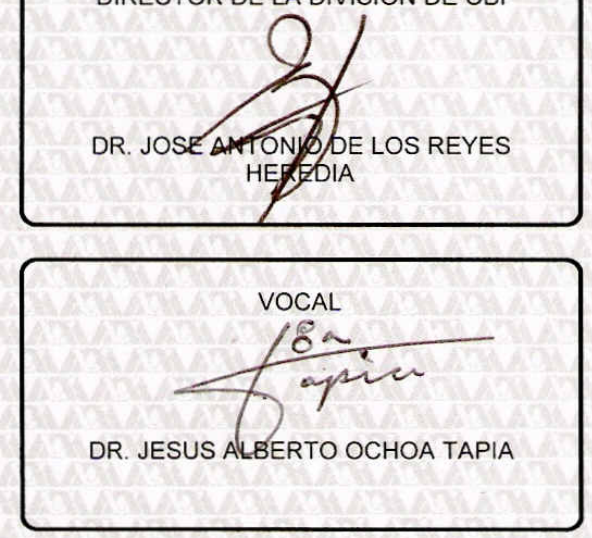

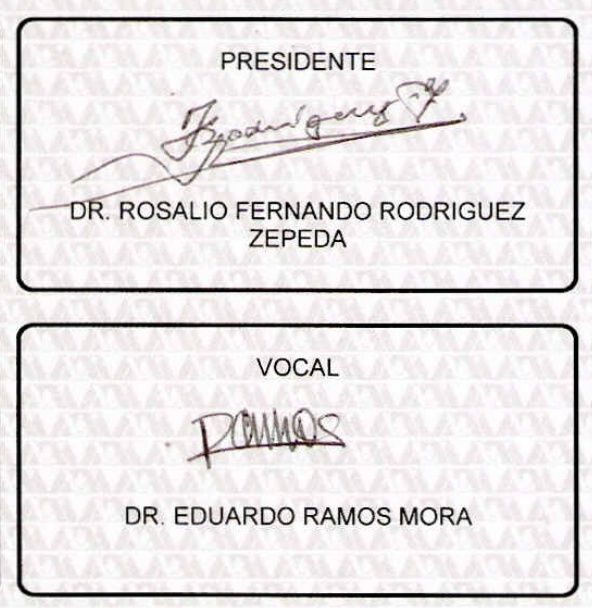

DR. JOSE DEJ J SUS AL NAREZ RAMIREZ 


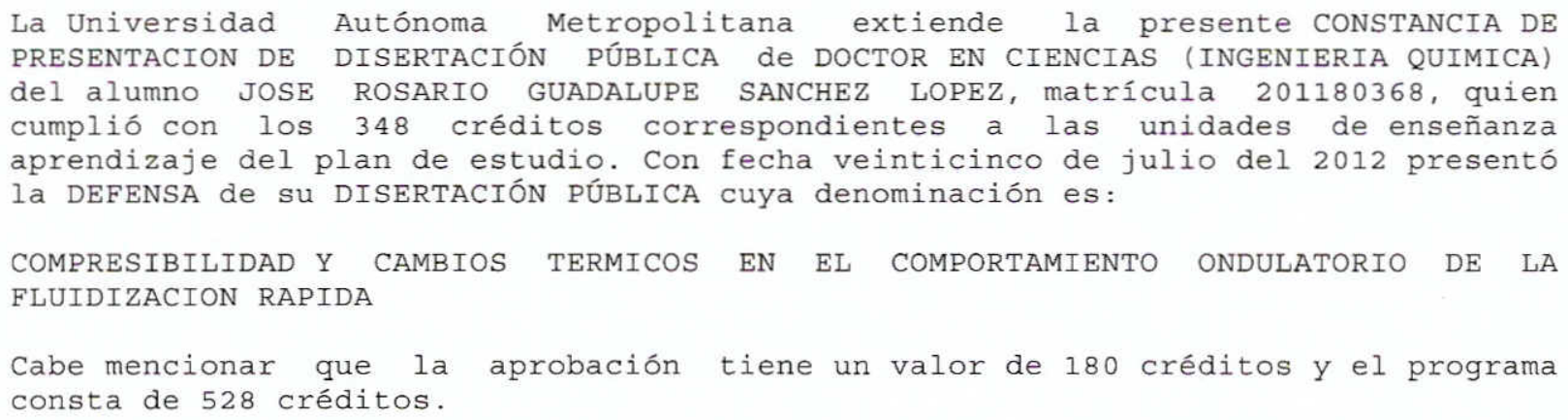

El jurado del examen ha tenido a bien otorgarle la calificación de:

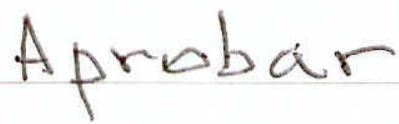

\section{JURADO}

Presidente

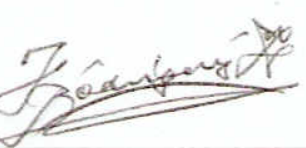

DR. ROSALIO FERNANDO RODRIGUEZ ZEPEDA
DR. ALBERTO

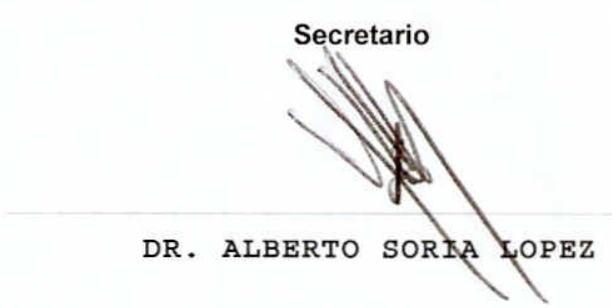

Vocal

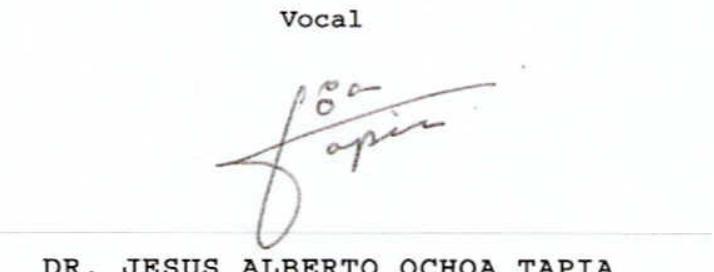

DR. JESUS ALBERTO OCHOA TAPIA
DR. JOSE DE JESUS ALVAREZ RAMIREZ

Vocal

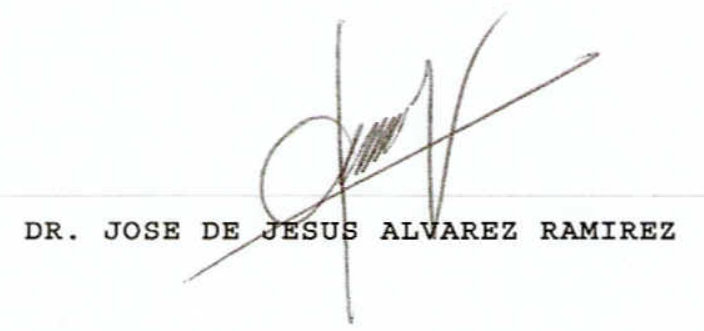

Vocal

Returos

DR. EDUARDO RAMOS MORA

\section{UNIDAD IZTAPALAPA}




\section{Agradecimientos}

Esta tesis doctoral, si bien ha requerido de esfuerzo y mucha dedicación por parte del autor y su director de tesis, su finalización no hubiese sido posible sin la cooperación desinteresada de todas y cada una de las personas que a continuación citaré y muchas de las cuales han sido un soporte muy fuerte en momentos de angustia y desesperación.

Primero y antes que nada quiero agradecer a Dios, por estar conmigo en cada paso que doy, por fortalecer mi corazón e iluminar mi mente y por haber puesto en mi camino a todas aquellas personas que han sido mi columna y compañía durante todo el periodo de estudio.

También deseo expresar mi agradecimiento hoy y siempre a mi familia porque a pesar de no estar presentes físicamente, se que procuran mi bienestar desde mi ciudad, Los Mochis, Sinaloa y está claro que si no fuese por el esfuerzo realizado por ellos, mis estudios de doctorado no hubiesen sido posibles. A mis padres Agustín y Rosa, a mis hermanos mayores Mónica Beatriz y Agustín Enrique y a mis hermanos menores Samuel Christian, Daniel Abraham, Jéssica María y Juan Carlos, a mis sobrinos Carlos Enrique, Grecia y José Emilio porque a pesar de la distancia, el ánimo, apoyo y alegría que me brindan me dan la fortaleza necesaria para seguir adelante.

De igual manera mi más sincero agradecimiento a mi director de tesis, Dr. Alberto Soria López quien ha tenido un papel fundamental en mi formación profesional y con quien siempre estaré agradecido, a la Dra. Elizabeth Salinas Rodríguez por motivarme y por ser una persona que ha influido mucho también en mi formación profesional. Al Coordinador del Posgrado en Ingeniería Química, Dr. José de Jesús Álvarez Ramírez quien siempre me ha apoyado y con quien estoy profundamente agradecido. Al Dr. Jesús Alberto Ochoa Tapia, quien ha sabido escucharme y aconsejarme en momentos en que realmente lo necesitaba. A todas estas personas por permitirme realizar el doctorado en una universidad tan prestigiosa como lo es la Universidad Autónoma Metropolitana-Iztapalapa, alrededor de gente brillante y muy talentosa. 
A las secretarias Martita del área de ingeniería química y a María Elena de la jefatura de IPH de la División de CBI quienes me han facilitado fotocopias, cañón de proyecciones y me han ayudado con trámites administrativos siempre que así lo he necesitado y que me han brindado siempre una sonrisa.

En general quisiera agradecer a todas y cada una de las personas que han vivido conmigo la realización de esta tesis doctoral, con sus altos y bajos porque tanto ellas como yo sabemos que desde lo más profundo de mi corazón les agradezco el haberme brindado todo el apoyo, colaboración, ánimo y sobre todo cariño y amistad. Son tantas personas a las cuales debo parte de este triunfo, de lograr alcanzar mi culminación académica, la cual es el anhelo de todos los que así lo deseamos.

A Salvador por ayudarme con un programa en FORTRAN, Centilio, Omar, David, Gustavo, Claudia F., al Sr. Arnulfo, Saúl V., Max, Sergio, Alicia, Fausto, Carlos Alberto y muy en especial a Xóchitl por todo su apoyo.

A los profesores que me han brindado sus enseñanzas:

- Dr. José de Jesús Álvarez Ramírez por el apoyo económico otorgado al ser su Ayudante de Investigador SNI Nivel III.

- Dr. Jesús Alberto Ochoa Tapia del Depto. de IPH de la UAM-I.

- Dr. Leonardo Dagdug Lima del Depto. de Física de la UAM-I, por sus sabias palabras y por demostrarme que además de ser mi profesor es mi amigo. Por creer en mí e invitarme a participar en la escritura de un libro con el reconocido profesor de esta casa de estudios, el Dr. Leopoldo García Colín-Scherer, lo cual significó para mí un gran honor y que después de poco más de 5 años de trabajo duro, hoy es una realidad.

- Dr. Emmanuel Haro Poniatowski del Depto. de Física de la UAM-I a quien conocí porque impartimos un curso juntos y por brindarme su confianza, motivarme y apoyarme. 
- Dr. Gustavo Izquierdo Buenrostro del Depto. de Matemáticas de la UAM-I quien sin conocerme me apoyó en la demostración de los casos asintóticos del promedio espaciotemporal que están incluidos en el Apéndice $A$ de esta tesis.

- Dr. Lorenzo Héctor Juárez Valencia del Depto. de Matemáticas de la UAM-I quien además de enseñarme métodos numéricos, me brindó su amistad, motivación y confianza, demostrando siempre una alta calidad humana.

- Ing. Uriel Aréchiga Viramontes del Depto. de IPH de la UAM-I con quien di un curso en licenciatura y me enseño acerca de la humildad y paciencia que debe tener un profesor.

En general, quisiera dar un agradecimiento muy especial a mi comité de revisión de tesis, por sus valiosas correcciones y comentarios que ayudaron a perfeccionar este trabajo:

* Dr. Alberto Soria López, del Depto. de IPH de la UAM-I

* Dr. José de Jesús Álvarez Ramírez, del Depto. de IPH de la UAM-I

* Dr. Jesús Alberto Ochoa Tapia, del Depto. de IPH de la UAM-I

* Dr. Rosalío Fernando Rodríguez Zepeda del Instituto de Física de la UNAM en CU

- Dr. Eduardo Ramos Mora del Depto. de Termociencias del Centro de Investigación en Energía de la UNAM en Temixco, Morelos.

Con esto llego al término de los agradecimientos, no sin antes agradecer también al Consejo Nacional de Ciencia y Tecnología (Conacyt) por el apoyo económico otorgado a través de la beca 162476. 
" 


\section{Resumen}

Se modela un flujo de dos fases gas-sólido en el régimen de fluidización rápida mediante un conjunto de ecuaciones con promedio espacio-temporal. Se define el promedio espacio-temporal y se obtienen las ecuaciones de conservación y de balance, de masa, de cantidad de movimiento, de energía y de entropía con promedio espacio-temporal. Estas ecuaciones se obtuvieron sistemáticamente a partir de sus correspondientes ecuaciones locales instantáneas. El promedio espacio-temporal contiene al promedio volumétrico y al promedio temporal como casos asintóticos. Se demuestran y discuten las condiciones bajo las cuales se obtienen los casos asintóticos del promedio espacio-temporal. Se estudia el balance de entropía y al aplicar la segunda ley de la termodinámica se obtienen restricciones sobre los términos fuente generados al promediar y que requieren de cerradura. Estas restricciones sirven como criterio para deducir formas cuadráticas positivas definidas, que cumplan con la segunda ley de la termodinámica.

El enfoque del promedio espacio-temporal permite obtener una ecuación de estado en donde se incorporan correcciones a primer orden o a órdenes más elevados en las desviaciones de las variables de estado. Esta es una diferencia sustantiva entre la descripción con promedios espacio-temporales de sistemas multifásicos y la descripción en términos de la teoría cinética extendida a medios granulares que aplica Gidaspow (1994). No obstante, en este primer estudio no se consideraron y se utilizó la ecuación de estado del gas ideal para representar la compresibilidad de la fase gaseosa, mientras que a la fase sólida se le consideró incompresible.

Se evalúa la hipótesis de incompresibilidad en los modelos de lechos fluidizados mediante la comparación de los modelos (1-D) incompresible y compresible. Todos los términos compresibles aparecen multiplicados por la velocidad de propagación del sonido, $s$ al cuadrado. En los dos modelos propuestos, se recupera la parte incompresible al aplicar el límite, $s \rightarrow \infty$. Se ha extendido el análisis de estabilidad de Liu (1982), para estimar la contribución de la compresibilidad. Se desarrolla un criterio basado en las rapideces de propagación y en el número de onda. Este método se aplica a dos sistemas físicos cuyas propiedades del sólido difieren ampliamente: catalizador de FCC-vapor de agua y arena-aire. 
Se identifica a dos rapideces de propagación de tercer orden con la propagación de la onda de presión. Todos los eigenvalores del modelo de cuarto orden coinciden con las rapideces de propagación de cuarto orden. Se muestra que el efecto de compresibilidad del fluido es tan importante como el efecto del módulo de compresibilidad del sólido. La onda de segundo orden es incompleta, ya que hay un sólo operador de onda actuando en una derivada espacial. Se muestra que al considerar la fricción en la pared, se incluye un modo de propagación asociado a la pared completándose la onda de segundo orden, pasando de parabólica a hiperbólica. Se separa la dinámica de la pared de la del centro del tubo y se obtiene un criterio de Liu generalizado al incorporar este efecto y se estudia cómo es que la pared del tubo aumenta la región de estabilidad basado en el criterio del número de onda.

Se desarrolla un modelo no-isotérmico cuya cerradura es acorde con la segunda ley de la termodinámica, en el que los efectos dominantes están en la región interfacial y no en el volumen. En este modelo se separaron los términos asociados a los dos efectos considerados: compresibilidad y termicidad. Todos los términos asociados a la parte térmica aparecen multiplicados por el coeficiente de expansión volumétrica, $\beta_{V}$. Se recupera el modelo isotérmico al tomar el límite, $\beta_{V} \rightarrow 0$. Se sigue con los dos casos anteriores FCC y CFB, pero con su extensión no-isotérmica a nivel de los coeficientes, encontrando cómo los efectos térmicos modulan a los coeficientes isotérmicos. Se ha demostrado que al considerar los efectos térmicos, un operador ondulatorio de quinto orden gobierna las perturbaciones de fracción volumen, de presión y de temperatura del gas. Mientras que en el caso de la temperatura del sólido, es el operador de quinto orden actuando en una onda de primer orden de la temperatura de las partículas sólidas.

Finalmente, se incorpora el efecto de la pared adiabática y se analiza cómo este modelo es el más general y permite estudiar un conjunto de ocho casos que están dados por la combinación de los efectos: compresibilidad, termicidad y pared del tubo. La metodología diseñada en este trabajo permite estudiar el impacto que tiene cada efecto en la dinámica y en la estabilidad, así como en las estrategias de modelado. 


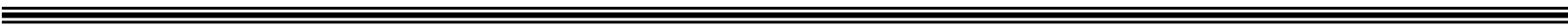

\section{Abstract}

Fast fluidization of a two-phase gas-solid flow was modeled by a set of space-time averaged equations. The space-time average is defined and the conservation and balance equations of mass, momentum, energy and entropy with space-time averaging were obtained. These equations were systematically obtained from the corresponding local-instantaneous equations. Space-time averaging contains volume averaging and time averaging as asymptotic cases. The conditions under which this occurs is demonstrated and discussed. The entropy balance is studied and when the second law of thermodynamics is applied, constraints on the source terms resulting from averaging were found which require closure. These restrictions provide a criterion to deduce positive definite quadratic forms that satisfy the second law of thermodynamics.

The space-time averaging approach allows obtaining a state equation in which first or higher order corrections on state variables deviations are incorporated. This is a substantial difference between the space-time averaging of multiphase systems and the extended kinetic theory approach to granular media of Gidaspow (1994). Nevertheless, in this first study this correction is not considered and the ideal gas state equation was used to represent the gas phase compressibility, whereas the solid phase was assumed incompressible.

The incompressibility hypothesis in fluidized beds models is evaluated by comparing the incompressible and compressible 1-D models. All compressible terms appear multiplied by the squared sound propagation speed, $s$. In both proposed models, the incompressible part was retrieved in the limit $s \rightarrow \infty$. Liu's (1982) linear stability analysis was extended to estimate the compressibility contribution. A criterion based on the propagation speeds and the wave number was developed. This method was applied to two physical systems whose solid properties differ widely: FCC catalyst-water vapor and CFB sand-air. 
Two third-order propagation speeds were identified with pressure wave propagation. All the fourth-order model eigenvalues coincide with the fourth-order propagation speeds. It was shown that the effect of the fluid compressibility is as important as the effect of the solid compressibility modulus. The second-order wave is incomplete, since there is only one wave operator acting in a space derivative. It is shown that when wall friction is considered, a propagation mode associated to the wall is included. This completes the second-order wave, passing from parabolic to hyperbolic. The wall dynamics is separated from the tube core thus resulting in a generalized Liu's criterion. In addition, it is shown how the wall enhances the stability region based on the wave number criterion.

A non-isothermal model was developed which closure is in agreement with the second law of thermodynamics assuming that the interfacial effects overcome the volumetric ones. In this model we separate the terms associated to both considered effects: compressibility and thermicity. All thermal-associated terms appear multiplied by the volumetric expansion coefficient, $\beta_{V}$. The isothermal model is retrieved by the limit, $\beta_{V} \rightarrow 0$. It was continued with FCC and CFB systems, but with its non-isothermal extension at the level of the coefficients finding how thermal effects module the isothermal coefficients. It was demonstrated that the void fraction, pressure and gas temperature are governed by the same fifth order wave operator, since the solid temperature is governed by the fifth wave operator acting in a first order wave of the solid particles temperature.

Finally, the adiabatic wall effect is incorporated and it was shown how this model is the most general and allows studying an eight cases set. These cases are given by the combination of the effects: incompressibility, compressibility, thermal effects and tube wall. The methodology designed in this work allows studying the impact of each effect over the dynamic and the stability, as well as in the modeling strategies. 


\section{O N T E N I D O}

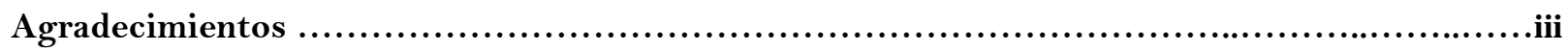

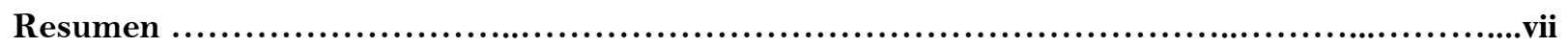

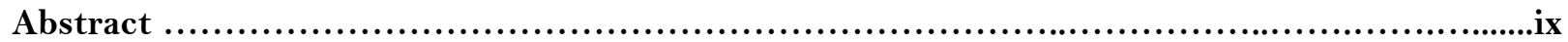

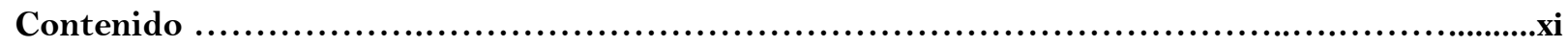

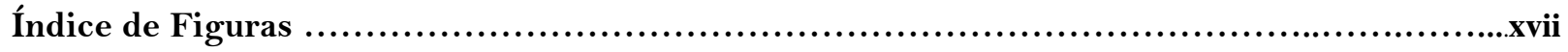

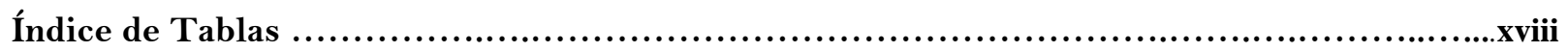

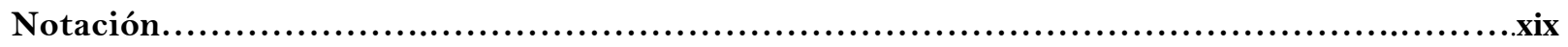

INTRODUCCIÓN. Antecedentes y justificación.............................................

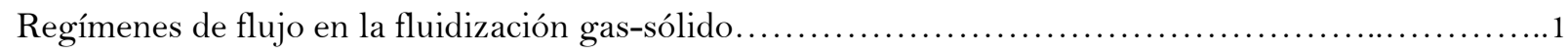

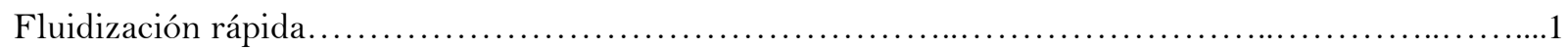

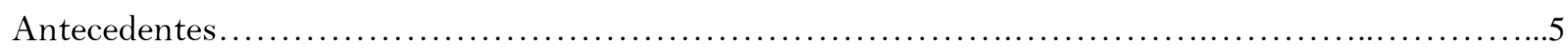

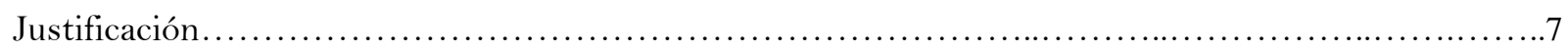

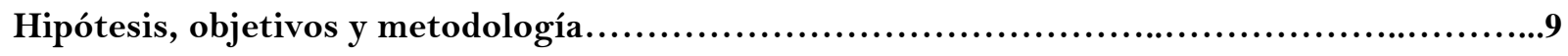

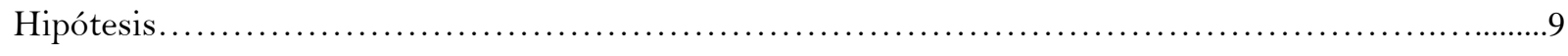

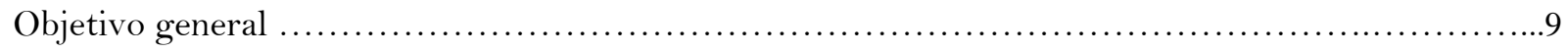

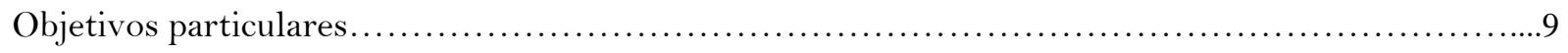

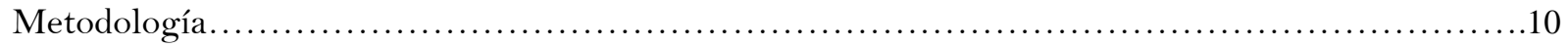

Capítulo 1. Ecuaciones de balance con promedio espacio-temporal ............................11

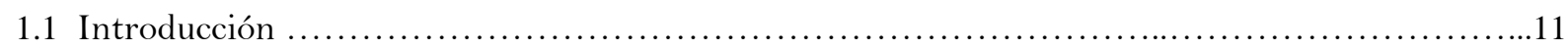

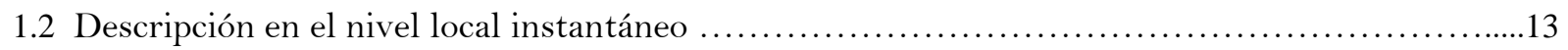

1.2.1 Ecuación de conservación de la masa ...............................................13

1.2.2 Ecuación de balance de cantidad de movimiento.............................................. 


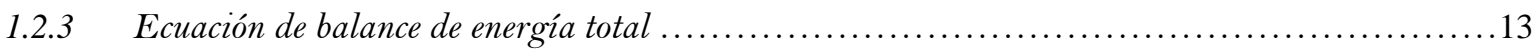

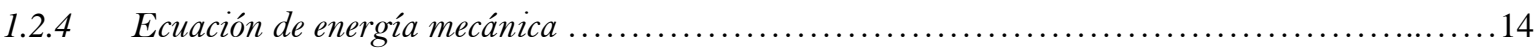

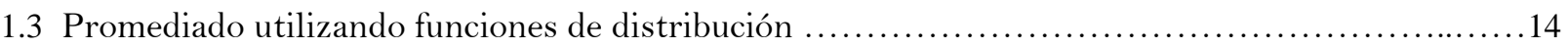

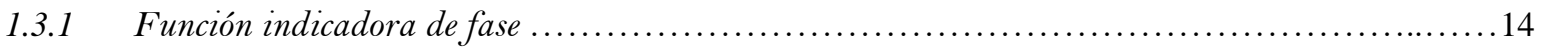

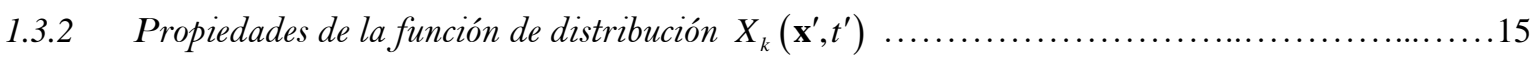

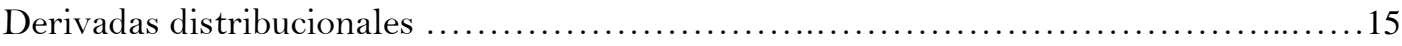

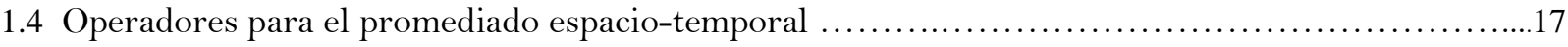

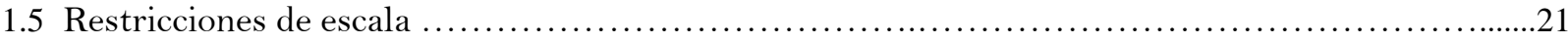

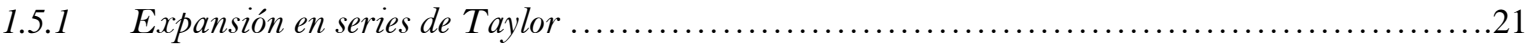

1.6 Teoremas fundamentales para el desarrollo de las ecuaciones con promedio espacio-temporal ....23

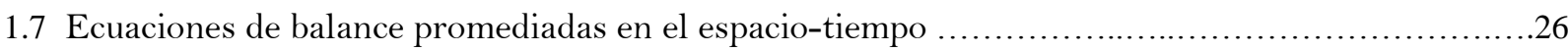

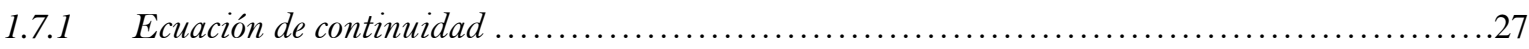

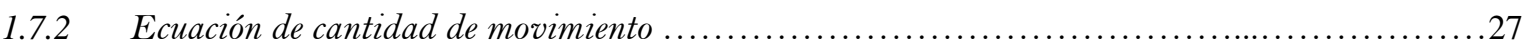

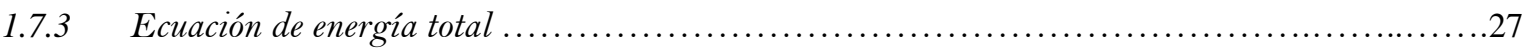

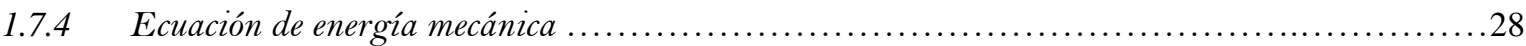

\section{Capítulo 2. Segunda ley de la termodinámica y su aplicación a la cerradura de}

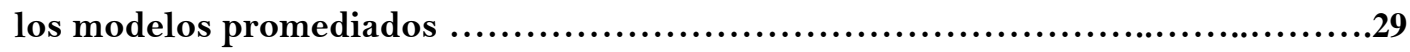

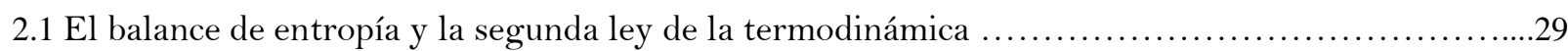

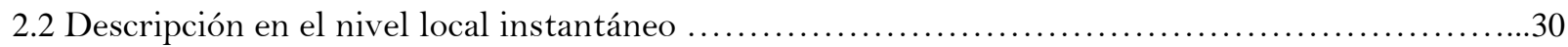

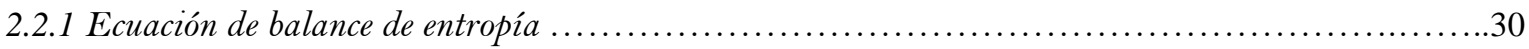

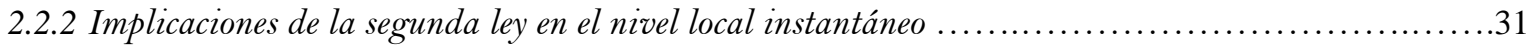

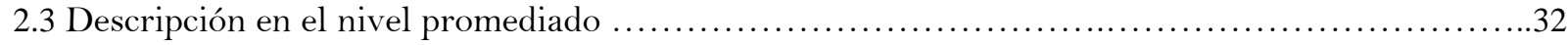

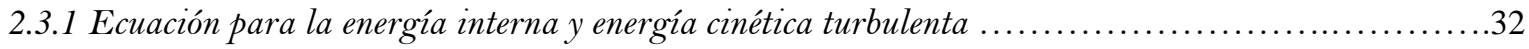

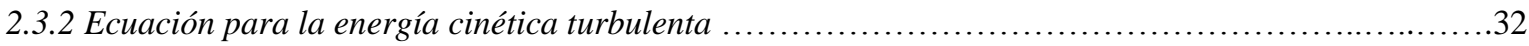

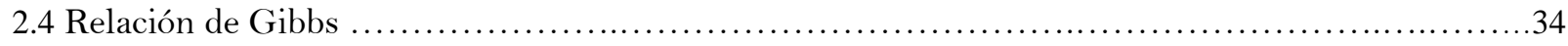


2.5 Restricción a escala promedio de la segunda ley de la termodinámica para modelos

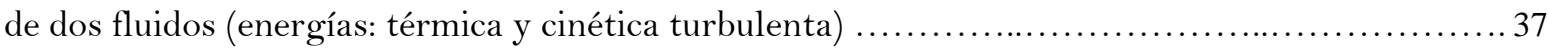

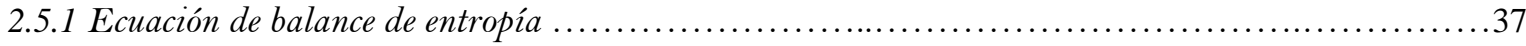

2.6 Restricción de la segunda ley de la termodinámica para modelos de dos fluidos (energía

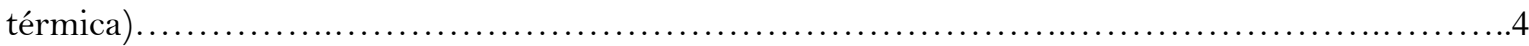

2.7 Restricción de la segunda ley de la termodinámica para modelos de dos fluidos (energía

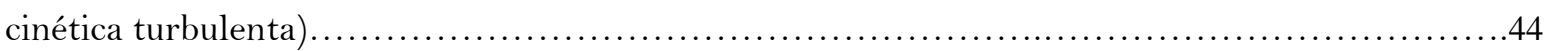

2.8 Segunda ley de la termodinámica en el nivel local y en el nivel promedio ......................45

2.9 Forma de aplicar las restricciones encontradas con la segunda ley de la termodinámica .............46

Capítulo 3. Modelo isotérmico unidimensional con promedio espacio-temporal ....................47

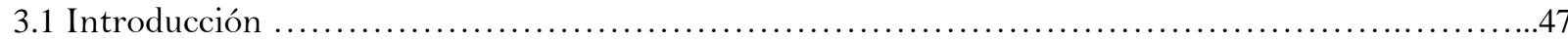

3.2 Modelamiento del flujo gas-sólido en el tubo elevador (riser) $\ldots \ldots \ldots \ldots \ldots \ldots \ldots \ldots \ldots \ldots \ldots \ldots . . .48$

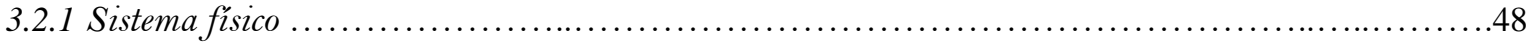

3.2.2 Ecuaciones promediadas en el espacio-tiempo .............................................48

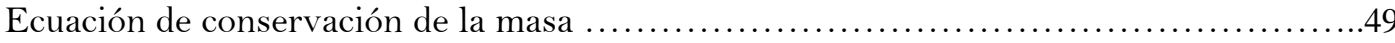

Ecuación de cantidad de movimiento ..........................................49

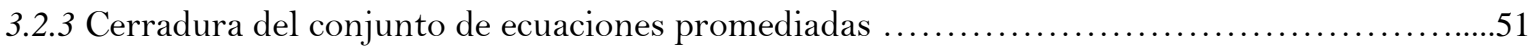

3.3 Validación de las cerraduras con la segunda ley de la termodinámica ..........................52

3.3.1 Cerraduras propuestas y su consistencia con las restricciones impuestas por la segunda ley de la

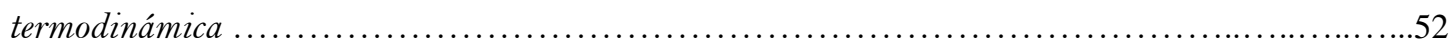

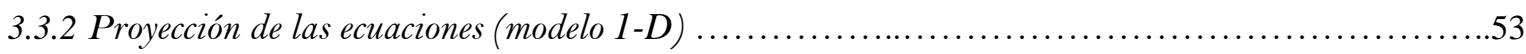

Capítulo 4. Propagación de ondas isotérmicas compresibles e incompresibles con modelos de lechos fluidizados rápidos 1-D linealizados ........................57

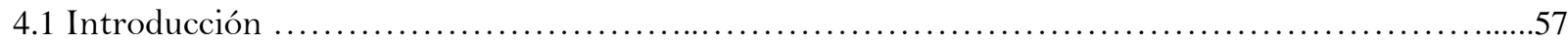

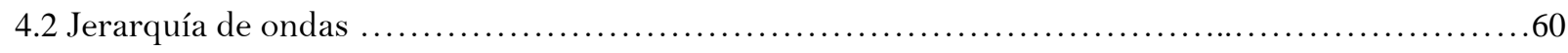

4.2.1 Ecuaciones del modelo .................................................................60 
4.2.2 Enunciado del problema ..........................................................62

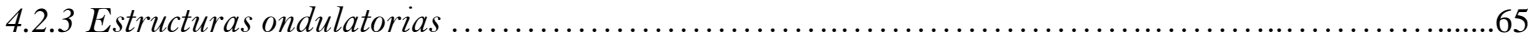

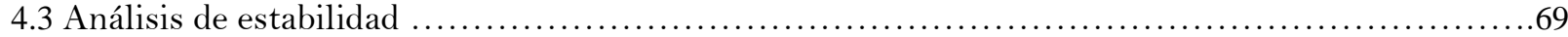

4.3.1 Análisis de estabilidad de Whitham por aproximación de velocidades de fase .....................69

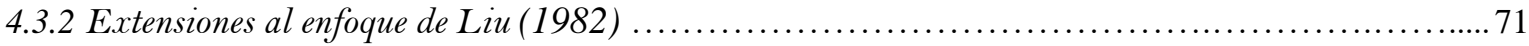

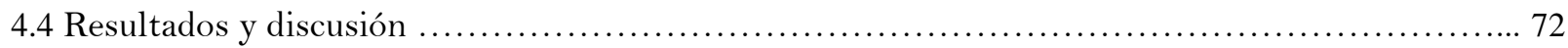

4.4.1 Valores de los parámetros y de las condiciones de operación de un sistema catalizador-vapor en una unidad de FCC y un sistema arena-aire en un CFB .....................................72

4.4.2 Comparación de los coeficientes ondulatorios y significado de las jerarquías de onda ................ 73

4.4.3 Análisis de las rapideces de propagación .............................................. 77

4.4.4 Condiciones de estabilidad de Whitham ...................................................

Capítulo 5. Incorporación del efecto de la pared en la propagación de ondas isotérmicas compresibles e incompresibles con modelos de lechos fluidizados rápidos 1-D

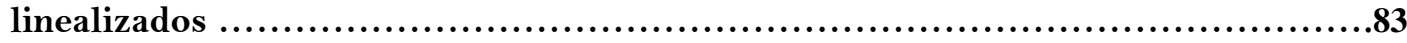

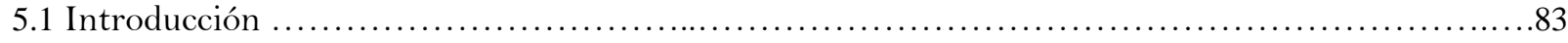

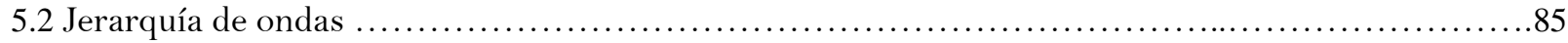

5.2.1 Ecuaciones del modelo ............................................................... 85

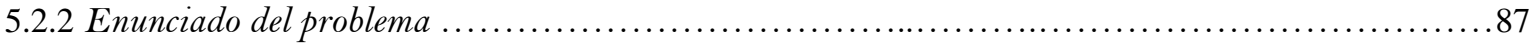

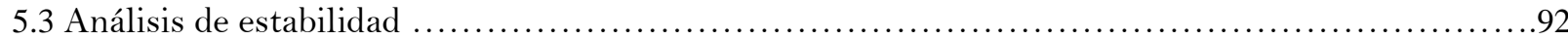

5.3.1 Análisis de estabilidad de Whitham por aproximación de velocidades de fase .....................92

5.3.2 Extensiones al enfoque de Liu (1982) .................................................94

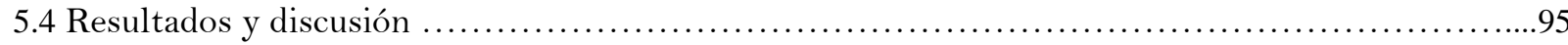

5.4.1 Valores de los parámetros y de las condiciones de operación de un sistema catalizador-vapor en una unidad de FCC y un sistema arena-aire en un CFB .......................................96

5.4.2 Comparación de los coeficientes ondulatorios y significado de las jerarquías de onda .................96

5.4 .3 Análisis de las rapideces de propagación ................................................ 101

5.4.4 Condiciones de estabilidad ............................................................... 


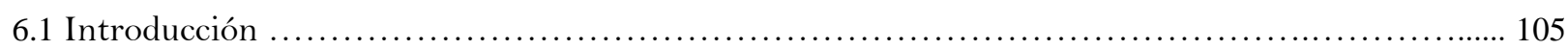

6.2 Modelamiento del flujo no-isotérmico gas-sólido en el riser ...............................105

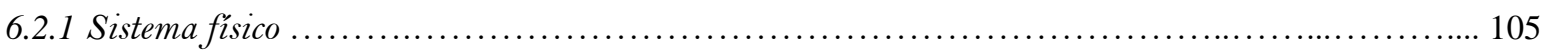

6.2.2 Ecuaciones promediadas en el espacio-tiempo ......................................... 105

Ecuaciones de conservación de la masa ............................................. 106

Ecuación de balance de cantidad de movimiento ........................................... 106

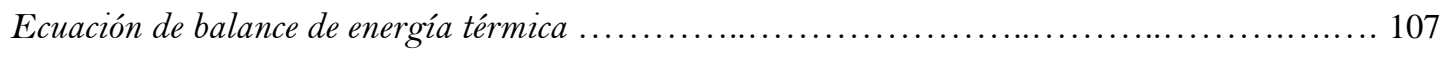

6.2 .3 Cerradura del conjunto de ecuaciones promedio ...................................... 108

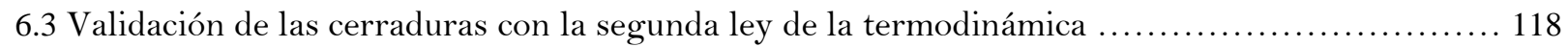

6.3.1 Cerraduras propuestas y su consistencia con las restricciones impuestas por la segunda ley de la

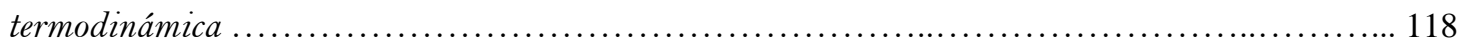

6.3.2 Proyección de las ecuaciones (modelo 1-D) ............................................ 127

\section{Capítulo 7. Propagación de ondas térmicas compresibles e incompresibles con modelos 1-D}

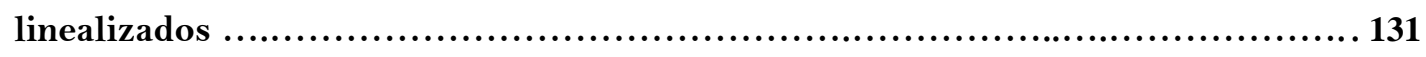

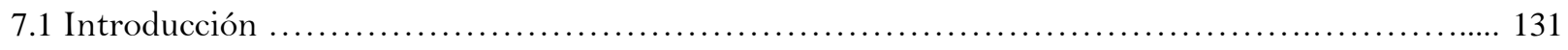

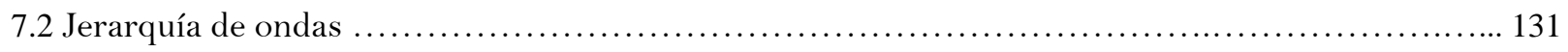

7.2.1 Ecuaciones del modelo ................................................................. 131

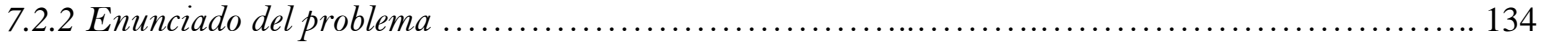

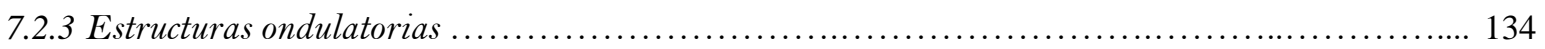

7.2.4 Análisis de términos del operador entre llaves en la Ec. (7.32) ....................................141

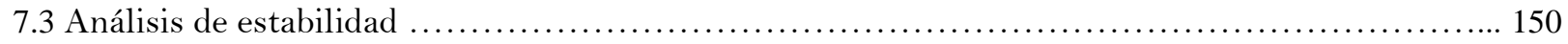

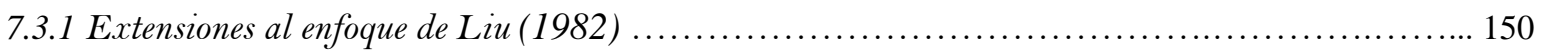

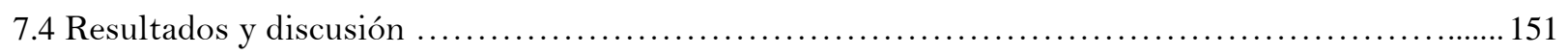

7.4.1 Valores de los parámetros y de las condiciones de operación de un sistema catalizador-vapor en una unidad de FCC y un sistema arena-aire en un CFB, donde están incorporados los efectos térmicos

7.4.2 Comparación de los coeficientes ondulatorios y significado de las jerarquías de onda 
Capítulo 8. Incorporación de la transferencia de calor en la pared en la propagación de ondas térmicas compresibles e incompresibles con modelos 1-D linealizados ............. 155

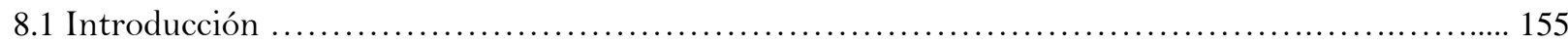

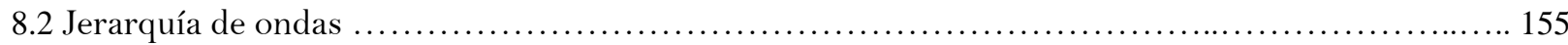

8.2.1 Ecuaciones del modelo ............................................................. 155

8.2.2 Enunciado del problema ........................................................... 157

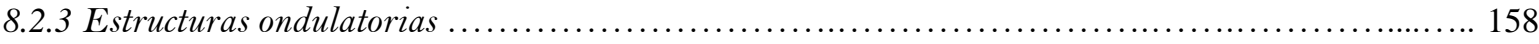

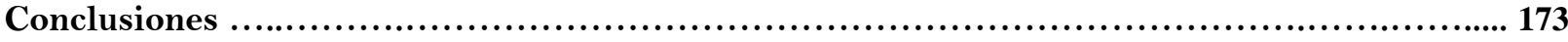

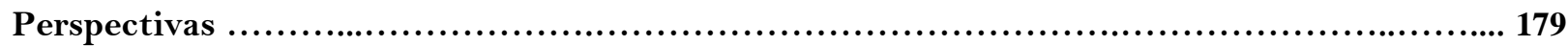

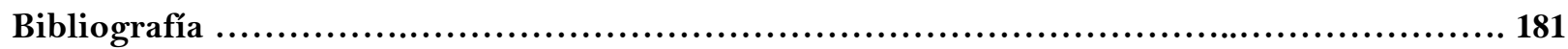

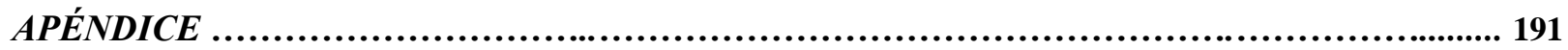

$A$. El promedio volumétrico y el promedio temporal como casos asintóticos del promedio

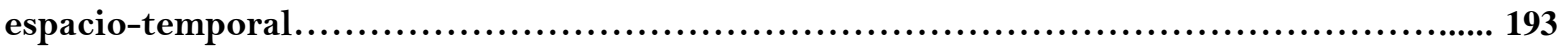

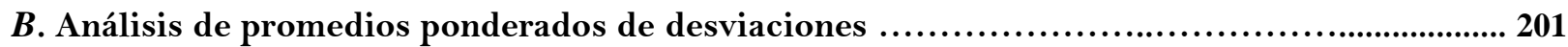

C. Ecuaciones promediadas en el espacio-tiempo ......................................... 203

D. Derivación del conjunto de ecuaciones representado en la Ec. (2.40) ....................... 211

E. Evaluación de la derivada del módulo de compresibilidad del sólido .......................... 219

F. Expresiones explícitas de las matrices B, C y D dadas en la Ec. (4.6)......................... 221

G. Sistemas de ecuaciones cuyas raíces son las rapideces de propagación del Capítulo 4 .......... 223

H. Expresiones explícitas de los componentes de la matriz $D^{*}$ dadas en la Ec. (5.11) ............. 227

I. Coeficientes de la parte de segundo y tercer orden compresible del modelo del Capítulo 5 ..229

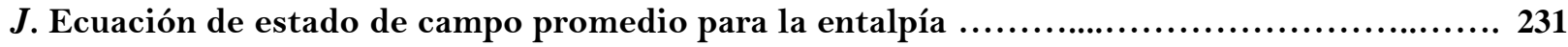

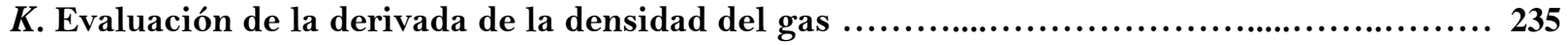

L. Expresiones explícitas de las matrices B, C y D dadas en la Ec. (7.8) .................... 237

M. Sistemas de ecuaciones cuyas raíces son las rapideces de propagación de la Ec. (7.34)...... 239

$N$. Sistemas de ecuaciones cuyas raíces son las rapideces de propagación de la Ec. (7.43) ..... 243

$O$. Expresiones explícitas de los componentes de la matriz $D^{* *}$ dadas en la Ec. (8.16)............ 249 


\section{Índice de Figuras}

Página

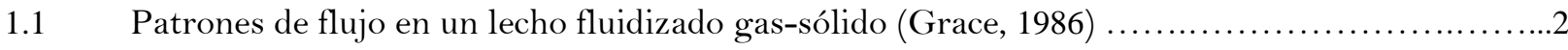

1.2 Lecho fluidizado rápido de donde se extrae el volumen que se toma como sistema para aplicar el método del promedio espacio-temporal con tres vistas en perspectiva

2.1 Metodología para obtener las restricciones que surgen de aplicar la segunda ley de la termodinámica a las ecuaciones promediadas

2.2 Metodología para aplicar las restricciones de la segunda ley en los términos de cerradura de las ecuaciones promediadas

4.1 (a) Tiempo de relajación adimensional $\tau^{\prime}$ del operador diferencial de segundo orden del modelo incompresible para el FCC (línea gris) y Arena (línea negra)

4.2 (a) Coeficientes adimensionales $\tau^{\prime}$ y $C^{\prime}$ para los sistemas: (a) granos de catalizador de FCC y (b) Arena

4.3 Coeficientes adimensionales de la onda compresible de segundo orden (degenerada) para el FCC (línea gris) y para la arena (línea negra)

4.4 Rapidez de propagación de primer orden incompresible $a$ (línea negra), rapideces de propagación de segundo orden incompresibles $c_{1}$ y $c_{2}$ (líneas grises) y rapidez de propagación de segundo orden compresible incompleta $\eta_{21}$ (línea punteada), para los sistemas: (a) FCC y (b) Arena

4.5 Rapideces de propagación de tercer orden del modelo compresible $\alpha_{1}, \alpha_{2}$ y $\alpha_{3}$ en la Ec. (4.29), para los sistemas: (a) FCC y (b) Arena 78

4.6 Rapidez de propagación de la onda de presión para el sistema de la arena.

- Datos experimentales de van der Schaaf y col. (1998). Línea continua: raíz exacta de la Ec. (4.30). Línea punteada: solución aproximada, Ec. (4.47) con $U_{\text {flow }}=0$

$4.7 \quad \kappa_{0}$ para estabilidad lineal, como una función de $\varepsilon_{s 0}$ para los sistemas:

(a) FCC y (b) Arena

4.8 Aumento de la región de estabilidad debido al efecto de compresibilidad del fluido, expresado como porcentaje por las funciones $H_{1}$ y $H_{2}$, para los sistemas: (a) FCC y (b) Arena ....

5.1 Coeficiente $A$ para los sistemas: Arena (línea gris) y FCC (línea negra).....................97

5.2 Coeficiente de tercer orden $C_{w}^{\prime}$ para los sistemas: Arena (línea gris) y FCC (línea negra)......98 
5.3 Coeficiente de segundo orden $B^{\prime}$ para los sistemas: Arena (línea gris) y FCC (línea negra)......99

5.4 Coeficiente de segundo orden para los sistemas: Arena (línea gris) y FCC (línea negra)........100

5.5 Contribución de segundo orden de los efectos de pared a las rapideces de propagación, como función de la fracción volumen de sólido para los sistemas: (a) FCC y (b) Arena

5.6 Rapideces de propagación de segundo orden, para los sistemas:

(a) FCC y (b) Arena 102

5.7 Rapideces de propagación $\gamma_{2}$ y $\alpha_{3 w}$ como función de la fracción volumen

de sólido, para los sistemas: (a) FCC y (b) Arena

5.8 Número de onda para estabilidad lineal, como función de la fracción

volumen de sólido para el sistema de la Arena .......................................104

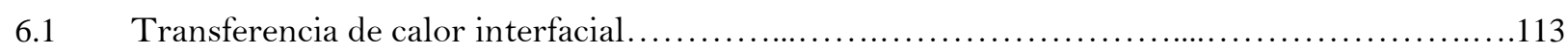

6.2 Transferencia de calor con la pared del tubo ........................................ 116

7.1 Coeficientes adimensionales $\tau^{\prime}$ y $C^{\prime}$ considerando los efectos térmicos y sin considerarlos para los sistemas: (a) granos de catalizador de FCC y (b) Arena

7.2 Coeficientes adimensionales de la onda compresible de segundo orden (degenerada) para el FCC (línea gris) y para la arena (línea negra) 154

\section{Índice de Tablas}

4.1 Valores de los parámetros y condiciones de operación en estado base.......................................72

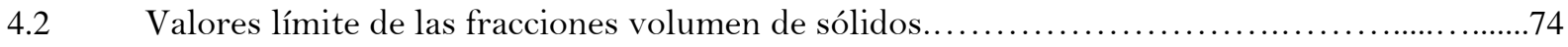

4.3 Errores porcentuales de las rapideces de propagación de las ondas de presión

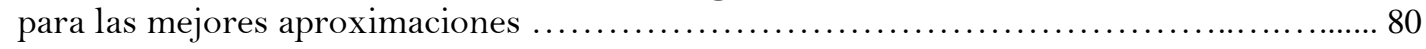

5.1 Valores de los parámetros y de las condiciones de operación en estado base ...................95

7.1 Ecuaciones de perturbación obtenidas al eliminar una variable con el modelo no-isotérmico sin efectos de pared ...........................................139

7.2 Valores de los parámetros y de las condiciones de operación en estado base ................152

8.1 Ecuaciones de perturbación obtenidas al eliminar una variable con el modelo

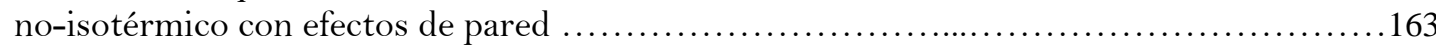

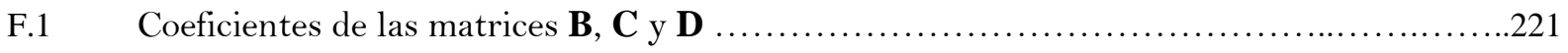

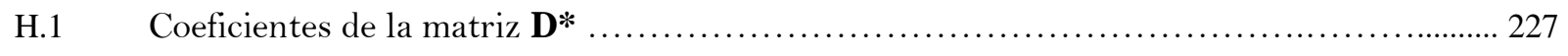

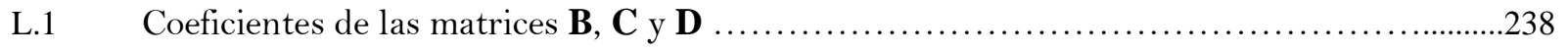

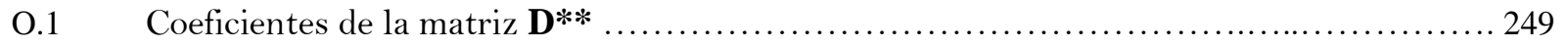




\section{Notación}

En esta Sección definimos las variables que aparecen dentro de la tesis e indicamos las dimensiones que tienen mediante la simbología: $\mathrm{M}$ (masa), $\mathrm{L}$ (longitud), $\mathrm{t}$ (tiempo), $\mathrm{T}$ (temperatura). Las variables en negritas, indican carácter vectorial o tensorial. Salvo que se indique otra cosa, los números entre paréntesis se refieren a las ecuaciones en las que aparecen los símbolos por primera vez o se da su definición.

\begin{tabular}{|c|c|}
\hline$a$ & velocidad de la onda incompresible de primer orden, Ec. $(4.22), \mathrm{L}^{-\mathrm{t}^{-1}}$. \\
\hline$a^{\prime}$ & velocidad adimensional de la onda incompresible de primer orden, adimen. \\
\hline$a_{w}$ & $\begin{array}{l}\text { rapidez de propagación de la onda incompresible de primer orden, } \\
\text { modificada por los efectos de pared, Ec. }(5.29), \mathrm{L} \cdot \mathrm{t}^{-1} \text {. }\end{array}$ \\
\hline$a_{f}$ & $\begin{array}{l}\text { rapidez de la onda incompresible de tercer orden (dinámica de la pared } \\
\text { exclusivamente), definida en la Ec. }(5.26), \mathrm{L} \cdot \mathrm{t}^{-1} \text {. }\end{array}$ \\
\hline$a_{k \ell}$ & área interfacial específica, formada por las fases $k \mathrm{y} \ell$, Ec. $(1.41), \mathrm{L}^{-1}$. \\
\hline$a_{\gamma \sigma}=a_{\sigma \gamma}$ & área interfacial específica, entre la fase continua y la dispersa, $\mathrm{L}^{-1}$. \\
\hline$a_{k W}=a_{W k}$ & área interfacial de la fase $k$-ésima con la pared del tubo, $\mathrm{L}^{-1}$. \\
\hline d & coeficiente de la onda plana, pág. 69. \\
\hline$A$ & coeficiente de la onda incompresible de tercer orden asociada a la dinámica \\
\hline & de la pared exclusivamente, definida en la Ec. (5.23), adimensional. \\
\hline$A_{2}$ & $\begin{array}{l}\text { coeficiente de la onda de segundo orden del modelo no-isotérmico } \\
\text { aglutinado, Ec. (7.43) y que se define en la pág. } 147, \mathrm{M} \cdot \mathrm{L}^{-2} \cdot \mathrm{t}^{-3} \cdot \mathrm{T}^{-1} \text {. }\end{array}$ \\
\hline$A_{3}$ & coeficiente de la onda de tercer orden del modelo no-isotérmico aglutinado, \\
\hline & Ec. (7.43) y que se define en la pág. $147, \mathrm{M} \cdot \mathrm{L}^{-3} \cdot \mathrm{t}^{-1} \cdot \mathrm{T}^{-1}$. \\
\hline$A_{4}$ & coeficiente de la onda de cuarto orden del modelo no-isotérmico aglutinado, \\
\hline & Ec. (7.43) y que se define en la pág. $147, \mathrm{M} \cdot \mathrm{L}^{-3} \cdot \mathrm{T}^{-1}$. \\
\hline$A_{5}$ & coeficiente de la onda de quinto orden del modelo no-isotérmico aglutinado, \\
\hline & Ec. (7.43) y que se define en la pág. $147, \mathrm{M} \cdot \mathrm{L}^{-3}$. \\
\hline$A_{g s}$ & área interfacial dentro de $\mathscr{T}(\mathbf{x}) \times T$, Ec. $(1.24), \mathrm{L}^{2}$. \\
\hline
\end{tabular}




\begin{tabular}{|c|c|}
\hline$B$ & $\begin{array}{l}\text { coeficiente de la onda compresible de segundo orden asociada a la dinámica } \\
\text { de la pared exclusivamente, definida en la Ec. }(5.25), \mathrm{t}^{-1} \text {. }\end{array}$ \\
\hline$B^{\prime}$ & $\begin{array}{l}B^{\prime} \doteq B L / U_{0} C \text {, coeficiente adimensional de la onda compresible de segundo } \\
\text { orden asociada a la dinámica de la pared exclusivamente, aparece por } \\
\text { primera vez en la Ec. }(5.48) \text {, adimensional. }\end{array}$ \\
\hline B & matriz de $4 \times 4$, cuyos elementos son constantes, Ec. (F.1). \\
\hline $\mathbf{B}$ & matriz de $6 \times 6$, cuyos elementos son constantes, Ec. (L.1). \\
\hline $\mathrm{Bi}_{\mathrm{s}}$ & $\mathrm{Bi}_{\mathrm{s}} \doteq \frac{\bar{h}_{i} d_{p}}{k_{k}}$, número de Biot de calor de las partículas sólidas, adimensional. \\
\hline $\begin{array}{l}c_{1}, c_{2} \\
c_{21 T}, c_{22 T}\end{array}$ & $\begin{array}{l}\text { velocidades de la onda incompresible de segundo orden, Ec. }(4.21), \mathrm{L}^{-\mathrm{t}^{-1}} \text {. } \\
\text { velocidades de la onda no-isotérmica incompresible de segundo orden, } \\
\text { pág. } 143, \mathrm{~L} \cdot \mathrm{t}^{-1} \text {. }\end{array}$ \\
\hline$c_{41 T} c_{42 T} c_{43 T} c_{44 T}$ & $\begin{array}{l}\text { velocidades de la onda no-isotérmica incompresible de cuarto orden, } \\
\text { Apéndice } M, \mathrm{~L} \cdot \mathrm{t}^{-1} \text {. }\end{array}$ \\
\hline$c_{5 i T}, i=1, \ldots, 5$ & $\begin{array}{l}\text { velocidades de la onda no-isotérmica incompresible de quinto orden, } \\
\text { Apéndice } M, \mathrm{~L} \cdot \mathrm{t}^{-1} \text {. }\end{array}$ \\
\hline$c_{p}$ & capacidad calorífica a presión constante por unidad de masa, $\mathrm{L}^{2} \cdot \mathrm{t}^{-2} \cdot \mathrm{T}^{-1}$. \\
\hline$c_{p k}$ & capacidad calorífica a presión constante de la fase $k$, Ec. $(\mathrm{J} .2), \mathrm{L}^{2} \cdot \mathrm{t}^{-2} \cdot \mathrm{T}^{-1}$. \\
\hline$C$ & representa a la ecuación de continuidad en la Fig. 2.1. \\
\hline$C$ & coeficiente de la onda compresible de tercer orden, Ec. (4.20), adimensional. \\
\hline$C^{\prime}$ & $\begin{array}{l}C^{\prime} \doteq\left(U_{0} / s\right)^{2} C \text {, coeficiente adimensional de la onda compresible de tercer } \\
\text { orden, aparece por primera vez en la Ec. (4.26), adimensional. }\end{array}$ \\
\hline $\mathbf{C}$ & matriz de $4 \times 4$, cuyos elementos son constantes, Ec. (F.2). \\
\hline $\mathbf{C}$ & matriz de $6 \times 6$, cuyos elementos son constantes, Ec. (L.2). \\
\hline$C_{w}$ & $\begin{array}{l}\text { coeficiente de la onda compresible de tercer orden asociada a la dinámica de } \\
\text { la pared exclusivamente, Ec. (5.24), adimensional. }\end{array}$ \\
\hline$C_{w}^{\prime}$ & $\begin{array}{l}C_{w}^{\prime}=C_{w} / C \text {, coeficiente adimensional de la onda compresible de tercer } \\
\text { orden asociado a la dinámica de la pared exclusivamente, aparece por } \\
\text { primera vez en la Ec. }(5.42) \text {, adimensional. }\end{array}$ \\
\hline
\end{tabular}




\begin{tabular}{|c|c|}
\hline$C_{D}$ & $\begin{array}{l}\text { coeficiente de arrastre (de las siglas en inglés: Drag Coefficient), } \\
\text { adimensional. }\end{array}$ \\
\hline $\mathrm{CFB}$ & Circulated Fluidized Bed (lecho fluidizado circulante). \\
\hline CFD & Computational Fluid Dynamics (Dinámica de Fluidos Computacional). \\
\hline $\mathscr{D}$ & región volumétrica, en el Apéndice $A$, pág. 196. \\
\hline $\mathbf{D}$ & matriz de $4 \times 4$, cuyos elementos son constantes, Ec. (F.3). \\
\hline D & matriz de $6 \times 6$, cuyos elementos son constantes, Ec. (L.3). \\
\hline $\mathbf{D}^{*}$ & matriz de $4 \times 4$, cuyos elementos son constantes, Ec. (H.1). \\
\hline $\mathbf{D}^{* *}$ & matriz de $6 \times 6$, cuyos elementos son constantes, Ec. $(\mathrm{O} .1)$. \\
\hline $\mathbf{D}_{k}$ & tensor de rapidez de deformación de la fase $k, \mathrm{t}^{-1}$. \\
\hline$D_{t}$ & diámetro del tubo elevador (considerado constante), L . \\
\hline$d_{p}$ & diámetro promedio de las partículas sólidas, L. \\
\hline $\mathrm{E}$ & error cuadrático medio, Tabla 4.3, adimensional. \\
\hline$E$ & representa a la ecuación de balance de la energía total en la Fig. 2.1. \\
\hline$E_{k}$ & $E_{k} \doteq\left\langle\mathbf{q}_{k} \cdot \nabla^{\prime} X_{k}\right\rangle=-\sum_{k \ell \mid k} a_{k \ell}\left\langle\mathbf{n}_{k} \cdot \mathbf{q}_{k}\right\rangle_{k \ell}$, fuente de calor interfacial, $\mathrm{M} \cdot \mathrm{L}^{-1} \cdot \mathrm{t}^{-3}$ \\
\hline$E_{T g W}$ & transporte de calor de la fase gas con la pared, Ec. (8.13), $\mathrm{M} \cdot \mathrm{L}^{-1} \cdot \mathrm{t}^{-3}$. \\
\hline$E_{T s W}$ & transporte de calor de la fase sólida con la pared, Ec. (8.14), $\mathrm{M} \cdot \mathrm{L}^{-1} \cdot \mathrm{t}^{-3}$. \\
\hline EDP & Ecuación Diferencial Parcial. \\
\hline $\mathbf{e}_{r}$ & vector unitario en la dirección $r$, adimensional. \\
\hline $\mathbf{e}_{z}$ & vector unitario en la dirección $z$, adimensional. \\
\hline$E S$ & representa a la ecuación de balance de la entropía en la Fig. 2.1. \\
\hline$F$ & fuerza promedio local ejercida por el fluido en las partículas, $\mathrm{M} \cdot \mathrm{L}^{-2} \cdot \mathrm{t}^{-2}$. \\
\hline$f_{T}$ & función auxiliar en la demostración del Apéndice $A$, Ec. (A.3). \\
\hline$f_{V}$ & función auxiliar en la demostración del Apéndice $A$, Ec. (A.18). \\
\hline$f_{k F}$ & factor de fricción asociado a la fase $k$, adimensional. \\
\hline$f_{k W}$ & factor de fricción asociado a la fase $k$ con la pared del tubo, adimensional. \\
\hline$F_{k W}$ & fuerza de arrastre de la fase $k$ en la pared del tubo, $\mathrm{M} \cdot \mathrm{L}^{-2} \cdot \mathrm{t}^{-2}$. \\
\hline FCC & Fluid Catalytic Cracking (Desintegración catalítica en medio fluido). \\
\hline
\end{tabular}




\begin{tabular}{|c|c|}
\hline $\mathbf{g}_{k}$ & campo vectorial de la aceleración gravitacional actuando en la fase $k, \mathrm{~L} \cdot \mathrm{t}^{-2}$. \\
\hline$g$ & magnitud del campo de aceleración gravitacional, $\mathrm{L} \cdot \mathrm{t}^{-2}$. \\
\hline$g_{1}, g_{2}$ & $\begin{array}{l}\text { funciones que sirven de cota superior para las funciones } f_{T} \text { y } f_{V} \text {, aparecen } \\
\text { por primera vez en la Ec. (A.3) y en la Ec. (A.18), respectivamente. }\end{array}$ \\
\hline$G^{\prime}$ & $\begin{array}{l}G^{\prime} \doteq L g / U_{0}{ }^{2} \text {, coeficiente adimensional de la onda compresible de segundo } \\
\text { orden, aparece por primera vez en la Ec. }(4.26) \text {, adimensional. }\end{array}$ \\
\hline$h_{i}$ & coeficiente de transferencia de calor asociado a la interfaz, $\mathrm{M} \cdot \mathrm{t}^{-3} \cdot \mathrm{T}^{-1}$. \\
\hline$\overline{h_{i}}$ & $\begin{array}{l}\text { coeficiente promedio de transferencia de calor asociado a la interfaz } s g \text {, } \\
\mathrm{M} \cdot \mathrm{t}^{-3} \cdot \mathrm{T}^{-1} \text {. }\end{array}$ \\
\hline $\bar{h}_{i 0}$ & $\begin{array}{l}\text { coeficiente promedio de transferencia de calor asociado a la interfaz } s g \\
\text { evaluado en el estado base, } \mathrm{M} \cdot \mathrm{t}^{-3} \cdot \mathrm{T}^{-1} \text {. }\end{array}$ \\
\hline$h_{k W}$ & $\begin{array}{l}\text { coeficiente de transferencia de calor asociado a la interfaz entre la fase } k \text { y } \\
\text { la pared, } \mathrm{M} \cdot \mathrm{t}^{-3} \cdot \mathrm{T}^{-1} \text {. }\end{array}$ \\
\hline $\bar{h}_{k W}$ & $\begin{array}{l}\text { coeficiente promedio de transferencia de calor asociado a la interfaz entre la } \\
\text { fase } k \text { y la pared, } \mathrm{M} \cdot \mathrm{t}^{-3} \cdot \mathrm{T}^{-1} \text {. }\end{array}$ \\
\hline$h_{1}, h_{2}$ & $\begin{array}{l}\text { funciones que definen la parte que se amplía el intervalo de estabilidad por } \\
\text { el efecto de la compresibilidad del fluido: } \\
h_{2,1}= \pm \frac{C}{s^{2}}\left(c_{j}^{3}-P_{1} c_{j}^{2}+P_{2} c_{j}-P_{3}\right), j=1,2 \text {, Ec. (4.45), L· } \mathrm{t}^{-1} \text {. }\end{array}$ \\
\hline h.o.t. & high order terms (términos de alto orden). \\
\hline$H_{1}, H_{2}$ & funciones de estabilidad definidas en la Ec. (4.49)-(4.50), adimensionales. \\
\hline$H_{k}$ & entalpía local específica, se trata en el Apéndice $J, \mathrm{~L}^{2} \cdot \mathrm{t}^{-2}$. \\
\hline$i$ & número imaginario que se define como $\sqrt{-1}$. \\
\hline IGT-DOE & Institute of Gas Technology. \\
\hline$k$ & indica la fase $k$-ésima. \\
\hline $\mathrm{K}$ & grados Kelvin, $\mathrm{T}$. \\
\hline$k_{k}$ & coeficiente de conductividad térmica, $\mathrm{M} \cdot \mathrm{L} \cdot \mathrm{t}^{-3} \cdot \mathrm{T}^{-1}$. \\
\hline$K_{1}, K_{2}$ & $\begin{array}{l}\text { operadores diferenciales de segundo y primer orden, respectivamente, } \\
\text { Ec. (4.12)-(4.13). }\end{array}$ \\
\hline
\end{tabular}




\begin{tabular}{|c|c|}
\hline$K_{k}^{\mathrm{Re}}$ & energía cinética turbulenta, aparece por primera vez en la Ec. (1.49), $\mathrm{L}^{2} \cdot \mathrm{t}^{-2}$. \\
\hline$K E$ ó $M E$ & $\begin{array}{l}\text { representa a la ecuación de balance de la energía cinética o energía } \\
\text { mecánica en la Fig. } 2.1 \text { (se tomaron las siglas en inglés de: Kinetic Energy o }\end{array}$ \\
\hline & Mechanical Energy, respectivamente). \\
\hline$L$ & longitud del lecho fluidizado, L. \\
\hline$L_{s}$ & longitud característica de la estructura de flujo, L. \\
\hline $\mathscr{L}$ & $\begin{array}{l}\text { operador local instantáneo general que describe el comportamiento } \\
\text { dinámico del fluido en la región } \mathscr{\mathscr { V }} \text {, aparece por primera vez en la Ec. (1.43). }\end{array}$ \\
\hline$L_{1}, L_{2}, L_{3}$ & operadores diferenciales de segundo orden, Ec. (4.9)-(4.11). \\
\hline$L_{4}, L_{9}$ & operadores diferenciales de orden cero, Ec. (7.13) y Ec. (7.16). \\
\hline$L_{6}, L_{8}, L_{10}$ & operadores diferenciales de primer orden, Ec. (7.14), Ec. (7.15) y Ec. (7.17). \\
\hline$L^{(5)}$ & operador ondulatorio de quinto orden con efectos térmicos, Ec. (7.31). \\
\hline$L_{W}^{(5)}$ & operador ondulatorio de quinto orden (con termicidad y pared), Ec. (8.36). \\
\hline$l_{s}$ & longitud característica de las fases dispersas, $\mathrm{L}$. \\
\hline$\ell$ & longitud característica de las ecuaciones locales-instantáneas, L. \\
\hline$\ell_{\gamma}$ & longitud característica de la fase continua (Fig. 1.2), L. \\
\hline$\ell_{\sigma}$ & longitud característica de la fase dispersa (Fig. 1.2), L. \\
\hline$M_{21}, M_{22}$ & $\begin{array}{l}\text { coeficientes polinomiales de la onda compresible de pared de segundo } \\
\text { orden, Ec. (5.22) y están definidos en el Apéndice } I \text {. }\end{array}$ \\
\hline$M_{31}, M_{32}, M_{33}$ & $\begin{array}{l}\text { coeficientes polinomiales de la onda compresible de pared de tercer orden, } \\
\text { Ec. (5.22) y están definidos en el Apéndice } I \text {. }\end{array}$ \\
\hline$M_{1 \gamma}, M_{2 \gamma}$ & $\begin{array}{l}\text { coeficientes polinomiales asociados a la onda no-isotérmica de tercer orden, } \\
\text { Ec. (7.34) y están definidos en el Apéndice } M \text {, Ec. (M.3) y Ec. (M.4). }\end{array}$ \\
\hline $\mathbf{M}_{k}$ & $\begin{array}{l}\mathbf{M}_{k} \doteq-\left\langle\boldsymbol{\Pi}_{k} \cdot \nabla^{\prime} X_{k}\right\rangle, \text { fuente de cantidad de movimiento interfacial, } \\
\text { Ec. }(1.48), \mathrm{M} \cdot \mathrm{L}^{-2} \cdot \mathrm{t}^{-2} \text {. }\end{array}$ \\
\hline $\mathbf{M}_{k p}$ & $\begin{array}{l}\mathbf{M}_{k p} \doteq\left\langle\nabla^{\prime} X_{k} \cdot\left(P_{k}-\left\langle P_{k}\right\rangle_{i}\right) \mathbf{1}\right\rangle, \text { fuerza de presión interfacial, Ec. (2.30), } \\
\mathbf{M} \cdot \mathrm{L}^{-2} \cdot \mathrm{t}^{-2}\end{array}$ \\
\hline
\end{tabular}




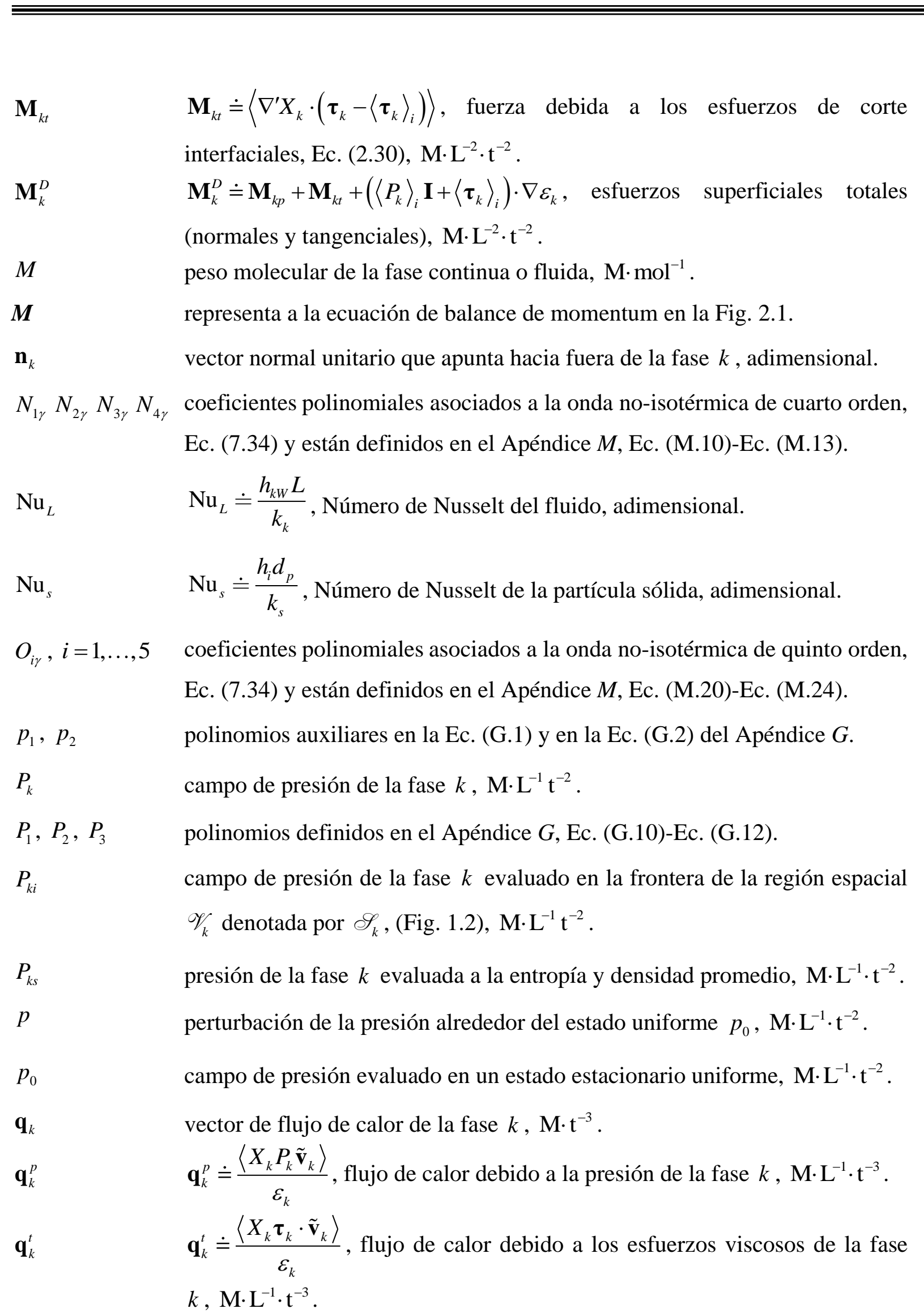




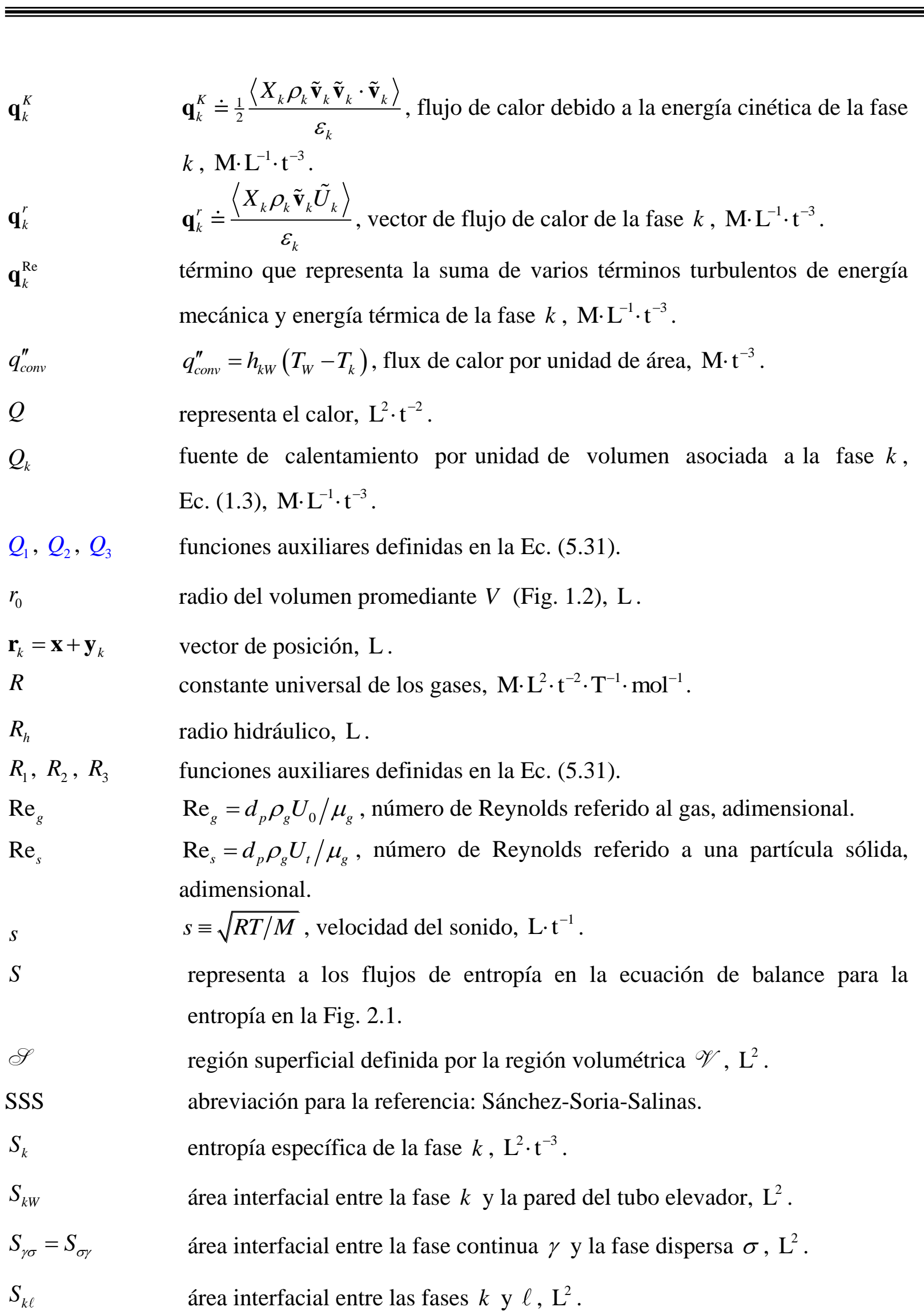




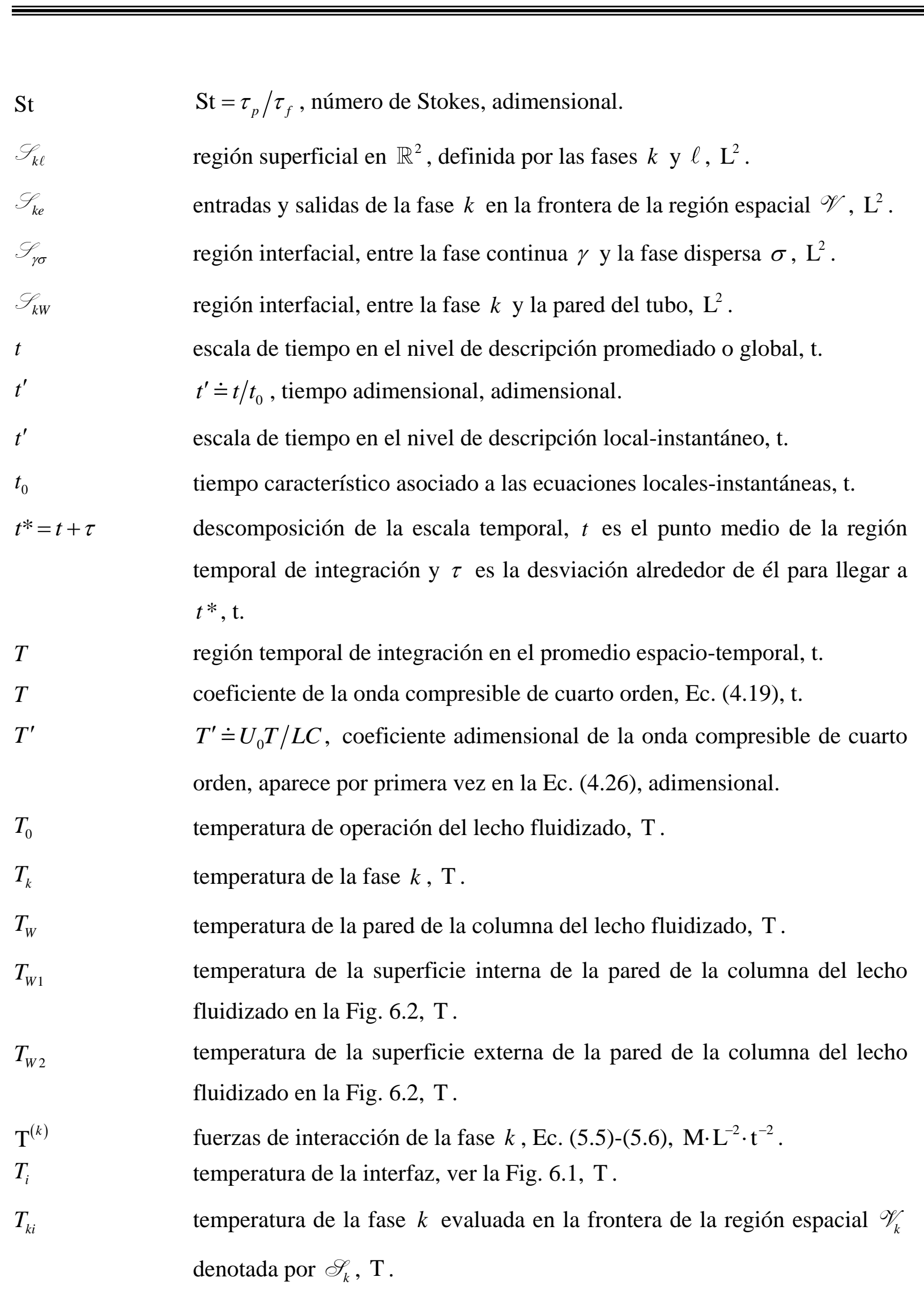




\begin{tabular}{|c|c|}
\hline$T_{k s}$ & temperatura de la fase $k$ evaluada a la entropía y densidad promedio, $\mathrm{T}$. \\
\hline$T_{T_{g}}$ & fuerzas de interacción de la fase gas con termicidad, Ec. (8.7), $\mathrm{M} \cdot \mathrm{L}^{-2} \cdot \mathrm{t}^{-2}$. \\
\hline$T_{T_{s}}$ & fuerzas de interacción de la fase sólida (termicidad), Ec. (8.8), $\mathrm{M} \cdot \mathrm{L}^{-2} \cdot \mathrm{t}^{-2}$. \\
\hline$T E$ & representa a la ecuación de balance de la energía térmica en la Fig. 2.1 (se \\
\hline & tomaron las siglas en inglés de: Thermal Energy). \\
\hline$T K E$ & representa a la ecuación de balance de la energía cinética turbulenta en la \\
\hline & Fig. 2.1 (se tomaron las siglas en inglés de: Turbulent Kinetic Energy). \\
\hline$T S L$ & representa a la segunda ley de la termodinámica en la Fig. 2.1 (se tomaron \\
\hline & las siglas en inglés de: Thermodynamic Second Law). \\
\hline $\mathbf{u}$ & vector columna de $1 \times 4$, de variables perturbadas en la Ec. (4.5) y (5.10). \\
\hline $\mathbf{u}$ & vector columna de $1 \times 6$, de variables perturbadas en la Ec. (7.7) y (8.15). \\
\hline$U$ & representa a la ecuación de la energía interna específica en la Fig. 2.1. \\
\hline$U_{\text {flow }}$ & velocidad de flujo característica, Ec. (4.47) y Ec. (4.48), $\mathrm{L} \cdot \mathrm{t}^{-1}$. \\
\hline$U_{m}$ & velocidad de la mezcla promedio, $\mathrm{L} \cdot \mathrm{t}^{-1}$. \\
\hline$U_{0}$ & $U_{0}=\left(v_{g 0}+v_{s 0}\right) / 2$, velocidad promedio aritmético, $\mathrm{L} \cdot \mathrm{t}^{-1}$. \\
\hline$U_{k}$ & energía interna específica de la fase $k, \mathrm{M} \cdot \mathrm{L}^{2} \cdot \mathrm{t}^{-2}$. \\
\hline$U_{k s}$ & $\begin{array}{l}\text { energía interna específica de la fase } k \text { evaluada a la entropía y densidad } \\
\text { promedio, } \mathrm{M} \cdot \mathrm{L}^{2} \cdot \mathrm{t}^{-2}\end{array}$ \\
\hline$U_{g}$ & velocidad promedio de la fase gas, $\mathrm{L} \cdot \mathrm{t}^{-1}$. \\
\hline$U_{s}$ & velocidad superficial de los sólidos, Ec. (4.46), L· t ${ }^{-1}$. \\
\hline$U_{S k}$ & velocidad superficial de la fase $k, \mathrm{~L} \cdot \mathrm{t}^{-1}$. \\
\hline$U_{S g}$ & velocidad superficial del gas, $\mathrm{L} \cdot \mathrm{t}^{-1}$. \\
\hline$U_{S s}$ & velocidad superficial de las partículas sólidas, $\mathrm{L} \cdot \mathrm{t}^{-1}$. \\
\hline$U_{t}$ & $\begin{array}{l}U_{t} \doteq\left|V_{s}-U_{m}\right|=\varepsilon_{g}\left(V_{g}-V_{s}\right) \text {, magnitud de la velocidad terminal de las } \\
\text { partículas en un medio estancado, } \mathrm{L} \cdot \mathrm{t}^{-1} \text {. }\end{array}$ \\
\hline $\mathbf{U}_{t}$ & $\begin{array}{l}\text { velocidad terminal de las partículas en un medio estancado, pág. } 110 \text {, } \\
\mathrm{L} \cdot \mathrm{t}^{-1} \text {. }\end{array}$ \\
\hline
\end{tabular}




\begin{tabular}{|c|c|}
\hline $\mathbf{v}$ & representa al campo de velocidad local en la Fig. 2.1. \\
\hline $\mathbf{v}_{k}$ & velocidad de flujo de la fase $k$ dentro de $\mathscr{T}, \mathrm{L} \cdot \mathrm{t}^{-1}$. \\
\hline$\tilde{\mathbf{v}}_{k}$ & $\begin{array}{l}\text { desviación del campo de velocidad de la fase } k \text { con respecto a un } \\
\text { promedio ponderado en la masa, } \mathrm{L} \cdot \mathrm{t}^{-1} \text {. }\end{array}$ \\
\hline $\mathbf{v}_{k}^{\prime}$ & $\begin{array}{l}\text { desviación del campo de velocidad de la fase } k \text { con respecto a un } \\
\text { promedio espacio-temporal, } \mathrm{L} \cdot \mathrm{t}^{-1} \text {. }\end{array}$ \\
\hline$v_{k}$ & velocidad promedio de la fase $k, \mathrm{~L} \cdot \mathrm{t}^{-1}$. \\
\hline$v_{k i}$ & $\begin{array}{l}\text { velocidad promedio de la fase } k \text { en el límite cuando nos acercamos a la } \\
\text { interfaz desde el interior de la fase } k, \mathrm{~L} \cdot \mathrm{t}^{-1} \text {. }\end{array}$ \\
\hline$v_{g 0}$ & $\begin{array}{l}\text { velocidad promedio de la fase continua o fluida evaluada en un estado } \\
\text { estacionario uniforme, } \mathrm{L} \cdot \mathrm{t}^{-1} \text {. }\end{array}$ \\
\hline$v_{s 0}$ & $\begin{array}{l}\text { velocidad promedio de la fase dispersa o sólida evaluada en un estado } \\
\text { estacionario uniforme, } \mathrm{L} \cdot \mathrm{t}^{-1} \text {. }\end{array}$ \\
\hline$V_{k}$ & velocidad intersticial de la fase $k, \mathrm{~L} \cdot \mathrm{t}^{-1}$. \\
\hline $\mathbf{V}$ & vector que representa las propiedades termodinámicas en $\mathscr{V}$, Ec. (1.43). \\
\hline$V$ & volumen promediante, definido por la región tridimensional $\mathscr{T}, \mathrm{L}^{3}$. \\
\hline$V_{k}$ & volumen ocupado por la fase $k$ en $V, \mathrm{~L}^{3}$. \\
\hline $\mathscr{V}$ & región en $\mathbb{R}^{3}$ (Fig. 1.2), $\mathrm{L}^{3}$. \\
\hline $\mathscr{T}_{k}$ & región ocupada por la fase $k$ en $\mathscr{V}$; es decir, $\mathscr{T}_{k} \subset \mathscr{V}, \mathrm{L}^{3}$. \\
\hline $\mathbf{w}_{k \ell}$ & velocidad de la interfaz $k \ell, \mathrm{L} \cdot \mathrm{t}^{-1}$. \\
\hline $\mathbf{w}_{\gamma \sigma}=\mathbf{w}_{\sigma \gamma}$ & velocidad de la interfaz gas-sólido, $\mathrm{L} \cdot \mathrm{t}^{-1}$. \\
\hline$W_{k i}$ & $W_{k i} \doteq-\left\langle\nabla^{\prime} X_{k} \cdot\left(\boldsymbol{\Pi}_{k} \cdot \mathbf{v}_{k}\right)\right\rangle$, trabajo interfacial, $\mathrm{M} \cdot \mathrm{L}^{-1} \cdot \mathrm{t}^{-3}$ \\
\hline$W_{k i}^{\prime \prime}$ & $\begin{array}{l}W_{k i}^{\prime \prime} \doteq\left\langle\nabla^{\prime} X_{k} \cdot\left(P_{k} \mathbf{1}+\boldsymbol{\tau}_{k}\right) \cdot\left(\mathbf{v}_{k}-\left\langle\mathbf{v}_{k}\right\rangle_{i}\right)\right\rangle \text {, trabajo “extra” interfacial, aparece } \\
\text { por primera vez en la Ec. }(2.30), \mathrm{M} \cdot \mathrm{L}^{-1} \cdot \mathrm{t}^{-3} \text {. }\end{array}$ \\
\hline $\mathbf{x}$ & vector de posición al centroide de la región $\mathscr{V}, \mathrm{L}$. \\
\hline $\mathbf{x}^{\prime}$ & vector de posición dirigido a cualquier punto de la región $\mathscr{V}, \mathrm{L}$. \\
\hline $\mathbf{x}_{k}^{\prime}$ & $\begin{array}{l}\text { vector de posición característico de la escala de descripción local } \\
\text { instantánea, dirigido desde el sistema coordenado de referencia a un punto } \\
\text { de la fase } k \text { en la región espacial } \mathscr{C} \text {, definida en la Fig. } 1.2, \mathrm{~L} \text {. }\end{array}$ \\
\hline
\end{tabular}




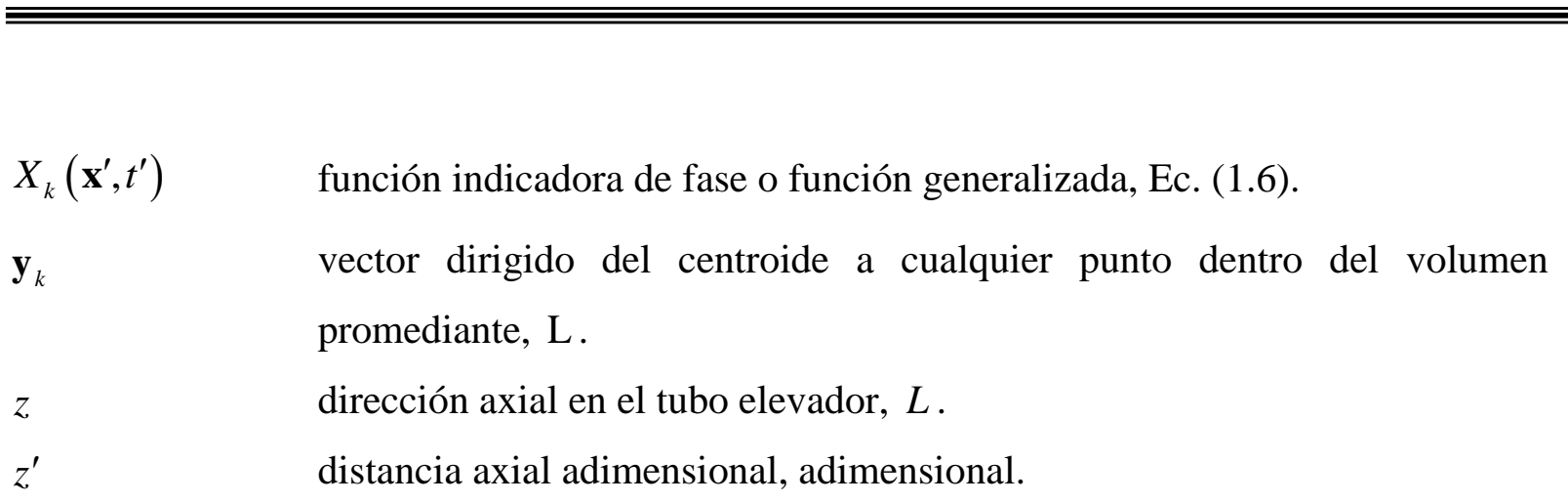

Símbolos griegos

$\alpha_{1}, \alpha_{2}, \alpha_{3} \quad$ rapideces de propagación del modelo aglutinado y que toman en cuenta tanto el comportamiento compresible como el incompresible, resultan de la

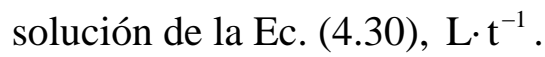

$\alpha_{1 w}, \alpha_{2 w}, \alpha_{3 w}$ rapideces de propagación del modelo aglutinado y que toman en cuenta el comportamiento compresible, el incompresible y los efectos de pared, aparecen por primera vez en la Ec. $(5.30), L \cdot t^{-1}$.

$\beta$ $\beta \equiv \frac{3}{4 d_{p}} C_{D} \rho_{g} U_{t}$, grupo de constantes que es proporcional al arrastre interfacial, $\mathrm{M} \cdot \mathrm{L}^{-3} \cdot \mathrm{t}^{-1}$.

$\beta_{V} \quad \beta_{V} \doteq-\rho_{g}^{-1}\left(\partial \rho_{g} / \partial T_{g}\right)_{P_{g}}$, coeficiente de expansión volumétrica, $\mathrm{T}^{-1}$.

$\beta_{k W} \quad \beta_{k W} \equiv \frac{2}{D_{t}} \varepsilon_{k} f_{k W}$, grupo de constantes que representa la fricción de la fase $k$ con la pared del tubo, $\mathrm{L}^{-1}$.

$\gamma \quad$ fase continua.

$\gamma \quad \gamma \doteq c_{p} / c_{V}$, índice adiabático o razón de capacidades caloríficas, adimensional.

$\gamma_{1}, \gamma_{2} \quad$ rapideces de propagación de la onda compresible de segundo orden, donde están aglutinados los efectos de pared, Ec. (5.30), L· $\mathrm{t}^{-1}$.

$\Gamma \quad$ operador de onda asociado a la pared del tubo, Ec. (5.16), $\mathrm{t}^{-2}$. 


\begin{tabular}{|c|c|}
\hline$\delta$ & representa una derivada inexacta. \\
\hline$\delta_{j}$ & error porcentual, que aparece en la Tabla 4.3, adimensional. \\
\hline$\delta_{V}$ & volumen ocupado por la región $\mathscr{D}, \mathrm{L}^{3}$. \\
\hline$\delta_{k \ell}$ & $\begin{array}{l}\text { representa la distribución delta de Dirac, asociada a la interfaz } k \ell \text {, en el } \\
\text { contexto de las funciones generalizadas, adimensional. }\end{array}$ \\
\hline$\Delta$ & $\begin{array}{l}\text { representa a los términos de generación de entropía en la ecuación de } \\
\text { balance para la entropía en la Fig. } 2.1 \text {. }\end{array}$ \\
\hline$\Delta_{k}$ & $\begin{array}{l}\text { representa a los términos de generación de entropía en la ecuación de } \\
\text { balance para la entropía, Ec. (2.3). }\end{array}$ \\
\hline$\varepsilon_{k}$ & fracción volumen de la fase $k$, adimensional. \\
\hline$\varepsilon=\varepsilon_{g}$ & fracción volumen de la fase continua, adimensional. \\
\hline$\varepsilon_{s}$ & fracción volumen de la fase sólida, adimensional. \\
\hline$\varepsilon_{0}$ & $\begin{array}{l}\text { fracción volumen de la fase continua, evaluada en el estado estacionario } \\
\text { uniforme, adimensional. }\end{array}$ \\
\hline$\varepsilon_{s 0}$ & $\begin{array}{l}\text { fracción volumen de la fase sólida o dispersa, evaluada en un estado } \\
\text { estacionario uniforme, adimensional. }\end{array}$ \\
\hline$\varepsilon_{s 0, \text { min }}$ & $\begin{array}{l}\text { valor crítico a partir del cual puede despreciarse la onda de cuarto orden en } \\
\text { relación a los términos de segundo y tercer orden, Ec. (4.27), adimensional. }\end{array}$ \\
\hline$\varepsilon_{s 0, I C}$ & $\begin{array}{l}\text { valor crítico de fracción volumen de sólido, para el cual la onda } \\
\text { incompresible de segundo orden tiene un mismo coeficiente que la onda } \\
\text { compresible de tercer orden; esto es, } \tau^{\prime}=C^{\prime} \text {, adimensional. }\end{array}$ \\
\hline$\eta_{21}$ & velocidad de la onda compresible de segundo orden, Ec. (4.23), $\mathrm{L} \cdot \mathrm{t}^{-1}$. \\
\hline$\eta_{31}, \eta_{32}, \eta_{33}$ & $\begin{array}{l}\text { velocidades de las ondas compresibles de tercer orden, aparecen por } \\
\text { primera vez en la Ec. }(4.17), \mathrm{L} \cdot \mathrm{t}^{-1} \text {. }\end{array}$ \\
\hline$\eta_{41}, \eta_{42}, \eta_{43}, \eta_{44}$ & velocidades de la onda compresible de cuarto orden, Ec. (4.24)-(4.25), $\mathrm{L}^{-\mathrm{t}^{-1}}$. \\
\hline$\varphi$ & representa una perturbación general; esto es, $\varphi$ es: $\left\{\varepsilon, p, v_{g}, v_{s}, T_{g}, T_{s}\right\}$. \\
\hline$\Phi$ & módulo de compresibilidad de la fase sólida, aparece por primera vez en la \\
\hline & Ec. (3.6) y el Apéndice $E$ está dedicado a su estudio, $\mathrm{M} \cdot \mathrm{L}^{-1} \cdot \mathrm{t}^{-2}$. \\
\hline
\end{tabular}




\begin{tabular}{|c|c|}
\hline$\Gamma_{k}$ & flujo másico interfacial, $\mathrm{M} \cdot \mathrm{L}^{-3} \cdot \mathrm{t}^{-1}$. \\
\hline$\zeta_{k i}$ & $\begin{array}{l}\text { valor de la propiedad } \zeta_{k} \text { en el límite cuando nos acercamos a la interfaz } \\
\text { desde el interior de la fase } k \text {, definido en la pág. } 28 \text {. }\end{array}$ \\
\hline$\theta$ & $\begin{array}{l}\text { ángulo formado entre el vector gravedad y la dirección axial ascendente en } \\
\text { el tubo dada por el vector unitario } \mathbf{e}_{z} \text {, radianes. }\end{array}$ \\
\hline$\kappa$ & número de onda, $\mathrm{L}^{-1}$. \\
\hline$\kappa_{0}$ & número de onda, Ec. (4.43), $\mathrm{L}^{-1}$. \\
\hline$\Lambda_{1}, \Lambda_{2}$ & $\begin{array}{l}\text { operadores de onda asociados a la pared del tubo, Ec. (5.14)-(5.15), } \\
\mathrm{M} \cdot \mathrm{L}^{-3} \cdot \mathrm{t}^{-2} \text {. }\end{array}$ \\
\hline$\lambda_{k}$ & $\begin{array}{l}\text { segundo coeficiente de viscosidad de la fase continua, viscosidad } \\
\text { volumétrica o de bulto, } \mathrm{M} \cdot \mathrm{L}^{-1} \cdot \mathrm{t}^{-1} \text {. }\end{array}$ \\
\hline$\mu_{k}$ & viscosidad dinámica o de corte de la fase $k, \mathrm{M} \cdot \mathrm{L}^{-1} \cdot \mathrm{t}^{-1}$. \\
\hline$\xi_{21}^{\prime}, \xi_{22}^{\prime}$ & $\begin{array}{l}\text { rapideces adimensionales de las ondas de pared de segundo orden, } \\
\text { aparecen por primera vez en la Ec. (5.48), i.e., } \xi_{21}^{\prime} \doteq \xi_{21} / U_{0} \text {, adimensional. }\end{array}$ \\
\hline$\xi_{31}^{\prime}, \xi_{32}^{\prime}, \xi_{33}^{\prime}$ & $\begin{array}{l}\text { rapideces adimensionales de las ondas de pared de tercer orden, aparecen } \\
\text { por primera vez en la Ec. (5.48), i.e., } \xi_{31}^{\prime} \doteq \xi_{31} / U_{0} \text {, adimensional. }\end{array}$ \\
\hline$\xi_{21}, \xi_{22}$ & $\begin{array}{l}\text { rapideces de las ondas de pared de segundo orden, aparecen por primera } \\
\text { vez en la Ec. }(5.27), \mathrm{L} \cdot \mathrm{t}^{-1} \text {. }\end{array}$ \\
\hline$\xi_{31}, \xi_{32}, \xi_{33}$ & $\begin{array}{l}\text { rapideces de las ondas de pared de tercer orden, aparecen por primera vez } \\
\text { en la Ec. }(5.27), \mathrm{L} \cdot \mathrm{t}^{-1} \text {. }\end{array}$ \\
\hline $\boldsymbol{\Pi}_{k}$ & $\boldsymbol{\Pi}_{k} \doteq-P_{k} \mathbf{1}-\boldsymbol{\tau}_{k}$, tensor de presiones de la fase $k, \mathrm{M} \cdot \mathrm{L}^{-1} \cdot \mathrm{t}^{-2}$. \\
\hline $\boldsymbol{\Pi}_{k}^{\mathrm{Re}}$ & $\boldsymbol{\Pi}_{k}^{\mathrm{Re}} \doteq-\left\langle X_{k} \rho_{k} \tilde{\mathbf{v}}_{k} \tilde{\mathbf{v}}_{k}\right\rangle / \varepsilon_{k}$, esfuerzos de Reynolds, $\mathrm{M} \cdot \mathrm{L}^{-1} \cdot \mathrm{t}^{-2}$ \\
\hline$\rho$ & densidad del fluido, $M \cdot L^{-3}$. \\
\hline$\rho_{k}$ & densidad de la fase $k, \mathrm{M} \cdot \mathrm{L}^{-3}$. \\
\hline$\rho_{g}$ & densidad de la fase continua o fluida, $\mathrm{M} \cdot \mathrm{L}^{-3}$. \\
\hline$\rho_{s}$ & densidad de las partículas, $\mathrm{M} \cdot \mathrm{L}^{-3}$. \\
\hline$\sigma$ & fase dispersa. \\
\hline
\end{tabular}




\begin{tabular}{|c|c|}
\hline$\Sigma_{k W}$ & área interfacial entre la fase $k$ y la pared del tubo. \\
\hline$\sum_{k=\gamma, \sigma}$ & suma sobre las dos fases, continua y dispersa. \\
\hline$\sum_{k \ell \mid k}$ & suma sobre todas las interfaces del tipo $k \ell$ tal que la fase $k$ este presente. \\
\hline$\tau$ & tiempo de relajación de la onda dinámica incompresible, Ec. (4.18), t . \\
\hline$\tau^{\prime}$ & $\tau^{\prime} \doteq U_{0} \tau / L$, coeficiente adimensional en la Ec. (4.26), adimensional. \\
\hline$\tau_{f}$ & escala de tiempo característica de la turbulencia del fluido, $\mathrm{t}$. \\
\hline$\tau_{p}$ & tiempo de relajación de las partículas, t . \\
\hline $\boldsymbol{\tau}_{k}$ & tensor de esfuerzos viscosos de la fase $k, \mathrm{M} \cdot \mathrm{L}^{-1} \cdot \mathrm{t}^{-2}$. \\
\hline $\boldsymbol{\tau}_{k}^{(t)}$ & tensor de esfuerzos turbulentos de la fase $k, \mathrm{M} \cdot \mathrm{L}^{-1} \cdot \mathrm{t}^{-2}$. \\
\hline $\boldsymbol{\Phi}_{k}$ & flux de $\boldsymbol{\Psi}_{k}$ dentro de $\mathscr{T}_{k}$. \\
\hline$\chi$ & $\begin{array}{l}\text { variable auxiliar que puede tomar el valor de } \chi=t, z \text {, aparece por primera } \\
\text { vez en la Ec. (K.1). }\end{array}$ \\
\hline$\Psi_{k}$ & $\begin{array}{l}\text { densidad volumétrica de una cantidad termodinámica de interés, aparece } \\
\text { por primera vez en la Ec. (1.11). }\end{array}$ \\
\hline$\omega$ & frecuencia, $\mathrm{Hz}$. \\
\hline$\omega_{I}$ & parte imaginaria de la frecuencia, Hz. \\
\hline$\omega_{R}$ & parte real de la frecuencia, $\mathrm{Hz}$. \\
\hline$\bullet$ & $\begin{array}{l}\text { representa cualquier propiedad termodinámica, con carácter tensorial en } \\
\text { general. }\end{array}$ \\
\hline 1 & tensor métrico o unitario, adimensional. \\
\hline
\end{tabular}




\section{Otros símbolos}

$\Upsilon_{3}$ coeficiente de la onda no-isotérmica de tercer orden, aparece por primera vez en la Ec. (7.34), $\mathrm{M} \cdot \mathrm{L}^{-2} \cdot \mathrm{t}^{-3}$.

$\Upsilon_{4}$ coeficiente de la onda no-isotérmica de cuarto orden, aparece por primera vez en la Ec. (7.34), $\mathrm{M} \cdot \mathrm{L}^{-3} \cdot \mathrm{t}^{-1}$.

$\Upsilon_{5}$ coeficiente de la onda no-isotérmica de quinto orden, aparece por primera vez en la Ec. (7.34), M. $\mathrm{L}^{-3}$.

$\Upsilon_{3}^{\prime} \quad$ coeficiente adimensional de la onda no-isotérmica de tercer orden, aparece por primera vez en la Ec. (7.41) y se define en la Ec. (7.42), adimensional.

$\Upsilon_{4}^{\prime} \quad$ coeficiente adimensional de la onda no-isotérmica de cuarto orden, aparece por primera vez en la Ec. (7.41) y se define en la Ec. (7.42), adimensional.

$\Upsilon_{5}^{\prime} \quad$ coeficiente adimensional de la onda no-isotérmica de quinto orden, aparece por primera vez en la Ec. (7.41) y se define en la Ec. (7.42), adimensional.

$\Psi \quad \Psi=\left(\varepsilon, p, T_{g}, L_{10} T_{s}\right)$, aparece por primera vez en la Ec. (7.32).

$\Psi_{W} \quad \Psi_{W}=\left[\varepsilon, p, T_{g},\left(L_{10}+\Xi_{1}\right) T_{s}\right]$, aparece por primera vez en la Ec. (8.37). 


\section{Símbolos matemáticos}

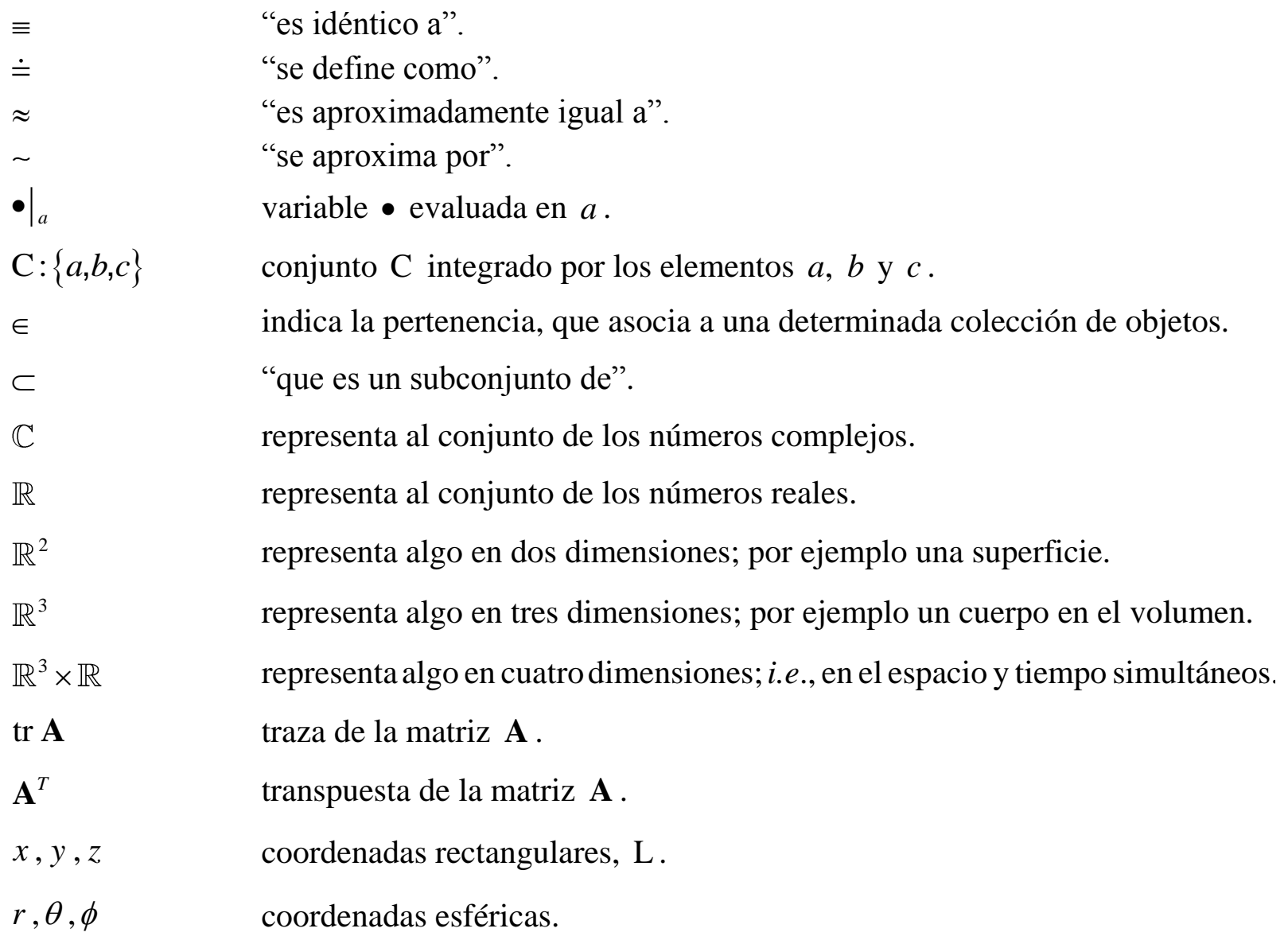

\section{Líneas superiores}

desviación de un promedio ponderado en la masa.

. desviación de un promedio espacio-temporal.

$\therefore \quad$ valor adimensional.

\section{Subíndices}

$\lambda$ correspondiente a la fase líquida.

$\gamma, g \quad$ correspondiente a la fase continua o fluida.

$\sigma, s \quad$ correspondiente a la fase dispersa o sólida.

$k$ correspondiente a la fase $k$.

\section{Superíndices}

$T$ transpuesto. 


\section{Operadores matemáticos}

\langle\rangle$\quad$ operador promedio integral, aparece por primera vez en la Ec. (1.17).

\langle\rangle$_{k \ell} \quad$ operador promedio de superficie intrínseco, Ec. (1.40).

\langle\rangle$_{\gamma \sigma}=\langle\rangle_{\sigma \gamma}$ operador promedio intrínseco en la superficie de las partículas de

\langle\rangle$^{W}$ catalizador.

\langle\rangle$^{k W}$ operador promedio intrínseco en la superficie del tubo elevador.

$\left\langle\bullet{ }_{k}\right\rangle$ operador promedio intrínseco en la superficie del tubo elevador.

$\left\langle\bullet_{k}\right\rangle_{i}$ operador promedio espacio-temporal de $\bullet_{k}$, Ec. (1.17).

$\left\langle\bullet_{k}\right\rangle^{k}$ campo $\bullet_{k}$ con promedio interfacial.

$\left\langle\bullet_{k}\right\rangle^{k, g s}$ operador promedio intrínseco de fase, Ec. (1.20).

$\left\langle\bullet_{k}\right\rangle^{g s}$ operador promedio interfacial, Ec. (1.22).

$\left\{\bullet_{k}\right\}^{k}$ operador promedio interfacial intrínseco, Ec. (1.23).

$\frac{D_{k}}{D t^{\prime}}$ promedio ponderado en la masa del campo $\bullet_{k}$, Ec. (1.25).

$\frac{D_{k}}{D t^{\prime}} \doteq \frac{\partial}{\partial t^{\prime}}+\mathbf{v}_{k} \cdot \nabla^{\prime}$, derivada material cuyo movimiento está referido a la velocidad de la fase $k, \mathrm{t}^{-1}$.

$\frac{D_{k \ell}}{D t}$ $\frac{D_{k \ell}}{D t} \doteq \frac{\partial}{\partial t}+\mathbf{w}_{k \ell} \cdot \nabla$, derivada material cuyo movimiento está referido a la interfaz $k \ell, \mathrm{t}^{-1}$.

$\frac{\partial}{\partial t}=\partial_{t} \quad$ derivada temporal a posición fija, $\mathrm{t}^{-1}$.

$\frac{\partial}{\partial z}=\partial_{z} \quad$ derivada espacial a tiempo fijo, $\mathrm{L}^{-1}$.

$\frac{\partial}{\partial n_{k}} \quad$ derivada normal, $\mathrm{L}^{-1}$.

$\nabla^{\prime}$

Operador gradiente, nabla o del en la escala local, $\mathrm{L}^{-1}$.

$\nabla$ Operador gradiente, nabla o del en la escala promedio o global, $\mathrm{L}^{-1}$.

$\lim _{x \rightarrow a} f(x) \quad$ límite de la función $f(x)$ cuando $\mathbf{x}$ tiende al valor de $a$. 



\section{INTRODUCCIÓN. Antecedentes y justificación}

Regímenes de flujo en la fluidización gas-sólido

Los regímenes de flujo en una sola fase pueden clasificarse de acuerdo a su movimiento interno en: laminar, de transición y turbulento. Sin embargo, los flujos en dos o más fases no pueden clasificarse de esta manera y los distintos regímenes se clasifican de acuerdo a la geometría de la interfaz (Ishii, 1990). Parte de la definición del régimen de flujo es una descripción del arreglo morfológico de las fases o patrón de flujo (Wallis, 1969).

La introducción de gas por el fondo de una columna que contiene partículas sólidas mediante un distribuidor de gas puede causar que las partículas se fluidicen. Se han identificado diversos patrones de flujo/regímenes (Figura 1.1). Con un incremento en la velocidad del gas, los regímenes en lechos fijos son: burbujeante retardado o fluidización libre de burbujas, fluidización burbujeante, fluidización en flujo tapón, fluidización turbulenta, fluidización rápida y convección neumática diluida. Sin embargo, continúa habiendo problemas para identificar la transición de un régimen a otro; por ejemplo, el cambio de la fluidización turbulenta a la rápida no está bien definido (Andreux y col., 2005).

\section{Fluidización rápida}

La fluidización rápida es un régimen para poner en contacto íntimo gas a altas velocidades con sólidos finos en una corriente densa caracterizada por turbulencia extrema y reflujo extenso de paquetes concentrados de partículas. Este régimen se ha utilizado como una técnica que se orienta principalmente hacia aplicaciones en reactores tipo gas-sólido, catalíticos o no-catalíticos, en donde ofrece muchas ventajas (Yerushalmi y col., 1976). Este régimen de fluidización es muy importante porque en él ocurren muchos procesos industriales, tales como la desintegración catalítica de hidrocarburos o mejor conocida como FCC por sus siglas en inglés. 


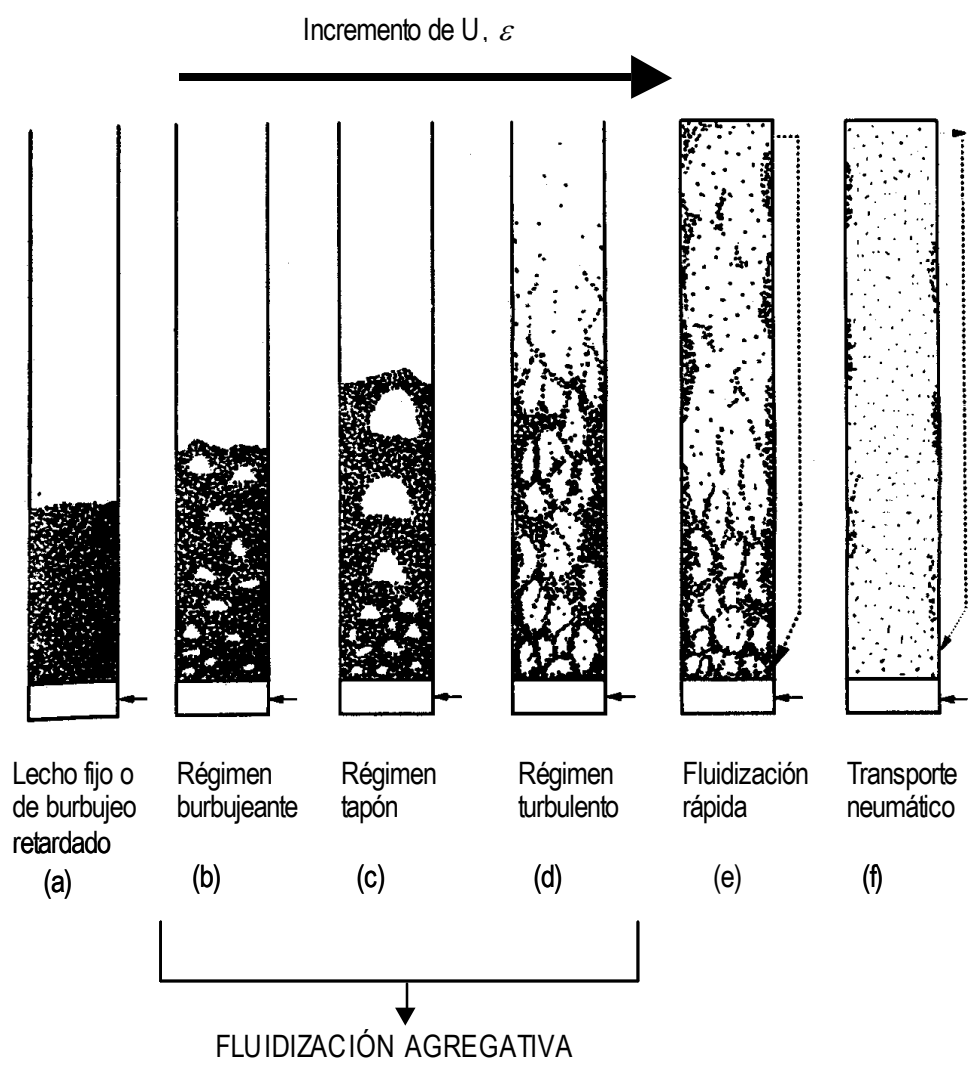

Figura 1.1. Patrones de flujo en un lecho fluidizado gas-sólido (Grace, 1986).

Existen diversos modelos para representar la estructura turbulenta en un flujo, Prandtl (1925) suponiendo que los remolinos se mueven en un fluido de igual forma que lo hacen las moléculas en un gas, sugirió una ecuación diferencial parcial para la longitud de mezcla; posteriormente, Rotta (1951) mejoró el modelo de Prandtl al sugerir uno que incluía dos ecuaciones para dos escalas de la turbulencia en vez de una. Launder y Spalding (1972) propusieron un modelo de dos ecuaciones y que es el que más se utiliza. Este modelo consiste de una ecuación para la energía cinética turbulenta $\mathrm{k}$ y otra para la tasa de disipación de la energía turbulenta epsilon. El modelo k-epsilon, aunque originalmente se propuso para una sola fase, su aplicación se ha extendido a sistemas de dos fases dando buenos resultados. Algunos autores han utilizado este modelo para representar la turbulencia, en columnas de burbujas (Troshko y Hassan, 2001; Lahey, 2005), en lechos fluidizados (Abou-Arab y Elghobashi, 1983; Chen y Wood, 1985; Dasgupta y col., 1994), en medios porosos (Pedras y de Lemos, 2001). A pesar del hecho de que la validez del modelo k-epsilon no es universal, presenta un buen compromiso entre simplicidad y generalidad. 
Elghobashi (1994) clasificó los flujos del tipo gas-sólido en dos regímenes: diluido y denso. En el régimen diluido, donde la fracción volumen de sólido es menor al 0.1\%, las colisiones entre partículas tienen un efecto despreciable en la turbulencia del gas acarreador. En tanto que en el régimen denso, la fracción volumen de sólido es mayor al $0.1 \%$ y las colisiones entre partículas empiezan a jugar un papel importante en los flujos.

Zhang y Reese (2001) estudiaron flujos turbulentos del tipo gas-sólido, con un modelo que incorpora la influencia de la turbulencia del gas en el movimiento aleatorio de las partículas por medio de la teoría cinética generalizada, sus resultados indicaron que no debe ignorarse la influencia de la turbulencia del gas en el comportamiento del flujo microscópico de partículas para flujos relativamente diluidos, especialmente con partículas pequeñas.

Zheng y col. (2001) hicieron simulaciones numéricas utilizado modelos tipo k-epsilon-k(p)epsilon(p)-Theta para modelar la turbulencia en un flujo isotérmico incompresible en un tubo elevador. Encontraron que la interacción de la turbulencia del gas con las partículas sólidas es un factor crucial que afecta los resultados que ellos obtuvieron.

Diversos autores han modelado procesos de la fluidización rápida; entre ellos Gidaspow (1994), Neri y Gidaspow (2000), y Valencia (2001). Ding y Gidaspow (1990) pudieron predecir la formación de burbujas de gas en un lecho fluidizado utilizando modelos obtenidos con teoría cinética extendida a medios granulares; Theologos y Markatos (1993), por otra parte, propusieron un modelo muy completo para reactores del tipo FCC, que incorpora la reacción de cracking catalítico del gasóleo y que resolvieron numéricamente (Theologos y col., 1997). Con este modelo ellos lograron predecir los aspectos ingenieriles más importantes en el tubo elevador como son: la caída de presión, la retención de catalizador, la velocidad de deslizamiento interfacial, la zona de aceleración del catalizador, la distribución de temperaturas en ambas fases y de rendimientos en el reactor. Este modelo constituyó una buena aproximación a estos sistemas, pero no se tomó en consideración el efecto de la compresibilidad del flujo de vapor; además, la turbulencia del vapor se incluyó a través de un modelo muy simple tipo Boussinesq, con la viscosidad turbulenta. Posteriormente, como un acercamiento más a los procesos que tienen lugar en los sistemas de FCC, Theologos y col. (1999) incorporaron en su modelo los efectos de alimentación de gasóleo y su tasa de evaporación. 
Jinsen y col. (1999) propusieron un modelo similar al de Theologos y Markatos (1993). En este modelo se incorporaron esquemas de reacción más complejos y la turbulencia se representó con un modelo tipo k-epsilon. En sus resultados encontraron que el régimen de flujo reactivo-turbulento de gas en los tubos elevadores de las unidades de FCC es extremadamente complejo, especialmente en la zona de alimentación. Jinsen y col. (2001) resolvieron numéricamente el modelo y lo utilizaron como herramienta de estudio para la operación y modificación del proceso de FCC. Sugirieron que la selectividad de la reacción para producir gasolina puede mejorarse al reducir el diámetro de la gota de gasóleo alimentada a la mitad; es decir, de 80 a $40 \mu \mathrm{m}$. Estos estudios muestran la importancia que tiene la obtención de modelos confiables en la optimización y mejoramiento del proceso de FCC.

Los flujos multifásicos tienen más fuentes de disipación que las situaciones de flujo en una fase equivalentes. Se incluyen fuentes adicionales de disipación tales como la disipación de energía turbulenta entre las fases y la disipación interfacial. Al aplicar la técnica de promediado se elimina información detallada del sistema que después debe introducirse adecuadamente en los términos fuente mediante una cerradura acorde a la situación física específica. Estos términos deben ser consistentes con la segunda ley de la termodinámica. Tener un modelo consistente con la segunda ley de la termodinámica, implica que la tasa de cambio de entropía y el flujo de entropía sean positivos definidos. Si todos los efectos están representados adecuadamente, entonces deberíamos encontrar que la segunda ley de la termodinámica siempre se satisface. De otra forma, si las expresiones de cerradura que se obtengan nos conducen a que el balance de entropía no siempre satisface la desigualdad de Clausius-Duhem, entonces sabemos que los términos de disipación en la generación de entropía no se están modelando correctamente. Al verificar la congruencia del modelo con la segunda ley de la termodinámica puede encontrarse que la forma de cerrar el problema sobre-estima o sub-estima la disipación, dependiendo de la situación de flujo (Arnold y col., 1990). La segunda ley de la termodinámica no es capaz de decirnos cuáles relaciones de comportamiento son correctas, sólo puede detectar cuál conjunto de relaciones de comportamiento es incompatible con requerimientos físicos específicos. 
Por ello, un conjunto de relaciones de comportamiento aceptable debería (Arnold y col., 1990):

- Incluir todos los efectos importantes,

- ser congruente con la segunda ley de la termodinámica,

- ser compatible con el principio de objetividad (Drew y Lahey, 1979),

- llevar a un modelo promediado bien planteado, y

- predecir los datos disponibles.

\section{Antecedentes}

En un trabajo anterior (Sánchez-López, 2003), considerando un sistema de dos fases gassólido, de un flujo homogéneo e isotérmico de partículas sólidas rígidas e iguales, se obtuvo un modelo hidrodinámico de dos fluidos con promedio espacio-temporal. Esto es, uno que no considera transferencia de masa, ni reacciones químicas y por lo tanto, no incorpora balances de especies químicas. En el modelo se incorporaron los efectos de compresibilidad de la fase fluida (gas o vapor) y se calculó el número de Stokes utilizando datos de dos sistemas físicos: (1) catalizador de FCC-vapor de agua (Kellogg, 1984) y (2) arena-aire (van der Schaaf y col., 1998). El número de Stokes caracteriza el comportamiento de las partículas suspendidas en un flujo y se define como la razón de un tiempo característico de la relajación de las partículas en el flujo a un tiempo característico del flujo. Posteriormente, con este modelo se realizó un estudio ondulatorio, el cual consiste en obtener una ecuación de onda representativa del modelo linealizado en las variables de perturbación para luego analizar con ella sus coeficientes, rapideces de propagación y estabilidad lineal, con el fin de estudiar las ondas de presión en el lecho y de establecer la importancia de la compresibilidad en la estabilidad del modelo.

Más delante, se tomó como punto de partida el modelo obtenido por Sánchez-López (2003), en el cual se suprimieron los efectos asociados a la turbulencia, con la finalidad de estudiar solamente la hipótesis de incompresibilidad. Con este modelo se realizó un estudio de estabilidad lineal para estudiar el comportamiento dinámico del sistema mediante una representación de una ecuación de jerarquía de ondas.

Consideraremos que un operador ondulatorio de primer orden es representado por $\left(\partial_{t}+a \partial_{z}\right)$, donde $a$ es la velocidad de la onda de primer orden. 
De acuerdo con Whitham (1974), una ecuación de jerarquía de ondas es una ecuación en donde aparecen distintos operadores ondulatorios, los cuales se jerarquizan de acuerdo al orden del operador más alto presente en la ecuación. Con esta ecuación de jerarquía de ondas se hizo un estudio formal (Soria y col., 2008) donde se compararon los modelos compresible e incompresible asociado a través de un procedimiento sistemático, el cual consiste en utilizar la representación de operadores en el modelo, para luego hacer manipulaciones operacionales y representar los dos modelos en una misma base de comparación.

El estudio ondulatorio se llevó a cabo y se validó con los datos de dos sistemas, el flujo de vapor-catalizador en una unidad de FCC y el flujo de aire-arena en una unidad de CFB. Entre los resultados más importantes que se obtuvieron están: se predijeron ondas de presión al incluir la compresibilidad de la fase gaseosa, cosa que no se consigue al utilizar un modelo incompresible. Las ondas de presión están esencialmente vinculadas con la compresibilidad de la mezcla de las dos fases [Micaelli (1982)]. La propagación de la presión gobierna en un sistema específico; por ejemplo, en el tubo elevador de un lecho fluidizado [Sánchez-López (2003)]. Su velocidad de propagación es menor o igual a la velocidad del sonido sobre las dos fases [Nguyen y col. (1981)]. Con el modelo incompresible sólo se obtienen ondas de fracción volumen de primero y segundo orden, mientras que con el compresible se encontraron ondas de segundo a cuarto orden; sin embargo, la onda de cuarto orden tiene un coeficiente sensiblemente inferior al de tercer orden, lo cual justifica despreciarla en relación a las otras. Al incluir la compresibilidad, el modelo ondulatorio incompresible aumenta en un orden, de segundo a tercer orden.

Las rapideces de propagación de las ondas de segundo orden del modelo incompresible resultaron ser complejas conjugadas, con parte real del orden de 10 y parte imaginaria del orden de $10^{-4}$. Bouré (1988) asocia la ocurrencia de rapideces complejas a modelos de dos fluidos rígidos que consideran un campo único de presión. En el caso de burbujas de aire en un medio continuo como agua, este problema se soluciona con presiones distintas basadas en la ecuación de Young-Laplace, pero para sistemas formados de partículas sólidas pequeñas del orden de $\mu \mathrm{m}$ en un medio continuo, el campo de presión del sólido no está definido. Para definir la presión en los granos sólidos se propuso una hipótesis básica en la obtención de los teoremas de Arquímedes de la hidrostática, que es considerar que el campo de presión del fluido se continúa al interior del sólido más una función de la aglomeración de sólidos que depende de la fracción volumen de sólido (Gidaspow, 1994). 
La función de aglomeración o módulo de compresibilidad del sólido que utilizamos por ser adecuada para los casos de estudio de esta tesis, es la propuesta por Jiradilok y col. (2006). Con esta modificación se logró remover las partes imaginarias en el modelo ondulatorio incompresible. Estas razones motivan el deseo de profundizar en el tema, con el fin de desentrañar aspectos fundamentales acerca del papel que juegan los efectos de compresibilidad y de termicidad en los modelos de la fluidización rápida, los cuales tienen un carácter no-homogéneo en general. Así como tener una descripción termodinámica completa al incluir los efectos térmicos, incorporando el balance de energía y de entropía.

En particular, se modelará el comportamiento ante pulsos de calor a la entrada, introduciendo la fase gaseosa a diferente temperatura que las partículas sólidas. Con la finalidad de evaluar las expresiones de cerradura que se obtengan, se analizará la consistencia del modelo con la segunda ley de la termodinámica (Arnold y col., 1990).

\section{Justificación}

Como se hizo notar en la revisión bibliográfica, existe una cantidad variada de trabajos que reportan modelos de lechos fluidizados rápidos, que consideran las fases incompresibles e incorporan modelos de la turbulencia. Otros modelos consideran los efectos térmicos del sistema y algunos más incorporan reacciones químicas y efectos de transferencia de masa. Los enfoques y propósitos son diversos. Entre éstos, el énfasis en el comportamiento hidrodinámico del sistema requiere elaborar modelos que representen adecuadamente la respuesta dinámica, primero a un nivel del análisis del movimiento de las fases, para luego incorporar, utilizando procedimientos compatibles, los efectos térmicos y de transporte de componentes y reacciones en el modelo.

El propósito de este trabajo es desarrollar un modelo hidrodinámico de dos fluidos, en el que las fases sólida y fluida sólo interactúan a través de las fuerzas de fricción interfacial y de los efectos térmicos y de disipación de energía mecánica, de modo que las perturbaciones en el sistema induzcan movimientos ondulatorios que sean correctamente representados por el modelo. Esto implica aplicar principios locales de conservación y de balance para dos materiales puros (los granos de sólido y el fluido continuo) y extender estos balances aplicando técnicas de promediado y resolviendo las cerraduras requeridas, bajo condiciones específicas plausibles. 
Se ha demostrado en trabajos anteriores (Soria y col., 2008) que la inclusión de la compresibilidad del fluido afecta la respuesta de los modelos ante pequeñas perturbaciones. En efecto, el modelo compresible que se obtuvo es de cuarto orden en tanto que el incompresible es sólo de segundo. Además, las rapideces de propagación del modelo compresible incorporan los modos de propagación relacionados con la velocidad del sonido.

La consideración de la hipótesis de incompresibilidad implica que las ondas de presión viajan en el sistema con velocidad infinita (Bouré, 1988). Asimismo se estudiará el comportamiento no-isotérmico porque deseamos representar adecuadamente la disipación de la energía entre las fases. Considerar isotermicidad implica que la respuesta térmica del sistema, incluidos los efectos de disipación viscosa, se propagan en el sistema con velocidad infinita de tal manera que son transferidos instantáneamente a los alrededores y no hay un incremento en la temperatura. La inclusión de la segunda ley de la termodinámica es necesaria para analizar si la forma en la que se cierra el problema cumple con el postulado de Clausius-Duhem.

Aunque la turbulencia generada por el flujo de gas es muy importante en el flujo de partículas en el tubo elevador en el régimen de fluidización rápida, ya que gobierna el movimiento de los sólidos en el tubo, su efecto no se considerará en los modelos que se estudiaran en este trabajo. Sin embargo, se estudia su efecto en las restricciones implicadas por la segunda ley de la termodinámica. La importancia del efecto de compresibilidad de la fase fluida debe ser evaluada, así como la propagación de ondas no-isotérmicas, que determinan el flujo de calor debido a la disipación de energía en el sistema y es necesaria para establecer el balance de entropía, que se requiere para aplicar la segunda ley de la termodinámica. Los efectos de transferencia de masa y los efectos asociados a reacciones químicas quedan fuera del alcance de este proyecto, aunque la sistematización de procedimientos útiles para la extensión a estos problemas quedará establecida.

Las aportaciones originales están en el modelar adecuadamente un sistema no-isotérmicocompresible, la evaluación de los efectos de compresibilidad, así como en el estudio de las ondas térmicas y la congruencia de las cerraduras con el postulado de Clausius-Duhem. 


\section{Hipótesis, objetivos y metodología}

\section{Hipótesis}

Los efectos de compresibilidad y de termicidad determinan ampliamente el comportamiento de los lechos fluidizados rápidos. Su inclusión apropiada en los modelos de dicha operación debe tener impacto en los resultados, como podrá observarse en los análisis de estabilidad de ondas de fracción volumen, térmicas y de presión.

\section{Objetivo general}

Elaborar un modelo hidrodinámico, que incluya promedios espacio-temporales y que sea consistente con la segunda ley de la termodinámica, que permita evaluar los efectos de compresibilidad y la evolución de ondas térmicas en la fluidización rápida en ausencia de turbulencia.

\section{Objetivos particulares}

- Obtener un modelo para el régimen de la fluidización rápida usando técnicas de promedio espacio-temporal.

- Cerrar el conjunto de ecuaciones utilizando modelos de celda y ecuaciones de estado para flujos de gas o vapor compresible.

- Verificar la consistencia del modelo aplicando la segunda ley de la termodinámica.

- Estudiar la dinámica y la estabilidad lineal del modelo.

- Analizar la importancia de los efectos considerados en el modelo. 


\section{Metodología}

Primero, partiendo de las ecuaciones de conservación de la masa y de la energía, ecuaciones de balance de momentum y de entropía, se procede a aplicarles promedio espacio-temporal (Drew, 1983; Sánchez-López, 2003). Posteriormente para cerrar el modelo, se utiliza la ecuación de estado del gas ideal (sin embargo, como se hará notar en la sección 2.4, en esta descripción en términos de variables con promedio espacio-temporal, es posible obtener una ecuación de estado que describa el comportamiento termostático del gas más real que la del gas ideal, pero en esta primera aproximación no se utilizará) para incorporar la compresibilidad de la fase fluida y los términos interfaciales se cierran mediante un modelo de celda. Se estudia la consistencia del modelo con la segunda ley de la termodinámica, siguiendo la metodología establecida por Arnold y col. (1990). A continuación se linealiza el modelo respecto a las perturbaciones y se eliminan las velocidades de las fases mediante las ecuaciones de conservación de la masa, obteniendo así un sistema de ecuaciones para (a) la fracción volumen y para la presión en el caso isotérmico, y para (b) la fracción volumen, la presión y la temperatura de cada una de las fases, para el caso no-isotérmico. Luego, mediante la aplicación de operadores diferenciales a las ecuaciones, podemos obtener una sola ecuación diferencial de orden superior que represente al sistema original, en esta ecuación están separados los efectos tomados en cuenta en el modelo; es decir, la compresibilidad, la pared del tubo y los efectos térmicos. Esta metodología nos permite evaluar cada efecto por separado y medir su importancia en la dinámica y en la estabilidad del sistema. Con este modelo se estudian las rapideces de propagación y se analiza la estabilidad lineal mediante la relación de dispersión asociada. Por último, se comparan los resultados del modelo incompresible con los del compresible y los del isotérmico con los del no-isotérmico. Se confrontan distintos casos de estudio; por ejemplo, para el caso isotérmico se comparan los datos experimentales de FCC reportados por Kellogg (1984) con los reportados en un trabajo experimental realizado por van der Schaaf y col. (1998) sobre un lecho fluidizado circulante CFB de partículas de arena arrastradas con aire a temperatura ambiente, donde se dan mediciones de la velocidad del sonido. Con los datos de este trabajo se obtuvo un número de Stokes de 2.15, lo que implica que las partículas sólidas tienen un movimiento más independiente del gas que en el FCC, donde el número de Stokes resultó ser de 0.13. El estudio ondulatorio con estos dos sistemas se vuelve atractivo por las diferencias importantes entre las propiedades de ambos, las cuales se presentan y discuten más adelante. 


\section{Capítulo 1}

\section{Ecuaciones de balance con promedio espacio-temporal}

\subsection{Introducción}

La principal dificultad en el modelamiento del flujo multifásico es que involucra muchas escalas características de longitud y tiempo diferentes del sistema, la geometría compleja y las condiciones a la frontera. La forma apropiada de modelar estos sistemas tan complejos debe basarse en un método riguroso en el que se establezca una conexión entre el nivel local instantáneo y la escala global. Por lo tanto, es importante desarrollar un método matemático que pueda utilizarse sistemáticamente para obtener las ecuaciones promediadas a partir de las ecuaciones locales instantáneas y que las relaciones de cerradura que se requieran sean bien definidas y calculables (Zhang, 1993).

Prosperetti y Tryggvason (2007) señalan que: "El problema del promediado en el flujo multifásico es una complicación de muchos años, que se extiende en la historia tan lejos como a la turbulencia monofásica. Hay sin embargo, una desventaja con este caso: mientras que las ideas relativamente simples que se aplican a la turbulencia en una sola fase, tales como la hipótesis de la longitud de mezclado, da buenos resultados, los enfoques simples aplicados al promediado multifásico han tenido un éxito mucho más limitado. En realidad, hablando en términos generales, es justo decir que los modelos de ecuaciones promediadas para el flujo multifásico son mucho menos desarrollados y mucho menos fieles a la realidad física, que los de la turbulencia monofásica. Estas dificultades son todavía considerables pues afectan el modelamiento de muchos términos y las ecuaciones a menudo parecen ser algo deficientes aún en el nivel puramente matemático.”

A partir de 1967 el promedio volumétrico fue usado para obtener las ecuaciones de medio efectivo que implican dos fases. Sin embargo, el promedio volumétrico es adecuado y suficiente para medios porosos. Si ambas fases están en movimiento, los métodos estadísticos o de promedio espacio-temporal son más adecuados. 
Los procedimientos de promediado que se aplican a sistemas de dos fases pueden clasificarse en tres grupos principales basados en los conceptos físicos utilizados para formular sistemas dinámicos (Ishii, 1975). Estos son, el promedio:

(1) Euleriano,

(2) Lagrangiano y

(3) Estadístico de Boltzmann.

El primero se sigue tradicionalmente ya que las ecuaciones locales instantáneas están formuladas en un marco de referencia Euleriano, por lo que éste se adopta normalmente para desarrollar el promediado. El promedio Lagrangiano sigue una determinada trayectoria; por ejemplo, la trayectoria de una partícula sólida. En el tercero, el promedio se toma sobre una función estadística de distribución, comúnmente la distribución de Maxwell-Boltzmann. Otro método estadístico propuesto para desarrollar modelos es el promedio de ensamble (Zhang, 1993; Zhang y Prosperetti, 1994; Drew y Passman, 1999). En flujos multifásicos, pensaremos en el ensamble correspondiente a un movimiento dado como un conjunto muy grande de realizaciones con variaciones que podemos observar. En efecto, el conjunto puede (teóricamente) contener rearreglos de los átomos dentro de las fases; sin embargo, estas distintas realizaciones no son diferentes en el contexto de la mecánica de medios continuos y por lo tanto no las consideraremos. Una diferencia importante entre el promedio volumétrico y el promedio de ensamble, es que el promedio de ensamble no requiere que el volumen de control contenga un gran número de partículas en cada realización como lo requiere el promedio volumétrico. Por otro lado, el promedio de ensamble es más general que el promedio temporal y que el promedio volumétrico, ya que se vuelve equivalente al promedio volumétrico para flujos que son estadísticamente uniformes u homogéneos espacialmente. Similarmente, cuando el número de muestras tiende a infinito, lo cual implica la ergodicidad del proceso, el promedio de ensamble es equivalente al promedio temporal (Drew y Passman, 1999). En este trabajo se demuestran las condiciones bajo las cuales el promedio espacio-temporal contiene al promedio volumétrico y al promedio temporal, como casos asintóticos. La selección de una descripción de promediado para un determinado problema depende del fenómeno que se desea describir. La fluidización rápida es un régimen altamente turbulento donde las fases gas-sólido están en movimiento, entonces para obtener un modelo en este régimen, se necesitan un promedio temporal y un promedio volumétrico. Con la finalidad de conseguir variables suavizadas; primeramente, se debe tomar un promedio temporal y después un promedio volumétrico o viceversa. Sin embargo, en este trabajo se propone un promedio espaciotemporal para obtener variables suavizadas en el tiempo y en el espacio en un sólo paso. 


\subsection{Descripción en el nivel local instantáneo ${ }^{1}$}

Las ecuaciones de balance a nivel local instantáneo para la fase $k$ son:

\subsubsection{Ecuación de conservación de la masa}

$$
\frac{\partial}{\partial t^{\prime}} \rho_{k}+\nabla^{\prime} \cdot\left(\rho_{k} \mathbf{v}_{k}\right)=0
$$

\subsubsection{Ecuación de balance de cantidad de movimiento}

$$
\frac{\partial}{\partial t^{\prime}}\left(\rho_{k} \mathbf{v}_{k}\right)+\nabla^{\prime} \cdot\left(\rho_{k} \mathbf{v}_{k} \mathbf{v}_{k}\right)-\rho_{k} \mathbf{g}_{k}-\nabla^{\prime} \cdot \boldsymbol{\Pi}_{k}=\mathbf{0} .
$$

\subsubsection{Ecuación de balance de energía total}

$$
\begin{aligned}
\frac{\partial}{\partial t^{\prime}}\left[\rho_{k}\left(U_{k}+\frac{1}{2} \mathbf{v}_{k} \cdot \mathbf{v}_{k}\right)\right] & +\nabla^{\prime} \cdot\left[\rho_{k} \mathbf{v}_{k}\left(U_{k}+\frac{1}{2} \mathbf{v}_{k} \cdot \mathbf{v}_{k}\right)\right] \\
& -\rho_{k} \mathbf{g}_{k} \cdot \mathbf{v}_{k}-\nabla^{\prime} \cdot\left(\boldsymbol{\Pi}_{k} \cdot \mathbf{v}_{k}\right)+\nabla^{\prime} \cdot \mathbf{q}_{k}-Q_{k}=0 .
\end{aligned}
$$

En estas ecuaciones, $t^{\prime}$ y $\nabla^{\prime}$ son el tiempo y el operador gradiente en la escala local instantánea, respectivamente. $\mathbf{v}_{k}$ es la velocidad de la fase $k, \mathbf{g}_{k}$ es un campo vectorial externo por unidad de volumen actuando en la fase $k, \Pi_{k}$ es el tensor de presiones; definido por: $\boldsymbol{\Pi}_{k} \doteq-P_{k} \mathbf{1}-\boldsymbol{\tau}_{k}$, donde $P_{k}$, es la presión termodinámica, $\mathbf{1}$ es el tensor métrico y $\boldsymbol{\tau}_{k}$ es el tensor de esfuerzos viscosos, $U_{k}$ es la energía interna específica o por unidad de masa, $\mathbf{q}_{k}$ es el vector de flujo de calor y $Q_{k}$ es una fuente de calentamiento por unidad de volumen asociada a la fase $k$.

El conjunto dado por la Ec. (1.1) a la Ec. (1.3) debe complementarse por las siguientes relaciones de comportamiento:

- Ley de Newton de la viscosidad: $\boldsymbol{\tau}_{k}=-2 \mu_{k} \mathbf{D}_{k}-\lambda_{k}\left(\nabla^{\prime} \cdot \mathbf{v}_{k}\right) \mathbf{1}$.

- Ley de Fourier de la conducción del calor: $\mathbf{q}_{k}=-k_{k} \nabla^{\prime} T_{k}$.

\footnotetext{
${ }^{1}$ Los vectores y tensores los representamos con negritas y el subíndice $k$ indica que pertenece a la fase $k$ ésima. Cabe mencionar que no utilizamos la notación indicial, sino la notación de Gibbs, por lo cual esperamos que esto no genere ambigüedad o confusión. J.W. Gibbs fue quien introdujo la notación del punto para el producto escalar y del $\times$ para el producto vectorial; así como nociones más complejas, tales como los operadores vectoriales: gradiente, divergencia y rotacional.
} 
Donde $\mathbf{D}_{k}$ es el tensor de rapidez de deformación, definido por: $\mathbf{D}_{k} \doteq \frac{1}{2}\left[\nabla^{\prime} \mathbf{v}_{k}+\left(\nabla^{\prime} \mathbf{v}_{k}\right)^{T}\right]$. También deben incluirse las siguientes condiciones a la frontera:
C.F.1
$P_{k}=P_{k i}$
en $\mathscr{\mathscr { P }}_{k \ell}(\mathbf{r}, t)$,
C.F.2
$\mathbf{v}_{k} \cdot \mathbf{n}_{k}=0$
en $\mathscr{O}_{k \ell}(\mathbf{r}, t)$,
C.F.3
$T_{k}=T_{k i}$
en $\mathscr{O}_{k \ell}(\mathbf{r}, t)$,
C.F.4
$\mathbf{n}_{s} \cdot \mathbf{q}_{s}=h_{i}\left(T_{s i}-T_{g}\right)$
en $\mathscr{\mathscr { S }}_{s g}(\mathbf{r}, t)$.

En estas ecuaciones $P_{k i}$ y $T_{k i}$, son la presión y la temperatura evaluadas en la frontera de la región espacial $\mathscr{T}_{k}(\mathbf{r}, t)$ denotada por $\mathscr{O}_{k \ell}(\mathbf{r}, t)$ (ver Figura 1.2), $h_{i}$ es un coeficiente de transferencia de calor asociado a la interfaz, $\mathbf{n}_{k}$ representa un vector normal unitario asociado a la fase $k$ y que apunta hacia afuera de la misma.

\subsubsection{Ecuación de energía mecánica}

La ecuación de energía mecánica se obtiene al tomar el producto punto de la ecuación de movimiento Ec. (1.2) con la velocidad.

$$
\rho_{k} \frac{D_{k}}{D t^{\prime}}\left(\frac{1}{2} \mathbf{v}_{k} \cdot \mathbf{v}_{k}\right)-\rho_{k}\left(\mathbf{v}_{k} \cdot \mathbf{g}_{k}\right)-\nabla^{\prime} \cdot\left(\boldsymbol{\Pi}_{k} \cdot \mathbf{v}_{k}\right)+\boldsymbol{\Pi}_{k}: \nabla^{\prime} \mathbf{v}_{k}=0
$$

Donde $\frac{D_{k}}{D t^{\prime}} \doteq \frac{\partial}{\partial t^{\prime}}+\mathbf{v}_{k} \cdot \nabla^{\prime}$, es la derivada material siguiendo a una partícula de fluido con velocidad $\mathbf{v}_{k}$. Restando la ecuación de energía mecánica resultante Ec. (1.4) de la ecuación de energía total Ec. (1.3), se obtiene la ecuación para la energía interna:

$$
\rho_{k} \frac{D_{k}}{D t^{\prime}} U_{k}+\nabla^{\prime} \cdot \mathbf{q}_{k}-Q_{k}-\boldsymbol{\Pi}_{k}: \nabla^{\prime} \mathbf{v}_{k}=0
$$

El conjunto de ecuaciones representado en la Ec. (1.1) a la Ec. (1.5), corresponde a un sistema cerrado en la escala de descripción local instantánea y se toma como punto de partida para obtener los modelos promediados. 


\subsection{Promediado utilizando funciones de distribución}

\subsubsection{Función indicadora de fase}

El dominio de definición de las ecuaciones locales instantáneas puede extenderse a toda la región espacial ơ utilizando una función de distribución apropiada (conocida como función generalizada) $X_{k}\left(\mathbf{x}^{\prime}, t^{\prime}\right)$. Esta función está definida en todas partes y tiene soporte compacto. Una función tiene soporte compacto si los valores en donde no es nula conforman un conjunto cerrado y acotado. La función indicadora de fase se define por (Gel'fand y Shilov, 1964; Drew, 1971; Soria y de Lasa, 1991):

$$
X_{k}\left(\mathbf{x}^{\prime}, t^{\prime}\right) \doteq\left\{\begin{array}{l}
1, \text { si } \mathbf{x}^{\prime} \text { está en la fase } k \text { al tiempo } t^{\prime} \\
0, \text { de otra manera }
\end{array}\right.
$$

donde el subíndice $k$ es una indicación de la fase gas o sólida, i.e., $k=\{g, s\}$ y $\mathbf{x}^{\prime}$ es un vector de posición. Esta función debe aplicarse a las ecuaciones locales instantáneas en el sentido de las funciones generalizadas. En Análisis matemático, una distribución o función generalizada es un objeto matemático que generaliza la noción de función y la de medida. La delta de Dirac es una distribución y como distribución, define un funcional en forma de integral sobre un cierto espacio de funciones (c. fr. Gel'fand y Shilov, 1964).

En el espacio-tiempo $X_{k}\left(\mathbf{x}^{\prime}, t^{\prime}\right)$ es una función definida en todas partes. Por lo tanto:

$$
\sum_{k=g, s} X_{k}\left(\mathbf{x}^{\prime}, t^{\prime}\right) \equiv 1 .
$$

Esto es, en cada punto del espacio-tiempo la función indicadora de fase está bien definida.

\subsubsection{Propiedades de la función de distribución $X_{k}\left(\mathbf{x}^{\prime}, t^{\prime}\right)$}

Derivadas distribucionales

El concepto de derivada distribucional o derivada en el sentido de las distribuciones generaliza el concepto de derivada ordinaria a distribuciones y funciones no-continuas. La función indicadora de fase tiene las siguientes propiedades importantes (Drew, 1983; Soria y de Lasa, 1991):

$$
\begin{aligned}
\frac{\partial X_{k}}{\partial t^{\prime}} & =\mathbf{n}_{k} \cdot \mathbf{w}_{k \ell} \delta_{k \ell} \\
\nabla^{\prime} X_{k} & =-\mathbf{n}_{k} \delta_{k \ell}
\end{aligned}
$$


donde

$\mathbf{w}_{k \ell}$, es la velocidad de la interfaz $k \ell$,

$\delta_{k \ell}$, es la función de distribución delta de Dirac asociada a la interfaz $k \ell$

(Gel'fand y Shilov, 1964; Zemanian, 1965; Estrada y Kanwal, 1980).

Tomando el producto punto de la Ec. (1.9) con $\mathbf{w}_{k \ell}$ y sumando el resultado a la Ec. (1.8) se obtiene la siguiente ecuación (Drew, 1983; Soria y de Lasa, 1991):

$$
\begin{aligned}
\frac{\partial X_{k}}{\partial t^{\prime}}+\mathbf{w}_{k \ell} \cdot \nabla^{\prime} X_{k} & =0 \\
& =\frac{D_{k \ell} X_{k}}{D t^{\prime}} .
\end{aligned}
$$

La Ec. (1.10) es conocida como la ecuación topológica para la función indicadora de fase y tiene una interpretación simple dada por Drew y Lahey (1993): Si se toma un punto fuera de la interfaz, entonces $X_{k}=1$ ó $X_{k}=0$. En ambos casos las derivadas parciales son cero y la Ec. (1.10) es igual a cero. Por otro lado, si se considera un punto que pertenezca a la interfaz, entonces el movimiento es con ella y se observa a la función $X_{k}$ como un salto constante. Por lo tanto, su derivada es cero.

Si $\Psi_{k}$ es la densidad volumétrica de una cantidad termodinámica de interés, pueden obtenerse relaciones importantes con la función indicadora, donde ésta se introduce a los operadores derivada temporal y divergencia:

$$
\begin{gathered}
X_{k} \frac{\partial \boldsymbol{\Psi}_{k}}{\partial t^{\prime}}=\frac{\partial}{\partial t^{\prime}}\left(X_{k} \boldsymbol{\Psi}_{k}\right)-\frac{\partial X_{k}}{\partial t^{\prime}} \boldsymbol{\Psi}_{k}, \\
X_{k} \nabla^{\prime} \cdot\left(\mathbf{v}_{k} \boldsymbol{\Psi}_{k}\right)=\nabla^{\prime} \cdot\left(X_{k} \mathbf{v}_{k} \boldsymbol{\Psi}_{k}\right)-\nabla^{\prime} X_{k} \cdot \mathbf{v}_{k} \Psi_{k} .
\end{gathered}
$$

Sustituyendo en estas ecuaciones las derivadas de la función indicadora de fase dadas en la Ec. (1.8) y en la Ec. (1.9):

$$
\begin{gathered}
X_{k} \frac{\partial \boldsymbol{\Psi}_{k}}{\partial t^{\prime}}=\frac{\partial}{\partial t^{\prime}}\left(X_{k} \boldsymbol{\Psi}_{k}\right)-\mathbf{n}_{k} \cdot \mathbf{w}_{k \ell} \delta_{k \ell} \boldsymbol{\Psi}_{k}, \\
X_{k} \nabla^{\prime} \cdot\left(\mathbf{v}_{k} \boldsymbol{\Psi}_{k}\right)=\nabla^{\prime} \cdot\left(X_{k} \mathbf{v}_{k} \boldsymbol{\Psi}_{k}\right)+\delta_{k \ell} \mathbf{n}_{k} \cdot \mathbf{v}_{k} \boldsymbol{\Psi}_{k} .
\end{gathered}
$$

Estas ecuaciones serán el punto de partida para el desarrollo de los teoremas del promedio espacio-temporal. 


\subsection{Operadores para el promedio espacio-temporal}

La aplicación del método de promediado requiere que se satisfagan ciertas condiciones importantes. Soria y de Lasa (1991) señalan las siguientes:

a) Una condición de escala que indica que el volumen promediante $V$ definido por la región espacial $\mathscr{O}$ debe ser seleccionado tal que:

$$
\ell_{s} \ll r_{0} \ll L_{s}
$$

donde

$$
\begin{aligned}
& \ell_{s} \text {, es una longitud característica de las fases dispersas y } \\
& L_{s} \text {, es una longitud característica de la estructura de flujo. }
\end{aligned}
$$

b) En un punto fijo $\mathbf{x}^{\prime}$ representado en $\mathscr{Y}\left(\mathbf{x}^{\prime}\right)$ puede haber alguna de las siguientes regiones:

$$
\mathscr{V}\left(\mathbf{x}^{\prime}\right):\left\{\mathscr{W}_{g}, \mathscr{T}_{s}, \mathscr{S}_{g s}, \mathscr{S}_{g W}, \mathscr{S}_{s W}\right\}
$$

Puesto que los efectos de pared son considerados, en la Ec. (1.16) se incluyen dos

regiones más $\mathscr{S}_{k W}(k=g, s)$. Estas regiones definen el área interfacial entre la fase $k \mathrm{y}$ la pared del tubo $\Sigma_{k W}$. (ver la Figura 1.2)

Un operador promedio de fase espacio-temporal para campos locales instantáneos $\bullet_{k}\left(\mathbf{x}^{\prime}, t^{\prime}\right)$, se define por (Sánchez-López, 2003):

$$
\left\langle\bullet_{k}\right\rangle(\mathbf{x}, t) \doteq \frac{1}{V T} \iiint_{\mathscr{V}(\mathbf{x}) t-\frac{T}{2}}^{t+\frac{T}{2}} \int_{k}\left(X_{k} \bullet_{k}\right)\left(\mathbf{x}^{\prime}, t^{\prime}\right) d t^{\prime} d V^{\prime}
$$

donde la región espacial de integración está referida a su centroide $\mathbf{x}$ y el intervalo de tiempo $\left[t-\frac{T}{2}, t+\frac{T}{2}\right]$ está referido al punto medio del intervalo $t$. El orden de integración puede intercambiarse de acuerdo a un teorema para funciones generalizadas, similar al de Fubini (Sánchez-López, 2003). En el Apéndice $A$ se presentan los casos asintóticos del promedio espacio-temporal. El promedio temporal se recupera al colapsar la región espacial de integración y el promedio volumétrico al hacer tender a cero la región temporal. 


\section{Lecho fluidizado rápido}

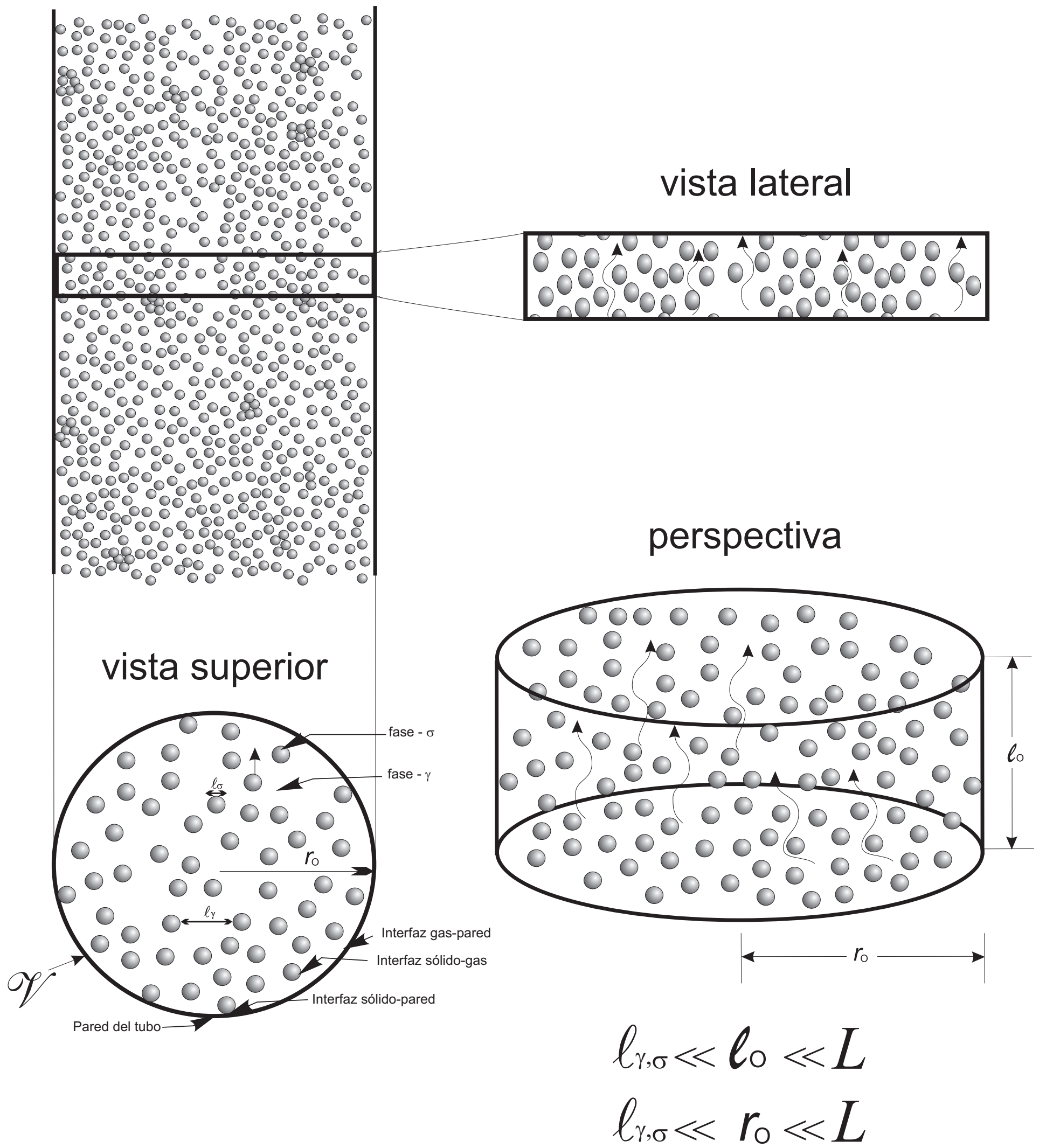

Figura 1.2. Lecho fluidizado rápido de donde se extrae el volumen que se toma como sistema para aplicar el método del promedio espacio-temporal con tres vistas en perspectiva. 
Promediando la Ec. (1.7) se obtiene el siguiente resultado importante:

$$
\sum_{k=g, s}\left\langle X_{k}\right\rangle=\sum_{k=g, s} \varepsilon_{k} \equiv 1
$$

El promedio espacio-temporal de la función indicadora de fase es la aplicación de la Ec. (1.17) a la unidad:

$$
\langle 1\rangle(\mathbf{x}, t)=\frac{1}{V T} \iiint_{\mathscr{V}(\mathbf{x})}^{t+\frac{T}{2}} \int_{t-\frac{T}{2}}^{2} X_{k} d t^{\prime} d V^{\prime}=\frac{V_{k}}{V} \equiv \varepsilon_{k}(\mathbf{x}, t) .
$$

También, el operador promedio intrínseco de fase está definido por

$$
\left\langle\bullet_{k}\right\rangle^{k}(\mathbf{x}, t) \doteq \frac{1}{V_{k} T} \iiint_{\mathscr{Y}(\mathbf{x})}^{t+\frac{T}{2}} \int_{t-\frac{T}{2}}^{2}\left(X_{k} \bullet\right)\left(\mathbf{x}^{\prime}, t^{\prime}\right) d t^{\prime} d V^{\prime}
$$

Con la Ec. (1.20), puede establecerse una relación entre el promedio de fase y el promedio intrínseco de fase:

$$
\left\langle\bullet_{k}\right\rangle=\varepsilon_{k}\left\langle\bullet_{k}\right\rangle^{k}
$$

Hay otros dos operadores promedio llamados el operador promedio interfacial y el operador promedio interfacial intrínseco y están definidos por

$$
\left\langle\bullet_{k}\right\rangle^{k, g s}(\mathbf{x}, t) \doteq \frac{1}{V T} \iiint_{\mathscr{V}(\mathbf{x})} \int_{t-\frac{T}{2}}^{t+\frac{T}{2}}\left[-\left.\frac{\partial X_{k}}{\partial n_{k}}\left(\mathbf{x}^{\prime}, t^{\prime}\right) \bullet_{k}\right|_{k}\left(\mathbf{x}^{\prime}, t^{\prime}\right)\right] d t^{\prime} d V^{\prime}
$$

y

$$
\left\langle\bullet_{k}\right\rangle^{g s}(\mathbf{x}, t) \doteq \frac{1}{A_{g s} T} \iiint_{\mathscr{\mathscr { V }}(\mathbf{x})} \int_{t-\frac{T}{2}}^{t+\frac{T}{2}}\left[-\left.\frac{\partial X_{k}}{\partial n_{k}}\left(\mathbf{x}^{\prime}, t^{\prime}\right) \bullet_{k}\right|_{k}\left(\mathbf{x}^{\prime}, t^{\prime}\right)\right] d t^{\prime} d V^{\prime},
$$

respectivamente. Aquí $n_{k}$ es una coordenada normal local a la interfaz, apuntando hacia afuera de la fase $k$. La derivada negativa de la función indicadora de fase es equivalente a la función de distribución delta de Dirac con soporte en la interfaz [Ec. (1.9)], tal que las integrales se desarrollan en la interfaz para los valores límites de la función desde adentro de la fase $\left.k \quad \bullet_{k}\right|_{k}$. 
Cuando la Ec. (1.22) se aplica a la unidad se obtiene el siguiente resultado

$$
\langle 1\rangle^{g, g s}(\mathbf{x}, t)=\langle 1\rangle^{s, g s}(\mathbf{x}, t)=\frac{1}{V T} \iiint_{\mathscr{\mathscr { V }}(\mathbf{x})} \int_{t-\frac{T}{2}}^{t+\frac{T}{2}}\left[-\frac{\partial X_{k}}{\partial n_{k}}\left(\mathbf{x}^{\prime}, t^{\prime}\right)\right] d t^{\prime} d V^{\prime}=\frac{A_{g s}}{V} \equiv a_{g s}(\mathbf{x}, t)
$$

que es el área interfacial específica, siendo $A_{g s}$ el área interfacial dentro de $\mathscr{V}(\mathbf{x}) \times T$. Para fluidos compresibles, se prefiere el promedio ponderado en la masa, el cual se define de acuerdo con Cebeci y Smith (1974) como

$$
\left\{\bullet_{k}\right\}^{k} \doteq \frac{\left\langle\rho_{k} \bullet_{k}\right\rangle^{k}}{\left\langle\rho_{k}\right\rangle^{k}}
$$

Para fluidos incompresibles, el promedio ponderado en la masa coincide con el promedio intrínseco de fase. Una variable local instantánea general, representada simbólicamente por $\bullet_{k}$, puede descomponerse como la suma de su valor promedio intrínseco de fase más una desviación $\bullet{ }_{k}^{\prime}$ alrededor de su promedio:

$$
\bullet_{k} \doteq\left\langle\bullet_{k}\right\rangle^{k}+\bullet_{k}^{\prime} .
$$

Las variables: velocidad, temperatura, energía interna, entalpía, entropía, pueden descomponerse de acuerdo con Cebeci y Smith (1974), como

$$
\bullet_{k} \doteq\left\{\bullet_{k}\right\}^{k}+\tilde{\bullet}_{k}
$$

El promedio de la desviación satisface las condiciones

$$
\left\langle\bullet_{k}^{\prime}\right\rangle^{k}=\mathbf{0} \mathbf{y}\left\{\tilde{\bullet}_{k}\right\}^{k}=\mathbf{0}
$$

Es interesante examinar la relación existente entre estos dos tipos de descripciones de promediado en términos del significado de sus desviaciones (Apéndice $B$ ).

Para analizar esto, tratemos de contestar la siguiente pregunta:

¿Bajo qué condiciones es válida o qué limitaciones tiene la siguiente igualdad?

$$
\left\{\rho_{k}^{\prime}\right\}^{k}=0
$$




\subsection{Restricciones de escala}

\subsubsection{Expansión en series de Taylor}

Para poder extraer adecuadamente cantidades promediadas de las integrales y de analizar las restricciones que esto implica, utilizamos un desarrollo en serie de Taylor alrededor del centroide de la región de integración espacio-temporal mediante un procedimiento similar al de Whitaker (1999):

$$
\begin{aligned}
\left\langle\bullet_{k}\right\rangle^{k}\left(\mathbf{r}_{k}, t^{*}\right)=\left.\left\langle\bullet_{k}\right\rangle^{k}\right|_{\mathbf{x}, t}+\left[\frac{\partial}{\partial \mathbf{r}_{k}}\left\langle\bullet_{k}\right\rangle^{k}\right]_{\mathbf{x}, t} \cdot\left(\mathbf{r}_{k}-\mathbf{x}\right) & +\left[\frac{\partial}{\partial t}\left\langle\bullet_{k}\right\rangle^{k}\right]_{\mathbf{x}, t}\left(t^{*}-t\right) \\
& +\frac{1}{2}\left[\frac{\partial}{\partial \mathbf{r}_{k}}\left(\frac{\partial}{\partial \mathbf{r}_{k}}\left\langle\bullet_{k}\right\rangle^{k}\right)\right]_{\mathbf{x}, t}:\left(\mathbf{r}_{k}-\mathbf{x}\right)\left(\mathbf{r}_{k}-\mathbf{x}\right) \\
& +\left[\frac{\partial}{\partial t *}\left(\frac{\partial}{\partial \mathbf{r}_{k}}\left\langle\bullet_{k}\right\rangle^{k}\right)\right]_{\mathbf{x}, t} \cdot\left(\mathbf{r}_{k}-\mathbf{x}\right)\left(t^{*}-t\right) \\
& +\frac{1}{2}\left[\frac{\partial}{\partial t *}\left(\frac{\partial}{\partial t^{*}}\left\langle\bullet_{k}\right\rangle^{k}\right)\right]_{\mathbf{x}, t}\left(t^{*}-t\right)^{2}+\cdots
\end{aligned}
$$

donde $\mathbf{r}_{k}=\mathbf{x}+\mathbf{y}_{k}$ y $t^{*}=t+\tau$. Por lo que sustituyendo, simplificando y escribiendo el resultado en notación de Gibbs, se tiene que:

$$
\begin{aligned}
\left.\left\langle\bullet_{k}\right\rangle^{k}\right|_{\mathbf{x}+\mathbf{y}_{k}, t+\tau}=\left.\left\langle\bullet_{k}\right\rangle^{k}\right|_{\mathbf{x}, t} & +\mathbf{y}_{k} \cdot\left[\nabla_{\mathbf{y}_{k}}\left\langle\bullet_{k}\right\rangle^{k}\right]_{\mathbf{x}, t}+\left[\frac{\partial}{\partial t}\left\langle\bullet_{k}\right\rangle^{k}\right]_{\mathbf{x}, t} \tau+\frac{1}{2} \mathbf{y}_{k} \mathbf{y}_{k}:\left[\nabla_{\mathbf{y}_{k}} \nabla_{\mathbf{y}_{k}}\left\langle\bullet_{k}\right\rangle^{k}\right]_{\mathbf{x}, t} \\
& +\mathbf{y}_{k} \cdot\left[\frac{\partial}{\partial \tau}\left(\nabla_{\mathbf{y}_{k}}\left\langle\bullet_{k}\right\rangle^{k}\right)\right]_{\mathbf{x}, t} \tau+\frac{1}{2}\left[\frac{\partial^{2}}{\partial \tau^{2}}\left\langle\bullet_{k}\right\rangle^{k}\right]_{\mathbf{x}, t} \tau^{2}+\cdots,
\end{aligned}
$$

donde $\mathbf{x}$ es el centroide de la región $\mathscr{Q}$ y $t$ es el punto medio de la región temporal de integración y tienen valores constantes. Sustituyendo este resultado en la Ec. (1.29):

$$
\begin{aligned}
\left\langle\rho_{k}\right\rangle^{k}\left\{\rho_{k}^{\prime}\right\}^{k}=\left\langle X_{k} \rho_{k} \rho_{k}^{\prime}\right\rangle & \\
\left.\left\langle\rho_{k}\right\rangle^{k}\left\{\rho_{k}^{\prime}\right\}^{k}\right|_{\mathbf{x}+\mathbf{y}_{k}, t+\tau}= & \left.\left\langle X_{k} \rho_{k} \rho_{k}^{\prime}\right\rangle\right|_{\mathbf{x}, t}+\mathbf{y}_{k} \cdot\left[\nabla_{\mathbf{y}_{k}}\left\langle X_{k} \rho_{k} \rho_{k}^{\prime}\right\rangle\right]_{\mathbf{x}, t} \\
& +\left[\frac{\partial}{\partial t}\left\langle X_{k} \rho_{k} \rho_{k}^{\prime}\right\rangle\right]_{\mathbf{x}, t} \tau+\frac{1}{2} \mathbf{y}_{k} \mathbf{y}_{k}:\left[\nabla_{\mathbf{y}_{k}} \nabla_{\mathbf{y}_{k}}\left\langle X_{k} \rho_{k} \rho_{k}^{\prime}\right\rangle\right]_{\mathbf{x}, t} \\
& +\mathbf{y}_{k} \cdot\left[\frac{\partial}{\partial \tau}\left(\nabla_{\mathbf{y}_{k}}\left\langle X_{k} \rho_{k} \rho_{k}^{\prime}\right\rangle\right)\right]_{\mathbf{x}, t} \tau+\frac{1}{2}\left[\frac{\partial^{2}}{\partial \tau^{2}}\left\langle X_{k} \rho_{k} \rho_{k}^{\prime}\right\rangle\right]_{\mathbf{x}, t} \tau^{2}+\cdots
\end{aligned}
$$


introduciendo la descomposición espacio-temporal de la densidad dada en la Ec. (1.26), desarrollando términos, dividiendo por $\left\langle\rho_{k}\right\rangle^{k}\left\langle\rho_{k}\right\rangle^{k}$ y eliminando los promedios de desviaciones mediante la Ec. (1.28):

$$
\begin{aligned}
\frac{\left\{\rho_{k}^{\prime}\right\}^{k} \mid{ }_{\mathbf{x}+\mathbf{y}_{k}, t+\tau}}{\left\langle\rho_{k}\right\rangle^{k}}= & \frac{\left.\left\langle\rho_{k}^{\prime} \rho_{k}^{\prime}\right\rangle^{k}\right|_{\mathbf{x}, t}}{\left\langle\rho_{k}\right\rangle^{k}\left\langle\rho_{k}\right\rangle^{k}}+\mathbf{y}_{k} \cdot \frac{\left[\nabla_{\mathbf{y}_{k}}\left\langle\rho_{k}^{\prime} \rho_{k}^{\prime}\right\rangle^{k}\right]_{\mathbf{x}, t}}{\left\langle\rho_{k}\right\rangle^{k}\left\langle\rho_{k}\right\rangle^{k}}+\tau \frac{\left[\frac{\partial}{\partial t}\left\langle\rho_{k}^{\prime} \rho_{k}^{\prime}\right\rangle^{k}\right]_{\mathbf{x}, t}}{\left\langle\rho_{k}\right\rangle^{k}\left\langle\rho_{k}\right\rangle^{k}} \\
& +\frac{1}{2} \mathbf{y}_{k} \mathbf{y}_{k}: \frac{\left[\nabla_{\mathbf{y}_{k}} \nabla_{\mathbf{y}_{k}}\left\langle\rho_{k}^{\prime} \rho_{k}^{\prime}\right\rangle^{k}\right]_{\mathbf{x}, t}}{\left\langle\rho_{k}\right\rangle^{k}\left\langle\rho_{k}\right\rangle^{k}}+\tau \mathbf{y}_{k} \cdot \frac{\left[\frac{\partial}{\partial \tau} \nabla_{\mathbf{y}_{k}}\left\langle\rho_{k}^{\prime} \rho_{k}^{\prime}\right\rangle^{k}\right]_{\mathbf{x}, t}}{\left\langle\rho_{k}\right\rangle^{k}\left\langle\rho_{k}\right\rangle^{k}} \\
& +\frac{1}{2} \tau^{2} \frac{\left[\frac{\partial^{2}}{\partial \tau^{2}}\left\langle\rho_{k}^{\prime} \rho_{k}^{\prime}\right\rangle^{k}\right]_{\mathbf{x}, t}+\cdots}{\left\langle\rho_{k}\right\rangle^{k}\left\langle\rho_{k}\right\rangle^{k}}
\end{aligned}
$$

utilizando estimados de órdenes de magnitud hasta primer orden en las desviaciones, se obtiene que:

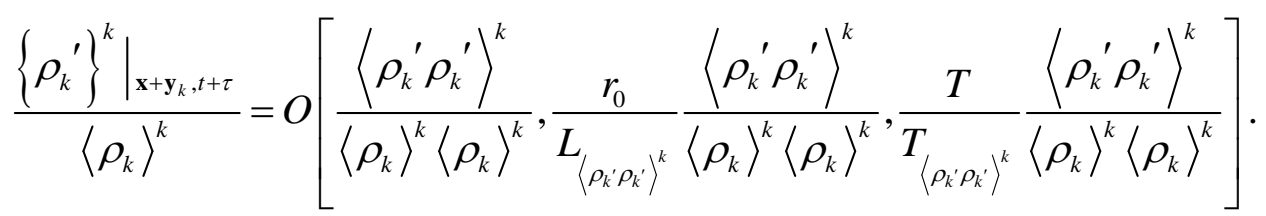

Donde $\mathbf{y}_{k}$ varía en distancias del orden del radio $r_{0}$ de la región espacial $\mathscr{V}$ y $\tau$ varía en tiempos del orden de la región temporal de integración $T$, mientras que $L_{\left\langle\rho_{k^{\prime}} \rho_{k^{\prime}}\right\rangle^{k}}$ y $T_{\left\langle\rho_{k^{\prime}} \rho_{k^{\prime}}\right\rangle^{k}}$ varían en distancias observables en una escala mayor; es decir, a escala del equipo.

Podemos sacar las cantidades promediadas de las integrales y a primer orden en las desviaciones se verifica la relación dada en la Ec. (1.29), pero teniendo en cuenta que esto trae consigo las siguientes restricciones de escala:

$$
\frac{\left\langle\rho_{k}^{\prime} \rho_{k}^{\prime}\right\rangle^{k}}{\left\langle\rho_{k}\right\rangle^{k}\left\langle\rho_{k}\right\rangle^{k}} \ll 1, \quad \frac{r_{0}}{L} \ll 1, \quad \frac{T}{\left\langle\rho_{k^{\prime}} \rho^{\prime}\right\rangle^{k}} \ll 1 .
$$

Más adelante se utilizará este resultado. 
1.6 Teoremas fundamentales para el desarrollo de las ecuaciones con promedio espacio-temporal

Los teoremas fundamentales para desarrollar las ecuaciones con promedio espaciotemporal se obtienen aplicando el operador promedio espacio-temporal dado en la Ec. (1.17) a la Ec. (1.13) y a la Ec. (1.14):

$$
\begin{gathered}
\left\langle X_{k} \frac{\partial \boldsymbol{\Psi}_{k}}{\partial t^{\prime}}\right\rangle=\left\langle\frac{\partial}{\partial t^{\prime}}\left(X_{k} \boldsymbol{\Psi}_{k}\right)-\mathbf{n}_{k} \cdot \mathbf{w}_{k \ell} \delta_{k \ell} \boldsymbol{\Psi}_{k}\right\rangle, \\
\left\langle X_{k} \nabla^{\prime} \cdot\left(\mathbf{v}_{k} \boldsymbol{\Psi}_{k}\right)\right\rangle=\left\langle\nabla^{\prime} \cdot\left(X_{k} \mathbf{v}_{k} \boldsymbol{\Psi}_{k}\right)+\delta_{k \ell} \mathbf{n}_{k} \cdot \mathbf{v}_{k} \boldsymbol{\Psi}_{k}\right\rangle .
\end{gathered}
$$

Puesto que el operador promedio espacio-temporal [Ec. (1.17)] tiene una representación integral, entonces es lineal y el promedio de una suma es la suma del promedio de los sumandos. Así, la Ec. (1.31) y la Ec. (1.32) pueden reescribirse en la forma:

$$
\begin{gathered}
\left\langle X_{k} \frac{\partial \boldsymbol{\Psi}_{k}}{\partial t^{\prime}}\right\rangle=\left\langle\frac{\partial}{\partial t^{\prime}}\left(X_{k} \boldsymbol{\Psi}_{k}\right)\right\rangle-\left\langle\mathbf{n}_{k} \cdot \mathbf{w}_{k \ell} \delta_{k \ell} \boldsymbol{\Psi}_{k}\right\rangle, \\
\left\langle X_{k} \nabla^{\prime} \cdot\left(\mathbf{v}_{k} \boldsymbol{\Psi}_{k}\right)\right\rangle=\left\langle\nabla^{\prime} \cdot\left(X_{k} \mathbf{v}_{k} \boldsymbol{\Psi}_{k}\right)\right\rangle+\left\langle\delta_{k \ell} \mathbf{n}_{k} \cdot \mathbf{v}_{k} \boldsymbol{\Psi}_{k}\right\rangle .
\end{gathered}
$$

La integración se realiza sobre la región espacial con centroide en $\mathbf{x}$, de tal manera que la región está fija. Además, la región temporal está centrada en el punto medio $t$. Por lo tanto, el operador derivada temporal y el operador divergencia pueden conmutar con el operador promedio, con lo que se obtienen los siguientes dos teoremas.

Teorema general de transporte (Gray, 1975; Drew, 1983; Soria y de Lasa, 1991; Drew y Lahey, 1993; Sánchez-López, 2003):

$$
\left\langle X_{k} \frac{\partial \boldsymbol{\Psi}_{k}}{\partial t^{\prime}}\right\rangle=\frac{\partial}{\partial t}\left\langle X_{k} \boldsymbol{\Psi}_{k}\right\rangle-\left\langle\mathbf{n}_{k} \cdot \mathbf{w}_{k \ell} \delta_{k \ell} \boldsymbol{\Psi}_{k}\right\rangle .
$$

Teorema del promedio volumétrico:

$$
\left\langle X_{k} \nabla^{\prime} \cdot\left(\mathbf{v}_{k} \boldsymbol{\Psi}_{k}\right)\right\rangle=\nabla \cdot\left\langle X_{k} \mathbf{v}_{k} \boldsymbol{\Psi}_{k}\right\rangle+\left\langle\delta_{k \ell} \mathbf{n}_{k} \cdot \mathbf{v}_{k} \boldsymbol{\Psi}_{k}\right\rangle .
$$

Este teorema representa una versión tridimensional de la regla de Leibniz para intercambiar diferenciación con integración. 
Este teorema es conocido como teorema del promedio volumétrico y fue obtenido en forma independiente en 1967 por Marle estudiando el flujo monofásico en medios porosos, por Anderson y Jackson estudiando lechos fluidizados, por Slattery estudiando el flujo bifásico en medios porosos y por Whitaker estudiando la dispersión en medios porosos.

Estos son los dos teoremas esenciales para el desarrollo de las ecuaciones promediadas (Drew, 1983; Soria y de Lasa, 1991; Drew y Lahey, 1993).

Para simplificar el procedimiento de promediado de las ecuaciones locales instantáneas se obtiene un tercer teorema, llamado teorema de transporte combinado, al sumar la Ec. (1.35) y la Ec. (1.36) para tener:

$$
\left\langle X_{k}\left(\frac{\partial \boldsymbol{\Psi}_{k}}{\partial t^{\prime}}+\nabla^{\prime} \cdot\left(\mathbf{v}_{k} \boldsymbol{\Psi}_{k}\right)\right)\right\rangle=\frac{\partial}{\partial t}\left\langle X_{k} \boldsymbol{\Psi}_{k}\right\rangle+\nabla \cdot\left\langle X_{k} \mathbf{v}_{k} \boldsymbol{\Psi}_{k}\right\rangle+\left\langle\delta_{k \ell} \mathbf{n}_{k} \cdot\left(\mathbf{v}_{k}-\mathbf{w}_{k \ell}\right) \boldsymbol{\Psi}_{k}\right\rangle \cdot(1
$$

Al deducir esta ecuación, se tuvo cuidado en el hecho de que $\boldsymbol{\Psi}_{k}$ puede ser un tensor. Utilizando la Ec. (1.21) se obtiene la siguiente ecuación:

$$
\left\langle X_{k}\left(\frac{\partial \boldsymbol{\Psi}_{k}}{\partial t^{\prime}}+\nabla^{\prime} \cdot\left(\mathbf{v}_{k} \boldsymbol{\Psi}_{k}\right)\right)\right\rangle=\frac{\partial}{\partial t}\left(\varepsilon_{k}\left\langle\boldsymbol{\Psi}_{k}\right\rangle^{k}\right)+\nabla \cdot\left(\varepsilon_{k}\left\langle\mathbf{v}_{k} \boldsymbol{\Psi}_{k}\right\rangle^{k}\right)+\left\langle\delta_{k \ell} \mathbf{n}_{k} \cdot\left(\mathbf{v}_{k}-\mathbf{w}_{k \ell}\right) \boldsymbol{\Psi}_{k}\right\rangle
$$

Analizando el término fuente correspondiente al último término en la Ec. (1.38), donde la función de distribución delta de Dirac $\delta_{k \ell}$ sirve de filtro a la integral dejando pasar la información a la interfaz,

$$
\begin{aligned}
\left\langle\delta_{k \ell} \mathbf{n}_{k} \cdot\left(\mathbf{v}_{k}-\mathbf{w}_{k \ell}\right) \boldsymbol{\Psi}_{k}\right\rangle & \equiv \frac{1}{V T} \iiint_{\mathscr{V}(\mathbf{x})}^{t+\frac{T}{2}} \int_{t-\frac{T}{2}} \delta_{k \ell} \mathbf{n}_{k} \cdot\left(\mathbf{v}_{k}-\mathbf{w}_{k \ell}\right) \boldsymbol{\Psi}_{k} d t^{\prime} d V \\
& =\sum_{k \ell \mid k} \frac{1}{V T} \iint_{k \ell} \int_{(\mathbf{x})}^{t+\frac{T}{2}} \mathbf{n}_{t-\frac{T}{2}} \cdot\left(\mathbf{v}_{k}-\mathbf{w}_{k \ell}\right) \boldsymbol{\Psi}_{k} d t^{\prime} d S
\end{aligned}
$$

donde el producto $V T$ se ha introducido a la sumatoria, debido a que es constante. La suma en la Ec. (1.39) debe leerse como: "suma sobre todas las interfaz kl tal que la fase $k$ esté presente" (Soria y de Lasa, 1991). 
En la Ec. (1.39) la región interfacial $\mathscr{S}_{k \ell}$ define el área $S_{k \ell}$. Esta ecuación debe reescribirse como un promedio superficial intrínseco de fase:

$$
\begin{aligned}
\left\langle\delta_{k \ell} \mathbf{n}_{k} \cdot\left(\mathbf{v}_{k}-\mathbf{w}_{k \ell}\right) \boldsymbol{\Psi}_{k}\right\rangle & =\sum_{k \ell \mid k} \frac{S_{k \ell}}{V} \frac{1}{S_{k \ell} T} \iint_{k \ell} \int_{t=\frac{T}{2}}^{t+\frac{T}{2}} \mathbf{n}_{k} \cdot\left(\mathbf{v}_{k}-\mathbf{w}_{k \ell}\right) \boldsymbol{\Psi}_{k} d t^{\prime} d S \\
& =\sum_{k \ell \mid k} a_{k \ell}\left\langle\mathbf{n}_{k} \cdot\left(\mathbf{v}_{k}-\mathbf{w}_{k \ell}\right) \boldsymbol{\Psi}_{k}\right\rangle_{k \ell}
\end{aligned}
$$

donde el área interfacial específica $a_{k \ell}$ fue introducida (Soria y de Lasa, 1991):

$$
a_{k \ell}=\left\langle\delta_{k \ell}\right\rangle=\frac{S_{k \ell}}{V} .
$$

Por lo tanto, la Ec. (1.38) puede reescribirse finalmente como:

$$
\left\langle X_{k}\left(\frac{\partial \boldsymbol{\Psi}_{k}}{\partial t^{\prime}}+\nabla^{\prime} \cdot\left(\mathbf{v}_{k} \boldsymbol{\Psi}_{k}\right)\right)\right\rangle=\frac{\partial}{\partial t}\left(\varepsilon_{k}\left\langle\boldsymbol{\Psi}_{k}\right\rangle^{k}\right)+\nabla \cdot\left(\varepsilon_{k}\left\langle\mathbf{v}_{k} \boldsymbol{\Psi}_{k}\right\rangle^{k}\right)+\sum_{k \ell \mid k} a_{k \ell}\left\langle\mathbf{n}_{k} \cdot\left(\mathbf{v}_{k}-\mathbf{w}_{k \ell}\right) \boldsymbol{\Psi}_{k}\right\rangle_{k \ell} \cdot
$$

Pueden obtenerse expresiones similares a la Ec. (1.40) para los términos superficiales, representados por el segundo término en el miembro derecho de los primeros dos teoremas.

En términos generales, las ecuaciones de balance locales instantáneas pueden representarse por la siguiente estructura funcional:

$$
\mathscr{L}(\mathbf{V})=\mathbf{0}, \quad \operatorname{para} \mathbf{x}_{k}^{\prime} \in \mathscr{T}\left(\mathbf{x}^{\prime}\right)
$$

donde

$\mathbf{V}$, es un vector que representa las propiedades termodinámicas en $\mathscr{V}$,

$\mathscr{L}$, es un operador local instantáneo general que describe el comportamiento dinámico del fluido en la región $\mathscr{T}$.

La función $X_{k}(\mathbf{x}, t)$ permite extender el dominio de definición de la Ec. (1.43) a toda la región $\mathscr{T}$. Aplicando esta función en el sentido de función generalizada:

$$
\left\langle X_{k} \mathscr{L}(\mathbf{V})\right\rangle=\mathbf{0}
$$

donde \langle\rangle es un operador promedio en una región $\mathbb{R}^{3} \times \mathbb{R}$. Puesto que $\mathscr{L}$ involucra tanto derivadas espaciales como derivadas temporales, se considera un procedimiento general para establecer la conmutatividad entre los operadores; esto es, intercambiar estas derivadas con el operador de promediado. Este procedimiento utiliza sistemáticamente la definición y propiedades de las derivadas distribucionales (Soria y de Lasa, 1991). 
Las ecuaciones de transporte promediadas en la región $\mathscr{V}$ pueden obtenerse en la forma general:

$$
\mathscr{L}\left\langle X_{k} \mathbf{V}\right\rangle-\left\langle\mathbf{V} \mathscr{L}\left(X_{k}\right)\right\rangle=\mathbf{0}
$$

donde el primer término representa el comportamiento dinámico promedio dentro de $\mathscr{V}$. El segundo término es el flujo neto promedio proveniente de $\mathscr{W}$ hacia la superficie interfacial [ver la Ec. (1.40)]. Esta ecuación es una representación compacta de la simbólica dada en la Ec. (1.38), que se desarrolló al aplicar el procedimiento de promediado espaciotemporal. En el último término de la Ec. (1.45) $\mathscr{L}\left(X_{k}\right)$ es una función de distribución tal que (Drew, 1983; Soria y de Lasa, 1991):

$$
\mathbf{V} \mathscr{L}\left(X_{k}\right)=\mathscr{L}\left(X_{k} \mathbf{V}\right)-X_{k} \mathscr{L}(\mathbf{V})
$$

En la siguiente sección se aplicarán los teoremas del promediado espacio-temporal a las ecuaciones de conservación y de balance locales instantáneas.

\subsection{Ecuaciones de balance promediadas en el espacio-tiempo}

El conjunto dado por la Ec. (1.1) a la Ec. (1.3) es sólo válido en la región ocupada por la fase $k$. Sin embargo, se pueden obtener las ecuaciones promediadas válidas en toda la región espacial $\mathscr{Y}$. Esto se logra al ponderar las ecuaciones: (1.1) a la (1.3) con la función indicadora de fase e introducirla en los operadores diferenciales. Luego se aplica el operador promedio definido en la Ec. (1.20), después se utilizan los teoremas de promediado [Ec. (1.35) a la Ec. (1.38)] y la descomposición espacio-temporal dada en la Ec. (1.26) a la Ec. (1.28), con lo que obtenemos finalmente las ecuaciones de campo promedio. Este procedimiento puede seguirse en tres etapas, (1) en Drew (1971 y 1983): funciones generalizadas en el flujo en dos fases, (2) en Soria y de Lasa (1991): funciones generalizadas en flujos multifásicos dispersos y (3) en Sánchez-López (2003): teoremas de promediado para funciones generalizadas en el espacio-tiempo, entre otros.

Las ecuaciones resultantes son (ver el Apéndice $C$ ): 


\subsubsection{Ecuación de continuidad}

$$
\frac{D_{k}}{D t}\left(\varepsilon_{k}\left\langle\rho_{k}\right\rangle^{k}\right)+\varepsilon_{k}\left\langle\rho_{k}\right\rangle^{k} \nabla \cdot\left\{\mathbf{v}_{k}\right\}^{k}=\Gamma_{k},
$$

donde $\Gamma_{k} \doteq\left\langle\rho_{k} \mathbf{n}_{k} \cdot\left(\mathbf{w}_{k \ell}-\mathbf{v}_{k}\right) \delta_{k \ell}\right\rangle$ es el flujo másico interfacial y establece la continuidad de la masa en la interfaz.

\subsubsection{Ecuación de cantidad de movimiento}

$$
\varepsilon_{k}\left\langle\rho_{k}\right\rangle^{k} \frac{D_{k}}{D t}\left\{\mathbf{v}_{k}\right\}^{k}-\varepsilon_{k}\left\langle\rho_{k}\right\rangle^{k} \mathbf{g}_{k}-\nabla \cdot\left[\varepsilon_{k}\left(\left\langle\boldsymbol{\Pi}_{k}\right\rangle^{k}+\boldsymbol{\Pi}_{k}^{\mathrm{Re}}\right)\right]=\Gamma_{k}\left(\mathbf{v}_{k i}-\left\{\mathbf{v}_{k}\right\}^{k}\right)+\mathbf{M}_{k} \cdot
$$

Donde $\boldsymbol{\Pi}_{k}^{\mathrm{Re}} \doteq-\left\langle X_{k} \rho_{k} \tilde{\mathbf{v}}_{k} \tilde{\mathbf{v}}_{k}\right\rangle / \varepsilon_{k}$ son los esfuerzos de Reynolds que aquí incorporan los efectos de no-uniformidad del flujo, $\mathbf{M}_{k} \doteq-\left\langle\boldsymbol{\Pi}_{k} \cdot \nabla^{\prime} X_{k}\right\rangle$ es la fuente de cantidad de movimiento interfacial.

\subsubsection{Ecuación de energía total}

$$
\begin{aligned}
\varepsilon_{k}\left\langle\rho_{k}\right\rangle^{k} \frac{D_{k}}{D t}\left(\left\{U_{k}\right\}^{k}\right. & \left.+\frac{1}{2}\left\{\mathbf{v}_{k}\right\}^{k} \cdot\left\{\mathbf{v}_{k}\right\}^{k}+K_{k}^{\mathrm{Re}}\right)=\nabla \cdot\left[\varepsilon_{k}\left(\left\langle\boldsymbol{\Pi}_{k}\right\rangle^{k}+\boldsymbol{\Pi}_{k}^{\mathrm{Re}}\right) \cdot\left\{\mathbf{v}_{k}\right\}^{k}\right] \\
& -\nabla \cdot\left[\varepsilon_{k}\left(\left\langle\mathbf{q}_{k}\right\rangle^{k}+\mathbf{q}_{k}^{\mathrm{Re}}\right)\right]+\varepsilon_{k}\left\langle Q_{k}\right\rangle^{k}+\varepsilon_{k}\left\langle\rho_{k}\right\rangle^{k} \mathbf{g}_{k} \cdot\left\{\mathbf{v}_{k}\right\}^{k}+E_{k} \\
& +W_{k i}+\Gamma_{k}\left\{U_{k i}-\left\{U_{k}\right\}^{k}+\frac{1}{2}\left[\left(\mathbf{v}_{k} \cdot \mathbf{v}_{k}\right)_{i}-\left\{\mathbf{v}_{k}\right\}^{k} \cdot\left\{\mathbf{v}_{k}\right\}^{k}\right]-K_{k}^{\mathrm{Re}}\right\} .
\end{aligned}
$$

Donde:

$E_{k} \doteq\left\langle\mathbf{q}_{k} \cdot \nabla^{\prime} X_{k}\right\rangle$ es la fuente de calor interfacial.

$W_{k i} \doteq-\left\langle\nabla^{\prime} X_{k} \cdot\left(\boldsymbol{\Pi}_{k} \cdot \mathbf{v}_{k}\right)\right\rangle=-\left\langle\boldsymbol{\Pi}_{k}: \mathbf{v}_{k} \nabla^{\prime} X_{k}\right\rangle$ es el trabajo interfacial.

$K_{k}^{\mathrm{Re}} \doteq \frac{1}{2}\left\langle X_{k} \rho_{k} \tilde{\mathbf{v}}_{k} \cdot \tilde{\mathbf{v}}_{k}\right\rangle / \varepsilon_{k}\left\langle\rho_{k}\right\rangle^{k}$ es la energía cinética turbulenta y

$\mathbf{q}_{k}^{\mathrm{Re}}$, está constituido por varios términos turbulentos de energía mecánica y térmica:

$\mathbf{q}_{k}^{\mathrm{Re}} \doteq \mathbf{q}_{k}^{p}+\mathbf{q}_{k}^{t}+\mathbf{q}_{k}^{K}+\mathbf{q}_{k}^{r}$, en donde: 


$$
\begin{aligned}
\mathbf{q}_{k}^{p} \doteq\left\langle X_{k} P_{k} \tilde{\mathbf{v}}_{k}\right\rangle / \varepsilon_{k} \\
\mathbf{q}_{k}^{t} \doteq\left\langle X_{k} \boldsymbol{\tau}_{k} \cdot \tilde{\mathbf{v}}_{k}\right\rangle / \varepsilon_{k} \\
\mathbf{q}_{k}^{K} \doteq \frac{1}{2}\left\langle X_{k} \rho_{k} \tilde{\mathbf{v}}_{k} \tilde{\mathbf{v}}_{k} \cdot \tilde{\mathbf{v}}_{k}\right\rangle / \varepsilon_{k} \\
\mathbf{q}_{k}^{r} \doteq\left\langle X_{k} \rho_{k} \tilde{\mathbf{v}}_{k} \tilde{U}_{k}\right\rangle / \varepsilon_{k} .
\end{aligned}
$$

Los términos de la forma $\Gamma_{k} \zeta_{k i}$ son promedios ponderados en el flujo másico interfacial: $\Gamma_{k} \zeta_{k i} \doteq\left\langle\rho_{k} \zeta_{k} \mathbf{n}_{k} \cdot\left(\mathbf{w}_{k \ell}-\mathbf{v}_{k}\right) \delta_{k \ell}\right\rangle$. Cuyo valor $\zeta_{k i}$ es el valor de la propiedad $\zeta_{k}$ en el límite cuando nos acercamos a la interfaz desde el interior de la fase $k$ y se define por:

$$
\zeta_{k i} \doteq \lim _{\mathbf{x}_{k} \rightarrow \mathbf{x}_{i}} \zeta_{k}, \quad \text { donde } \mathbf{x}_{k} \in \mathscr{Y} \quad \text { y } \quad \mathbf{x}_{i} \in \mathscr{\mathscr { P }}
$$

Como puede notarse en la Ec. (1.49), la ecuación de energía total es la suma de tres tipos de energías: energía interna, energía cinética y energía cinética turbulenta.

\subsubsection{Ecuación de energía mecánica}

La ecuación de energía mecánica se obtiene al tomar el producto punto de la velocidad promedio con la ecuación de movimiento promedio dada en la Ec. (1.48).

$$
\begin{array}{r}
\varepsilon_{k}\left\langle\rho_{k}\right\rangle^{k}\left\{\mathbf{v}_{k}\right\}^{k} \cdot \frac{D_{k}}{D t}\left\{\mathbf{v}_{k}\right\}^{k}-\varepsilon_{k}\left\langle\rho_{k}\right\rangle^{k}\left\{\mathbf{v}_{k}\right\}^{k} \cdot \mathbf{g}_{k}-\left\{\mathbf{v}_{k}\right\}^{k} \cdot \nabla \cdot\left[\varepsilon_{k}\left(\left\langle\boldsymbol{\Pi}_{k}\right\rangle^{k}+\boldsymbol{\Pi}_{k}^{\mathrm{Re}}\right)\right]= \\
\Gamma_{k}\left\{\mathbf{v}_{k}\right\}^{k} \cdot\left(\mathbf{v}_{k i}-\left\{\mathbf{v}_{k}\right\}^{k}\right)+\left\{\mathbf{v}_{k}\right\}^{k} \cdot \mathbf{M}_{k} .
\end{array}
$$

Donde $\frac{D_{k}}{D t} \doteq \frac{\partial}{\partial t}+\left\{\mathbf{v}_{k}\right\}^{k} \cdot \nabla$. El primer término puede expresarse como

$$
\varepsilon_{k}\left\langle\rho_{k}\right\rangle^{k}\left\{\mathbf{v}_{k}\right\}^{k} \cdot \frac{D_{k}}{D t}\left\{\mathbf{v}_{k}\right\}^{k}=\varepsilon_{k}\left\langle\rho_{k}\right\rangle^{k} \frac{D_{k}}{D t}\left(\frac{1}{2}\left\{\mathbf{v}_{k}\right\}^{k} \cdot\left\{\mathbf{v}_{k}\right\}^{k}\right)
$$

ya que $\frac{D_{k}}{D t}\left(\left\{\mathbf{v}_{k}\right\}^{k} \cdot\left\{\mathbf{v}_{k}\right\}^{k}\right)=2\left\{\mathbf{v}_{k}\right\}^{k} \cdot \frac{D_{k}}{D t}\left\{\mathbf{v}_{k}\right\}^{k}$. La ecuación de energía mecánica queda:

$$
\begin{array}{r}
\varepsilon_{k}\left\langle\rho_{k}\right\rangle^{k} \frac{D_{k}}{D t}\left(\frac{1}{2}\left\{\mathbf{v}_{k}\right\}^{k} \cdot\left\{\mathbf{v}_{k}\right\}^{k}\right)-\varepsilon_{k}\left\langle\rho_{k}\right\rangle^{k}\left\{\mathbf{v}_{k}\right\}^{k} \cdot \mathbf{g}_{k}-\left\{\mathbf{v}_{k}\right\}^{k} \cdot \nabla \cdot\left[\varepsilon_{k}\left(\left\langle\boldsymbol{\Pi}_{k}\right\rangle^{k}+\boldsymbol{\Pi}_{k}^{\mathrm{Re}}\right)\right]= \\
\Gamma_{k}\left\{\mathbf{v}_{k}\right\}^{k} \cdot\left(\mathbf{v}_{k i}-\left\{\mathbf{v}_{k}\right\}^{k}\right)+\left\{\mathbf{v}_{k}\right\}^{k} \cdot \mathbf{M}_{k} .
\end{array}
$$

Esta ecuación toma en consideración a la energía cinética promedio solamente.

El contenido de este Capítulo es fundamental para la obtención y el análisis de las ecuaciones promediadas en el espacio-tiempo, así como para la elaboración de las restricciones en los términos de cerradura que se obtienen de aplicar la segunda ley de la termodinámica a las ecuaciones promediadas. 


\section{Capítulo 2}

\section{Segunda ley de la termodinámica y su aplicación a la cerradura de los modelos promediados}

\subsection{El balance de entropía y la segunda ley de la termodinámica}

La relación de Gibbs relaciona las variables termodinámicas de estado: energía interna y entropía. Por tal razón, utilizando la relación de Gibbs, podemos expresar la primera ley de la termodinámica o ecuación de balance de energía, en términos de la entropía y separar en ella los flujos de las fuentes, luego al restar el balance de entropía, podemos obtener las fuentes de entropía (ver Figura 2.1).

La segunda ley de la termodinámica establece que los términos de generación o fuentes de entropía deben ser iguales a cero en los procesos reversibles y mayores a cero en los procesos irreversibles.
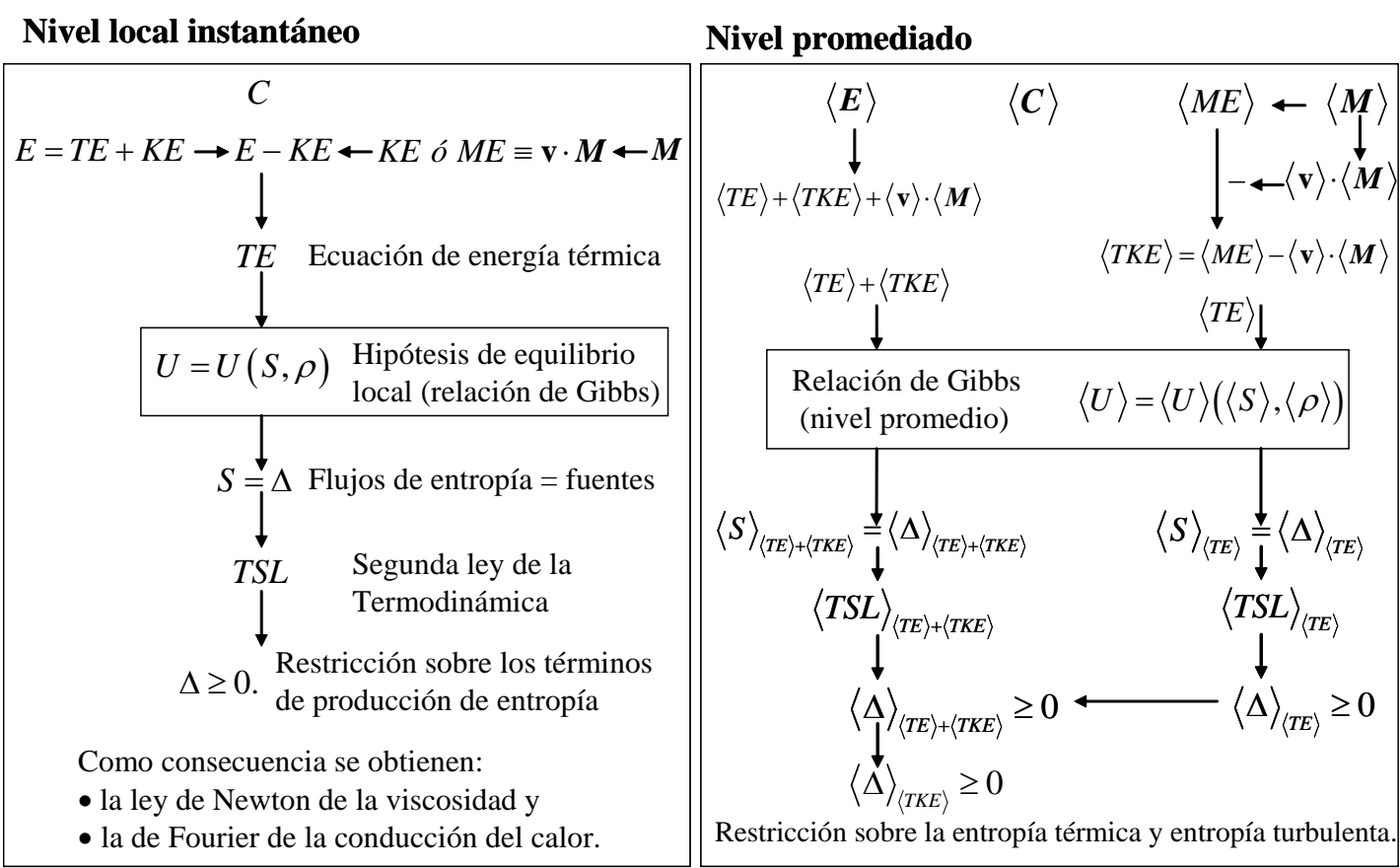

Figura 2.1. Metodología para obtener las restricciones que surgen de aplicar la segunda ley de la termodinámica a las ecuaciones promediadas. 
Notación:

$C$ : Ecuación de continuidad

$\boldsymbol{M}$ : Ecuación de movimiento

$E$ : Ecuación de energía total

$M E$ : Ecuación de energía mecánica

$T E$ : Ecuación de energía térmica

$E S$ : Ecuación de entropía

$T S L$ : Segunda ley de la termodinámica
$K E$ : Energía cinética

$T K E$ : Energía cinética turbulenta

$U$ : Energía interna específica

$S$ : Entropía específica

$\rho$ : Densidad de masa

$\Delta$ : Generación de entropía

\langle\rangle : Operador promedio.

En este Capítulo, siguiendo un procedimiento paralelo a como se hace en el nivel local instantáneo, donde las restricciones implicadas por la segunda ley de la termodinámica sobre los términos de generación de entropía son bien conocidas (Sección 2.2.2), aplicaremos esa metodología, pero a escala promedio para obtener información acerca del buen planteamiento de las cerraduras de los modelos de dos fluidos. Las restricciones que se encuentran a nivel promedio, las utilizaremos para verificar la validez de los modelos cerrados de dos fluidos. Para lograr esto, primero se generan las restricciones que se obtienen de aplicar la segunda ley a las ecuaciones en el nivel local instantáneo. Posteriormente, se derivan las restricciones que surgen de aplicar la segunda ley de la termodinámica a las ecuaciones de campo promedio (ver Figura 2.1).

\subsection{Descripción en el nivel local instantáneo}

\subsubsection{Ecuación de balance de entropía}

Partiendo de la primera ley de la termodinámica (para un sistema cerrado): $\delta Q=d U+\delta W$, donde $\delta W$ es el trabajo reversible efectuado durante el proceso y que puede reescribirse como: $-\left(P / \rho^{2}\right) d \rho$, junto con la relación termodinámica para un proceso reversible: $\delta Q=T d S$, podemos finalmente escribir para un proceso reversible: $d U=T d S-\delta W$ y expresar la ecuación de Gibbs en términos de derivadas materiales de la energía interna, la entropía y la densidad que al combinar con la ecuación de continuidad se obtiene que 


$$
\frac{D_{k}}{D t^{\prime}} U_{k}=T_{k} \frac{D_{k}}{D t^{\prime}} S_{k}-\frac{P_{k}}{\rho_{k}}\left(\nabla^{\prime} \cdot \mathbf{v}_{k}\right)
$$

Combinando la Ec. (2.1) con la ecuación de balance para la energía interna Ec. (1.5) y haciendo uso de la ecuación de continuidad Ec. (1.1), obtenemos la siguiente ecuación para la entropía:

$$
\rho_{k} \frac{D_{k}}{D t^{\prime}} S_{k}+\nabla^{\prime} \cdot\left(\frac{1}{T_{k}} \mathbf{q}_{k}\right)-\nabla^{\prime}\left(\frac{1}{T_{k}}\right) \cdot \mathbf{q}_{k}-\frac{Q_{k}}{T_{k}}+\frac{1}{T_{k}} \boldsymbol{\tau}_{k}: \nabla^{\prime} \mathbf{v}_{k}=0
$$

Donde se ha hecho uso de la definición de los esfuerzos: $\boldsymbol{\Pi}_{k}=-P_{k} \mathbf{1}-\boldsymbol{\tau}_{k}$. En esta ecuación podemos separar los flujos de las fuentes de entropía, para tener:

$$
\frac{\partial}{\partial t^{\prime}}\left(\rho_{k} S_{k}\right)+\nabla^{\prime} \cdot\left(\rho_{k} S_{k} \mathbf{v}_{k}\right)+\nabla^{\prime} \cdot\left(\frac{\mathbf{q}_{k}}{T_{k}}\right)-\frac{Q_{k}}{T_{k}}=\Delta_{k}
$$

En esta ecuación, los términos de producción están representados por $\Delta_{k} \mathrm{y}$ deben satisfacer la segunda ley de la termodinámica, es decir:

$$
\Delta_{k} \geq 0
$$

La Ec. (2.3) nos dice que la tasa de cambio de entropía asociada con la fase $k$ más la tasa a la cual se transfiere entropía de la fase a través de sus superficies limitantes por transmisión de energía por contacto más la transmisión de energía externa mutua, es igual a la tasa de generación de entropía.

\subsubsection{Implicaciones de la segunda ley en el nivel local instantáneo}

Podemos combinar la Ec. (2.2) y la Ec. (2.3), junto con la ecuación de continuidad para obtener

$$
\frac{1}{T_{k}}\left(-\boldsymbol{\tau}_{k}: \nabla^{\prime} \mathbf{v}_{k}-\frac{1}{T_{k}} \mathbf{q}_{k} \cdot \nabla^{\prime} T_{k}\right) \geq 0
$$

donde la tasa de generación de entropía se da en cada punto de la fase por disipación viscosa y por disipación térmica. 
De esta expresión, al tomar cada sumando como positivo definido (lo cual es más restrictivo) cerca de equilibrio permite obtener formas cuadráticas con las relaciones funcionales de Newton y Fourier (Slattery, 1999):

$$
\frac{\lambda_{k}}{T_{k}}\left(\operatorname{tr} \mathbf{D}_{k}\right)^{2}+\frac{2 \mu_{k}}{T_{k}}\left(\operatorname{tr} \mathbf{D}_{k}^{2}\right)+\frac{k_{k}}{T_{k}^{2}}\left(\nabla^{\prime} T_{k}\right)^{2} \geq 0,
$$

con los coeficientes de transporte $\lambda_{k}, \mu_{k}$ y $k_{k}$ no-negativos.

- $\quad$ Ley de Newton de la viscosidad: $\boldsymbol{\tau}_{k}=-2 \mu_{k} \mathbf{D}_{k}-\lambda_{k}\left(\nabla^{\prime} \cdot \mathbf{v}_{k}\right) \mathbf{1}$.

- Ley de Fourier de la conducción del calor: $\mathbf{q}_{k}=-k_{k} \nabla^{\prime} T_{k}$.

Donde $\mathbf{D}_{k}$ es el tensor de rapidez de deformación, definido por: $\mathbf{D}_{k} \doteq \frac{1}{2}\left[\nabla^{\prime} \mathbf{v}_{k}+\left(\nabla^{\prime} \mathbf{v}_{k}\right)^{T}\right]$.

Físicamente, esto significa que los esfuerzos viscosos realizan un trabajo de resistencia a los movimientos del fluido y que los flujos de calor sólo se dan en la dirección de menor temperatura; es decir, en la dirección contraria a la del gradiente de temperatura.

\subsection{Descripción en el nivel promediado}

\subsubsection{Ecuación para la energía interna y energía cinética turbulenta}

Para obtener esta ecuación, restamos la ecuación de la energía cinética promedio Ec. (1.50) de la ecuación de la energía total Ec. (1.49).

$$
\begin{aligned}
& \varepsilon_{k}\left\langle\rho_{k}\right\rangle^{k} \frac{D_{k}}{D t}\left(\left\{U_{k}\right\}^{k}+K_{k}^{\mathrm{Re}}\right)-\varepsilon_{k}\left(\left\langle\boldsymbol{\Pi}_{k}\right\rangle^{k}+\boldsymbol{\Pi}_{k}^{\mathrm{Re}}\right): \nabla\left\{\mathbf{v}_{k}\right\}^{k} \\
& -W_{k i}+\mathbf{M}_{k} \cdot\left\{\mathbf{v}_{k}\right\}^{k}-\varepsilon_{k}\left\langle Q_{k}\right\rangle^{k}-E_{k}+\nabla \cdot\left[\varepsilon_{k}\left(\left\langle\mathbf{q}_{k}\right\rangle^{k}+\mathbf{q}_{k}^{\mathrm{Re}}\right)\right] \\
& -\Gamma_{k}\left[\left(U_{k i}-\left\{U_{k}\right\}^{k}\right)+\frac{1}{2}\left(\mathbf{v}_{k} \cdot \mathbf{v}_{k}\right)_{i}-\left\{\mathbf{v}_{k}\right\}^{k} \cdot \mathbf{v}_{k i}+\frac{1}{2}\left(\left\{\mathbf{v}_{k}\right\}^{k} \cdot\left\{\mathbf{v}_{k}\right\}^{k}\right)-K_{k}^{\mathrm{Re}}\right]=0 .
\end{aligned}
$$

\subsubsection{Ecuación para la energía cinética turbulenta}

La ecuación para la energía cinética turbulenta puede obtenerse al restar la ecuación de la energía interna Ec. (C.21) de la Ec. (2.7), para obtener: 


$$
\begin{aligned}
& \varepsilon_{k}\left\langle\rho_{k}\right\rangle^{k} \frac{D_{k}}{D t} K_{k}^{\mathrm{Re}}-\varepsilon_{k}\left(\left\langle\boldsymbol{\Pi}_{k}\right\rangle^{k}+\boldsymbol{\Pi}_{k}^{\mathrm{Re}}\right): \nabla\left\{\mathbf{v}_{k}\right\}^{k} \\
& -W_{k i}+\mathbf{M}_{k} \cdot\left\{\mathbf{v}_{k}\right\}^{k}+\nabla \cdot\left[\varepsilon_{k}\left(\mathbf{q}_{k}^{p}+\mathbf{q}_{k}^{t}+\mathbf{q}_{k}^{K}\right)\right]+\left\langle X_{k} \boldsymbol{\Pi}_{k}: \nabla^{\prime} \mathbf{v}_{k}\right\rangle \\
& -\Gamma_{k}\left[\frac{1}{2}\left(\mathbf{v}_{k} \cdot \mathbf{v}_{k}\right)_{i}-\left\{\mathbf{v}_{k}\right\}^{k} \cdot \mathbf{v}_{k i}+\frac{1}{2}\left(\left\{\mathbf{v}_{k}\right\}^{k} \cdot\left\{\mathbf{v}_{k}\right\}^{k}\right)-K_{k}^{\mathrm{Re}}\right]=0 .
\end{aligned}
$$

Esta ecuación puede expresarse de otra manera si reescribimos el término $\left\langle X_{k} \boldsymbol{\Pi}_{k}: \nabla^{\prime} \mathbf{v}_{k}\right\rangle$ al descomponer el campo de velocidad, $\mathbf{v}_{k} \doteq\left\{\mathbf{v}_{k}\right\}^{k}+\tilde{\mathbf{v}}_{k}$ :

$$
\begin{aligned}
\left\langle X_{k} \boldsymbol{\Pi}_{k}: \nabla^{\prime} \mathbf{v}_{k}\right\rangle & =\left\langle X_{k} \boldsymbol{\Pi}_{k}: \nabla^{\prime}\left(\left\{\mathbf{v}_{k}\right\}^{k}+\tilde{\mathbf{v}}_{k}\right)\right\rangle \\
& =\left\langle X_{k} \boldsymbol{\Pi}_{k}: \nabla\left\{\mathbf{v}_{k}\right\}^{k}\right\rangle+\left\langle X_{k} \boldsymbol{\Pi}_{k}: \nabla^{\prime} \tilde{\mathbf{v}}_{k}\right\rangle \\
& =\left\langle X_{k} \boldsymbol{\Pi}_{k}\right\rangle: \nabla\left\{\mathbf{v}_{k}\right\}^{k}+\left\langle X_{k} \boldsymbol{\Pi}_{k}: \nabla^{\prime} \tilde{\mathbf{v}}_{k}\right\rangle \\
& =\varepsilon_{k}\left\langle\boldsymbol{\Pi}_{k}\right\rangle^{k}: \nabla\left\{\mathbf{v}_{k}\right\}^{k}+\left\langle X_{k} \boldsymbol{\Pi}_{k}: \nabla^{\prime} \tilde{\mathbf{v}}_{k}\right\rangle .
\end{aligned}
$$

Introduciendo esta descomposición en la Ec. (2.8)

$$
\begin{aligned}
& \varepsilon_{k}\left\langle\rho_{k}\right\rangle^{k} \frac{D_{k}}{D t} K_{k}^{\mathrm{Re}}-\varepsilon_{k}\left(\left\langle\boldsymbol{\Pi}_{k}\right\rangle^{k}+\boldsymbol{\Pi}_{k}^{\mathrm{Re}}\right): \nabla\left\{\mathbf{v}_{k}\right\}^{k} \\
& -W_{k i}+\mathbf{M}_{k} \cdot\left\{\mathbf{v}_{k}\right\}^{k}+\nabla \cdot\left[\varepsilon_{k}\left(\mathbf{q}_{k}^{p}+\mathbf{q}_{k}^{t}+\mathbf{q}_{k}^{K}\right)\right]+\varepsilon_{k}\left\langle\boldsymbol{\Pi}_{k}\right\rangle^{k}: \nabla\left\{\mathbf{v}_{k}\right\}^{k}+\left\langle X_{k} \boldsymbol{\Pi}_{k}: \nabla^{\prime} \tilde{\mathbf{v}}_{k}\right\rangle \\
- & \Gamma_{k}\left[\frac{1}{2}\left(\mathbf{v}_{k} \cdot \mathbf{v}_{k}\right)_{i}-\left\{\mathbf{v}_{k}\right\}^{k} \cdot \mathbf{v}_{k i}+\frac{1}{2}\left(\left\{\mathbf{v}_{k}\right\}^{k} \cdot\left\{\mathbf{v}_{k}\right\}^{k}\right)-K_{k}^{\mathrm{Re}}\right]=0 .
\end{aligned}
$$

Analizando el término $\left\langle X_{k} \boldsymbol{\Pi}_{k}: \nabla^{\prime} \tilde{\mathbf{v}}_{k}\right\rangle$ :

$$
\begin{aligned}
\left\langle X_{k} \boldsymbol{\Pi}_{k}: \nabla^{\prime} \tilde{\mathbf{v}}_{k}\right\rangle & =\left\langle X_{k} \nabla^{\prime} \cdot\left(\boldsymbol{\Pi}_{k} \cdot \tilde{\mathbf{v}}_{k}\right)\right\rangle-\left\langle X_{k} \tilde{\mathbf{v}}_{k} \cdot\left(\nabla^{\prime} \cdot \boldsymbol{\Pi}_{k}\right)\right\rangle \\
& =\left\langle\nabla^{\prime} \cdot\left(X_{k} \boldsymbol{\Pi}_{k} \cdot \tilde{\mathbf{v}}_{k}\right)\right\rangle-\left\langle\nabla^{\prime} X_{k} \cdot\left(\boldsymbol{\Pi}_{k} \cdot \tilde{\mathbf{v}}_{k}\right)\right\rangle-\left\langle X_{k} \tilde{\mathbf{v}}_{k} \cdot\left(\nabla^{\prime} \cdot \boldsymbol{\Pi}_{k}\right)\right\rangle \\
& =\nabla \cdot\left\langle X_{k} \boldsymbol{\Pi}_{k} \cdot \tilde{\mathbf{v}}_{k}\right\rangle-\left\langle\nabla^{\prime} X_{k} \cdot\left[\boldsymbol{\Pi}_{k} \cdot\left(\mathbf{v}_{k}-\left\{\mathbf{v}_{k}\right\}^{k}\right)\right]\right\rangle-\left\langle X_{k} \tilde{\mathbf{v}}_{k} \cdot\left(\nabla^{\prime} \cdot \boldsymbol{\Pi}_{k}\right)\right\rangle \\
& =\nabla \cdot\left\langle X_{k} \boldsymbol{\Pi}_{k} \cdot \tilde{\mathbf{v}}_{k}\right\rangle-\left\langle\nabla^{\prime} X_{k} \cdot \boldsymbol{\Pi}_{k} \cdot \mathbf{v}_{k}\right\rangle+\left\langle\nabla^{\prime} X_{k} \cdot \boldsymbol{\Pi}_{k} \cdot\left\{\mathbf{v}_{k}\right\}^{k}\right\rangle-\left\langle X_{k} \tilde{\mathbf{v}}_{k} \cdot\left(\nabla^{\prime} \cdot \boldsymbol{\Pi}_{k}\right)\right\rangle
\end{aligned}
$$

por lo tanto,

$$
\begin{aligned}
\left\langle X_{k} \boldsymbol{\Pi}_{k}: \nabla^{\prime} \tilde{\mathbf{v}}_{k}\right\rangle= & \nabla \cdot\left\langle X_{k} \boldsymbol{\Pi}_{k} \cdot \tilde{\mathbf{v}}_{k}\right\rangle-\left\langle\boldsymbol{\Pi}_{k}: \mathbf{v}_{k} \nabla^{\prime} X_{k}\right\rangle \\
& +\left\langle\nabla^{\prime} X_{k} \cdot \boldsymbol{\Pi}_{k}\right\rangle \cdot\left\{\mathbf{v}_{k}\right\}^{k}-\left\langle X_{k} \tilde{\mathbf{v}}_{k} \cdot\left(\nabla^{\prime} \cdot \boldsymbol{\Pi}_{k}\right)\right\rangle .
\end{aligned}
$$


El argumento de la divergencia en el primer término puede reescribirse con ayuda de la descomposición del tensor de presiones y de las definiciones de $\mathbf{q}^{p}$ y $\mathbf{q}^{t}$, como:

$$
\left\langle X_{k} \boldsymbol{\Pi}_{k} \cdot \tilde{\mathbf{v}}_{k}\right\rangle=\left\langle X_{k}\left(-P_{k} \mathbf{1}-\boldsymbol{\tau}_{k}\right) \cdot \tilde{\mathbf{v}}_{k}\right\rangle=-\left\langle X_{k} P_{k} \mathbf{1} \cdot \tilde{\mathbf{v}}_{k}\right\rangle-\left\langle X_{k} \boldsymbol{\tau}_{k} \cdot \tilde{\mathbf{v}}_{k}\right\rangle=-\varepsilon_{k}\left(\mathbf{q}^{p}+\mathbf{q}^{t}\right)
$$

y utilizando las definiciones dadas anteriormente:

$$
\begin{aligned}
& W_{k i} \doteq-\left\langle\boldsymbol{\Pi}_{k}: \mathbf{v}_{k} \nabla^{\prime} X_{k}\right\rangle \\
& \mathbf{M}_{k} \doteq-\left\langle\boldsymbol{\Pi}_{k} \cdot \nabla^{\prime} X_{k}\right\rangle .
\end{aligned}
$$

Sustituyendo la Ec. (2.13) y la Ec. (2.14) en la Ec. (2.12) y el resultado en la Ec. (2.10), se obtiene:

$$
\begin{aligned}
\varepsilon_{k}\left\langle\rho_{k}\right\rangle^{k} \frac{D_{k}}{D t} K_{k}^{\mathrm{Re}} & -\varepsilon_{k} \boldsymbol{\Pi}_{k}^{\mathrm{Re}}: \nabla\left\{\mathbf{v}_{k}\right\}^{k}+\nabla \cdot\left(\varepsilon_{k} \mathbf{q}_{k}^{K}\right)-\left\langle X_{k} \tilde{\mathbf{v}}_{k} \cdot\left(\nabla^{\prime} \cdot \boldsymbol{\Pi}_{k}\right)\right\rangle \\
& -\Gamma_{k}\left[\frac{1}{2}\left(\mathbf{v}_{k} \cdot \mathbf{v}_{k}\right)_{i}-\left\{\mathbf{v}_{k}\right\}^{k} \cdot \mathbf{v}_{k i}+\frac{1}{2}\left(\left\{\mathbf{v}_{k}\right\}^{k} \cdot\left\{\mathbf{v}_{k}\right\}^{k}\right)-K_{k}^{\mathrm{Re}}\right]=0
\end{aligned}
$$

Que es otra forma de la ecuación de la energía cinética turbulenta.

\subsection{Relación de Gibbs}

Sabemos que en general no tenemos una ecuación de estado simple en términos de variables promediadas (Ishii, 1975). La relación entre la energía interna, la entropía y la densidad está influenciada tanto por la transferencia interfacial como por los efectos de las desviaciones de las variables.

Expresaremos la ecuación fundamental de la termodinámica; esto es, la energía interna como una función de la entropía y de la densidad:

$$
U_{k}\left(S_{k}, \rho_{k}\right)=U_{k}\left(\left\{S_{k}\right\}^{k}+\tilde{S}_{k},\left\langle\rho_{k}\right\rangle^{k}+\rho_{k}^{\prime}\right),
$$

desarrollando el miembro derecho mediante una serie de Taylor (conservando solamente los términos de primer orden en las desviaciones) alrededor del punto $\left\{S_{k}\right\}^{k} \mathrm{y}\left\langle\rho_{k}\right\rangle^{k}$ : 


$$
\begin{aligned}
U_{k}\left(S_{k}, \rho_{k}\right)=U_{k}\left(\left\{S_{k}\right\}^{k},\left\langle\rho_{k}\right\rangle^{k}\right) & +\left[\left(\frac{\partial U_{k}}{\partial S_{k}}\right)_{\rho_{k}}\right]_{\left\{S_{k}\right\}^{k},\left\langle\rho_{k}\right\}^{k}}\left(S_{k}-\left\{S_{k}\right\}^{k}\right) \\
+ & {\left[\left(\frac{\partial U_{k}}{\partial \rho_{k}}\right)_{S_{k}}\right]_{\left\{S_{k}\right\}^{k},\left\langle\rho_{k}\right\rangle^{k}}\left(\rho_{k}-\left\langle\rho_{k}\right\rangle^{k}\right)+\cdots }
\end{aligned}
$$

o bien,

$$
U_{k}\left(S_{k}, \rho_{k}\right)=U_{k}\left(\left\{S_{k}\right\}^{k},\left\langle\rho_{k}\right\rangle^{k}\right)+\left[\left(\frac{\partial U_{k}}{\partial S_{k}}\right)_{\rho_{k}}\right]_{\left\{S_{k}\right\}^{k},\left\langle\rho_{k}\right\rangle^{k}} \tilde{S}_{k}+\left[\left(\frac{\partial U_{k}}{\partial \rho_{k}}\right)_{S_{k}}\right]_{\left\{S_{k}\right\}^{k},\left\langle\rho_{k}\right\rangle^{k}} \rho_{k}^{\prime}+\cdots
$$

introduciendo las definiciones de temperatura y presión termodinámicas (Callen, 1985):

$$
\begin{aligned}
& \left(\frac{\partial U_{k}}{\partial S_{k}}\right)_{\rho_{k}} \doteq T_{k} \\
& \left(\frac{\partial U_{k}}{\partial \rho_{k}}\right)_{S_{k}} \doteq \frac{P_{k}}{\rho_{k}^{2}},
\end{aligned}
$$

obtenemos

$$
U_{k}\left(S_{k}, \rho_{k}\right)=U_{k}\left(\left\{S_{k}\right\}^{k},\left\langle\rho_{k}\right\rangle^{k}\right)+\left[T_{k}\right]_{\left\{S_{k}\right\}^{k},\left\langle\rho_{k}\right\rangle^{k}} \tilde{S}_{k}+\left[\frac{P_{k}}{\rho_{k}^{2}}\right]_{\left\{S_{k}\right\}^{k},\left\langle\rho_{k}\right\}^{k}} \rho_{k}^{\prime}+\cdots .
$$

Puesto que las desviaciones de las propiedades termodinámicas son pequeñas en relación a su valor promedio, el producto de las mismas es aun más pequeño. Por lo tanto, podemos truncar la serie a primer orden en las desviaciones y tomar el promedio ponderado en la masa para obtener:

$$
\left\{U_{k}\left(S_{k}, \rho_{k}\right)\right\}^{k} \approx U_{k}\left(\left\{S_{k}\right\}^{k},\left\langle\rho_{k}\right\rangle^{k}\right)\{1\}^{k}+\left\{\left[T_{k}\right]_{\left\{S_{k}\right\}^{k},\left\langle\rho_{k}\right\rangle^{k}} \tilde{S}_{k}\right\}^{k}+\left\{\left[\frac{P_{k}}{\rho_{k}^{2}}\right]_{\left\{S_{k}\right\}^{k},\left\langle\rho_{k}\right\rangle^{k}} \rho_{k}^{\prime}\right\}^{k}
$$

sacando de las integrales las cantidades evaluadas en cantidades promedio:

$$
\begin{aligned}
\left\{U_{k}\left(S_{k}, \rho_{k}\right)\right\}^{k} & \approx U_{k}\left(\left\{S_{k}\right\}^{k},\left\langle\rho_{k}\right\rangle^{k}\right)+T_{k s}\left\{\tilde{S}_{k}\right\}^{k}+\frac{P_{k s}}{\left\langle\rho_{k}\right\rangle^{k}\left\langle\rho_{k}\right\rangle^{k}}\left\{\rho_{k}^{\prime}\right\}^{k} \\
& \approx U_{k}\left(\left\{S_{k}\right\}^{k},\left\langle\rho_{k}\right\rangle^{k}\right)+\frac{P_{k s}}{\left\langle\rho_{k}\right\rangle^{k}\left\langle\rho_{k}\right\rangle^{k}}\left\{\rho_{k}^{\prime}\right\}^{k}
\end{aligned}
$$


donde $T_{k s}$ y $P_{k s}$ están evaluadas a entropía y densidad promedio. Por otro lado, en la Sección 1.5 hemos dado los argumentos de escala necesarios para que en la Ec. (1.29): $\left\{\rho_{k}^{\prime}\right\}^{k}=0$. Por lo que finalmente encontramos que:

$$
\left\{U_{k}\right\}^{k} \sim U_{k}\left(\left\{S_{k}\right\}^{k},\left\langle\rho_{k}\right\rangle^{k}\right)=U_{k s} .
$$

Resulta entonces que es válida una relación simple, pero no igual a la de equilibrio entre estas variables promediadas si las desviaciones son suficientemente pequeñas para que los cambios macroscópicos de estas variables y así su expansión lineal de la ecuación de estado es una buena aproximación.

Tomando la derivada material de la Ec. (2.24) y utilizando la Ec. (1.47) se obtiene

$$
\frac{D_{k}}{D t}\left\{U_{k}\right\}^{k} \sim T_{k s} \frac{D_{k}}{D t}\left\{S_{k}\right\}^{k}+\frac{P_{k s}}{\left\langle\rho_{k}\right\rangle^{k}\left\langle\rho_{k}\right\rangle^{k}} \frac{D_{k}}{D t}\left\langle\rho_{k}\right\rangle^{k} .
$$

La descripción en términos de promedios espacio-temporales que aquí se presenta, permite obtener una ecuación de estado donde se incorporen correcciones a primer orden en las desviaciones de las variables termodinámicas [Ec. (2.23)] o a órdenes más elevados. La Ec. (2.23) es la ecuación fundamental de la termodinámica (Callen, 1985), en términos de variables de estado promediadas; por lo tanto, apartir de ella podemos obtener toda la información termodinámica del sistema; esto es: la capacidad calorífica a volumen constante, la capacidad calorífica a presión constante, la compresibilidad isotérmica e isentrópica, la expansión volumétrica, entre otras. No obstante, aunque es posible obtener la ecuación de estado con términos adicionales por la corrección a primer orden en las desviaciones de las variables de estado, en este primer estudio no se considerará y se utilizará la ecuación de estado del gas ideal. Dejando la descripción del comportamiento del gas en términos de descripciones termostáticas más complejas que la del gas ideal para estudios posteriores. 
2.5 Restricción a escala promedio de la segunda ley de la termodinámica para modelos de dos fluidos (energias: térmica y cinética turbulenta)

\subsubsection{Ecuación de balance de entropía}

La ecuación de campo promedio del balance de entropía viene dada por la Ec. (C.32):

$$
\begin{array}{r}
\varepsilon_{k}\left\langle\rho_{k}\right\rangle^{k} \frac{D_{k}}{D t}\left\{S_{k}\right\}^{k}+\nabla \cdot\left(\varepsilon_{k}\left\langle\frac{\mathbf{q}_{k}}{T_{k}}\right\rangle^{k}\right)+\nabla \cdot\left(\varepsilon_{k}\left\langle\rho_{k}\right\rangle^{k}\left\{\tilde{S} \tilde{\mathbf{v}}_{k}\right\}^{k}\right)-\left\langle X_{k} \frac{Q_{k}}{T_{k}}\right\rangle= \\
\left\langle\frac{\mathbf{q}_{k}}{T_{k}} \cdot \nabla^{\prime} X_{k}\right\rangle+\Gamma_{k}\left(S_{k i}-\left\{S_{k}\right\}^{k}\right)+\left\langle X_{k} \Delta_{k}\right\rangle .
\end{array}
$$

Donde sabemos de la Ec. (2.5) que:

$$
\left\langle X_{k} \Delta_{k}\right\rangle=\left\langle-X_{k}\left(\frac{1}{T_{k}}\right) \boldsymbol{\tau}_{k}: \nabla^{\prime} \mathbf{v}_{k}\right\rangle+\left\langle X_{k} \mathbf{q}_{k} \cdot \nabla^{\prime}\left(\frac{1}{T_{k}}\right)\right\rangle \geq 0 .
$$

Sustituyendo $D_{k}\left\{S_{k}\right\}^{k} / D t$ de la Ec. (2.26) en la Ec. (2.25) y el resultado en la Ec. (2.7), obtenemos la ecuación para la entropía promedio:

$$
\begin{aligned}
& \varepsilon_{k}\left(\left\langle\boldsymbol{\Pi}_{k}\right\rangle^{k}+\boldsymbol{\Pi}_{k}^{\mathrm{Re}}\right): \nabla\left\{\mathbf{v}_{k}\right\}^{k}+W_{k i}-\mathbf{M}_{k} \cdot\left\{\mathbf{v}_{k}\right\}^{k}+T_{k s} \nabla \cdot\left(\varepsilon_{k}\left\langle\frac{\mathbf{q}_{k}}{T_{k}}\right\rangle^{k}\right) \\
& -\nabla \cdot\left[\varepsilon_{k}\left(\left\langle\mathbf{q}_{k}\right\rangle^{k}+\mathbf{q}_{k}^{\mathrm{Re}}\right)\right]+T_{k s} \nabla \cdot\left(\varepsilon_{k}\left\langle\rho_{k}\right\rangle^{k}\left\{\tilde{S}_{k} \tilde{\mathbf{v}}_{k}\right\}^{k}\right) \\
& +\varepsilon_{k}\left\langle Q_{k}\right\rangle^{k}-T_{k s}\left\langle X_{k} \frac{Q_{k}}{T_{k}}\right\rangle+\left\langle\mathbf{q}_{k} \cdot \nabla^{\prime} X_{k}\right\rangle-T_{k s}\left\langle\frac{\mathbf{q}_{k}}{T_{k}} \cdot \nabla^{\prime} X_{k}\right\rangle \\
& -\varepsilon_{k} \frac{P_{k s}}{\left\langle\rho_{k}\right\rangle^{k}} \frac{D_{k}}{D t}\left\langle\rho_{k}\right\rangle^{k}-\varepsilon_{k}\left\langle\rho_{k}\right\rangle^{k} \frac{D_{k}}{D t} K_{k}^{\mathrm{Re}} \\
& +\Gamma_{k}\left[\begin{array}{c}
U_{k i}-\left\{U_{k}\right\}^{k}-T_{k s}\left(S_{k i}-\left\{S_{k}\right\}^{k}\right) \\
-K_{k}^{\mathrm{Re}}+\frac{1}{2}\left(\mathbf{v}_{k} \cdot \mathbf{v}_{k}\right)_{i}-\left\{\mathbf{v}_{k}\right\}^{k} \cdot \mathbf{v}_{k i}+\frac{1}{2}\left(\left\{\mathbf{v}_{k}\right\}^{k} \cdot\left\{\mathbf{v}_{k}\right\}^{k}\right)
\end{array}\right]= \\
& T_{k s}\left\langle X_{k} \Delta_{k}\right\rangle \geq 0 \text {. }
\end{aligned}
$$

Donde hemos reintroducido la definición del transporte de calor interfacial $E_{k} \doteq\left\langle\mathbf{q}_{k} \cdot \nabla^{\prime} X_{k}\right\rangle$. 
Conviene ahora considerar el agrupamiento de algunos términos y la disociación de otros. Si se utiliza la definición del tensor de esfuerzos: $\boldsymbol{\Pi}_{k}=-P_{k} \mathbf{1}-\boldsymbol{\tau}_{k}$, el trabajo interfacial puede reescribirse al sumar y restar en el miembro derecho las cantidades: $\left\langle\nabla^{\prime} X_{k} \cdot\left(P_{k} \mathbf{1}+\boldsymbol{\tau}_{k}\right) \cdot\left\langle\mathbf{v}_{k}\right\rangle_{i}\right\rangle$ y $\left\langle\nabla^{\prime} X_{k} \cdot\left(\left\langle P_{k}\right\rangle_{i} \mathbf{1}+\left\langle\boldsymbol{\tau}_{k}\right\rangle_{i}\right) \cdot\left\langle\mathbf{v}_{k}\right\rangle_{i}\right\rangle$, como

$$
\begin{aligned}
W_{k i}= & \left\langle\nabla^{\prime} X_{k} \cdot\left(\boldsymbol{\tau}_{k}+P_{k} \mathbf{1}\right) \cdot \mathbf{v}_{k}\right\rangle-\left\langle\nabla^{\prime} X_{k} \cdot\left(P_{k} \mathbf{1}+\boldsymbol{\tau}_{k}\right) \cdot\left\langle\mathbf{v}_{k}\right\rangle_{i}\right\rangle \\
& +\left\langle\nabla^{\prime} X_{k} \cdot\left(P_{k} \mathbf{1}+\boldsymbol{\tau}_{k}\right) \cdot\left\langle\mathbf{v}_{k}\right\rangle_{i}\right\rangle-\left\langle\nabla^{\prime} X_{k} \cdot\left(\left\langle P_{k}\right\rangle_{i} \mathbf{1}+\left\langle\boldsymbol{\tau}_{k}\right\rangle_{i}\right) \cdot\left\langle\mathbf{v}_{k}\right\rangle_{i}\right\rangle \\
& +\left\langle\nabla^{\prime} X_{k} \cdot\left(\left\langle P_{k}\right\rangle_{i} \mathbf{1}+\left\langle\boldsymbol{\tau}_{k}\right\rangle_{i}\right) \cdot\left\langle\mathbf{v}_{k}\right\rangle_{i}\right\rangle
\end{aligned}
$$

por lo tanto,

$$
W_{k i}=\nabla \varepsilon_{k} \cdot\left(\left\langle P_{k}\right\rangle_{i} \mathbf{1}+\left\langle\boldsymbol{\tau}_{k}\right\rangle_{i}\right) \cdot\left\langle\mathbf{v}_{k}\right\rangle_{i}+W_{k i}^{\prime \prime}+\left(\mathbf{M}_{k p}+\mathbf{M}_{k t}\right) \cdot\left\langle\mathbf{v}_{k}\right\rangle_{i}
$$

donde:

$\mathbf{M}_{k p} \doteq\left\langle\nabla^{\prime} X_{k} \cdot\left(P_{k}-\left\langle P_{k}\right\rangle_{i}\right) \mathbf{1}\right\rangle$, es la fuerza de presión interfacial $\mathbf{M}_{k t} \doteq\left\langle\nabla^{\prime} X_{k} \cdot\left(\boldsymbol{\tau}_{k}-\left\langle\boldsymbol{\tau}_{k}\right\rangle_{i}\right)\right\rangle$, es la fuerza debida a los esfuerzos de corte interfaciales $\mathrm{y}$ $W_{k i}^{\prime \prime} \doteq\left\langle\nabla^{\prime} X_{k} \cdot\left(P_{k} \mathbf{1}+\boldsymbol{\tau}_{k}\right) \cdot\left(\mathbf{v}_{k}-\left\langle\mathbf{v}_{k}\right\rangle_{i}\right)\right\rangle$, es el término de trabajo "extra" interfacial.

Similarmente, el término de transporte de cantidad de movimiento interfacial $\mathbf{M}_{k} \doteq-\left\langle\boldsymbol{\Pi}_{k} \cdot \nabla^{\prime} X_{k}\right\rangle$ puede expresarse al sumar y restar el término $\left\langle\left(\left\langle P_{k}\right\rangle_{i} \mathbf{1}+\left\langle\boldsymbol{\tau}_{k}\right\rangle_{i}\right) \cdot \nabla^{\prime} X_{k}\right\rangle$, como

$$
\mathbf{M}_{k}=\left(\left\langle P_{k}\right\rangle_{i} \mathbf{1}+\left\langle\boldsymbol{\tau}_{k}\right\rangle_{i}\right) \cdot \nabla \varepsilon_{k}+\left\langle\left(P_{k} \mathbf{1}-\left\langle P_{k}\right\rangle_{i} \mathbf{1}\right) \cdot \nabla^{\prime} X_{k}\right\rangle+\left\langle\left(\boldsymbol{\tau}_{k}-\left\langle\boldsymbol{\tau}_{k}\right\rangle_{i}\right) \cdot \nabla^{\prime} X_{k}\right\rangle
$$

o bien,

$$
\mathbf{M}_{k}=\mathbf{M}_{k p}+\mathbf{M}_{k t}+\left(\left\langle P_{k}\right\rangle_{i} \mathbf{1}+\left\langle\boldsymbol{\tau}_{k}\right\rangle_{i}\right) \cdot \nabla \varepsilon_{k}
$$

El trabajo interfacial $W_{k i}$ no es objetivo, mientras que en esta disociación, los términos $\mathbf{M}_{k}$ y $W_{k i}^{\prime \prime}$ son objetivos. 
Por otro lado, la Ec. (2.28) contiene todavía un término de entropía: $S_{k i}-\left\{S_{k}\right\}^{k}$, el cual trabajaremos apoyándonos en la Ec. (2.21) y en el hecho que hemos demostrado al promediar la Ec. (2.21); es decir, la Ec. (2.24), por lo que podemos expresar lo siguiente:

$$
U_{k}-\left\{U_{k}\right\}^{k}-\left(S_{k}-\left\{S_{k}\right\}^{k}\right) T_{k s} \approx \frac{\rho_{k}-\left\langle\rho_{k}\right\rangle^{k}}{\left\langle\rho_{k}\right\rangle^{k}} \frac{P_{k s}}{\left\langle\rho_{k}\right\rangle^{k}} .
$$

Multiplicando ambos lados por $\rho_{k}\left(\mathbf{v}_{k}-\mathbf{w}_{k \ell}\right) \cdot \nabla^{\prime} X_{k}$ y promediando:

$$
\begin{aligned}
\left\langle\rho_{k}\left(\mathbf{v}_{k}-\mathbf{w}_{k \ell}\right) \cdot \nabla^{\prime} X_{k}\left(U_{k}-\left\{U_{k}\right\}^{k}\right)\right\rangle & -\left\langle\rho_{k}\left(\mathbf{v}_{k}-\mathbf{w}_{k \ell}\right) \cdot \nabla^{\prime} X_{k}\left(S_{k}-\left\{S_{k}\right\}^{k}\right) T_{k s}\right\rangle \approx \\
& \left\langle\rho_{k}\left(\mathbf{v}_{k}-\mathbf{w}_{k \ell}\right) \cdot \nabla^{\prime} X_{k} \frac{\rho_{k}-\left\langle\rho_{k}\right\rangle^{k}}{\left\langle\rho_{k}\right\rangle^{k}} \frac{P_{k s}}{\left\langle\rho_{k}\right\rangle^{k}}\right\rangle
\end{aligned}
$$

de otra forma:

$$
\Gamma_{k}\left(U_{k i}-\left\{U_{k}\right\}^{k}\right)-\Gamma_{k}\left(S_{k i}-\left\{S_{k}\right\}^{k}\right) T_{k s} \approx \Gamma_{k} \frac{\rho_{k i}-\left\langle\rho_{k}\right\rangle^{k}}{\left\langle\rho_{k}\right\rangle^{k}} \frac{P_{k s}}{\left\langle\rho_{k}\right\rangle^{k}}
$$

o bien

$$
U_{k i}-\left\{U_{k}\right\}^{k}-\left(S_{k i}-\left\{S_{k}\right\}^{k}\right) T_{k s} \approx \frac{\rho_{k i}-\left\langle\rho_{k}\right\rangle^{k}}{\left\langle\rho_{k}\right\rangle^{k}} \frac{P_{k s}}{\left\langle\rho_{k}\right\rangle^{k}}
$$

Sustituyendo las Ecs. (2.30), (2.32) y (2.36) en la Ec. (2.28), así como también la definición del tensor de esfuerzos: $\boldsymbol{\Pi}_{k}=-P_{k} \mathbf{1}-\boldsymbol{\tau}_{k}$, obtenemos:

$$
\begin{aligned}
& \varepsilon_{k}\left(P_{k s}-\left\langle P_{k}\right\rangle^{k}\right) \operatorname{tr}\left\{\mathbf{D}_{k}\right\}^{k}+\varepsilon_{k}\left(-\left\langle\boldsymbol{\tau}_{k}\right\rangle^{k}+\mathbf{\Pi}_{k}^{\mathrm{Re}}\right):\left\{\mathbf{D}_{k}\right\}^{k} \\
& +\left(\mathbf{M}_{k p}+\mathbf{M}_{k t}\right) \cdot\left(\left\langle\mathbf{v}_{k}\right\rangle_{i}-\left\{\mathbf{v}_{k}\right\}^{k}\right)+W_{k i}^{\prime \prime}+\left(\left\langle\mathbf{v}_{k}\right\rangle_{i}-\left\{\mathbf{v}_{k}\right\}^{k}\right) \cdot\left(\left\langle P_{k}\right\rangle_{i} \mathbf{1}+\left\langle\boldsymbol{\tau}_{k}\right\rangle_{i}\right) \cdot \nabla \varepsilon_{k} \\
& +T_{k s} \nabla \cdot\left(\varepsilon_{k}\left\langle\frac{\mathbf{q}_{k}}{T_{k}}\right\rangle^{k}\right)+T_{k s} \nabla \cdot\left(\varepsilon_{k}\left\langle\rho_{k}\right\rangle^{k}\left\{\tilde{S}_{k} \tilde{\mathbf{v}}_{k}\right\}^{k}\right) \\
& -\nabla \cdot\left[\varepsilon_{k}\left(\left\langle\mathbf{q}_{k}\right\rangle^{k}+\mathbf{q}_{k}^{\mathrm{Re}}\right)\right]+\varepsilon_{k}\left\langle Q_{k}\right\rangle^{k}-T_{k s}\left\langle X_{k} \frac{Q_{k}}{T_{k}}\right\rangle \\
& +\left\langle\mathbf{q}_{k} \cdot \nabla^{\prime} X_{k}\right\rangle-T_{k s}\left\langle\frac{\mathbf{q}_{k}}{T_{k}} \cdot \nabla^{\prime} X_{k}\right\rangle-\varepsilon_{k}\left\langle\rho_{k}\right\rangle^{k} \frac{D_{k}}{D t} K_{k}^{\mathrm{Re}}+P_{k s} \frac{D_{k}}{D t} \varepsilon_{k} \\
& +\Gamma_{k}\left[\frac{\rho_{k i}-2\left\langle\rho_{k}\right\rangle^{k}}{\left\langle\rho_{k}\right\rangle^{k}\left\langle\rho_{k}\right\rangle^{k}} P_{k s}-K_{k}^{\mathrm{Re}}+\frac{1}{2}\left(\mathbf{v}_{k} \cdot \mathbf{v}_{k}\right)_{i}-\left\{\mathbf{v}_{k}\right\}^{k} \cdot \mathbf{v}_{k i}+\frac{1}{2}\left(\left\{\mathbf{v}_{k}\right\}^{k} \cdot\left\{\mathbf{v}_{k}\right\}^{k}\right)\right]= \\
& T_{k s}\left\langle X_{k} \Delta_{k}\right\rangle \geq 0 .
\end{aligned}
$$


Donde, $\left\{\mathbf{D}_{k}\right\}^{k}$ es el tensor de rapidez de deformación promedio, definido por

$$
\left\{\mathbf{D}_{k}\right\}^{k} \doteq \frac{1}{2}\left[\nabla\left\{\mathbf{v}_{k}\right\}^{k}+\left(\nabla\left\{\mathbf{v}_{k}\right\}^{k}\right)^{T}\right]
$$

$\mathrm{y} \operatorname{tr}\left\{\mathbf{D}_{k}\right\}^{k}$ es su traza, que es igual a: $\operatorname{tr}\left\{\mathbf{D}_{k}\right\}^{k}=\nabla \cdot\left\{\mathbf{v}_{k}\right\}^{k}$. Al obtener esta ecuación hemos utilizado la ecuación de continuidad promediada en la forma:

$$
\frac{\varepsilon_{k}}{\left\langle\rho_{k}\right\rangle^{k}} \frac{D_{k}}{D t}\left\langle\rho_{k}\right\rangle^{k}=\frac{\Gamma_{k}}{\left\langle\rho_{k}\right\rangle^{k}}-\varepsilon_{k} \nabla \cdot\left\{\mathbf{v}_{k}\right\}^{k}-\frac{D_{k}}{D t} \varepsilon_{k} .
$$

Bajo condiciones de flujo normales, se espera que las desviaciones en el estado del fluido sean relativamente pequeñas; i.e. la desviación de cualquier variable de estado dividida por el promedio de esa variable es pequeño.Las propiedades del fluidoque dependen de las variables de estado tendrán así también pequeñas desviaciones relativas. De esta manera, el producto de dos variables de estado, una variable de estado y una propiedad del fluido o dos propiedades del fluido pueden considerarse como suficientemente pequeñas para ignorarse. Sin embargo, los productos de desviaciones de la velocidad no necesariamente son pequeños. Ciertamente, una desviación instantánea en la velocidad de un fluido puede ser mayor que la velocidad promedio. Así, todos los productos de desviaciones de la velocidad se retendrán. Análogamente, los productos de desviaciones de la velocidad con desviaciones de variables de estado o del fluido también se conservarán.

Eliminando los términos de segundo orden en las desviaciones de las propiedades del fluido se puede demostrar que (Arnold, 1988; ver Apéndice $D$ ):

$$
\begin{aligned}
& P_{k s}-\left\langle P_{k}\right\rangle^{k} \approx 0 \\
& \left\{T_{k}\right\}^{k}-T_{k s} \approx 0 \\
& \left\{T_{k}\right\}^{k}-\left\langle T_{k}\right\rangle^{k} \approx 0 \\
& \left\langle X_{k} Q_{k}\right\rangle-\left\{T_{k}\right\}^{k}\left\langle X_{k} \frac{Q_{k}}{T_{k}}\right\rangle \approx 0 \\
& \left\{T_{k}\right\}^{k} \nabla \cdot\left(\varepsilon_{k}\left\langle\rho_{k}\right\rangle^{k}\left\{\tilde{S}_{k} \tilde{\mathbf{v}}_{k}\right\}^{k}\right)-\nabla \cdot\left(\varepsilon_{k} \mathbf{q}_{k}^{r}\right) \approx-\varepsilon_{k}\left\langle\rho_{k}\right\rangle^{k}\left\{\tilde{S}_{k} \tilde{\mathbf{v}}_{k}\right\}^{k} \cdot \nabla\left\{T_{k}\right\}^{k} \\
& \left\{T_{k}\right\}^{k} \nabla \cdot\left(\varepsilon_{k}\left\langle\frac{\mathbf{q}_{k}}{T_{k}}\right\rangle^{k}\right)-\nabla \cdot\left(\varepsilon_{k}\left\langle\mathbf{q}_{k}\right\rangle^{k}\right) \approx-\varepsilon_{k} \frac{\left\langle\mathbf{q}_{k}\right\rangle^{k}}{\left\{T_{k}\right\}^{k}} \cdot \nabla\left\{T_{k}\right\}^{k} \\
& \left\langle\mathbf{q}_{k} \cdot \nabla^{\prime} X_{k}\right\rangle-\left\{T_{k}\right\}^{k}\left\langle\frac{\mathbf{q}_{k}}{T_{k}} \cdot \nabla^{\prime} X_{k}\right\rangle \approx 0 .
\end{aligned}
$$


Utilizando el conjunto de ecuaciones dado por las Ec. (2.40) en la Ec. (2.37), obtenemos:

$$
\begin{aligned}
& \varepsilon_{k}\left(-\left\langle\boldsymbol{\tau}_{k}\right\rangle^{k}+\boldsymbol{\Pi}_{k}^{\mathrm{Re}}\right):\left\{\mathbf{D}_{k}\right\}^{k}+\left(\mathbf{M}_{k p}+\mathbf{M}_{k t}\right) \cdot\left(\left\langle\mathbf{v}_{k}\right\rangle_{i}-\left\{\mathbf{v}_{k}\right\}^{k}\right)+W_{k i}^{\prime \prime} \\
& +\left(\left\langle\mathbf{v}_{k}\right\rangle_{i}-\left\{\mathbf{v}_{k}\right\}^{k}\right) \cdot\left(\left\langle P_{k}\right\rangle_{i} \mathbf{1}+\left\langle\boldsymbol{\tau}_{k}\right\rangle_{i}\right) \cdot \nabla \varepsilon_{k}-\varepsilon_{k} \frac{\left\langle\mathbf{q}_{k}\right\rangle^{k}}{\left\{T_{k}\right\}^{k}} \cdot \nabla\left\{T_{k}\right\}^{k}+\left\langle P_{k}\right\rangle^{k} \frac{D_{k}}{D t} \varepsilon_{k} \\
& -\nabla \cdot\left[\varepsilon_{k}\left(\mathbf{q}_{k}^{p}+\mathbf{q}_{k}^{t}+\mathbf{q}_{k}^{K}\right)\right]-\varepsilon_{k}\left\langle\rho_{k}\right\rangle^{k}\left\{\tilde{S}_{k} \tilde{\mathbf{v}}_{k}\right\}^{k} \cdot \nabla\left\{T_{k}\right\}^{k}-\varepsilon_{k}\left\langle\rho_{k}\right\rangle^{k} \frac{D_{k}}{D t} K_{k}^{\mathrm{Re}} \\
& +\Gamma_{k}\left[\frac{\rho_{k i}-2\left\langle\rho_{k}\right\rangle^{k}}{\left\langle\rho_{k}\right\rangle^{k}} \frac{\left\langle P_{k}\right\rangle^{k}}{\left\langle\rho_{k}\right\rangle^{k}}-K_{k}^{\mathrm{Re}}+\frac{1}{2}\left(\mathbf{v}_{k} \cdot \mathbf{v}_{k}\right)_{i}-\left\{\mathbf{v}_{k}\right\}^{k} \cdot \mathbf{v}_{k i}+\frac{1}{2}\left(\left\{\mathbf{v}_{k}\right\}^{k} \cdot\left\{\mathbf{v}_{k}\right\}^{k}\right)\right]= \\
& \left\{T_{k}\right\}^{k}\left\langle X_{k} \Delta_{k}\right\rangle \geq 0 \text {. }
\end{aligned}
$$

Consideremos ahora la forma Lagrangiana de la ecuación de balance de entropía local instantánea Ec. (2.2) dada por

$$
\rho_{k} \frac{D_{k}}{D t^{\prime}} S_{k}+\nabla^{\prime} \cdot\left(\frac{\mathbf{q}_{k}}{T_{k}}\right)-\frac{Q_{k}}{T_{k}}=\Delta_{k},
$$

donde se han ubicado los términos de entropía correspondiente a la tasa de cambio, el flujo y la fuente externa, en tanto que del lado derecho se incorporan los términos de generación de entropía debidos a disipación, estos términos constituyen la fuente de entropía que cumple la segunda ley de la termodinámica Ec. (2.4):

$$
\begin{aligned}
& \Delta_{k} \geq 0 \\
& \frac{1}{T_{k}}\left(-\boldsymbol{\tau}_{k}: \nabla^{\prime} \mathbf{v}_{k}-\frac{1}{T_{k}} \mathbf{q}_{k} \cdot \nabla^{\prime} T_{k}\right) \geq 0 .
\end{aligned}
$$

Por otro lado, la Ec. (2.41) contiene cantidades promediadas en el miembro izquierdo, en tanto que del lado derecho incorpora los términos de disipación local promediados $\left\langle X_{k} \Delta_{k}\right\rangle$. Esto nos dice que debe haber transferencia de energía de la macroescala a la microescala que está en exceso; es decir, la tasa de cambio de la entropía promedio debe exceder las fuentes de entropía que dan origen a calentamiento $\left[T\left(d S / d t^{\prime}\right) \geq \dot{Q}\right.$ (calentamiento), desigualdad de Clausius-Planck], las cuales están expresadas en términos de parámetros promediados. Si todos los términos en esta ecuación están constituidos apropiadamente, entonces debemos encontrar que la ecuación de entropía resultante siempre se satisface. 
Alternativamente, si las relaciones de comportamiento no siempre satisfacen la ecuación de la entropía, entonces sabemos que la disipación (i.e. transferencia de energía a la microescala) no está siendo predecida correctamente.

En un sistema de ecuaciones de balance promediadas, la solución de la ecuación de la energía será sólo una solución válida para la partición de la energía del sistema si todos los términos dentro de la ecuación de la energía modelan en forma precisa el fenómeno deseado. Si las relaciones de comportamiento utilizadas para obtener las cerraduras para el sistema de ecuaciones no modelan en forma precisa la disipación, entonces ellos llevarán a una distribución imprecisa de la energía.

En consecuencia, con las restricciones que se obtienen de la segunda ley de la termodinámica aplicada a las ecuaciones promediadas, uno podría esperar que la incapacidad de las relaciones de cerradura de satisfacer la ecuación de la entropía implique que la disipación está siendo subestimada. Sin embargo, puede mostrarse (Arnold, 1988 y Arnold y col., 1990) que la imposibilidad de satisfacer la ecuación de la entropía puede llevar a sobreestimar o subestimar la disipación, dependiendo de la situación de flujo.

Es importante notar que la Ec. (2.41) toma en cuenta la energía cinética turbulenta recuperable y no-recuperable. En los flujos en dos fases, una de las fuentes primarias de energía cinética turbulenta es el trabajo interfacial. Estos términos de trabajo aparecen en la Ec. (2.41) como términos con la forma general, $\left\langle\boldsymbol{\Pi}_{k}: \mathbf{v}_{k} \nabla^{\prime} X_{k}\right\rangle$. Muchos de estos términos aparecen debido a que la Ec. (2.41) ha sido particionada en términos más reconocibles.

Cuando el trabajo interfacial se realiza en la fase continua, estos términos de trabajo son una fuente positiva de disipación. Sin embargo, la energía cinética turbulenta aparece en la ecuación en la forma de: $-\varepsilon_{k}\left\langle\rho_{k}\right\rangle^{k} D_{k} K_{k}^{\mathrm{Re}} / D t$, que durante la producción de energía cinética turbulenta actúa para reducir la fuente de disipación. Así podemos ver que la energía cinética turbulenta actúa para almacenar energía temporalmente. 
Si más tarde el trabajo interfacial actúa para reducir la energía cinética turbulenta, entonces aquella energía puede recuperarse en forma reversible y alguna parte aparece en realidad como disipación.

Sin embargo, la turbulencia es reducida por difusión $\left[-\nabla \cdot\left(\varepsilon_{k} \mathbf{q}_{k}^{K}\right)\right]$, la tasa de reducción de energía cinética turbulenta $\left[-\varepsilon_{k}\left\langle\rho_{k}\right\rangle^{k} D_{k} K_{k}^{\mathrm{Re}} / D t\right]$ puede resultar en disipación. Esta disipación representa el exceso de ese producto menos del que se difunde lejos.

2.6 Restricción de la segunda ley de la termodinámica para modelos de dos fluidos (energía térmica)

Combinando la ecuación de la energía interna Ec. (C.21) y la relación de Gibbs Ec. (2.25), junto con el balance de entropía Ec. (2.26), obtenemos:

$$
\begin{aligned}
\varepsilon_{k}\left\langle\rho_{k}\right\rangle^{k} T_{k s} \frac{D_{k}}{D t}\left\{S_{k}\right\}^{k} & +\varepsilon_{k} \frac{P_{k s}}{\left\langle\rho_{k}\right\rangle^{k}} \frac{D_{k}}{D t}\left\langle\rho_{k}\right\rangle^{k}+\nabla \cdot\left[\varepsilon_{k}\left(\left\langle\mathbf{q}_{k}\right\rangle^{k}+\mathbf{q}_{k}^{r}\right)\right] \\
& -\varepsilon_{k}\left\langle Q_{k}\right\rangle^{k}-\left\langle X_{k} \boldsymbol{\Pi}_{k}: \nabla^{\prime} \mathbf{v}_{k}\right\rangle=\Gamma_{k}\left(U_{k i}-\left\{U_{k}\right\}^{k}\right)+E_{k} .
\end{aligned}
$$

Expresando la velocidad local instantánea como la suma de su promedio ponderado en la masa más una desviación alrededor de este valor; esto es, $\mathbf{v}_{k} \doteq\left\{\mathbf{v}_{k}\right\}^{k}+\tilde{\mathbf{v}}_{k}$ y considerando también la definición del tensor de los esfuerzos: $\boldsymbol{\Pi}_{k}=-P_{k} \mathbf{1}-\boldsymbol{\tau}_{k}$, se obtiene:

$$
\begin{aligned}
& \varepsilon_{k}\left\langle\rho_{k}\right\rangle^{k} T_{k s} \frac{D_{k}}{D t}\left\{S_{k}\right\}^{k}+\varepsilon_{k} \frac{P_{k s}}{\left\langle\rho_{k}\right\rangle^{k}} \frac{D_{k}}{D t}\left\langle\rho_{k}\right\rangle^{k}+\nabla \cdot\left[\varepsilon_{k}\left(\left\langle\mathbf{q}_{k}\right\rangle^{k}+\mathbf{q}_{k}^{r}\right)\right] \\
& -\varepsilon_{k}\left\langle Q_{k}\right\rangle^{k}+\varepsilon_{k}\left\langle P_{k}\right\rangle^{k} \nabla \cdot\left\{\mathbf{v}_{k}\right\}^{k}+\varepsilon_{k}\left\langle\boldsymbol{\tau}_{k}\right\rangle^{k}: \nabla\left\{\mathbf{v}_{k}\right\}^{k} \\
& +\varepsilon_{k}\left\langle P_{k} \nabla^{\prime} \cdot \tilde{\mathbf{v}}_{k}\right\rangle^{k}+\varepsilon_{k}\left\langle\boldsymbol{\tau}_{k}: \nabla^{\prime} \tilde{\mathbf{v}}_{k}\right\rangle^{k}=\Gamma_{k}\left(U_{k i}-\left\{U_{k}\right\}^{k}\right)+E_{k} .
\end{aligned}
$$

Sustituyendo la Ec. (2.26) en la Ec. (2.45), utilizando las Ecs. (2.36), (2.39) y el conjunto de ecuaciones dadas en la Ec. (2.40), obtenemos una restricción para los términos provenientes de la ecuación de energía interna: 


$$
\begin{aligned}
& \left\langle P_{k}\right\rangle^{k} \frac{D_{k}}{D t} \varepsilon_{k}+\Gamma_{k} \frac{\rho_{k i}-2\left\langle\rho_{k}\right\rangle^{k}}{\left\langle\rho_{k}\right\rangle^{k}} \frac{\left\langle P_{k}\right\rangle^{k}}{\left\langle\rho_{k}\right\rangle^{k}}-\varepsilon_{k}\left\langle P_{k} \nabla^{\prime} \cdot \tilde{\mathbf{v}}_{k}\right\rangle^{k} \\
& -\varepsilon_{k} \frac{\left\langle\mathbf{q}_{k}\right\rangle^{k}}{\left\{T_{k}\right\}^{k}} \cdot \nabla\left\{T_{k}\right\}^{k}-\varepsilon_{k}\left\langle\rho_{k}\right\rangle^{k}\left\{\tilde{S}_{k} \tilde{\mathbf{v}}_{k}\right\}^{k} \cdot \nabla\left\{T_{k}\right\}^{k} \\
& -\varepsilon_{k}\left\langle\boldsymbol{\tau}_{k}\right\rangle^{k}: \nabla\left\{\mathbf{v}_{k}\right\}^{k}-\varepsilon_{k}\left\langle\boldsymbol{\tau}_{k}: \nabla^{\prime} \tilde{\mathbf{v}}_{k}\right\rangle^{k}=\left\{T_{k}\right\}^{k}\left\langle X_{k} \Delta_{k}\right\rangle \geq 0 .
\end{aligned}
$$

Esta es la restricción que se obtiene de aplicar la segunda ley de la termodinámica a la ecuación de la energía térmica.

2.7 Restricción de la segunda ley de la termodinámica para modelos de dos fluidos (energía cinética turbulenta)

Esta última restricción puede obtenerse fácilmente al restar la Ec. (2.46) de la Ec. (2.41)

$$
\begin{aligned}
& \varepsilon_{k} \mathbf{\Pi}_{k}^{\mathrm{Re}}:\left\langle\mathbf{D}_{k}\right\rangle_{k}+\left(\mathbf{M}_{k p}+\mathbf{M}_{k t}\right) \cdot\left(\left\langle\mathbf{v}_{k}\right\rangle_{i}-\left\{\mathbf{v}_{k}\right\}^{k}\right)+W_{k i}^{\prime \prime} \\
& +\left(\left\langle\mathbf{v}_{k}\right\rangle_{i}-\left\{\mathbf{v}_{k}\right\}^{k}\right) \cdot\left(\left\langle P_{k}\right\rangle_{i} \mathbf{1}+\left\langle\boldsymbol{\tau}_{k}\right\rangle_{i}\right) \cdot \nabla \varepsilon_{k}-\nabla \cdot\left[\varepsilon_{k}\left(\mathbf{q}_{k}^{p}+\mathbf{q}_{k}^{t}+\mathbf{q}_{k}^{K}\right)\right] \\
& -\varepsilon_{k}\left\langle\rho_{k}\right\rangle^{k} \frac{D_{k}}{D t} K_{k}^{\mathrm{Re}}+\Gamma_{k}\left[-K_{k}^{\mathrm{Re}}+\frac{1}{2}\left(\mathbf{v}_{k} \cdot \mathbf{v}_{k}\right)_{i}-\left\{\mathbf{v}_{k}\right\}^{k} \cdot \mathbf{v}_{k i}+\frac{1}{2}\left(\left\{\mathbf{v}_{k}\right\}^{k} \cdot\left\{\mathbf{v}_{k}\right\}^{k}\right)\right] \\
& +\varepsilon_{k}\left\langle P_{k} \nabla^{\prime} \cdot \tilde{\mathbf{v}}_{k}\right\rangle^{k}+\varepsilon_{k}\left\langle\boldsymbol{\tau}_{k}: \nabla^{\prime} \tilde{\mathbf{v}}_{k}\right\rangle^{k}=0 .
\end{aligned}
$$

Puede demostrarse que la Ec. (2.47) es la ecuación de balance para la energía cinética turbulenta representada en la Ec. (2.8). Para demostrar esto, agruparemos los siguientes términos $\varepsilon_{k}\left\langle P_{k} \nabla^{\prime} \cdot \tilde{\mathbf{v}}_{k}\right\rangle^{k}+\varepsilon_{k}\left\langle\boldsymbol{\tau}_{k}: \nabla^{\prime} \tilde{\mathbf{v}}_{k}\right\rangle^{k}$ en $-\varepsilon_{k}\left\langle\Pi_{k}: \nabla^{\prime} \tilde{\mathbf{v}}_{k}\right\rangle^{k}$ para tener:

$$
\begin{aligned}
& \varepsilon_{k} \boldsymbol{\Pi}_{k}^{\mathrm{Re}}:\left\langle\mathbf{D}_{k}\right\rangle_{k}+\left(\mathbf{M}_{k p}+\mathbf{M}_{k t}\right) \cdot\left(\left\langle\mathbf{v}_{k}\right\rangle_{i}-\left\{\mathbf{v}_{k}\right\}^{k}\right)+W_{k i}^{\prime \prime} \\
& +\left(\left\langle\mathbf{v}_{k}\right\rangle_{i}-\left\{\mathbf{v}_{k}\right\}^{k}\right) \cdot\left(\left\langle P_{k}\right\rangle_{i} \mathbf{1}+\left\langle\boldsymbol{\tau}_{k}\right\rangle_{i}\right) \cdot \nabla \varepsilon_{k}-\nabla \cdot\left[\varepsilon_{k}\left(\mathbf{q}_{k}^{p}+\mathbf{q}_{k}^{t}+\mathbf{q}_{k}^{K}\right)\right] \\
& -\varepsilon_{k}\left\langle\rho_{k}\right\rangle^{k} \frac{D_{k}}{D t} K_{k}^{\mathrm{Re}}+\Gamma_{k}\left[-K_{k}^{\mathrm{Re}}+\frac{1}{2}\left(\mathbf{v}_{k} \cdot \mathbf{v}_{k}\right)_{i}-\left\{\mathbf{v}_{k}\right\}^{k} \cdot \mathbf{v}_{k i}+\frac{1}{2}\left(\left\{\mathbf{v}_{k}\right\}^{k} \cdot\left\{\mathbf{v}_{k}\right\}^{k}\right)\right] \\
& -\varepsilon_{k}\left\langle\boldsymbol{\Pi}_{k}: \nabla^{\prime} \tilde{\mathbf{v}}_{k}\right\rangle^{k}=0 .
\end{aligned}
$$


Luego reescribimos el último término de esta ecuación:

$$
\begin{aligned}
& \left\langle X_{k} \boldsymbol{\Pi}_{k}: \nabla^{\prime} \tilde{\mathbf{v}}_{k}\right\rangle=\left\langle X_{k} \boldsymbol{\Pi}_{k}: \nabla^{\prime} \mathbf{v}_{k}\right\rangle-\left\langle X_{k} \boldsymbol{\Pi}_{k}: \nabla\left\{\mathbf{v}_{k}\right\}^{k}\right\rangle, \\
& \left\langle X_{k} \boldsymbol{\Pi}_{k}: \nabla^{\prime} \tilde{\mathbf{v}}_{k}\right\rangle=\left\langle X_{k} \boldsymbol{\Pi}_{k}: \nabla^{\prime} \mathbf{v}_{k}\right\rangle-\varepsilon_{k}\left\langle\boldsymbol{\Pi}_{k}\right\rangle^{k}: \nabla\left\{\mathbf{v}_{k}\right\}^{k} .
\end{aligned}
$$

Recordando las definiciones dadas previamente:

$$
\begin{aligned}
& W_{k i}^{\prime \prime}=W_{k i}-\nabla \varepsilon_{k} \cdot\left(\left\langle P_{k}\right\rangle_{i} \mathbf{1}+\left\langle\boldsymbol{\tau}_{k}\right\rangle_{i}\right) \cdot\left\langle\mathbf{v}_{k}\right\rangle_{i}-\left(\mathbf{M}_{k p}+\mathbf{M}_{k t}\right) \cdot\left\langle\mathbf{v}_{k}\right\rangle_{i}, \\
& \mathbf{M}_{k p}+\mathbf{M}_{k t}=\mathbf{M}_{k}-\left(\left\langle P_{k}\right\rangle_{i} \mathbf{1}+\left\langle\boldsymbol{\tau}_{k}\right\rangle_{i}\right) \cdot \nabla \varepsilon_{k} .
\end{aligned}
$$

Introduciendo estas definiciones junto con la Ec. (2.49) en la Ec. (2.48) y simplificando encontramos que:

$$
\begin{aligned}
& \varepsilon_{k}\left(\left\langle\boldsymbol{\Pi}_{k}\right\rangle^{k}+\boldsymbol{\Pi}_{k}^{\mathrm{Re}}\right): \nabla\left\{\mathbf{v}_{k}\right\}^{k}-\mathbf{M}_{k} \cdot\left\{\mathbf{v}_{k}\right\}^{k}+W_{k i}-\nabla \cdot\left[\varepsilon_{k}\left(\mathbf{q}_{k}^{p}+\mathbf{q}_{k}^{t}+\mathbf{q}_{k}^{K}\right)\right] \\
& -\varepsilon_{k}\left\langle\rho_{k}\right\rangle^{k} \frac{D_{k}}{D t} K_{k}^{\mathrm{Re}}+\Gamma_{k}\left[-K_{k}^{\mathrm{Re}}+\frac{1}{2}\left(\mathbf{v}_{k} \cdot \mathbf{v}_{k}\right)_{i}-\left\{\mathbf{v}_{k}\right\}^{k} \cdot \mathbf{v}_{k i}+\frac{1}{2}\left(\left\{\mathbf{v}_{k}\right\}^{k} \cdot\left\{\mathbf{v}_{k}\right\}^{k}\right)\right] \\
& -\left\langle X_{k} \boldsymbol{\Pi}_{k}: \nabla^{\prime} \mathbf{v}_{k}\right\rangle=0 .
\end{aligned}
$$

Que es la ecuación de balance de la energía cinética turbulenta [Ec. (2.8)]. Por lo tanto, en realidad son sólo tres formas equivalentes de la misma restricción, que dependen de la partición de términos. Posteriormente utilizaremos la Ec. (2.41) ó la Ec. (2.46) según convenga para validar nuestros modelos de dos fluidos ya cerrados.

2.8 Segunda ley de la termodinámica en el nivel local y en el nivel promedio

La ecuación de entropía promedio tiene una gran ventaja sobre la ecuación local instantánea. En general no es posible expresar $\rho_{k} D_{k} S_{k} / D t^{\prime}$ analíticamente. Por lo que no es posible evaluar analíticamente el miembro izquierdo de la ecuación de entropía a nivel local. Sin embargo, al obtener la ecuación de entropía a nivel promedio, hemos sido capaces de eliminar las derivadas de la entropía al escribirla en términos de parámetros que se expresan más fácilmente en forma analítica. Por lo que dado un conjunto de relaciones de cerradura es posible evaluar la ecuación de la entropía para muchas situaciones de flujo. 
La ecuación de entropía promedio es significativamente más compleja que la ecuación local instantánea. Esto se debe en parte a las dificultades involucradas al remplazar la derivada de la entropía y también a la complejidad de los términos interfaciales que surgen en las ecuaciones de campo promedio. Si la ecuación de la entropía, expresada como una desigualdad se infringe, entonces se espera que nuestro sistema de ecuaciones sobre prediga la energía de esa fase. Sin información adicional no podemos saber qué forma toma el exceso de energía. Puede aparecer como un incremento en la energía interna, en la energía cinética, en la energía cinética turbulenta o cualquier otra forma de energía.

2.9 Forma de aplicar las restricciones encontradas con la segunda ley de la termodinámica

La Figura 2.2 muestra la metodología que debe seguirse para utilizar las restricciones encontradas en la Ec. (2.28), en la Ec. (2.41) y en la Ec. (2.46) al aplicarse a modelos promediados de dos fluidos.

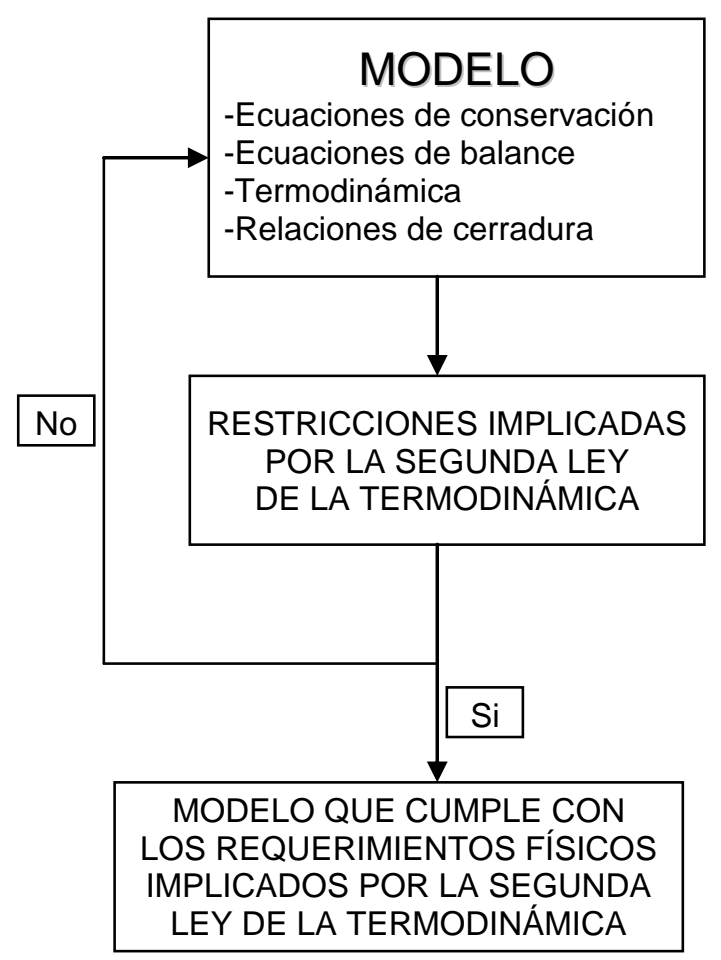

Figura 2.2. Metodología para aplicar las restricciones de la segunda ley en los términos de cerradura de las ecuaciones promediadas. 


\section{Capítulo 3}

\section{Modelo isotérmico unidimensional \\ con promedio espacio-temporal}

\subsection{Introducción}

El régimen de fluidización rápida es significativo ya que en él se lleva a cabo el proceso de FCC, el cual es un proceso muy importante para la producción de gasolina en todo el mundo. En el libro de Gidaspow (1994) se ofrece una revisión de los principales modelos para la fluidización en diversos regímenes de flujo. Se consideran tres modelos para lechos fluidizados circulantes. En el primero, el Modelo A, la presión del gas se toma como parte de la presión del sólido, complementada por el módulo de compresibilidad del sólido el cual es un efecto de fuerza normal no-local debido al movimiento de los granos sólidos. Mientras que en el segundo, el Modelo B, la presión del sólido se toma como el módulo de compresibilidad solamente. Finalmente, el Modelo $\mathrm{C}$ es un modelo de velocidad relativa conocido comúnmente como una ecuación constitutiva para la mezcla, este modelo no contiene a la presión.

En los 70's se resolvió la discusión de los modelos incompresibles mal planteados al incluir el módulo de compresibilidad del sólido como parte del campo de presión del mismo. Se introduce este término para producir velocidades de propagación de segundo orden reales. Ransom y Hicks (1984) discuten la hiperbolicidad de las ecuaciones y los problemas bien planteados. Ellos analizan modelos compresibles de dos fases con un mismo y con diferente campo de presión centrándose en el criterio de estabilidad de von Neumann, el cual establece que para un sistema de ecuaciones diferenciales parciales de primer orden: $\partial_{t} \mathbf{u}=\mathbf{C}(\mathbf{u}) \partial_{z} \mathbf{u}+\mathbf{B}(\mathbf{u})$, la matriz $\mathbf{C}$ (de tamaño $m \times m$ ) es hiperbólica en $\mathbf{u}$ (de tamaño $m \times 1)$ si y sólo si $\mathbf{C}(\mathbf{u})$ tiene un conjunto de valores propios que son reales y un conjunto de vectores característicos que es completo. 
Esta ecuación es estable en el sentido de von Neumann si para toda matriz B (de tamaño $m \times 1)$ si y sólo si $\mathbf{C}(\mathbf{u})$ es hiperbólica. Concluyen que los modelos con un distinto campo de presión son estables en el sentido de von Neumann.

Song e Ishii (2001) proponen un criterio diferente de estabilidad para el modelo incompresible, el cual se basa en la evaluación de los parámetros del flujo de momento y obtienen estabilidad sin necesidad del módulo de compresibilidad del sólido.

En este Capítulo utilizaremos un modelo de dos fluidos, describiremos el sistema físico, las ecuaciones de conservación de la masa y de balance de cantidad de movimiento promediadas, la cerradura de las ecuaciones promediadas, se desarrollan las ecuaciones linealizadas, se analizarán las cerraduras con las restricciones encontradas con la segunda ley de la termodinámica promedio y finalmente, proyectaremos el modelo en la dirección $z$.

\subsection{Modelamiento del flujo isotérmico gas-sólido en el riser}

\subsubsection{Sistema físico}

El sistema físico está formado por un conjunto monodisperso de partículas esféricas rígidas de diámetro medio $d_{p}$, moviéndose con una corriente de gas o vapor en un tubo vertical. El fluido es considerado un gas Newtoniano compresible de densidad $\rho_{g}$ y viscosidad constante $\mu_{g}$.

La dispersión de partículas es isotérmica y diluida con fracción volumen de sólido $\varepsilon_{s 0} \ll 1$. El promedio espacio-temporal se toma sobre la sección transversal del riser incluyendo la pared del tubo (ver la Figura 1.2).

\subsubsection{Ecuaciones promediadas en el espacio-tiempo}

En esta Sección retomaremos las ecuaciones promediadas de masa y de cantidad de movimiento del Capítulo 1, Ec. (1.47) y Ec. (1.48), respectivamente. 
Ecuación de conservación de la masa

Aplicando la C.F.1 y la C.F.4 (dadas en la pág. 14) en la Ec. (1.47), se elimina el flujo de masa en la interfaz $\Gamma_{k}=0$, obteniéndose en términos de la notación de la derivada parcial:

$$
\frac{\partial}{\partial t}\left(\varepsilon_{k}\left\langle\rho_{k}\right\rangle^{k}\right)+\nabla \cdot\left(\varepsilon_{k}\left\langle\rho_{k}\right\rangle^{k}\left\{\mathbf{v}_{k}\right\}^{k}\right)=0
$$

Esta ecuación implica que los materiales no cambian de fase; es decir, ni el vapor se absorbe en las partículas ni las partículas se subliman en el vapor. Esta es la ecuación de conservación de la masa de campo promedio para la fase $k$. Las fases pueden ser compresibles o incompresibles.

\section{Ecuación de cantidad de movimiento}

Aplicando la C.F.1 y la C.F.4 en la Ec. (1.48), se elimina el flujo de masa en la interfaz $\Gamma_{k}=0$, obteniéndose (en términos de la notación de la derivada parcial):

$$
\begin{array}{r}
\frac{\partial}{\partial t}\left(\varepsilon_{k}\left\langle\rho_{k}\right\rangle^{k}\left\{\mathbf{v}_{k}\right\}^{k}\right)+\nabla \cdot\left(\varepsilon_{k}\left\langle\rho_{k}\right\rangle^{k}\left\{\mathbf{v}_{k}\right\}^{k}\left\{\mathbf{v}_{k}\right\}^{k}\right)+\varepsilon_{k} \nabla \cdot\left\langle\boldsymbol{\Pi}_{k}\right\rangle^{k}-\nabla \cdot\left(\varepsilon_{k} \boldsymbol{\Pi}_{k}^{\mathrm{Re}}\right) \\
+\sum_{k \ell \mid k} a_{k \ell}\left\langle\mathbf{n}_{k} \cdot \tilde{\boldsymbol{\Pi}}_{k}\right\rangle_{k \ell}-\varepsilon_{k}\left\langle\rho_{k}\right\rangle^{k} \mathbf{g}=\mathbf{0} .
\end{array}
$$

Puede demostrarse que: $-\nabla \cdot\left(\varepsilon_{k}\left\langle\boldsymbol{\Pi}_{k}\right\rangle^{k}\right)-\mathbf{M}_{k}=\varepsilon_{k} \nabla \cdot\left\langle\boldsymbol{\Pi}_{k}\right\rangle^{k}+\sum_{k \ell \mid k} a_{k \ell}\left\langle\mathbf{n}_{k} \cdot \tilde{\boldsymbol{\Pi}}_{k}\right\rangle_{k \ell}$, al utilizar la Ec. (1.39) y la Ec. (1.40). Debe enfatizarse la importancia del área interfacial en la determinación de la interacción entre las fases.

Básicamente, los efectos geométricos de primer orden en el término interfacial que son tomados en cuenta por la concentración de área interfacial $a_{k \ell}$ de tal forma que la transferencia de cantidad de movimiento interfacial tiende hacia el equilibrio mecánico (Ishii y Kocamustafaogullari, 1983).

La fracción volumen y el área interfacial específica caracterizan la configuración geométrica del flujo de dos fases (Soria y de Lasa, 1992). 
Este conjunto promediado no está cerrado. El promedio espacio-temporal ha introducido muchos términos fuente que deben analizarse para cerrar el conjunto de EDP.

En resumen, el conjunto de EDP está dado por:

$$
\underbrace{\frac{\partial}{\partial t}\left(\varepsilon_{k}\left\langle\rho_{k}\right\rangle^{k}\right)}_{\text {Acumulación de masa }}+\underbrace{\nabla \cdot\left(\varepsilon_{k}\left\langle\rho_{k}\right\rangle^{k}\left\{\mathbf{v}_{k}\right\}^{k}\right)}_{\text {Transporte convectivo de masa }}=0,
$$

$$
\begin{aligned}
& \underbrace{\frac{\partial}{\partial t}\left(\varepsilon_{k}\left\langle\rho_{k}\right\rangle^{k}\left\{\mathbf{v}_{k}\right\}^{k}\right)}_{\text {Evolución temporal de cantidad de movimiento }}+\underbrace{\nabla \cdot\left(\varepsilon_{k}\left\langle\rho_{k}\right\rangle^{k}\left\{\mathbf{v}_{k}\right\}^{k}\left\{\mathbf{v}_{k}\right\}^{k}\right)}_{\text {Transporte convectivo de cantidad de movimiento }} \\
& +\underbrace{\varepsilon_{k} \nabla \cdot\left\langle\boldsymbol{\Pi}_{k}\right\rangle^{k}}_{\text {Esfuerzos moleculares totales }}+\underbrace{\sum_{k \ell \mid k} a_{k \ell}\left\langle\mathbf{n}_{k} \cdot \tilde{\boldsymbol{\Pi}}_{k}\right\rangle_{k \ell}}_{\text {Transporte interfacial de cantidad de movimiento }}-\underbrace{\varepsilon_{k}\left\langle\rho_{k}\right\rangle^{k} \mathbf{g}}_{\text {Fuerzas gravitacionales }}=\mathbf{0},
\end{aligned}
$$

donde los esfuerzos turbulentos suavizados $\boldsymbol{\Pi}_{k}^{\mathrm{Re}}$ están relacionados a los parámetros de flujo de cantidad de movimiento considerados por Song e Ishii (2001) pero que no se toman en cuenta en esta descripción de primer orden.

$\left\langle\boldsymbol{\Pi}_{k}\right\rangle^{k}$ es el promedio intrínseco del tensor de esfuerzos de la fase $k$, mientras que $\sum_{k \ell \mid k} a_{k \ell}\left\langle\mathbf{n}_{k} \cdot \tilde{\boldsymbol{\Pi}}_{k}\right\rangle^{k \ell}$ es el transporte promedio intrínseco interfacial de cantidad de movimiento de la fase $k$ debido a las desviaciones de contacto en la interfaz $(k \ell=g s)$ y en la pared del tubo $(k \ell=k W ; k=g, s)$.

Para un fluido Newtoniano $\left\langle\boldsymbol{\Pi}_{g}\right\rangle^{g}=\left\langle P_{g}\right\rangle^{g} \mathbf{1}+\left\langle\boldsymbol{\tau}_{g}\right\rangle^{g}$, donde $\mathbf{1}$ es el tensor métrico y $\left\langle\boldsymbol{\tau}_{g}\right\rangle^{g}$ es el tensor de esfuerzos viscosos promedio (intrínseco). g es la fuerza gravitacional por unidad de masa. 


\subsubsection{Cerradura del conjunto de ecuaciones promediadas}

El flujo de las fases se mueve con la velocidad de la mezcla promedio $U_{m}=\varepsilon_{s} V_{s}+\varepsilon_{g} V_{g}$, donde $V_{g}$ y $V_{s}$ son las velocidades intersticiales del gas y del sólido, respectivamente. Se considera a la mezcla altamente turbulenta, tal que los efectos viscosos son significativos sólo cerca de las superficies del gránulo y de las paredes del tubo. El número de Reynolds de partícula, dado por $\operatorname{Re}_{s}=d_{p} \rho_{g} U_{t} / \mu_{g}$, donde $U_{t}=\left|V_{s}-U_{m}\right|=\varepsilon_{g}\left(V_{g}-V_{s}\right)$ es la velocidad terminal. El valor del $\operatorname{Re}_{s}$ define la expresión del coeficiente de arrastre $C_{D}$ que debe seleccionarse. En este modelo de primer orden, los efectos de la viscosidad del bulto y los esfuerzos turbulentos no son considerados.

En la Ec. (3.4), los términos que requieren de una cerradura son los esfuerzos totales y los términos de transporte de cantidad de movimiento interfacial para ambas fases. Simplifiquemos primero los esfuerzos promedio para la fase gas: puesto que las fuerzas viscosas están concentradas en una capa delgada cercana a la interfaz y a las paredes del tubo, es posible satisfacer la desigualdad $\varepsilon_{g} \nabla \cdot\left\langle\boldsymbol{\tau}_{g}\right\rangle^{g} \ll a_{g s}\left\langle\mathbf{n}_{g} \cdot \tilde{\boldsymbol{\tau}}_{g}\right\rangle^{g s}$, cuando se satisface la restricción de escala de longitud $L \gg \ell$ y la restricción de escala temporal $T \gg t_{0}$.

Más aún, el tensor de esfuerzos del sólido debe equilibrarse mecánicamente en las interfaz con los esfuerzos del gas, entonces $\left\langle\mathbf{n}_{s} \cdot \tilde{\boldsymbol{\Pi}}_{s}\right\rangle^{g s}=-\left\langle\mathbf{n}_{g} \cdot \tilde{\boldsymbol{\Pi}}_{g}\right\rangle^{g s}$. Finalmente, los esfuerzos promedio en el sólido $\left\langle\boldsymbol{\Pi}_{s}\right\rangle^{s}$ deben ser considerados como dependientes de los esfuerzos promedio en el fluido; por lo tanto una primera aproximación a la cerradura podría ser

$$
\left\langle\boldsymbol{\Pi}_{s}\right\rangle^{s}=\left\langle P_{g}\right\rangle^{g} \mathbf{1}
$$

la cual es consistente con la consideración de la continuación del campo de presión del gas dentro de la localización de los sólidos en mezclas homogéneas. Esta es una consideración clave para la obtención de los teoremas de Arquímedes en la hidrostática (Slattery, 1999) y su extensión a las condiciones de flujo es directa como una primera aproximación. 
Sin embargo, la no-homogeneidad requiere que se involucre otra función, el llamado módulo de compresibilidad $\Phi\left(\varepsilon_{s}\right)$ (Gidaspow, 1994), en una forma aditiva:

$$
\left\langle\boldsymbol{\Pi}_{s}\right\rangle^{s}=\left(\left\langle P_{g}\right\rangle^{g}+\Phi\left(\varepsilon_{s}\right)\right) \mathbf{1}
$$

Tomando en cuenta todas las consideraciones enunciadas anteriormente, la Ec. (3.4) puede expresarse para la fase gas como

$$
\begin{gathered}
\frac{\partial}{\partial t}\left(\varepsilon_{g}\left\langle\rho_{g}\right\rangle^{g}\left\{\mathbf{v}_{g}\right\}^{g}\right)+\nabla \cdot\left(\varepsilon_{g}\left\langle\rho_{g}\right\rangle^{g}\left\{\mathbf{v}_{g}\right\}^{g}\left\{\mathbf{v}_{g}\right\}^{g}\right) \\
+\varepsilon_{g} \nabla\left\langle\boldsymbol{P}_{g}\right\rangle^{g}+a_{g s}\left\langle\mathbf{n}_{g} \cdot \tilde{\boldsymbol{\Pi}}_{g}\right\rangle^{g s}+a_{g W}\left\langle\mathbf{n}_{g} \cdot \tilde{\boldsymbol{\Pi}}_{g}\right\rangle^{g W}-\varepsilon_{g}\left\langle\rho_{g}\right\rangle^{g} \mathbf{g}=\mathbf{0}
\end{gathered}
$$

y para la fase sólida como

$$
\begin{gathered}
\frac{\partial}{\partial t}\left(\varepsilon_{s}\left\langle\rho_{s}\right\rangle^{s}\left\{\mathbf{v}_{s}\right\}^{s}\right)+\nabla \cdot\left(\varepsilon_{s}\left\langle\rho_{s}\right\rangle^{s}\left\{\mathbf{v}_{s}\right\}^{s}\left\{\mathbf{v}_{s}\right\}^{s}\right) \\
+\varepsilon_{s} \nabla\left(\left\langle P_{g}\right\rangle^{g}+\Phi\left(\varepsilon_{s}\right)\right)-a_{g s}\left\langle\mathbf{n}_{g} \cdot \tilde{\boldsymbol{\Pi}}_{g}\right\rangle^{g s}+a_{s W}\left\langle\mathbf{n}_{s} \cdot \tilde{\boldsymbol{\Pi}}_{s}\right\rangle^{s W}-\varepsilon_{s}\left\langle\rho_{s}\right\rangle^{s} \mathbf{g}=\mathbf{0}
\end{gathered}
$$

3.3 Validación de las cerraduras con la segunda ley de la termodinámica

3.3.1 Cerraduras propuestas y su consistencia con las restricciones impuestas por la segunda ley de la termodinámica

La restricción de la segunda ley de la termodinámica para modelos de dos fluidos está dada por la Ec. (2.41). Para el caso de que el sistema sea isotérmico, no haya cambio de fase $\left(\Gamma_{k}=0\right)$, de que no consideremos la turbulencia y en ausencia de trabajo virtual, esta ecuación se reduce a:

$$
-\varepsilon_{k}\left\langle\boldsymbol{\tau}_{k}\right\rangle^{k}:\left\{\mathbf{D}_{k}\right\}^{k}+\mathbf{M}_{k} \cdot\left(\left\langle\mathbf{v}_{k}\right\rangle_{i}-\left\{\mathbf{v}_{k}\right\}^{k}\right)+\left\langle P_{k}\right\rangle^{k} \frac{D_{k}}{D t} \varepsilon_{k} \geq 0
$$


donde hemos considerado la Ec. (2.32). El primer término de la Ec. (3.9) sugiere una forma de modelar los esfuerzos viscosos de campo promedio; es decir, para que $-\varepsilon_{k}\left\langle\boldsymbol{\tau}_{k}\right\rangle^{k}:\left\{\mathbf{D}_{k}\right\}^{k} \geq 0$, se requiere que $\left\langle\boldsymbol{\tau}_{k}\right\rangle^{k} \propto\left\{\mathbf{D}_{k}\right\}^{k}$, o bien que: $\left\langle\boldsymbol{\tau}_{k}\right\rangle^{k}=-2 \mu\left\{\mathbf{D}_{k}\right\}^{k}$, que es una relación de comportamiento similar a la de Newton, pero a nivel promedio, donde $\left\{\mathbf{D}_{k}\right\}^{k}$ está definida en la Ec. (2.38).

El segundo término, $\mathbf{M}_{k} \cdot\left(\left\langle\mathbf{v}_{k}\right\rangle_{i}-\left\{\mathbf{v}_{k}\right\}^{k}\right) \geq 0$, implica que $\mathbf{M}_{k} \propto\left\langle\mathbf{v}_{k}\right\rangle_{i}-\left\{\mathbf{v}_{k}\right\}^{k}$, con lo que la cerradura que utilizaremos más adelante, Ec. (3.13) está en acuerdo, los esfuerzos interfaciales deben ser expresados en términos de la velocidad relativa entre las fases la cual es objetiva al igual que $\mathbf{M}_{k}$. Finalmente, el último término en la Ec. (3.9), requiere que $D_{k} \varepsilon_{k} / D t \propto\left\langle P_{k}\right\rangle^{k}$.

\subsubsection{Proyección de las ecuaciones (modelo 1-D)}

Se obtendrá un modelo unidimensional del conjunto de cuatro ecuaciones dado por la Ec. (3.3) $(k=g, s)$, la Ec. (3.7) y la Ec. (3.8). Para este propósito se considera que $\left\{\mathbf{v}_{k}\right\}^{k}=V_{k} \mathbf{e}_{z}$ en un sistema coordenado de referencia $(r, \theta, z)$, donde $V_{k}$ es una notación abreviada para la magnitud de la velocidad $\left\{\mathbf{v}_{k}\right\}^{k}$ y desarrollando la proyección de las ecuaciones de movimiento dadas por la Ec. (3.7) y la Ec. (3.8) en la dirección axial $\mathbf{e}_{z}$ :

$$
\begin{gathered}
\frac{\partial}{\partial t}\left(\varepsilon_{g} \rho_{g} V_{g}\right)+\frac{\partial}{\partial z}\left(\varepsilon_{g} \rho_{g} V_{g}^{2}\right)+\varepsilon_{g} \frac{\partial}{\partial z} P_{g} \\
+a_{g s}\left\langle\mathbf{n}_{g} \cdot \tilde{\boldsymbol{\Pi}}_{g} \cdot \mathbf{e}_{z}\right\rangle^{g s}+a_{g W}\left\langle\mathbf{e}_{r} \cdot \tilde{\boldsymbol{\Pi}}_{g} \cdot \mathbf{e}_{z}\right\rangle^{g W}+\varepsilon_{g} \rho_{g} g=0 \\
\frac{\partial}{\partial t}\left(\varepsilon_{s} \rho_{s} V_{s}\right)+\frac{\partial}{\partial z}\left(\varepsilon_{s} \rho_{s} V_{s}^{2}\right)+\varepsilon_{s} \frac{\partial}{\partial z}\left[P_{g}+\Phi\left(\varepsilon_{s}\right)\right] \\
-a_{g s}\left\langle\mathbf{n}_{g} \cdot \tilde{\boldsymbol{\Pi}}_{g} \cdot \mathbf{e}_{z}\right\rangle^{g s}+a_{s W}\left\langle\mathbf{e}_{r} \cdot \tilde{\boldsymbol{\Pi}}_{s} \cdot \mathbf{e}_{z}\right\rangle^{s W}+\varepsilon_{s} \rho_{s} g=0 .
\end{gathered}
$$


Por simplicidad, de aquí en adelante se omitirán todos los símbolos de promedio. Considerando granos esféricos sólidos, el área interfacial específica es $a_{g s}=\left(6 / d_{p}\right) \varepsilon_{s}$. También, la fuerza interfacial promedio $\left\langle\mathbf{n}_{g} \cdot \tilde{\boldsymbol{\Pi}}_{g} \cdot \mathbf{e}_{z}\right\rangle^{g s}$ puede modelarse mediante un modelo de celda, utilizando un coeficiente de arrastre apropiado, de tal forma que

$$
a_{g s}\left\langle\mathbf{n}_{g} \cdot \tilde{\boldsymbol{\Pi}}_{g} \cdot \mathbf{e}_{z}\right\rangle^{g s}=\varepsilon_{s} \rho_{g} g+C_{D} \frac{3}{4 d_{p}} \varepsilon_{s} \rho_{g} U_{t}{ }^{2} .
$$

La contribución cinética a esta fuerza en una capa laminar alrededor de los granos sólidos es linealmente proporcional a la velocidad relativa entre las fases; por lo tanto, la Ec. (3.12) puede escribirse como

$$
a_{g s}\left\langle\mathbf{n}_{g} \cdot \tilde{\boldsymbol{\Pi}}_{g} \cdot \mathbf{e}_{z}\right\rangle^{g s}=\varepsilon_{s} \rho_{g} g+\beta \varepsilon_{s} \varepsilon_{g}\left(V_{g}-V_{s}\right),
$$

donde $\beta \equiv \frac{3}{4 d_{p}} C_{D} \rho_{g} U_{t}$. Se necesitan expresiones de cerradura también para los términos de interacción con la pared en la Ec. (3.10) y en la Ec. (3.11). Esto puede resolverse al introducir el enfoque del factor de fricción (Gidaspow, 1994). Así, para la fase $k=\{g, s\}$

$$
a_{k W}\left\langle\mathbf{e}_{r} \cdot \tilde{\boldsymbol{\Pi}}_{k} \cdot \mathbf{e}_{z}\right\rangle^{k W}=\frac{4}{D_{t}} \varepsilon_{k} f_{k W} \frac{1}{2} \rho_{k} V_{k}^{2}=\beta_{k W} \rho_{k} V_{k}^{2}
$$

donde $\beta_{k W} \equiv \frac{2}{D_{t}} \varepsilon_{k} f_{k W}$. En la interacción del flujo en la mezcla turbulenta con la pared, la fuerza cinética es aproximadamente proporcional al cuadrado de las velocidades.

Después de sustituir la Ec. (3.13) y la Ec. (3.14) en la Ec. (3.10) y en la Ec. (3.11), el conjunto de ecuaciones en 1-D junto con la ecuación de continuidad dada por la Ec. (3.3) es: 
Ecuación de continuidad para la fase gas:

$$
\frac{\partial}{\partial t}\left(\varepsilon_{g} \rho_{g}\right)+\frac{\partial}{\partial z}\left(\varepsilon_{g} \rho_{g} V_{g}\right)=0 \text {. }
$$

Ecuación de continuidad para la fase sólida:

$$
\frac{\partial}{\partial t}\left(\varepsilon_{s} \rho_{s}\right)+\frac{\partial}{\partial z}\left(\varepsilon_{s} \rho_{s} V_{s}\right)=0
$$

Ecuación de movimiento para la fase gas:

$$
\begin{aligned}
& \frac{\partial}{\partial t}\left(\varepsilon_{g} \rho_{g} V_{g}\right)+\frac{\partial}{\partial z}\left(\varepsilon_{g} \rho_{g} V_{g}^{2}\right)+\varepsilon_{g} \frac{\partial P_{g}}{\partial z} \\
+ & \beta \varepsilon_{s} \varepsilon_{g}\left(V_{g}-V_{s}\right)+\beta_{g W} \rho_{g} V_{g}^{2}+\left(\varepsilon_{s}+\varepsilon_{g}\right) \rho_{g} g=0 .
\end{aligned}
$$

Ecuación de movimiento para la fase sólida:

$$
\begin{gathered}
\frac{\partial}{\partial t}\left(\varepsilon_{s} \rho_{s} V_{s}\right)+\frac{\partial}{\partial z}\left(\varepsilon_{s} \rho_{s} V_{s}^{2}\right)+\varepsilon_{s} \frac{\partial P_{g}}{\partial z}+\Phi^{\prime} \varepsilon_{s} \frac{\partial \varepsilon_{s}}{\partial z} \\
-\beta \varepsilon_{s} \varepsilon_{g}\left(V_{g}-V_{s}\right)+\beta_{s W} \rho_{s} V_{s}^{2}+\varepsilon_{s}\left(\rho_{s}-\rho_{g}\right) g=0
\end{gathered}
$$

donde $\Phi^{\prime}=d \Phi / d \varepsilon_{s}$. En la Ec. (3.15) a la Ec. (3.18) hay ocho cantidades desconocidas. Para tener un conjunto cerrado para las variables $\left\{\varepsilon_{g}, P_{g}, V_{g}, V_{s}\right\}$, es necesario considerar diferentes condiciones:

(1) la función $\Phi^{\prime}$ debe especificarse, tal que las rapideces de propagación de segundo orden del modelo incompresible sean reales [ver el Apéndice $E$ y la Ec. (4.21)];

(2) El sistema sólido-fluido (gas o vapor) es saturado, i.e., $\varepsilon_{s}+\varepsilon_{g}=1$;

(3) los granos sólidos son incompresibles, $\rho_{s}=\rho_{s 0}$ es una constante y

(4) la densidad promedio de la fase gas o vapor es una función de su presión promedio, de acuerdo a una ecuación de estado isotérmica, $\rho_{g}=\rho_{g}\left(P_{g}\right)$.

(5) Además $\beta, \beta_{g W}$ y $\beta_{s W}$ pueden calcularse en el estado base por métodos estándar, así como $U_{t}$. 
Por lo tanto, la Ec. (3.15) a la Ec. (3.18) se reducen al conjunto de ecuaciones no-lineales (Sánchez-López, 2003; Soria y col., 2008):

$$
\begin{aligned}
& \frac{\partial}{\partial t}\left[\varepsilon_{g} \rho_{g}\left(P_{g}\right)\right]+\frac{\partial}{\partial z}\left[\varepsilon_{g} \rho_{g}\left(P_{g}\right) V_{g}\right]=0, \\
& -\rho_{s 0} \frac{\partial}{\partial t} \varepsilon_{g}+\rho_{s 0} \frac{\partial}{\partial z}\left[\left(1-\varepsilon_{g}\right) V_{s}\right]=0, \\
& \frac{\partial}{\partial t}\left[\varepsilon_{g} \rho_{g}\left(P_{g}\right) V_{g}\right]+\frac{\partial}{\partial z}\left[\varepsilon_{g} \rho_{g}\left(P_{g}\right) V_{g}^{2}\right]+\varepsilon_{g} \frac{\partial}{\partial z} P_{g} \\
& +\beta \varepsilon_{g}\left(1-\varepsilon_{g}\right)\left(V_{g}-V_{s}\right)+\beta_{g W} \rho_{g}\left(P_{g}\right) V_{g}^{2}+\rho_{g}\left(P_{g}\right) g=0, \\
& \rho_{s 0} \frac{\partial}{\partial t}\left[\left(1-\varepsilon_{g}\right) V_{s}\right]+\rho_{s 0} \frac{\partial}{\partial z}\left[\left(1-\varepsilon_{g}\right) V_{s}^{2}\right]+\left(1-\varepsilon_{g}\right) \frac{\partial}{\partial z} P_{g}-\Phi^{\prime}\left(1-\varepsilon_{g}\right) \frac{\partial}{\partial z} \varepsilon_{g} \\
& -\beta \varepsilon_{g}\left(1-\varepsilon_{g}\right)\left(V_{g}-V_{s}\right)+\beta_{s W} \rho_{s 0} V_{s}^{2}+\left(1-\varepsilon_{g}\right)\left[\rho_{s 0}-\rho_{g}\left(P_{g}\right)\right] g=0 .
\end{aligned}
$$

La Ec. (3.19) a la Ec. (3.22) pueden linealizarse alrededor de un estado base uniforme, denotado por un subíndice cero, tal que $\varepsilon_{g}=\varepsilon_{0}+\varepsilon, P_{g}=P_{0}+p, \quad V_{g}=v_{g 0}+v_{g} \quad \mathrm{y}$ $V_{s}=v_{s 0}+v_{s}$. Una vez que la linealización se lleva a cabo los símbolos para las velocidades $v_{g} \mathrm{y} v_{s}$ se utilizan para las correspondientes perturbaciones.

También puede obtenerse un modelo que no considere los efectos de la pared, a partir del conjunto de ecuaciones (3.19)-(3.22), al tomar los límites: $\beta_{g W} \rightarrow 0$ y $\beta_{s W} \rightarrow 0$. El conjunto linealizado de la Ec. (3.19) a la Ec. (3.22) será el punto de partida para el estudio de propagación de ondas en los Capítulos 4 y 5. 


\section{Capítulo 4}

\section{Propagación de ondas isotérmicas compresibles e incompresibles con modelos de lechos fluidizados rápidos 1-D linealizados ${ }^{2}$}

\subsection{Introducción}

Los lechos fluidizados circulantes (CFB) usualmente operan bajo el régimen de fluidización rápida donde las partículas sólidas son acarreadas por un fluido. En este régimen, la velocidad terminal de las partículas individuales es sobrepasada por la velocidad del fluido acarreador que fluye ascendentemente en el tubo; por lo tanto, tiene lugar un flujo continuo de la mezcla de dos fases en la parte superior. La recirculación de sólidos es una forma conveniente de mantener la operación por un largo tiempo. La recirculación aumenta la superficie de contacto entre las fases y reduce las resistencias a la transferencia de masa y a la transferencia de calor interfacial, minimizando cambios grandes en las variables de estado.

El efecto de la presión en los CFB fue estudiado por Gidaspow en 1994 para muchos regímenes de flujo. Después van der Schaaf y col. (1998) miden la velocidad de propagación de ondas de presión en un CFB con la fracción volumen de sólido dependiente de la posición vertical en el lecho. Al colocar un conjunto de transductores de presión en diversas alturas, ellos miden localmente la rapidez de propagación de la presión a diferentes fracciones volumen de sólido.

Finalmente, De Wilde y col. (2007) proponen una ecuación para estimar la velocidad del sonido en la mezcla mediante algún tipo de promediado de los primeros dos eigenvalores del determinante característico.

\footnotetext{
${ }^{2}$ Los resultados de este Capítulo están publicados en: Sánchez-López, J.R.G., Soria, A. y Salinas-Rodríguez, E. (2011). Compressible and incompressible 1-D linear wave propagation assessment in fast fluidized beds. AIChE J. 57 (11), 2965-2976.
} 
También exploran un método de filtrado, incorporando expresiones para el efecto de masa agregada y el término histórico para una partícula esférica, en el nivel de descripción promedio.

La velocidad del sonido en la mezcla que reportan con el modelo no-filtrado ajusta mejor los resultados de van der Schaaf y col. (1998) que con el modelo filtrado.

Algunos estudios de ondas de presión en lechos fluidizados involucran tanto el régimen denso como el régimen diluido. Bi y col. (1995) estudiaron la velocidad de propagación de ondas de presión mediante la teoría ondulatoria compresible pseudo-homogénea y la teoría ondulatoria del flujo separado compresible para partículas de los Grupos A y B, con diferencias insignificantes en los resultados. Musmarra y col. (1995) utilizaron una expresión simplificada pseudo-homogénea para ondas de presión obtenida por Wallis (1969) y una expresión para el flujo separado de Gregor y Rumpf (1976). Consideran la rapidez de propagación de las ondas de presión como una función de la frecuencia como Atkinson y Kytömaa (1992) lo hicieron antes. De sus resultados (para fracción volumen > 0.35), Musmarra y col. (1995) asumen que las ondas dinámicas observadas en sus experimentos son más elásticas que las ondas de compresión.

Jones y Prosperetti (1987) obtienen criterios de estabilidad lineal para modelos de flujo en dos fases incompresibles unidimensionales (1-D), al incorporar diferentes efectos tales como la tensión superficial, viscosidad, efectos de correlación y masa agregada. Song e Ishii (2001) proponen un criterio de estabilidad diferente para el modelo incompresible, que se basa en la evaluación de los parámetros de flujo de cantidad de movimiento.

Recientemente, Bi (2007) desarrolló una revisión de las fuentes de perturbaciones de la presión, refiriendo a la expresión de Gregor y Rumpf (1976) para la propagación de la onda de presión pseudo-homogénea y a la expresión de flujos separados de Ryzhkov y Tolmachev (1983). Bi (2007) también reporta una dependencia significativa de la frecuencia para la velocidad de propagación de la onda en flujos de fase densa fluidizados. 
Ryzhkov y Tolmachev (1983) obtuvieron las velocidades de propagación de las perturbaciones y estudiaron la resonancia en lechos fluidizados vibrantes. Actualmente, los métodos acústicos están siendo activamente investigados, debido a que son métodos para la adquisición de datos, que prometen ser efectivos y económicos en sistemas granulares (Weir, 2001).

Debe mencionarse que existen muchos trabajos en la literatura que analizan modelos compresibles. En particular, Ransom y Hicks (1984) discuten modelos transitorios unidimensionales, con dos campos de presión para el flujo separado de dos fases compresibles. Estos modelos conducen a sistemas de ecuaciones hiperbólicas con mayor significado físico que los modelos con un único campo de presión. Hay otros artículos (Needham y Merkin, 1983; 1984; 1986; Göz, 1992; 1993; Göz y Sundaresan, 1998; Glasser y col., 1996; 1997; Johri y Glasser, 2002; Hirayama y Takaki, 2000) donde se estudia la estabilidad lineal y no-lineal en lechos fluidizados en el régimen homogéneo o uniforme con la teoría de bifurcaciones.

Liu (1982) estudió la condición de estabilidad en lechos fluidizados siguiendo a Whitham (1974), quien mostró que la velocidad de propagación relevante del movimiento de más alto orden debe ser mayor que la rapidez de propagación del movimiento de más bajo orden. Liu encontró que la condición de estabilidad no se satisface cuando las ondas de más alto orden tienen rapideces menores que las ondas de órdenes más bajos.

En este Capítulo se revisa la consideración de incompresibilidad, construyendo una metodología que permite la comparación entre los modelos compresible e incompresible. Para alcanzar este propósito, en la Sección 4.2 de Jerarquía de ondas se presenta el desarrollo teórico utilizado para obtener los modelos de perturbaciones linealizados incompresible y compresible. Luego en la Sección 4.3 de Estabilidad, se desarrolla el análisis de estabilidad de Whitham por aproximación de velocidades de fase y extendiendo el enfoque de Liu en lechos fluidizados. En la Sección de Resultados, se aplica este método a dos sistemas, el primero es catalizador de FCC arrastrado por vapor de agua en condiciones isotérmicas y el segundo es un sistema de arena-aire reportado por van der Schaaf y col. (1998). 


\subsection{Jerarquía de ondas}

\subsubsection{Ecuaciones del modelo}

El sistema físico consiste de una dispersión monodispersa de partículas pequeñas (del orden de micrómetros) esféricas y rígidas de diámetro medio $d_{p}$ y densidad intrínseca $\rho_{s}$, moviéndose dentro de una corriente de gas en un tubo vertical a temperatura constante. El fluido se considera un gas newtoniano y compresible de densidad intrínseca $\rho_{g}$ y viscosidad $\mu_{g}$. La fracción volumen de sólido $\varepsilon_{s}$, se encuentra entre 0 y 0.5 y satisface la condición $\varepsilon_{s}+\varepsilon_{g}=1$, donde $\varepsilon_{g}$ es la fracción volumen de gas. La mezcla se mueve con una velocidad promedio $U_{m}=\varepsilon_{s} V_{s}+\varepsilon_{g} V_{g}$, donde $V_{g}$ y $V_{s}$ son los valores de las velocidades ascendentes con promedio ponderado en la masa del gas y sólido, respectivamente. Debido a que la mezcla se considera en un régimen altamente turbulento, los efectos viscosos son sólo significativos en una región cercana a la superficie de los gránulos. El número de Reynolds de las partículas es $\operatorname{Re}_{s}=d_{p} \rho_{g} U_{t} / \mu_{g}$, donde la velocidad terminal se define como $U_{t} \doteq\left|V_{s}-U_{m}\right|=\varepsilon_{g}\left(V_{g}-V_{s}\right)$. En este modelo, despreciamos los efectos viscosos en las fases, así como los esfuerzos turbulentos.

Partiremos del modelo promediado y cerrado constituido por las ecuaciones de conservación de la masa y de movimiento para las fases gas y sólida, representado en la Ec. (3.19) a la Ec. (3.22). No consideraremos los efectos de pared, por lo que $\beta_{g W} \rightarrow 0 \mathrm{y}$ $\beta_{s W} \rightarrow 0$. El conjunto de ecuaciones para las variables dependientes $\left\{\varepsilon_{g}, P_{g}, V_{g}, V_{s}\right\}$ es:

$$
\begin{aligned}
& \frac{\partial}{\partial t}\left[\varepsilon_{g} \rho_{g}\left(P_{g}\right)\right]+\frac{\partial}{\partial z}\left[\varepsilon_{g} \rho_{g}\left(P_{g}\right) V_{g}\right]=0, \\
& -\rho_{s} \frac{\partial}{\partial t} \varepsilon_{g}+\rho_{s} \frac{\partial}{\partial z}\left[\left(1-\varepsilon_{g}\right) V_{s}\right]=0, \\
& \frac{\partial}{\partial t}\left[\varepsilon_{g} \rho_{g}\left(P_{g}\right) V_{g}\right]+\frac{\partial}{\partial z}\left[\varepsilon_{g} \rho_{g}\left(P_{g}\right) V_{g}^{2}\right]+\varepsilon_{g} \frac{\partial}{\partial z} P_{g}+F+\rho_{g}\left(P_{g}\right) g=0, \\
& \rho_{s} \frac{\partial}{\partial t}\left[\left(1-\varepsilon_{g}\right) V_{s}\right]+\rho_{s} \frac{\partial}{\partial z}\left[\left(1-\varepsilon_{g}\right) V_{s}^{2}\right]+\left(1-\varepsilon_{g}\right) \frac{\partial}{\partial z} P_{g} \\
& -\Phi^{\prime}\left(1-\varepsilon_{g}\right) \frac{\partial}{\partial z} \varepsilon_{g}-F+\left(1-\varepsilon_{g}\right)\left[\rho_{s}-\rho_{g}\left(P_{g}\right)\right] g=0 .
\end{aligned}
$$


Aquí $z$ y $t$ son la coordenada vertical y el tiempo, respectivamente, $g$ es la fuerza gravitacional por unidad de masa y $P_{g}$ es la presión de la fase gas promediada; la densidad de los granos sólidos es constante, $\rho_{s}=\rho_{s 0}$. La presión promedio de la fase sólida $P_{s}$ se modeló a través de la cerradura $P_{s}=P_{g}+\Phi\left(\varepsilon_{s}\right)$ en la Ec. (4.4), donde la función $\Phi\left(\varepsilon_{s}\right)$ es el módulo de compresibilidad del sólido y $\Phi^{\prime}=d \Phi / d \varepsilon_{s}$. La cerradura simple $P_{s}=\Phi\left(\varepsilon_{s}\right)$ reduce el acoplamiento entre los balances de momentum a únicamente la fuerza de interacción hidrodinámica $F$ entre el fluido y las partículas. Esta cerradura fue utilizada por Jackson (1985) y Liu (1982) entre otros y fue recomendada por Gidaspow (1994).

Por otro lado, Jackson (1985) consideró dos contribuciones a $F$ : una fuerza de arrastre dominante y un efecto de masa agregada. Aquí sólo consideramos el primer efecto en $F$, tal que $F=\beta \varepsilon_{g}\left(1-\varepsilon_{g}\right)\left(V_{g}-V_{s}\right)$, donde $\beta=\left(3 / 4 d_{p}\right) C_{D} \rho_{g} U_{t}$ es un parámetro de interación. También, Jackson (1985), Liu (1982) y Homsy y col. (1980) consideraron un efecto de viscosidad efectiva en el régimen de fluidización denso, el cual no ha sido tomado en cuenta aquí. La principal diferencia entre los resultados presentados en este Capítulo y los trabajos previamente citados, es la estructura de las ecuaciones obtenidas y la forma en que se muestra explícitamente el efecto de compresibilidad sobre el efecto de incompresibilidad. Además, la compresibilidad se introduce explícitamente a través de una ecuación de estado de la fase gas en el régimen de fluidización rápida.

Para obtener la jerarquía ondulatoria de las perturbaciones para los modelos incompresible y compresible, se proponen soluciones ondulatorias para las ecuaciones linealizadas, Ec. (4.1) a la Ec. (4.4). La linealización se realizó alrededor de un estado base (denotado por un subíndice cero) más una pequeña perturbación tal que $\varepsilon_{g}=\varepsilon_{0}+\varepsilon, p_{g}=p_{0}+p$, $V_{g}=v_{g 0}+v_{g} \mathrm{y} V_{s}=v_{s 0}+v_{s}$.

En la siguiente subsección representamos las ecuaciones linealizadas en forma matricial y después presentamos el análisis comparativo de coeficientes entre los modelos. 


\subsubsection{Enunciado del problema}

Sea

$$
\mathbf{u}=\left(\begin{array}{llll}
\varepsilon & p & v_{g} & v_{s}
\end{array}\right)^{T}
$$

el vector de perturbaciones para el sistema linealizado de EDP de primer orden:

$$
\mathbf{B} \frac{\partial \mathbf{u}}{\partial t}+\mathbf{C} \frac{\partial \mathbf{u}}{\partial z}+\mathbf{D u}=\mathbf{0}
$$

donde las matrices B, C y $\mathbf{D}$ de tamaño $4 \times 4$, están dadas en el Apéndice $F$. Las derivadas espaciales de las perturbaciones de velocidad $v_{g} \mathrm{y} v_{s}$ pueden obtenerse de las ecuaciones de continuidad, luego se sustituyen en las ecuaciones de momentum para llegar así a dos ecuaciones linealizadas de segundo orden para las perturbaciones de la fracción volumen $(\varepsilon)$ y para las perturbaciones de la presión $(p)$,

$$
\begin{aligned}
& L_{1} \varepsilon+\left[L_{3}+\left(\frac{d \rho_{g}}{d P_{g}}\right)_{0} K_{1}\right] p=0, \\
& L_{2} \varepsilon+\left[L_{3}+\left(\frac{d \rho_{g}}{d P_{g}}\right)_{0} K_{2}\right] p=0 .
\end{aligned}
$$

Los operadores diferenciales de segundo orden $L_{1}, L_{2}, L_{3}$ y $K_{1}$, así como el operador de primer orden $K_{2}$, están dados por

$$
\begin{aligned}
& L_{1} \doteq-\frac{\rho_{g 0}}{\varepsilon_{0}}\left(\frac{\partial}{\partial t}+v_{g 0} \frac{\partial}{\partial z}\right)^{2}-\beta\left(\frac{\partial}{\partial t}+v_{g 0} \frac{\partial}{\partial z}\right)-\frac{1-\varepsilon_{0}}{\varepsilon_{0}} \beta\left(\frac{\partial}{\partial t}+v_{s 0} \frac{\partial}{\partial z}\right) \\
& L_{2} \doteq \frac{\rho_{s 0}}{1-\varepsilon_{0}}\left(\frac{\partial}{\partial t}+v_{s 0} \frac{\partial}{\partial z}\right)^{2}+\frac{\varepsilon_{0}}{1-\varepsilon_{0}} \beta\left(\frac{\partial}{\partial t}+v_{g 0} \frac{\partial}{\partial z}\right)+\beta\left(\frac{\partial}{\partial t}+v_{s 0} \frac{\partial}{\partial z}\right) \\
& -\Phi_{0}^{\prime} \frac{\partial^{2}}{\partial z^{2}}-\frac{\rho_{s 0}-\rho_{g 0}}{1-\varepsilon_{0}} g \frac{\partial}{\partial z} \\
& L_{3} \doteq \frac{\partial^{2}}{\partial z^{2}}, \\
& K_{1} \doteq-\left(\frac{\partial}{\partial t}+v_{g 0} \frac{\partial}{\partial z}\right)^{2}-\left(1-\varepsilon_{0}\right) \frac{\beta}{\rho_{g 0}}\left(\frac{\partial}{\partial t}+v_{g 0} \frac{\partial}{\partial z}\right)+\frac{g}{\varepsilon_{0}} \frac{\partial}{\partial z} \\
& K_{2} \doteq \varepsilon_{0} \frac{\beta}{\rho_{g 0}}\left(\frac{\partial}{\partial t}+v_{g 0} \frac{\partial}{\partial z}\right)-g \frac{\partial}{\partial z} .
\end{aligned}
$$


El factor $\left(d \rho_{g} / d P_{g}\right)_{0}$ toma en cuenta el cambio de la densidad del fluido como una función de la presión en el mismo, a través de una ecuación de estado isotérmica $\rho_{g}=\rho_{g}\left(P_{g}\right)$. La velocidad del sonido en un gas homogéneo puede introducirse al considerar la relación $\left(d \rho_{g} / d P_{g}\right)_{0}=s^{-2}$, donde $s$ es la velocidad del sonido en el gas puro. De la Ec. (4.7) y de la Ec. (4.8), puede recuperarse la aproximación de incompresibilidad al tomar el límite $\left(d \rho_{g} / d P_{g}\right)_{0}=0$ o en forma equivalente, cuando $s \rightarrow \infty$. Bajo la aproximación de incompresibilidad puede encontrarse en forma directa una EDP de segundo orden para $\varepsilon$ al restar la Ec. (4.7) a la Ec. (4.8), obteniendo $\left(L_{2}-L_{1}\right) \varepsilon=0$. También, puede obtenerse alternativamente una EDP de cuarto orden para $p$ mediante la aplicación de los operadores $L_{2}$ y $L_{1}$ en la Ec. (4.7) y en la Ec. (4.8), respectivamente y restando se obtiene $\left(L_{2}-L_{1}\right) L_{3} p=0$. Entonces, mientras la dinámica de la fracción volumen se representa por una EDP de segundo orden, la dinámica de las perturbaciones de presión es el mismo operador aplicado a una derivada espacial de segundo orden. Más aún, si la cerradura de la presión del sólido, tal como $P_{s}=\Phi\left(\varepsilon_{s}\right)$, permite el desacoplamiento del término de presión, el balance de momentum de la fase sólida da la forma reducida $L_{2} \varepsilon=0$. El comportamiento completo puede también ser expresado como una ecuación de onda para $\varepsilon$ o para $p$. Entonces, la aplicación cruzada de los operadores $L_{2}$ y $L_{1}$ a la Ec. (4.7) y a la Ec. (4.8) y restando obtenemos una EDP de cuarto orden para las perturbaciones de presión. Un procedimiento alternativo da la misma EDP para las perturbaciones de fracción volumen,

$$
\left[\left(L_{2}-L_{1}\right) L_{3}-\frac{1}{s^{2}}\left(L_{1} K_{2}-L_{2} K_{1}\right)\right] \varphi=0,
$$

donde $\varphi$ es $\varepsilon$ ó $p$. El comportamiento ondulatorio de las perturbaciones de $\varepsilon$ del modelo incompresible es representado por el operador diferencial $\left(L_{2}-L_{1}\right)$, el cual puede descomponerse y analizarse en forma similar a como lo hizo Liu (1982) para un lecho fluidizado, incorporando las diferencias en el régimen de fluidización y en los efectos físicos tomados en cuenta. 
En esta forma obtenemos

$$
\begin{aligned}
L_{2}-L_{1}= & \frac{\rho_{g 0}}{\varepsilon_{0}}\left(\frac{\partial}{\partial t}+v_{g 0} \frac{\partial}{\partial z}\right)^{2}+\frac{\rho_{s 0}}{1-\varepsilon_{0}}\left(\frac{\partial}{\partial t}+v_{s 0} \frac{\partial}{\partial z}\right)^{2}+\frac{\beta}{1-\varepsilon_{0}}\left(\frac{\partial}{\partial t}+v_{g 0} \frac{\partial}{\partial z}\right) \\
& +\frac{\beta}{\varepsilon_{0}}\left(\frac{\partial}{\partial t}+v_{s 0} \frac{\partial}{\partial z}\right)-\Phi_{0}^{\prime} \frac{\partial^{2}}{\partial z^{2}}-\frac{\rho_{s 0}-\rho_{g 0}}{1-\varepsilon_{0}} g \frac{\partial}{\partial z}
\end{aligned}
$$

donde los términos ondulatorios de segundo orden representan los efectos inerciales del fluido y de las partículas, mientras que los términos ondulatorios de primer orden provienen de las perturbaciones de la fuerza de arrastre interfacial. Los términos de segundo y de primer orden están relacionados, respectivamente, al módulo de compresibilidad de la fase sólida y a las perturbaciones de la fuerza de flotación. El operador diferencial $\left(L_{2}-L_{1}\right)$ se modifica adicionalmente por $L_{3} p$, un operador de segundo orden aplicado a $p$. Esto puede visualizarse como un efecto de difusión en la estructura ondulatoria de segundo orden. También, este término da lugar a términos diferenciales de tercero y de cuarto orden que gobiernan la dinámica de la perturbación de la presión en la aproximación de incompresibilidad. Por otro lado, el operador $\left(L_{2} K_{1}-L_{1} K_{2}\right)$ es la contribución dinámica de la densidad del fluido a todas las fuerzas mencionadas, dando sus correspondientes efectos de interacción. Este operador está ponderado por el factor $s^{-2}$ y está dado por

$$
\begin{aligned}
& -\left(L_{2} K_{1}-L_{1} K_{2}\right) \doteq\left[-\frac{\rho_{s 0}}{1-\varepsilon_{0}}\left(\frac{\partial}{\partial t}+v_{s 0} \frac{\partial}{\partial z}\right)^{2}+\Phi_{0}^{\prime} \frac{\partial^{2}}{\partial z^{2}}\right]\left(\frac{\partial}{\partial t}+v_{g 0} \frac{\partial}{\partial z}\right)^{2} \\
& +\left[\begin{array}{c}
\frac{1-2 \varepsilon_{0}}{1-\varepsilon_{0}} \beta\left(\frac{\partial}{\partial t}+v_{g 0} \frac{\partial}{\partial z}\right) \\
-\beta\left(\frac{\partial}{\partial t}+v_{s 0} \frac{\partial}{\partial z}\right)+\frac{\varepsilon_{0} \rho_{s 0}-\rho_{g 0}}{\varepsilon_{0}\left(1-\varepsilon_{0}\right)} g \frac{\partial}{\partial z}
\end{array}\right]\left(\frac{\partial}{\partial t}+v_{g 0} \frac{\partial}{\partial z}\right)^{2} \\
& +\left[-\beta \frac{\rho_{s 0}}{\rho_{g 0}}\left(\frac{\partial}{\partial t}+v_{g 0} \frac{\partial}{\partial z}\right)+\frac{\rho_{s 0} g}{\varepsilon_{0}\left(1-\varepsilon_{0}\right)} \frac{\partial}{\partial z}\right]\left(\frac{\partial}{\partial t}+v_{s 0} \frac{\partial}{\partial z}\right)^{2} \\
& +\left(1-\varepsilon_{0}\right) \Phi_{0}^{\prime} \frac{\beta}{\rho_{g 0}}\left(\frac{\partial}{\partial t}+v_{g 0} \frac{\partial}{\partial z}\right) \frac{\partial^{2}}{\partial z^{2}}-\frac{1}{\varepsilon_{0}} \Phi_{0}^{\prime} g \frac{\partial^{3}}{\partial z^{3}} \\
& +\left(\frac{\rho_{s 0}}{\rho_{g 0}}-\frac{1-2 \varepsilon_{0}}{1-\varepsilon_{0}}\right) \beta g\left(\frac{\partial}{\partial t}+v_{g 0} \frac{\partial}{\partial z}\right) \frac{\partial}{\partial z} \\
& +\beta g\left(\frac{\partial}{\partial t}+v_{s 0} \frac{\partial}{\partial z}\right) \frac{\partial}{\partial z}-\frac{\rho_{s 0}-\rho_{g 0}}{\varepsilon_{0}\left(1-\varepsilon_{0}\right)} g^{2} \frac{\partial^{2}}{\partial z^{2}} \text {. }
\end{aligned}
$$


En la Ec. (4.16), están tomadas en cuenta las interacciones ondulatoria y difusiva, así como interacciones espaciales. Estas están asociadas a las fuerzas inerciales del fluido y del sólido, compresibilidad del sólido, arrastre viscoso interfacial y flotación. Ninguna de estas fuerzas aparece sola en la Ec. (4.16), sino que aparecen como pares de interacciones con algún otro efecto. Cada uno de estos pares representa el aumento debido a los pares de fuerza considerados, debidos al cambio de la densidad del fluido. El punto aquí es cuantificar la magnitud de tal aumento al compararlo con el modelo incompresible.

\subsubsection{Estructuras ondulatorias}

Al agrupar los operadores diferenciales dados por la Ec. (4.15) y la Ec. (4.16) en una estructura ondulatoria y sustituirlos en la Ec. (4.14), se obtiene la siguiente forma equivalente

$$
\begin{aligned}
&\left(L_{2}-L_{1}\right) L_{3}-\frac{1}{s^{2}}\left(L_{1} K_{2}-L_{2} K_{1}\right)= {\left[\tau\left(\frac{\partial}{\partial t}+c_{1} \frac{\partial}{\partial z}\right)\left(\frac{\partial}{\partial t}+c_{2} \frac{\partial}{\partial z}\right)+\left(\frac{\partial}{\partial t}+a \frac{\partial}{\partial z}\right)\right] \frac{\partial^{2}}{\partial z^{2}} } \\
&-\frac{1}{s^{2}}\left[\begin{array}{r}
T\left(\frac{\partial}{\partial t}+\eta_{41} \frac{\partial}{\partial z}\right)\left(\frac{\partial}{\partial t}+\eta_{42} \frac{\partial}{\partial z}\right)\left(\frac{\partial}{\partial t}+\eta_{43} \frac{\partial}{\partial z}\right)\left(\frac{\partial}{\partial t}+\eta_{44} \frac{\partial}{\partial z}\right) \\
+C\left(\frac{\partial}{\partial t}+\eta_{31} \frac{\partial}{\partial z}\right)\left(\frac{\partial}{\partial t}+\eta_{32} \frac{\partial}{\partial z}\right)\left(\frac{\partial}{\partial t}+\eta_{33} \frac{\partial}{\partial z}\right) \\
-g C\left(\frac{\partial}{\partial t}+\eta_{21} \frac{\partial}{\partial z}\right) \frac{\partial}{\partial z}
\end{array}\right] .
\end{aligned}
$$

Aquí hemos utilizado la notación establecida por Liu (1982) para denotar los parámetros correspondientes al comportamientobásicoincompresible.Los tiempos derelajación $\tau$ y $T$ son

$$
\begin{aligned}
& \tau \doteq \frac{\left(1-\varepsilon_{0}\right) \rho_{g 0}+\varepsilon_{0} \rho_{s 0}}{\beta} \\
& T \doteq \frac{\varepsilon_{0} \rho_{s 0}}{\beta}
\end{aligned}
$$

y el coeficiente adimensional $C$, se define como

$$
C \doteq \varepsilon_{0} \frac{\varepsilon_{0} \rho_{g 0}+\left(1-\varepsilon_{0}\right) \rho_{s 0}}{\rho_{g 0}}
$$


Las rapideces de propagación se encontraron al resolver diferentes conjuntos de ecuaciones algebraicas para los casos incompresible y compresible, como se discute a continuación. Las rapideces de propagación de segundo orden incompresibles $c_{1}$ y $c_{2}$ se encuentran como soluciones de la Ec. (G.1) y de la Ec. (G.2) del Apéndice G, obteniéndose

$$
c_{1}, c_{2} \doteq \frac{\left(1-\varepsilon_{0}\right) \rho_{g 0} v_{g 0}+\varepsilon_{0} \rho_{s 0} v_{s 0}}{\left(1-\varepsilon_{0}\right) \rho_{g 0}+\varepsilon_{0} \rho_{s 0}} \pm \frac{\sqrt{\varepsilon_{0}\left(1-\varepsilon_{0}\right) \rho_{g 0} \rho_{s 0}\left[\left(\frac{1-\varepsilon_{0}}{\rho_{s 0}}+\frac{\varepsilon_{0}}{\rho_{g 0}}\right) \Phi_{0}^{\prime}-\left(v_{g 0}-v_{s 0}\right)^{2}\right]}}{\left(1-\varepsilon_{0}\right) \rho_{g 0}+\varepsilon_{0} \rho_{s 0}} .
$$

Las rapideces de propagación para el modelo compresible se obtuvieron de conjuntos de ecuaciones algebraicas construidas al ajustar la forma expandida de los polinomios de la Ec. (4.17) y en variables originales, Ec. (4.15) y Ec. (4.16) (ver Apéndice G). Las expresiones para las rapideces de propagación de la onda de primer orden del modelo incompresible $a$ y de la onda compresible de segundo orden $\eta_{21}$ son

$$
\begin{aligned}
& a=\varepsilon_{0} v_{g 0}+\left(1-\varepsilon_{0}\right) v_{s 0}-\varepsilon_{0}\left(\rho_{s 0}-\rho_{g 0}\right) \frac{g}{\beta}, \\
& \eta_{21}=\frac{\left(2 \varepsilon_{0}-1\right) \rho_{g 0} v_{g 0}+\left(1-\varepsilon_{0}\right) \rho_{s 0} v_{g 0}+\left(1-\varepsilon_{0}\right) \rho_{g 0} v_{s 0}+\rho_{g 0}\left(\rho_{s 0}-\rho_{g 0}\right) \frac{g}{\varepsilon_{0} \beta}}{\varepsilon_{0} \rho_{g 0}+\left(1-\varepsilon_{0}\right) \rho_{s 0}} .
\end{aligned}
$$

Las expresiones análogas para las rapideces compresibles de cuarto orden $\eta_{4 k}, k=1,2,3,4$ son

$$
\begin{aligned}
& \eta_{41}=\eta_{42}=v_{g 0}, \\
& \eta_{43}, \eta_{44}=v_{s 0} \pm \sqrt{\frac{1-\varepsilon_{0}}{\rho_{s 0}} \Phi_{0}^{\prime}} .
\end{aligned}
$$

Estas raíces son reales y coinciden con los eigenvalores del polinomio característico del sistema de EDP linealizado de primer orden, Ec. (4.6). Por lo tanto, el sistema es estable en el sentido de ser hiperbólico, pero no totalmente hiperbólico ya que $\eta_{41}=\eta_{42}$.

Las expresiones para las rapideces de propagación de tercer orden $\eta_{31}, \eta_{32}$ y $\eta_{33}$ se encuentran de la Ec. (G.7) a la Ec. (G.9). Puesto que las expresiones completas en términos de parámetros originales son demasiado largas y complejas, no las incluiremos aquí, sino que presentaremos cálculos numéricos. 
Para simplificar aun más la Ec. (4.17), consideraremos su forma adimensional que se obtiene al utilizar la longitud de la columna $L$ y la velocidad promedio aritmético $U_{0}=\left(v_{g 0}+v_{s 0}\right) / 2$ como variables características. Las variables adimensionales son $z^{\prime} \doteq z / L, \quad a^{\prime} \doteq a / U_{0}, t^{\prime} \doteq t U_{0} / L$ y todas las rapideces de propagación adimensionales pueden definirse en forma similar a $a^{\prime}$. Por lo tanto, la Ec. (4.17) puede reescribirse como

$$
\begin{gathered}
{\left[\tau^{\prime}\left(\frac{\partial}{\partial t^{\prime}}+c_{1}^{\prime} \frac{\partial}{\partial z^{\prime}}\right)\left(\frac{\partial}{\partial t^{\prime}}+c_{2}^{\prime} \frac{\partial}{\partial z^{\prime}}\right) \frac{\partial^{2}}{\partial z^{\prime 2}}+\left(\frac{\partial}{\partial t^{\prime}}+a^{\prime} \frac{\partial}{\partial z^{\prime}}\right) \frac{\partial^{2}}{\partial z^{\prime 2}}\right] \varphi} \\
-C^{\prime}\left[\begin{array}{r}
\left.T^{\prime}\left(\frac{\partial}{\partial t^{\prime}}+\eta_{41}^{\prime} \frac{\partial}{\partial z^{\prime}}\right)\left(\frac{\partial}{\partial t^{\prime}}+\eta_{42}^{\prime} \frac{\partial}{\partial z^{\prime}}\right)\left(\frac{\partial}{\partial t^{\prime}}+\eta_{43}^{\prime} \frac{\partial}{\partial z^{\prime}}\right)\left(\frac{\partial}{\partial t^{\prime}}+\eta_{44}^{\prime} \frac{\partial}{\partial z^{\prime}}\right)\right] \\
+\left(\frac{\partial}{\partial t^{\prime}}+\eta_{31}^{\prime} \frac{\partial}{\partial z^{\prime}}\right)\left(\frac{\partial}{\partial t^{\prime}}+\eta_{32}^{\prime} \frac{\partial}{\partial z^{\prime}}\right)\left(\frac{\partial}{\partial t^{\prime}}+\eta_{33}^{\prime} \frac{\partial}{\partial z^{\prime}}\right) \\
\left.-G^{\prime}\left(\frac{\partial}{\partial t^{\prime}}+\eta_{21}^{\prime} \frac{\partial}{\partial z^{\prime}}\right) \frac{\partial}{\partial z^{\prime}}\right]
\end{array}\right] \varphi=0
\end{gathered}
$$

donde los índices prima denotan coeficientes adimensionales, $\tau^{\prime} \doteq U_{0} \tau / L, T^{\prime} \doteq U_{0} T / L C$, $C^{\prime} \doteq\left(U_{0} / s\right)^{2} C$ y $G^{\prime} \doteq L g / U_{0}{ }^{2}$. Debemos señalar que la onda de cuarto orden del modelo compresible puede despreciarse, al compararla con la de tercer orden, si $T^{\prime} \ll 1$. Puesto que $\rho_{s 0} \gg \rho_{g 0}$, esta condición se cumple si $\tau^{\prime} \ll \frac{\rho_{s 0}}{\rho_{g 0}}\left(1-\varepsilon_{0}\right) \varepsilon_{0}$, la cual se satisface en una zona delgada cercana al límite cuando $\varepsilon_{0} \rightarrow 1$. Fuera de esta zona, donde

$$
\varepsilon_{s 0}>\varepsilon_{s 0, \min } \approx \frac{1}{2}-\sqrt{\frac{1}{4}-100 \frac{\rho_{g 0}}{\rho_{s 0}} \tau^{\prime}}
$$

el efecto de compresibilidad asociado a los términos de cuarto orden es despreciable comparado con los términos de segundo y de tercer orden. Así, una forma simplificada de la Ec. (4.17) es

$$
\begin{gathered}
{\left[\tau\left(\frac{\partial}{\partial t}+c_{1} \frac{\partial}{\partial z}\right)\left(\frac{\partial}{\partial t}+c_{2} \frac{\partial}{\partial z}\right)+\left(\frac{\partial}{\partial t}+a \frac{\partial}{\partial z}\right)\right] \frac{\partial^{2}}{\partial z^{2}} \varphi} \\
-\frac{C}{s^{2}}\left[\left(\frac{\partial}{\partial t}+\eta_{31} \frac{\partial}{\partial z}\right)\left(\frac{\partial}{\partial t}+\eta_{32} \frac{\partial}{\partial z}\right)\left(\frac{\partial}{\partial t}+\eta_{33} \frac{\partial}{\partial z}\right)-g\left(\frac{\partial}{\partial t}+\eta_{21} \frac{\partial}{\partial z}\right) \frac{\partial}{\partial z}\right] \varphi=0
\end{gathered}
$$


O

$$
\tau\left(\frac{\partial}{\partial t}+c_{1} \frac{\partial}{\partial z}\right)\left(\frac{\partial}{\partial t}+c_{2} \frac{\partial}{\partial z}\right) \frac{\partial^{2}}{\partial z^{2}} \varphi-\frac{C}{s^{2}}\left[\begin{array}{r}
\left(\frac{\partial}{\partial t}+\alpha_{1} \frac{\partial}{\partial z}\right)\left(\frac{\partial}{\partial t}+\alpha_{2} \frac{\partial}{\partial z}\right)\left(\frac{\partial}{\partial t}+\alpha_{3} \frac{\partial}{\partial z}\right) \\
-g\left(\frac{\partial}{\partial t}+\eta_{21} \frac{\partial}{\partial z}\right) \frac{\partial}{\partial z}
\end{array}\right] \varphi=0 .
$$

Aquí $\alpha_{i}, i=1,2,3$ son las rapideces de propagación que toman en cuenta tanto el comportamiento compresible como el incompresible y son las raíces de

$$
\alpha^{3}-P_{1} \alpha^{2}+\left(P_{2}-\frac{s^{2}}{C}\right) \alpha-\left(P_{3}-\frac{s^{2} a}{C}\right)=0
$$

donde los polinomios $P_{k}, \quad k=1,2,3$ están definidos en el Apéndice $G$. Aunque la Ec. (4.28) y la Ec. (4.29) son equivalentes, en ellas pueden observarse diferentes aspectos correspondientes al comportamiento físico. En el primer corchete de la Ec. (4.28), aparece la estructura original del modelo incompresible, como fue reportada por Liu (1982). Sin embargo, las ondas de primer y de segundo orden en este corchete están aplicadas a una derivada espacial de segundo orden. Por otro lado, en la Ec. (4.29), se presenta la estructura de la forma ondulatoria completa con un sólo término correspondiente a cada jerarquía de onda. En la Ec. (4.28), se expresa completamente el efecto de compresibilidad en el segundo corchete con un coeficiente proporcional a $s^{-2}$, llevando al modelo incompresible al tomar el límite $s \rightarrow \infty$ y con rapideces de propagación, $\eta_{i j}$, que son independientes de la velocidad del sonido $s$. En contraste, en la Ec. (4.29) las rapideces de propagación $\alpha_{1}$ y $\alpha_{3}$ son altamente dependientes de $s$ y el proceso de límite para obtener el modelo incompresible $s \rightarrow \infty$, debe hacerse con cuidado de los valores límite de estas rapideces de propagación $\alpha_{j} \rightarrow \pm \infty, j=1,3$. Esto puede obtenerse cuando el producto de operadores hiperbólicos genera un comportamiento parabólico expresado por el operador $\partial^{2} / \partial z^{2}$, junto con el hecho de que $\alpha_{2} \rightarrow a$. Además, el término compresible de segundo orden, en la Ec. (4.28) o en la Ec. (4.29), es una onda degenerada de segundo orden asociada a los efectos de gravedad con sólo una rapidez de propagación finita. 


\subsection{Análisis de estabilidad}

\subsubsection{Análisis de estabilidad de Whitham por aproximaciones de velocidades de fase}

Para obtener un criterio de estabilidad, asumimos soluciones para $\varphi(z, t)$ en la Ec. (4.29) dadas por funciones de onda (Whitham, 1974) $\varphi(z, t)=\mathscr{C A} e^{i(\kappa z-\omega t)}$, donde $\kappa \in \mathbb{R}$ es el número de onda y $\omega=\omega_{R}+i \omega_{I} \in \mathbb{C}$ está compuesta por la frecuencia $\omega_{R}$ y por el factor de atenuación de onda $\omega_{I}$. Por lo tanto, debe satisfacerse que $\omega_{I}<0$ para obtener una condición de estabilidad. La aplicación de esta solución a la Ec. (4.29) nos lleva a

$$
\tau\left(c_{1} \kappa-\omega\right)\left(c_{2} \kappa-\omega\right) \kappa^{2}-\frac{C}{s^{2}}\left[\begin{array}{r}
-i\left(\alpha_{1} \kappa-\omega\right)\left(\alpha_{2} \kappa-\omega\right)\left(\alpha_{3} \kappa-\omega\right) \\
+g\left(\eta_{21} \kappa-\omega\right) \kappa
\end{array}\right]=0 .
$$

Ahora, es posible analizar el comportamiento de la rapidez de la onda cercana a cada una de las velocidades de fase, siguiendo el criterio de Whitham (1974) en las jerarquías de onda. Para obtener algunas desigualdades que se utilizarán más adelante, asumamos que el tamaño relativo de las rapideces de propagación es tal que: (1) $c_{1}>c_{2}$ y (2) $\alpha_{1}>\alpha_{2}>\alpha_{3}$. Por lo tanto, analizaremos la Ec. (4.31) viajando a una velocidad de fase cercana a $\alpha_{1}$; es decir, bajo la acción ondulatoria $\omega \rightarrow \alpha_{1} \kappa$ tal que

$$
\tau\left(c_{1}-\alpha_{1}\right)\left(c_{2}-\alpha_{1}\right) \kappa^{2}+i \frac{C}{s^{2}}\left(\alpha_{1} \kappa-\omega\right)\left(\alpha_{2}-\alpha_{1}\right)\left(\alpha_{3}-\alpha_{1}\right)-\frac{C g}{s^{2}}\left(\eta_{21}-\alpha_{1}\right)=0 .
$$

Que implica que

$$
\omega=\alpha_{1} \kappa+i \frac{g\left(\eta_{21}-\alpha_{1}\right)-\frac{s^{2} \kappa^{2}}{C} \tau\left(c_{1}-\alpha_{1}\right)\left(c_{2}-\alpha_{1}\right)}{\left(\alpha_{2}-\alpha_{1}\right)\left(\alpha_{3}-\alpha_{1}\right)},
$$

donde su parte imaginaria debe satisfacer la desigualdad

$$
\omega_{I}=\frac{g\left(\eta_{21}-\alpha_{1}\right)-\frac{s^{2} \kappa^{2}}{C} \tau\left(c_{1}-\alpha_{1}\right)\left(c_{2}-\alpha_{1}\right)}{\left(\alpha_{2}-\alpha_{1}\right)\left(\alpha_{3}-\alpha_{1}\right)}<0,
$$

para ser estable. Considerando que $c_{1}>c_{2}$ y $\alpha_{1}>\alpha_{2}>\alpha_{3}$, dados arriba, la Ec. (4.33) puede simplificarse para dar la condición

$$
-\left(\alpha_{1}-c_{1}\right)\left(\alpha_{1}-c_{2}\right)<-\frac{C g}{\tau s^{2} \kappa^{2}}\left(\eta_{21}-\alpha_{1}\right) .
$$


Un procedimiento similar para $\omega \rightarrow \alpha_{2} \kappa, \omega \rightarrow \alpha_{3} \kappa, \omega \rightarrow \eta_{21} \kappa, \omega \rightarrow c_{1} \kappa$ y $\omega \rightarrow c_{2} \kappa$, nos da que

$$
\begin{gathered}
\left(\alpha_{2}-c_{1}\right)\left(\alpha_{2}-c_{2}\right)<\frac{C g}{\tau s^{2} \kappa^{2}}\left(\eta_{21}-\alpha_{2}\right), \\
-\left(\alpha_{3}-c_{1}\right)\left(\alpha_{3}-c_{2}\right)<-\frac{C g}{\tau s^{2} \kappa^{2}}\left(\eta_{21}-\alpha_{3}\right), \\
\left(\eta_{21}-\alpha_{1}\right)\left(\eta_{21}-\alpha_{2}\right)\left(\eta_{21}-\alpha_{3}\right)<0, \\
-\left(\alpha_{1}-c_{1}\right)\left(\alpha_{2}-c_{1}\right)\left(\alpha_{3}-c_{1}\right)<0
\end{gathered}
$$

$\mathrm{y}$

$$
\left(\alpha_{1}-c_{2}\right)\left(\alpha_{2}-c_{2}\right)\left(\alpha_{3}-c_{2}\right)<0
$$

respectivamente. Puesto que $\alpha_{1}>\alpha_{2}>\alpha_{3}$ la desigualdad de la Ec. (4.37) implica que $\eta_{21}$ debe ser tal que $\alpha_{1}>\eta_{21}>\alpha_{2}$ ó $\alpha_{3}>\eta_{21}$. Similarmente, la desigualdad de la Ec. (4.38) requiere que $c_{1}$ deba ser tal que $\alpha_{1}>c_{1}>\alpha_{2}$ ó $\alpha_{3}>c_{1}$ y la desigualdad de la Ec. (4.39) que $c_{2}$ debe ser tal que $\alpha_{2}>c_{2}>\alpha_{3}$. Por lo tanto, encontramos que ambas secuencias $\alpha_{1}>c_{1}>\alpha_{2}>c_{2}>\alpha_{3}$ y $\alpha_{1}>\eta_{21}>\alpha_{2}$ deben satisfacerse o $\alpha_{1}>c_{1}>\alpha_{2}>c_{2}>\alpha_{3}>\eta_{21}$ para que el sistema sea estable. Sin embargo, en la Sección 4.4 de Resultados y Discusión se mostrará que $\eta_{21}$ no es la rapidez de propagación menor, por lo que la desigualdad $\alpha_{1}>c_{1}>\alpha_{2}>c_{2}>\alpha_{3}>\eta_{21}$ no se desarrollará más. Si tenemos $\alpha_{1}>c_{1}>\alpha_{2}>c_{2}>\alpha_{3}$ y $\alpha_{1}>\eta_{21}>\alpha_{2}$, las desigualdades de la Ec. (4.34) a la Ec. (4.36) nos conducen a que

$$
\begin{aligned}
& \frac{\left(\alpha_{1}-c_{1}\right)\left(\alpha_{1}-c_{2}\right)}{\left(c_{1}-\alpha_{3}\right)\left(c_{2}-\alpha_{3}\right)}>-\frac{\left(\alpha_{1}-\eta_{21}\right)}{\left(\eta_{21}-\alpha_{3}\right)}, \\
& \frac{\left(c_{1}-\alpha_{2}\right)\left(\alpha_{2}-c_{2}\right)}{\left(c_{1}-\alpha_{3}\right)\left(c_{2}-\alpha_{3}\right)}>-\frac{\left(\eta_{21}-\alpha_{2}\right)}{\left(\eta_{21}-\alpha_{3}\right)}
\end{aligned}
$$

$\mathrm{y}$

$$
\frac{\tau s^{2} \kappa^{2}}{C g}\left(c_{2}-\alpha_{3}\right)>\frac{\left(\eta_{21}-\alpha_{3}\right)}{\left(c_{1}-\alpha_{3}\right)},
$$

como otras formas de las mismas condiciones de estabilidad. Como todos los términos en las Ec. (4.40) y en la Ec. (4.41) son positivos, se sigue que no hay restricciones adicionales obtenidas de estas dos condiciones. 
Más aún, la Ec. (4.42) puede ser rearreglada como una condición límite en el número de onda,

$$
\kappa>\kappa_{0}=\sqrt{\frac{C g}{\tau s^{2}} \frac{\left(\eta_{21}-\alpha_{3}\right)}{\left(c_{1}-\alpha_{3}\right)\left(c_{2}-\alpha_{3}\right)}}
$$

para que el lecho fluidizado sea linealmente estable.

\subsubsection{Extensiones al enfoque de Liu (1982)}

El análisis previo mostró que la compresibilidad de la fase gas aumenta la región de estabilidad cuando se compara con el comportamiento incompresible. Sin embargo, para desarrollar un análisis de estabilidad siguiendo el enfoque de Liu (1982), comenzamos con la transformada de Fourier de la Ec. (4.28)

$$
\left[\tau\left(c_{1} \kappa-\omega\right)\left(c_{2} \kappa-\omega\right)-i(a \kappa-\omega)\right] \kappa^{2}-\frac{C}{s^{2}}\left[\begin{array}{r}
-i\left(\eta_{31} \kappa-\omega\right)\left(\eta_{32} \kappa-\omega\right)\left(\eta_{33} \kappa-\omega\right) \\
+g\left(\eta_{21} \kappa-\omega\right) \kappa
\end{array}\right]=0
$$

En esta ecuación, las condiciones de estabilidad pueden encontrarse al considerar que las ondas se mueven con rapideces cercanas a las de las ondas de segundo orden incompresibles y luego tomando los límites cuando $\omega \rightarrow c_{1} \kappa$ y $\omega \rightarrow c_{2} \kappa$. De la Ec. (4.44) se sigue que

$$
c_{1}+h_{1}=c_{1}-\frac{C}{s^{2}}\left(c_{1}^{3}-P_{1} c_{1}^{2}+P_{2} c_{1}-P_{3}\right)>a>c_{2}-\frac{C}{s^{2}}\left(c_{2}^{3}-P_{1} c_{2}^{2}+P_{2} c_{2}-P_{3}\right)=c_{2}-h_{2},
$$

donde $P_{k}, k=1,2,3$ están dados en el Apéndice $G$.

Este criterio se reduce a la condición bien conocida $c_{1}>a>c_{2}$ en el límite de incompresibilidad y clarifica cómo los efectos de compresibilidad modifican estos límites de estabilidad a través de las funciones $h_{1} \mathrm{y} h_{2}$. 


\subsection{Resultados y discusión}

El procedimiento desarrollado en la Sección 4.3 de análisis de estabilidad se aplicó a dos sistemas. El primer sistema es un catalizador de FCC acarreado verticalmente por un flujo turbulento de vapor de agua, en una unidad típica (riser) industrial, a las condiciones de operación similares en el riser (Kellogg, 1984). El segundo es un sistema experimental arena-aire en un CFB, reportado por van der Schaaf y col. (1998).

4.4.1. Valores de los parámetros y de las condiciones de operación de un sistema catalizador-vapor en una unidad de FCC y un sistema de arena-aire en un CFB

En la Tabla 4.1 se reportan las propiedades físicas del sólido y del fluido, así como también los parámetros geométricos relevantes y las condiciones de operación en un estado base homogéneo para ambos sistemas.

Tabla 4.1. Valores de los parámetros y de las condiciones de operación en estado base.

\begin{tabular}{lll}
\hline \multicolumn{1}{c}{ Parámetro } & Catalizador-vapor & Arena-aire \\
\hline $\begin{array}{l}\text { Velocidad del sonido en el fluido } \\
\text { puro, } s(\mathrm{~m} / \mathrm{s})\end{array}$ & 655 & 346 \\
Densidad del sólido, $\rho_{s 0}\left(\mathrm{~kg} / \mathrm{m}^{3}\right)$ & 1300 & 2650 \\
Densidad del fluido, $\rho_{g 0}\left(\mathrm{~kg} / \mathrm{m}^{3}\right)$ & 0.7065 & 0.9340 \\
Diámetro del grano sólido, $d_{p}(\mu \mathrm{m})$ & 60 & 310 \\
Velocidad terminal, $U_{t}(\mathrm{~m} / \mathrm{s})$ & 0.085 & 2.231 \\
Velocidad superficial del fluido, $U_{g}$ & & \\
$(\mathrm{~m} / \mathrm{s})$ & 7.000 & 3.200 \\
Diámetro de la columna, $D_{t}(\mathrm{~m})$ & 1.168 & 0.960 \\
Longitud de la columna, $L(\mathrm{~m})$ & 22 & 9 \\
Número de Reynolds del fluido, $\mathrm{Re}_{g}$ & $1.93 \times 10^{5}$ & $1.43 \times 10^{5}$ \\
Número de Reynolds del sólido, $\mathrm{Re}_{s}$ & 0.12 & 32.30 \\
Número de Stokes, St & 0.13 & 2.15 \\
\hline
\end{tabular}

Note que el flujo de catalizador de FCC está gobernado por el arrastre de gas en una mayor extensión que el movimiento de granos de arena, como se indica por los valores del número de Stokes. 
También, sus velocidades terminales son muy diferentes y esta es una indicación de la diferencia entre las velocidades de flujo del fluido y del sólido, la cual es mucho menor para el catalizador que para la arena. Más aún, se asume que los sólidos son retardados, con respecto a la velocidad media de la mezcla, en una cantidad que corresponde a la velocidad terminal. Considerando esta suposición, la fracción volumen de sólido en el estado base homogéneo está dada por

$$
\varepsilon_{s 0}=\frac{U_{s}}{U_{m}-U_{t}} .
$$

Para desarrollar los cálculos, se considera que la velocidad superficial del sólido $U_{s}$ o la fracción volumen de sólido, es dada. Debe seleccionarse una expresión conveniente para el parámetro de interacción sólido-fluido $\beta$ de acuerdo con el valor del número de Reynolds del sólido, ya que los granos en el FCC están en el régimen de Stokes, mientras que los granos de arena son descritos mejor por un coeficiente de arrastre de acuerdo con la ecuación de Ergun.

El módulo de compresibilidad del sólido se calculó mediante la expresión de Jiradilok y col. (2006), $\varepsilon_{s 0} \Phi_{0}^{\prime}=10^{6.837-2.475 \varepsilon_{0}}$. Más adelante se examinaran ambos ejemplos para apreciar sus similitudes y diferencias.

\subsubsection{Comparación de los coeficientes ondulatorios y significado de las jerarquías de onda}

Los coeficientes adimensionales de la Ec. (4.26), están denotados por índices prima, tal que $\quad \tau^{\prime} \doteq U_{0} \tau / L, \quad T^{\prime} \doteq U_{0} T / L C, \quad C^{\prime} \doteq\left(U_{0} / s\right)^{2} C \quad$ y $\quad G^{\prime} \doteq L g / U_{0}{ }^{2}$. Comparando estos coeficientes con respecto a la unidad, que es el coeficiente de la onda de primer orden del modelo incompresible, da una idea de la importancia de cada uno de ellos a medida que cambia la fracción volumen de sólido. Así, puede apreciarse en la Figura 4.1 el tiempo de relajación de la onda de segundo orden del modelo incompresible. Debemos señalar que para las partículas del FCC $\tau^{\prime}$ está cercano al valor constante de 0.0027. Por otro lado,

para el sistema de la arena $\tau^{\prime}$ es una función que decae a partir de un valor inicial de 0.0507, que es 18 veces mayor que el valor del FCC. 


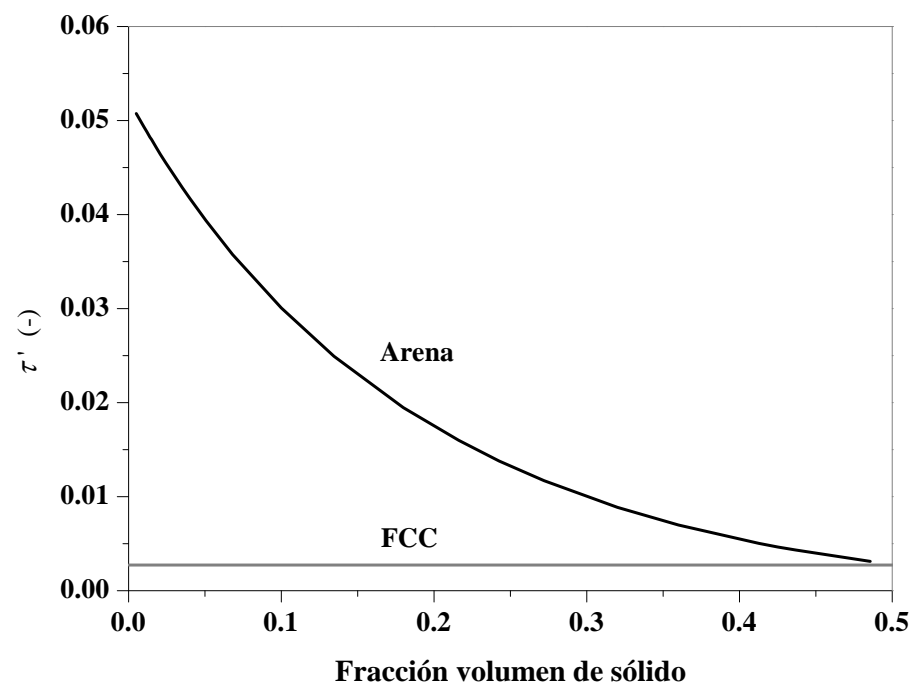

Figura 4.1. Tiempo de relajación adimensional $\tau^{\prime}$ del operador diferencial de segundo orden del modelo incompresible para el FCC (línea gris) y Arena (línea negra).

Si $T^{\prime}$ se escoge de tal manera que $T^{\prime}<0.01$, que se satisface para $\varepsilon_{s 0}<\varepsilon_{s 0 \text {,min }}$ de acuerdo con la Ec. (4.27), puede despreciarse el coeficiente de la onda de cuarto orden al compararse con el coeficiente de la onda de tercer orden cuyo valor se fija igual a la unidad. En la Tabla 4.2 se dan los valores de $\varepsilon_{s 0 \text {,min }}$ para los dos sistemas, FCC y la arena.

Tabla 4.2. Valores límite de las fracciones volumen de sólido.

\begin{tabular}{|c|c|c|}
\hline Sistema & $\varepsilon_{s 0, \min }$ & $\varepsilon_{s 0, I C}$ \\
\hline $\begin{array}{c}\text { Catalizador de } \\
\text { FCC-vapor }\end{array}$ & 0.0002 & 0.0125 \\
\hline Arena-aire & 0.0050 & 0.1680 \\
\hline
\end{tabular}

En la Figura 4.2, se grafican $\tau^{\prime}$ y $C^{\prime}$ como función de $\varepsilon_{s 0}$. Aparece un cruce entre ambas gráficas en el valor $\varepsilon_{s 0, I C}$, donde $\tau^{\prime}=C^{\prime}$. Así, para $\varepsilon_{s 0}<\varepsilon_{s 0, I C}$, el término incompresible de más alto orden, gobernado por el efecto del módulo de compresibilidad del sólido, es mayor que el efecto de compresibilidad del fluido; sucede lo opuesto para $\varepsilon_{s 0}>\varepsilon_{s 0, I C}$. 
En la Tabla 4.2 se muestran los valores de $\varepsilon_{s 0, I C}$ para ambos sistemas. Para el sistema catalizador de FCC-vapor, puede observarse en la Figura 4.2a, que la consideración de la compresibilidad del fluido es mucho más importante que la relajación del sólido, debido al módulo de compresibilidad, en la mayor parte del intervalo de $\varepsilon_{s 0}$. Sin embargo, ambos efectos son importantes para el sistema arena-aire, como puede verse en la Figura 4.2b. Estos comportamientos están de acuerdo con los números de Stokes para ambos sistemas; para los polvos de FCC, la dinámica del sólido es altamente dependiente de la dinámica del fluido, pero ambas dinámicas se vuelven más independientes para el sistema arena-aire.
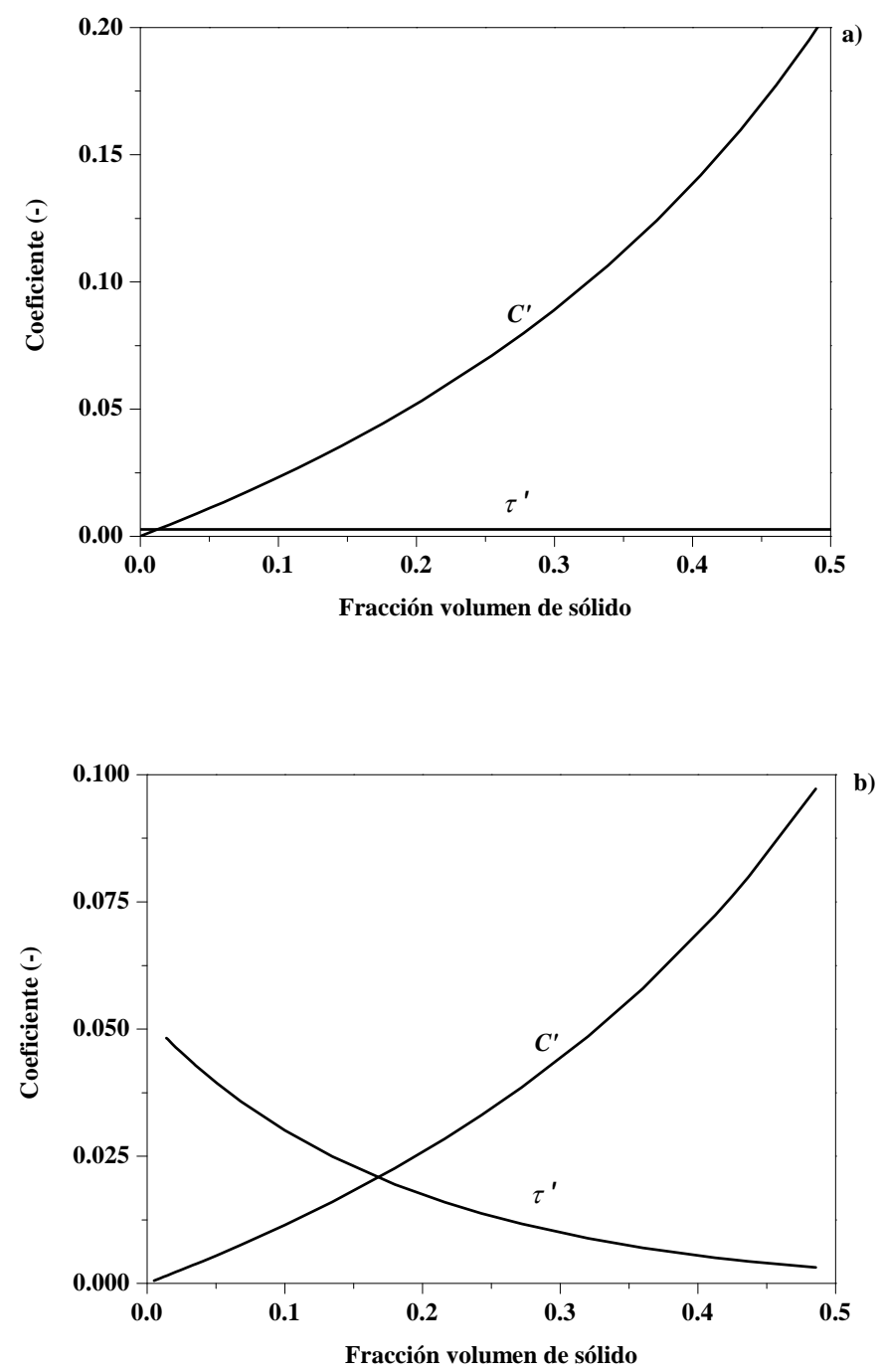

Figura 4.2. Coeficientes adimensionales $\tau^{\prime}$ y $C^{\prime}$ para los sistemas: (a) granos de catalizador de FCC y (b) Arena. 
Más aún, los valores de los coeficientes pueden compararse directamente con el coeficiente ondulatorio básico de primer orden cuyo coeficiente es uno. Así, para el sistema de FCC, el significado de la compresibilidad del fluido es cerca del $20 \%$ de la onda básica incompresible de primer orden, para un valor de fracción volumen de sólido de 0.5 ; en contraste, el comportamiento incompresible de alto orden es sólo cerca del $0.3 \%$. Por otro lado, para el sistema de la arena, la compresibilidad del fluido da un máximo de cerca del $10 \%$ del comportamiento básico, mientras que el efecto incompresible de alto orden tiene un valor máximo aproximado de 5\%. Por lo tanto, ambos efectos son significativos para este sistema. También, los valores típicos del coeficiente local $G^{\prime}$ están en el intervalo de 1.09 a 4.46 para el FCC y de 5.38 a 20.12 para el sistema de la arena.

En la Figura 4.3, se muestra la gráfica de la función $\left(C^{\prime} G^{\prime}\right)$, que puede ser comparada directamente con el comportamiento básico incompresible de primer orden cuyo valor es la unidad. Esta función toma en cuenta los efectos de gravedad en la compresibilidad del fluido y su importancia es cercana al 52\% del comportamiento básico incompresible para el sistema de la arena y alrededor del $21 \%$ para el polvo de FCC, ambas para fracciones volumen de sólido cercanas a 0.5 .

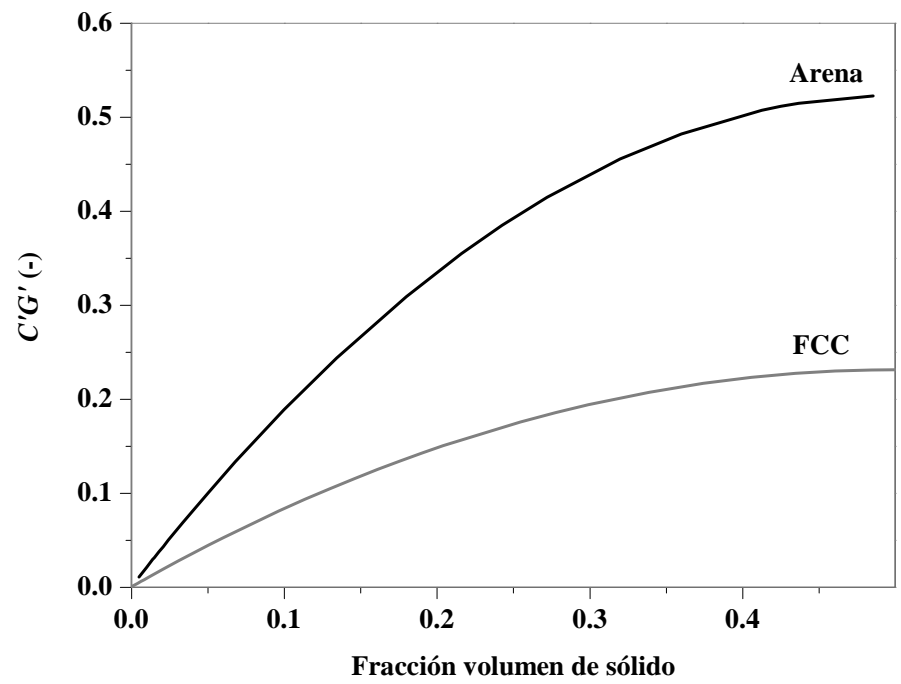

Figura 4.3. Coeficientes adimensionales de la onda compresible de segundo orden (degenerada) para el FCC (línea gris) y para la arena (línea negra). 


\subsubsection{Análisis de las rapideces de propagación}

En la Figura 4.4 se grafica la rapidez de propagación básica $a$ de la onda incompresible de primer orden para ambos sistemas, junto con las rapideces de propagación incompresibles de alto orden $c_{1}$ y $c_{2}$. También la rapidez de propagación incompleta del modelo compresible de segundo orden $\eta_{21}$, que está en el mismo orden de magnitud.
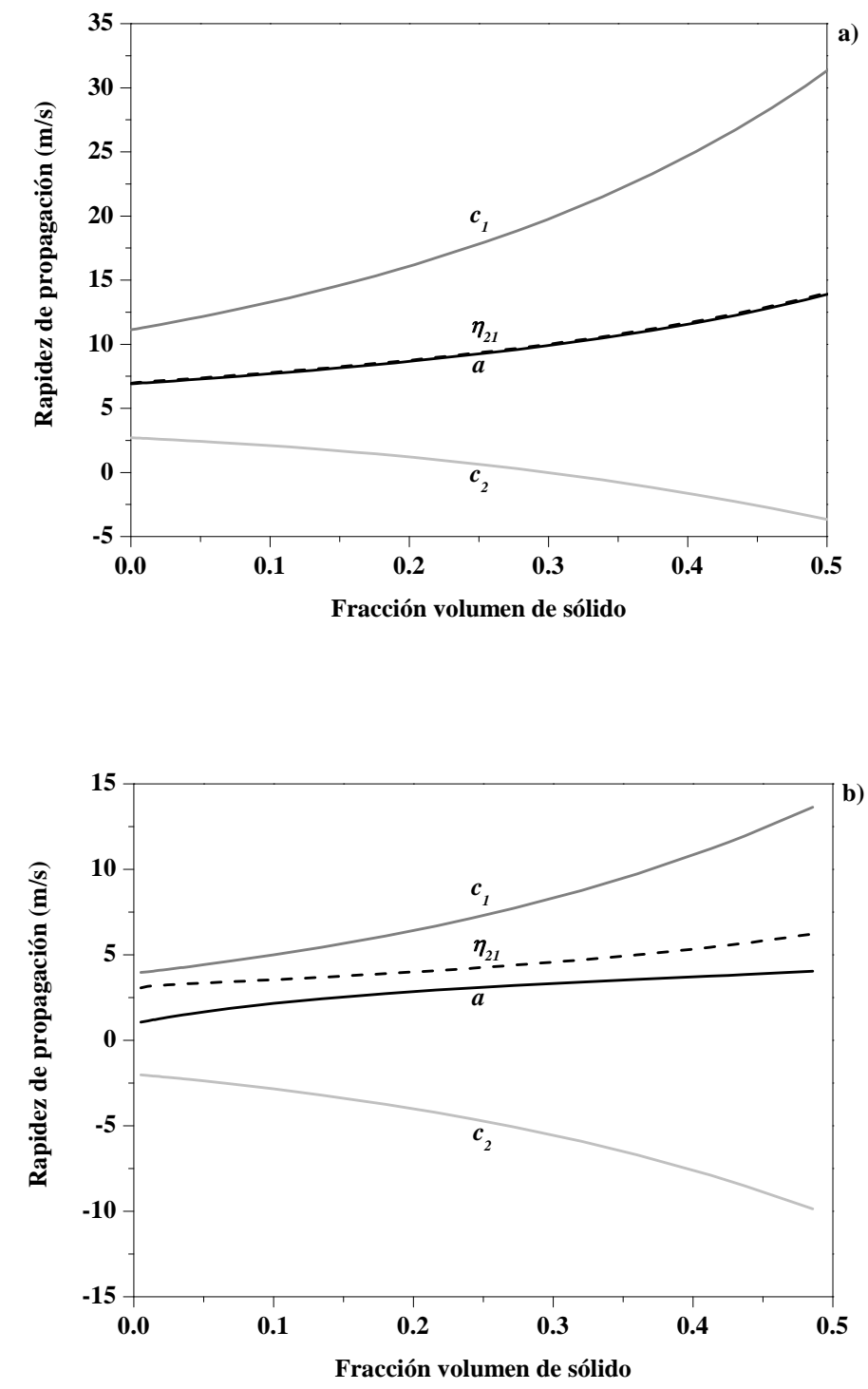

Figura 4.4. Rapidez de propagación de primer orden incompresible $a$ (línea negra), rapideces de propagación de segundo orden incompresibles $c_{1}$ y $c_{2}$ (líneas grises) y rapidez de propagación de segundo orden compresible incompleta $\eta_{21}$ (línea punteada), para los sistemas: (a) FCC y (b) Arena. 
Estas velocidades se ordenaron de acuerdo con la secuencia $c_{1}>\eta_{21}>a>c_{2}$ en ambos sistemas, incluso si la diferencia de $\eta_{21}$ a $a$ es muy pequeña para el sistema del FCC. Las rapideces de propagación de tercer orden $\alpha_{1}, \alpha_{2}$ y $\alpha_{3}$, como se muestra en la Ec. (4.29), son dependientes de la velocidad de propagación del sonido en el fluido $s$ y son expresadas aquí por la secuencia $\alpha_{1}>\alpha_{2}>\alpha_{3}$.
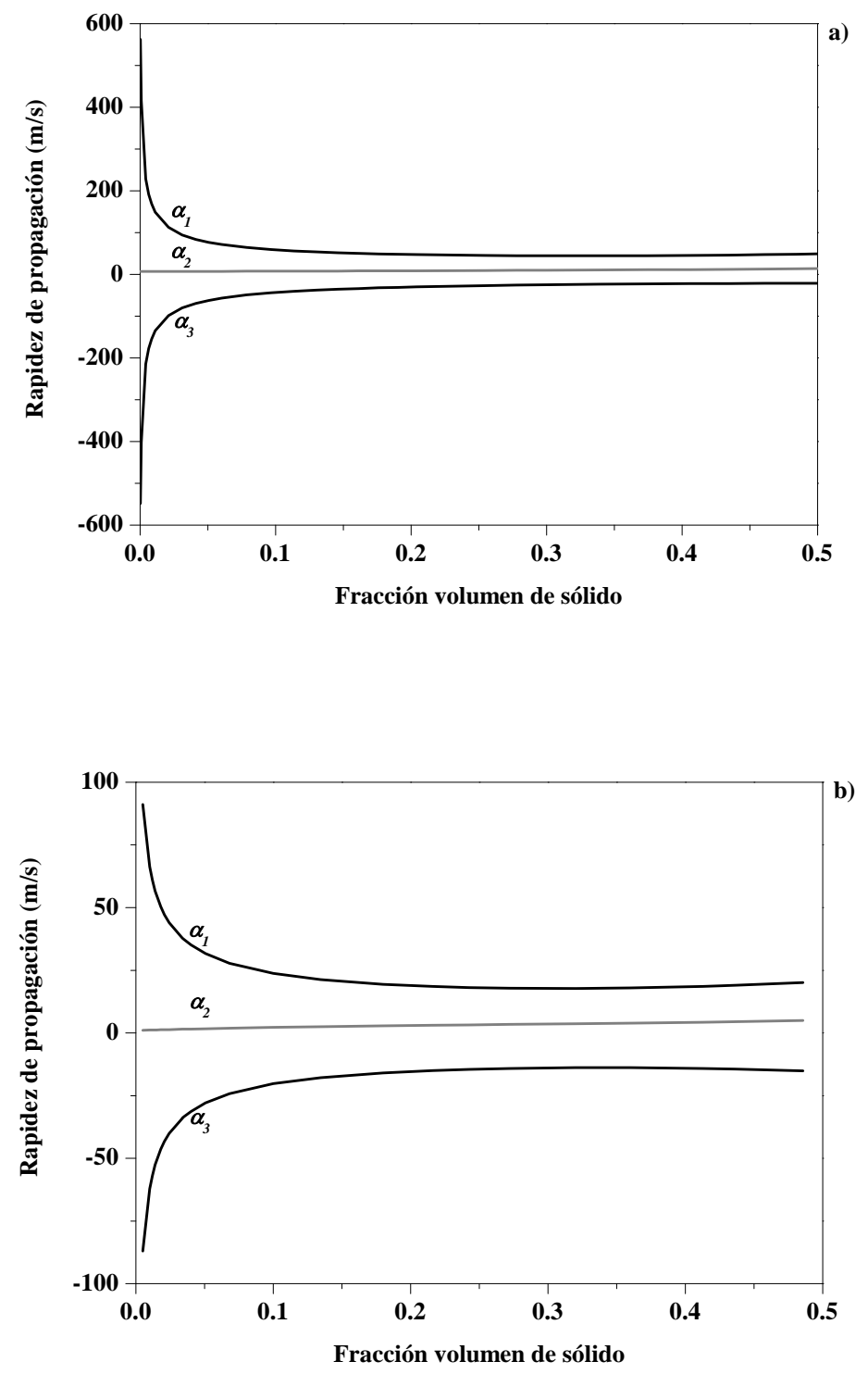

Figura 4.5. Rapideces de propagación de tercer orden del modelo compresible $\alpha_{1}, \alpha_{2}$ y $\alpha_{3}$ en la Ec. (4.29), para los sistemas: (a) FCC y (b) Arena. 
Se encontraron las raíces de la ecuación de tercer grado dada en la Ec. (4.30) y están graficadas en la Figura 4.5 , de $\varepsilon_{s 0 \text {,min }}$ en adelante.

La raíz más grande y más pequeña $\alpha_{1}$ y $\alpha_{3}$, respectivamente, pueden asociarse directamente a las rapideces ondulatorias de propagación acústicas (Gregor y Rumpf, 1975), expandiéndose a partir de la velocidad de flujo característica, denotada en general como $U_{\text {flow }}$.

Estas raíces están dadas por

$$
\begin{aligned}
& \tilde{\alpha}_{1} \doteq U_{\text {flow }}+s \sqrt{\frac{\rho_{g 0}}{\varepsilon_{0}\left(\varepsilon_{0} \rho_{g 0}+\varepsilon_{s 0} \rho_{s 0}\right)}}, \\
& \tilde{\alpha}_{3} \doteq U_{\text {flow }}-s \sqrt{\frac{\rho_{g 0}}{\varepsilon_{0}\left(\varepsilon_{0} \rho_{g 0}+\varepsilon_{s 0} \rho_{s 0}\right)}} .
\end{aligned}
$$

La elección particular de la velocidad de flujo $U_{\text {flow }}$ ó $U_{m}$, puede verse en Gregor y Rumpf (1975), con la que se obtienen mejores aproximaciones que con las raíces exactas de la Ec. (4.30).

Sin embargo, para el sistema de la arena, la elección $U_{\text {flow }}=0$ fue la mejor para ajustar los valores experimentales de van der Schaaf y col. (1998).

En la Figura 4.6 se comparó la raíz exacta $\alpha_{1}$ de la Ec. (4.30), así como la expresión aproximada dada en la Ec. (4.47) con $U_{\text {flow }}=0$ con los valores experimentales de la rapidez de propagación ondulatoria reportada por van der Schaaf y col. (1998).

En la Tabla 4.3 se presenta el error cuadrático medio de las mejores aproximaciones a la raíz exacta y a los valores experimentales, expresados en porcentaje, así como también los errores porcentuales menor y mayor. 
Tabla 4.3. Errores porcentuales de las rapideces de propagación de las ondas de presión para las mejores aproximaciones

\begin{tabular}{|c|c|c|c|c|}
\hline $\begin{array}{c}\text { Error porcentual y } \\
\text { sistema } \\
\delta_{j}=100\left|\frac{\alpha_{j}-\tilde{\alpha}_{j}}{\alpha_{j}}\right|\end{array}$ & $\begin{array}{c}\text { Valor de referencia } \\
\alpha_{j} / U_{\text {flow }} \text { en la } \\
\text { Ec. }(4.47)\end{array}$ & $\begin{array}{c}\text { Error } \\
\text { mínimo }\end{array}$ & $\begin{array}{c}\text { Error } \\
\text { máximo }\end{array}$ & $\begin{array}{c}\text { Error cuadrático } \\
\text { medio } \\
\sqrt{\frac{\sum_{i=1}^{N} \delta_{j, i}^{2}}{N}}\end{array}$ \\
\hline$\delta_{1}$ para el FCC & $\begin{array}{c}\text { Raíz exacta } \\
\text { Ec. }(4.30)\end{array}$ & 0.1 & 9.7 & 3.6 \\
\hline$\delta_{3}$ para el FCC & $\begin{array}{c}\text { Raíz exacta } \\
\text { Ec. }(4.30)\end{array}$ & 0.1 & 22.8 & 7.9 \\
\hline$\delta_{1}$ para la Arena & $\begin{array}{c}\text { Raíz exacta } \\
\text { Ec. }(4.30)\end{array}$ & 1.2 & 15.1 & 5.2 \\
\hline$\delta_{3}$ para la Arena & $\begin{array}{c}\text { Raíz exacta } \\
\text { Ec. }(4.30)\end{array}$ & 1.4 & 41.3 & 18.5 \\
\hline$\delta_{1}$ para la Arena & Experimental $/ U_{\text {flow }}=0$ & 0.4 & 48.8 & 23.4 \\
\hline
\end{tabular}

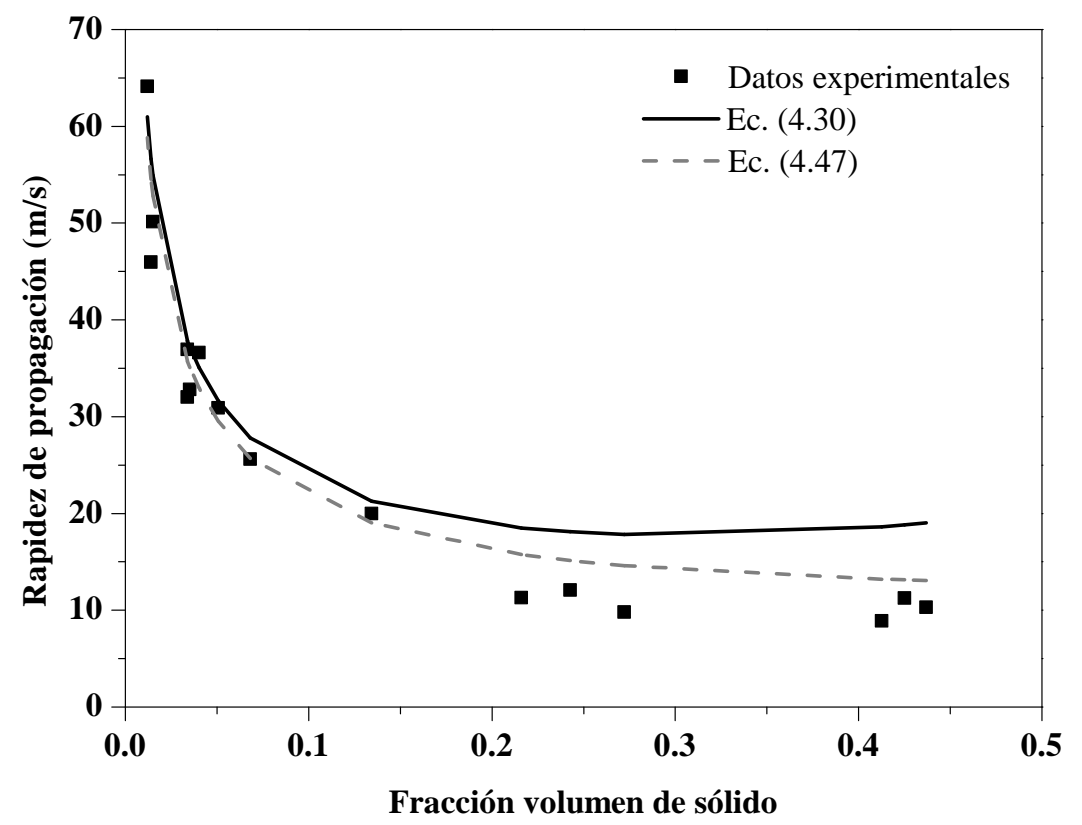

Figura 4.6. Rapidez de propagación de la onda de presión para el sistema de la arena. $\mathbf{m}$ : Datos experimentales de van der Schaaf y col. (1998). Línea continua: raíz exacta de la Ec. (4.30). Línea punteada: solución aproximada, Ec. (4.47) con $U_{\text {flow }}=0$. 


\subsubsection{Condiciones de estabilidad de Whitham}

La evaluación de las rapideces de propagación mostró que sus órdenes están en acuerdo con el criterio de estabilidad desarrollado anteriormente; así, las condiciones $\alpha_{1}>c_{1}>\alpha_{2}>c_{2}>\alpha_{3}$ y $\alpha_{1}>\eta_{21}>\alpha_{2}$ se satisfacen por ambos sistemas. Más aún, el límite inferior del número de onda para estabilidad lineal $\kappa_{0}$, dado en la Ec. (4.43), se grafica para los sistemas de FCC y la arena en la Figura 4.7.
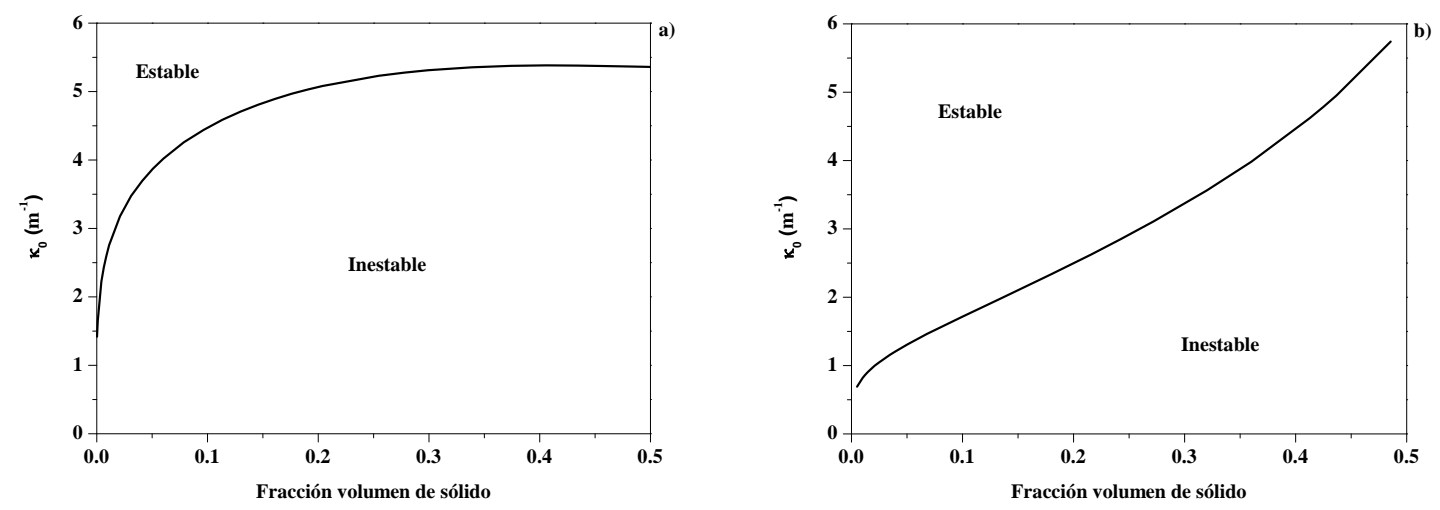

Figura 4.7. $\kappa_{0}$ para estabilidad lineal, como una función de $\varepsilon_{s 0}$ para los sistemas: (a) FCC y (b) Arena.

En la Ec. (4.45), se consideró la estabilidad lineal como una extensión al estudio desarrollado por Liu (1982) sobre la base de la consideración de incompresibilidad.

Por lo tanto, puede mostrarse que el efecto de la compresibilidad aumenta la región de estabilidad, pero sólo marginalmente, como puede verse al graficar las funciones $h_{1}$ y $h_{2}$.

Este criterio puede expresarse como porcentaje, al definir nuevas funciones adimensionales $H_{1}$ y $H_{2}$ así como el criterio equivalente:

$$
\begin{aligned}
& H_{1}=\frac{100 h_{1}}{\left(c_{1}-a\right)}=-\frac{100 C}{\left(c_{1}-a\right) s^{2}}\left(c_{1}^{3}-P_{1} c_{1}^{2}+P_{2} c_{1}-P_{3}\right)>-100, \\
& H_{2}=\frac{100 h_{2}}{\left(a-c_{2}\right)}=\frac{100 C}{\left(a-c_{2}\right) s^{2}}\left(c_{2}^{3}-P_{1} c_{2}^{2}+P_{2} c_{2}-P_{3}\right)>-100 .
\end{aligned}
$$


En la Figura 4.8 se grafican las funciones $H_{1}$ y $H_{2}$, en ella se puede observar que el aumento debido al efecto de compresibilidad es menor al $0.01 \%$ para el sistema de FCC y menor al $0.03 \%$ para el sistema de la arena.
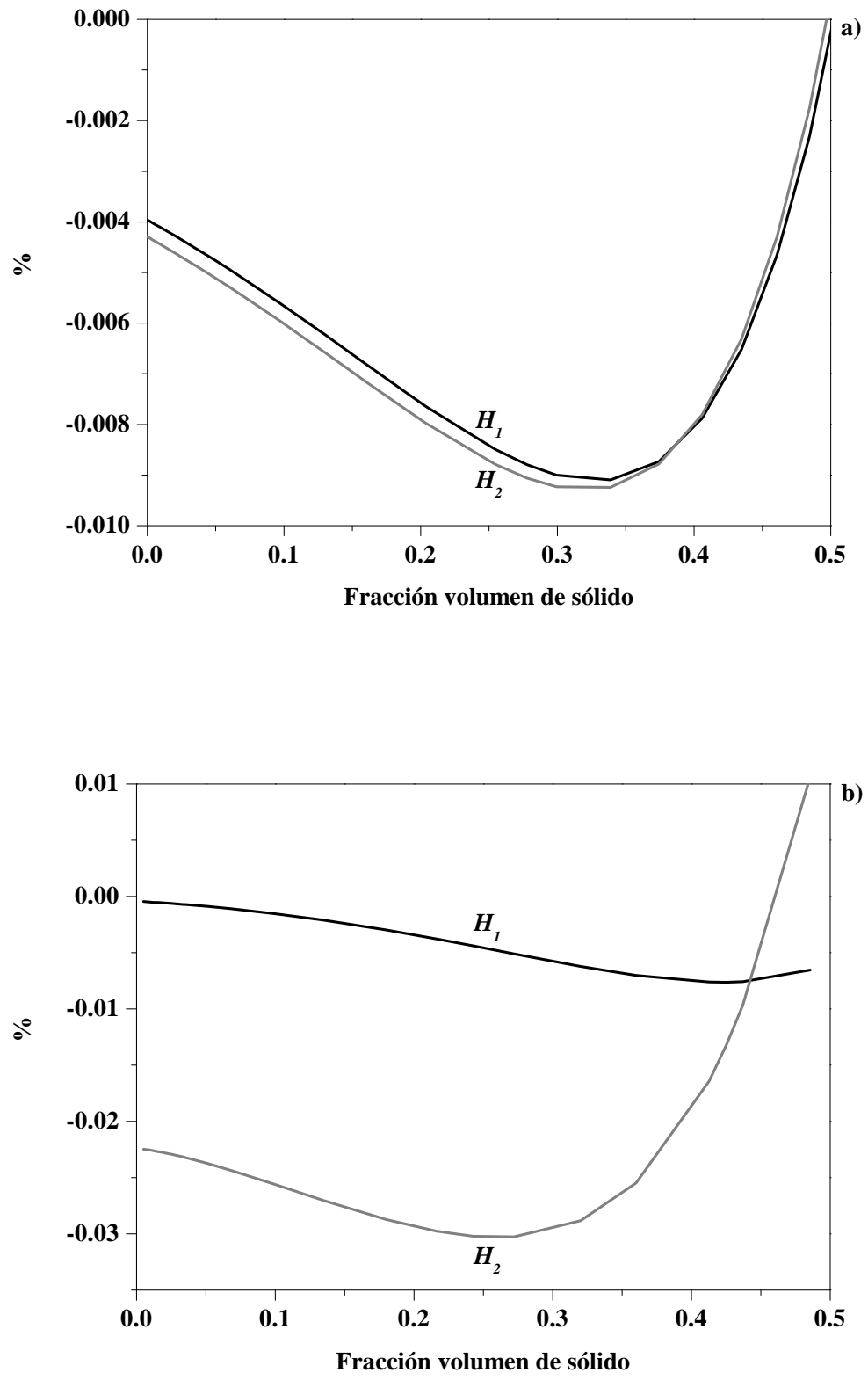

Figura 4.8. Aumento de la región de estabilidad debido al efecto de compresibilidad del fluido, expresado como porcentaje por las funciones $H_{1}$ y $H_{2}$, para los sistemas: (a) FCC y (b) Arena. 


\section{Capítulo 5}

\section{Incorporación del efecto de la pared en la propagación de ondas isotérmicas compresibles e incompresibles con modelos de lechos fluidizados rápidos 1-D linealizados ${ }^{3}$}

\subsection{Introducción}

Las columnas de tipo gas-líquido o de burbujeo, lechos fluidizados sólido-líquido, lechos fluidizados tipo gas-sólido, son usados ampliamente en la industria como reactores multifásicos. Estos reactores operan en uno de los dos siguientes regímenes característicos: régimen particulado o homogéneo y régimen agregativo o heterogéneo.

Las tasas de transferencia de calor y de masa, transporte de momentum y mezclado son muy diferentes en estos dos regímenes. Por lo tanto, es importante conocer el intervalo de operación y los parámetros de diseño en los cuales prevalece cada régimen y las condiciones bajo las cuales ocurre la transición. Este tema ha sido investigado extensivamente durante los pasados 60 años y se han reportado numerosos enfoques fundamentales, semi-empíricos y empíricos para la predicción de la transición.

Uno de los enfoques hace una clasificación en dos categorías. En la primera, se considera que el sistema multifásico no tiene fronteras. Por lo tanto, este enfoque no considera la existencia del distribuidor o de la pared de la columna. Tales lechos han sido llamados "unbounded beds". En el segundo enfoque, se considera la presencia del distribuidor o de la pared de la columna y se les llama "bounded beds". En ambos casos, se ha utilizado el método de estabilidad lineal para obtener un criterio de transición de uno a otro (Joshi y col., 2001).

\footnotetext{
${ }^{3}$ Los resultados de este Capítulo corresponden al artículo: Soria, A., Salinas-Rodríguez, E. y Sánchez-López, J.R.G. (2012). Wall effects assessment on Compressible and incompressible 1-D linear wave propagation assessment in fast fluidized beds. AIChE J. (en revision).
} 
En el régimen homogéneo (espacialmente) las perturbaciones que se suponen pequeñas, se extinguen con el paso del tiempo, dando como resultado que el lecho sea estable ante pequeñas perturbaciones.

Si el régimen de operación es heterogéneo, entonces las perturbaciones crecen exponencialmente con el tiempo y aparecen no-uniformidades. Esto hace al lecho heterogéneo, dando como resultado que los lechos sean inestables. Los términos, lecho inestable y régimen heterogéneo, son entonces utilizados como sinónimos.

Los lechos fluidizados reales no tienen una extensión infinita, por lo que debe tomarse en cuenta la longitud finita de sus fronteras en la superficie superior e inferior, así como también la presencia de las paredes que rodean lateralmente al lecho. Por lo tanto, el caso "unbounded" es una simplificación de los sistemas reales y la mayor parte de los criterios basados en su enfoque tienen limitaciones.

En este Capítulo presentaremos un modelo más elaborado que toma en cuenta la presencia de las paredes laterales. Joshi y col. (2001) encontraron que reducir el diámetro de la columna tiene un efecto estabilizador y que la velocidad superficial crítica del gas se incrementa con una disminución en el diámetro de la columna $D_{t}$.

De la comparación de un análisis de estabilidad lineal para un sistema "unbounded" y uno "bounded", concluyen que la aplicabilidad del análisis "unbounded" es limitada a resistencias bajas en el distribuidor y que en el caso de resistencias elevadas, debe utilizarse el análisis "bounded” para tener predicciones más realistas.

En este Capítulo nos concentraremos en estudiar el efecto de la pared del tubo en el modelo, en la dinámica ondulatoria y en la estabilidad lineal.

Por lo tanto, compararemos los resultados que aquí encontramos con los del caso "unbounded" estudiado en el Capítulo anterior. 


\subsection{Jerarquía de ondas}

\subsubsection{Ecuaciones del modelo}

Una dispersión monodispersa de partículas pequeñas esféricas sólidas de diámetro medio $d_{p}$ y densidad intrínseca $\rho_{s}$ se mueve dentro de una corriente de gas o vapor en un tubo vertical de diámetro interno $D_{t}$ a temperatura constante. Se considera que el fluido es un gas newtoniano compresible con densidad $\rho_{g}$ y viscosidad constante $\mu_{g}$. La fracción volumen de sólido es $\varepsilon_{s}$, se fija entre 0 y 0.5 y satisface la relación $\varepsilon_{g}+\varepsilon_{s}=1$, donde $\varepsilon_{g}$ es la fracción volumen de gas. La mezcla se mueve con una velocidad promedio $U_{m}=\varepsilon_{s} V_{s}+\varepsilon_{g} V_{g}$, donde $V_{g}$ y $V_{s}$ son los valores de las velocidades ascendentes con promedio ponderado en la masa del gas y sólido, respectivamente. Debido a que la mezcla se considera en un régimen altamente turbulento, los efectos viscosos son sólo significativos en una región cercana a la superficie de los gránulos.

El número de Reynolds de las partículas es $\operatorname{Re}_{s}=d_{p} \rho_{g} U_{t} / \mu_{g}$, donde la velocidad terminal

se define como $U_{t} \doteq\left|V_{s}-U_{m}\right|=\varepsilon_{g}\left(V_{g}-V_{s}\right)$. En este modelo, despreciamos los efectos viscosos en las fases, así como los esfuerzos turbulentos.

Siguiendo el trabajo de Sánchez, Soria y Salinas, citado como SSS (2011), se aplica el método de promediado espacio-temporal a las ecuaciones locales de continuidad y movimiento para las fases gaseosa y sólida. Entonces obtenemos el siguiente conjunto cerrado de ecuaciones promediadas, dado por la Ec. (3.19) a la Ec. (3.22) en estado transitorio, no-lineal en una-dimensión para las variables dependientes $\left\{\varepsilon_{g}, P_{g}, V_{g}, V_{s}\right\}$ :

$$
\begin{aligned}
& \frac{\partial}{\partial t}\left[\varepsilon_{g} \rho_{g}\left(P_{g}\right)\right]+\frac{\partial}{\partial z}\left[\varepsilon_{g} \rho_{g}\left(P_{g}\right) V_{g}\right]=0 \\
& -\rho_{s} \frac{\partial}{\partial t} \varepsilon_{g}+\rho_{s} \frac{\partial}{\partial z}\left[\left(1-\varepsilon_{g}\right) V_{s}\right]=0 \\
& \frac{\partial}{\partial t}\left[\varepsilon_{g} \rho_{g}\left(P_{g}\right) V_{g}\right]+\frac{\partial}{\partial z}\left[\varepsilon_{g} \rho_{g}\left(P_{g}\right) V_{g}^{2}\right]+\varepsilon_{g} \frac{\partial}{\partial z} P_{g}+\mathrm{T}^{(g)}+F_{g W}=0 \\
& \rho_{s} \frac{\partial}{\partial t}\left[\left(1-\varepsilon_{g}\right) V_{s}\right]+\rho_{s} \frac{\partial}{\partial z}\left[\left(1-\varepsilon_{g}\right) V_{s}^{2}\right]+\left(1-\varepsilon_{g}\right) \frac{\partial}{\partial z} P_{g}-\Phi^{\prime}\left(1-\varepsilon_{g}\right) \frac{\partial}{\partial z} \varepsilon_{g}+\mathrm{T}^{(s)}+F_{s W}=0
\end{aligned}
$$


Aquí $z$ y $t$ son la coordenada vertical y el tiempo, respectivamente. $P_{g}$ es la presión de la fase gas promediada. La presión promedio de la fase sólida es $P_{s}=P_{g}+\Phi\left(\varepsilon_{s}\right)$, donde la función $\Phi\left(\varepsilon_{s}\right)$ es el módulo de compresibilidad del sólido y $\Phi^{\prime}=d \Phi / d \varepsilon_{s}$ su derivada. Las fuerzas de interacción hidrodinámica son

$$
\begin{aligned}
& \mathrm{T}^{(g)}=F+\rho_{g}\left(p_{g}\right) g \\
& \mathrm{~T}^{(s)}=-F+\left(1-\varepsilon_{g}\right)\left[\rho_{s}-\rho_{g}\left(P_{g}\right)\right] g,
\end{aligned}
$$

donde $F$ es fuerza promedio local ejercida por el fluido en las partículas y $g$ es la fuerza gravitacional por unidad de masa. $F_{g W}$ y $F_{s W}$ corresponden a la fuerza de arrastre del fluido y de las partículas sólidas en la pared del tubo, respectivamente. Estas fuerzas, junto con $F$ son modeladas de tal forma que sus principales dependencias permanezcan explícitas:

$$
\begin{aligned}
& F=\beta \varepsilon_{g} \varepsilon_{s}\left(V_{g}-V_{s}\right) \\
& F_{g W}=\beta_{g W} \rho_{g}\left(P_{g}\right) V_{g}^{2} \\
& F_{s W}=\beta_{s W} \rho_{s} V_{s}^{2}
\end{aligned}
$$

donde $\beta=\left(3 / 4 d_{p}\right) C_{D} \rho_{g} U_{t}$ es un parámetro de interacción.

En este Capítulo no se tomaron en cuenta el efecto de masa agregada (Jackson, 1985) y el efecto de viscosidad efectiva en el régimen de fluidización denso (Jackson, 1985; Liu, 1982 y Homsy y col., 1980). Los coeficientes $\beta_{k W}=\left(2 \varepsilon_{k} / D_{t}\right) f_{k W}$ son las funciones de arrastre en la pared con $k=\{g, s\}$, que involucran el factor de fricción de arrastre $f_{k W}$ y el diámetro interno del tubo $D_{t}$. Los granos sólidos son incompresibles, entonces $\rho_{s}=\rho_{s 0}$ es una constante. La principal característica del presente Capítulo es la evaluación del efecto de la pared en las estrategias de modelado. 


\subsubsection{Enunciado del problema}

La Ec. (5.1) a la Ec. (5.4) son linealizadas alrededor de un estado base (subíndice cero) más una pequeña perturbación tal que $\varepsilon_{g}=\varepsilon_{0}+\varepsilon, \quad P_{g}=p_{0}+p, \quad V_{g}=v_{g 0}+v_{g} \quad \mathrm{y}$ $V_{s}=v_{s 0}+v_{s}$.

Para obtener jerarquías de onda de perturbaciones para ambos modelos, el incompresible y el compresible, se proponen soluciones ondulatorias para las ecuaciones linealizadas de la Ec. (5.1) a la Ec. (5.4). Estas ecuaciones son representadas en forma matricial como:

$$
\mathbf{u}=\left(\begin{array}{llll}
\varepsilon & p & v_{g} & v_{s}
\end{array}\right)^{T},
$$

el vector de perturbaciones para el sistema linealizado de EDP de primer orden:

$$
\mathbf{B} \frac{\partial \mathbf{u}}{\partial t}+\mathbf{C} \frac{\partial \mathbf{u}}{\partial z}+\mathbf{D}^{*} \mathbf{u}=\mathbf{0}
$$

donde las matrices $\mathbf{B}, \mathbf{C}$ y $\mathbf{D}^{*}$ de tamaño $4 \times 4$, son los coeficientes linealizados de la Ec. (5.1) a la Ec. (5.4), están dadas en los Apéndices $F(\mathbf{B}, \mathbf{C})$ y $H\left(\mathbf{D}^{*}\right)$. Las derivadas espaciales de las perturbaciones de velocidad $v_{g}$ y $v_{s}$ pueden obtenerse de las ecuaciones de continuidad, luego se sustituyen en las ecuaciones de momentum para llegar así a dos ecuaciones linealizadas de segundo orden para las perturbaciones de la fracción volumen $(\varepsilon)$ y para las perturbaciones de la presión $(p)$ :

$$
\begin{aligned}
& \left(L_{1}+\Lambda_{1}\right) \varepsilon+\left[L_{3}+\left(\frac{d \rho_{g}}{d P_{g}}\right)_{0}\left(K_{1}+\Gamma\right)\right] p=0, \\
& \left(L_{2}+\Lambda_{2}\right) \varepsilon+\left[L_{3}+\left(\frac{d \rho_{g}}{d P_{g}}\right)_{0} K_{2}\right] p=0,
\end{aligned}
$$

donde los operadores diferenciales $L_{1}, L_{2}, L_{3}, K_{1}$ y $K_{2}$ están definidos en la Ec. (4.9) a la Ec. (4.13) y la influencia de la pared está dada por tres funciones adicionales:

$$
\begin{aligned}
& \Lambda_{1}=-\frac{2 \beta_{g W} \rho_{g 0} v_{g 0}}{\varepsilon_{0}^{2}}\left(\frac{\partial}{\partial t}+v_{g 0} \frac{\partial}{\partial z}\right), \\
& \Lambda_{2}=\frac{2 \beta_{s W} \rho_{s 0} v_{s 0}}{\left(1-\varepsilon_{0}\right)^{2}}\left(\frac{\partial}{\partial t}+v_{s 0} \frac{\partial}{\partial z}\right),
\end{aligned}
$$




$$
\Gamma=-\frac{2 \beta_{g W} v_{g 0}}{\varepsilon_{0}}\left(\frac{\partial}{\partial t}+\frac{v_{g 0}}{2} \frac{\partial}{\partial z}\right)
$$

El factor $\left(d \rho_{g} / d P_{g}\right)_{0}$ toma en cuenta el cambio de densidad del fluido como una función de su presión, a través de una ecuación de estado isotérmica $\rho_{g}=\rho_{g}\left(P_{g}\right)$. La velocidad del sonido en un gas homogéneo puede introducirse aquí, al considerar la relación $\left(d \rho_{g} / d P_{g}\right)_{0}=s^{-2}$, donde $s$ es la velocidad del sonido en el gas puro. La aproximación de incompresibilidad del fluido puede recuperarse de la Ec. (5.12) y Ec. (5.13) al tomar el límite $\left(d \rho_{g} / d P_{g}\right)_{0}=0$ o en forma equivalente cuando $s \rightarrow \infty$. Bajo la aproximación de incompresibilidad puede encontrarse directamente una EDP de segundo orden para $\varepsilon$ al restar la Ec. (5.12) de la Ec. (5.13), dando $\left[\left(L_{2}-L_{1}\right)+\left(\Lambda_{2}-\Lambda_{1}\right)\right] \varepsilon=0$. También, puede obtenerse una EDP de cuarto orden para $p$, obtenida alternativamente mediante la aplicación cruzada de los operadores $\left(L_{2}+\Lambda_{2}\right)$ y $\left(L_{1}+\Lambda_{1}\right)$ a las ecuaciones Ec. (5.12) y Ec. (5.13), respectivamente y restando posteriormente, para obtener:

$$
\left[\left(L_{2}-L_{1}\right)+\left(\Lambda_{2}-\Lambda_{1}\right)\right] L_{3} p=0 .
$$

Entonces, mientras la dinámica de la fracción volumen está representada por una EDP de segundo orden, la dinámica de las perturbaciones de presión es el mismo operador aplicado a una derivada espacial de segundo orden. Más aún, si la cerradura del campo de presión del sólido es tal que $P_{s}=\Phi\left(\varepsilon_{s}\right)$, permite un desacoplamiento del término de presión, el balance de momentum del sólido da la forma reducida

$$
\left(L_{2}+\Lambda_{2}\right) \varepsilon=0
$$

El comportamiento compresible global puede también ser expresado como una ecuación de onda para $\varepsilon$ ó para $p$. Así, la aplicación cruzada de los operadores $\left(L_{2}+\Lambda_{2}\right)$ y $\left(L_{1}+\Lambda_{1}\right)$ a la Ec. (5.12) y a la Ec. (5.13), respectivamente y posteriormente restando se obtiene una EDP de cuarto orden para las perturbaciones de la presión. Un procedimiento alternativo da la misma EDP para las perturbaciones de la fracción volumen. Esto es 


$$
\begin{aligned}
& {\left[\left(L_{2}-L_{1}\right) L_{3}+\frac{1}{s^{2}}\left(L_{2} K_{1}-L_{1} K_{2}\right)\right] \varphi } \\
+ & {\left[\left(\Lambda_{2}-\Lambda_{1}\right) L_{3}+\frac{1}{s^{2}}\left(\Lambda_{2} K_{1}+L_{2} \Gamma+\Lambda_{2} \Gamma-\Lambda_{1} K_{2}\right)\right] \varphi=0, }
\end{aligned}
$$

donde $\varphi$ es $\varepsilon$ ó $p$. El comportamiento principal, fue reportado en la Ec. (4.14) y está dado por el primer corchete de la Ec. (5.19), mientras que el segundo corchete contiene términos adicionales que representan la dinámica debida a los efectos de pared. Puesto que $L_{1}, L_{2}$, $L_{3}$ y $K_{1}$ son operadores diferenciales de segundo orden, en tanto que $K_{2}, \Lambda_{1}, \Lambda_{2}$ y $\Gamma$ son de primer orden, los efectos de pared pueden pensarse como una estructura ondulatoria de tercer orden superpuesta al comportamiento básico de la corriente libre. En esta estructura ondulatoria puede encontrarse un comportamiento incompresible de tercer orden:

$$
\left(\Lambda_{2}-\Lambda_{1}\right) L_{3}=\left[\frac{2 \beta_{s W} \rho_{s 0} v_{s 0}}{\left(1-\varepsilon_{0}\right)^{2}}\left(\frac{\partial}{\partial t}+v_{s 0} \frac{\partial}{\partial z}\right)+\frac{2 \beta_{g W} \rho_{g 0} v_{g 0}}{\varepsilon_{0}^{2}}\left(\frac{\partial}{\partial t}+v_{g 0} \frac{\partial}{\partial z}\right)\right] \frac{\partial^{2}}{\partial z^{2}}
$$

así como también términos compresibles de interacción de tercer y de segundo orden:

$$
\begin{array}{r}
\Lambda_{2} K_{1}+L_{2} \Gamma+\Lambda_{2} \Gamma-\Lambda_{1} K_{2}= \\
-\frac{2 \beta_{s W} \rho_{s 0} v_{s 0}}{\left(1-\varepsilon_{0}\right)^{2}}\left(\frac{\partial}{\partial t}+v_{g 0} \frac{\partial}{\partial z}\right)^{2}\left(\frac{\partial}{\partial t}+v_{s 0} \frac{\partial}{\partial z}\right)-2 \frac{\beta_{g W} v_{g 0}}{\varepsilon_{0}} \frac{\rho_{s 0}}{1-\varepsilon_{0}}\left(\frac{\partial}{\partial t}+\frac{v_{g 0}}{2} \frac{\partial}{\partial z}\right)\left(\frac{\partial}{\partial t}+v_{s 0} \frac{\partial}{\partial z}\right)^{2} \\
+2 \frac{\beta_{g W} v_{g 0}}{\varepsilon_{0}} \Phi_{0}^{\prime}\left(\frac{\partial}{\partial t}+\frac{v_{g 0}}{2} \frac{\partial}{\partial z}\right) \frac{\partial^{2}}{\partial z^{2}}-\frac{\beta}{\rho_{g 0}} \frac{2 \beta_{s W} \rho_{s 0} v_{s 0}}{1-\varepsilon_{0}}\left(\frac{\partial}{\partial t}+v_{g 0} \frac{\partial}{\partial z}\right)\left(\frac{\partial}{\partial t}+v_{s 0} \frac{\partial}{\partial z}\right) \\
-2 \frac{\beta_{g W} v_{g 0}}{1-\varepsilon_{0}}\left(\frac{\partial}{\partial t}+v_{g 0} \frac{\partial}{\partial z}\right)\left(\frac{\partial}{\partial t}+\frac{v_{g 0}}{2} \frac{\partial}{\partial z}\right)-2 \frac{\beta_{g W} v_{g 0}}{\varepsilon_{0}} \beta\left(\frac{\partial}{\partial t}+\frac{v_{g 0}}{2} \frac{\partial}{\partial z}\right)\left(\frac{\partial}{\partial t}+v_{s 0} \frac{\partial}{\partial z}\right) \\
+\frac{2 \beta_{g W} v_{g 0}}{1-\varepsilon_{0}} \frac{g}{\varepsilon_{0}}\left(\rho_{s 0}-\rho_{g 0}\right)\left(\frac{\partial}{\partial t}+\frac{v_{g 0}}{2} \frac{\partial}{\partial z}\right) \frac{\partial}{\partial z}+\frac{2 \beta_{s W} \rho_{s 0} v_{s 0}}{\left(1-\varepsilon_{0}\right)^{2}} \frac{\beta_{g W}}{\varepsilon_{0}}\left(\frac{\partial}{\partial t}+v_{s 0} \frac{\partial}{\partial z}\right) \frac{\partial}{\partial z} \\
+2 \frac{\beta_{s 0} v_{g 0} v_{s 0}}{\varepsilon_{0}\left(1-\varepsilon_{0}\right)^{2}}\left(\frac{\partial}{\partial t}+\frac{v_{g 0}}{2} \frac{\partial}{\partial z}\right)\left(\frac{\partial}{\partial t}+v_{s 0} \frac{\partial}{\partial z}\right) \\
\left.\frac{\partial}{\partial t}+v_{g 0} \frac{\partial}{\partial z}\right)\left[\left(\frac{\partial}{\partial t}+v_{g 0} \frac{\partial}{\partial z}\right)-\frac{\rho_{g 0} g}{\beta \varepsilon_{0}} \frac{\partial}{\partial z}\right] .
\end{array}
$$


Los términos de interacción asociados con la pared en la Ec. (5.19) pueden rearreglarse para obtener:

$$
\begin{array}{r}
\left(\Lambda_{2}-\Lambda_{1}\right) L_{3}+\frac{1}{s^{2}}\left(\Lambda_{2} K_{1}+L_{2} \Gamma+\Lambda_{2} \Gamma-\Lambda_{1} K_{2}\right)=A\left(\frac{\partial}{\partial t}+a_{f} \frac{\partial}{\partial z}\right) \frac{\partial^{2}}{\partial z^{2}} \\
-\frac{1}{s^{2}}\left[\begin{array}{r}
C_{w}\left(\frac{\partial^{3}}{\partial t^{3}}+M_{31} \frac{\partial^{3}}{\partial t^{2} \partial z}+M_{32} \frac{\partial^{3}}{\partial t \partial z^{2}}+M_{33} \frac{\partial^{3}}{\partial z^{3}}\right) \\
\left.+B\left(\frac{\partial^{2}}{\partial t^{2}}+M_{21} \frac{\partial^{2}}{\partial t \partial z}+M_{22} \frac{\partial^{2}}{\partial z^{2}}\right)\right]
\end{array}\right]
\end{array}
$$

con coeficientes

$$
\begin{aligned}
A & =2 \frac{\beta_{g W}\left(1-\varepsilon_{0}\right)^{2} \rho_{g 0} v_{g 0}+\beta_{s W} \varepsilon_{0}^{2} \rho_{s 0} v_{s 0}}{\beta \varepsilon_{0}\left(1-\varepsilon_{0}\right)}, \\
C_{w} & =\frac{2 \rho_{s 0}}{\beta\left(1-\varepsilon_{0}\right)}\left[\beta_{s W} \varepsilon_{0} v_{s 0}+\beta_{g W}\left(1-\varepsilon_{0}\right) v_{g 0}\right], \\
B & =2 \varepsilon_{0} \rho_{s 0}\left[\frac{\beta_{g W} v_{g 0}}{\rho_{s 0}}+\frac{\beta_{s W} v_{s 0}}{\rho_{g 0}}+2 \frac{\beta_{g W} \beta_{s W}}{\beta} \frac{v_{g 0} v_{s 0}}{\varepsilon_{0}\left(1-\varepsilon_{0}\right)}\right],
\end{aligned}
$$

la cual toma en cuenta el factor de escala $\frac{\varepsilon_{0}\left(1-\varepsilon_{0}\right)}{\beta}$ manejado al obtener la Ec. (4.17) para obtener la unidad como coeficiente del término de la onda incompresible de primer orden. En esta forma los coeficientes propuestos están en la misma escala que los de la contribución previa. También la rapidez de propagación de primer orden:

$$
a_{f}=\frac{\beta_{g W}\left(1-\varepsilon_{0}\right)^{2} \rho_{g 0} v_{g 0}{ }^{2}+\beta_{s W} \varepsilon_{0}^{2} \rho_{s 0} v_{s 0}{ }^{2}}{\beta_{g W}\left(1-\varepsilon_{0}\right)^{2} \rho_{g 0} v_{g 0}+\beta_{s W} \varepsilon_{0}{ }^{2} \rho_{s 0} v_{s 0}} .
$$

Se definieron y se utilizaron los coeficientes $M_{j k}$, que están dados en el Apéndice $I$. Los operadores de tercer y segundo orden pueden expresarse en forma equivalente con rapideces de propagación explícitas, como sigue:

$$
\begin{aligned}
& \left(\Lambda_{2}-\Lambda_{1}\right) L_{3}+\frac{1}{s^{2}}\left(\Lambda_{2} K_{1}+L_{2} \Gamma+\Lambda_{2} \Gamma-\Lambda_{1} K_{2}\right)=A\left(\frac{\partial}{\partial t}+a_{f} \frac{\partial}{\partial z}\right) \frac{\partial^{2}}{\partial z^{2}} \\
& -\frac{1}{s^{2}}\left[C_{w}\left(\frac{\partial}{\partial t}+\xi_{31} \frac{\partial}{\partial z}\right)\left(\frac{\partial}{\partial t}+\xi_{32} \frac{\partial}{\partial z}\right)\left(\frac{\partial}{\partial t}+\xi_{33} \frac{\partial}{\partial z}\right)+B\left(\frac{\partial}{\partial t}+\xi_{21} \frac{\partial}{\partial z}\right)\left(\frac{\partial}{\partial t}+\xi_{22} \frac{\partial}{\partial z}\right)\right]
\end{aligned}
$$


la cual, junto con la dinámica de la corriente libre dada en la Ec. (4.17) da la ecuación completa con efectos de pared:

$$
\begin{array}{r}
{\left[\tau\left(\frac{\partial}{\partial t}+c_{1} \frac{\partial}{\partial z}\right)\left(\frac{\partial}{\partial t}+c_{2} \frac{\partial}{\partial z}\right)+(1+A)\left(\frac{\partial}{\partial t}+a_{w} \frac{\partial}{\partial z}\right)\right] \frac{\partial^{2}}{\partial z^{2}}} \\
-\frac{C}{s^{2}}\left[\left(\frac{\partial}{\partial t}+\eta_{31} \frac{\partial}{\partial z}\right)\left(\frac{\partial}{\partial t}+\eta_{32} \frac{\partial}{\partial z}\right)\left(\frac{\partial}{\partial t}+\eta_{33} \frac{\partial}{\partial z}\right)+\frac{C_{w}}{C}\left(\frac{\partial}{\partial t}+\xi_{31} \frac{\partial}{\partial z}\right)\left(\frac{\partial}{\partial t}+\xi_{32} \frac{\partial}{\partial z}\right)\left(\frac{\partial}{\partial t}+\xi_{33} \frac{\partial}{\partial z}\right)\right]=0 \\
\left.-g\left(\frac{\partial}{\partial t}+\eta_{21} \frac{\partial}{\partial z}\right) \frac{\partial}{\partial z}+\frac{B}{C}\left(\frac{\partial}{\partial t}+\xi_{21} \frac{\partial}{\partial z}\right)\left(\frac{\partial}{\partial t}+\xi_{22} \frac{\partial}{\partial z}\right)\right]
\end{array}
$$

donde la rapidez de propagación incompresible de primer orden, modificada por los efectos de pared es

$$
a_{w}=\frac{a+A a_{f}}{1+A} .
$$

La importancia de los efectos de pared puede evaluarse para el modelo compresible e incompresible al estimar la importancia de los coeficientes $A, C_{w}$ y $B$ en la Ec. (5.28). Por otro lado, para fines de comparaciones con valores experimentales, conviene reescribir la Ec. (5.28) en forma tal que queden agrupadas las ondas del mismo orden, para obtener:

$$
\tau\left(\frac{\partial}{\partial t}+c_{1} \frac{\partial}{\partial z}\right)\left(\frac{\partial}{\partial t}+c_{2} \frac{\partial}{\partial z}\right) \frac{\partial^{2}}{\partial z^{2}}-\frac{C}{s^{2}}\left[\begin{array}{r}
\left(1+\frac{C_{w}}{C}\right)\left(\frac{\partial}{\partial t}+\alpha_{1 w} \frac{\partial}{\partial z}\right)\left(\frac{\partial}{\partial t}+\alpha_{2 w} \frac{\partial}{\partial z}\right)\left(\frac{\partial}{\partial t}+\alpha_{3 w} \frac{\partial}{\partial z}\right) \\
+\frac{B}{C}\left(\frac{\partial}{\partial t}+\gamma_{1} \frac{\partial}{\partial z}\right)\left(\frac{\partial}{\partial t}+\gamma_{2} \frac{\partial}{\partial z}\right)
\end{array}\right]=0
$$

donde $\alpha_{j w}, j=1,2,3$ son las raíces de la ecuación: $\alpha_{w}^{3}-R_{1} \alpha_{w}^{2}+R_{2} \alpha_{w}-R_{3}=0$, cuyos coeficientes son

$$
\begin{aligned}
& R_{1}=\frac{C}{C+C_{w}}\left[P_{1}+\frac{C_{w}}{C} Q_{1}\right], P_{1}=\eta_{33}+\eta_{32}+\eta_{31}, \\
& P_{2}=\eta_{33} \eta_{31}+\eta_{31} \eta_{32}+\eta_{32} \eta_{33}, \\
& R_{2}=\frac{C}{C+C_{w}}\left[P_{2}+\frac{C_{w}}{C} Q_{2}-\frac{s^{2}}{C}(1+A)\right], P_{3}=\eta_{31} \eta_{32} \eta_{33}, \\
& Q_{1}=\xi_{31}+\xi_{32}+\xi_{33}, \\
& R_{3}=\frac{C}{C+C_{w}}\left[P_{3}+\frac{C_{w}}{C} Q_{3}-\frac{s^{2}}{C}(1+A) a_{w}\right], Q_{32}+\xi_{32} \xi_{33}+\xi_{33} \xi_{31}, \\
& Q_{3}=\xi_{31} \xi_{32} \xi_{33} .
\end{aligned}
$$


Estos coeficientes incluyen la influencia de la compresibilidad del fluido a través de la velocidad de propagación del sonido en el fluido puro $s$ y son similares a los reportados en el Apéndice $G$ y en SSS (2011), pero considerando la influencia de la pared, a través de los polinomios $Q_{1}, Q_{2}$ y $Q_{3}$, así como también $A$ y. $a_{w} \cdot \gamma_{j}, j=1,2$ son las raíces de la ecuación:

$$
\gamma^{2}-\left(\xi_{21}+\xi_{22}-\frac{C}{B} g\right) \gamma+\left(\xi_{21} \xi_{22}-\frac{C}{B} g \eta_{21}\right)=0 .
$$

Debe señalarse que la única diferencia estructural entre la Ec. (5.30) y la Ec. (4.29), es el hecho de que ahora la onda compresible de segundo orden es una onda completa (con dos rapideces de propagación), mientras que era una onda incompleta (con una sola rapidez de propagación) en la Ec. (4.28) o en la Ec. (4.29), con un sólo operador ondulatorio de primer orden junto con un operador difusivo. Más aún, tomando los límites: $\beta_{s W} \rightarrow 0$ y $\beta_{g W} \rightarrow 0$, es fácil ver como la Ec. (5.28) se transforma en la Ec. (4.28), puesto que $A, C_{w}$, $B$ son cero.

\subsection{Análisis de estabilidad}

\subsubsection{Análisis de estabilidad de Whitham por aproximaciones de velocidades de fase}

Consideraremos que $\varphi(z, t)=\mathscr{A} e^{i(\kappa z-\omega t)}$ son las soluciones a la Ec. (5.30), donde $\kappa \in \mathbb{R}$ es el número de onda y $\omega=\omega_{R}+i \omega_{I} \in \mathbb{C}$ está formado por la frecuencia $\omega_{R}$ y por el factor de atenuación de onda $\omega_{I}$. Por lo tanto, para obtener estabilidad se requiere que $\omega_{I}<0$. Esto es equivalente a tomar la transformada de Fourier $(\partial / \partial t \rightarrow-i \omega$ y $\partial / \partial z \rightarrow i \kappa)$ de la Ec. (5.30), obteniendo que

$$
\tau\left(\omega-c_{1} \kappa\right)\left(\omega-c_{2} \kappa\right) \kappa^{2}-\frac{C}{s^{2}}\left[\begin{array}{r}
i\left(1+\frac{C_{w}}{C}\right)\left(\omega-\alpha_{1 W} \kappa\right)\left(\omega-\alpha_{2 W} \kappa\right)\left(\omega-\alpha_{3 W} \kappa\right) \\
-\frac{B}{C}\left(\omega-\gamma_{1} \kappa\right)\left(\omega-\gamma_{2} \kappa\right)
\end{array}\right]=0
$$

De acuerdo con el criterio de Whitham de las jerarquías de onda, debe ser posible analizar el comportamiento de la velocidad de la onda viajando con cada una de las velocidades de fase. Proponemos que: (1) $c_{1}>c_{2}$, (2) $\alpha_{1 w}>\alpha_{2 w}>\alpha_{3 w}$ y (3) $\gamma_{1}>\gamma_{2}$, para analizar algunas desigualdades. 
Por lo tanto, considerando que $\omega \rightarrow \alpha_{1 w} \kappa, \omega \rightarrow \alpha_{2 w} \kappa$ y $\omega \rightarrow \alpha_{3 w} \kappa$, se obtiene respectivamente que:

$$
\begin{gathered}
\tau \kappa^{2} s^{2}\left(\alpha_{1 w}-c_{1}\right)\left(\alpha_{1 w}-c_{2}\right)+B\left(\alpha_{1 w}-\gamma_{1}\right)\left(\alpha_{1 w}-\gamma_{2}\right)>0, \\
\tau \kappa^{2} s^{2}\left(\alpha_{2 w}-c_{1}\right)\left(\alpha_{2 w}-c_{2}\right)+B\left(\alpha_{2 w}-\gamma_{1}\right)\left(\alpha_{2 w}-\gamma_{2}\right)<0, \\
\tau \kappa^{2} s^{2}\left(\alpha_{3 w}-c_{1}\right)\left(\alpha_{3 w}-c_{2}\right)+B\left(\alpha_{3 w}-\gamma_{1}\right)\left(\alpha_{3 w}-\gamma_{2}\right)>0 .
\end{gathered}
$$

En forma similar, los resultados para $\omega \rightarrow c_{1} \kappa \quad$ y $\quad \omega \rightarrow c_{2} \kappa$, son $\left(c_{1}-\alpha_{1 w}\right)\left(c_{1}-\alpha_{2 w}\right)\left(c_{1}-\alpha_{3 w}\right)<0 \quad$ y $\quad\left(c_{2}-\alpha_{1 w}\right)\left(c_{2}-\alpha_{2 w}\right)\left(c_{2}-\alpha_{3 w}\right)>0$, respectivamente. Como $c_{1}>c_{2}$ y $\alpha_{1 w}>\alpha_{2 w}>\alpha_{3 w}$, entonces, ambas desigualdades implican que para que haya estabilidad se requiere que:

$$
\alpha_{1 w}>c_{1}>\alpha_{2 w}>c_{2}>\alpha_{3 w}
$$

Finalmente, para las rapideces de propagación $\omega \rightarrow \gamma_{1} \kappa$ y $\omega \rightarrow \gamma_{2} \kappa$, las condiciones de estabilidad son respectivamente:

$$
\left(\gamma_{1}-\alpha_{1 w}\right)\left(\gamma_{1}-\alpha_{2 w}\right)\left(\gamma_{1}-\alpha_{3 w}\right)<0 \text { y }\left(\gamma_{2}-\alpha_{1 w}\right)\left(\gamma_{2}-\alpha_{2 w}\right)\left(\gamma_{2}-\alpha_{3 w}\right)>0
$$

Puesto que $\gamma_{1}>\gamma_{2}$ y $\alpha_{1 w}>\alpha_{2 w}>\alpha_{3 w}$, estas desigualdades implican que

$$
\alpha_{1 w}>\gamma_{1}>\alpha_{2 w}>\gamma_{2}>\alpha_{3 w}
$$

Las desigualdades dadas en la Ec. (5.34) y en la Ec. (5.35) siempre se satisfacen al considerar la Ec. (5.37) y la Ec. (5.40). De la Ec. (5.36) y la Ec. (5.37) se sigue que

$$
\left(\alpha_{3 w}-c_{1}\right)\left(\alpha_{3 w}-c_{2}\right)>-\frac{B}{\tau \kappa^{2} s^{2}}\left(\alpha_{3 w}-\gamma_{1}\right)\left(\alpha_{3 w}-\gamma_{2}\right)
$$

Por lo tanto, la condición de estabilidad puede ubicarse como la condición límite en el número de onda, de la siguiente manera

$$
\kappa>\sqrt{-\frac{B}{\tau s^{2}} \frac{\left(\alpha_{3 w}-\gamma_{1}\right)\left(\alpha_{3 w}-\gamma_{2}\right)}{\left(\alpha_{3 w}-c_{1}\right)\left(\alpha_{3 w}-c_{2}\right)}}
$$




\subsubsection{Extensiones al enfoque de Liu (1982)}

El análisis previo mostró que la compresibilidad y el efecto de la pared aumentan la región de estabilidad cuando se comparan con el comportamiento incompresible sin pared. Sin embargo, para desarrollar un análisis de estabilidad siguiendo el enfoque de Liu (1982), comenzamos con la transformada de Fourier de la Ec. (5.28),

$$
-\frac{C}{s^{2}}\left[\begin{array}{r}
{\left[\tau\left(\omega-c_{1} \kappa\right)\left(\omega-c_{2} \kappa\right)+i(1+A)\left(\omega-a_{w} \kappa\right)\right] \kappa^{2}} \\
i\left(\omega-\eta_{31} \kappa\right)\left(\omega-\eta_{32} \kappa\right)\left(\omega-\eta_{33} \kappa\right)+i \frac{C_{w}}{C}\left(\omega-\xi_{31} \kappa\right)\left(\omega-\xi_{32} \kappa\right)\left(\omega-\xi_{33} \kappa\right) \\
-g\left(\omega-\eta_{21} \kappa\right) \kappa-\frac{B}{C}\left(\omega-\xi_{21} \kappa\right)\left(\omega-\xi_{22} \kappa\right)
\end{array}\right]=0 .
$$

En esta ecuación, las condiciones de estabilidad pueden encontrarse al considerar que las ondas se mueven con rapideces cercanas a las de las ondas de segundo orden incompresibles y luego tomando los límites cuando $\omega \rightarrow c_{1} \kappa$ y $\omega \rightarrow c_{2} \kappa$. De la Ec. (5.43):

$$
\begin{array}{r}
a<c_{1}+\left[\begin{array}{r}
A c_{1}-A a_{f}-\frac{C_{w}}{s^{2}}\left(c_{1}-\xi_{31}\right)\left(c_{1}-\xi_{32}\right)\left(c_{1}-\xi_{33}\right) \\
-\frac{C}{s^{2}}\left(c_{1}-\eta_{31}\right)\left(c_{1}-\eta_{32}\right)\left(c_{1}-\eta_{33}\right)
\end{array}\right], \\
a>c_{2}+\left[\begin{array}{r}
A c_{2}-A a_{f}-\frac{C_{w}}{s^{2}}\left(c_{2}-\xi_{31}\right)\left(c_{2}-\xi_{32}\right)\left(c_{2}-\xi_{33}\right) \\
-\frac{C}{s^{2}}\left(c_{2}-\eta_{31}\right)\left(c_{2}-\eta_{32}\right)\left(c_{2}-\eta_{33}\right)
\end{array}\right] .
\end{array}
$$

Cuando en este criterio se eliminan los efectos de pared, se obtiene la condición de Liu que toma en cuenta la compresibilidad de la fase gas, obtenida en el Capítulo 4 [Ec. (4.45)]:

$c_{1}+h_{1}=c_{1}-\frac{C}{s^{2}}\left(c_{1}-\eta_{31}\right)\left(c_{1}-\eta_{32}\right)\left(c_{1}-\eta_{33}\right)>a>c_{2}-\frac{C}{s^{2}}\left(c_{2}-\eta_{31}\right)\left(c_{2}-\eta_{32}\right)\left(c_{2}-\eta_{33}\right)=c_{2}-h_{2}$

y a la bien conocida condición $c_{1}>a>c_{2}$ en el límite de incompresibilidad y clarifica cómo los efectos de pared modifican estos límites de estabilidad a través de las funciones $A$ y $C_{w}$. Puede observarse en la Ec. (5.45) como hay una parte incompresible de la pared $A$ y una compresible de la pared $C_{w}$ que modifican el criterio. 


\subsection{Resultados y discusión}

Se estudiaron dos casos de estudio, un catalizador de FCC arrastrado por un flujo turbulento de vapor de agua y un sistema experimental CFB de arena-aire. En la Tabla 5.1 se dan las propiedades físicas del sólido y del fluido, así como los parámetros geométricos y las condiciones de operación en un estado base homogéneo para cada sistema.

En esta sección, se analizan ambos sistemas, enfatizando sus similitudes y diferencias. Se considera como en el Capítulo anterior, que la fracción volumen de sólido en estado base está dada por:

$$
\varepsilon_{s 0}=\frac{U_{s}}{U_{m}-U_{t}},
$$

donde se calcula la velocidad superficial del sólido $U_{s}$, dada la fracción volumen de sólido.

Tabla 5.1. Valores de los parámetros y de las condiciones de operación en estado base.

\begin{tabular}{lll}
\hline \multicolumn{1}{c}{ Parámetro } & Catalizador-vapor & Arena-aire \\
\hline Velocidad del sonido en el fluido & & \\
puro, $s(\mathrm{~m} / \mathrm{s})$ & 655 & 346 \\
Densidad del sólido, $\rho_{s 0}\left(\mathrm{~kg} / \mathrm{m}^{3}\right)$ & 1300 & 2650 \\
Densidad del fluido, $\rho_{g 0}\left(\mathrm{~kg} / \mathrm{m}^{3}\right)$ & 0.7065 & 0.9340 \\
Diámetro del grano sólido, $d_{p}(\mu \mathrm{m})$ & 60 & 310 \\
Factor de fricción del fluido & 0.0158 & 0.01670 \\
Factor de fricción del sólido & $0.0285 \sqrt{g D_{t}} / v_{s 0}$ & $0.0285 \sqrt{g D_{t}} / v_{s 0}$ \\
Velocidad terminal, $U_{t}(\mathrm{~m} / \mathrm{s})$ & 0.085 & 2.231 \\
Velocidad superficial del fluido, $U_{g}$ & & \\
(m/s) & 7.000 & 3.200 \\
Diámetro de la columna, $D_{t}(\mathrm{~m})$ & 1.168 & 0.960 \\
Longitud de la columna, $L(\mathrm{~m})$ & 22 & 9 \\
Número de Reynolds del fluido, $\mathrm{Re}_{g}$ & $1.93 \times 10^{5}$ & $1.43 \times 10^{5}$ \\
Número de Reynolds del sólido, $\mathrm{Re}_{s}$ & 0.12 & 32.30 \\
Número de Stokes, St & 0.13 & 2.15 \\
\hline
\end{tabular}

${ }^{4}$ de acuerdo con la fórmula del factor de fricción, ${ }^{5}$ de acuerdo con la fórmula de Konno y Saito (1969). 
5.4.1. Valores de los parámetros y de las condiciones de operación de un sistema catalizador-vapor en una unidad de FCC y un sistema de arena-aire en un CFB Utilizamos el módulo de compresibilidad del sólido reportado por Jiradilok y col. (2006) dado por la expresión, $\varepsilon_{s 0} \Phi_{0}^{\prime}=10^{6.837-2.475 \varepsilon_{0}}$. El parámetro de interacción $\beta$ se seleccionó para partículas sólidas de catalizador de FCC de acuerdo al régimen de Stokes. Para los granos, el mejor coeficiente de arrastre está dado por la ecuación de Ergun.

\subsubsection{Comparación de los coeficientes ondulatorios y significado de las jerarquías de onda}

Para comparar el modelo con y sin efectos de pared (Capítulo 4 y SSS, 2011), se obtienen las ecuaciones adimensionales equivalentes a la Ec. (5.28) y a la Ec. (5.30), considerando la longitud de la columna $L$ y la velocidad media de la mezcla $U_{0}$ como parámetros de escalamiento. Las variables adimensionales son $z^{\prime} \doteq z / L, a_{w}{ }^{\prime}=a_{w} / U_{0}, t^{\prime}=t U_{0} / L$ y todas las rapideces de propagación adimensionales pueden definirse en forma similar a $a_{w}^{\prime}$. Obteniendo que,

$$
\begin{aligned}
& {\left[\tau^{\prime}\left(\frac{\partial}{\partial t^{\prime}}+c_{1}^{\prime} \frac{\partial}{\partial z^{\prime}}\right)\left(\frac{\partial}{\partial t^{\prime}}+c_{2}^{\prime} \frac{\partial}{\partial z^{\prime}}\right)+(1+A)\left(\frac{\partial}{\partial t^{\prime}}+a_{w}^{\prime} \frac{\partial}{\partial z^{\prime}}\right)\right] \frac{\partial^{2}}{\partial z^{\prime 2}}} \\
& -C^{\prime}\left[\begin{array}{r}
\left(\frac{\partial}{\partial t^{\prime}}+\eta_{31}^{\prime} \frac{\partial}{\partial z^{\prime}}\right)\left(\frac{\partial}{\partial t^{\prime}}+\eta_{32}^{\prime} \frac{\partial}{\partial z^{\prime}}\right)\left(\frac{\partial}{\partial t^{\prime}}+\eta_{33}^{\prime} \frac{\partial}{\partial z^{\prime}}\right)+C_{w}^{\prime}\left(\frac{\partial}{\partial t^{\prime}}+\xi_{31}^{\prime} \frac{\partial}{\partial z^{\prime}}\right)\left(\frac{\partial}{\partial t^{\prime}}+\xi_{32}^{\prime} \frac{\partial}{\partial z^{\prime}}\right)\left(\frac{\partial}{\partial t^{\prime}}+\xi_{33}^{\prime} \frac{\partial}{\partial z^{\prime}}\right) \\
+B^{\prime}\left[-\frac{G^{\prime}}{B^{\prime}}\left(\frac{\partial}{\partial t^{\prime}}+\eta_{21}^{\prime} \frac{\partial}{\partial z^{\prime}}\right) \frac{\partial}{\partial z^{\prime}}+\left(\frac{\partial}{\partial t^{\prime}}+\xi_{21}^{\prime} \frac{\partial}{\partial z^{\prime}}\right)\left(\frac{\partial}{\partial t^{\prime}}+\xi_{22}^{\prime} \frac{\partial}{\partial z^{\prime}}\right)\right]
\end{array}\right]=0
\end{aligned}
$$

$\mathrm{y}$

$$
\begin{aligned}
& \tau^{\prime}\left(\frac{\partial}{\partial t^{\prime}}+c_{1}^{\prime} \frac{\partial}{\partial z^{\prime}}\right)\left(\frac{\partial}{\partial t^{\prime}}+c_{2}^{\prime} \frac{\partial}{\partial z^{\prime}}\right) \frac{\partial^{2}}{\partial z^{\prime 2}} \varphi \\
& -C^{\prime}\left[\begin{array}{r}
\left(1+C_{w}^{\prime}\right)\left(\frac{\partial}{\partial t^{\prime}}+\alpha_{1 w}^{\prime} \frac{\partial}{\partial z^{\prime}}\right)\left(\frac{\partial}{\partial t^{\prime}}+\alpha_{2 w}^{\prime} \frac{\partial}{\partial z^{\prime}}\right)\left(\frac{\partial}{\partial t^{\prime}}+\alpha_{3 w}^{\prime} \frac{\partial}{\partial z^{\prime}}\right) \\
\left.+B^{\prime}\left(\frac{\partial}{\partial t^{\prime}}+\gamma_{21}^{\prime} \frac{\partial}{\partial z^{\prime}}\right)\left(\frac{\partial}{\partial t^{\prime}}+\gamma_{22}^{\prime} \frac{\partial}{\partial z^{\prime}}\right)\right] \varphi=0
\end{array}\right]
\end{aligned}
$$

donde los coeficientes adimensionales también se denotan con índices primados, entonces $\tau^{\prime} \doteq U_{0} \tau / L, C^{\prime} \doteq\left(U_{0} / s\right)^{2} C, C_{w}^{\prime}=C_{w} / C, G^{\prime}=g L / U_{0}^{2}$ y $B^{\prime} \doteq B L / U_{0} C$. 
Los coeficientes $A, C_{w}^{\prime}\left(=C_{w} / C\right), B^{\prime}$ y $G^{\prime} / B^{\prime}$, representan los efectos de pared y se grafican como función de la fracción volumen de sólido.

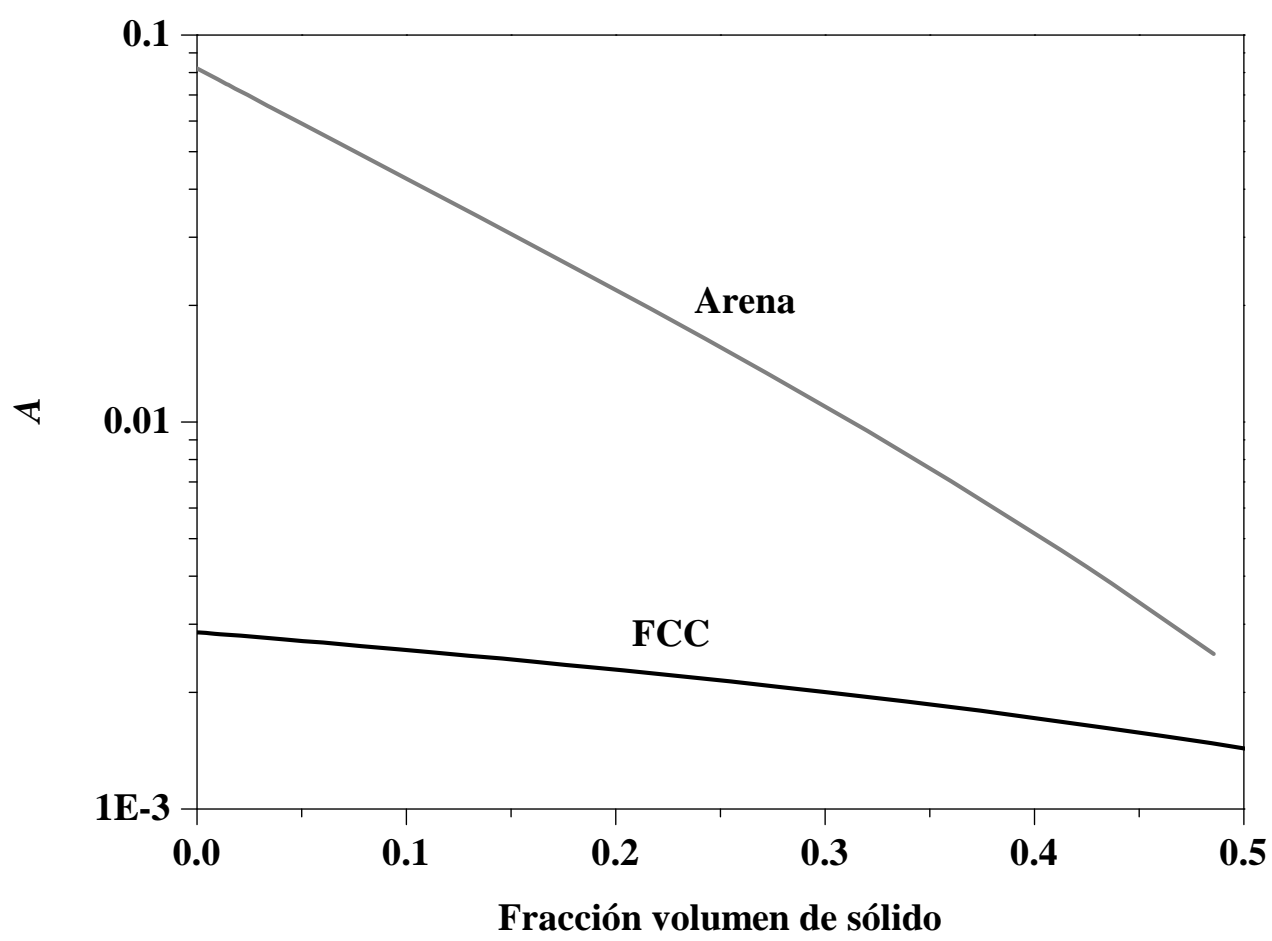

Figura 5.1. Coeficiente $A$ para los sistemas: Arena (línea gris) y FCC (línea negra).

La Figura 5.1 ilustra la importancia del efecto de la pared en la onda de primer orden del modelo incompresible, representado por el coeficiente $A$ comparado con la unidad.

Como podemos observar, para la Arena tiene un valor de 0.08 a fracciones de sólido cercanas a cero y decae hasta $3 \times 10^{-3}$ para una fracción volumen de sólido cercana a 0.5 , en tanto que para el FCC, el valor mayor es de $3 \times 10^{-3}$ y decae hasta $1.5 \times 10^{-3}$.

Por lo tanto, notamos que el efecto adicional obtenido de considerar los efectos de pared en la onda de primer orden de la parte incompresible es menor al $8 \%$. 


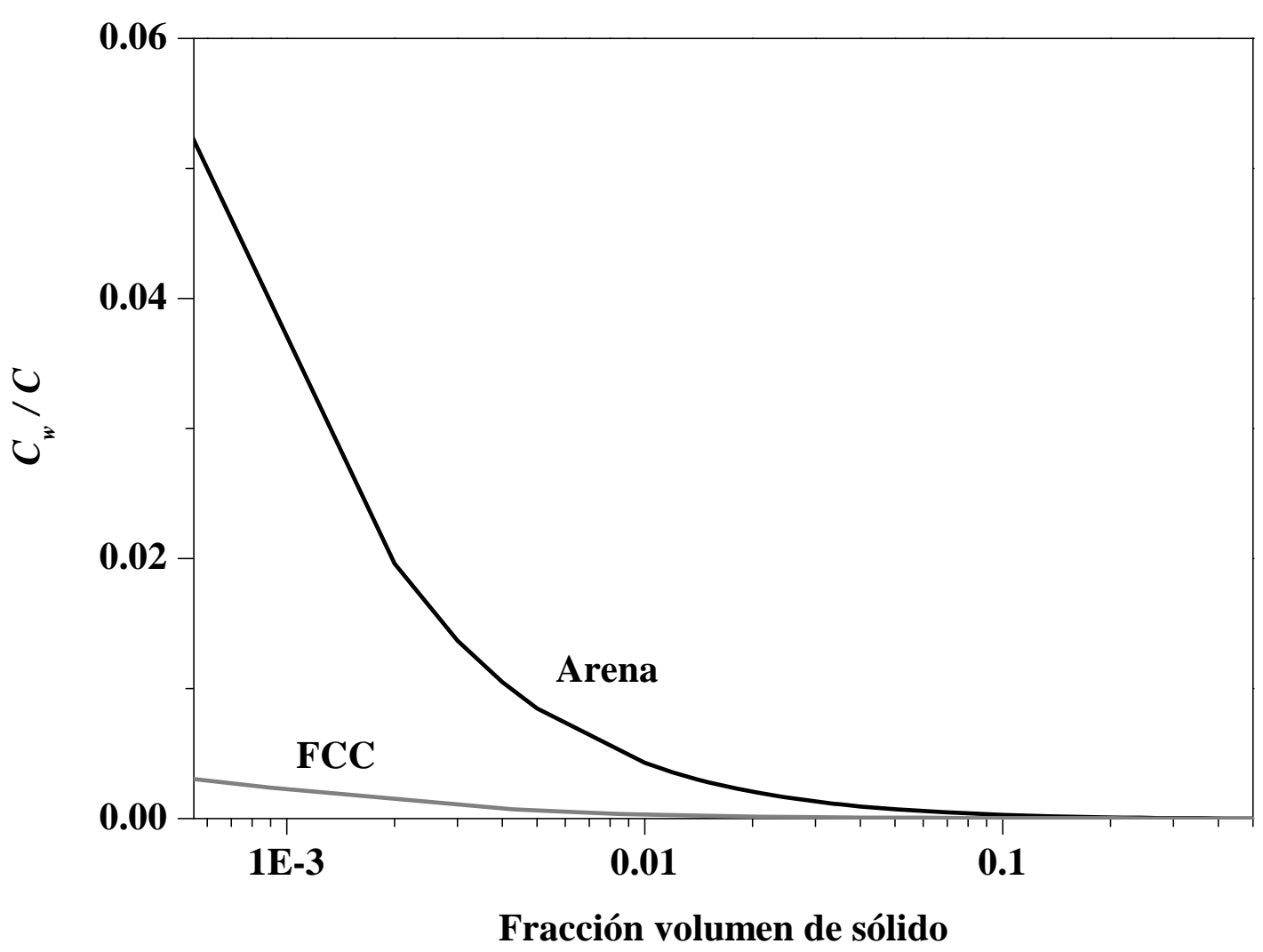

Figura 5.2. Coeficiente de tercer orden $C_{w}^{\prime}$ para los sistemas: Arena (línea gris) y FCC (línea negra).

El coeficiente compresible de tercer orden con efecto de pared normalizado con el coeficiente compresible de tercer orden sin pared, está graficado en la Figura 5.2.

En la gráfica podemos observar que para la Arena éstos van de 0.05 y decaen a cero al aumentar la fracción volumen de sólido, mientras que para el FCC, el valor máximo es de 0.003 y decaen rápidamente.

Esto es, tienen un valor de alrededor del 5\% del efecto correspondiente compresible sin pared. Por lo que a este nivel tienen un valor cualitativo más que cuantitativo. 


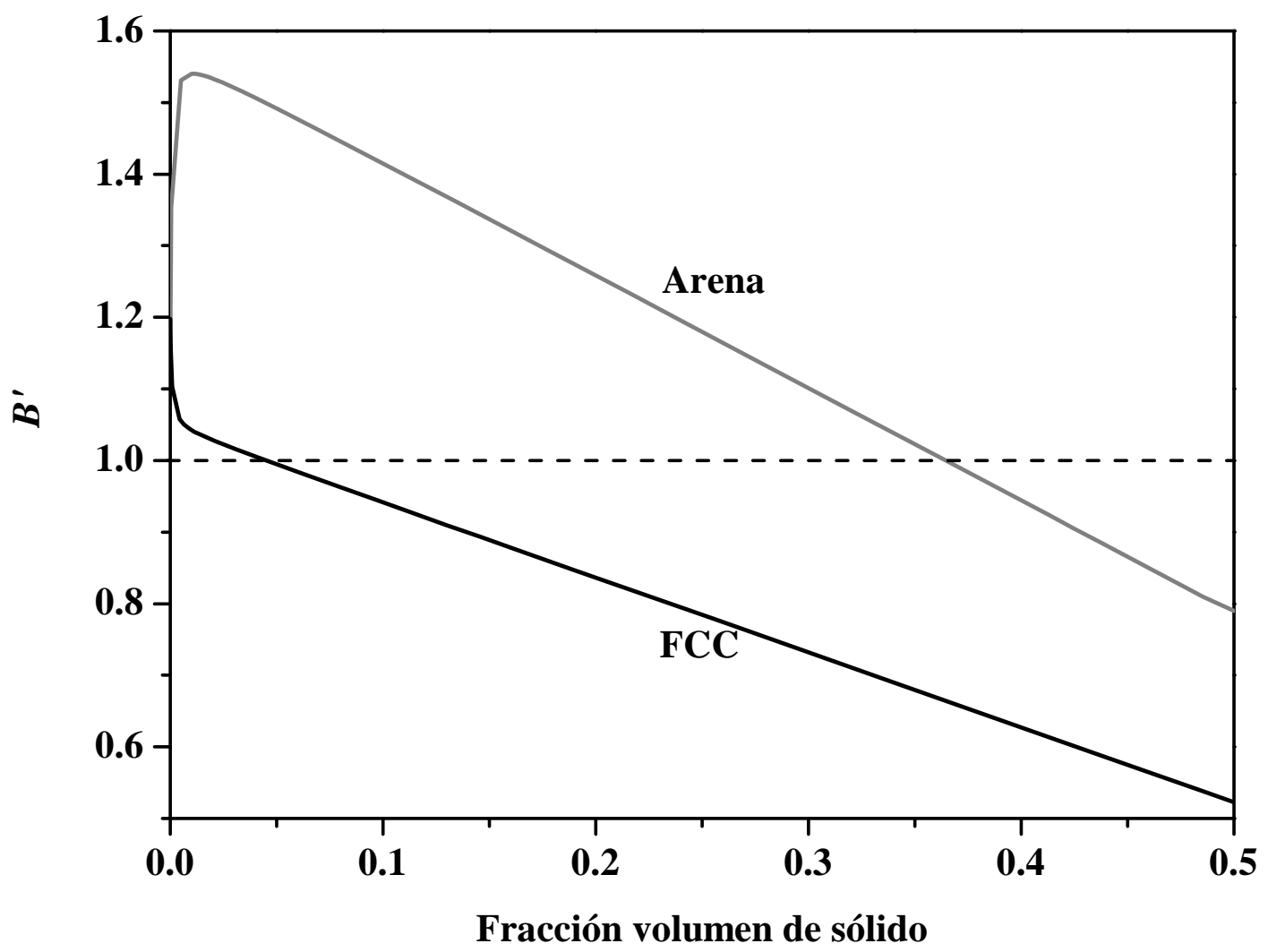

Figura 5.3. Coeficiente de segundo orden $B^{\prime}$ para los sistemas: Arena (línea gris) y FCC (línea negra).

El efecto de pared en la onda de segundo orden compresible se ilustra en la Figura 5.3. Como podemos notar, este efecto tiene valor cuantitativo ya que va de 1.5 a 0.8 para la Arena y de 1.1 a 0.5 para el FCC, que es comparable con el coeficiente sin efecto de pared que es unitario.

Además, tiene valor cualitativo porque ahora la onda de segundo orden se completa con dos rapideces de propagación, dejando de tener un comportamiento hiperbólico degenerado (parabólico) a hiperbólico completo. 


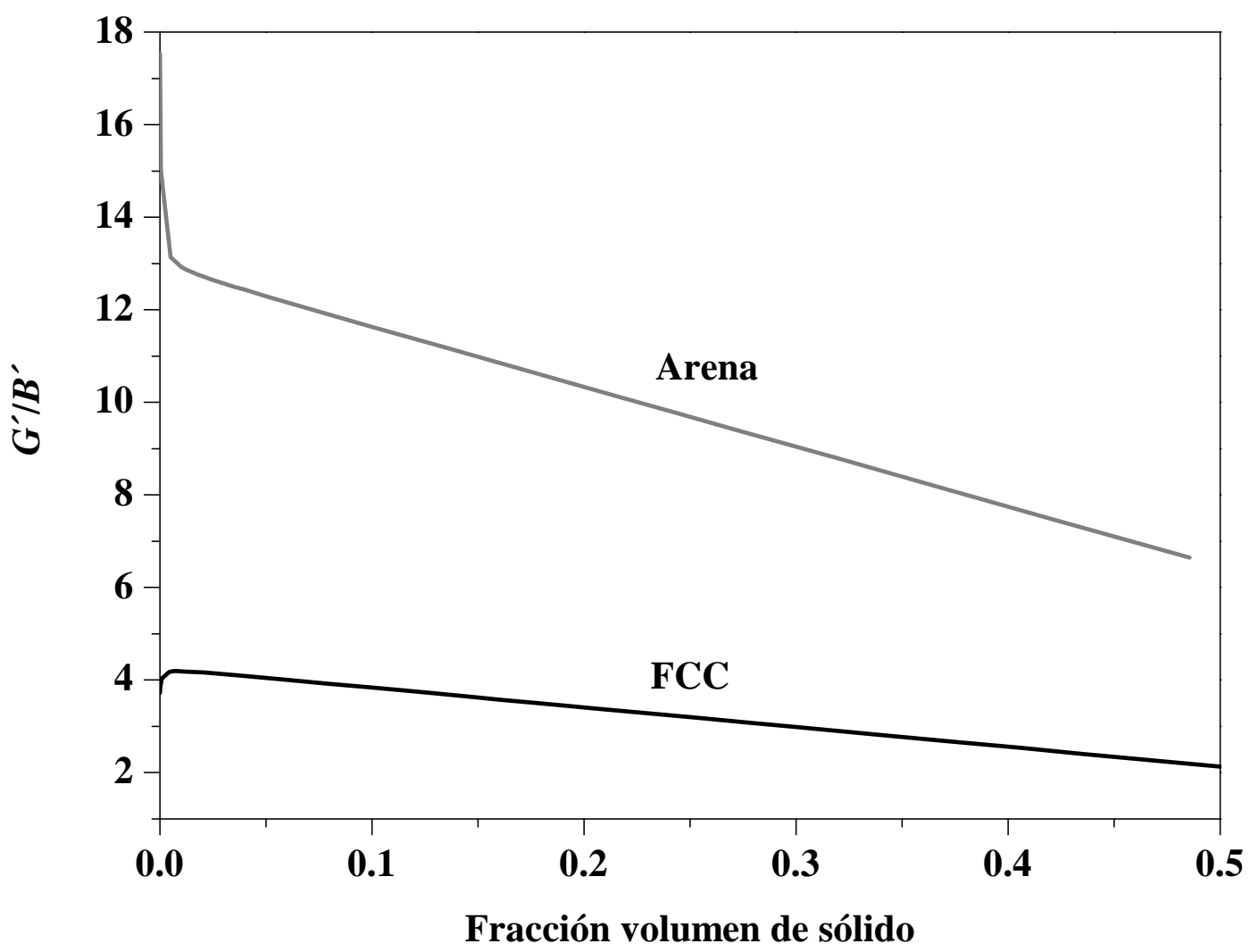

Figura 5.4. Coeficiente de segundo orden para los sistemas: Arena (línea gris) y FCC (línea negra).

En la Figura 5.4, se reporta el coeficiente de la onda compresible de segundo orden que para el caso de la Arena decae de 14 a 7, en tanto que para el FCC decae de 4 a 2.5, lo cual está en el mismo orden que la contribución de la onda asociada a la pared que tiene un coeficiente unitario.

En este caso la onda de segundo orden de efectos de pared que se superpone a la onda degenerada compresible de segundo orden, tiene un valor cualitativo importante porque permite volver hiperbólico el operador parabólico (hiperbólico degenerado) con una rapidez adicional asociada a la pared (Soria y col., 2008). 


\subsubsection{Análisis de las rapideces de propagación}

Las rapideces $\xi_{21}$ y $\xi_{22}$ están graficadas en la Figura 5.5. Notamos que tienen magnitudes similares a las velocidades de flujo.
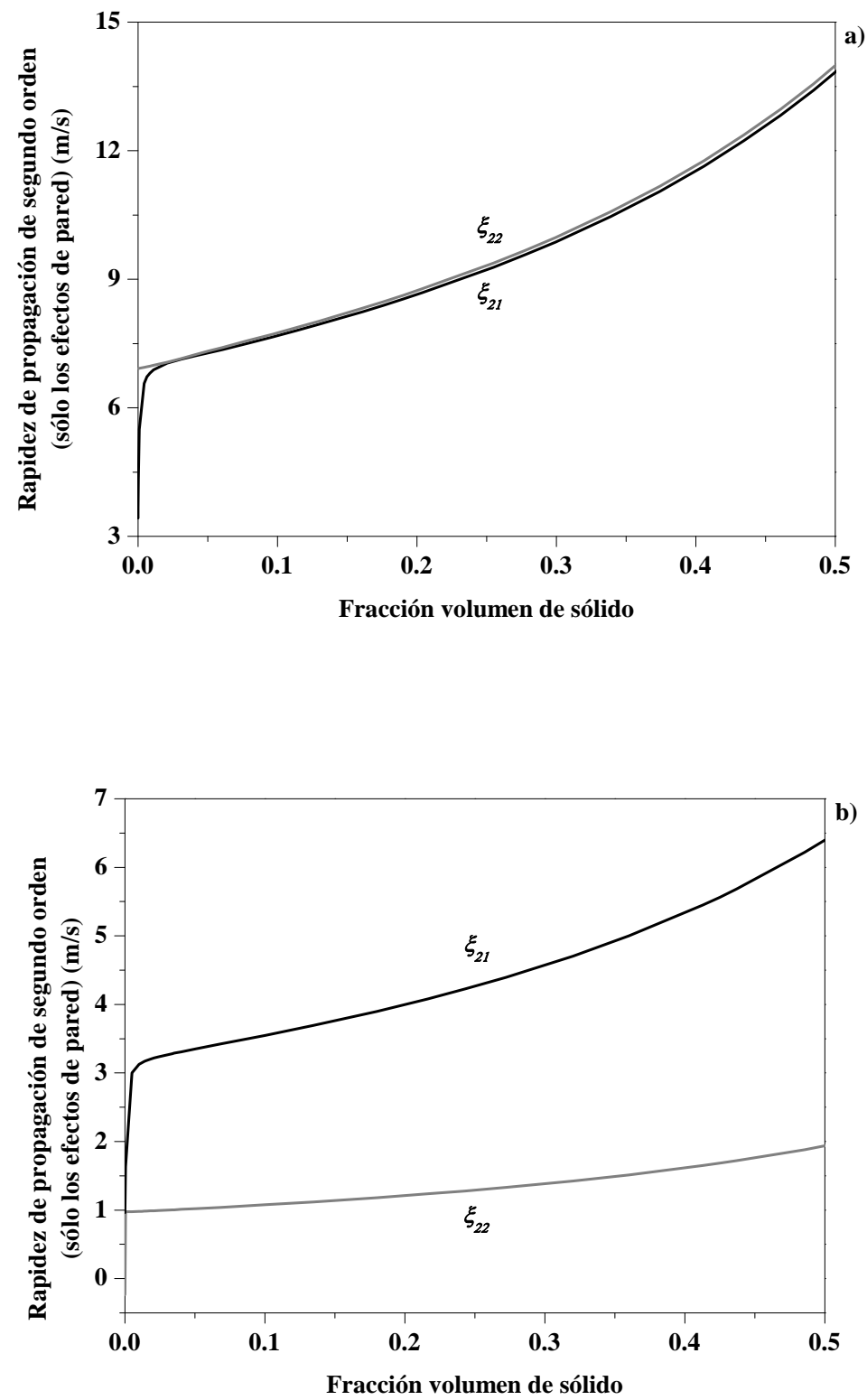

Figura 5.5. Contribución de segundo orden de los efectos de pared a las rapideces de propagación, como función de la fracción volumen de sólido para los sistemas: (a) FCC y (b) Arena. 

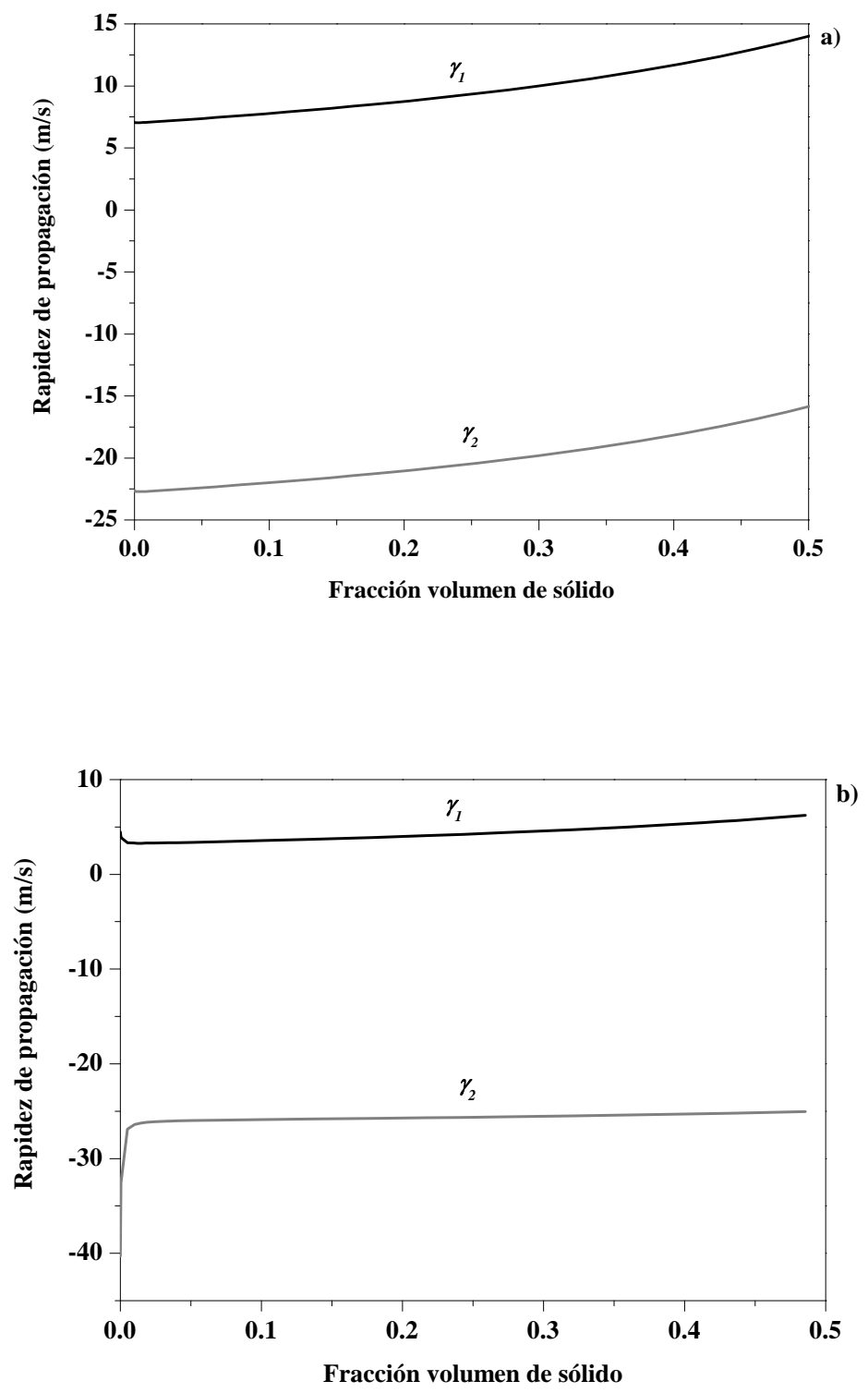

Figura 5.6. Rapideces de propagación de segundo orden, para los sistemas: (a) FCC y (b) Arena.

La gráfica de $\gamma_{1}$ y $\gamma_{2}$ se reporta en la Figura 5.6. $\gamma_{1}$ corresponde a $\eta_{21}$ en la onda de segundo orden degenerada del modelo aglutinado [Ec. (4.23)]. Note como los efectos de pared completaron la onda y aparece una velocidad adicional $\gamma_{2}$, la cual se debe a la fricción en la pared y cuya expresión aproximada es (Soria y col., 2008):

$$
\gamma_{2} \approx \frac{v_{g 0}+v_{s 0}}{2}-\frac{\varepsilon_{s 0} g}{2 v_{s 0} \beta_{s W}}
$$



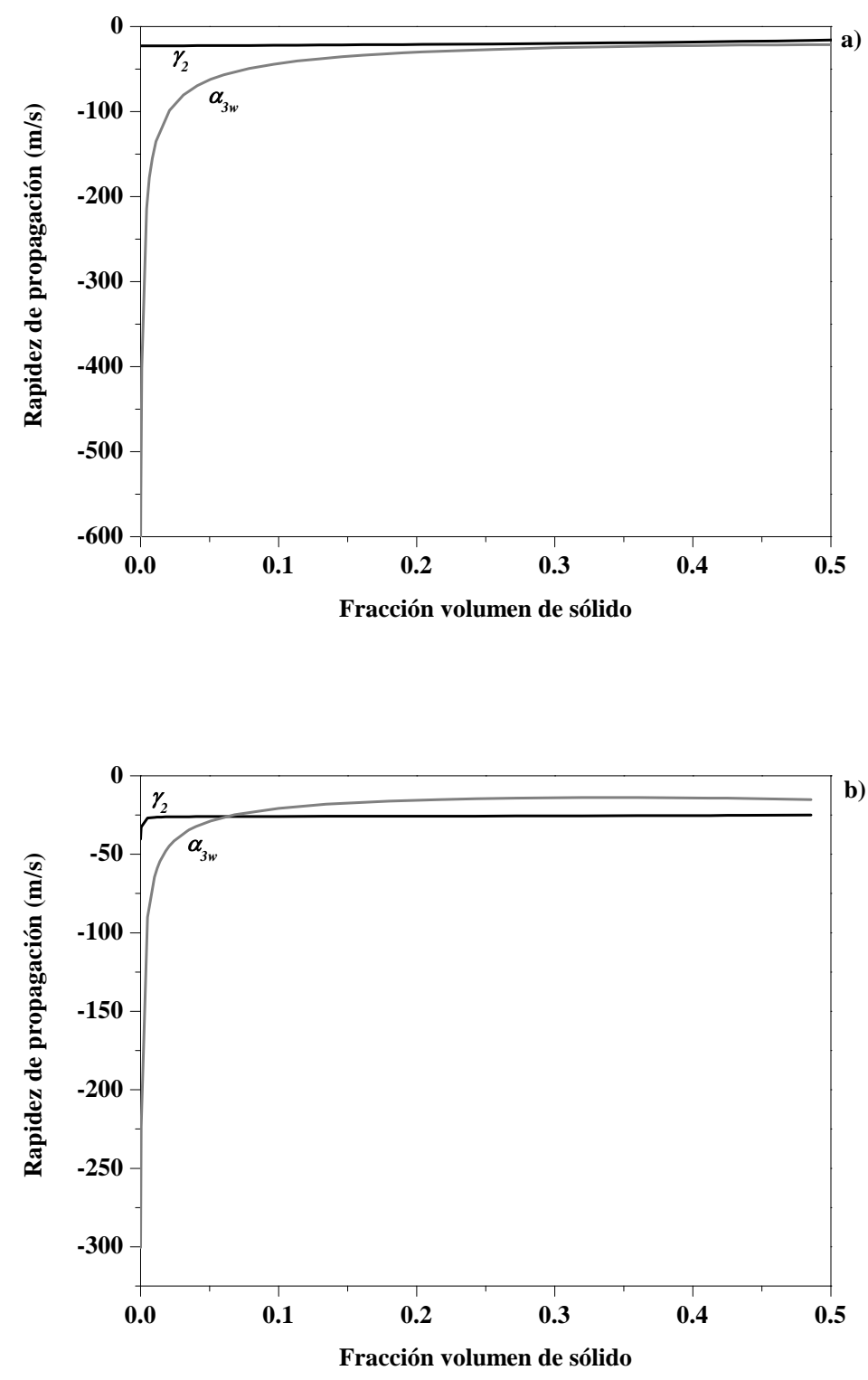

Figura 5.7. Rapideces de propagación $\gamma_{2}$ y $\alpha_{3 w}$ como función de la fracción volumen de sólido, para los sistemas: (a) FCC y (b) Arena.

En la Figura 5.7 reportamos la rapidez de segundo orden incorporada por el efecto de pared junto con la rapidez de tercer orden de la parte compresible. En el FCC [caso (a)], podemos ver como no hay ningún entrecruzamiento, en tanto que para la Arena [caso (b)], ocurre un entrecruzamiento alrededor de un valor de 0.07 de fracción volumen de sólido. Dando lugar a una inestabilidad. 


\subsubsection{Condiciones de estabilidad}

En la Figura 5.8 mostramos como el efecto de la pared amplía la zona de estabilidad encontrada en el Capítulo 4 al incorporar la compresibilidad de la fase gas.

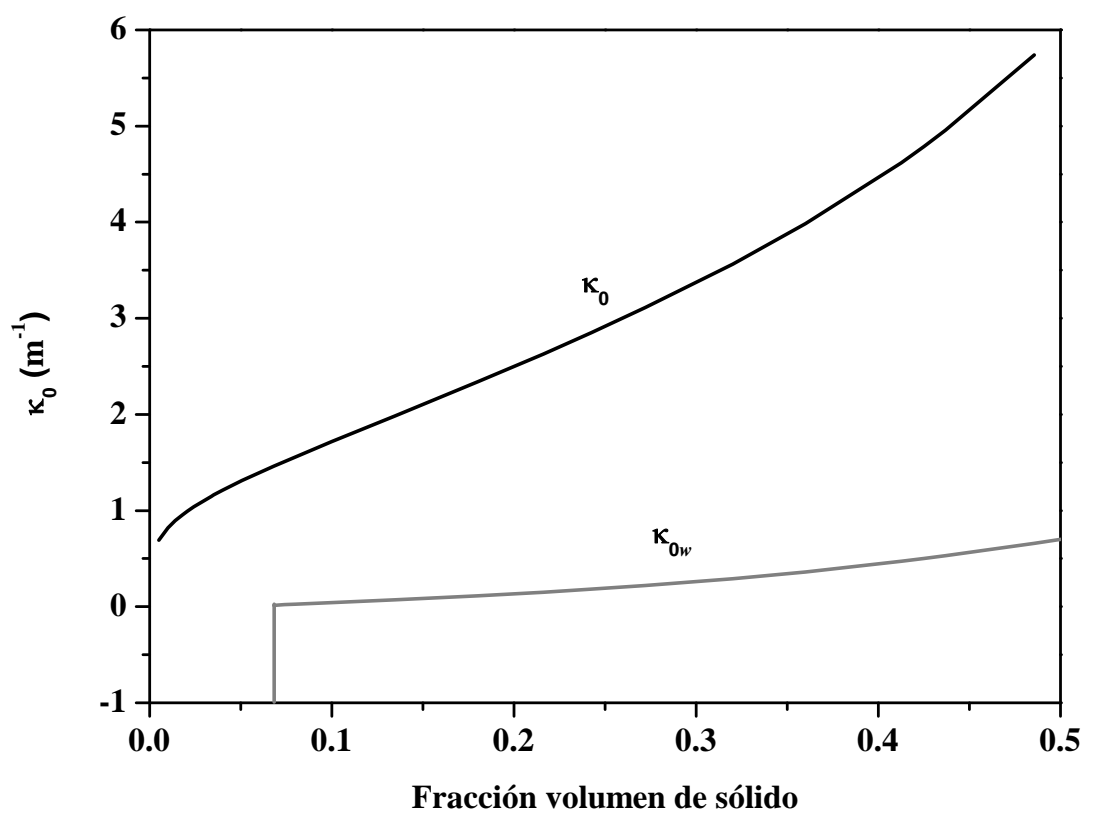

Figura 5.8. Número de onda para estabilidad lineal, como función de la fracción volumen de sólido para la Arena. 


\section{Capítulo 6}

\section{Modelo no-isotérmico unidimensional con promedio espacio-temporal}

\subsection{Introducción}

El modelo en el que ahora nos centraremos está constituido por las ecuaciones de conservación de la masa, las ecuaciones de balance de cantidad de movimiento y las ecuaciones de balance de energía térmica, de campo promedio. Obtendremos un modelo no-isotérmico cerrado que considere los efectos de pared.

\subsection{Modelamiento del flujo no-isotérmico gas-sólido en el riser}

\subsubsection{Sistema físico}

El sistema físico está formado por un conjunto monodisperso de partículas esféricas rígidas de diámetro medio $d_{p}$, moviéndose con una corriente de gas o vapor en un tubo vertical. El fluido es un gas Newtoniano compresible de densidad $\rho_{g}$ y viscosidad constante $\mu_{g}$. La dispersión de partículas es no-isotérmica y diluida con fracción volumen de sólido $\varepsilon_{s 0} \ll 1$.

Las partículas son alimentadas al tubo a una temperatura mayor que la temperatura promedio del gas. El promedio espacio-temporal se toma sobre la sección transversal del riser incluyendo la pared del tubo (ver la Figura 1.2).

\subsubsection{Ecuaciones promediadas en el espacio-tiempo}

En esta Sección retomaremos las ecuaciones promediadas de masa y de cantidad de movimiento del Capítulo 1, Ec. (1.47) y Ec. (1.48), respectivamente. Además, por tratarse de un sistema no-isotérmico, debe incorporarse la ecuación promediada de energía térmica dada por la Ec. (C.20) del Apéndice $C$. 
Ecuación de conservación de la masa

Aplicando la C.F.1 y la C.F.4 en la Ec. (1.47) se elimina el flujo de masa en la interfaz $\Gamma_{k}=0$, obteniéndose:

$$
\varepsilon_{k} \frac{D_{k}}{D t}\left\langle\rho_{k}\right\rangle^{k}+\left\langle\rho_{k}\right\rangle^{k}\left(\frac{D_{k}}{D t} \varepsilon_{k}+\varepsilon_{k} \nabla \cdot\left\{\mathbf{v}_{k}\right\}^{k}\right)=0,
$$

o bien, en términos de la derivada parcial,

$$
\frac{\partial}{\partial t}\left(\varepsilon_{k}\left\langle\rho_{k}\right\rangle^{k}\right)+\nabla \cdot\left[\varepsilon_{k}\left\langle\rho_{k}\right\rangle^{k}\left\{\mathbf{v}_{k}\right\}^{k}\right]=0
$$

Esta ecuación implica que los materiales no cambian de fase; es decir, ni el vapor se absorbe en las partículas ni las partículas se subliman en el vapor. Esta es la ecuación de conservación de la masa de campo promedio para la fase $k$. Las fases pueden ser compresibles o incompresibles.

Ecuación de balance de cantidad de movimiento

Aplicando la C.F.1 y la C.F.4 en la Ec. (1.48) se elimina el flujo de masa en la interfaz $\Gamma_{k}=0$,

$$
\begin{aligned}
\varepsilon_{k}\left\langle\rho_{k}\right\rangle^{k} \frac{D_{k}}{D t}\left\{\mathbf{v}_{k}\right\}^{k}-\varepsilon_{k}\left\langle\rho_{k}\right\rangle^{k} \mathbf{g}_{k}+\varepsilon_{k} \nabla\left\langle P_{k}\right\rangle^{k} & +\left(\left\langle P_{k}\right\rangle^{k}-\left\langle P_{k}\right\rangle_{i}\right) \nabla \varepsilon_{k} \\
& -\nabla \cdot\left[\varepsilon_{k}\left(-\left\langle\boldsymbol{\tau}_{k}\right\rangle^{k}+\mathbf{\Pi}_{k}^{\mathrm{Re}}\right)\right]=\Gamma_{k}\left(\mathbf{v}_{k i}-\left\{\mathbf{v}_{k}\right\}^{k}\right)+\mathbf{M}_{k}^{D}
\end{aligned}
$$

donde $\quad \mathbf{M}_{k}^{D} \doteq \mathbf{M}_{k p}+\mathbf{M}_{k t}+\left(\left\langle P_{k}\right\rangle_{i} \mathbf{1}+\left\langle\boldsymbol{\tau}_{k}\right\rangle_{i}\right) \cdot \nabla \varepsilon_{k}$, son los esfuerzos superficiales totales (normales y tangenciales). También podemos expresar esta ecuación en términos de la derivada parcial,

$$
\begin{array}{r}
\frac{\partial}{\partial t}\left(\varepsilon_{k}\left\langle\rho_{k}\right\rangle^{k}\left\{\mathbf{v}_{k}\right\}^{k}\right)+\nabla \cdot\left(\varepsilon_{k}\left\langle\rho_{k}\right\rangle^{k}\left\{\mathbf{v}_{k}\right\}^{k}\left\{\mathbf{v}_{k}\right\}^{k}\right)+\varepsilon_{k} \nabla \cdot\left\langle\boldsymbol{\Pi}_{k}\right\rangle^{k}-\nabla \cdot\left(\varepsilon_{k} \mathbf{\Pi}_{k}^{\mathrm{Re}}\right) \\
+\sum_{k \ell \mid k} a_{k \ell}\left\langle\mathbf{n}_{k} \cdot \tilde{\boldsymbol{\Pi}}_{k}\right\rangle_{k \ell}-\varepsilon_{k}\left\langle\rho_{k}\right\rangle^{k} \mathbf{g}=\mathbf{0} .
\end{array}
$$

Puede demostrarse que: $-\nabla \cdot\left(\varepsilon_{k}\left\langle\boldsymbol{\Pi}_{k}\right\rangle^{k}\right)-\mathbf{M}_{k}=\varepsilon_{k} \nabla \cdot\left\langle\boldsymbol{\Pi}_{k}\right\rangle^{k}+\sum_{k \ell \mid k} a_{k \ell}\left\langle\mathbf{n}_{k} \cdot \tilde{\boldsymbol{\Pi}}_{k}\right\rangle_{k \ell}$, al utilizar la Ec. (1.39) y la Ec. (1.40). 
Ecuación de balance de energía térmica

Aplicando la C.F.1 y la C.F.4 en la Ec. (C.21) se elimina el flujo de energía calorífica en la interfaz debido a cambios de fase, además no hay generación de calor en el volumen por lo que $Q_{k}=0$,

$$
\begin{aligned}
\varepsilon_{k}\left\langle\rho_{k}\right\rangle^{k} \frac{D_{k}}{D t}\left\{U_{k}\right\}^{k}+\nabla \cdot\left[\varepsilon_{k}\left(\left\langle\mathbf{q}_{k}\right\rangle^{k}+\mathbf{q}_{k}^{r}\right)\right]-\varepsilon_{k}\left\langle Q_{k}\right\rangle^{k} & +\left\langle X_{k} P_{k}\left(\nabla^{\prime} \cdot \mathbf{v}_{k}\right)\right\rangle \\
& +\left\langle X_{k} \boldsymbol{\tau}_{k}: \nabla^{\prime} \mathbf{v}_{k}\right\rangle=\Gamma_{k}\left(U_{k i}-\left\{U_{k}\right\}^{k}\right)+E_{k},
\end{aligned}
$$

o bien,

$$
\begin{aligned}
\varepsilon_{k}\left\langle\rho_{k}\right\rangle^{k} \frac{D_{k}}{D t}\left\{U_{k}\right\}^{k} & +\nabla \cdot\left[\varepsilon_{k}\left(\left\langle\mathbf{q}_{k}\right\rangle^{k}+\mathbf{q}_{k}^{r}\right)\right]+\left\langle X_{k} P_{k}\left(\nabla^{\prime} \cdot \mathbf{v}_{k}\right)\right\rangle \\
& +\left\langle X_{k} \boldsymbol{\tau}_{k}: \nabla^{\prime} \mathbf{v}_{k}\right\rangle+\sum_{k \ell \mid k} a_{k \ell}\left\langle\mathbf{n}_{k} \cdot \mathbf{q}_{k}\right\rangle_{k \ell}=0
\end{aligned}
$$

Donde podemos reconocer $E_{k} \doteq\left\langle\mathbf{q}_{k} \cdot \nabla^{\prime} X_{k}\right\rangle=-\sum_{k \ell \mid k} a_{k \ell}\left\langle\mathbf{n}_{k} \cdot \mathbf{q}_{k}\right\rangle_{k \ell}$, como el flux de energía calorífica interfacial. En esta ecuación, también se ha introducido el tensor de esfuerzos totales y $\mathbf{q}_{k}^{r} \doteq\left\langle X_{k} \rho_{k} \tilde{\mathbf{v}}_{k} \tilde{U}_{k}\right\rangle / \varepsilon_{k}$ que es el flujo convectivo de calor debido a fluctuaciones de la energía.

Esta última ecuación puede ser reescrita en términos de la entalpía, la cual es una forma más común y útil en ingeniería, pues estamos más relacionados con variaciones en la temperatura dentro de un cuerpo que con variaciones en la densidad de energía interna. Del Apéndice $C$, Ec. (C.27) se tiene:

$$
\begin{aligned}
\varepsilon_{k}\left\langle\rho_{k}\right\rangle^{k} \frac{D_{k}}{D t}\left\{H_{k}\right\}^{k}+\nabla \cdot\left[\varepsilon_{k}\left(\left\langle\mathbf{q}_{k}\right\rangle^{k}+\hat{\mathbf{q}}_{k}^{r}\right)\right]-\varepsilon_{k} \frac{D_{k}}{D t}\left\langle P_{k}\right\rangle^{k}-\left(\left\langle P_{k}\right\rangle^{k}-\left\langle P_{k}\right\rangle_{i}\right) \frac{D_{k}}{D t} \varepsilon_{k} \\
+\sum_{k \ell \mid k} a_{k \ell}\left\langle\mathbf{n}_{k} \cdot \mathbf{q}_{k}\right\rangle_{k \ell}+\left\langle X_{k} \boldsymbol{\tau}_{k}: \nabla^{\prime} \mathbf{v}_{k}\right\rangle=0 .
\end{aligned}
$$

El coeficiente del término $\frac{D_{k}}{D t} \varepsilon_{k}$, puede eliminarse al considerar $\left\langle P_{k}\right\rangle^{k}-\left\langle P_{k}\right\rangle_{i} \approx 0$, de la Ec. (2.40). Este conjunto promediado no está cerrado. El promedio espacio-temporal ha introducido muchos términos fuente que deben analizarse para cerrar el sistema de EDP. 
En resumen, el conjunto de EDP está dado por:

$$
\begin{aligned}
& \underbrace{\frac{\partial}{\partial t}\left(\varepsilon_{k}\left\langle\rho_{k}\right\rangle^{k}\right)}_{\text {Acumulación de masa }}+\underbrace{\nabla \cdot\left(\varepsilon_{k}\left\langle\rho_{k}\right\rangle^{k}\left\{\mathbf{v}_{k}\right\}^{k}\right)}_{\text {Transporte convectivo de masa }}=0, \\
& \underbrace{\frac{\partial}{\partial t}\left(\varepsilon_{k}\left\langle\rho_{k}\right\rangle^{k}\left\{\mathbf{v}_{k}\right\}^{k}\right)}_{\text {Evolución de cantidad de movimiento }}+\underbrace{\nabla \cdot\left(\varepsilon_{k}\left\langle\rho_{k}\right\rangle^{k}\left\{\mathbf{v}_{k}\right\}^{k}\left\{\mathbf{v}_{k}\right\}^{k}\right)}_{\text {Transporte convectivo de cantidad de movimiento }} \\
& +\underbrace{\varepsilon_{k} \nabla \cdot\left\langle\boldsymbol{\Pi}_{k}\right\rangle^{k}}_{\text {Esfuerzos moleculares totales }}+\underbrace{\sum_{k \ell \mid k} a_{k \ell}\left\langle\mathbf{n}_{k} \cdot \tilde{\mathbf{\Pi}}_{k}\right\rangle_{k \ell}}_{\text {Transporte interfacial de cantidad de movimiento }}-\underbrace{\varepsilon_{k}\left\langle\rho_{k}\right\rangle^{k} \mathbf{g}}_{\text {Fuerzas gravitacionales }}=\mathbf{0}, \\
& \underbrace{\varepsilon_{k}\left\langle\rho_{k}\right\rangle^{k} \frac{D_{k}}{D t}\left\{H_{k}\right\}^{k}}_{\text {Evolución de la energía térmica }}+\underbrace{\nabla \cdot\left[\varepsilon_{k}\left(\left\langle\mathbf{q}_{k}\right\rangle^{k}+\hat{\mathbf{q}}_{k}^{r}\right)\right]}_{\begin{array}{c}
\text { Transporte convectivo de calor debido a } \\
\text { conducción y a fluctuaciones de energía }
\end{array}}-\underbrace{\varepsilon_{k} \frac{D_{k}}{D t}\left\langle P_{k}\right\rangle^{k}}_{\text {Evolución del campo de presión promedio }} \\
& +\underbrace{\sum_{k \ell \mid k} a_{k \ell}\left\langle\mathbf{n}_{k} \cdot \mathbf{q}_{k}\right\rangle_{k \ell}}_{\text {Transporte interfacial de energía térmica }}+\underbrace{\left\langle X_{k} \boldsymbol{\tau}_{k}: \nabla^{\prime} \mathbf{v}_{k}\right\rangle}_{\text {Disipasión viscosa promedio }}=0 .
\end{aligned}
$$

En la Ec. (6.6) y en la Ec. (6.7), $\sum_{k \ell \mid k} a_{k \ell}\left\langle\mathbf{n}_{k} \cdot \tilde{\boldsymbol{\Pi}}_{k}\right\rangle_{k \ell} \mathrm{y} \sum_{k \ell \mid k} a_{k \ell}\left\langle\mathbf{n}_{k} \cdot \mathbf{q}_{k}\right\rangle_{k \ell}$; son respectivamente, el transporte interfacial de cantidad de movimiento y de energía, en las interfaz sólido-gas y de cada una de las fases con la pared del tubo.

\subsubsection{Cerradura del conjunto de ecuaciones promedio}

Simplifiquemos primero los esfuerzos promedio para la fase gas: puesto que las fuerzas viscosas están concentradas en una capa delgada cercana a la interfaz y a las paredes del tubo, es posible satisfacer la desigualdad $\varepsilon_{g} \nabla \cdot\left\langle\boldsymbol{\tau}_{g}\right\rangle^{g} \ll a_{g s}\left\langle\mathbf{n}_{g} \cdot \tilde{\boldsymbol{\tau}}_{g}\right\rangle^{g s}$, cuando se satisface la restricción de longitud de escala $L \gg \ell$ y la restricción temporal $T \gg t_{0}$. Además, si consideramos que las partículas sólidas son rígidas, entonces $\nabla^{\prime} \cdot \mathbf{v}_{s}=0$. Por otro lado, se considerará también que la fuerza externa por unidad de volumen $\mathbf{g}_{k}$ es la fuerza de gravedad $\mathbf{g}$ y se propone en esta aproximación que $a_{g s}\left\langle\mathbf{n}_{s} \cdot \mathbf{q}_{s}\right\rangle^{g s}>\nabla \cdot\left(\varepsilon_{k} \hat{\mathbf{q}}_{k}^{r}\right)$. 
Por último, como más adelante se demuestra en la subsección 6.3.1, despreciaremos el transporte de calor y de esfuerzos viscosos en el volumen en comparación con el transporte de calor y de esfuerzos viscosos en la interfaz. Más aún, el tensor de esfuerzos del sólido debe equilibrarse mecánicamente en las interfaz con los esfuerzos del fluido, entonces $\left\langle\mathbf{n}_{s} \cdot \tilde{\boldsymbol{\Pi}}_{s}\right\rangle^{g s}=-\left\langle\mathbf{n}_{g} \cdot \tilde{\boldsymbol{\Pi}}_{g}\right\rangle^{g s}$; así como, el flujo de calor en las interfaz debe equilibrarse térmicamente; es decir, $\left\langle\mathbf{n}_{s} \cdot \mathbf{q}_{s}\right\rangle^{g s}=-\left\langle\mathbf{n}_{g} \cdot \mathbf{q}_{g}\right\rangle^{g s}$. Finalmente, los esfuerzos promedio en el sólido $\left\langle\boldsymbol{\Pi}_{s}\right\rangle^{s}$ deben ser considerados como dependientes de los esfuerzos promedio en el fluido; por lo tanto una primera aproximación a la cerradura podría ser

$$
\left\langle\boldsymbol{\Pi}_{s}\right\rangle^{s}=\left\langle\boldsymbol{P}_{g}\right\rangle^{g} \mathbf{1}
$$

la cual es consistente con la consideración de la continuación del campo de presión del gas dentro de la localización de los sólidos en mezclas homogéneas. Esta es una consideración clave para la obtención de los teoremas de Arquímedes en la hidrostática (Slattery, 1999) y su extensión a las condiciones de flujo es directa como una primera aproximación. Sin embargo, la no-homogeneidad requiere que se involucre otra función, el llamado módulo de compresibilidad $\Phi\left(\varepsilon_{s}\right)$ (Gidaspow, 1994), en una forma aditiva:

$$
\left\langle\boldsymbol{\Pi}_{s}\right\rangle^{s}=\left(\left\langle P_{g}\right\rangle^{g}+\Phi\left(\varepsilon_{s}\right)\right) \mathbf{1}
$$

Por lo tanto, la Ec. (6.5), la Ec. (6.6) y la Ec. (6.7) se reducen a (en términos de la derivada material):

Ecuaciones de conservación de la masa

$$
\begin{aligned}
& \varepsilon_{g} \frac{D_{g}}{D t}\left\langle\rho_{g}\right\rangle^{g}+\left\langle\rho_{g}\right\rangle^{g}\left(\frac{D_{g}}{D t} \varepsilon_{g}+\varepsilon_{g} \nabla \cdot\left\{\mathbf{v}_{g}\right\}^{g}\right)=0, \\
& \varepsilon_{s} \frac{D_{s}}{D t}\left\langle\rho_{s}\right\rangle^{s}+\left\langle\rho_{s}\right\rangle^{s}\left(\frac{D_{s}}{D t} \varepsilon_{s}+\varepsilon_{s} \nabla \cdot\left\{\mathbf{v}_{s}\right\}^{s}\right)=0 .
\end{aligned}
$$


Ecuaciones de balance de cantidad de movimiento

$$
\begin{gathered}
\varepsilon_{g}\left\langle\rho_{g}\right\rangle^{g} \frac{D_{g}}{D t}\left\{\mathbf{v}_{g}\right\}^{g}-\varepsilon_{g}\left\langle\rho_{g}\right\rangle^{g} \mathbf{g}+\varepsilon_{g} \nabla\left\langle P_{g}\right\rangle^{g}+a_{g W}\left\langle\mathbf{n}_{g} \cdot \tilde{\boldsymbol{\Pi}}_{g}\right\rangle^{g W}=\mathbf{M}_{g}^{D}, \\
\varepsilon_{s}\left\langle\rho_{s}\right\rangle^{s} \frac{D_{s}}{D t}\left\{\mathbf{v}_{s}\right\}^{s}-\varepsilon_{s}\left\langle\rho_{s}\right\rangle^{s} \mathbf{g}+\varepsilon_{s} \nabla\left\langle P_{s}\right\rangle^{s}+a_{s W}\left\langle\mathbf{n}_{s} \cdot \tilde{\boldsymbol{\Pi}}_{s}\right\rangle^{s W}=\mathbf{M}_{s}^{D},
\end{gathered}
$$

donde $\mathbf{M}_{s}^{D}$ y $\mathbf{M}_{g}^{D}$ son los esfuerzos superficiales totales en la fase sólida (normales y tangenciales), que pueden cerrarse mediante la siguiente relación obtenida con un modelo de celda (Sánchez-López, 2003):

$$
\mathbf{M}_{s}^{D}=\left\langle\mathbf{n}_{s} \cdot \tilde{\boldsymbol{\Pi}}_{s}\right\rangle^{g s}=-\varepsilon_{s}\left\langle\rho_{g}\right\rangle^{g} \mathbf{g}+\beta \varepsilon_{s} \varepsilon_{g}\left(\left\{\mathbf{v}_{g}\right\}^{g}-\left\{\mathbf{v}_{s}\right\}^{s}\right)
$$

con $\beta$ definido por $\beta \doteq \frac{3}{4 d_{p}} C_{D} \rho_{g} U_{t}$, donde $C_{D}$ es el coeficiente de arrastre, que depende fuertemente del número de Reynolds de la partícula y $U_{t}$ es la velocidad terminal de la partícula (velocidad intersticial de la partícula menos la velocidad de la mezcla), la cual viene expresada como: $\mathbf{U}_{t}=\varepsilon_{g}\left(\left\{\mathbf{v}_{g}\right\}^{g}-\left\{\mathbf{v}_{s}\right\}^{s}\right)$.

Los esfuerzos en la fase sólida deben ser equilibrados mecánicamente en las interfaz con los esfuerzos en el gas, entonces: $\mathbf{M}_{s}^{D}=-\mathbf{M}_{g}^{D}$ (Drew, 1983).

Por otro lado, los esfuerzos de las fases con la pared del tubo se modelan por:

$$
a_{k W}\left\langle\mathbf{e}_{r} \cdot \tilde{\boldsymbol{\Pi}}_{k}\right\rangle^{k W}=\frac{4}{D_{t}} \varepsilon_{k} f_{k W} \frac{1}{2} \rho_{k} V_{k}^{2} \mathbf{e}_{z}=\beta_{k W} \rho_{k} V_{k}^{2} \mathbf{e}_{z},
$$

donde $\beta_{k W} \equiv \frac{2}{D_{t}} \varepsilon_{k} f_{k W}$. 
El término de flujo viscoso promedio puede analizarse al utilizar la relación de comportamiento de Newton:

$$
\varepsilon_{k}\left\langle\boldsymbol{\tau}_{k}\right\rangle^{k}=\left\langle X_{k} \boldsymbol{\tau}_{k}\right\rangle
$$

Introduciendo la relación de comportamiento de Newton, considerando a la viscosidad como constante, expresando el promedio de fase en el miembro derecho y utilizando la Ec. (1.9) se obtiene:

$$
\varepsilon_{k}\left\langle\boldsymbol{\tau}_{k}\right\rangle^{k}=-2 \mu_{k} \varepsilon_{k}\left\langle\mathbf{D}_{k}\right\rangle^{k}-\lambda_{k}\left[\nabla \cdot\left(\varepsilon_{k}\left\langle\mathbf{v}_{k}\right\rangle^{k}\right)\right] \mathbf{1}-\lambda_{k}\left\langle\mathbf{v}_{k} \cdot \mathbf{n}_{k} \delta_{k \ell}\right\rangle \mathbf{1}
$$

Aplicando la condición a la frontera:

$$
\mathbf{v}_{k} \cdot \mathbf{n}_{k}=0 \quad \text { en } \quad \mathscr{Q}_{k \ell}(\mathbf{r}, t)
$$

y proponiendo que $\left\langle\mathbf{D}_{k}\right\rangle^{k} \doteq\left\{\mathbf{D}_{k}\right\}^{k}$, lo cual implica la restricción $\frac{\rho_{k}^{\prime}}{\left\langle\rho_{k}\right\rangle^{k}} \ll 1$

$$
\left\{\mathbf{D}_{k}\right\}^{k} \doteq \frac{1}{2}\left[\nabla\left\{\mathbf{v}_{k}\right\}^{k}+\left(\nabla\left\{\mathbf{v}_{k}\right\}^{k}\right)^{T}\right]
$$

se tiene finalmente que:

$$
\left\langle\boldsymbol{\tau}_{k}\right\rangle^{k}=-\mu_{k}\left[\nabla\left\{\mathbf{v}_{k}\right\}^{k}+\left(\nabla\left\{\mathbf{v}_{k}\right\}^{k}\right)^{T}\right]-\lambda_{k}\left(\nabla \cdot\left\langle\mathbf{v}_{k}\right\rangle^{k}+\frac{\nabla \varepsilon_{k}}{\varepsilon_{k}} \cdot\left\langle\mathbf{v}_{k}\right\rangle^{k}\right) \mathbf{1} .
$$

Ecuaciones de balance de energía térmica

$$
\begin{gathered}
\varepsilon_{g}\left\langle\rho_{g}\right\rangle^{g} \frac{D_{g}}{D t}\left\{H_{g}\right\}^{g}-\varepsilon_{g} \frac{D_{g}}{D t}\left\langle P_{g}\right\rangle^{g}+a_{g W}\left\langle\mathbf{n}_{g} \cdot \mathbf{q}_{g}\right\rangle^{g W}=E_{g}=-a_{s g}\left\langle\mathbf{n}_{g} \cdot \mathbf{q}_{g}\right\rangle^{g s}, \\
\varepsilon_{s}\left\langle\rho_{s}\right\rangle^{s} \frac{D_{s}}{D t}\left\{H_{s}\right\}^{s}-\varepsilon_{s} \frac{D_{s}}{D t}\left\langle P_{s}\right\rangle^{s}+a_{s W}\left\langle\mathbf{n}_{s} \cdot \mathbf{q}_{s}\right\rangle^{s W}=E_{s}=-a_{s g}\left\langle\mathbf{n}_{s} \cdot \mathbf{q}_{s}\right\rangle^{g s} .
\end{gathered}
$$

El término de flujo de calor promedio puede analizarse al utilizar la relación de comportamiento de Fourier (Drew y Lahey, 1993; Drew y Passman, 1999):

$$
\varepsilon_{k}\left\langle\mathbf{q}_{k}\right\rangle^{k}=\left\langle X_{k} \mathbf{q}_{k}\right\rangle
$$


Introduciendo la relación de comportamiento de Fourier:

$$
\varepsilon_{k}\left\langle\mathbf{q}_{k}\right\rangle^{k}=\left\langle-k_{k} X_{k} \nabla^{\prime} T_{k}\right\rangle
$$

considerando a la conductividad calorífica de la fase $k\left(k_{k}\right)$ como constante y expresando el promedio de fase:

$$
\varepsilon_{k}\left\langle\mathbf{q}_{k}\right\rangle^{k}=-k_{k}\left[\nabla\left(\varepsilon_{k}\left\langle T_{k}\right\rangle^{k}\right)-\left\langle T_{k} \nabla^{\prime} X_{k}\right\rangle\right]
$$

Utilizando la Ec. (1.9) se obtiene:

$$
\varepsilon_{k}\left\langle\mathbf{q}_{k}\right\rangle^{k}=-k_{k}\left[\nabla\left(\varepsilon_{k}\left\langle T_{k}\right\rangle^{k}\right)+\left\langle\mathbf{n}_{k} T_{k} \delta_{k \ell}\right\rangle\right]
$$

Aplicando las propiedades de la función delta de Dirac, desarrollando la derivada del producto en $\nabla\left(\varepsilon_{k}\left\langle T_{k}\right\rangle^{k}\right)$ y reagrupando se obtiene que

$$
\varepsilon_{k}\left\langle\mathbf{q}_{k}\right\rangle^{k}=-k_{k} \varepsilon_{k}\left[\nabla\left\langle T_{k}\right\rangle^{k}+\frac{1}{\varepsilon_{k}}\left(\sum_{k \ell \mid k} a_{k \ell}\left\langle\mathbf{n}_{k} T_{k}\right\rangle_{k \ell}+\left\langle T_{k}\right\rangle^{k} \nabla \varepsilon_{k}\right)\right]
$$

utilizando la condición de frontera:

$$
T_{k}=T_{k i} \quad \text { en } \quad \mathscr{Q}_{k \ell}(\mathbf{r}, t),
$$

considerando que $T_{k i} \sim\left\langle T_{k}\right\rangle_{k \ell}$ y recordando además que:

$$
\begin{aligned}
\sum_{k \ell \mid k} a_{k \ell}\left\langle\mathbf{n}_{k}\right\rangle_{k \ell} & =\frac{1}{\mathscr{Y}} \int_{\mathscr{S}} \mathbf{n}_{k} d S \\
& =-\nabla \varepsilon_{k}
\end{aligned}
$$

obtenemos finalmente:

$$
\left\langle\mathbf{q}_{k}\right\rangle^{k}=-k_{k}\left[\nabla\left\langle T_{k}\right\rangle^{k}-\left(T_{k i}-\left\langle T_{k}\right\rangle^{k}\right) \frac{\nabla \varepsilon_{k}}{\varepsilon_{k}}\right]
$$


Utilizando la condición a la frontera dada por la ley de enfriamiento de Newton aplicada a una partícula sólida en contacto con un fluido (C.F.4 de la pág. 14), junto con un balance de energía en la interfaz, en el que el flujo de calor que pierde la partícula es igual al que gana el gas; esto es, $-\mathbf{n}_{s} \cdot \mathbf{q}_{s}=\mathbf{n}_{g} \cdot \mathbf{q}_{g}$ y considerando equilibrio térmico en la misma; es decir, $T_{s i}=T_{g i} \equiv T_{i}$, donde $\mathbf{n}_{g}=-\mathbf{n}_{s}$ (ver Figura 6.1).

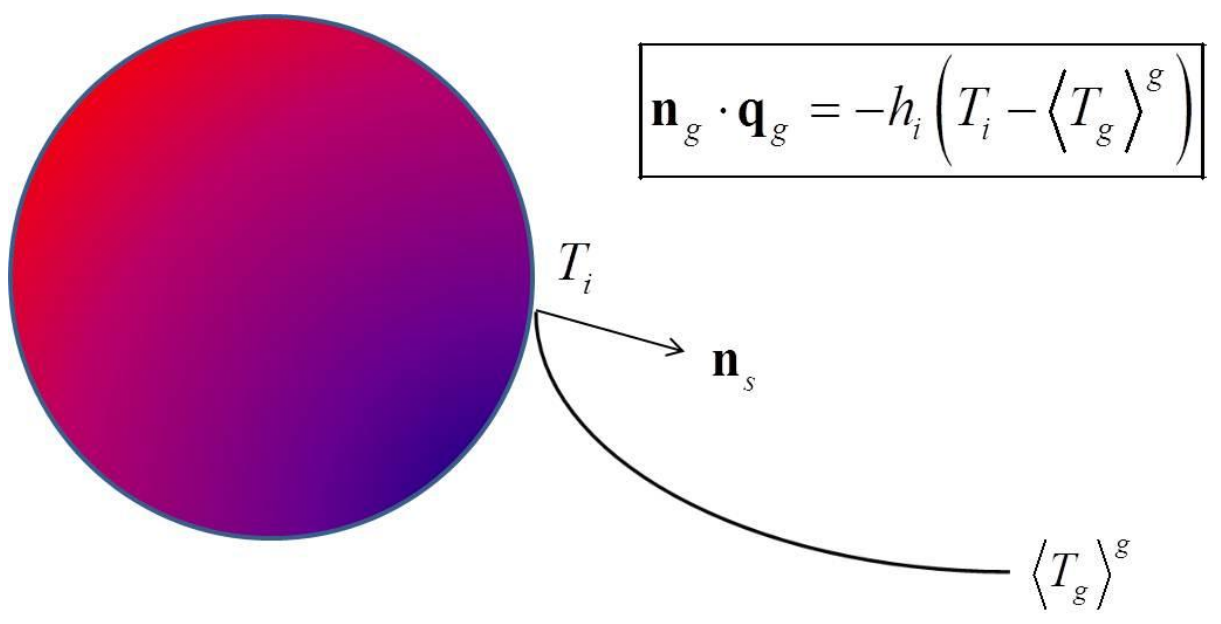

Figura 6.1. Transferencia de calor interfacial.

Tenemos la condición:

$$
\mathbf{n}_{s} \cdot \mathbf{q}_{s}=h_{i}\left(T_{i}-\left\langle T_{g}\right\rangle^{g}\right) \quad \text { en } \quad \mathscr{O}_{s g}(\mathbf{r}, t)
$$

Donde hemos considerado que la temperatura del fluido lejos de la partícula puede aproximarse por la temperatura promedio. Podemos cerrar el transporte interfacial de energía al sustituir la Ec. (6.20) en el término $a_{s g}\left\langle\mathbf{n}_{s} \cdot \mathbf{q}_{s}\right\rangle^{g s}$,

$$
E_{s}=a_{s g}\left\langle\mathbf{n}_{s} \cdot \mathbf{q}_{s}\right\rangle^{g s}=a_{s g}\left\langle h_{i}\left(T_{i}-\left\langle T_{g}\right\rangle^{g}\right)\right\rangle^{s g}
$$

sacando el coeficiente de la integral y aplicando el operador promedio a cada sumando

$$
E_{s}=a_{s g}\left\langle\mathbf{n}_{s} \cdot \mathbf{q}_{s}\right\rangle^{g s}=a_{s g} \bar{h}_{i}\left(\left\langle T_{i}\right\rangle^{s g}-\left\langle T_{g}\right\rangle^{g}\right)
$$


donde $\bar{h}_{i}$ es un coeficiente promedio de transferencia de calor asociado a la interfaz $s g$. Considerando partículas esféricas de diámetro constante $d_{p}$ podemos evaluar el área interfacial específica $a_{s g}$ como sigue:

$$
a_{g s}=a_{s g}=\frac{S_{s g}}{V}=\frac{S_{s g}}{V} \frac{V_{s}}{V_{s}}=\frac{6}{d_{p}} \varepsilon_{s},
$$

quedando finalmente

$$
E_{s}=a_{s g}\left\langle\mathbf{n}_{s} \cdot \mathbf{q}_{s}\right\rangle^{g s}=\frac{6}{d_{p}} \varepsilon_{s} \bar{h}_{i}\left(\left\langle T_{i}\right\rangle_{i}-\left\langle T_{g}\right\rangle^{g}\right)
$$

El flujo de calor promedio en la fase sólida debe ser equilibrado térmicamente en las interfaz con el flujo de calor en el gas, entonces: $\left\langle\mathbf{n}_{s} \cdot \mathbf{q}_{s}\right\rangle^{g s}=-\left\langle\mathbf{n}_{g} \cdot \mathbf{q}_{g}\right\rangle^{g s}$, que es equivalente a la condición: $E_{s}=-E_{g}$.

El transporte interfacial de calor en la pared $a_{s W}\left\langle\mathbf{n}_{s} \cdot \mathbf{q}_{s}\right\rangle^{s W}$ (ver Figura 6.2) puede evaluarse de forma similar al transporte de energía en la interfaz al considerar la condición a la frontera (Gidaspow, 1994):

$$
\mathbf{n}_{k} \cdot \mathbf{q}_{k}=h_{k W}\left(T_{W}-\left\langle T_{k}\right\rangle^{k}\right) \quad \text { en } \quad \mathscr{O}_{k W}(\mathbf{r}, t) .
$$

Consideramos en este primer acercamiento que podemos despreciar la conducción térmica en la pared; es decir, $T_{W 1}=T_{W 2}=T_{W}$. Por lo que,

$$
a_{k W}\left\langle\mathbf{n}_{k} \cdot \mathbf{q}_{k}\right\rangle^{k W}=a_{k W}\left\langle h_{k W}\left(T_{W}-\left\langle T_{k}\right\rangle^{k}\right)\right\rangle^{k W}
$$

sacando el coeficiente de la integral y aplicando el operador promedio a cada sumando

$$
a_{k W}\left\langle\mathbf{n}_{k} \cdot \mathbf{q}_{k}\right\rangle^{k W}=a_{k W} \bar{h}_{k W}\left(\left\langle T_{W}\right\rangle^{W}-\left\langle T_{k}\right\rangle^{k}\right),
$$

donde $\bar{h}_{k W}$ es un coeficiente promedio de transferencia de calor asociado a la interfaz $k W$. Como se obtuvo anteriormente, $a_{k W}=\left(4 / D_{t}\right) \varepsilon_{k}$. Por lo tanto:

$$
a_{k W}\left\langle\mathbf{n}_{k} \cdot \mathbf{q}_{k}\right\rangle^{k W}=\frac{4}{D_{t}} \varepsilon_{k} \bar{h}_{k W}\left(\left\langle T_{W}\right\rangle^{W}-\left\langle T_{k}\right\rangle^{k}\right) .
$$

Que es una expresión muy similar a la que representa al transporte interfacial de calor. 
Ecuación de estado para un gas ideal

$$
P_{g}=\frac{R}{M_{g}} \rho_{g} T_{g}
$$

multiplicando por la función indicadora de fase,

$$
X_{g} P_{g}=\frac{R}{M_{g}} X_{g} \rho_{g} T_{g}
$$

tomando el promedio de esta ecuación se obtiene

$$
\left\langle X_{g} P_{g}\right\rangle=\frac{R}{M_{g}}\left\langle X_{g} \rho_{g} T_{g}\right\rangle
$$

reexpresando los promedios en ambos miembros de la ecuación

$$
\varepsilon_{g}\left\langle P_{g}\right\rangle^{g}=\frac{R}{M_{g}} \varepsilon_{g}\left\langle\rho_{g}\right\rangle^{g}\left\{T_{g}\right\}^{g}
$$

dividiendo finalmente por la fracción volumen de gas se obtiene que

$$
\left\langle P_{g}\right\rangle^{g}=\frac{R}{M_{g}}\left\langle\rho_{g}\right\rangle^{g}\left\{T_{g}\right\}^{g}
$$

Que es la ecuación de estado a nivel promedio que utilizaremos en los desarrollos posteriores. 


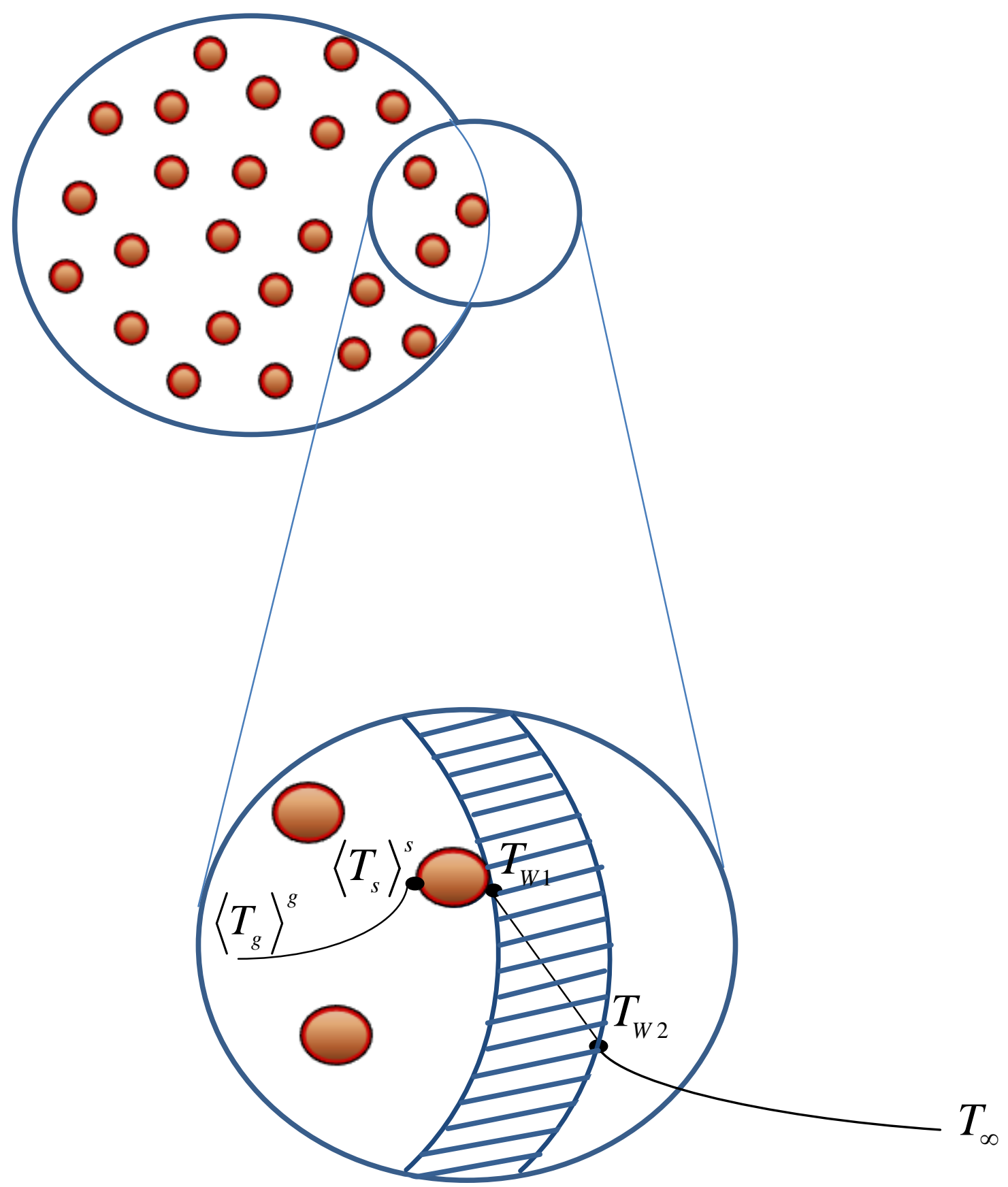

Figura 6.2. Transferencia de calor con la pared del tubo. 
Resumen del modelo

$$
\begin{aligned}
& \varepsilon_{g} \frac{D_{g}}{D t}\left\langle\rho_{g}\right\rangle^{g}+\left\langle\rho_{g}\right\rangle^{g}\left(\frac{D_{g}}{D t} \varepsilon_{g}+\varepsilon_{g} \nabla \cdot\left\{\mathbf{v}_{g}\right\}^{g}\right)=0, \\
& \varepsilon_{s} \frac{D_{s}}{D t}\left\langle\rho_{s}\right\rangle^{s}+\left\langle\rho_{s}\right\rangle^{s}\left(\frac{D_{s}}{D t} \varepsilon_{s}+\varepsilon_{s} \nabla \cdot\left\{\mathbf{v}_{s}\right\}^{s}\right)=0, \\
& \varepsilon_{g}\left\langle\rho_{g}\right\rangle^{g} \frac{D_{g}}{D t}\left\{\mathbf{v}_{g}\right\}^{g}-\varepsilon_{g}\left\langle\rho_{g}\right\rangle^{g} \mathbf{g}+\varepsilon_{g} \nabla\left\langle P_{g}\right\rangle^{g}+a_{g W}\left\langle\mathbf{n}_{g} \cdot \tilde{\mathbf{\Pi}}_{g}\right\rangle^{g W}=-\mathbf{M}_{s}^{D}, \\
& \varepsilon_{s}\left\langle\rho_{s}\right\rangle^{s} \frac{D_{s}}{D t}\left\{\mathbf{v}_{s}\right\}^{s}-\varepsilon_{s}\left\langle\rho_{s}\right\rangle^{s} \mathbf{g}+\varepsilon_{s} \nabla\left\langle P_{s}\right\rangle^{s}+a_{s W}\left\langle\mathbf{n}_{s} \cdot \tilde{\mathbf{\Pi}}_{s}\right\rangle^{s W}=\mathbf{M}_{s}^{D}, \\
& \varepsilon_{g}\left\langle\rho_{g}\right\rangle^{g} \frac{D_{g}}{D t}\left\{H_{g}\right\}^{g}-\varepsilon_{g} \frac{D_{g}}{D t}\left\langle P_{g}\right\rangle^{g}+a_{g W}\left\langle\mathbf{n}_{g} \cdot \mathbf{q}_{g}\right\rangle^{g W}=-E_{s}=-a_{s g}\left\langle\mathbf{n}_{g} \cdot \mathbf{q}_{g}\right\rangle^{g s}, \\
& \varepsilon_{s}\left\langle\rho_{s}\right\rangle^{s} \frac{D_{s}}{D t}\left\{H_{s}\right\}^{s}-\varepsilon_{s} \frac{D_{s}}{D t}\left\langle P_{s}\right\rangle^{s}+a_{s W}\left\langle\mathbf{n}_{s} \cdot \mathbf{q}_{s}\right\rangle^{s W}=E_{s}=-a_{s g}\left\langle\mathbf{n}_{s} \cdot \mathbf{q}_{s}\right\rangle^{g s} .
\end{aligned}
$$

Relaciones adicionales que cierran el modelo

$$
\begin{gathered}
\left\langle P_{s}\right\rangle^{s}=\left\langle P_{g}\right\rangle^{g}+\Phi\left(\varepsilon_{s}\right) \\
\varepsilon_{g}+\varepsilon_{s}=1 .
\end{gathered}
$$

Además, mediante un procedimiento similar a como se obtuvo la relación de Gibbs de campo promedio en la Sección 2.4, se puede demostrar que (ver Apéndice J):

$$
\begin{array}{ll}
\left\{H_{g}\right\}^{g}=\left\{H_{g}\right\}^{g}\left(\left\{T_{g}\right\}^{g}\right): & \frac{D_{g}}{D t}\left\{H_{g}\right\}^{g}=c_{p g} \frac{D_{g}}{D t}\left\{T_{g}\right\}^{g} \\
\left\{H_{s}\right\}^{s}=\left\{H_{s}\right\}^{s}\left(\left\{T_{s}\right\}^{s},\left\langle P_{s}\right\rangle^{s}\right): & \frac{D_{s}}{D t}\left\{H_{s}\right\}^{s}=c_{p s} \frac{D_{s}}{D t}\left\{T_{s}\right\}^{s}+\frac{D_{s}}{D t}\left(\frac{1}{\rho_{s}}\left\langle P_{s}\right\rangle^{s}\right)
\end{array}
$$

donde $c_{p k}$ es la capacidad calorífica de gas ideal de la fase $k(k=g, s)$.

$$
\begin{gathered}
\left\langle P_{g}\right\rangle^{g}=\frac{R}{M_{g}}\left\langle\rho_{g}\right\rangle^{g}\left\{T_{g}\right\}^{g} \\
\mathbf{M}_{s}^{D}=-\varepsilon_{s}\left\langle\rho_{g}\right\rangle^{g} \mathbf{g}+\beta \varepsilon_{s} \varepsilon_{g}\left(\left\{\mathbf{v}_{g}\right\}^{g}-\left\{\mathbf{v}_{s}\right\}^{s}\right)
\end{gathered}
$$


$\operatorname{con} \beta=\frac{3}{4 d_{p}} C_{D}\left\langle\rho_{g}\right\rangle^{g} \varepsilon_{g}\left(\left\{v_{g}\right\}^{g}-\left\{v_{s}\right\}^{s}\right)>0$.

$$
E_{s}=\frac{6}{d_{p}} \varepsilon_{s} \bar{h}_{i}\left(\left\langle T_{i}\right\rangle_{i}-\left\{T_{g}\right\}^{g}\right)
$$

$\mathrm{y}$

$$
a_{k W}\left\langle\mathbf{n}_{k} \cdot \mathbf{q}_{k}\right\rangle^{k W}=\frac{4}{D_{t}} \varepsilon_{k} \bar{h}_{k W}\left(\left\langle T_{W}\right\rangle^{W}-\left\langle T_{k}\right\rangle^{k}\right)
$$

Las variables dependientes principales a considerar serán:

$$
\left\{\varepsilon_{g},\left\langle P_{g}\right\rangle^{g},\left\{\mathbf{v}_{g}\right\}^{g},\left\{\mathbf{v}_{s}\right\}^{s},\left\{T_{g}\right\}^{g},\left\{T_{s}\right\}^{s}\right\}
$$

6.3 Validación de las cerraduras con la segunda ley de la termodinámica

6.3.1 Cerraduras propuestas y su consistencia con las restricciones impuestas por la segunda ley de la termodinámica

Mostraremos cómo puede utilizarse la restricción de la entropía para evaluar modelos cerrados, para lo cual valoraremos el conjunto propuesto en la Sección 6.2.3, junto con las relaciones mostradas abajo que son congruentes con la situación física descrita en el modelo.

- No hay transporte de masa en la interfaz, $\Gamma_{k}=0$.

- Podemos despreciar el flujo de calor en el volumen $\nabla \cdot\left(\varepsilon_{k}\left\langle\mathbf{q}_{k}\right\rangle^{k}\right)$ en comparación con el flujo de calor en la interfaz $E_{s}$ :

$$
\nabla \cdot\left(\varepsilon_{k}\left\langle\mathbf{q}_{k}\right\rangle^{k}\right)=\varepsilon_{k} \nabla \cdot\left\langle\mathbf{q}_{k}\right\rangle^{k}+\nabla \varepsilon_{k} \cdot\left\langle\mathbf{q}_{k}\right\rangle^{k}=O\left[\left(\frac{\varepsilon_{k}}{L_{\left\langle\mathbf{q}_{k}\right\rangle^{k}}}+\frac{\Delta \varepsilon_{k}}{L_{\varepsilon_{k}}}\right)\left\langle\mathbf{q}_{k}\right\rangle^{k}\right]
$$


de la Ec. (6.19),

$$
\left\langle\mathbf{q}_{k}\right\rangle^{k}=O\left[-k_{k}\left(\frac{1}{L_{\left\langle T_{k}\right\rangle^{k}}}\left\langle T_{k}\right\rangle^{k}-\frac{1}{L_{\varepsilon_{k}}} \frac{\Delta \varepsilon_{k}}{\varepsilon_{k}}\left(T_{k i}-\left\langle T_{k}\right\rangle^{k}\right)\right]\right]
$$

y ya que $\left\langle T_{k}\right\rangle^{k} \approx\left\{T_{k}\right\}^{k}$ :

$$
\begin{aligned}
& \nabla \cdot\left(\varepsilon_{k}\left\langle\mathbf{q}_{k}\right\rangle^{k}\right)=O\left[-k_{k}\left(\frac{\varepsilon_{k}}{L_{\left\langle\mathbf{q}_{k}\right\rangle^{k}}}+\frac{\Delta \varepsilon_{k}}{L_{\varepsilon_{k}}}\right)\left(\frac{1}{L_{\left\langle T_{k}\right\rangle^{k}}} \Delta\left\{T_{k}\right\}^{k}-\frac{1}{L_{\varepsilon_{k}}} \frac{\Delta \varepsilon_{k}}{\varepsilon_{k}}\left(T_{k i}-\left\{T_{k}\right\}^{k}\right)\right]\right. \\
& E_{s}=\frac{6}{d_{p}} \varepsilon_{s} \bar{h}_{i}\left(\left\langle T_{s}\right\rangle_{i}-\left\{T_{g}\right\}^{g}\right)=O\left[6 \frac{\varepsilon_{s}}{d_{p}} \bar{h}_{i}\left(T_{s i}-\left\{T_{g}\right\}^{g}\right)\right]
\end{aligned}
$$

$\frac{\text { flujo de calor en el volumen }}{\text { flujo de calor en la superficie }}=\frac{\nabla \cdot\left(\varepsilon_{k}\left\langle\mathbf{q}_{k}\right\rangle^{k}\right)}{E_{s}}$.

Sustituyendo $\nabla \cdot\left(\varepsilon_{k}\left\langle\mathbf{q}_{k}\right\rangle^{k}\right)$ y $E_{s}$ :

$$
\frac{\nabla \cdot\left(\varepsilon_{k}\left\langle\mathbf{q}_{k}\right\rangle^{k}\right)}{E_{s}}=\frac{O\left[-k_{k}\left(\frac{\varepsilon_{k}}{L_{\left\langle\mathbf{q}_{k}\right\rangle^{k}}}+\frac{\Delta \varepsilon_{k}}{L_{\varepsilon_{k}}}\right)\left(\frac{1}{L_{\left\langle T_{k}\right\rangle^{k}}} \Delta\left\{T_{k}\right\}^{k}-\frac{1}{L_{\varepsilon_{k}}} \frac{\Delta \varepsilon_{k}}{\varepsilon_{k}}\left(T_{k i}-\left\{T_{k}\right\}^{k}\right)\right]\right.}{O\left[6 \frac{\varepsilon_{s}}{d_{p}} \bar{h}_{i}\left(T_{s i}-\left\{T_{g}\right\}^{g}\right)\right]}
$$

para que $\nabla \cdot\left(\varepsilon_{k}\left\langle\mathbf{q}_{k}\right\rangle^{k}\right) \ll E_{s}$, se requiere que $\frac{\nabla \cdot\left(\varepsilon_{k}\left\langle\mathbf{q}_{k}\right\rangle^{k}\right)}{E_{s}} \ll 1$.

Reagrupando tenemos:

$=O\left\{-\frac{1}{6}\left(\frac{d_{p}}{L_{\left\langle\mathbf{q}_{k}\right\rangle^{k}}} \frac{\varepsilon_{k}}{\varepsilon_{s}}+\frac{d_{p}}{L_{\varepsilon_{k}}} \frac{\Delta \varepsilon_{k}}{\varepsilon_{s}}\right)\left[\frac{d_{p}}{L_{\left\{T_{k}\right\}^{k}}} \frac{\Delta\left\{T_{k}\right\}^{k}}{T_{s i}-\left\{T_{g}\right\}^{g}}-\frac{d_{p}}{L_{\varepsilon_{k}}} \frac{\Delta \varepsilon_{k}}{\varepsilon_{k}} \frac{T_{k i}-\left\{T_{k}\right\}^{k}}{T_{s i}-\left\{T_{g}\right\}^{g}}\right]\right\} \ll \mathrm{Bi}_{s}$

donde $\mathrm{Bi}_{s}=\frac{\bar{h}_{i}}{k_{k}} d_{p}$. Simplificando se tiene que: $\left(\frac{d_{p}}{L}\right)^{2} \ll \mathrm{Bi}_{s}$,

$d_{p}=O\left(10^{-6}\right)$ y $L=O(10)$. Por lo que: $10^{-14} \ll \mathrm{Bi}_{s}$. Con los datos de la

Tabla 7.1, encontramos que para el $\mathrm{FCC}, \mathrm{Bi}_{s}=733 . \overline{3}$ y para la arena, $\mathrm{Bi}_{s}=79839$. Esto implica que las partículas tienen temperatura uniforme $T_{s}=T_{s}(t)$. Por lo tanto, podemos descartar el flujo de calor en el volumen en comparación con el flujo de calor en la interfaz. 
- También podemos despreciar el transporte de esfuerzos viscosos en el volumen $\nabla \cdot\left(\varepsilon_{k}\left\langle\boldsymbol{\tau}_{k}\right\rangle^{k}\right)$ en comparación con el transporte de esfuerzos viscosos en la interfaz $\mathbf{M}_{s}$.

$$
\begin{aligned}
& \nabla \cdot\left(\varepsilon_{k}\left\langle\boldsymbol{\tau}_{k}\right\rangle^{k}\right)=\varepsilon_{k} \nabla \cdot\left\langle\boldsymbol{\tau}_{k}\right\rangle^{k}+\nabla \varepsilon_{k} \cdot\left\langle\boldsymbol{\tau}_{k}\right\rangle^{k} \\
& =O\left[\left(\frac{1}{L_{\left\langle\tau_{k}\right\rangle^{k}}} \varepsilon_{k}+\frac{1}{L_{\varepsilon_{k}}} \Delta \varepsilon_{k}\right)\left\langle\boldsymbol{\tau}_{k}\right\rangle^{k}\right] \\
& \left\langle\boldsymbol{\tau}_{k}\right\rangle^{k}=O\left[-\mu_{k} \frac{\left\{\mathbf{v}_{k}\right\}^{k}}{L_{\left\{\mathbf{v}_{k}\right\}^{k}}}-\mu_{k} \frac{\left(\left\{\mathbf{v}_{k}\right\}^{k}\right)^{T}}{L_{\left(\left\{\mathbf{v}_{k}\right\}^{k}\right)^{T}}}-\lambda_{k}\left(\frac{1}{L_{\left\langle\mathbf{v}_{k}\right\rangle^{k}}}\left\langle\mathbf{v}_{k}\right\rangle^{k}+\frac{1}{L_{\varepsilon_{k}}} \frac{\Delta \varepsilon_{k}}{\varepsilon_{k}}\left\langle\mathbf{v}_{k}\right\rangle^{k}\right)\right] \\
& \mathbf{M}_{s}=\frac{3}{4 d_{p}} C_{D}\left\langle\rho_{g}\right\rangle^{g} \varepsilon_{g}\left|\left\{\mathbf{v}_{g}\right\}^{g}-\left\{\mathbf{v}_{s}\right\}^{s}\right| \varepsilon_{s} \varepsilon_{g}\left(\left\{\mathbf{v}_{g}\right\}^{g}-\left\{\mathbf{v}_{s}\right\}^{s}\right) \\
& =O\left[\frac{3}{4} \varepsilon_{g} C_{D}\left|\left\{\mathbf{v}_{g}\right\}^{g}-\left\{\mathbf{v}_{s}\right\}^{s}\right| \frac{\left\langle\rho_{g}\right\rangle^{g}}{d_{p}} \varepsilon_{s} \varepsilon_{g}\left(\left\{\mathbf{v}_{g}\right\}^{g}-\left\{\mathbf{v}_{s}\right\}^{s}\right)\right] \\
& \frac{\text { transporte de esfuerzos viscosos en el volumen }}{\text { transporte de esfuerzos viscosos en la superficie }}=\frac{\nabla \cdot\left(\varepsilon_{k}\left\langle\boldsymbol{\tau}_{k}\right\rangle^{k}\right)}{\mathbf{M}_{s}}
\end{aligned}
$$

Sustituyendo $\nabla \cdot\left(\varepsilon_{k}\left\langle\boldsymbol{\tau}_{k}\right\rangle^{k}\right)$ y $\mathbf{M}_{s}$ :

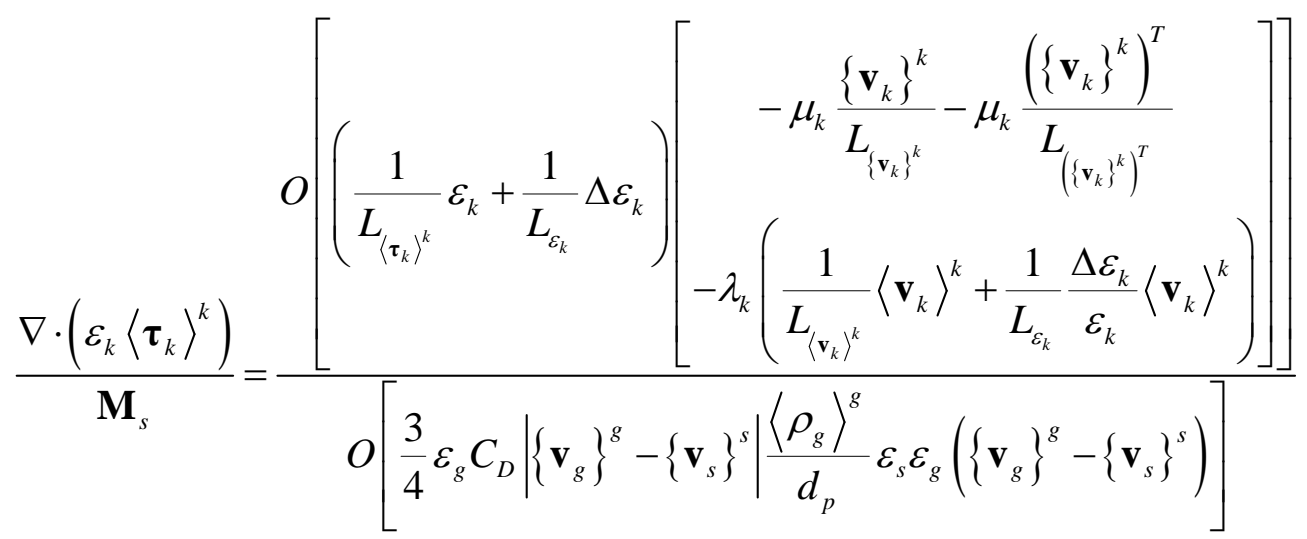

$$
\begin{aligned}
& \text { para que } \nabla \cdot\left(\varepsilon_{k}\left\langle\boldsymbol{\tau}_{k}\right\rangle^{k}\right) \ll \mathbf{M}_{s} \text {, se requiere que } \frac{\nabla \cdot\left(\varepsilon_{k}\left\langle\boldsymbol{\tau}_{k}\right\rangle^{k}\right)}{\mathbf{M}_{s}} \ll 1 \text {. }
\end{aligned}
$$


Reagrupando tenemos:

$$
=O\left[-\frac{1}{\frac{3}{4} C_{D}}\left\{\left[\begin{array}{c}
\frac{d_{p}}{L_{\left\langle\tau_{k}\right\rangle^{k}}} \frac{\varepsilon_{k}}{\varepsilon_{s}} \\
+\frac{d_{p}}{L_{\varepsilon_{k}}} \frac{\Delta \varepsilon_{k}}{\varepsilon_{g}}
\end{array}\right]\left[\begin{array}{c}
\frac{d_{p}}{L_{\left\{\mathbf{v}_{k}\right\}^{k}}} \frac{\left\{\mathbf{v}_{k}\right\}^{k}}{\mathbf{U}_{t}}+\frac{d_{p}}{L_{\left(\left\{\mathbf{v}_{k}\right\}^{k}\right)^{T}}} \frac{\left(\left\{\mathbf{v}_{k}\right\}^{k}\right)^{T}}{\mathbf{U}_{t}} \\
+\frac{\lambda_{k}}{\mu_{k}}\left(\frac{d_{p}}{L_{\left\langle\mathbf{v}_{k}\right\rangle^{k}}} \frac{\left\langle\mathbf{v}_{k}\right\rangle^{k}}{\mathbf{U}_{t}}+\frac{d_{p}}{L_{\varepsilon_{k}}} \frac{\Delta \varepsilon_{k}}{\varepsilon_{k}} \frac{\left\langle\mathbf{v}_{k}\right\rangle^{k}}{\mathbf{U}_{t}}\right)
\end{array}\right] \cdot \mathbf{1}\right]\right\} \ll \operatorname{Re}_{s} .
$$

Donde $\operatorname{Re}_{s} \doteq \frac{U_{t}\left\langle\rho_{g}\right\rangle^{g} d_{p}}{\mu_{k}}$, con $U_{t} \doteq \varepsilon_{\gamma}\left|\left\{\mathbf{v}_{\gamma}\right\}^{\gamma}-\left\{\mathbf{v}_{\sigma}\right\}^{\sigma}\right|$. Simplificando: $\left(\frac{d_{p}}{L}\right)^{2} \ll \operatorname{Re}_{s}, d_{p}=O\left(10^{-6}\right)$ y $L=O(10)$. Por lo que: $10^{-14} \ll \operatorname{Re}_{s}$.

En lechos fluidizados rápidos Sánchez-López y col. (2011) encontraron que para el flujo de vapor-catalizador en una unidad de FCC $\operatorname{Re}_{s}=O\left(10^{-1}\right)$, en tanto que para el sistema aire-arena de van der Schaaf y col. (1998) $\operatorname{Re}_{s}=O(10)$. Por lo tanto, podemos descartar el transporte de esfuerzos viscosos en el volumen en comparación con el término equivalente en la interfaz.

- Los términos asociados a la estructura turbulenta del flujo: $K_{k}^{\mathrm{Re}}$ y $\boldsymbol{\Pi}_{k}^{\mathrm{Re}}$, los despreciaremos debido a que consideramos un flujo altamente turbulento o tapón en una sola dirección, representado por $\left\{\mathbf{v}_{k}\right\}^{k}=V_{k} \mathbf{e}_{z}$.

- Por último, despreciamos los flujos convectivos turbulentos, $\mathbf{q}_{k}^{p}+\mathbf{q}_{k}^{t}+\mathbf{q}_{k}^{K}=\mathbf{0}$.

Sin embargo, debe reconocerse que estas relaciones de cerradura son muy simples y que pueden mejorarse. Simplificando con estas relaciones la Ec. (2.41) y recordando que:

$$
\begin{aligned}
& W_{k i}^{\prime \prime} \doteq\left\langle\nabla^{\prime} X_{k} \cdot\left(P_{k} \mathbf{1}+\boldsymbol{\tau}_{k}\right) \cdot\left(\mathbf{v}_{k}-\left\langle\mathbf{v}_{k}\right\rangle_{i}\right)\right\rangle, \\
& \mathbf{M}_{k}^{D} \doteq \mathbf{M}_{k p}+\mathbf{M}_{k t}+\left(\left\langle P_{k}\right\rangle_{i} \mathbf{1}+\left\langle\boldsymbol{\tau}_{k}\right\rangle_{i}\right) \cdot \nabla \varepsilon_{k},
\end{aligned}
$$

se obtiene:

$$
\left[\left\langle\mathbf{v}_{k}\right\rangle_{i}-\left\{\mathbf{v}_{k}\right\}^{k}\right] \cdot \mathbf{M}_{k}^{D}+W_{k i}^{\prime \prime}-\varepsilon_{k}\left\langle\rho_{k}\right\rangle^{k}\left\{\tilde{S}_{k} \tilde{\mathbf{v}}_{k}\right\}^{k} \cdot \nabla\left\{T_{k}\right\}^{k}+\left\langle P_{k}\right\rangle^{k} \frac{D_{k}}{D t} \varepsilon_{k} \geq 0 .
$$


Analizando ahora cada término como semipositivo definido:
a) $\left[\left\langle\mathbf{v}_{k}\right\rangle_{i}-\left\{\mathbf{v}_{k}\right\}^{k}\right] \cdot \mathbf{M}_{k}^{D} \geq 0$,
b) $W_{k i}^{\prime \prime} \geq 0$,
c) $-\varepsilon_{k}\left\langle\rho_{k}\right\rangle^{k}\left\{\tilde{S}_{k} \tilde{\mathbf{v}}_{k}\right\}^{k} \cdot \nabla\left\{T_{k}\right\}^{k} \geq 0$,
d) $\left\langle P_{k}\right\rangle^{k} \frac{D_{k}}{D t} \varepsilon_{k} \geq 0$.

Examinando la primera restricción a), para lo cual sustituimos la cerradura propuesta:

$$
\mathbf{M}_{s}^{D}=-\varepsilon_{s}\left\langle\rho_{g}\right\rangle^{g} \mathbf{g}+\beta \varepsilon_{s} \varepsilon_{g}\left(\left\{\mathbf{v}_{g}\right\}^{g}-\left\{\mathbf{v}_{s}\right\}^{s}\right)
$$

encontramos que:

$$
\begin{array}{r}
-\left[\left\langle\mathbf{v}_{g}\right\rangle_{i}-\left\{\mathbf{v}_{g}\right\}^{g}\right] \cdot \mathbf{M}_{s}^{D} \geq 0, \\
{\left[\left\langle\mathbf{v}_{s}\right\rangle_{i}-\left\{\mathbf{v}_{s}\right\}^{s}\right] \cdot \mathbf{M}_{s}^{D} \geq 0 .}
\end{array}
$$

$\underline{\text { Para el vapor: }}$

$$
-\varepsilon_{s}\left\langle\rho_{g}\right\rangle^{g}\left[\left\{\mathbf{v}_{g}\right\}^{g}-\left\langle\mathbf{v}_{g}\right\rangle_{i}\right] \cdot \mathbf{g}+\beta \varepsilon_{s} \varepsilon_{g}\left[\left\{\mathbf{v}_{g}\right\}^{g}-\left\langle\mathbf{v}_{g}\right\rangle_{i}\right] \cdot\left(\left\{\mathbf{v}_{g}\right\}^{g}-\left\{\mathbf{v}_{s}\right\}^{s}\right) \geq 0 .
$$

Para el sistema físico: $\left|\left\{\mathbf{v}_{g}\right\}^{g}-\left\{\mathbf{v}_{s}\right\}^{s}\right|>0 \&\left|\left\{\mathbf{v}_{g}\right\}^{g}-\left\langle\mathbf{v}_{g}\right\rangle_{i}\right|>0, \mathbf{g}=-g \mathbf{e}_{z}$ (puesto que $z$ se toma en la dirección ascendente en el tubo).

$$
\varepsilon_{s}\left\langle\rho_{g}\right\rangle^{g} g\left[\left\{\mathbf{v}_{g}\right\}^{g}-\left\langle\mathbf{v}_{g}\right\rangle_{i}\right] \cdot \mathbf{e}_{z}+\beta \varepsilon_{s} \varepsilon_{g}\left[\left\{\mathbf{v}_{g}\right\}^{g}-\left\langle\mathbf{v}_{g}\right\rangle_{i}\right] \cdot\left(\left\{\mathbf{v}_{g}\right\}^{g}-\left\{\mathbf{v}_{s}\right\}^{s}\right) \geq 0 .
$$

Para el sólido:

$\left\langle\mathbf{v}_{s}\right\rangle_{i}-\left\{\mathbf{v}_{s}\right\}^{s}=\mathbf{0}$ (por ser $s$ un sólido rígido), por lo tanto:

$$
\begin{gathered}
{\left[\left\langle\mathbf{v}_{s}\right\rangle_{i}-\left\{\mathbf{v}_{s}\right\}^{s}\right] \cdot\left[-\varepsilon_{s}\left\langle\rho_{g}\right\rangle^{g} \mathbf{g}+\beta \varepsilon_{s} \varepsilon_{g}\left(\left\{\mathbf{v}_{g}\right\}^{g}-\left\{\mathbf{v}_{s}\right\}^{s}\right)\right] \geq 0,} \\
0 \equiv 0 .
\end{gathered}
$$


b) $W_{g i}{ }^{\prime \prime} \geq 0$

$$
W_{s i}^{\prime \prime} \geq 0 .
$$

Ahora analizamos la restricción dada en c):

$$
\begin{gathered}
-\varepsilon_{g}\left\langle\rho_{g}\right\rangle^{g}\left\{\tilde{S}_{g} \tilde{\mathbf{v}}_{g}\right\}^{g} \cdot \nabla\left\{T_{g}\right\}^{g} \geq 0, \\
-\varepsilon_{s}\left\langle\rho_{s}\right\rangle^{s}\left\{\tilde{S}_{s} \tilde{\mathbf{v}}_{s}\right\}^{s} \cdot \nabla\left\{T_{s}\right\}^{s} \geq 0, \\
-\varepsilon_{k}\left\langle\rho_{k}\right\rangle^{k}\left\{\tilde{S}_{k} \tilde{\mathbf{v}}_{k}\right\}^{k} \cdot \nabla\left\{T_{k}\right\}^{k} .
\end{gathered}
$$

Arnold y col. (1990) proponen que:

$$
\left\{\tilde{S}_{k} \tilde{\mathbf{v}}_{k}\right\}^{k} \doteq-k_{s k} \nabla\left\{S_{k}\right\}^{k},
$$

donde $k_{s k}$ es un coeficiente positivo de difusividad turbulenta para el transporte de entropía en la fase $k$.

Especializando esta ecuación para cada fase:

Para el vapor:

$$
\left\{\tilde{S}_{g} \tilde{\mathbf{v}}_{g}\right\}^{g} \doteq-k_{s g} \nabla\left\{S_{g}\right\}^{g},
$$

la ecuación de Gibbs para un campo de presión uniforme puede representarse como

$$
d H_{g}=T_{g} d S_{g} .
$$

Utilizando la relación $d H_{g}=c_{p g} d T_{g}$, podemos expresar esta versión de la ecuación de Gibbs en términos de la temperatura y del gradiente de entropía como

$$
\nabla S=\frac{c_{p}}{T} \nabla T .
$$

Por lo que tenemos

$$
-\varepsilon_{g}\left\langle\rho_{g}\right\rangle^{g}\left\{\tilde{S}_{g} \tilde{\mathbf{v}}_{g}\right\}^{g} \cdot \nabla\left\{T_{g}\right\}^{g}=k_{s g} c_{p g} \frac{\varepsilon_{g}\left\langle\rho_{g}\right\rangle^{g}}{\left\{T_{g}\right\}^{g}} \nabla\left\{T_{g}\right\}^{g} \cdot \nabla\left\{T_{g}\right\}^{g} .
$$

El cual es obviamente positivo. 
Para el sólido:

$$
\left\{\tilde{S}_{s} \tilde{\mathbf{v}}_{s}\right\}^{s} \doteq-k_{s s} \nabla\left\{S_{s}\right\}^{s}
$$

la ecuación de Gibbs puede representarse como

$$
d H_{s}=T_{s} d S_{s}+\frac{1}{\rho_{s}} d P_{s} .
$$

Utilizando la relación $d H_{s}=c_{p s} d T_{s}+\frac{1}{\rho_{s}} d P_{s}$, podemos expresar esta versión de la ecuación de Gibbs en términos de la temperatura y del gradiente de entropía como

$$
\nabla S=\frac{c_{p}}{T} \nabla T
$$

Por lo que tenemos

$$
-\varepsilon_{s}\left\langle\rho_{s}\right\rangle^{s}\left\{\tilde{S}_{s} \tilde{\mathbf{v}}_{s}\right\}^{s} \cdot \nabla\left\{T_{s}\right\}^{s}=k_{s s} c_{p s} \frac{\varepsilon_{s}\left\langle\rho_{s}\right\rangle^{s}}{\left\{T_{s}\right\}^{s}} \nabla\left\{T_{s}\right\}^{s} \cdot \nabla\left\{T_{s}\right\}^{s},
$$

el cual es positivo definido. Por último, la restricción dada en d) acerca de la derivada material de la fracción volumen:

$$
\left\langle P_{g}\right\rangle^{g} \frac{D_{g}}{D t} \varepsilon_{g} \geq 0 \&\left\langle P_{s}\right\rangle^{s} \frac{D_{s}}{D t} \varepsilon_{s} \geq 0
$$

puede ser positiva o negativa. Para analizar este término, estudiamos lo siguiente:

$$
\frac{\left\langle P_{k}\right\rangle^{k}}{\left\langle\rho_{k}\right\rangle^{k}} \Gamma_{k} \doteq \frac{\left\langle P_{k}\right\rangle^{k}}{\left\langle\rho_{k}\right\rangle^{k}}\left\langle\rho_{k}\left(\mathbf{v}_{k}-\mathbf{w}_{k \ell}\right) \cdot \nabla^{\prime} X_{k}\right\rangle
$$

descomponiendo la densidad, sumando y restando la velocidad promedio, obtenemos

$$
\begin{gathered}
\frac{\left\langle P_{k}\right\rangle^{k}}{\left\langle\rho_{k}\right\rangle^{k}} \Gamma_{k} \doteq \frac{\left\langle P_{k}\right\rangle^{k}}{\left\langle\rho_{k}\right\rangle^{k}}\left\langle\rho_{k}\left(\mathbf{v}_{k}-\mathbf{w}_{k \ell}\right) \cdot \nabla^{\prime} X_{k}\right\rangle, \\
\frac{\left\langle P_{k}\right\rangle^{k}}{\left\langle\rho_{k}\right\rangle^{k}} \Gamma_{k}=\frac{\left\langle P_{k}\right\rangle^{k}}{\left\langle\rho_{k}\right\rangle^{k}}\left\langle\left(\left\langle\rho_{k}\right\rangle^{k}+\rho_{k}^{\prime}\right)\left(\mathbf{v}_{k}-\mathbf{w}_{k \ell}\right) \cdot \nabla^{\prime} X_{k}\right\rangle, \\
\frac{\left\langle P_{k}\right\rangle^{k}}{\left\langle\rho_{k}\right\rangle^{k}} \Gamma_{k}=\left\langle P_{k}\right\rangle^{k}\left\langle\left(\mathbf{v}_{k}-\left\{\mathbf{v}_{k}\right\}^{k}+\left\{\mathbf{v}_{k}\right\}^{k}-\mathbf{w}_{k \ell}\right) \cdot \nabla^{\prime} X_{k}\right\rangle+\left\langle P_{k}\right\rangle^{k}\left\langle\frac{\rho_{k}^{\prime}}{\left\langle\rho_{k}\right\rangle^{k}}\left(\mathbf{v}_{k}-\mathbf{w}_{k \ell}\right) \cdot \nabla^{\prime} X_{k}\right\rangle, \\
\frac{\left\langle P_{k}\right\rangle^{k}}{\left\langle\rho_{k}\right\rangle^{k}} \Gamma_{k}=\left\langle P_{k}\right\rangle^{k}\left\langle\tilde{\mathbf{v}}_{k} \cdot \nabla^{\prime} X_{k}\right\rangle+\left\langle P_{k}\right\rangle^{k}\left\{\mathbf{v}_{k}\right\}^{k} \cdot \nabla\left\langle X_{k}\right\rangle-\left\langle P_{k}\right\rangle^{k}\left\langle\mathbf{w}_{k \ell} \cdot \nabla^{\prime} X_{k}\right\rangle,
\end{gathered}
$$




$$
\frac{\left\langle P_{k}\right\rangle^{k}}{\left\langle\rho_{k}\right\rangle^{k}} \Gamma_{k}=\left\langle P_{k}\right\rangle^{k}\left\langle\tilde{\mathbf{v}}_{k} \cdot \nabla^{\prime} X_{k}\right\rangle+\left\langle P_{k}\right\rangle^{k}\left\{\mathbf{v}_{k}\right\}^{k} \cdot \nabla \varepsilon_{k}-\left\langle P_{k}\right\rangle^{k}\left\langle\mathbf{w}_{k \ell} \cdot \nabla^{\prime} X_{k}\right\rangle
$$

y utilizamos la ecuación:

$$
\frac{\partial X_{k}}{\partial t^{\prime}}=-\mathbf{w}_{k \ell} \cdot \nabla^{\prime} X_{k}
$$

Para tener,

$$
\begin{gathered}
\frac{\left\langle P_{k}\right\rangle^{k}}{\left\langle\rho_{k}\right\rangle^{k}} \Gamma_{k}=\left\langle P_{k}\right\rangle^{k}\left\langle\tilde{\mathbf{v}}_{k} \cdot \nabla^{\prime} X_{k}\right\rangle+\left\langle P_{k}\right\rangle^{k}\left\{\mathbf{v}_{k}\right\}^{k} \cdot \nabla \varepsilon_{k}+\left\langle P_{k}\right\rangle^{k} \frac{\partial}{\partial t}\left\langle X_{k}\right\rangle, \\
\frac{\left\langle P_{k}\right\rangle^{k}}{\left\langle\rho_{k}\right\rangle^{k}} \Gamma_{k}=\left\langle P_{k}\right\rangle^{k}\left\langle\tilde{\mathbf{v}}_{k} \cdot \nabla^{\prime} X_{k}\right\rangle+\left\langle P_{k}\right\rangle^{k}\left\{\mathbf{v}_{k}\right\}^{k} \cdot \nabla \varepsilon_{k}+\left\langle P_{k}\right\rangle^{k} \frac{\partial}{\partial t} \varepsilon_{k},
\end{gathered}
$$

por lo tanto,

$$
\frac{\left\langle P_{k}\right\rangle^{k}}{\left\langle\rho_{k}\right\rangle^{k}} \Gamma_{k}=\left\langle P_{k}\right\rangle^{k}\left\langle\tilde{\mathbf{v}}_{k} \cdot \nabla^{\prime} X_{k}\right\rangle+\left\langle P_{k}\right\rangle^{k} \frac{D_{k}}{D t} \varepsilon_{k}
$$

donde se ha utilizado la relación $\left(\rho_{k}^{\prime} /\left\langle\rho_{k}\right\rangle^{k}\right) \ll 1$.

Podemos ver que la inclusión de este término de cambio de fase causa la cancelación del término $\left\langle P_{k}\right\rangle^{k} D_{k} \varepsilon_{k} / D t$ y evita que se infrinja la segunda ley de la termodinámica. Para analizar esto sustituyamos esta ecuación en la segunda ley de la termodinámica [Ec. (2.41)]:

$$
\begin{aligned}
& \varepsilon_{k}\left(-\left\langle\boldsymbol{\tau}_{k}\right\rangle^{k}+\mathbf{\Pi}_{k}^{\mathrm{Re}}\right):\left\langle\mathbf{D}_{k}\right\rangle_{k}+\left(\mathbf{M}_{k p}+\mathbf{M}_{k t}\right) \cdot\left(\left\langle\mathbf{v}_{k}\right\rangle_{i}-\left\{\mathbf{v}_{k}\right\}^{k}\right)+W_{k i}^{\prime \prime} \\
& +\left(\left\langle\mathbf{v}_{k}\right\rangle_{i}-\left\{\mathbf{v}_{k}\right\}^{k}\right) \cdot\left(\left\langle p_{k}\right\rangle_{i} \mathbf{1}+\left\langle\boldsymbol{\tau}_{k}\right\rangle_{i}\right) \cdot \nabla \varepsilon_{k}-\varepsilon_{k} \frac{\left\langle\mathbf{q}_{k}\right\rangle^{k}}{\left\{T_{k}\right\}^{k}} \cdot \nabla\left\{T_{k}\right\}^{k}+\left\langle P_{k}\right\rangle^{k} \frac{D_{k}}{D t} \varepsilon_{k} \\
& -\nabla \cdot\left[\varepsilon_{k}\left(\mathbf{q}_{k}^{p}+\mathbf{q}_{k}^{t}+\mathbf{q}_{k}^{K}\right)\right]-\varepsilon_{k}\left\langle\rho_{k}\right\rangle^{k}\left\{\tilde{S}_{k} \tilde{\mathbf{v}}_{k}\right\}^{k} \cdot \nabla\left\{T_{k}\right\}^{k}-\varepsilon_{k}\left\langle\rho_{k}\right\rangle^{k} \frac{D_{k}}{D t} K_{k}^{\mathrm{Re}} \\
& -\left[\Gamma_{k} \frac{\left\langle P_{k}\right\rangle^{k}}{\left\langle\rho_{k}\right\rangle^{k}}=\left\langle P_{k}\right\rangle^{k}\left\langle\tilde{\mathbf{v}}_{k} \cdot \nabla^{\prime} X_{k}\right\rangle+\left\langle P_{k}\right\rangle^{k} \frac{D_{k}}{D t} \varepsilon_{k}\right] \\
& +\Gamma_{k}\left[\frac{\rho_{k i}-\left\langle\rho_{k}\right\rangle^{k}}{\left\langle\rho_{k}\right\rangle^{k}} \frac{\left\langle\rho_{k}\right\rangle^{k}}{\left\langle\rho_{k}\right.}-K_{k}^{\mathrm{Re}}+\frac{1}{2}\left(\mathbf{v}_{k} \cdot \mathbf{v}_{k}\right)_{i}-\left\{\mathbf{v}_{k}\right\}^{k} \cdot \mathbf{v}_{k i}+\frac{1}{2}\left(\left\{\mathbf{v}_{k}\right\}^{k} \cdot\left\{\mathbf{v}_{k}\right\}^{k}\right)\right]= \\
& \left\{T_{k}\right\}^{k}\left\langle X_{k} \Delta_{k}\right\rangle \geq 0,
\end{aligned}
$$


quedando:

$$
\begin{aligned}
& \varepsilon_{k}\left(-\left\langle\boldsymbol{\tau}_{k}\right\rangle^{k}+\mathbf{\Pi}_{k}^{\mathrm{Re}}\right):\left\langle\mathbf{D}_{k}\right\rangle_{k}+\left(\mathbf{M}_{k p}+\mathbf{M}_{k t}\right) \cdot\left(\left\langle\mathbf{v}_{k}\right\rangle_{i}-\left\{\mathbf{v}_{k}\right\}^{k}\right)+W_{k i}^{\prime \prime} \\
& +\left(\left\langle\mathbf{v}_{k}\right\rangle_{i}-\left\{\mathbf{v}_{k}\right\}^{k}\right) \cdot\left(\left\langle P_{k}\right\rangle_{i} \mathbf{1}+\left\langle\boldsymbol{\tau}_{k}\right\rangle_{i}\right) \cdot \nabla \varepsilon_{k}-\varepsilon_{k} \frac{\left\langle\mathbf{q}_{k}\right\rangle^{k}}{\left\{T_{k}\right\}^{k}} \cdot \nabla\left\{T_{k}\right\}^{k}-\left\langle P_{k}\right\rangle^{k}\left\langle\tilde{\mathbf{v}}_{k} \cdot \nabla^{\prime} X_{k}\right\rangle \\
& -\nabla \cdot\left[\varepsilon_{k}\left(\mathbf{q}_{k}^{p}+\mathbf{q}_{k}^{t}+\mathbf{q}_{k}^{K}\right)\right]-\varepsilon_{k}\left\langle\rho_{k}\right\rangle^{k}\left\{\tilde{S}_{k} \tilde{\mathbf{v}}_{k}\right\}^{k} \cdot \nabla\left\{T_{k}\right\}^{k}-\varepsilon_{k}\left\langle\rho_{k}\right\rangle^{k} \frac{D_{k}}{D t} K_{k}^{\mathrm{Re}} \\
& +\Gamma_{k}\left[\frac{\rho_{k i}-\left\langle\rho_{k}\right\rangle^{k}}{\left\langle\rho_{k}\right\rangle^{k}} \frac{\left\langle P_{k}\right\rangle^{k}}{\left\langle\rho_{k}\right\rangle^{k}}-K_{k}^{\mathrm{Re}}+\frac{1}{2}\left(\mathbf{v}_{k} \cdot \mathbf{v}_{k}\right)_{i}-\left\{\mathbf{v}_{k}\right\}^{k} \cdot \mathbf{v}_{k i}+\frac{1}{2}\left(\left\{\mathbf{v}_{k}\right\}^{k} \cdot\left\{\mathbf{v}_{k}\right\}^{k}\right)\right]= \\
& \left\{T_{k}\right\}^{k}\left\langle X_{k} \Delta_{k}\right\rangle \geq 0 .
\end{aligned}
$$

La cual, contiene el término:

$$
\left(\left\langle\mathbf{v}_{k}\right\rangle_{i}-\left\{\mathbf{v}_{k}\right\}^{k}\right) \cdot\left\langle P_{k}\right\rangle_{i} \mathbf{1} \cdot \nabla \varepsilon_{k}=\left\langle P_{k}\right\rangle_{i}\left(\left\langle\mathbf{v}_{k}\right\rangle_{i}-\left\{\mathbf{v}_{k}\right\}^{k}\right) \cdot \nabla \varepsilon_{k}
$$

Por ejemplo, para un flujo de líquido $\lambda$ que se evapora, podemos modelar este término considerando que para condensación la velocidad interfacial es el promedio de la velocidad de la fase vapor $(g)$ y podemos constituir el término $\left\langle\tilde{\mathbf{v}}_{\lambda} \cdot \nabla^{\prime} X_{\lambda}\right\rangle$ por el negativo de $\left\langle P_{\lambda}\right\rangle_{i}\left(\left\langle\mathbf{v}_{g}\right\rangle_{i}-\left\{\mathbf{v}_{\lambda}\right\}^{\lambda}\right) \cdot \nabla \varepsilon_{\lambda}$ tal que en esta forma no se viola la segunda ley de la termodinámica.

Para ser consistentes, modelaremos este término como:

$$
\left\langle\tilde{\mathbf{v}}_{\lambda} \cdot \nabla^{\prime} X_{\lambda}\right\rangle= \begin{cases}-\left(\left\langle\mathbf{v}_{g}\right\rangle_{i}-\left\{\mathbf{v}_{\lambda}\right\}^{\lambda}\right) \cdot \nabla \varepsilon_{\lambda}, & \text { para condensación, } \\ 0, & \text { para evaporación. }\end{cases}
$$

En nuestro caso no hay cambio de fase y modelamos el término $\left\langle\tilde{\mathbf{v}}_{k} \cdot \nabla^{\prime} X_{k}\right\rangle$ como cero, lo cual cumple con la segunda ley de la termodinámica. 


\subsubsection{Proyección de las ecuaciones (modelo 1-D)}

Se obtendrá un modelo unidimensional del conjunto de seis ecuaciones dado por la Ec. (6.25) a la Ec. (6.30) con $k=g, s$. Para este propósito se considera que $\left\{\mathbf{v}_{k}\right\}^{k}=V_{k} \mathbf{e}_{z}$ en un sistema coordenado de referencia $(r, \theta, z)$, donde $V_{k}$ es una notación corta para la magnitud de la velocidad $\left\{\mathbf{v}_{k}\right\}^{k}$ y desarrollando la proyección de las ecuaciones de movimiento dadas por la Ec. (3.7) y la Ec. (3.8) en la dirección axial $\mathbf{e}_{z}$ :

$$
\begin{aligned}
& \varepsilon_{g}\left\langle\rho_{g}\right\rangle^{g} \frac{D_{g}}{D t} V_{g}-\varepsilon_{g}\left\langle\rho_{g}\right\rangle^{g} g+\varepsilon_{g} \frac{\partial}{\partial z} P_{g}+a_{g W}\left\langle\mathbf{e}_{r} \cdot \tilde{\boldsymbol{\Pi}}_{g} \cdot \mathbf{e}_{z}\right\rangle^{g W}=-M_{s z}^{D}, \\
& \varepsilon_{s}\left\langle\rho_{s}\right\rangle^{s} \frac{D_{s}}{D t} V_{s}-\varepsilon_{s}\left\langle\rho_{s}\right\rangle^{s} g+\varepsilon_{s} \frac{\partial}{\partial z}\left[P_{g}+\Phi\left(\varepsilon_{s}\right)\right]+a_{s W}\left\langle\mathbf{e}_{r} \cdot \tilde{\boldsymbol{\Pi}}_{s} \cdot \mathbf{e}_{z}\right\rangle^{s W}=M_{s z}^{D}
\end{aligned}
$$

Por simplicidad, de aquí en adelante se omitirán todos los símbolos de promediado. Considerando granos esféricos sólidos, el área interfacial específica es $a_{g s}=\left(6 / d_{p}\right) \varepsilon_{s}$.

También, la fuerza interfacial promedio $\left\langle\mathbf{n}_{g} \cdot \tilde{\boldsymbol{\Pi}}_{g} \cdot \mathbf{e}_{z}\right\rangle^{g s}$ puede modelarse mediante un modelo de celda, utilizando un coeficiente de arrastre apropiado, de tal forma que (Sánchez-López, 2003):

$$
-M_{s z}^{D}=a_{g s}\left\langle\mathbf{n}_{g} \cdot \tilde{\boldsymbol{\Pi}}_{g} \cdot \mathbf{e}_{z}\right\rangle^{g s}=\varepsilon_{s} \rho_{g} g+C_{D} \frac{3}{4 d_{p}} \varepsilon_{s} \rho_{g} U_{t}^{2} .
$$

La contribución cinética a esta fuerza en una capa laminar alrededor de los granos sólidos es linealmente proporcional a la velocidad relativa entre las fases; por lo tanto la Ec. (6.42) puede escribirse como

$$
-M_{s z}^{D}=a_{g s}\left\langle\mathbf{n}_{g} \cdot \tilde{\mathbf{\Pi}}_{g} \cdot \mathbf{e}_{z}\right\rangle^{g s}=\varepsilon_{s} \rho_{g} g+\beta \varepsilon_{s} \varepsilon_{g}\left(V_{g}-V_{s}\right),
$$

donde $\beta \equiv \frac{3}{4 d_{p}} C_{D} \rho_{g} U_{t}$. Se necesitan también expresiones de cerradura para los términos de interacción con la pared en la Ec. (6.40) y en la Ec.(6.41). Esto puede resolverse al introducir el enfoque del factor de fricción (Gidaspow, 1994). Así, para la fase $k=\{g, s\}$ 


$$
a_{k W}\left\langle\mathbf{e}_{r} \cdot \tilde{\boldsymbol{\Pi}}_{k} \cdot \mathbf{e}_{z}\right\rangle^{k W}=\frac{4}{D_{t}} \varepsilon_{k} f_{k W} \frac{1}{2} \rho_{k} V_{k}^{2}=\beta_{k W} \rho_{k} V_{k}^{2},
$$

donde $\beta_{k W} \equiv\left(2 / D_{t}\right) \varepsilon_{k} f_{k W}$. En la interacción del flujo en la mezcla turbulenta con la pared, la fuerza cinética es aproximadamente proporcional al cuadrado de las velocidades.

Después de sustituir en la Ec. (6.25) a la Ec. (6.30), las relaciones de cerradura, las relaciones del Apéndice $K$ para la derivada de la densidad de la fase gas y la proyección de las ecuaciones de movimiento, se obtiene el conjunto cerrado:

$$
\begin{aligned}
& \rho_{g}\left(P_{g}, T_{g}\right) \frac{\partial}{\partial t} \varepsilon_{g}+\rho_{g}\left(P_{g}, T_{g}\right) V_{g} \frac{\partial}{\partial z} \varepsilon_{g}+\varepsilon_{g} \rho_{g}\left(P_{g}, T_{g}\right) \frac{\partial}{\partial z} V_{g} \\
& +\varepsilon_{g}\left[\left(\frac{\partial \rho_{g}}{\partial P_{g}}\right)_{T_{g}} \frac{\partial P_{g}}{\partial t}+\left(\frac{\partial \rho_{g}}{\partial T_{g}}\right)_{P_{g}} \frac{\partial T_{g}}{\partial t}\right]+\varepsilon_{g} V_{g}\left[\left(\frac{\partial \rho_{g}}{\partial P_{g}}\right)_{T_{g}} \frac{\partial P_{g}}{\partial z}+\left(\frac{\partial \rho_{g}}{\partial T_{g}}\right)_{P_{g}} \frac{\partial T_{g}}{\partial z}\right]=0 \\
& -\rho_{s} \frac{\partial}{\partial t} \varepsilon_{g}-\rho_{s} V_{s} \frac{\partial}{\partial z} \varepsilon_{g}+\rho_{s} \varepsilon_{s} \frac{\partial}{\partial z} V_{s}=0 \\
& \varepsilon_{g} \rho_{g}\left(P_{g}, T_{g}\right) \frac{\partial}{\partial t} V_{g}+\varepsilon_{g} \rho_{g}\left(P_{g}, T_{g}\right) V_{g} \frac{\partial}{\partial z} V_{g}+\rho_{g}\left(P_{g}, T_{g}\right) g+\varepsilon_{g} \frac{\partial}{\partial z} P_{g}+\beta_{g W} \rho_{g}\left(P_{g}, T_{g}\right) V_{g}^{2}= \\
& -\beta \varepsilon_{s} \varepsilon_{g}\left(V_{g}-V_{s}\right) \\
& \varepsilon_{s} \rho_{s} \frac{\partial}{\partial t} V_{s}+\varepsilon_{s} \rho_{s} V_{s} \frac{\partial}{\partial z} V_{s}+\varepsilon_{s}\left[\rho_{s}-\rho_{g}\left(P_{g}, T_{g}\right)\right] g+\varepsilon_{s} \frac{\partial}{\partial z} P_{g}-\Phi^{\prime} \varepsilon_{s} \frac{\partial}{\partial z} \varepsilon_{g}+\beta_{s W} \rho_{s 0} V_{s}^{2}= \\
& \beta \varepsilon_{s} \varepsilon_{g}\left(V_{g}-V_{s}\right) \\
& \varepsilon_{g} \rho_{g}\left(P_{g}, T_{g}\right) c_{p g} \frac{\partial}{\partial t} T_{g}+\varepsilon_{g} \rho_{g}\left(P_{g}, T_{g}\right) c_{p g} V_{g} \frac{\partial}{\partial z} T_{g}-\varepsilon_{g} \frac{\partial}{\partial t} P_{g}-\varepsilon_{g} V_{g} \frac{\partial}{\partial z} P_{g} \\
& +\frac{4}{D_{t}} \varepsilon_{g} \bar{h}_{g W}\left(T_{W}-T_{g}\right)=-\frac{6}{d_{p}} \bar{h}_{i} \varepsilon_{s}\left(T_{i}-T_{g}\right) \\
& \varepsilon_{s} \rho_{s} c_{p s} \frac{\partial}{\partial t} T_{s}+\varepsilon_{s} \rho_{s} c_{p s} V_{s} \frac{\partial}{\partial z} T_{s}+\frac{4}{D_{t}} \varepsilon_{s} \bar{h}_{s W}\left(T_{W}-T_{s}\right)=\frac{6}{d_{p}} \bar{h}_{i} \varepsilon_{s}\left(T_{i}-T_{g}\right) .
\end{aligned}
$$

Donde $\Phi^{\prime} \equiv d \Phi / d \varepsilon_{s}, \beta \equiv \frac{3}{4 d_{p}} C_{D} \rho_{g} U_{t}$ y $\beta_{k W} \equiv \frac{2}{D_{t}} \varepsilon_{k} f_{k W}$. En la Ec. (6.45) a la Ec. (6.50) hay ocho cantidades desconocidas, $\left\{\varepsilon_{g}, \varepsilon_{s}, \rho_{g}, P_{g}, V_{g}, V_{s}, T_{g}, T_{s}\right\}$. 
Para tener un conjunto cerrado para las variables $\left\{\varepsilon_{g}, \varepsilon_{s}, \rho_{g}, P_{g}, V_{g}, V_{s}, T_{g}, T_{s}\right\}$, es necesario considerar diferentes condiciones:

(1) la función $\Phi^{\prime}$ debe especificarse, tal que las rapideces de propagación de segundo orden del modelo incompresible sean reales [ver el Apéndice $E$ y la Ec. (4.21)];

(2) El sistema sólido-gas es saturado, i.e., $\varepsilon_{s}+\varepsilon_{g}=1$;

(3) los granos sólidos son incompresibles, $\rho_{s}=\rho_{s 0}$ es una constante y

(4) la densidad promedio de la fase gas o vapor es una función de su presión promedio y de su temperatura promedio, de acuerdo a una ecuación de estado $\rho_{g}=\rho_{g}\left(P_{g}, T_{g}\right)$.

(5) Además $\beta, \beta_{g W}, \beta_{s W}, \bar{h}_{i}, \bar{h}_{g W}$ y $\bar{h}_{s W}$ pueden calcularse en el estado base por métodos estándar, así como $U_{t}$.

El conjunto dado en la Ec. (6.45) a la Ec. (6.50) puede linealizarse alrededor de un estado base uniforme, denotado por un subíndice cero, tal que $\varepsilon_{g}=\varepsilon_{0}+\varepsilon, P_{g}=P_{0}+p$, $V_{g}=v_{g 0}+v_{g}, V_{s}=v_{s 0}+v_{s}, T_{g}=T_{g 0}+T_{g}$ y $T_{s}=T_{s 0}+T_{s}$. Una vez que la linealización se lleva a cabo, los símbolos para las velocidades $v_{g} \mathrm{y} v_{s}$, así como para las temperaturas $T_{g}$ y $T_{s}$, se utilizan para las correspondientes perturbaciones.

Puede obtenerse un modelo que no considere los efectos de la pared, a partir del conjunto de ecuaciones (6.45)-(6.50), al tomar los límites: $\beta_{g W} \rightarrow 0, \quad \beta_{s W} \rightarrow 0, \bar{h}_{g W} \rightarrow 0$ y $\bar{h}_{s W} \rightarrow 0$.

El conjunto linealizado de la Ec. (6.45) a la Ec. (6.50) será el punto de partida para el estudio de propagación de ondas térmicas en los Capítulos 7 y 8. 


\section{Capítulo 7}

\section{Propagación de ondas térmicas compresibles e incompresibles con modelos 1-D linealizados}

\subsection{Introducción}

Para llevar a cabo los análisis y estudios de este Capítulo es indispensable contar con un modelo térmico cerrado, lineal y unidimensional para las variables de perturbación $\left\{\varepsilon, p, v_{g}, v_{s}, T_{g}, T_{s}\right\}$.

En este Capítulo no se considerarán los efectos de pared, por lo que este modelo no contendrá tanto al transporte interfacial de cantidad de movimiento con la pared del tubo, como al transporte interfacial de energía con la pared del tubo. Dejando el análisis de estos efectos para el Capítulo siguiente.

El modelo en el que nos concentraremos ya ha sido desarrollado en el Capítulo 6. Con este modelo no-isotérmico se obtendrá una ecuación de jerarquía de ondas con una metodología similar a la seguida en el Capítulo 4, que represente al modelo no-isotérmico y al isotérmico en una misma base de comparación, para estudiar sus coeficientes, rapideces de propagación y estabilidad lineal.

\subsection{Jerarquía de ondas}

\subsubsection{Ecuaciones del modelo}

El sistema físico consiste de una dispersión monodispersa de partículas pequeñas esféricas y rígidas de diámetro medio $d_{p}$, densidad intrínseca $\rho_{s}$ y temperatura $T_{s}$, moviéndose dentro de una corriente de gas con temperatura $T_{g}$ en un tubo vertical, donde $T_{s}>T_{g}$. El fluido se considera un gas newtoniano, compresible, con densidad intrínseca $\rho_{g}$ asociada a una ecuación de estado $\rho_{g}\left(P_{g}, T_{g}\right)$ y viscosidad constante $\mu_{g}$. 
La fracción volumen de sólido $\varepsilon_{s}$, se encuentra entre 0 y 0.5 y satisface la condición $\varepsilon_{s}+\varepsilon_{g}=1$, donde $\varepsilon_{g}$ es la fracción volumen de gas. La mezcla se mueve con una velocidad promedio $U_{m}=\varepsilon_{s} V_{s}+\varepsilon_{g} V_{g}$, donde $V_{g}$ y $V_{s}$ son los valores de las velocidades ascendentes con promedio ponderado en la masa del gas y sólido, respectivamente. Debido a que la mezcla se considera en un régimen altamente turbulento, los efectos viscosos son sólo significativos en una región cercana a la superficie de los gránulos. El número de Reynolds de las partículas es $\operatorname{Re}_{s}=d_{p} \rho_{g} U_{t} / \mu_{g}$, donde la velocidad terminal se define como $U_{t} \doteq\left|V_{s}-U_{m}\right|=\varepsilon_{g}\left(V_{g}-V_{s}\right)$. En este modelo, despreciamos los efectos viscosos en las fases, así como los esfuerzos turbulentos.

El conjunto dado en la Ec. (6.45) a la Ec. (6.50) puede linealizarse alrededor de un estado base uniforme, denotado por un subíndice cero, tal que $\varepsilon_{g}=\varepsilon_{0}+\varepsilon, P_{g}=P_{0}+p$, $v_{g}=v_{g 0}+v_{g}, v_{s}=v_{s 0}+v_{s}, T_{g}=T_{g 0}+T_{g}$ y $T_{s}=T_{s 0}+T_{s}$. Una vez que la linealización se lleva a cabo los símbolos para las velocidades $v_{g} \mathrm{y} v_{s}$, así como para las temperaturas $T_{g} \mathrm{y}$ $T_{s}$ se utilizan para las correspondientes perturbaciones. El conjunto linealizado de la Ec. (6.45) a la Ec. (6.50) está dado por:

$$
\begin{aligned}
& \rho_{g 0}\left(\frac{\partial}{\partial t}+v_{g 0} \frac{\partial}{\partial z}\right) \varepsilon+\varepsilon_{0} \rho_{g 0} \frac{\partial}{\partial z} v_{g}+\varepsilon_{0} \frac{\gamma}{s^{2}}\left(\frac{\partial}{\partial t}+v_{g 0} \frac{\partial}{\partial z}\right) p-\varepsilon_{0} \rho_{g 0} \beta_{V}\left(\frac{\partial}{\partial t}+v_{g 0} \frac{\partial}{\partial z}\right) T_{g}=0 \\
& -\rho_{s 0}\left(\frac{\partial}{\partial t}+v_{s 0} \frac{\partial}{\partial z}\right) \varepsilon+\rho_{s 0}\left(1-\varepsilon_{0}\right) \frac{\partial}{\partial z} v_{s}=0 \\
& \varepsilon_{0} \rho_{g 0}\left(\frac{\partial}{\partial t}+v_{g 0} \frac{\partial}{\partial z}\right) v_{g}+\varepsilon_{0} \frac{\partial}{\partial z} p+g\left(-\rho_{g 0} \beta_{V} T_{g}+\frac{\gamma}{s^{2}} p\right)+\beta\left(1-2 \varepsilon_{0}\right)\left(v_{g 0}-v_{s 0}\right) \varepsilon \\
& +\beta\left(1-\varepsilon_{0}\right) \varepsilon_{0}\left(v_{g}-v_{s}\right)=0 \\
& \left(1-\varepsilon_{0}\right) \rho_{s 0}\left(\frac{\partial}{\partial t}+v_{s 0} \frac{\partial}{\partial z}\right) v_{s}+\left(1-\varepsilon_{0}\right) \frac{\partial}{\partial z} p-\left(1-\varepsilon_{0}\right) g\left(-\rho_{g 0} \beta_{V} T_{g}+\frac{\gamma}{s^{2}} p\right) \\
& -\left(1-\varepsilon_{0}\right) \Phi_{0}^{\prime} \frac{\partial \varepsilon}{\partial z}-\left[\left(\rho_{s 0}-\rho_{g 0}\right) g+\beta\left(1-2 \varepsilon_{0}\right)\left(v_{g 0}-v_{s 0}\right)\right] \varepsilon-\beta\left(1-\varepsilon_{0}\right) \varepsilon_{0}\left(v_{g}-v_{s}\right)=0
\end{aligned}
$$




$$
\begin{aligned}
& \varepsilon_{0} \rho_{g 0} c_{p g}\left(\frac{\partial}{\partial t}+v_{g 0} \frac{\partial}{\partial z}\right) T_{g}-\varepsilon_{0}\left(\frac{\partial}{\partial t}+v_{g 0} \frac{\partial}{\partial z}\right) p-\frac{6}{d_{p}} \bar{h}_{i 0}\left(1-\varepsilon_{0}\right) T_{g}-\frac{6}{d_{p}} \bar{h}_{i 0}\left(T_{i}-T_{g 0}\right) \varepsilon=0,(7.5) \\
& \left(1-\varepsilon_{0}\right) \rho_{s 0} c_{p s}\left(\frac{\partial}{\partial t}+v_{s 0} \frac{\partial}{\partial z}\right) T_{s}+\frac{6}{d_{p}} \bar{h}_{i 0}\left(1-\varepsilon_{0}\right) T_{g}+\frac{6}{d_{p}} \bar{h}_{i 0}\left(T_{i}-T_{g 0}\right) \varepsilon=0
\end{aligned}
$$

La linealización de $\rho_{g}=\rho_{g}\left(P_{g}, T_{g}\right)$ puede verse en la sección K.2 del Apéndice K. Aquí $z$ y $t$ son la coordenada vertical y el tiempo, respectivamente, $g$ es la fuerza gravitacional por unidad de masa y $P_{g}$ es la presión de la fase gas promediada; la densidad de los granos sólidos es constante $\rho_{s}=\rho_{s 0}$. La presión promedio de la fase sólida $P_{s}$ se modeló a través de la cerradura $P_{s}=P_{g}+\Phi\left(\varepsilon_{s}\right)$ en la Ec. (7.4), donde la función $\Phi\left(\varepsilon_{s}\right)$ es el módulo de compresibilidad del sólido y $\Phi^{\prime}=d \Phi / d \varepsilon_{s}$. La cerradura simple $P_{s}=\Phi\left(\varepsilon_{s}\right)$ reduce el acoplamiento entre los balances de momentum a únicamente la fuerza de interacción $F$ entre el fluido y las partículas. Esta cerradura fue utilizada por Jackson (1985) y Liu (1982) entre otros y fue recomendada por Gidaspow (1994).

\section{$\underline{\text { Discusión de la linealización del modelo no-isotérmico-compresible }}$}

Las ecuaciones (7.1) a la (7.6) presentan un proceso de linealización que consiste en dos etapas. La primera donde se determina cuales funcionalidades son fundamentales, las que no son fundamentales se evalúan directamente en el estado base. La segunda etapa del proceso de linealización se lleva a cabo al linealizar las funcionalidades fundamentales.

Para discriminar las funcionalidades fundamentales de las que no lo son, se utiliza la física del problema, por ejemplo: al linealizar la fricción en la pared del tubo, se deja como dependencia fundamental la velocidad del fluido al cuadrado en consistencia con el régimen de Newton de fricción para esferas. El coeficiente de arrastre, que de acuerdo a los datos de este trabajo se modeló con la correlación de Ergun, no se linealizó porque al hacerlo se introducirían variables artificialmente. La importancia del proceso de linealización reside en que si no se determinan las funcionalidades fundamentales, se pueden inducir funcionalidades espurias o artificiales al linealizar por ejemplo cerraduras que contienen correlaciones de variables.

En la siguiente subsección representamos las ecuaciones linealizadas en forma matricial y después presentamos el análisis comparativo de coeficientes entre los modelos. 


\subsubsection{Enunciado del problema}

Sea

$$
\mathbf{u} \doteq\left(\begin{array}{llllll}
\varepsilon & p & v_{g} & v_{s} & T_{g} & T_{s}
\end{array}\right)^{T}
$$

el vector de perturbaciones para el sistema linealizado de EDP de primer orden:

$$
\mathbf{B} \frac{\partial \mathbf{u}}{\partial t}+\mathbf{C} \frac{\partial \mathbf{u}}{\partial z}+\mathbf{D u}=\mathbf{0}
$$

donde B, C y $\mathbf{D}$ son matrices de tamaño $6 \times 6$, definidas en el Apéndice $L$. Las derivadas espaciales de las perturbaciones de velocidad $v_{g} \mathrm{y} v_{s}$ pueden obtenerse de las ecuaciones de continuidad, luego se sustituyen en las ecuaciones de momentum y en las ecuaciones de energía para llegar así a cuatro ecuaciones linealizadas, dos de segundo orden Ec. (7.9) y Ec. (7.10) y dos de primer orden Ec. (7.11) y Ec. (7.12) para las perturbaciones de la fracción volumen $(\varepsilon)$, para las perturbaciones de la presión $(p)$, para las perturbaciones

de la temperatura de la fase gaseosa $\left(T_{g}\right)$ y para las perturbaciones de la temperatura de la fase sólida $\left(T_{s}\right)$.

\subsubsection{Estructuras ondulatorias}

$$
\begin{array}{rrrl}
L_{1} \varepsilon & +\left(L_{3}+\frac{\gamma}{s^{2}} K_{1}\right) p & -\rho_{g 0} \beta_{V} K_{1} T_{g} & =0, \\
L_{2} \varepsilon & +\left(L_{3}+\frac{\gamma}{s^{2}} K_{2}\right) p & -\rho_{g 0} \beta_{V} K_{2} T_{g} & =0, \\
L_{4} \varepsilon & +L_{6} p & -L_{8} T_{g} & =0, \\
L_{4} \varepsilon & +L_{9} T_{g}+L_{10} T_{s} & =0 .
\end{array}
$$

Los operadores diferenciales $L_{1}, L_{2}, L_{3}, K_{1}$ y $K_{2}$ están definidos en el Capítulo 4, Ec. (4.9) a la Ec. (4.13). 
Por otra parte, los operadores de primer orden $L_{6}, L_{8}$ y $L_{10}$, así como los operadores de orden cero $L_{4}$ y $L_{9}$, están dados por

$$
\begin{aligned}
& L_{4} \doteq \frac{6}{d_{p}} \bar{h}_{i 0}\left(T_{i}-T_{g 0}\right), \\
& L_{6} \doteq \varepsilon_{0}\left(\frac{\partial}{\partial t}+v_{g 0} \frac{\partial}{\partial z}\right), \\
& L_{8} \doteq \varepsilon_{0} \rho_{g 0} c_{p g}\left(\frac{\partial}{\partial t}+v_{g 0} \frac{\partial}{\partial z}\right)-\frac{6}{d_{p}} \bar{h}_{i 0}\left(1-\varepsilon_{0}\right), \\
& L_{9} \doteq \frac{6}{d_{p}} \bar{h}_{i 0}\left(1-\varepsilon_{0}\right), \\
& L_{10} \doteq\left(1-\varepsilon_{0}\right) \rho_{s 0} c_{p s}\left(\frac{\partial}{\partial t}+v_{s 0} \frac{\partial}{\partial z}\right) .
\end{aligned}
$$

Donde $\beta_{V} \doteq-\rho_{g}{ }^{-1}\left(\partial \rho_{g} / \partial T_{g}\right)_{P_{g}}$ (que se define en el Apéndice $K$ ), es el coeficiente de expansión volumétrica. El factor $\left(\partial \rho_{g} / \partial P_{g}\right)_{0}$ toma en cuenta el cambio de la densidad del fluido como una función de la presión en el mismo, a través de una ecuación de estado $\rho_{g}=\rho_{g}\left(P_{g}, T_{g}\right)$. La velocidad del sonido en un gas homogéneo puede introducirse al considerar la relación $\left(\partial \rho_{g} / \partial P_{g}\right)_{0}=s^{-2}$, donde $s$ es la velocidad del sonido en el gas puro.

Se desarrollarán cuatro modelos:

(1) El primero para el flujo isotérmico $\left(T_{g}=T_{s}=T_{0}\right)$ compresible $(s \neq 0)$.

(2) El segundo para el flujo isotérmico incompresible $(s \rightarrow \infty)$. Por lo tanto, $\beta_{V} \rightarrow 0$ y los coeficientes $\left\{L_{4}, L_{6}, L_{8}, L_{9}, L_{10}\right\}$ se vuelven cero.

(3) El tercer modelo a desarrollarse es para el flujo no-isotérmico $\left(T_{s}>T_{g}\right)$ de gas o vapor compresible representado en la Ec. (7.32) y

(4) El cuarto modelo es para el flujo no-isotérmico incompresible. 
El procedimiento que seguiremos a continuación nos permite obtener una EDP para estudiar los cuatro modelos siguientes:

$$
\begin{aligned}
& \text { modelo no-isotérmico }\left\{\begin{array}{c}
\text { compresible } \\
\text { incompresible }
\end{array}\right. \\
& \text { modelo isotérmico }\left\{\begin{array}{c}
\text { compresible } \\
\text { incompresible. }
\end{array}\right.
\end{aligned}
$$

Puesto que el conjunto dado por la Ec. (7.9) a la Ec. (7.11) es independiente de la Ec. (7.12); esto es, podemos resolver el sistema de EDP para $\left\{\varepsilon, p, T_{g}\right\}$ y con estas soluciones resolver para $T_{s}$ en la Ec. (7.12). Primero eliminaremos a $\varepsilon$ en la Ec. (7.9) a la Ec. (7.11) para obtener un sistema de dos ecuaciones para las variables: $\left\{p, T_{g}\right\}$.

Luego entonces, despejamos el término que tiene a $T_{s}$ en la Ec. (7.12),

$$
L_{10} T_{s}=-L_{4} \varepsilon-L_{9} T_{g}
$$

Esta ecuación gobierna las perturbaciones de la temperatura del sólido, una vez que se conocen las perturbaciones de la fracción volumen de gas y de la temperatura del gas. Resolvemos en la Ec. (7.11) para $\varepsilon$,

$$
L_{4} \varepsilon=-L_{6} p+L_{8} T_{g}
$$

Ahora aplicamos el operador $L_{4}$ a la Ec. (7.9) y a la Ec. (7.10) para luego sustituir la Ec. (7.19) y obtener dos ecuaciones para $\left\{p, T_{g}\right\}$ :

$$
\begin{aligned}
& \left(L_{3} L_{4}-L_{1} L_{6}+\frac{\gamma}{s^{2}} L_{4} K_{1}\right) p+\left(L_{1} L_{8}-\rho_{g 0} \beta_{V} L_{4} K_{1}\right) T_{g}=0, \\
& \left(L_{3} L_{4}-L_{2} L_{6}+\frac{\gamma}{s^{2}} L_{4} K_{2}\right) p+\left(L_{2} L_{8}-\rho_{g 0} \beta_{V} L_{4} K_{2}\right) T_{g}=0 .
\end{aligned}
$$


Posteriormente aplicamos el operador $\left(L_{3} L_{4}-L_{1} L_{6}+\frac{\gamma}{s^{2}} L_{4} K_{1}\right)$ a la Ec. (7.21) y el operador $\left(L_{3} L_{4}-L_{2} L_{6}+\frac{\gamma}{s^{2}} L_{4} K_{2}\right)$ a la Ec. (7.20), luego restamos la segunda de la primera para tener la siguiente ecuación para $T_{g}$ :

$$
-\left\{\begin{array}{l}
{\left[\left(L_{2}-L_{1}\right) L_{3}-\frac{\gamma}{s^{2}}\left(L_{1} K_{2}-L_{2} K_{1}\right)\right] L_{8}} \\
-\rho_{g 0} \beta_{V}\left[\left(K_{2}-K_{1}\right) L_{3} L_{4}-\left(L_{1} K_{2}-L_{2} K_{1}\right) L_{6}\right]
\end{array}\right\} L_{4} T_{g}=0 .
$$

Puede obtenerse la ecuación para $p$ al aplicar el operador $\left(L_{2} L_{8}-\rho_{g 0} \beta_{V} L_{4} K_{2}\right)$ a la Ec. (7.20) y el operador $\left(L_{1} L_{8}-\rho_{g 0} \beta_{V} L_{4} K_{1}\right)$ a la Ec. (7.21) y restando la segunda de la primera se obtiene una ecuación equivalente a la Ec. (7.22) pero para la perturbación en $p$.

$\underline{\text { Si eliminamos a }} p$ de la Ec. (7.9) a la Ec. (7.11), obtenemos un sistema de dos EDP para $\left\{\varepsilon, T_{g}\right\}$. Para lo cual, primero resolvemos en la Ec. (7.11) para $L_{6} p:$

$$
L_{6} p=L_{8} T_{g}-L_{4} \varepsilon
$$

y sustituimos en la Ec. (7.9) y en la Ec. (7.10), a las cuales se les ha aplicado previamente el operador $L_{6}$ para obtener un par de ecuaciones en $\left\{\varepsilon, T_{g}\right\}$ :

$$
\begin{aligned}
& \left(L_{1} L_{6}-L_{3} L_{4}-\frac{\gamma}{s^{2}} L_{4} K_{1}\right) \varepsilon+\left(L_{3} L_{8}+\frac{\gamma}{s^{2}} L_{8} K_{1}-\rho_{g 0} \beta_{V} L_{6} K_{1}\right) T_{g}=0 \\
& \left(L_{2} L_{6}-L_{3} L_{4}-\frac{\gamma}{s^{2}} L_{4} K_{2}\right) \varepsilon+\left(L_{3} L_{8}+\frac{\gamma}{s^{2}} L_{8} K_{2}-\rho_{g 0} \beta_{V} L_{6} K_{2}\right) T_{g}=0 .
\end{aligned}
$$

Puede obtenerse la ecuación para $T_{g}$ al aplicar el operador $\left(L_{2} L_{6}-L_{3} L_{4}-\frac{\gamma}{s^{2}} L_{4} K_{2}\right)$ a la Ec. (7.24) y el operador $\left(L_{1} L_{6}-L_{3} L_{4}-\frac{\gamma}{s^{2}} L_{4} K_{1}\right)$ a la Ec. (7.25) y restando la segunda de la primera se obtiene, 


$$
\left\{\begin{array}{l}
{\left[\left(L_{2}-L_{1}\right) L_{3}-\frac{\gamma}{s^{2}}\left(L_{1} K_{2}-L_{2} K_{1}\right)\right] L_{8}} \\
-\rho_{g 0} \beta_{V}\left[\left(K_{2}-K_{1}\right) L_{3} L_{4}-\left(L_{1} K_{2}-L_{2} K_{1}\right) L_{6}\right]
\end{array}\right\} L_{6} T_{g}=0 .
$$

Asimismo, podemos encontrar la ecuación para $\varepsilon$ al aplicar el operador $\left(L_{3} L_{8}+\frac{\gamma}{s^{2}} L_{8} K_{2}-\rho_{g 0} \beta_{V} L_{6} K_{2}\right)$ a la Ec. (7.24) y el operador $\left(L_{3} L_{8}+\frac{\gamma}{s^{2}} L_{8} K_{1}-\rho_{g 0} \beta_{V} L_{6} K_{1}\right)$

a la Ec. (7.25) y restando la segunda de la primera se obtiene una ecuación equivalente a la Ec. (7.26) pero para $\varepsilon$.

Finalmente, eliminamos a $T_{g}$ de la Ec. (7.9) a la Ec. (7.11), con lo que obtenemos un $\underline{\text { sistema de dos EDP para }}\{\varepsilon, p\}$. Resolvemos para el término que tiene a $T_{g}$ en la Ec. (7.11),

$$
L_{8} T_{g}=L_{4} \varepsilon+L_{6} p
$$

Posteriormente, sustituimos la Ec. (7.27) en la Ec. (7.9) y en la Ec. (7.10), a las cuales previamente se les aplicó el operador diferencial de primer orden $L_{8}$ para obtener el conjunto de dos ecuaciones para $\{\varepsilon, p\}$ :

$$
\begin{aligned}
& \left(L_{1} L_{8}-\rho_{g 0} \beta_{V} L_{4} K_{1}\right) \varepsilon+\left[\left(L_{3}+\frac{\gamma}{s^{2}} K_{1}\right) L_{8}-\rho_{g 0} \beta_{V} L_{6} K_{1}\right] p=0, \\
& \left(L_{2} L_{8}-\rho_{g 0} \beta_{V} L_{4} K_{2}\right) \varepsilon+\left[\left(L_{3}+\frac{\gamma}{s^{2}} K_{2}\right) L_{8}-\rho_{g 0} \beta_{V} L_{6} K_{2}\right] p=0,
\end{aligned}
$$

ahora, le aplicamos el operador $\left(L_{2} L_{8}-\rho_{g 0} \beta_{V} L_{4} K_{2}\right)$ a la Ec. (7.28) y le aplicamos el operador $\left(L_{1} L_{8}-\rho_{g 0} \beta_{V} L_{4} K_{1}\right)$ a la Ec. (7.29). Luego restamos la última de la primera para tener:

$$
\left\{\begin{array}{c}
{\left[\left(L_{2}-L_{1}\right) L_{3}-\frac{\gamma}{s^{2}}\left(L_{1} K_{2}-L_{2} K_{1}\right)\right] L_{8}} \\
-\rho_{g 0} \beta_{V}\left[\left(K_{2}-K_{1}\right) L_{3} L_{4}-\left(L_{1} K_{2}-L_{2} K_{1}\right) L_{6}\right]
\end{array}\right\} L_{8} p=0
$$


Análogamente, puede obtenerse una ecuación para $\varepsilon$ al aplicar el operador $\left[\left(L_{3}+\frac{\gamma}{s^{2}} K_{2}\right) L_{8}-\rho_{g 0} \beta_{V} L_{6} K_{2}\right]$ a la Ec. (7.28) y el operador $\left[\left(L_{3}+\frac{\gamma}{s^{2}} K_{1}\right) L_{8}-\rho_{g 0} \beta_{V} L_{6} K_{1}\right]$ a la Ec. (7.29). Luego restamos la última de la primera para tener la misma Ec. (7.30) pero para $\varepsilon$.

Resumiendo los principales resultados encontrados al aplicar el procedimiento de eliminación de variables se tiene la siguiente Tabla:

Tabla 7.1. Ecuaciones de perturbación obtenidas al eliminar una variable con el modelo no-isotérmico sin efectos de pared.

\begin{tabular}{|c|c|c|}
\hline Variable eliminada & \multicolumn{2}{|c|}{ Ecuación para las perturbaciones } \\
\hline$\varepsilon$ & $L_{4} L^{(5)} p=0$ & $L_{4} L^{(5)} T_{g}=0$ \\
\hline$p$ & $L_{6} L^{(5)} \varepsilon=0$ & $L_{6} L^{(5)} T_{g}=0$ \\
\hline$T_{g}$ & $L_{8} L^{(5)} \varepsilon=0$ & $L_{8} L^{(5)} p=0$ \\
\hline
\end{tabular}

Al eliminar $\varepsilon$, tenemos las dos ecuaciones: $L_{4} L^{(5)} p=0$ y $L_{4} L^{(5)} T_{g}=0$. Como el operador $L_{4}$ es algebraico y además constante $\left(L_{4} \neq 0\right)$, al dividir por $L_{4}$ se obtiene el par de ecuaciones:

$$
L^{(5)} p=0
$$

$\mathrm{y}$

$$
L^{(5)} T_{g}=0
$$

donde $L^{(5)}$ es el operador de quinto orden definido por:

$$
L^{(5)} \equiv\left\{\begin{array}{c}
{\left[\left(L_{2}-L_{1}\right) L_{3}-\frac{\gamma}{s^{2}}\left(L_{1} K_{2}-L_{2} K_{1}\right)\right] L_{8}} \\
-\rho_{g 0} \beta_{V}\left[\left(K_{2}-K_{1}\right) L_{3} L_{4}-\left(L_{1} K_{2}-L_{2} K_{1}\right) L_{6}\right]
\end{array}\right\} .
$$

Luego al aplicar el operador $L^{(5)}$ a la Ec. (7.11):

$$
L_{4} L^{(5)} \varepsilon+L_{6}\left[L^{(5)} p\right]-L_{8}\left[L^{(5)} T_{g}\right]=0
$$

y utilizando los resultados encontrados anteriormente tenemos que:

$$
L_{4} L^{(5)} \varepsilon=0
$$


y ya que el operador $L_{4}$ es algebraico y además constante, se obtiene que:

$$
L^{(5)} \varepsilon=0
$$

Por último obtenemos la ecuación de las perturbaciones de $T_{s}$ al aplicar el operador $L^{(5)}$ a la Ec. (7.18),

$$
L_{10} L^{(5)} T_{s}=-L_{4}\left[L^{(5)} \varepsilon\right]-L_{9}\left[L^{(5)} T_{g}\right]
$$

Con lo que se obtiene:

$$
L_{10} L^{(5)} T_{s}=0
$$

Entonces, podemos escribir en forma general que:

$$
\left\{\begin{array}{l}
{\left[\left(L_{2}-L_{1}\right) L_{3}-\frac{\gamma}{s^{2}}\left(L_{1} K_{2}-L_{2} K_{1}\right)\right] L_{8}} \\
-\rho_{g 0} \beta_{V}\left[\left(K_{2}-K_{1}\right) L_{3} L_{4}-\left(L_{1} K_{2}-L_{2} K_{1}\right) L_{6}\right]
\end{array}\right\} \Psi=0,
$$

donde $\Psi=\left(\varepsilon, p, T_{g}, L_{10} T_{s}\right)$

El operador entre llaves es de quinto orden. Entonces la ecuación para $\varepsilon, p$ y $T_{g}$ es de quinto orden; mientras que la ecuación para $T_{s}$, está dada por el mismo operador de quinto orden pero aplicado a $L_{10}$ que es de primer orden. Por lo tanto, la ecuación para $T_{s}$ es de sexto orden.

Puesto que el operador de más bajo orden es el de las perturbaciones en $\left\{\varepsilon, p, T_{g}\right\}$, sólo analizaremos el operador entre llaves. El modelo isotérmico asociado se recupera al tomar el límite cuando el coeficiente de expansión volumétrica es cero; esto es, $\beta_{V} \rightarrow 0$ y las ecuaciones de las perturbaciones siguen siendo del mismo orden mencionado arriba. 
7.2.4 Análisis de términos del operador entre llaves en la Ec. (7.32)

El comportamiento ondulatorio de las perturbaciones de $\varepsilon$ del modelo incompresible representado por $\left(L_{2}-L_{1}\right) L_{3} L_{8}$, contiene al operador $\left(L_{2}-L_{1}\right) L_{3}$ que es exactamente el mismo que el que se vio en el Capítulo 4, Ec. (4.15), que en este caso está afectado por el operador externo $L_{8}$ que es un operador de primer orden asociado a los efectos térmicos. Por otro lado, el operador $\left(L_{2} K_{1}-L_{1} K_{2}\right)$ correspondiente a los efectos de compresibilidad, es el mismo que el analizado en el Capítulo 4, Ec. (4.16) que en este caso está afectado por la termicidad a través del operador $\gamma L_{8}$, donde $\gamma$ es el índice adiabático y que en el caso de que el sistema sea isotérmico tiende a la unidad, recuperándose el comportamiento isotérmico correspondiente. La dinámica de los efectos térmicos que está superpuesta a la de la incompresible y a la de la compresible, está gobernada por el operador $\left(K_{2}-K_{1}\right) L_{3} L_{4}-\left(L_{1} K_{2}-L_{2} K_{1}\right) L_{6}$,

$$
\begin{aligned}
& \left(K_{2}-K_{1}\right) L_{3} L_{4}-\left(L_{1} K_{2}-L_{2} K_{1}\right) L_{6} \doteq \\
& \frac{6}{d_{p}} \bar{h}_{i 0}\left(T_{i}-T_{g 0}\right)\left[\left(\frac{\partial}{\partial t}+v_{g 0} \frac{\partial}{\partial z}\right)^{2}+\frac{\beta}{\rho_{g 0}}\left(\frac{\partial}{\partial t}+v_{g 0} \frac{\partial}{\partial z}\right)-\frac{1+\varepsilon_{0}}{\varepsilon_{0}} g \frac{\partial}{\partial z}\right] \frac{\partial^{2}}{\partial z^{2}} \\
& \left.\left[-\frac{\rho_{s 0}}{1-\varepsilon_{0}}\left(\frac{\partial}{\partial t}+v_{s 0} \frac{\partial}{\partial z}\right)^{2}+\Phi_{0}^{\prime} \frac{\partial^{2}}{\partial z^{2}}\right]\left(\frac{\partial}{\partial t}+v_{g 0} \frac{\partial}{\partial z}\right)^{2}\right) \\
& +\left[\begin{array}{c}
\frac{1-2 \varepsilon_{0}}{1-\varepsilon_{0}} \beta\left(\frac{\partial}{\partial t}+v_{g 0} \frac{\partial}{\partial z}\right) \\
-\beta\left(\frac{\partial}{\partial t}+v_{s 0} \frac{\partial}{\partial z}\right)+\frac{\varepsilon_{0} \rho_{s 0}-\rho_{g 0}}{\varepsilon_{0}\left(1-\varepsilon_{0}\right)} g \frac{\partial}{\partial z}
\end{array}\right]\left(\frac{\partial}{\partial t}+v_{g 0} \frac{\partial}{\partial z}\right)^{2} \\
& -\left\{+\left[-\beta \frac{\rho_{s 0}}{\rho_{g 0}}\left(\frac{\partial}{\partial t}+v_{g 0} \frac{\partial}{\partial z}\right)+\frac{\rho_{s 0} g}{\varepsilon_{0}\left(1-\varepsilon_{0}\right)} \frac{\partial}{\partial z}\right]\left(\frac{\partial}{\partial t}+v_{s 0} \frac{\partial}{\partial z}\right)^{2}\right\} \varepsilon_{0}\left(\frac{\partial}{\partial t}+v_{g 0} \frac{\partial}{\partial z}\right) \text {. } \\
& +\left(1-\varepsilon_{0}\right) \Phi_{0}^{\prime} \frac{\beta}{\rho_{g 0}}\left(\frac{\partial}{\partial t}+v_{g 0} \frac{\partial}{\partial z}\right) \frac{\partial^{2}}{\partial z^{2}}-\frac{1}{\varepsilon_{0}} \Phi_{0}^{\prime} g \frac{\partial^{3}}{\partial z^{3}} \\
& +\left(\frac{\rho_{s 0}}{\rho_{g 0}}-\frac{1-2 \varepsilon_{0}}{1-\varepsilon_{0}}\right) \beta g\left(\frac{\partial}{\partial t}+v_{g 0} \frac{\partial}{\partial z}\right) \frac{\partial}{\partial z} \\
& \left.+\beta g\left(\frac{\partial}{\partial t}+v_{s 0} \frac{\partial}{\partial z}\right) \frac{\partial}{\partial z}-\frac{\rho_{s 0}-\rho_{g 0}}{\varepsilon_{0}\left(1-\varepsilon_{0}\right)} g^{2} \frac{\partial^{2}}{\partial z^{2}}\right)
\end{aligned}
$$


En la dinámica de los efectos térmicos están asociadas las fuerzas inerciales del fluido y del sólido, compresibilidad del sólido, arrastre viscoso interfacial, flotación; así como transporte interfacial de energía. Ninguna de estas fuerzas aparece sola, sino que aparecen como pares de interacciones con algún otro efecto. Cada uno de estos pares representa el aumento debido a los pares de fuerza considerados, debidos al cambio térmico. El punto aquí es cuantificar la magnitud de tal aumento al compararlo con los modelos isotérmico compresible e incompresible.

Al agrupar los operadores diferenciales dados por la Ec. (4.15), por la Ec. (4.16) y por la Ec. (7.33) en una estructura ondulatoria y sustituirlos en el operador encerrado entre llaves en la Ec. (7.32), se obtiene la siguiente forma equivalente

$$
\begin{aligned}
& {\left[\left(L_{2}-L_{1}\right) L_{3}-\frac{\gamma}{s^{2}}\left(L_{1} K_{2}-L_{2} K_{1}\right)\right] L_{8}} \\
& -\rho_{g 0} \beta_{V}\left[\left(K_{2}-K_{1}\right) L_{3} L_{4}-\left(L_{1} K_{2}-L_{2} K_{1}\right) L_{6}\right] \doteq \\
& \left.\frac{\beta}{\varepsilon_{0}\left(1-\varepsilon_{0}\right)}\left\{\begin{array}{r}
{\left[\tau\left(\frac{\partial}{\partial t}+c_{1} \frac{\partial}{\partial z}\right)\left(\frac{\partial}{\partial t}+c_{2} \frac{\partial}{\partial z}\right)+\left(\frac{\partial}{\partial t}+a \frac{\partial}{\partial z}\right)\right] \frac{\partial^{2}}{\partial z^{2}}} \\
-\frac{\gamma}{s^{2}}\left[\begin{array}{r}
T\left(\frac{\partial}{\partial t}+\eta_{41} \frac{\partial}{\partial z}\right)\left(\frac{\partial}{\partial t}+\eta_{42} \frac{\partial}{\partial z}\right)\left(\frac{\partial}{\partial t}+\eta_{43} \frac{\partial}{\partial z}\right)\left(\frac{\partial}{\partial t}+\eta_{44} \frac{\partial}{\partial z}\right) \\
+C\left(\frac{\partial}{\partial t}+\eta_{31} \frac{\partial}{\partial z}\right)\left(\frac{\partial}{\partial t}+\eta_{32} \frac{\partial}{\partial z}\right)\left(\frac{\partial}{\partial t}+\eta_{33} \frac{\partial}{\partial z}\right) \\
-g C\left(\frac{\partial}{\partial t}+\eta_{21} \frac{\partial}{\partial z}\right) \frac{\partial}{\partial z}
\end{array}\right]
\end{array}\right]\right\}\left[\begin{array}{c}
\varepsilon_{0} \rho_{g 0} c_{p g}\left(\frac{\partial}{\partial t}+v_{g 0} \frac{\partial}{\partial z}\right) \\
-\frac{6}{d_{p}} \bar{h}_{i 0}\left(1-\varepsilon_{0}\right)
\end{array}\right] \\
& -\rho_{g 0} \beta_{V}\left[\begin{array}{r}
\Upsilon_{5}\left(\frac{\partial^{5}}{\partial t^{5}}+O_{1 \gamma} \frac{\partial^{5}}{\partial t^{4} \partial z}+O_{2 \gamma} \frac{\partial^{5}}{\partial t^{3} \partial z^{2}}+O_{3 \gamma} \frac{\partial^{5}}{\partial t^{2} \partial z^{3}}+O_{4 \gamma} \frac{\partial^{5}}{\partial t \partial z^{4}}+O_{5 \gamma} \frac{\partial^{5}}{\partial z^{5}}\right) \\
+\Upsilon_{4}\left(\frac{\partial^{4}}{\partial t^{4}}+N_{1 \gamma} \frac{\partial^{4}}{\partial t^{3} \partial z}+N_{2 \gamma} \frac{\partial^{4}}{\partial t^{2} \partial z^{2}}+N_{3 \gamma} \frac{\partial^{4}}{\partial t \partial z^{3}}+N_{4 \gamma} \frac{\partial^{4}}{\partial z^{4}}\right) \\
+\Upsilon_{3}\left(\frac{\partial^{2}}{\partial t^{2}}+M_{1 \gamma} \frac{\partial^{2}}{\partial t \partial z}+M_{2 \gamma} \frac{\partial^{2}}{\partial z^{2}}\right) \frac{\partial}{\partial z}
\end{array}\right]
\end{aligned}
$$


reagrupando la parte no-isotérmica, encontramos que,

$$
\left.\mid \begin{array}{r}
{\left[\left(L_{2}-L_{1}\right) L_{3}-\frac{\gamma}{s^{2}}\left(L_{1} K_{2}-L_{2} K_{1}\right)\right] L_{8}} \\
-\rho_{g 0} \beta_{V}\left[\left(K_{2}-K_{1}\right) L_{3} L_{4}-\left(L_{1} K_{2}-L_{2} K_{1}\right) L_{6}\right]
\end{array}\right]
$$

donde,

$$
\begin{aligned}
& \Upsilon_{5} \doteq \frac{\varepsilon_{0}}{1-\varepsilon_{0}} \rho_{s 0}, \\
& \Upsilon_{4} \doteq \beta \varepsilon_{0}\left(\frac{\varepsilon_{0}}{1-\varepsilon_{0}}+\frac{\rho_{s 0}}{\rho_{g 0}}\right), \\
& \Upsilon_{3} \doteq-g \beta \varepsilon_{0}\left(\frac{\varepsilon_{0}}{1-\varepsilon_{0}}+\frac{\rho_{s 0}}{\rho_{g 0}}\right) .
\end{aligned}
$$


Multiplicando por $\frac{\varepsilon_{0}\left(1-\varepsilon_{0}\right)}{\beta}$ para tener en la misma escala que la Ec. (4.17) resulta que,

$$
\frac{\varepsilon_{0}\left(1-\varepsilon_{0}\right)}{\beta}\left\{\begin{array}{c}
{\left[\left(L_{2}-L_{1}\right) L_{3}-\frac{\gamma}{s^{2}}\left(L_{1} K_{2}-L_{2} K_{1}\right)\right] L_{8}} \\
-\rho_{g 0} \beta_{V}\left[\left(K_{2}-K_{1}\right) L_{3} L_{4}-\left(L_{1} K_{2}-L_{2} K_{1}\right) L_{6}\right]
\end{array}\right\} \dot{=}
$$

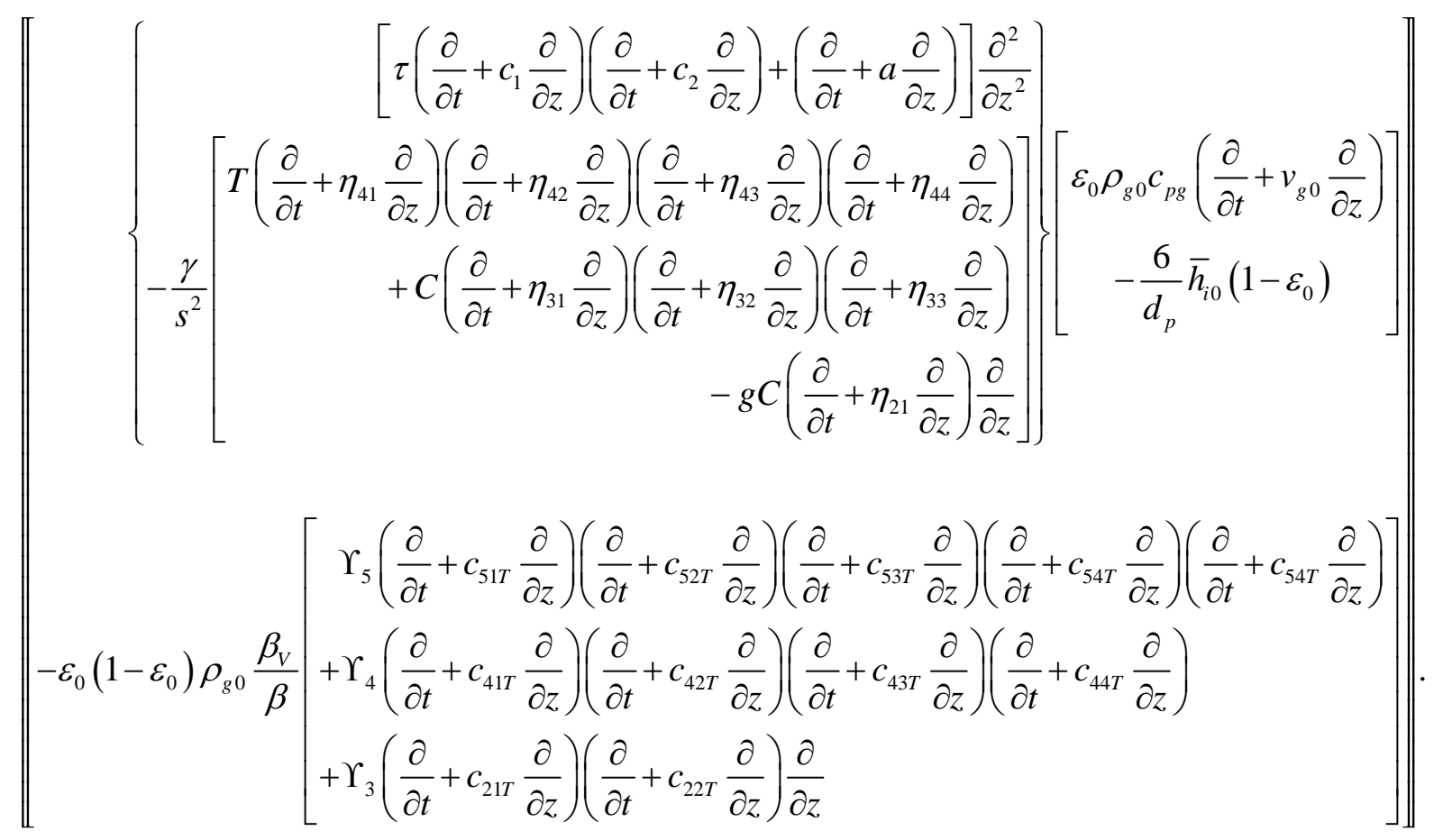

Aquí hemos seguido con la notación establecida en el Capítulo 4 para denotar los parámetros correspondientes al comportamiento incompresible y compresible; es decir, para los coeficientes $\tau, T, C$ y las rapideces $c_{1}, c_{2}, a, \eta_{41}, \eta_{42}, \eta_{43}, \eta_{44}, \eta_{31}, \eta_{32}, \eta_{33}$ у $\eta_{21}$

Por otro lado, los parámetros que caracterizan a las ondas de tercer y de cuarto orden, incorporadas por la parte térmica se definen en el Apéndice $M$. 
Las rapideces de quinto y de cuarto orden asociadas a la parte térmica incompresible $c_{51 T}$, $c_{52 T}, c_{53 T}, c_{54 T}, c_{55 T}$ y $c_{41 T}, c_{42 T}, c_{43 T}, c_{44 T}$, respectivamente, se obtuvieron de conjuntos de ecuaciones algebraicas construidas al ajustar la forma expandida de los polinomios de la Ec. (7.34) (ver Apéndice $M$ ) y ya que las expresiones completas en términos de parámetros originales son demasiado largas y complejas para incluirlas aquí, no las presentaremos.

Para simplificar aun más la Ec. (7.39), consideraremos su forma adimensional que se obtiene al utilizar la longitud de la columna $L$ y la velocidad promedio aritmético $U_{0}=\left(v_{g 0}+v_{s 0}\right) / 2$ como variables características. Las variables adimensionales son $z^{\prime} \doteq z / L, \quad a^{\prime}=a / U_{0}, \quad t^{\prime}=t U_{0} / L$ y todas las rapideces de propagación adimensionales pueden definirse en forma similar a $a^{\prime}$. Por lo tanto, la Ec. (7.39) puede reescribirse como

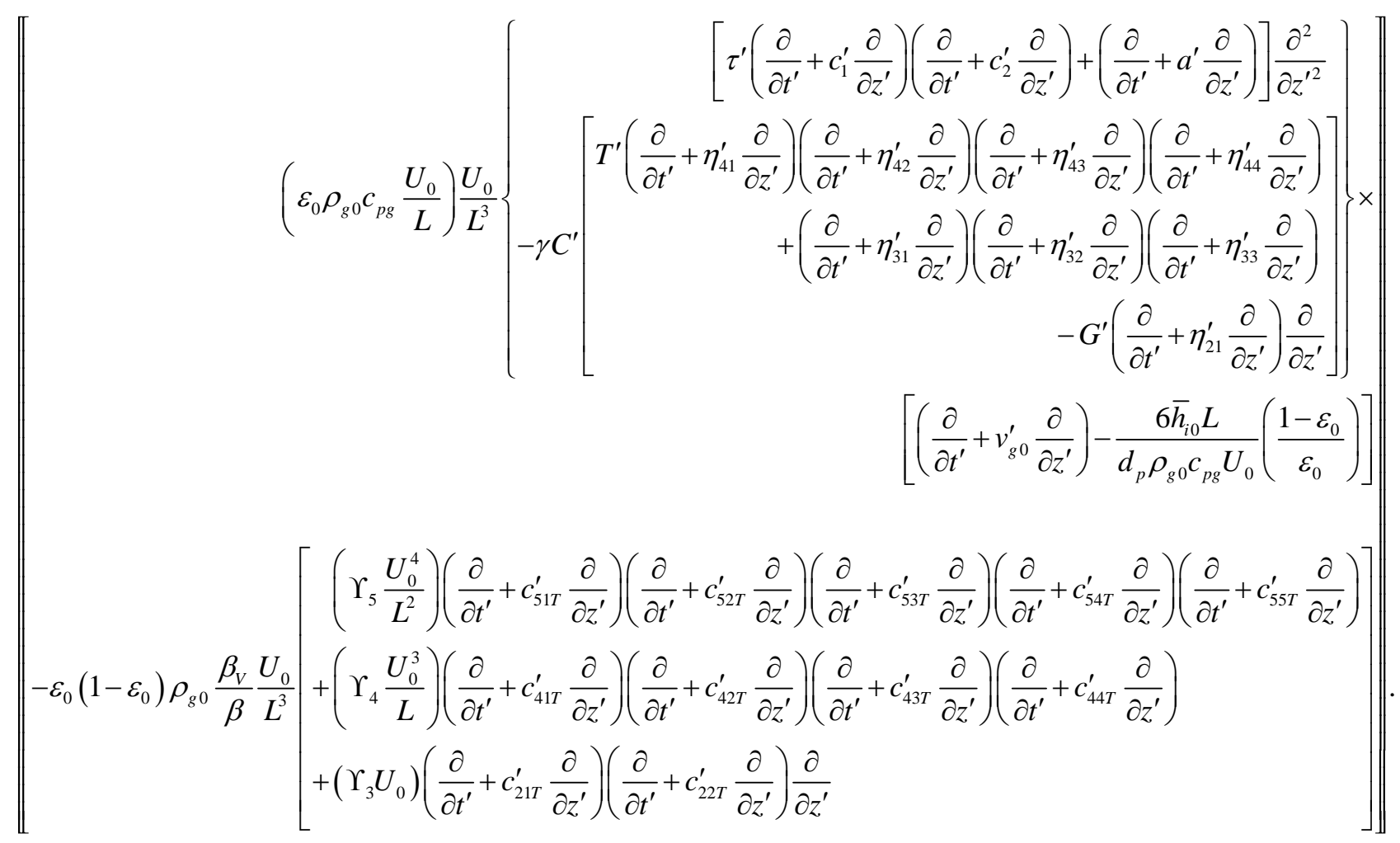


Dividiendo por $\left(\varepsilon_{0} \rho_{g 0} c_{p g} \frac{U_{0}}{L}\right) \frac{U_{0}}{L^{3}}$ obtenemos la forma adimensional,

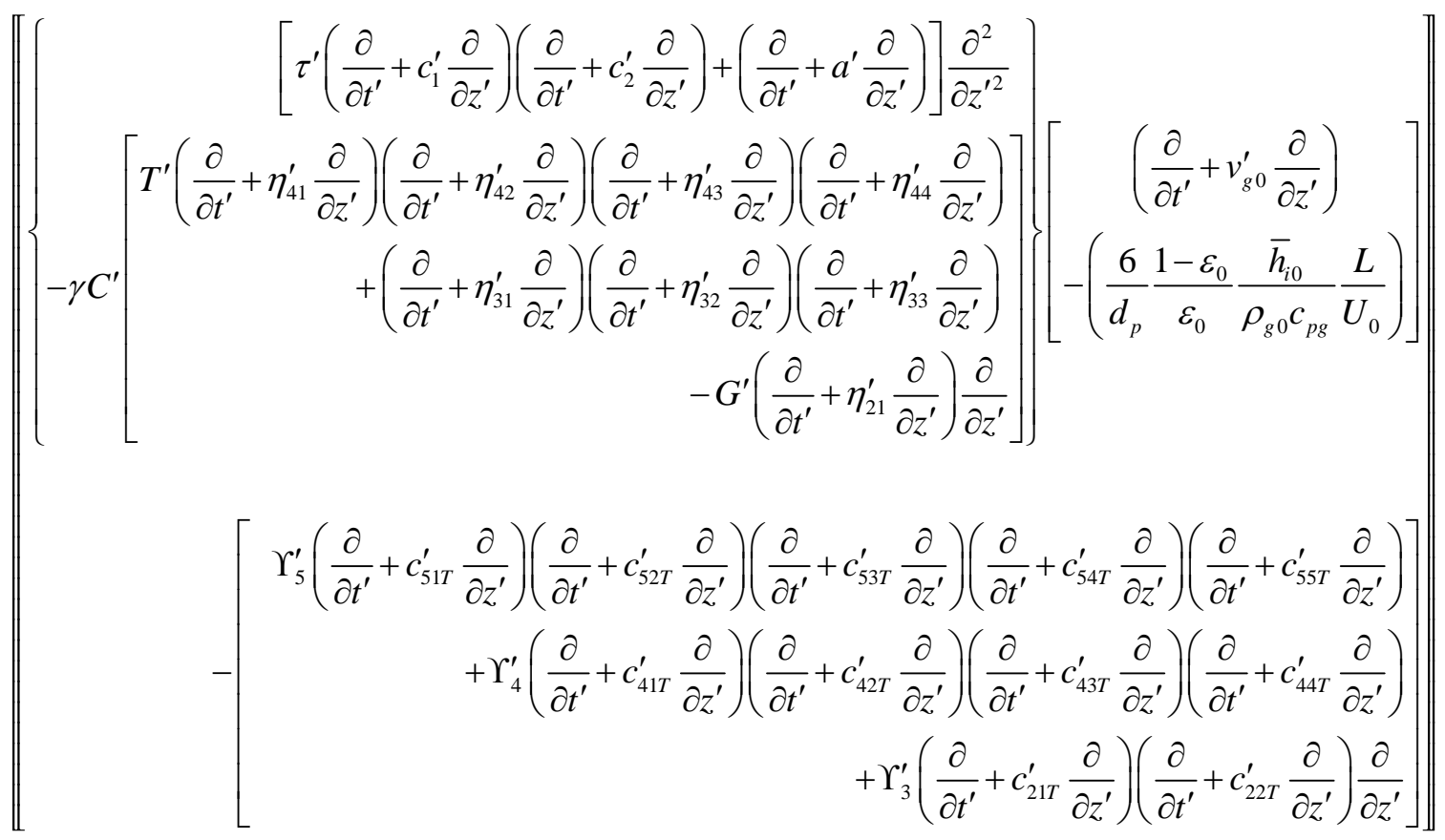

donde los índices prima denotan coeficientes adimensionales:

$$
\Upsilon_{5}^{\prime} \doteq\left(1-\varepsilon_{0}\right) \frac{\beta_{V}}{c_{p g} \beta} \frac{U_{0}^{3}}{L} \Upsilon_{5}, \quad \Upsilon_{4}^{\prime} \doteq\left(1-\varepsilon_{0}\right) \frac{\beta_{V}}{c_{p g} \beta} U_{0}^{2} \Upsilon_{4} \quad y \quad \Upsilon_{3}^{\prime} \doteq\left(1-\varepsilon_{0}\right) \frac{\beta_{V}}{c_{p g} \beta} L \Upsilon_{3}
$$

La forma en la que estructuramos la Ec. (7.39) es adecuada porque permite discriminar los efectos estudiados en el modelo; es decir, los efectos de termicidad, compresibilidad e incompresibilidad. Sin embargo, se puede obtener una ecuación equivalente en la cual aparezca una sola ecuación de onda y en ella estén estos efectos pero en forma combinada.

$$
\left[\begin{array}{r}
A_{5}\left(\frac{\partial}{\partial t}+\alpha_{5,1 T} \frac{\partial}{\partial z}\right)\left(\frac{\partial}{\partial t}+\alpha_{5,2 T} \frac{\partial}{\partial z}\right)\left(\frac{\partial}{\partial t}+\alpha_{5,3 T} \frac{\partial}{\partial z}\right)\left(\frac{\partial}{\partial t}+\alpha_{5,4 T} \frac{\partial}{\partial z}\right)\left(\frac{\partial}{\partial t}+\alpha_{5,5 T} \frac{\partial}{\partial z}\right) \\
+A_{4}\left(\frac{\partial}{\partial t}+\alpha_{4,1 T} \frac{\partial}{\partial z}\right)\left(\frac{\partial}{\partial t}+\alpha_{4,2 T} \frac{\partial}{\partial z}\right)\left(\frac{\partial}{\partial t}+\alpha_{4,3 T} \frac{\partial}{\partial z}\right)\left(\frac{\partial}{\partial t}+\alpha_{4,4 T} \frac{\partial}{\partial z}\right) \\
+A_{3}\left(\frac{\partial}{\partial t}+\alpha_{3,1 T} \frac{\partial}{\partial z}\right)\left(\frac{\partial}{\partial t}+\alpha_{3,2 T} \frac{\partial}{\partial z}\right) \frac{\partial}{\partial z} \\
+A_{2}\left(\frac{\partial}{\partial t}+\eta_{21} \frac{\partial}{\partial z}\right) \frac{\partial}{\partial z}
\end{array}\right]
$$


donde,

$$
\begin{aligned}
& A_{5} \doteq-\varepsilon_{0} \rho_{g 0}\left[c_{p g} \frac{\gamma}{s^{2}} T+\left(1-\varepsilon_{0}\right) \frac{\beta_{V}}{\beta} \Upsilon_{5}\right], \\
& A_{4} \doteq\left[-\frac{\gamma}{s^{2}} C \varepsilon_{0} \rho_{g 0} c_{p g}+\frac{6}{d_{p}} \bar{h}_{i 0}\left(1-\varepsilon_{0}\right) \frac{\gamma}{s^{2}} T-\varepsilon_{0}\left(1-\varepsilon_{0}\right) \rho_{g 0} \frac{\beta_{V}}{\beta} \Upsilon_{4}\right], \\
& A_{3} \doteq \frac{6}{d_{p}} \bar{h}_{i 0}\left(1-\varepsilon_{0}\right) \frac{\gamma}{s^{2}} C, \\
& A_{2} \doteq-\frac{6}{d_{p}} \bar{h}_{i 0}\left(1-\varepsilon_{0}\right) \frac{\gamma}{s^{2}} g C .
\end{aligned}
$$

Las $\alpha_{i, j T}, i=3,4,5$ y $j=1,2,3,4,5$ en la Ec. (7.43) son las rapideces de propagación que toman en cuenta los efectos térmicos, así como el comportamiento compresible y el incompresible. Los valores que caracterizan estas rapideces están dadas en el Apéndice $N$.

Por otro lado, trataremos si es posible, reexpresar los coeficientes de la parte no-isotérmica en términos de la isotérmica. Por tal razón, los reescribiremos aquí:

$$
\Upsilon_{5}^{\prime} \doteq\left(1-\varepsilon_{0}\right) \frac{\beta_{V}}{c_{p g} \beta} \frac{U_{0}^{3}}{L} \Upsilon_{5}, \quad \Upsilon_{4}^{\prime} \doteq\left(1-\varepsilon_{0}\right) \frac{\beta_{V}}{c_{p g} \beta} U_{0}^{2} \Upsilon_{4} \quad \text { y } \quad \Upsilon_{3}^{\prime} \doteq\left(1-\varepsilon_{0}\right) \frac{\beta_{V}}{c_{p g} \beta} L \Upsilon_{3},
$$

donde:

$$
\begin{aligned}
& \Upsilon_{5} \doteq \frac{\varepsilon_{0}}{1-\varepsilon_{0}} \rho_{s 0}, \\
& \Upsilon_{4} \doteq \frac{\varepsilon_{0} \beta C}{1-\varepsilon_{0}} \\
& \Upsilon_{3} \doteq-\frac{g \beta C}{1-\varepsilon_{0}}
\end{aligned}
$$

Sustituyendo y simplificando:

$$
\begin{gathered}
\Upsilon_{5}^{\prime} \doteq \frac{\beta_{V}}{c_{p g}} \frac{U_{0}^{3}}{L}\left(\frac{\varepsilon_{0} \rho_{s 0}}{\beta}\right), \\
\Upsilon_{4}^{\prime} \doteq \frac{\beta_{V}}{c_{p g}} \varepsilon_{0}\left(U_{0}^{2} C\right), \\
\Upsilon_{3}^{\prime} \doteq-\frac{\beta_{V}}{c_{p g}}\left(\frac{L g}{U_{0}^{2}}\right)\left(U_{0}^{2} C\right),
\end{gathered}
$$


ahora, los expresaremos en términos de $T^{\prime}, C^{\prime}$ y $G^{\prime}$ definidos en el Capítulo 4 para obtener finalmente que:

$$
\begin{aligned}
& \frac{\Upsilon_{5}^{\prime}}{C^{\prime}} \doteq\left(s^{2} \frac{\beta_{V}}{c_{p g}}\right) T^{\prime}, \\
& \frac{\Upsilon_{4}^{\prime}}{C^{\prime}} \doteq\left(s^{2} \frac{\beta_{V}}{c_{p g}}\right) \varepsilon_{0}, \\
& \frac{\Upsilon_{3}^{\prime}}{C^{\prime}} \doteq-\left(s^{2} \frac{\beta_{V}}{c_{p g}}\right) G^{\prime},
\end{aligned}
$$

ya que $\Upsilon_{5}^{\prime} \propto T^{\prime}$ y como $T^{\prime}$ se pudo eliminar por análisis de órdenes de magnitud en el Capítulo 4, podemos eliminarlo del modelo térmico representado en la Ec. (7.37) para tener el modelo térmico simplificado:

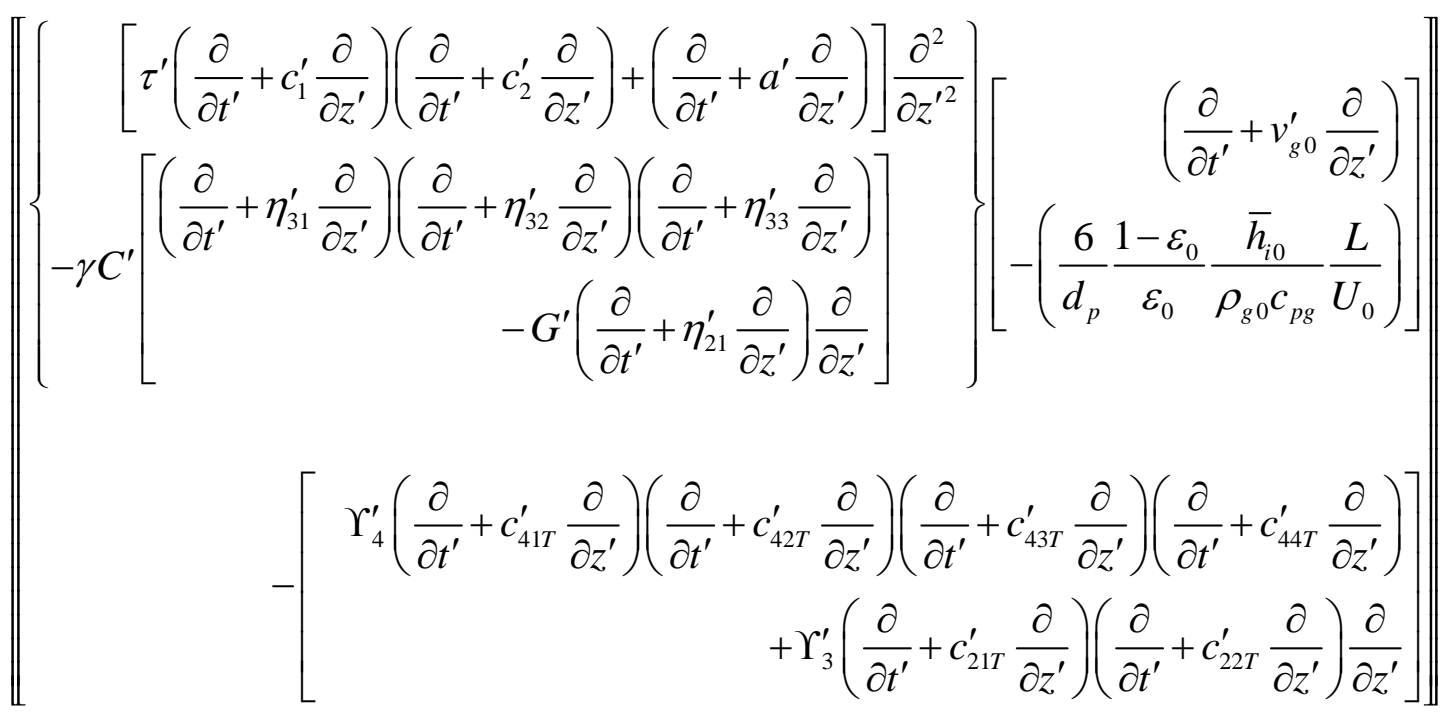


o bien, en forma dimensional

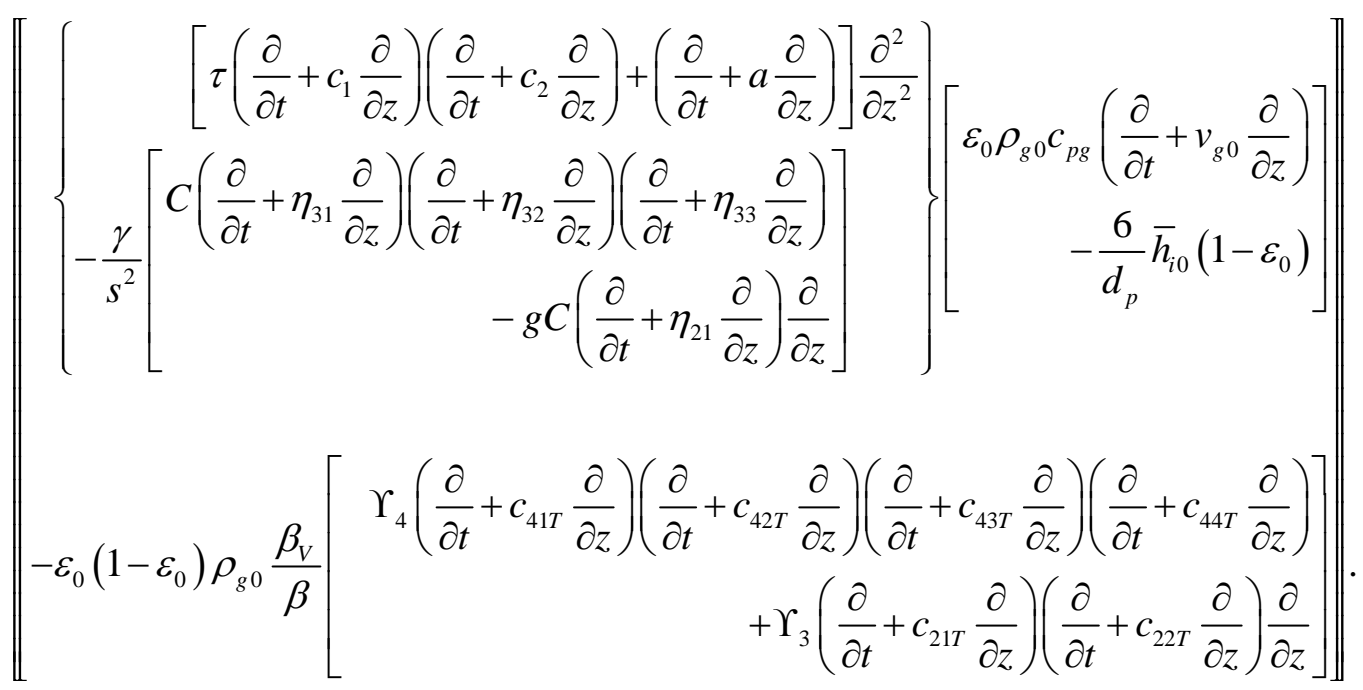

Esta ecuación será el punto de partida para el análisis de estabilidad que realizaremos en la siguiente sección. 


\subsection{Análisis de estabilidad}

\subsubsection{Extensiones al enfoque de Liu (1982)}

Los análisis de los Capítulos 4 y 5 mostraron que la compresibilidad y la pared del tubo aumentan la región de estabilidad cuando se compara con el comportamiento incompresible y sin la pared.

Sin embargo, para desarrollar un análisis de estabilidad siguiendo el enfoque de Liu (1982), comenzamos con la transformada de Fourier de la Ec. (7.47),

$$
\left.\begin{array}{c}
\left.\left\{\begin{array}{r}
\left.\tau\left(\omega-c_{1} \kappa\right)\left(\omega-c_{2} \kappa\right)+i(\omega-a \kappa)\right] \kappa^{2} \\
-\frac{\gamma}{s^{2}}\left[i C\left(\omega-\eta_{31} \kappa\right)\left(\omega-\eta_{32} \kappa\right)\left(\omega-\eta_{33} \kappa\right)\right. \\
-g C\left(\omega-\eta_{21} \kappa\right) \kappa
\end{array}\right]\right\}\left[\begin{array}{r}
i \varepsilon_{0} \rho_{g 0} c_{p g}\left(\omega-v_{g 0} \kappa\right) \\
+\frac{6}{d_{p}} \bar{h}_{i 0}\left(1-\varepsilon_{0}\right)
\end{array}\right] \\
-\|+\varepsilon_{0}\left(1-\varepsilon_{0}\right) \rho_{g 0} \frac{\beta_{V}}{\beta}\left[\begin{array}{r}
\Upsilon_{4}\left(\omega-c_{41 T} \kappa\right)\left(\omega-c_{42 T} \kappa\right)\left(\omega-c_{43 T} \kappa\right)\left(\omega-c_{44 T} \kappa\right) \\
-i \Upsilon_{3}\left(\omega-c_{21 T} \kappa\right)\left(\omega-c_{22 T} \kappa\right) \kappa
\end{array}\right]
\end{array}\right] .
$$

En esta ecuación, las condiciones de estabilidad pueden encontrarse al considerar que las ondas se mueven con rapideces cercanas a las de las ondas de segundo orden incompresibles y luego tomando los límites cuando $\omega \rightarrow c_{1} \kappa$ y $\omega \rightarrow c_{2} \kappa$.

De la Ec. (7.48) se sigue que,

$$
\begin{aligned}
& \begin{array}{l}
a<c_{1}-\frac{\gamma}{s^{2}} C\left(c_{1}-\eta_{31}\right)\left(c_{1}-\eta_{32}\right)\left(c_{1}-\eta_{33}\right) \\
-\frac{\beta_{V}}{\beta} \varepsilon_{0}\left(1-\varepsilon_{0}\right) \rho_{g 0} \frac{\left\{\begin{array}{l}
\Upsilon_{4} \varepsilon_{0} \rho_{g 0} c_{p g}\left(c_{1}-v_{g 0}\right)\left(c_{1}-c_{41 T}\right)\left(c_{1}-c_{42 T}\right)\left(c_{1}-c_{43 T}\right)\left(c_{1}-c_{44 T}\right) \kappa^{2} \\
+\frac{6}{d_{p}} \Upsilon_{3} \bar{h}_{i 0}\left(1-\varepsilon_{0}\right)\left(c_{1}-c_{21 T}\right)\left(c_{1}-c_{22 T}\right)
\end{array}\right\}}{\left[\varepsilon_{0}^{2} \rho_{g 0}^{2} c_{p g}^{2}\left(c_{1}-v_{g 0}\right)^{2} \kappa^{2}+\frac{36}{d_{p}^{2}} \bar{h}_{i 0}^{2}\left(1-\varepsilon_{0}\right)^{2}\right]},
\end{array}
\end{aligned}
$$




$$
\begin{aligned}
& a>c_{2}-\frac{\gamma}{s^{2}} C\left(c_{2}-\eta_{31}\right)\left(c_{2}-\eta_{32}\right)\left(c_{2}-\eta_{33}\right) \\
& -\frac{\beta_{V}}{\beta} \rho_{g 0} \varepsilon_{0}\left(1-\varepsilon_{0}\right) \frac{\left\{\begin{array}{l}
\Upsilon_{4} \varepsilon_{0} \rho_{g 0} c_{p g}\left(c_{2}-v_{g 0}\right)\left(c_{2}-c_{41 T}\right)\left(c_{2}-c_{42 T}\right)\left(c_{2}-c_{43 T}\right)\left(c_{2}-c_{44 T}\right) \kappa^{2} \\
+\frac{6}{d_{p}} \Upsilon_{3} \bar{h}_{i 0}\left(1-\varepsilon_{0}\right)\left(c_{2}-c_{21 T}\right)\left(c_{2}-c_{22 T}\right)
\end{array}\right.}{\left[\varepsilon_{0}^{2} \rho_{g 0}^{2} c_{p g}^{2}\left(c_{2}-v_{g 0}\right)^{2} \kappa^{2}+\frac{36}{d_{p}^{2}} \bar{h}_{i 0}^{2}\left(1-\varepsilon_{0}\right)^{2}\right]} .
\end{aligned}
$$

Las desigualdades dadas en la Ec. (7.49) y en la Ec. (7.50) corresponden a una generalización de la Ec. (4.45) obtenida en el Capítulo 4, puesto que incorporan el efecto térmico adicionado al efecto de compresibilidad previamente estudiado.

\subsection{Resultados y discusión}

Aquí analizaremos el impacto que tiene la parte no-isotérmica en los resultados encontrados en el Capítulo 4, para el modelo isotérmico, para lo cual continuaremos con los dos casos de estudio, incorporando solamente los datos de los efectos térmicos. Estos datos adicionales se tomaron del artículo de Gao y col. (2001), los cuales estudian un sistema de FCC industrial.

7.4.1. Valores de los parámetros y de las condiciones de operación de un sistema catalizador-vapor en una unidad de FCC y un sistema de arena-aire en un CFB, donde están incorporados los efectos térmicos

En la Tabla 7.2 se reportan las propiedades físicas del sólido y del fluido, así como también los parámetros geométricos relevantes y las condiciones de operación en un estado base homogéneo para ambos sistemas. Estos datos se utilizarán para analizar numéricamente los casos de estudio previamente citados. 
Tabla 7.2. Valores de los parámetros y de las condiciones de operación en estado base.

$\frac{\text { Parámetro }}{\text { Velocidad del sonido en el fluido }}$

Catalizador-vapor

Arena-aire puro, $s(\mathrm{~m} / \mathrm{s})$

Densidad del sólido, $\rho_{s 0}\left(\mathrm{~kg} / \mathrm{m}^{3}\right)$

Densidad del fluido, $\rho_{g 0}\left(\mathrm{~kg} / \mathrm{m}^{3}\right)$

Diámetro del grano sólido, $d_{p}(\mu \mathrm{m})$

Velocidad terminal, $U_{t}(\mathrm{~m} / \mathrm{s})$

655

1300

0.7065

60

0.085

7.000

1.168

22

$1.93 \times 10^{5}$

0.12

0.13

0.002

100

26613

$h_{i},\left(\frac{\mathrm{W}}{\mathrm{m}^{2} \cdot \mathrm{s}}\right)$

Calor específico a presión constante de

las partículas sólidas, $c_{p s},\left(\frac{\mathrm{J}}{\mathrm{kg} \cdot \mathrm{K}}\right)$

Calor específico a presión constante

de la fase gaseosa (líquido) $\left(300^{\circ} \mathrm{C}\right)$,

$c_{p g},\left(\frac{\mathrm{J}}{\mathrm{kg} \cdot \mathrm{K}}\right)$

2670

2670

Conductividad térmica de la fase

sólida, $k_{s},\left(\frac{\mathrm{W}}{\mathrm{m} \cdot \mathrm{s}}\right)^{7}$

Temperatura de la fase sólida, $T_{s},(\mathrm{~K})$

Temperatura de la fase gas, $T_{g},(\mathrm{~K})$

1097

1097

Coeficiente de expansión

volumétrica (para gas ideal),

\begin{tabular}{lll}
$\beta_{V}=T_{g}^{-1},\left(\mathrm{~K}^{-1}\right)$ & 0.001686 & 0.001776 \\
\hline
\end{tabular}

${ }^{6}$ Tomado de Gao y col. (2001): $\mathrm{Nu}_{s}=0.03 \operatorname{Re}_{s}^{1.3}$ para $0.1<\operatorname{Re}_{s}<100$, donde: $\mathrm{Nu}_{s} \doteq \frac{h_{i} d_{p}}{k_{s}}$.

${ }^{7}$ Tomado de McGaughey y Kaviany (2004)
3

840.6

875.4

593

563 


\subsubsection{Comparación de los coeficientes ondulatorios y significado de las jerarquías de onda}

Los coeficientes adimensionales de la Ec. (7.42), están denotados por índices prima, tal que $\tau^{\prime} \doteq U_{0} \tau / L, \quad C^{\prime} \doteq\left(U_{0} / s\right)^{2} C \quad$ y $\quad G^{\prime} \doteq L g / U_{0}^{2}$. Comparando estos coeficientes con respecto a la unidad, que es el coeficiente de la onda de primer orden del modelo incompresible, da una idea de la importancia de cada uno de ellos a medida que cambia la fracción volumen de sólido.
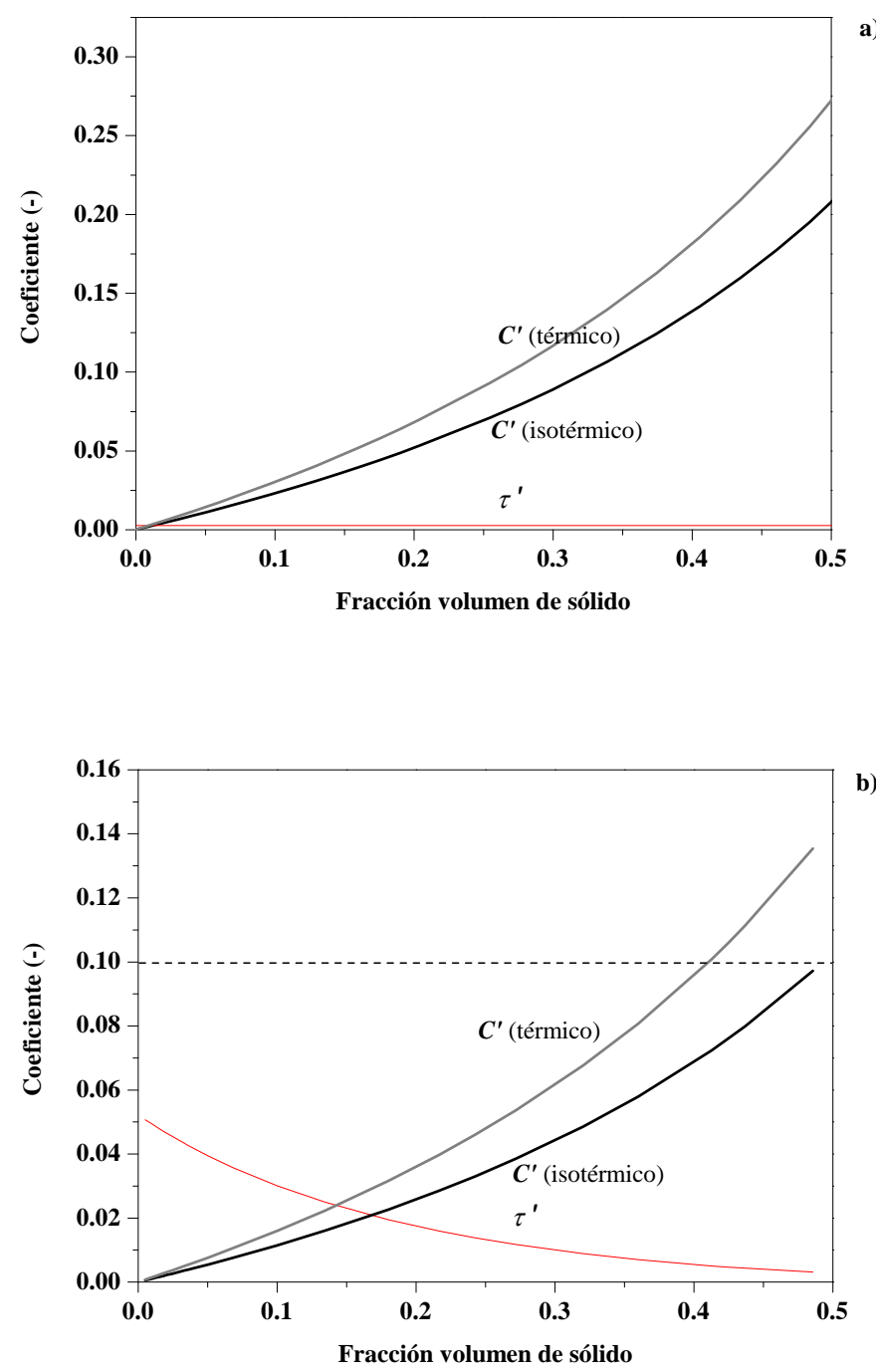

Figura 7.1. Coeficientes adimensionales $\tau^{\prime}$ y $C^{\prime}$ considerando los efectos térmicos y sin considerarlos para los sistemas: (a) granos de catalizador de FCC y (b) Arena. 
Así, puede apreciarse en la Figura 7.1 que considerar los efectos térmicos no afecta al tiempo de relajación de la onda de segundo orden del modelo incompresible. Mientras que si tienen un efecto de amplificar el coeficiente de la onda compresible de tercer orden a medida que aumentamos la fracción volumen de sólido. Para las partículas del FCC $C^{\prime}$ tiene un incremento máximo del $25 \%$, en tanto que para la Arena el incremento es aún mayor; esto es, del $40 \%$.

En la Figura 7.2, se muestra la gráfica de la función $\left(C^{\prime} G^{\prime}\right)$, que puede ser comparada directamente con el comportamiento básico incompresible de primer orden cuyo valor es la unidad. Esta función, que en el caso isotérmico toma en cuenta los efectos de gravedad en la compresibilidad del fluido y su importancia es cercana al 52\% del comportamiento básico incompresible para el sistema de la arena y alrededor del $21 \%$ para el polvo de FCC, ambas para fracciones volumen de sólido cercanas a 0.5. En el caso no-isotérmico, este coeficiente tiene un factor adicional que toma en cuenta la termicidad del sistema y que para el caso de la Arena reduce su valor relativo a la onda incompresible de primer orden pasando del $52 \%$ al $40 \%$, mientras que para el FCC, los efectos térmicos incrementan el valor relativo del coeficiente del $21 \%$ al $140 \%$.

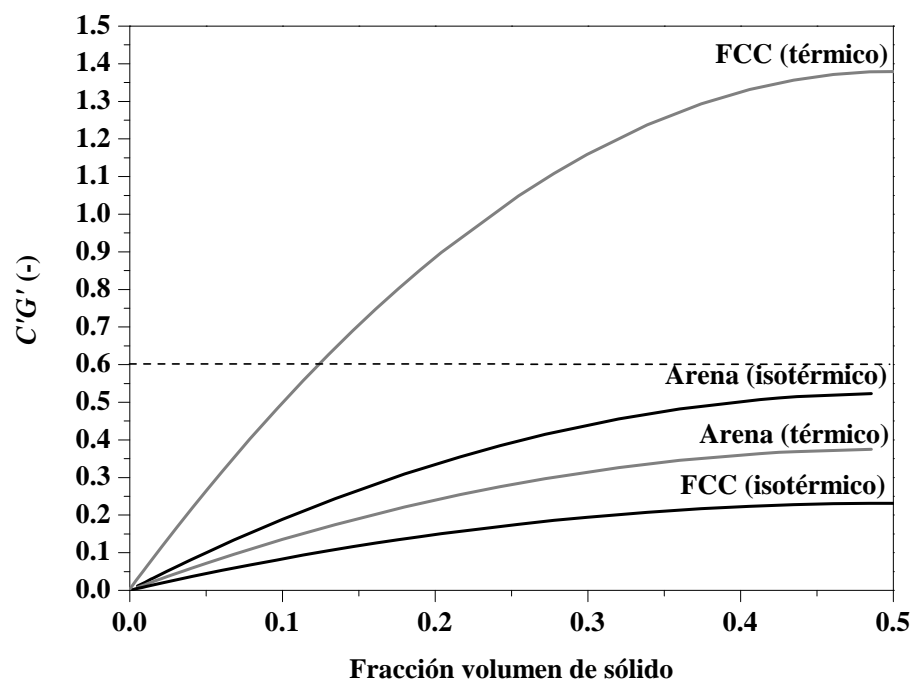

Figura 7.2. Coeficientes adimensionales de la onda compresible de segundo orden (degenerada) para el FCC (línea gris) y para la arena (línea negra). 


\section{Capítulo 8}

\section{Incorporación de la transferencia de calor en la pared en la propagación de ondas térmicas compresibles e incompresibles con modelos 1-D linealizados}

\subsection{Introducción}

En este Capítulo analizaremos el efecto de la pared del tubo en la propagación de ondas térmicas. El modelo que estudiaremos aquí es general, porque contiene al efecto de la compresibilidad, a la termicidad y al efecto de pared.

Con este modelo podemos obtener mediante límites adecuados correspondientes a la situación física particular, los modelos estudiados en los Capítulos 4, 5 y 7.

Hasta el momento sólo contamos con el estudio a nivel cualitativo, por lo que para hacer las comparaciones entre la propagación de ondas lineales no-isotérmicas con las isotérmicas con y sin efectos de pared, es necesario contar con datos experimentales y realizar las simulaciones numéricas.

\subsection{Jerarquía de ondas}

\subsubsection{Ecuaciones del modelo}

Se seguirán las mismas hipótesis descritas en las ecuaciones del modelo en los Capítulos precedentes, 4, 5 y 7 . Por lo que no las escribiremos nuevamente aquí. 
El conjunto cerrado de ecuaciones no-lineales promediadas dado por la Ec. (6.45) a la Ec. (6.50) para las variables dependientes $\left\{\varepsilon, p, V_{g}, V_{s}, T_{g}, T_{s}\right\}$,

$$
\begin{aligned}
\rho_{g}\left(P_{g}, T_{g}\right) \frac{\partial}{\partial t} \varepsilon_{g} & +\rho_{g}\left(P_{g}, T_{g}\right) V_{g} \frac{\partial}{\partial z} \varepsilon_{g}+\varepsilon_{g} \rho_{g}\left(P_{g}, T_{g}\right) \frac{\partial}{\partial z} V_{g} \\
& +\varepsilon_{g}\left[\left(\frac{\partial \rho_{g}}{\partial P_{g}}\right)_{T_{g}} \frac{\partial P_{g}}{\partial t}+\left(\frac{\partial \rho_{g}}{\partial T_{g}}\right)_{P_{g}} \frac{\partial T_{g}}{\partial t}\right]+\varepsilon_{g} V_{g}\left[\left(\frac{\partial \rho_{g}}{\partial P_{g}}\right)_{T_{g}} \frac{\partial P_{g}}{\partial z}+\left(\frac{\partial \rho_{g}}{\partial T_{g}}\right)_{P_{g}} \frac{\partial T_{g}}{\partial z}\right]=0,
\end{aligned}
$$

$-\rho_{s} \frac{\partial}{\partial t} \varepsilon_{g}-\rho_{s} V_{s} \frac{\partial}{\partial z} \varepsilon_{g}+\rho_{s} \varepsilon_{s} \frac{\partial}{\partial z} V_{s}=0$

$\varepsilon_{g} \rho_{g}\left(P_{g}, T_{g}\right) \frac{\partial}{\partial t} V_{g}+\varepsilon_{g} \rho_{g}\left(P_{g}, T_{g}\right) V_{g} \frac{\partial}{\partial z} V_{g}+\varepsilon_{g} \frac{\partial}{\partial z} P_{g}+T_{T g}+F_{T g W}=0$,

$\varepsilon_{s} \rho_{s} \frac{\partial}{\partial t} V_{s}+\varepsilon_{s} \rho_{s} V_{s} \frac{\partial}{\partial z} V_{s}+\varepsilon_{s} \frac{\partial}{\partial z} P_{g}-\Phi^{\prime} \varepsilon_{s} \frac{\partial}{\partial z} \varepsilon_{g}+T_{T s}+F_{T s W}=0$

$\varepsilon_{g} \rho_{g}\left(P_{g}, T_{g}\right) c_{p g} \frac{\partial}{\partial t} T_{g}+\varepsilon_{g} \rho_{g}\left(P_{g}, T_{g}\right) c_{p g} V_{g} \frac{\partial}{\partial z} T_{g}-\varepsilon_{g} \frac{\partial}{\partial t} P_{g}-\varepsilon_{g} V_{g} \frac{\partial}{\partial z} P_{g}+E_{T g}+E_{T g W}=0$,

$\varepsilon_{s} \rho_{s} c_{p s} \frac{\partial}{\partial t} T_{s}+\varepsilon_{s} \rho_{s} c_{p s} V_{s} \frac{\partial}{\partial z} T_{s}+E_{T s}+E_{T s W}=0$

Aquí aparecen las funciones $T_{T g}, T_{T s}, E_{T g}$ y $E_{T s}$, que se definen a continuación,

$$
\begin{aligned}
& T_{T g}=F+\rho_{g}\left(P_{g}, T_{g}\right) g, \\
& T_{T s}=-F+\left(1-\varepsilon_{g}\right)\left[\rho_{s}-\rho_{g}\left(P_{g}, T_{g}\right)\right] g, \\
& E_{T g}=-E_{T s}=\frac{6}{d_{p}} \bar{h}_{i} \varepsilon_{s}\left(T_{i}-T_{g}\right),
\end{aligned}
$$

donde $F$ es la fuerza promedio local ejercida por el fluido en las partículas y $g$ es la fuerza gravitacional por unidad de masa. $F_{T g W}$ y $F_{T s W}$ corresponden a la fuerza de arrastre del fluido y de las partículas sólidas en la pared del tubo, respectivamente. Estas fuerzas, junto con $F$ son modeladas de tal forma que sus principales dependencias permanezcan explícitas: 


$$
\begin{aligned}
& F=\beta \varepsilon_{g} \varepsilon_{s}\left(v_{g}-v_{s}\right), \\
& F_{T g W}=\beta_{g W} \rho_{g}\left(P_{g}, T_{g}\right) v_{g}{ }^{2}, \\
& F_{T s W}=\beta_{s W} \rho_{s 0} v_{s}^{2}, \\
& E_{T g W}=\theta_{g W}\left(T_{W}-T_{g}\right), \\
& E_{T s W}=\theta_{s W}\left(T_{W}-T_{s}\right),
\end{aligned}
$$

donde $\beta=\left(3 / 4 d_{p}\right) C_{D} \rho_{g} U_{t}$ es un parámetro de interacción. En este Capítulo no se tomaron en cuenta el efecto de masa agregada (Jackson, 1985) y el efecto de viscosidad efectiva en el régimen de fluidización denso (Jackson, 1985; Liu, 1982 y Homsy y col. 1980).

Los coeficientes $\beta_{k W}=\frac{2 \varepsilon_{k}}{D_{t}} f_{k W}$ y $\theta_{k W}=\frac{4 \varepsilon_{k}}{D_{t}} \bar{h}_{k W}$, son las funciones de arrastre en la pared y de la transferencia de calor de cada una de las fases con la pared, con $k=\{g, s\}$, que involucran tanto el factor de fricción de arrastre $f_{k W}$, como el coeficiente de transferencia de calor $\bar{h}_{k W}$ y el diámetro interno del tubo $D_{t}$. Los granos sólidos son incompresibles, entonces $\rho_{s}=\rho_{s 0}$ es una constante. La principal característica del presente Capítulo es la evaluación del efecto de la pared en las estrategias de modelado.

\subsubsection{Enunciado del problema}

La Ec. (8.1) a la Ec. (8.6) son linealizadas alrededor de un estado base (subíndice cero) más una pequeña perturbación tal que $\varepsilon_{g}=\varepsilon_{0}+\varepsilon, \quad p_{g}=p_{0}+p, \quad V_{g}=v_{g 0}+v_{g}$, $V_{s}=v_{s 0}+v_{s}, \quad T_{g}=T_{g 0}+T_{g} \quad \mathrm{y} \quad T_{s}=T_{s 0}+T_{s}$. Para obtener jerarquías de onda de perturbaciones para ambos modelos, el no-isotérmico-incompresible y el no-isotérmicocompresible. Estas ecuaciones son representadas en forma matricial como

$$
\mathbf{u}=\left(\begin{array}{llllll}
\varepsilon & p & v_{g} & v_{s} & T_{g} & T_{s}
\end{array}\right)^{T}
$$


el vector de perturbaciones para el sistema linealizado de EDP de primer orden:

$$
\mathbf{B} \frac{\partial \mathbf{u}}{\partial t}+\mathbf{C} \frac{\partial \mathbf{u}}{\partial z}+\mathbf{D}^{* *} \mathbf{u}=\mathbf{0}
$$

donde las matrices $\mathbf{B}, \mathbf{C}$ y $\mathbf{D}^{* *}$ de tamaño $6 \times 6$, son los coeficientes linealizados de la Ec. (8.1) a la Ec. (8.6) y están dadas en los Apéndices $L(\mathbf{B}, \mathbf{C})$ y $O\left(\mathbf{D}^{* *}\right)$. Las derivadas espaciales de las perturbaciones de velocidad $v_{g} \mathrm{y} v_{s}$ pueden obtenerse de las ecuaciones de continuidad, luego se sustituyen en las ecuaciones de momentum y en las ecuaciones de energía para llegar así a cuatro ecuaciones linealizadas, dos de segundo orden Ec. (8.17) y Ec. (8.18) y dos de primer orden Ec. (8.19) y Ec. (8.20) para las perturbaciones de la fracción volumen $(\varepsilon)$, para las perturbaciones de la presión $(p)$, para las perturbaciones de la temperatura del gas $\left(T_{g}\right)$ y para las perturbaciones de la temperatura del sólido $\left(T_{s}\right)$.

\subsubsection{Estructuras ondulatorias}

Partimos del conjunto de ecuaciones representado como:

$$
\begin{aligned}
& \left(L_{1}+\Lambda_{1}\right) \varepsilon+\left[L_{3}+\frac{\gamma}{s^{2}}\left(K_{1}+\Gamma\right)\right] p-\rho_{g 0} \beta_{V}\left(K_{1}+\Gamma\right) T_{g} \quad=0 \\
& \left(L_{2}+\Lambda_{2}\right) \varepsilon+\left[L_{3}+\frac{\gamma}{s^{2}}\left(K_{2}\right)\right] p-\rho_{g 0} \beta_{V}\left(K_{2}\right) T_{g} \quad=0 \\
& \begin{array}{llll}
L_{4} \varepsilon & +L_{6} p & -\left(L_{8}+\Pi_{1}\right) T_{g} & =0
\end{array} \\
& L_{4} \varepsilon \quad+L_{9} T_{g}+\left[L_{10}+\Xi_{1}\right] T_{s}=0
\end{aligned}
$$

donde los operadores diferenciales $L_{1}, L_{2}, L_{3}, K_{1}$ y $K_{2}$ están definidos en el Capítulo 4, Ec. (4.9) a la Ec. (4.13) y la influencia de la fricción en la pared dada por los operadores $\Lambda_{1}, \Lambda_{2}, \Gamma$, están definidos en el Capítulo 5, Ec. (5.14) a la Ec. (5.16). Los operadores $L_{4}$, $L_{6}, L_{8}, L_{9}, L_{10}$ están definidos en el Capítulo 7, Ec. (7.13) a la Ec. (7.17) y la influencia de la pared en el modelo no-isotérmico está dada por dos funciones adicionales:

$$
\Pi_{1}=\theta_{g W},
$$




$$
\Xi_{1}=-\theta_{s W}
$$

Que representan la ponderación del coeficiente de transferencia de calor entre la fase $g$ y $s$, con la pared del tubo respectivamente con el área interfacial específica, evaluadas en el estado base.

Seguiremos el mismo procedimiento de eliminación de variables de la subsección 7.2.3 para obtener las ecuaciones que describen las perturbaciones en $\left\{\varepsilon, p, T_{g}, T_{s}\right\}$.

El conjunto de ecuaciones dado por la Ec. (8.17) a la Ec. (8.19) es independiente de la Ec. (8.20); esto es, podemos resolver el sistema de EDP para $\left\{\varepsilon, p, T_{g}\right\}$ y con estas soluciones resolver para $T_{s}$ en la Ec. (8.20).

$\underline{\text { Primero eliminaremos a }} \varepsilon$ en la Ec. (8.17) a la Ec. (8.19) para obtener un sistema de dos ecuaciones para las variables: $\left\{p, T_{g}\right\}$.

Luego entonces, despejamos el término que tiene a $T_{s}$ en la Ec. (8.20),

$$
\left(L_{10}+\Xi_{1}\right) T_{s}=-L_{4} \varepsilon-L_{9} T_{g}
$$

Esta ecuación gobierna las perturbaciones de la temperatura del sólido, una vez que se conocen las perturbaciones de fracción volumen de gas y de la temperatura del gas. Resolvemos en la Ec. (8.19) para $\varepsilon$,

$$
L_{4} \varepsilon=-L_{6} p+\left(L_{8}+\Pi_{1}\right) T_{g}
$$

Ahora aplicamos el operador $L_{4}$ a la Ec. (8.17) y a la Ec. (8.18) para luego sustituir la Ec. (8.24) y obtener dos ecuaciones para $\left\{p, T_{g}\right\}$ : 


$$
\begin{aligned}
& {\left[L_{3} L_{4}-\left(L_{1}+\Lambda_{1}\right) L_{6}+\frac{\gamma}{s^{2}} L_{4}\left(K_{1}+\Gamma\right)\right] p+\left[\left(L_{1}+\Lambda_{1}\right)\left(L_{8}+\Pi_{1}\right)-\rho_{g 0} \beta_{V}\left(K_{1}+\Gamma\right) L_{4}\right] T_{g}=0,} \\
& {\left[L_{3} L_{4}-\left(L_{2}+\Lambda_{2}\right) L_{6}+\frac{\gamma}{s^{2}} L_{4} K_{2}\right] p+\left[\left(L_{2}+\Lambda_{2}\right)\left(L_{8}+\Pi_{1}\right)-\rho_{g 0} \beta_{V} L_{4} K_{2}\right] T_{g}=0 .}
\end{aligned}
$$

Posteriormente aplicamos el operador $\left[L_{3} L_{4}-\left(L_{1}+\Lambda_{1}\right) L_{6}+\frac{\gamma}{s^{2}} L_{4}\left(K_{1}+\Gamma\right)\right]$ a la Ec. (8.26) y el operador $\left[L_{3} L_{4}-\left(L_{2}+\Lambda_{2}\right) L_{6}+\frac{\gamma}{s^{2}} L_{4} K_{2}\right]$ a la Ec. (8.25), luego restamos la segunda de la primera para tener la siguiente ecuación para $T_{g}$ :

$$
\|\left\{\begin{array}{c}
\left.\left(L_{2}-L_{1}+\Lambda_{2}-\Lambda_{1}\right) L_{3}-\frac{\gamma}{s^{2}}\left[\left(L_{1}+\Lambda_{1}\right) K_{2}-\left(L_{2}+\Lambda_{2}\right)\left(\Gamma+K_{1}\right)\right]\right\}\left(L_{8}+\Pi_{1}\right) \\
-\rho_{g 0} \beta_{V}\left\{\left(K_{2}-K_{1}-\Gamma\right) L_{3} L_{4}-\left[\left(L_{1}+\Lambda_{1}\right) K_{2}-\left(L_{2}+\Lambda_{2}\right)\left(\Gamma+K_{1}\right)\right] L_{6}\right\}
\end{array} \| L_{4} T_{g}=0 .\right.
$$

Puede obtenerse la ecuación para $p$ al aplicar el operador $\left[\left(L_{2}+\Lambda_{2}\right)\left(L_{8}+\Pi_{1}\right)-\rho_{g 0} \beta_{V} L_{4} K_{2}\right]$ a $\quad$ la $\quad$ Ec. (8.25) y $\quad$ el operador $\left[\left(L_{1}+\Lambda_{1}\right)\left(L_{8}+\Pi_{1}\right)-\rho_{g 0} \beta_{V}\left(K_{1}+\Gamma\right) L_{4}\right]$ a la Ec. (8.26) y restando la segunda de la primera se obtiene una ecuación equivalente a la Ec. (8.27) pero para la perturbación en $p$.

$\underline{\text { Si eliminamos a }} p \underline{\text { de la Ec. (8.17) a la Ec. (8.19), obtenemos un sistema de dos EDP para }}$ $\left\{\varepsilon, T_{g}\right\}$. Para lo cual, primero resolvemos en la Ec. (8.19) para $L_{6} p:$

$$
L_{6} p=\left(L_{8}+\Pi_{1}\right) T_{g}-L_{4} \varepsilon
$$

y sustituimos en la Ec. (8.17) y en la Ec. (8.18), a las cuales se les ha aplicado previamente el operador $L_{6}$ para obtener un par de ecuaciones en $\left\{\varepsilon, T_{g}\right\}$ : 


$$
\begin{aligned}
& {\left[\left(L_{1}+\Lambda_{1}\right) L_{6}-L_{3} L_{4}-\frac{\gamma}{s^{2}} L_{4}\left(K_{1}+\Gamma\right)\right] \varepsilon} \\
& +\left[L_{3}\left(L_{8}+\Pi_{1}\right)+\frac{\gamma}{s^{2}}\left(L_{8}+\Pi_{1}\right)\left(K_{1}+\Gamma\right)-\rho_{g 0} \beta_{V} L_{6}\left(K_{1}+\Gamma\right)\right] T_{g}=0, \\
& {\left[\left(L_{2}+\Lambda_{2}\right) L_{6}-L_{3} L_{4}-\frac{\gamma}{s^{2}} L_{4} K_{2}\right] \varepsilon} \\
& +\left[L_{3}\left(L_{8}+\Pi_{1}\right)+\frac{\gamma}{s^{2}}\left(L_{8}+\Pi_{1}\right) K_{2}-\rho_{g 0} \beta_{V} L_{6} K_{2}\right] T_{g}=0 .
\end{aligned}
$$

Puede obtenerse la ecuación para $T_{g}$ al aplicar el operador $\left[\left(L_{2}+\Lambda_{2}\right) L_{6}-L_{3} L_{4}-\frac{\gamma}{s^{2}} L_{4} K_{2}\right]$ a la Ec. (8.29) y el operador $\left[\left(L_{1}+\Lambda_{1}\right) L_{6}-L_{3} L_{4}-\frac{\gamma}{s^{2}} L_{4}\left(K_{1}+\Gamma\right)\right]$ a la Ec. (8.30) y restando la segunda de la primera se obtiene,

$$
\|\left\{\begin{array}{c}
\left.\left(L_{2}-L_{1}+\Lambda_{2}-\Lambda_{1}\right) L_{3}-\frac{\gamma}{s^{2}}\left[\left(L_{1}+\Lambda_{1}\right) K_{2}-\left(L_{2}+\Lambda_{2}\right)\left(\Gamma+K_{1}\right)\right]\right\}\left(L_{8}+\Pi_{1}\right) \\
-\rho_{g 0} \beta_{V}\left\{\left(K_{2}-K_{1}-\Gamma\right) L_{3} L_{4}-\left[\left(L_{1}+\Lambda_{1}\right) K_{2}-\left(L_{2}+\Lambda_{2}\right)\left(\Gamma+K_{1}\right)\right] L_{6}\right\}
\end{array} \| L_{6} T_{g}=0\right.
$$

Asimismo, podemos encontrar la ecuación para $\varepsilon$ al aplicar el operador $\left[L_{3}\left(L_{8}+\Pi_{1}\right)+\frac{\gamma}{s^{2}}\left(L_{8}+\Pi_{1}\right) K_{2}-\rho_{g 0} \beta_{V} L_{6} K_{2}\right] \quad$ a $\quad$ la $\quad$ Ec. (8.29) y el operador $\left[L_{3}\left(L_{8}+\Pi_{1}\right)+\frac{\gamma}{s^{2}}\left(L_{8}+\Pi_{1}\right)\left(K_{1}+\Gamma\right)-\rho_{g 0} \beta_{V} L_{6}\left(K_{1}+\Gamma\right)\right]$ a la Ec. (8.30) y restando la segunda de la primera se obtiene una ecuación equivalente a la Ec. (8.31) pero para $\varepsilon$.

Finalmente, eliminamos a $T_{g}$ de la Ec. (8.17) a la Ec. (8.19), con lo que obtenemos un sistema de dos EDP para $\{\varepsilon, p\}$. Resolvemos para el término que tiene a $T_{g}$ en la Ec. (8.19),

$$
\left(L_{8}+\Pi_{1}\right) T_{g}=L_{4} \varepsilon+L_{6} p
$$


Posteriormente, sustituimos la Ec. (8.32) en la Ec. (8.17) y en la Ec. (8.18), a las cuales previamente se les aplicó el operador diferencial de primer orden $\left(L_{8}+\Pi_{1}\right)$ para obtener el conjunto de dos ecuaciones para $\{\varepsilon, p\}$ :

$$
\begin{aligned}
& {\left[\left(L_{1}+\Lambda_{1}\right)\left(L_{8}+\Pi_{1}\right)-\rho_{g 0} \beta_{V}\left(K_{1}+\Gamma\right) L_{4}\right] \varepsilon} \\
& +\left[\left(L_{3}+\frac{\gamma}{s^{2}}\left(K_{1}+\Gamma\right)\right)\left(L_{8}+\Pi_{1}\right)-\rho_{g 0} \beta_{V}\left(K_{1}+\Gamma\right) L_{6}\right] p=0, \\
& {\left[\left(L_{2}+\Lambda_{2}\right)\left(L_{8}+\Pi_{1}\right)-\rho_{g 0} \beta_{V} L_{4} K_{2}\right] \varepsilon} \\
& +\left[\left(L_{3}+\frac{\gamma}{s^{2}} K_{2}\right)\left(L_{8}+\Pi_{1}\right)-\rho_{g 0} \beta_{V} L_{6} K_{2}\right] p=0,
\end{aligned}
$$

ahora le aplicamos el operador $\left[\left(L_{2}+\Lambda_{2}\right)\left(L_{8}+\Pi_{1}\right)-\rho_{g 0} \beta_{V} L_{4} K_{2}\right]$ a la Ec. (8.33) y le aplicamos el operador $\left[\left(L_{1}+\Lambda_{1}\right)\left(L_{8}+\Pi_{1}\right)-\rho_{g 0} \beta_{V} L_{4}\left(K_{1}+\Gamma\right)\right]$ a la Ec. (8.34). Luego restamos la última de la primera para tener:

$$
\|\left\{\begin{array}{c}
\left.\left(L_{2}-L_{1}+\Lambda_{2}-\Lambda_{1}\right) L_{3}-\frac{\gamma}{s^{2}}\left[\left(L_{1}+\Lambda_{1}\right) K_{2}-\left(L_{2}+\Lambda_{2}\right)\left(\Gamma+K_{1}\right)\right]\right\}\left(L_{8}+\Pi_{1}\right) \\
-\rho_{g 0} \beta_{V}\left\{\left(K_{2}-K_{1}-\Gamma\right) L_{3} L_{4}-\left[\left(L_{1}+\Lambda_{1}\right) K_{2}-\left(L_{2}+\Lambda_{2}\right)\left(\Gamma+K_{1}\right)\right] L_{6}\right\}
\end{array} \|\left(L_{8}+\Pi_{1}\right) p=0 .\right.
$$

Análogamente, puede obtenerse una ecuación para $\varepsilon$ al aplicar el operador

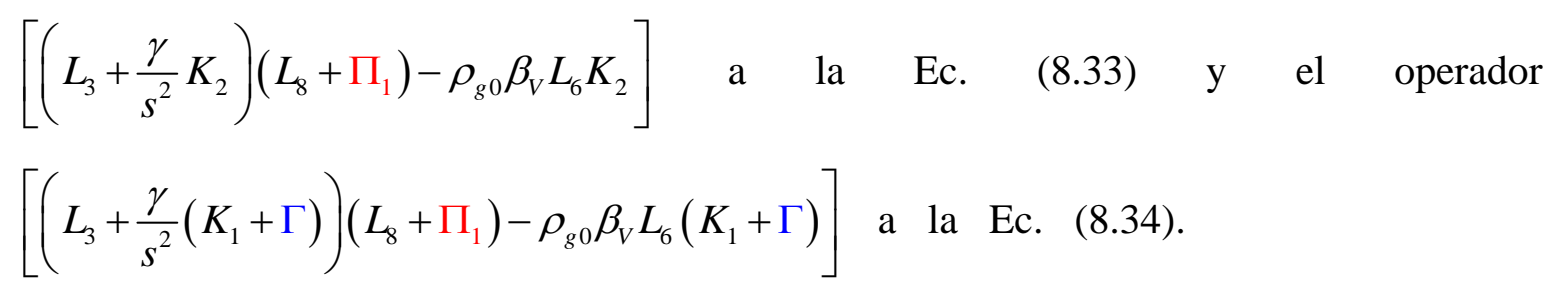

Luego restamos la última de la primera para tener la misma Ec. (8.35) pero aplicada a $\varepsilon$. 
En la siguiente tabla están concentrados los principales resultados encontrados al aplicar el procedimiento de eliminación de variables:

Tabla 8.1. Ecuaciones de perturbación obtenidas al eliminar una variable con el modelo no-isotérmico con efectos de pared.

\begin{tabular}{|c|c|c|}
\hline Variable eliminada & \multicolumn{2}{|c|}{ Ecuación para las perturbaciones } \\
\hline$\varepsilon$ & $L_{4} L_{W}^{(5)} p=0$ & $L_{4} L_{W}^{(5)} T_{g}=0$ \\
\hline$p$ & $L_{6} L_{W}^{(5)} \varepsilon=0$ & $L_{6} L_{W}^{(5)} T_{g}=0$ \\
\hline$T_{g}$ & $\left(L_{8}+\Pi_{1}\right) L_{W}^{(5)} \varepsilon=0$ & $\left(L_{8}+\Pi_{1}\right) L_{W}^{(5)} p=0$ \\
\hline
\end{tabular}

Al eliminar $\varepsilon$, tenemos las dos ecuaciones: $L_{4} L_{W}^{(5)} p=0$ y $L_{4} L_{W}^{(5)} T_{g}=0$ y ya que el operador $L_{4}$ es algebraico y además constante $\left(L_{4} \neq 0\right)$, dividiendo por $L_{4}$, se obtiene el par de ecuaciones simplificado:

$$
L_{W}^{(5)} p=0
$$

$\mathrm{y}$

$$
L_{W}^{(5)} T_{g}=0
$$

donde $L_{W}^{(5)}$ es el operador de quinto orden definido por:

$$
L_{W}^{(5)} \equiv\left\|\begin{array}{c}
\left.\left(L_{2}-L_{1}+\Lambda_{2}-\Lambda_{1}\right) L_{3}-\frac{\gamma}{s^{2}}\left[\left(L_{1}+\Lambda_{1}\right) K_{2}-\left(L_{2}+\Lambda_{2}\right)\left(\Gamma+K_{1}\right)\right]\right\}\left(L_{8}+\Pi_{1}\right) \| . \\
-\rho_{g 0} \beta_{V}\left\{\left(K_{2}-K_{1}-\Gamma\right) L_{3} L_{4}-\left[\left(L_{1}+\Lambda_{1}\right) K_{2}-\left(L_{2}+\Lambda_{2}\right)\left(\Gamma+K_{1}\right)\right] L_{6}\right\}
\end{array}\right\|
$$

Luego al aplicar el operador $L_{W}^{(5)}$ a la Ec. (8.23):

$$
\left(L_{10}+\Xi_{1}\right) L_{W}^{(5)} T_{s}=-L_{4}\left[L_{W}^{(5)} \varepsilon\right]-L_{9}\left[L_{W}^{(5)} T_{g}\right]
$$

y usando los resultados encontrados anteriormente tenemos que:

$$
\left(L_{10}+\Xi_{1}\right) L_{W}^{(5)} T_{s}=0
$$


Entonces, podemos escribir en forma general que:

$$
\|\left\{\begin{array}{c}
\left.\left(L_{2}-L_{1}+\Lambda_{2}-\Lambda_{1}\right) L_{3}-\frac{\gamma}{s^{2}}\left[\left(L_{1}+\Lambda_{1}\right) K_{2}-\left(L_{2}+\Lambda_{2}\right)\left(\Gamma+K_{1}\right)\right]\right\}\left(L_{8}+\Pi_{1}\right) \\
-\rho_{g 0} \beta_{V}\left\{\left(K_{2}-K_{1}-\Gamma\right) L_{3} L_{4}-\left[\left(L_{1}+\Lambda_{1}\right) K_{2}-\left(L_{2}+\Lambda_{2}\right)\left(\Gamma+K_{1}\right)\right] L_{6}\right\}
\end{array} \| \Psi_{W}=0,\right.
$$

donde $\Psi_{W}=\left[\varepsilon, p, T_{g},\left(L_{10}+\Xi_{1}\right) T_{s}\right]$.

Esta es una EDP de quinto orden para $\Psi_{W}$, siendo $\Psi_{W}$ alguna de las perturbaciones $\left\{\varepsilon, p, T_{g},\left(L_{10}+\Xi_{1}\right) T_{s}\right\}$ que aumentó de cuarto a quinto orden por los efectos térmicos. Esta ecuación puede escribirse en una forma en la que estén separados todos los efectos: incompresibilidad, compresibilidad, termicidad, pared-iso-térmica y pared-térmica:

$$
\begin{aligned}
& \| \begin{array}{c}
{\left[\left(L_{2}-L_{1}\right) L_{3}-\frac{\gamma}{s^{2}}\left(L_{1} K_{2}-L_{2} K_{1}\right)\right] L_{8}} \\
-\rho_{g 0} \beta_{V}\left\{\left(K_{2}-K_{1}\right) L_{3} L_{4}-\left[\left(L_{1} K_{2}-L_{2} K_{1}\right)\right] L_{6}\right\} \rrbracket
\end{array} \\
& +\|\left\{\left(\Lambda_{2}-\Lambda_{1}\right) L_{3}-\frac{\gamma}{s^{2}}\left[\left(\Lambda_{1} K_{2}-\Lambda_{2} K_{1}\right)-\left(L_{2}+\Lambda_{2}\right) \Gamma\right]\right\} L_{8} \rrbracket \Psi_{W} \\
& +\rho_{g 0} \beta_{V}\left\{-L_{3} L_{4} \Gamma-\left[\left(\Lambda_{1} K_{2}-\Lambda_{2} K_{1}\right)-\left(L_{2}+\Lambda_{2}\right) \Gamma\right] L_{6}\right\} \| \\
& +\llbracket\left\{\left[\left(L_{2}-L_{1}\right)+\left(\Lambda_{2}-\Lambda_{1}\right)\right] L_{3}-\frac{\gamma}{s^{2}}\left[\left(L_{1}+\Lambda_{1}\right) K_{2}-\left(L_{2}+\Lambda_{2}\right)\left(\Gamma+K_{1}\right)\right]\right\} \Pi_{1} \rrbracket \Psi_{W}
\end{aligned}
$$

$$
\left.d \begin{array}{l}
{\left[\begin{array}{l}
\left.\left(L_{2}-L_{1}\right) L_{3}-\frac{\gamma}{s^{2}}\left(L_{1} K_{2}-L_{2} K_{1}\right)\right] L_{8} \\
-\rho_{g 0} \beta_{V}\left\{\left(K_{2}-K_{1}\right) L_{3} L_{4}-\left[\left(L_{1} K_{2}-L_{2} K_{1}\right)\right] L_{6}\right\} \\
+\left\{\left(\Lambda_{2}-\Lambda_{1}\right) L_{3}-\frac{\gamma}{s^{2}}\left[\left(\Lambda_{1} K_{2}-\Lambda_{2} K_{1}\right)-\left(L_{2}+\Lambda_{2}\right) \Gamma\right]\right\}
\end{array}\right] L_{8}} \\
-\rho_{g 0} \beta_{V}\left\{-L_{3} L_{4} \Gamma-\left[\left(\Lambda_{1} K_{2}-\Lambda_{2} K_{1}\right)-\left(L_{2}+\Lambda_{2}\right) \Gamma\right] L_{6}\right\} \\
+\left\{\left[\left(L_{2}-L_{1}\right)+\left(\Lambda_{2}-\Lambda_{1}\right)\right] L_{3}-\frac{\gamma}{s^{2}}\left[\left(L_{1}+\Lambda_{1}\right) K_{2}-\left(L_{2}+\Lambda_{2}\right)\left(\Gamma+K_{1}\right)\right]\right\} \Pi_{1}
\end{array}\right] \Psi_{W}=0 .
$$


En la Ec. (8.38) se pueden apreciar todos los modelos estudiados hasta el momento en las siguientes combinaciones:
a) Isotérmico, incompresible y sin tomar en cuenta la pared-isotérmica.
b) Isotérmico compresible y sin tomar en cuenta la pared-isotérmica.
c) Isotérmico, incompresible y tomando en cuenta a la pared-isotérmica.
d) Isotérmico, compresible y tomando en cuenta a la pared-isotérmica.
e) No-isotérmico, incompresible y sin tomar en cuenta a la pared.
f) No-isotérmico, compresible y sin tomar en cuenta a la pared.
g) No-isotérmico, incompresible y tomando en cuenta a la pared-isotérmica.
h) No-isotérmico, compresible y tomando en cuenta a la pared-isotérmica.
i) No-isotérmico, incompresible y tomando en cuenta a la pared-térmica.
j) No-isotérmico, compresible y tomando en cuenta a la pared-térmica.

La metodología diseñada nos ha permitido generar modelos en los cuales se pueden discriminar las contribuciones de cada efecto y con esto, medir el impacto que éste tiene en la dinámica del sistema; esto es, obtuvimos un modelo compresible en el cual están separados los efectos de incompresibilidad y puede obtenerse el caso asintótico correspondiente a partir del compresible. También, al incorporar el efecto de la pared del tubo, separamos la dinámica de interacción de las fases con la pared del comportamiento en el centro del tubo. Además, al incorporar la termicidad, pudimos obtener un modelo más completo en el cual están separados los efectos en tres partes: parte incompresible, parte compresible y parte térmica, sin que haya efectos combinados, lo cual se debe al proceso de linealización.

Finalmente, hemos incorporado el efecto de la pared del tubo en el modelo no-isotérmico obteniendo así la Ec. (8.38), donde están separados los efectos también, pero es demasiado complicado expresar aquí el desarrollo en términos de parámetros originales como en los casos anteriores por la extensión de los operadores que intervienen.

Sin embargo, en esta ecuación pueden recuperarse como caso particular todos los casos estudiados anteriormente al tomar el límite apropiado. 
Incompresibilidad:

Para obtener el límite de incompresibilidad, termodinámicamente se requiere que la velocidad del sonido tienda a infinito. Esto es, si partimos de una ecuación de estado de la forma $\rho_{g}=\rho_{g}\left(P_{g}\right)$, la definición de la velocidad del sonido es:

$$
\frac{d \rho_{g}}{d P_{g}}=\frac{1}{s^{2}},
$$

donde $s$ es la velocidad del sonido en el medio puro, entonces si $s \rightarrow \infty$, se tiene que:

$$
\frac{d \rho_{g}}{d P_{g}}=0
$$

por lo que, $\rho_{g}$ es constante y sabemos que esta es la condición que debe satisfacer un fluido incompresible.

Isotérmico sin pared:

La condición de no tomar en cuenta la fricción en la pared del tubo equivale a tomar el factor de fricción de la fase $k$ con la pared del tubo como cero; esto es, $f_{k W} \rightarrow 0$ y ya que $\beta_{k W}=\frac{2 \varepsilon_{k}}{D_{t}} f_{k W}$, entonces $\beta_{k W} \rightarrow 0$. Los operadores de pared $\Lambda_{1}, \Lambda_{2}$ y $\Gamma$, definidos en la

Ec. (5.14) a la Ec. (5.16) son proporcionales a $\beta_{k W}$, lo cual implica que también puede tomarse el límite cuando $\Lambda_{2} \rightarrow 0, \Lambda_{1} \rightarrow 0$ y $\Gamma \rightarrow 0$.

Pared adiabática, aislante del calor o atérmica:

Para convección forzada de un fluido sujeto a diferencias de temperatura moderadas, el flux de calor por unidad de área $q_{c o n v}^{\prime \prime}$, puede considerarse como proporcional a la diferencia de temperatura $\Delta T=T_{W}-T_{k}$. Esto fue descubierto por Newton que infirió que $q_{c o n v}^{\prime \prime} \propto \Delta T$. Así llegamos a la ley de enfriamiento de Newton:

$$
q_{\text {conv }}^{\prime \prime}=h_{k W}\left(T_{W}-T_{k}\right),
$$

donde $h_{k W}$ se conoce como coeficiente de transferencia de calor, con unidades de $\mathrm{W} \cdot \mathrm{m}^{-2}$ $\mathrm{o} \mathrm{Btu} \cdot \mathrm{ft}^{-2} \cdot \mathrm{h}^{-1} \cdot{ }^{\mathrm{o}} \mathrm{F}^{-1}$. 
Entonces $h_{k W}$ es dimensional y así su valor depende de las unidades utilizadas. La forma adimensional tradicional de $h_{k W}$ es el número de Nusselt, el cual puede definirse como la razón del transporte de calor por convección en el fluido a la transferencia de calor por conducción bajo las mismas condiciones.

Considere una capa de fluido de espesor $L$ y diferencia de temperatura $T_{W}-T_{k}$. Suponga que la película se mueve en forma tal que ocurre la convección, el flux de calor sería,

$$
q_{\text {conv }}^{\prime \prime}=h_{k W}\left(T_{W}-T_{k}\right) \text {. }
$$

Si por otro lado, la película está estancada, el flux de calor sería completamente debido a la conducción del calor a través de la película:

$$
q_{W}^{\prime \prime}=\frac{k_{k}}{L}\left(T_{W}-T_{k}\right)=-k_{k} \frac{d T}{d z}
$$

Definimos al número de Nusselt como la razón entre las dos:

$$
\mathrm{Nu}_{L}=\frac{q_{W}^{\prime \prime}(\text { convección })}{q_{\text {conv }}^{\prime \prime}(\text { conducción })}=\frac{h_{k W} L}{k_{k}}
$$

Un número de Nusselt del orden de la unidad indicaría un movimiento lento poco más efectivo que la conducción en el fluido puro. Un número de Nusselt grande implica una convección muy eficiente.

Igualando la Ec. (8.41) con la Ec. (8.40), obtenemos que:

$$
-k_{k} \frac{d T}{d z}=h_{k W}\left(T_{W}-T_{k}\right),
$$

de aquí podemos estudiar dos casos de importancia:

- Pared adiabática, $h_{k W} \rightarrow 0$ y $\frac{d T}{d z}=0$.

- Pared isotérmica $h_{k W} \rightarrow \infty$ y $T_{k}=T_{W}$.

Como $\Pi_{1}$ y $\Xi_{1}$ son proporcionales a $h_{k W}$, entonces la condición física de tomar a la pared como adiabática $h_{k W} \rightarrow 0$ es equivalente a la condición de que $\Pi_{1}$ y $\Xi_{1}$ tiendan a cero. 
A continuación expresaremos los diez modelos obtenidos a partir de la Ec. (8.38).

Para ser sistemáticos, empezaremos de abajo hacia arriba de los mencionados anteriormente; esto es: i, h, g, f, e, d, c, b, a.

i) No-isotérmico, incompresible y tomando en cuenta la transferencia de calor en la pared (pared conductora del calor o pared térmica).

Límite cuando la velocidad del sonido es infinita: $s \rightarrow \infty$ (con esto se recupera la hipótesis de incompresibilidad).

$$
\begin{aligned}
& \llbracket \begin{array}{c}
\left(L_{2}-L_{1}\right) L_{3} L_{8} \\
-\rho_{g 0} \beta_{V}\left\{\left(K_{2}-K_{1}\right) L_{3} L_{4}-\left[\left(L_{1} K_{2}-L_{2} K_{1}\right)\right] L_{6}\right\}
\end{array} \Psi_{W} \\
& +\llbracket \begin{array}{c}
\left(\Lambda_{2}-\Lambda_{1}\right) L_{3} L_{8} \\
-\rho_{g 0} \beta_{V}\left\{-L_{3} L_{4} \Gamma-\left[\left(\Lambda_{1} K_{2}-\Lambda_{2} K_{1}\right)-\left(L_{2}+\Lambda_{2}\right) \Gamma\right] L_{6}\right\}
\end{array} \| \Psi_{W} \\
& +\llbracket\left[\left(L_{2}-L_{1}\right)+\left(\Lambda_{2}-\Lambda_{1}\right)\right] L_{3} \Pi_{1} \rrbracket \Psi_{W}
\end{aligned}
$$

$$
\left.+\| \begin{array}{l}
\left(L_{2}-L_{1}\right) L_{3} L_{8} \\
-\rho_{g 0} \beta_{V}\left\{\left(K_{2}-K_{1}\right) L_{3} L_{4}-\left[\left(L_{1} K_{2}-L_{2} K_{1}\right)\right] L_{6}\right\} \\
+\left(\Lambda_{2}-\Lambda_{1}\right) L_{3} L_{8} \\
-\rho_{g 0} \beta_{V}\left\{-L_{3} L_{4} \Gamma-\left[\left(\Lambda_{1} K_{2}-\Lambda_{2} K_{1}\right)-\left(L_{2}+\Lambda_{2}\right) \Gamma\right] L_{6}\right\} \\
+\left\{\left[\left(L_{2}-L_{1}\right)+\left(\Lambda_{2}-\Lambda_{1}\right)\right] L_{3}-\frac{\gamma}{s^{2}}\left[\left(L_{1}+\Lambda_{1}\right) K_{2}-\left(L_{2}+\Lambda_{2}\right)\left(\Gamma+K_{1}\right)\right]\right\} \Pi_{1}
\end{array}\right] \Psi_{W}=0 \cdot
$$


h) No-isotérmico, compresible y tomando en cuenta los efectos de fricción en la pared, pero no los efectos térmicos en la misma (pared adiabática) (pared aislante del calor o pared atérmica).

Límites cuando los coeficientes de transferencia de calor entre las fases y la pared tienden a cero: $\bar{h}_{s W 0} \rightarrow 0$ y $\bar{h}_{g W 0} \rightarrow 0$ (como $\Xi_{1}$ y $\Pi_{1}$ son proporcionales a $\bar{h}_{s W 0}$ y $\bar{h}_{g W 0}$, entonces, el límite que debe tomarse es: $\Xi_{1} \rightarrow 0$ y $\left.\Pi_{1} \rightarrow 0\right)$.

$$
\begin{aligned}
& \| \begin{array}{c}
{\left[\left(L_{2}-L_{1}\right) L_{3}-\frac{\gamma}{s^{2}}\left(L_{1} K_{2}-L_{2} K_{1}\right)\right] L_{8}} \\
-\rho_{g 0} \beta_{V}\left\{\left(K_{2}-K_{1}\right) L_{3} L_{4}-\left[\left(L_{1} K_{2}-L_{2} K_{1}\right)\right] L_{6}\right\} \|
\end{array} \\
& +\|\left\{\begin{array}{l}
\left.\left(\Lambda_{2}-\Lambda_{1}\right) L_{3}-\frac{\gamma}{s^{2}}\left[\left(\Lambda_{1} K_{2}-\Lambda_{2} K_{1}\right)-\left(L_{2}+\Lambda_{2}\right) \Gamma\right]\right\} L_{8} \\
-\rho_{g 0} \beta_{V}\left\{-L_{3} L_{4} \Gamma-\left[\left(\Lambda_{1} K_{2}-\Lambda_{2} K_{1}\right)-\left(L_{2}+\Lambda_{2}\right) \Gamma\right] L_{6}\right\}
\end{array} \| \Psi_{W}=0 .\right.
\end{aligned}
$$

g) No-isotérmico, incompresible y tomando en cuenta los efectos de fricción en la pared, pero no los efectos térmicos en la misma (pared adiabática) (pared aislante del calor o pared atérmica).

Límite cuando la velocidad del sonido es infinita: $s \rightarrow \infty$ (con esto se recupera la hipótesis de incompresibilidad). Límites cuando los coeficientes de transferencia de calor entre las fases y la pared tienden a cero: $\bar{h}_{s W 0} \rightarrow 0$ y $\bar{h}_{g W 0} \rightarrow 0$ (como $\Xi_{1}$ y $\Pi_{1}$ son proporcionales a $\bar{h}_{s W 0}$ y $\bar{h}_{g W 0}$, entonces, los límites que deben tomarse son: $\Xi_{1} \rightarrow 0$ y $\left.\Pi_{1} \rightarrow 0\right)$.

$$
\begin{gathered}
\| \begin{array}{c}
\left(L_{2}-L_{1}\right) L_{3} L_{8} \\
-\rho_{g 0} \beta_{V}\left\{\left(K_{2}-K_{1}\right) L_{3} L_{4}-\left[\left(L_{1} K_{2}-L_{2} K_{1}\right)\right] L_{6}\right\}
\end{array} \\
+\left\|\begin{array}{c}
\left(\Lambda_{2}-\Lambda_{1}\right) L_{3} L_{8} \\
-\rho_{g 0} \beta_{V}\left\{-L_{3} L_{4} \Gamma-\left[\left(\Lambda_{1} K_{2}-\Lambda_{2} K_{1}\right)-\left(L_{2}+\Lambda_{2}\right) \Gamma\right] L_{6}\right\}
\end{array}\right\| \Psi_{W}=0 .
\end{gathered}
$$


f) No-isotérmico, compresible y sin tomar en cuenta la pared (lecho "unbounded").

Por las razones físicas ya discutidas antes, deben tomarse los límites: $\Xi_{1} \rightarrow 0, \Pi_{1} \rightarrow 0$, $\Lambda_{2} \rightarrow 0, \Lambda_{1} \rightarrow 0$ y $\left.\Gamma \rightarrow 0\right)$. Además, $\Psi_{W} \rightarrow \Psi$.

$$
\left.\| \begin{array}{c}
{\left[\left(L_{2}-L_{1}\right) L_{3}-\frac{\gamma}{s^{2}}\left(L_{1} K_{2}-L_{2} K_{1}\right)\right] L_{8}} \\
-\rho_{g 0} \beta_{V}\left\{\left(K_{2}-K_{1}\right) L_{3} L_{4}-\left[\left(L_{1} K_{2}-L_{2} K_{1}\right)\right] L_{6}\right\}
\end{array}\right] \Psi=0 .
$$

Este modelo corresponde al estudiado en el Capítulo 7.

e) No-isotérmico, incompresible y sin tomar en cuenta la pared (lecho "unbounded").

Límites: $s \rightarrow \infty, \Xi_{1} \rightarrow 0, \Pi_{1} \rightarrow 0, \Lambda_{2} \rightarrow 0, \Lambda_{1} \rightarrow 0$ y $\Gamma \rightarrow 0$. También, $\Psi_{W} \rightarrow \Psi$.

$$
\llbracket \begin{gathered}
\left(L_{2}-L_{1}\right) L_{3} L_{8} \\
-\rho_{g 0} \beta_{V}\left\{\left(K_{2}-K_{1}\right) L_{3} L_{4}-\left[\left(L_{1} K_{2}-L_{2} K_{1}\right)\right] L_{6}\right\}
\end{gathered} \| \Psi=0 .
$$

Este modelo corresponde al estudiado en el Capítulo 7.

d) Isotérmico, compresible y tomando en cuenta la pared adiabática o atérmica.

Límite cuando la expansión térmica es nula: $\beta_{V} \rightarrow 0, \Xi_{1} \rightarrow 0$ y $\Pi_{1} \rightarrow 0$. Con $\Psi_{W} \rightarrow \varphi$.

$$
\begin{aligned}
& {\left[\left(L_{2}-L_{1}\right) L_{3}-\frac{\gamma}{s^{2}}\left(L_{1} K_{2}-L_{2} K_{1}\right)\right] \varphi} \\
& +\left[\left(\Lambda_{2}-\Lambda_{1}\right) L_{3}-\frac{\gamma}{s^{2}}\left[\left(\Lambda_{1} K_{2}-\Lambda_{2} K_{1}\right)-\left(L_{2}+\Lambda_{2}\right) \Gamma\right]\right] \varphi=0 .
\end{aligned}
$$

Este modelo corresponde al estudiado en el Capítulo 5.

c) Isotérmico, incompresible, tomando en cuenta la fricción en la pared y considerando a la pared como adiabática o atérmica. Límites: $\beta_{V} \rightarrow 0, s \rightarrow \infty, \Xi_{1} \rightarrow 0$ y $\Pi_{1} \rightarrow 0$. Con $\Psi_{W} \rightarrow \varphi$.

$$
\left[\left(L_{2}-L_{1}\right) L_{3}\right] \varphi+\left[\left(\Lambda_{2}-\Lambda_{1}\right) L_{3}\right] \varphi=0
$$

Este modelo corresponde al estudiado en el Capítulo 5. 
b) Isotérmico, compresible y sin tomar en cuenta la pared.

Límites: $\beta_{V} \rightarrow 0, s \rightarrow \infty, \Xi_{1} \rightarrow 0, \Pi_{1} \rightarrow 0, \Lambda_{2} \rightarrow 0, \Lambda_{1} \rightarrow 0$ у $\Gamma \rightarrow 0$. Con $\Psi_{W} \rightarrow \varphi$.

$$
\left[\left(L_{2}-L_{1}\right) L_{3}-\frac{\gamma}{s^{2}}\left(L_{1} K_{2}-L_{2} K_{1}\right)\right] \varphi=0 .
$$

Este modelo corresponde al estudiado en el Capítulo 4.

a) Isotérmico, incompresible y sin tomar en cuenta la pared.

Límites: $\beta_{V} \rightarrow 0, s \rightarrow \infty, \Xi_{1} \rightarrow 0, \Pi_{1} \rightarrow 0, \Lambda_{2} \rightarrow 0, \Lambda_{1} \rightarrow 0$ y $\Gamma \rightarrow 0$. Además, $\Psi_{W} \rightarrow \varphi$

$$
\left[\left(L_{2}-L_{1}\right) L_{3}\right] \varphi=0
$$

Este modelo corresponde al estudiado en el Capítulo 4.

Como puede verse en todos estos casos, están sepados los efectos incorporados en el modelo, lo cual proporciona muchas ventajas, pues permite realizar estudios de análisis de estabilidad lineal y medir el impacto de cada contribución del modelo en las estrategias de modelado. 


\section{Conclusiones}

Se desarrolló un modelo hidrodinámico de dos fluidos de un lecho fluidizado gas-sólido en el régimen de fluidización rápida mediante la aplicación de los principios locales de conservación y de balance para dos materiales puros (granos de sólido y fluido continuo) y se extendieron a cualquier lugar y tiempo al aplicarles técnicas de promediado espaciotemporal. Luego, con la aplicación de la segunda ley de la termodinámica a las ecuaciones promediadas se obtuvieron restricciones a los términos de cerradura generados en el proceso de promediado. Se separaron las contribuciones en estas restricciones debidas a los distintos tipos de energía; esto es, energía cinética turbulenta, energía mecánica y energía térmica.

Se planteó una solución al problema de cerradura mediante un modelo de celda al considerar que las fases sólida y fluida sólo interactúan a través de las fuerzas y de los efectos térmicos y de disipación de energía mecánica. Se revisó que las cerraduras encontradas cumplieran con las restricciones impuestas por la segunda ley de la termodinámica para modelos multifásicos.

Se mostró que la descripción en términos de promedios espacio-temporales que aquí se presentó, permite obtener una ecuación de estado en donde se incorporen correcciones a primer orden o a órdenes más elevados en las desviaciones de las variables de estado. Esta es una diferencia sustantiva entre la descripción con promedios espacio-temporales de sistemas multifásicos y la descripción en términos de la teoría cinética extendida a medios granulares que aplica Gidaspow (1994). La teoría de medios granulares presenta muchas ventajas, como el poder describir la generación de burbujas en un lecho fluidizado (Ding y Gidaspow, 1993), pero también hereda las desventajas y limitaciones de la teoría cinética de los gases, como lo es la posibilidad de obtener una ecuación de estado que describa un comportamiento más allá del gas ideal (García-Colín y col., 2011). No obstante, aunque es posible obtener la ecuación de estado con términos adicionales por la corrección a primer orden en las desviaciones de las variables de estado, en este primer estudio no se consideraron y se utilizó la ecuación de estado del gas ideal. Dejando la descripción del comportamiento del gas en términos de descripciones termostáticas más complejas que la del gas ideal para estudios posteriores. 
Con el estudio de la dinámica ondulatoria se confirmó la hipótesis de que la incompresibilidad implica que las ondas de presión viajan en el sistema con velocidad infinita, lo cual fue reportado también para sistemas multifásicos por Bouré (1988). Asimismo, se estudió el comportamiento no-isotérmico utilizando cerraduras consistentes con la segunda ley de la termodinámica para representar adecuadamente la disipación de la energía entre las fases. Estableciendo la analogía con los sistemas isotérmicos, se propone que considerar isotermicidad implica que la respuesta térmica del sistema, incluidos los efectos de disipación viscosa, se propagan en el sistema con velocidad infinita de tal manera que son transferidos instantáneamente a los alrededores y no hay un incremento en la temperatura.

Se estableció la estructura ondulatoria lineal en una dimensión en lechos fluidizados rápidos para dos modelos: uno incompresible y uno compresible. Se mostró que el modelo incompresible está embebido dentro del modelo compresible y se recupera en el límite $s \rightarrow \infty$. Este modelo, expresado para perturbaciones de la fracción volumen, tiene una estructura ondulatoria típica de segundo orden, aplicada a una derivada espacial de segundo orden de las perturbaciones de presión.

El modelo compresible es la suma de la misma estructura ondulatoria incompresible más la compresible. Esta última contribución consiste de operadores ondulatorios de cuarto y tercer orden, así como de una onda degenerada de segundo orden, todos ellos multiplicados por $s^{-2}$. Sin embargo, la onda de cuarto orden es muy pequeña y puede despreciarse excepto para fracciones volumen de sólido inferiores al valor límite $\varepsilon_{s 0 \text {,min }}$, que para el sistema de FCC es de 0.0002 y para la arena es de 0.0050. La onda compresible de tercer orden es del mismo orden de magnitud que el término de segundo orden incompresible y que el término degenerado de la onda de segundo orden del modelo incompresible, el cual incorpora el efecto de la gravedad, es de alrededor de un orden de magnitud mayor que la de tercer orden. 
El modelo con parte explícita incompresible y compresible está dado en la Ec. (4.28), pero también puede reagruparse para obtener una expresión equivalente con sólo un operador diferencial para cada uno de los órdenes de derivadas, Ec. (4.29). La derivada espacial de segundo orden en la onda de primer orden del operador incompresible, puede pensarse como una estructura ondulatoria degenerada, ya que se obtiene del operador de tercer orden compresible bajo la condición límite de que la velocidad de propagación del sonido se vuelva infinita.

Se desarrolló un análisis de estabilidad basado en el criterio de Whitham de jerarquías de onda. Se mostró que las rapideces de propagación son ordenadas de tal forma que $\alpha_{1}>c_{1}>\alpha_{2}>c_{2}>\alpha_{3}$ y $\alpha_{1}>\eta_{21}>\alpha_{2}$. Se obtuvo también un criterio para la frontera inferior del número de onda $\kappa_{0}$. Puesto que el efecto de compresibilidad aumenta la estabilidad del modelo incompresible, el criterio de Liu fue extendido para incorporar la parte compresible. Por lo tanto, el efecto de compresibilidad puede estimarse al extender la región de estabilidad, resultando despreciable esta extensión.

Los dos sistemas seleccionados para ilustrar esta evaluación del efecto de compresibilidad fueron un sistema de catalizador de FCC-vapor de agua y un sistema de arena-aire. $\mathrm{Su}$ comportamiento es muy diferente ya que sus partículas sólidas difieren ampliamente. El sistema de FCC es principalmente conducido por el fluido, mientras que las partículas sólidas más pesadas en el sistema de la arena se comportan más independientes.

Todas estas características muestran una gran influencia de la compresibilidad del gas para el FCC, expresado en el término de segundo orden incompresible por un coeficiente ondulatorio de tercer orden grande y un tiempo de relajación despreciablemente pequeño. En contraste, para fracciones volumen de sólido pequeñas, el tiempo de relajación en el sistema de la arena es un orden de magnitud mayor que para el sistema del FCC. En este caso el coeficiente de tercer orden compresible es sólo la mitad del de FCC y el coeficiente del efecto de la gravedad en el término de la compresibilidad del gas es más de dos veces importante. 
El comportamiento de las rapideces de propagación es afectado por las diferencias en la dinámica del FCC y de la arena. Para el FCC la rapidez de propagación de la onda de primer orden $a$ está localizada en medio de las rapideces de propagación de las ondas de segundo orden $c_{1}$ y $c_{2}$ mientras que para la arena, hay un desplazamiento de $c_{1} \mathrm{y} c_{2}$ hacia valores más pequeños e incluso negativos, con valor mayor o menor a los dos valores $c_{1} \mathrm{y}$ $c_{2}$. Las ondas de presión $\alpha_{1}$ y $\alpha_{3}$, en el límite $\varepsilon_{s 0} \rightarrow 0$, se vuelven coincidentes numéricamente a la velocidad de propagación del sonido en el fluido. Más aún, $\alpha_{3}$ está cerca de la rapidez de propagación de la presión experimental en todo el intervalo de $\varepsilon_{s 0} \mathrm{y}$ se obtiene el mejor ajuste cuando $U_{\text {flow }}=0$.

Ambos sistemas son linealmente estables de acuerdo con el criterio de Whitham, ya que las rapideces de propagación están ordenadas y satisfacen una condición decreciente sin entrecruzamientos. El número de onda mínimo para alcanzar estabilidad da una función que va de 1 a $6 \mathrm{~m}^{-1}$, para muy diferentes valores de $\varepsilon_{s 0}$.

Es muy importante enfatizar que el efecto de compresibilidad del fluido es un efecto tan importante como el módulo de compresibilidad del sólido en la estrategia del modelado. También, la fuerza de gravedad en el efecto de interacción de la compresibilidad del gas aparece con la forma de una onda degenerada de segundo orden con sólo una rapidez de propagación finita $\eta_{21}$.

Es importante tomar en cuenta el efecto de la pared en las estrategias de modelado, como se mostró en el Capítulo 5, su inclusión da más estructura a las ecuaciones, ya que la onda compresible de segundo orden pasó de ser degenerada (parabólica) a tener un operador hiperbólico con dos ondas de primer orden completas, donde la rapidez adicional que se obtiene es debida a los efectos de pared y es altamente dependiente de la fricción de las partículas sólidas en la pared del tubo. Otro resultado importante es que la zona de estabilidad lineal al considerar el efecto de la pared del tubo se amplía a diferencia de cuando no se considera este efecto. 
En el Capítulo 6 hemos obtenido un modelo no-isotérmico cerrado y cuyas relaciones de cerradura han sido validadas con la segunda ley de la termodinámica. La segunda ley no es capaz de decirnos cual conjunto de cerraduras es correcto, sólo puede detectar cuales conjuntos de cerraduras son incompatibles con requerimientos físicos específicos. Notamos que el modelo para el flujo no-isotérmico compresible cumple con los requerimientos físicos implicados por la segunda ley de la termodinámica Ec. (2.41). En el modelo obtenido se consideraron los efectos de la pared, lo cual permitió hacer análisis de estabilidad que consideren la compresibilidad, la termicidad y las fronteras laterales o la pared del tubo.

A partir de un modelo térmico cerrado linealizado se estableció la estructura ondulatoria lineal en una dimensión en lechos fluidizados rápidos. Se mostró que en el modelo pueden separarse las contribuciones de cada efecto. La parte incompresible, la parte compresible y la parte térmica. Se obtuvo una ecuación de onda de quinto orden donde están incorporados todos los efectos y se demostró que es la misma para las perturbaciones de fracción volumen, de la presión, de la temperatura del gas y en el caso de la temperatura del sólido es este operador ondulatorio de quinto orden actuando en una onda de primer orden de la temperatura del sólido.

Se extendió la estabilidad lineal con el criterio establecido por Liu (1982) al considerar efectos térmicos. Los efectos térmicos incrementaron la importancia relativa a la onda de primer orden de la parte compresible, volviéndola comparable para el FCC, mientras que para la Arena se redujo.

Finalmente, se estableció la estructura ondulatoria lineal en una dimensión en lechos fluidizados rápidos para modelos que consideran distintos efectos: la compresibilidad de la fase fluida, la termicidad y el efecto de la pared. Se mostró que al seguir la metodología desarrollada en esta tesis pudimos ser capaces de obtener un modelo lineal, representado por una ecuación de orden superior para las perturbaciones en la cual están separadas las contribuciones de los distintos efectos; esto es, la parte incompresible, de la parte compresible, de la parte térmica y de los efectos de pared isotérmicos de los térmicos. Esta metodología permite evaluar la importancia de cada efecto por separado en la estrategia de modelado. 
También puede estudiarse el impacto que tiene cada efecto en la dinámica y en la estabilidad lineal. Además puede observarse cómo es que pueden obtenerse los casos asintóticos correspondientes a los ocho modelos más sencillos representados en las dos tablas siguientes.

MODELOS SIN EFECTOS DE PARED

\begin{tabular}{|c|c|c|}
\hline EFECTOS & Isotermicidad & Termicidad \\
\hline \multirow{3}{*}{ Incompresibilidad } & Modelo & Modelo \\
& Isotérmico- & No-isotérmico- \\
& Incompresible & Incompresible \\
& (Cap. 4) & (Cap. 7) \\
\hline \multirow{3}{*}{ Compresibilidad } & Modelo & Modelo \\
& Isotérmico- & No-isotérmico- \\
& Compresible & Compresible \\
& (Cap.4) & (Cap. 7) \\
\hline
\end{tabular}

MODELOS CON EFECTOS DE PARED

\begin{tabular}{|c|c|c|}
\hline EFECTOS & Isotermicidad & Termicidad \\
\hline \multirow{3}{*}{ Incompresibilidad } & Modelo & Modelo \\
& Isotérmico- & No-isotérmico- \\
& Incompresible & Incompresible \\
& (Cap. 5) & (Cap. 8) \\
\hline \multirow{3}{*}{ Compresibilidad } & Modelo & Modelo \\
& Isotérmico- & No-isotérmico- \\
& Compresible & Compresible \\
& (Cap. 5) & (Cap. 8) \\
\hline
\end{tabular}




\section{Perspectivas}

Una perspectiva fundamental de este trabajo es incorporar la turbulencia y las reacciones químicas de cracking catalítico en una unidad de FCC. Hacer estudios de Dinámica de Fluidos Computacional (CFD) con software comercial como FLUENT (si es posible) o alternativamente, utilizar bibliotecas de software desarrolladas en universidades mexicanas, lo cual además serviría para enriquecer el software generado por nuestra comunidad. 


\section{Bibliografia}

- Abou-Arab, T.W. y Elghobashi, S.E. (1983). A two-equation turbulence model for two-phase flows. Phys. Fluids 26 (4), 931-938.

- Anderson, T.B. y Jackson, R. (1967). A fluid mechanical description of fluidized beds. Equations of motion. I \& EC Fundamentals 6 (4), 527-538.

- Andreux R., Gauthier T., Chaouki, J. y Simonin O. (2005). New description of fluidization regimes. AIChE J. 51 (4), 1125-1130.

- Arnold, G.S. (1988). Entropy and objectivity as constraints upon constitutive equations for two-fluid modeling of multiphase flow. Ph.D. Thesis. Rensselaer Polytechnic Institute. Troy, N.Y. E.U.A.

- Arnold, G.S., Drew, D.A. y Lahey (Jr), R.T. (1990). An assessment of multiphase flow models using the second law of thermodynamics. Int. J. of Multiphase Flow 16 (3), 481-494.

- Atkinson, C.M. y Kytömaa, H.K. (1992). Acoustic-wave speed and attenuation in suspensions. Int. J. Multiphase Flow 18, 577-592.

- Bi, H.T. (2007). A critical review of the complex pressure fluctuation phenomenon in gas-solids fluidized beds. Chem. Eng. Sci. 62, 3473-3493.

- Bi, H.T., Grace, J.R. y Zhu, J. (1995). Propagation of pressure waves and forced oscillations in gas-solid fluidized beds and their influence on diagnostics of local hydrodynamics. Powder Technol. 82, 239-253.

- Bouré, J.A. (1988). An alternative strategy for the development of averaged twophase flow models. Seminar on theoretical aspects of multiphase flow phenomena. Ithaca, N.Y., Oct. 22-25.

- Callen, H. (1985). Thermodynamics and an Introduction to Thermostatistics. Second edition, John Wiley \& Sons. E.U.A.

- Cebeci, T. y Smith, A.M.O. (1974). Analysis of Turbulent Boundary Layers. Academic Press. E.U.A. 
- Chen, C.P. y Wood, P.E. (1985). A turbulence closure model for dilute gas-particle flows. Can. J. Chem. Eng. 63, 349-360.

- Dasgupta, S., Jackson, R. y Sundaresan, S. (1994). Turbulent gas-particle flow in vertical risers. AIChE J. 40 (2), 215-228.

- De Wilde, J., Constales, D., Heynderickx, G.J. y Marin, G.B. (2007). Assessment of filtered gas-solid momentum transfer models via a linear wave propagation speed test. Int. J. Multiphase Flow 33, 616-637.

- Ding, J. y Gidaspow, D. (1990). A bubbling fluidization model using kinetic theory of granular flow. AIChE J. 36 (4), 523-538.

- Drew, D.A. (1971). Averaged field equations for two-phase media. Studies in Applied Mathematics L (2), 133-166.

- Drew, D.A. (1983). Mathematical modeling of two-phase flow. Ann. Rev. Fluid Mech. 15, 261-291.

- Drew, D.A. y Lahey (Jr), R.T. (1979). Application of general constitutive principles to the derivation of multidimensional two-phase flow equations. Int. J. Multiphase Flow 5, 243-264.

- Drew, D.A. y Lahey (Jr), R.T. (1993). Analytical modeling of multiphase flow. Particulate two-phase flow. (Editado por Roco, M.C.). ButterworthHeinemann Series in Chemical Engineering, 509-569. E.U.A.

- Drew, D.A. y Passman, S.L. (1999). Theory of Multicomponent Fluids. SpringerVerlag, N.Y. E.U.A.

- Elghobashi, S.E. (1994). On predicting particle-laden turbulent flows. Appl. Sci. Res. 52, 309-329.

- Estrada, R. y Kanwal, R.P. (1980). Applications of Distributional Derivatives to Wave Propagation. IMA J. Appl. Math. 26, 39-63.

- Firoozabadi, A. (1999). Thermodynamics of Hydrocarbon Reservoirs. McGrawHill. E.U.A. 
- Gao, J.; Xu, Ch.; Lin, Sh. y Yang, G. (2001). Simulations of gas-liquid-solid 3phase flow and reaction in FCC riser reactors. AIChE J. 47, 677-692.

- García-Colín, L.S., Dagdug, L. y Sánchez-López, J.R.G. (2011). Introducción a la Teoría Cinética. El Colegio Nacional, México.

- Gel'fand, I.M. y Shilov, G.E. (1964). Generalized Functions, vol. 1, Academic Press, N.Y. E.U.A.

- Gidaspow, D. (1994). Multiphase Flow and Fluidization Continuum and Kinetic Theory Descriptions. Academic Press. E.U.A.

- Glasser, B.J., Kevrekidis, I.G. y Sundaresan, S. (1996). One- and two-dimensional travelling wave solutions in gas-fluidized beds. J. Fluid Mech. 306, 183-221.

- Glasser, B.J., Kevrekidis, I.G. y Sundaresan, S. (1997). Fully developed travelling wave solutions and bubble formation in fluidized beds. J. Fluid Mech. 334, 157-188.

- Göz, M.F. (1992). On the origin of wave patterns in fluidized beds. J. Fluid Mech. 240, 379-404.

- Göz, M.F. (1993). Bifurcation of plane voidage waves in fluidized beds. Physica D. 65, 319-351.

- Göz, M.F. y Sundaresan, S. (1998). The growth, saturation, and scaling behaviour of one- and two-dimensional disturbances in fluidized beds. J. Fluid Mech. 362, 83-119.

- Grace, J.R. (1986). Contacting modes and behaviour classification of gas-solid and other two-phase suspensions. Can. J. of Chem. Eng. 64, 353-363.

- Gray, W.G. (1975). A derivation of the equations for multiphase transport. Chem. Eng. Sci. 30, 229-233.

- Gregor, W. y Rumpf, H. (1975). Velocity of sound in two-phase media, Int. J. Multiphase Flow 1, 753-769. 
- Gregor, W. y Rumpf, H. (1976). Attenuation of sound in gas-solid suspensions. Powder Technol. 15, 43-51.

- Hirayama, O. y Takaki, R. (2000). Linear stability of one-dimensional fluidized bed containing two kinds of solid particles. Fluid Dyn. Res. 27, 199-216.

- Homsy, G.M., El-Kaissy, M.M. y Didwinia, A. (1980). Instability waves and the origin of bubbles in fluidized beds-II. Comparison with theory. Int. J. Multiphase Flow 6, 305-318.

- Incropera, F.P. y DeWitt, D.P. (1996). Fundamentals of Heat and Mass Transfer. Fourth edition, John Wiley \& Sons. E.U.A.

- Ishii, M. (1975). Thermo-fluid Dynamic Theory of Two-phase Flow. Eyrolles, Paris.

- Ishii, M. (1990). Two-fluid model for two-phase flow. Multiphase Science and Technology 5. (Editado por Hewitt, G.F., Delhaye, J.M. y Zuber, N.). Hemisphere Publishing Corporation, 1-63.

- Ishii, M. y Kocamustafaogullari, G. (1983). Two-phase models and their limitations. Advances in Two-Phase Flow and Heat Transfer. vol. 1. Ed. Nato.

- Jackson, R. Hydrodynamic stability of fluid-particle systems. En: Davidson, J.F., Clift, R., Harrison, D., editores. Fluidization, 2da edición. Academic Press, Inc., 1985: 47-72.

- Jinsen, G., Chunming, X., Shixiong, L., Guanghua, Y. y Yincheng, G. (1999). Advanced model for turbulent gas-solid flow and reaction in FCC riser reactors, AIChE J. 45, 1095-1113.

- Jinsen, G., Chunming, X., Shixiong, L., Guanghua, Y. y Yincheng, G. (2001). Simulations of gas-liquid-solid 3-phase flow and reaction in FCC riser reactors. AIChE J. 47, 677-692.

- Jiradilok, V., Gidaspow, D., Damronglerd, S., Koves, W.J. y Mostofi, R. (2006). Kinetic theory based CFD simulation of turbulent fluidization of FCC particles in a riser. Chem. Eng. Sci. 61, 5544-5559. 
- Johri, J. y Glasser, B.J. (2002). Connections between density waves in fluidized beds and compressible flows. AIChE J. 48, 1645-1664.

- Jones, A.V. y Prosperetti, A. (1987). The linear stability of general two-phase flow models-II. Int. J. Multiphase Flow 13, 161-171.

- Joshi, J.B., Deshpande, N.S., Dinkar, M. y Phanikumar, D.V. (2001). Hydrodynamic stability of multiphase reactors. Advances in Chemical Engineering 26, 1-130.

- Kellogg Company M.W. (1981). Salina Cruz Oaxaca Refinery, Catalytic Plant Operation Manual. The Kellogg Company Pullman Incorporated. [in Spanish]

- Konno, H. y Saito, S. (1969). Pneumatic conveying of solids through straight pipes. J. Chem. Eng. Jpn. 2 (2), 211-217.

- Lahey (Jr), R.T. (2005). The simulation of multidimensional multiphase flows. Nuclear Engineering and Design 235, 1043-1060.

- Launder, B.E. y Spalding, D.B. (1972). Mathematical Models of Turbulence. Academic Press, Inc. (London).

- Liu, J.T.C. (1982). Note on a wave-hierarchy interpretation of fluidized bed instabilities. Proc. R. Soc. Lond. A 380, 229-239.

- Marle, C.M. (1967). Écoulements monophasique en milieu poreux. Rev. Inst. Français du Petrolé 22 (10), 1471-1509.

- McGaughey, A.J.H. y Kaviany, M. (2004). Thermal conductivity decomposition and analysis using molecular dynamics simulations Part II. Complex silica structures. Int. J. Heat and Mass Transfer 47, 1799-1816.

- Micaelli, J.C. (1982). Propagation d'ondes dans les écoulements disphasiques à bulles à deux constituants. Thèsis, Université Scientifique et Medicale et Institut National Polytechnique des Grenoble, France.

- Musmarra, D., Poletto, M., Vaccaro, S., Clift, R. (1995). Dynamic waves in fluidized-beds. Powder Technol. 82, 255-268. 
- Needham, D.J. y Merkin, J.H. (1983). The propagation of a voidage disturbance in a uniformly fluidized bed. J. Fluid Mech. 131, 427-454.

- Needham, D.J. y Merkin, J.H. (1984). A note on the stability and the bifurcation to periodic solutions for wave-hierarchy problems with dissipation. Acta Mechanica 54, 75-85.

- Needham, D.J. y Merkin, J.H. (1986). The existence and stability of quasi-steady periodic voidage waves in a fluidized bed. Zeitschrift für Angewandte Mathematik und Physik (ZAMP) 37, 322-339.

- Neri, A. y Gidaspow, D. (2000). Riser hydrodynamics: simulation using kinetic theory. AIChE J. 46 (1), 52-67.

- Nguyen, D.L., Winter, E.R.F. y Greiner, M. (1981). Sonic velocity in two-phase systems. Int. J. Multiphase Flow 7 (3), 311-320.

- Pedras, M.H.J. y de Lemos, M.J.S. (2001). Macroscopic turbulence modelling for incompressible flow through undeformable porous media. Int. J. Heat Mass Transfer 44, 1081-1093.

- Prandtl, L. (1925). Über die ausgebildete turbulenz. Zeitschrift für Angewandte Mathematik und Mechanik (ZAMM) 5, 136-139.

- Prosperetti, A. y Tryggvason, G. (2007). Computational Methods for Multiphase Flow. Cambridge University Press, New York.

- Ransom, V.H. y Hicks, D.L. (1984). Hyperbolic two-pressure models for two-phase flow. J. Comput. Phys. 53, 124-151.

- Rotta, J. (1951). Statistische theorie nichthomogener turbulenz. Z. Phys. 129, 547572 .

- Ryzhkov, A.F. y Tolmachev, E.M. (1983). Selection of optimal height for vibrofluidized bed. Teor. Osn. Khim. Tekhnol. 17, 140-147. 
- Sánchez-López, J.R.G. (2003). Fenómenos ondulatorios en un tubo elevador en el régimen de fluidización rápida y con efectos de compresibilidad. Tesis de Maestría en Ingeniería Química, Posgrado Divisional de CBI, UAMIztapalapa, México.

- Sánchez-López, J.R.G., Soria, A. y Salinas-Rodríguez, E. (2011). Compressible and incompressible 1-D linear wave propagation assessment in fast fluidized beds. AIChE J. 57 (11), 2965-2976.

- Slattery, J.C. (1967). Flow of viscoelastic fluids through porous media. AIChE J. 13 (6), 1066-1071.

- Slattery, J.C. (1999). Advanced Transport Phenomena. Cambridge University Press, E.U.A.

- Song, J.H. y Ishii, M. (2000). The well-posedness of incompressible onedimensional two-fluid model. Int. J. Heat Mass Transfer 43, 2221-2231.

- Soria, A. y de Lasa, H.I. (1991). Averaged transport equations for multiphase systems with interfacial effects. Chem. Eng. Sci. 46, 2093-2111.

- Soria, A. and de Lasa, H. I. (1992). Averaged topological equations for dispersed two-phase flows. Int. J. Multiphase Flow 18, 943-964.

- Soria, A., Sánchez-López, J.R.G. y Salinas-Rodríguez, E.M. (2008). The incompressibility assumption assessment in fast fluidized bed wave propagation. Proceedings of the 14th WASCOM Conference, 2007. World Scientific Pub., Singapore, 530-535.

- Soria, A., Salinas-Rodríguez, E.M. y Sánchez-López, J.R.G. (2012). Wall effects assessment on compressible and incompressible 1D linear wave propagation in fast fluidized beds. AIChE J. (en revisión)

- Stromberg, K.R. (1981). Introduction to Classical Real Analysis. Wadsworth International Group. E.U.A.

- Stuhmiller, J.H. (1977). The influence of interfacial pressure forces on the character of two-phase flow model equations. Int. J. Multiphase Flow 3, 551-560. 
- Theologos, K.N. y Markatos, N.C. (1993). Advanced modeling of fluid catalytic cracking riser-type reactors. AIChE J. 39 (6), 1007-1017.

- Theologos, K.N., Nikou, I.D., Lygeros, A.I. y Markatos, N.C. (1997). Simulation and design of fluid catalytic-cracking riser-type reactors. AIChE J. 43 (2), 486-494.

- Theologos, K.N., Nikou, I.D., Lygeros, A.I. y Markatos, N.C. (1999). Feedstock atomization effects on FCC riser reactors selectivity. Chem. Eng. Sci. 54, 5617-5625.

- Troshko, A.A. y Hassan, Y.A. (2001). A two-equation turbulence model of turbulent bubbly flows. Int. J. Multiphase Flow 27, 1965-2000.

- Valencia-Martínez, O. (2001). Modelo hidrodinámico para el tubo elevador de una unidad de FCC con evaporación de gasóleo. Tesis de maestría en Ingeniería Química, Posgrado Divisional de CBI, UAM-Iztapalapa, México.

- van der Schaaf, J., Schouten, J.C. y van den Bleek, C.M. (1998). Origin, propagation and attenuation of pressure waves in fluidized beds. Powder Technol. 95 (3), 220-233.

- Wallis, B. (1969). One-dimensional Two-Phase Flow. McGraw-Hill.

- Weir, G.J. (2001). Sound speed and attenuation in dense, non-cohesive air-granular systems. Chem. Eng. Sci. 56, 3699-3717.

- Whitaker, S. (1967). Diffusion and dispersion in porous media. AIChE J. 13, 420427.

- Whitaker, S. (1999). The Method of Volume Averaging. Kluwer Academic Publishers, Dordrecht, The Netherlands.

- Whitham, G.B. (1974). Linear and Nonlinear Waves. Wiley, N.Y., E.U.A.

- Yerushalmi, J., Turner, D.H. y Squires, A.M. (1976). The fast fluidized bed. Ind. Eng. Chem., Process Des. Dev. 15 (1), 47-54. 
- Zemanian, A.H. (1965). Distribution Theory and Transform Analysis: An Introduction to Generalized Functions, with Applications. Dover, N.Y., E.U.A.

- Zhang, D.Z. (1993). Ensemble phase averaged equations for multiphase flows. Ph.D. Thesis, Dep. of Mechanical Engineering, Johns Hopkins University.

- Zhang, D.Z. y Prosperetti, A. (1994). Averaged equations for inviscid disperse twophase flow. J. Fluid Mech. 267, 185-219.

- Zhang, Y. y Reese, J.M. (2001). Particle-gas turbulence interactions in a kinetic theory approach to granular flows. Int. J. Multiphase Flow 27, 1945-1965.

- Zheng, Y., Wan, X.T., Qian, Z., Wei, F. y Jin, Y. (2001). Numerical simulation of the gas-particle turbulent flow in riser reactor based on k-epsilon-k(p)epsilon(p)-Theta two-fluid model. Chem. Eng. Sci. 56 (24), 6813-6822. 


\section{Apéndice}

A. El promedio volumétrico y el promedio temporal como casos asintóticos del promedio espacio-temporal ............. 193

B. Análisis de promedios ponderados de desviaciones ......... 201

C. Ecuaciones promediadas en el espacio-tiempo .................. 203

D. Derivación del conjunto de ecuaciones representado en la Ec. (2.40)

E. Evaluación de la derivada del módulo de compresibilidad del sólido

$\boldsymbol{F}$. Expresiones explícitas de las matrices $\mathbf{B}, \mathbf{C}$ y $\mathbf{D}$ dadas en la Ec. (4.6)

G. Sistemas de ecuaciones cuyas raíces son las rapideces de propagación del Capítulo 4

H. Expresiones explícitas de los componentes de la matriz $\mathbf{D}^{*}$ dada en la Ec. (5.11)

I. Coeficientes de la parte de segundo y tercer orden compresible del modelo del Capítulo 5

J. Ecuación de estado de campo promedio para la entalpía

K. Evaluación de la derivada de la densidad del gas

L. Expresiones explícitas de las matrices B, C y D dadas en la Ec. (7.8)

M. Sistemas de ecuaciones cuyas raíces son las rapideces de propagación de la Ec. (7.34)

$N$. Sistemas de ecuaciones cuyas raíces son las rapideces de propagación de la Ec. (7.43)

o. Expresiones explícitas de los componentes de la matriz $\mathbf{D}^{* *}$ dada en la Ec. (8.16) 


\section{Apéndice $A$ \\ El promedio volumétrico y el promedio temporal \\ como casos asintóticos del promedio \\ espacio-temporal}

Se consideran dos casos particulares del promedio espacio-temporal, el primero es el promedio volumétrico (Whitaker, 1967, 1999; Slattery, 1967; Marle, 1967; Anderson y Jackson, 1967) el segundo caso es el promedio temporal o de Reynolds (métodos propuestos en 1883). Estos casos se recuperan como casos límite del promedio espaciotemporal definido en la Ec. (1.17).

\section{A.1. Promedio volumétrico}

La condición $T \rightarrow 0$ nos da el promedio volumétrico como un caso asintótico. Para probar esto, considere la Ec. (1.21) en este límite

$$
\lim _{T \rightarrow 0}\left\langle\bullet_{k}\right\rangle^{k}(\mathbf{x}, t)=\lim _{T \rightarrow 0}\left[\frac{1}{V_{k} T} \iiint_{\mathscr{\mathscr { V }}(\mathbf{x})}^{t+\frac{T}{2}} \int_{t-\frac{T}{2}}^{2}\left(X_{k} \bullet_{k}\right)\left(\mathbf{x}^{\prime}, t^{\prime}\right) d t^{\prime} d V^{\prime}\right] .
$$

Esto nos lleva a

$$
\left\langle\bullet_{k}\right\rangle^{k}(\mathbf{x}, t)=\lim _{T \rightarrow 0}\left[\frac{1}{V_{k}} \iiint_{\mathscr{V}(\mathbf{x})} f_{T}\left(\mathbf{x}^{\prime}, t^{\prime}\right) d V^{\prime}\right],
$$

donde

$$
f_{T} \doteq \frac{1}{T} \int_{t-\frac{T}{2}}^{t+\frac{T}{2}}\left(X_{k} \bullet \bullet_{k}\right)\left(\mathbf{x}^{\prime}, t^{\prime}\right) d t^{\prime}
$$

Para introducir el límite bajo el signo integral, la función $f_{T}$ debe analizarse en el límite cuando el tiempo de integración $T$ tiende a cero. Si pasa un intervalo infinitesimal de tiempo ( $T$ es muy pequeño) y la región $k$ se desplaza una distancia infinitesimal tal que, un punto seleccionado (que no pertenezca a la interfaz) permanezca en el interior o en el exterior de la fase $k$. 
Entonces la región temporal de integración $\left[t-\frac{1}{2} T, t+\frac{1}{2} T\right]$ y para $t^{\prime}$ cercano a $t$, que implica que $T \rightarrow 0$. Por lo tanto, $X_{k}\left(\mathbf{x}^{\prime}, t^{\prime}\right)$ pueda aproximarse por $X_{k}\left(\mathbf{x}^{\prime}, t\right)$. Así:

$$
\lim _{T \rightarrow 0} f_{T}\left(\mathbf{x}^{\prime}, t\right)=\lim _{T \rightarrow 0} \frac{1}{T} \int_{t-\frac{T}{2}}^{t+\frac{T}{2}} X_{k}\left(\mathbf{x}^{\prime}, t\right) \bullet_{k}\left(\mathbf{x}^{\prime}, t^{\prime}\right) d t^{\prime} .
$$

En esta forma $X_{k}\left(\mathbf{x}^{\prime}, t\right)$ no depende de la región de integración ni de la variable con la que se toma el límite. Por lo tanto, $X_{k}$ puede sacarse tanto del signo integral como del límite, para tener:

$$
\lim _{T \rightarrow 0} f_{T}\left(\mathbf{x}^{\prime}, t\right)=X_{k}\left(\mathbf{x}^{\prime}, t\right) \lim _{T \rightarrow 0} \frac{1}{T} \int_{t-\frac{T}{2}}^{t+\frac{T}{2}} \bullet_{k}\left(\mathbf{x}^{\prime}, t^{\prime}\right) d t^{\prime}
$$

$\mathrm{Si} \bullet_{k}$ es continua, entonces es Riemann integrable y puede aplicarse el Teorema fundamental del cálculo para tener

$$
\lim _{T \rightarrow 0} f_{T}\left(\mathbf{x}^{\prime}, t\right)=X_{k}\left(\mathbf{x}^{\prime}, t\right) \bullet_{k}\left(\mathbf{x}^{\prime}, t\right)
$$

Este resultado es válido en toda la región $k$, excepto en un conjunto de medida cero, el cual está formado por los puntos de la región interfacial.

El límite puede introducirse a la integral en la Ec. (A.2), si existe una función $g_{1}$ finita, que sea Lebesgue integrable y que acote el valor absoluto del promedio temporal de $\bullet_{k}\left(\mathbf{x}^{\prime}, t^{\prime}\right)$, así

$$
\left|f_{T}\left(\mathbf{x}^{\prime}, t\right)\right| \leq g_{1}\left(\mathbf{x}^{\prime}\right) \quad \text { y } \quad \frac{1}{V} \iiint_{\mathscr{V}(\mathbf{x})} g_{1}\left(\mathbf{x}^{\prime}\right) d V<\infty .
$$

El caso particular en que $\bullet_{k}=\rho_{g}$ y $\rho_{g}=P_{g} / s^{2}$ para un gas ideal, vapor de agua $\left(s=655 \mathrm{~m} \cdot \mathrm{s}^{-1}\right)$ el cual se inyecta en la base del tubo a $11 \mathrm{~atm}\left[1114630 \mathrm{~kg} \cdot\left(\mathrm{m} \cdot \mathrm{s}^{2}\right)^{-1}\right]$ y se expande a $3 \mathrm{~atm}\left[303990 \mathrm{~kg} \cdot\left(\mathrm{m} \cdot \mathrm{s}^{2}\right)^{-1}\right]$ en él. Si la presión a la entrada se toma como cota superior y se usa este valor para obtener $g_{1}$, 


$$
\begin{aligned}
\left|\frac{1}{T} \int_{t-\frac{T}{2}}^{t+\frac{T}{2}} \rho_{g}\left(\mathbf{x}^{\prime}, t^{\prime}\right) d t^{\prime}\right| & \leq \frac{1}{T} \int_{t-\frac{T}{2}}^{t+\frac{T}{2}} 2.5981 d t^{\prime} \\
& \leq 2.5981 \mathrm{~kg} \cdot \mathrm{m}^{-3}
\end{aligned}
$$

donde la función constante $g_{1}=2.5981 \mathrm{~kg} \cdot \mathrm{m}^{-3}$, acota a la densidad en todo el intervalo de operación, ya que a 3 atm la densidad será menor.

$\mathrm{Si}$ se satisfacen estas dos condiciones, puede utilizarse el Teorema de la convergencia dominada de Lebesgue (Stromberg, 1981):

$$
\lim _{T \rightarrow 0} \frac{1}{V} \iiint_{\mathscr{Q}(\mathbf{x})} f_{T}\left(\mathbf{x}^{\prime}, t\right) d V=\frac{1}{V} \iiint_{\mathscr{V}(\mathbf{x})} \lim _{T \rightarrow 0} f_{T}\left(\mathbf{x}^{\prime}, t\right) d V
$$

El cual es válido sí y sólo sí el $\lim _{T \rightarrow 0} f_{T}\left(\mathbf{x}^{\prime}, t\right)$ converge en cada punto, excepto en un conjunto de medida cero, como ocurre en este caso con la Ec. (A.6). Sustituyendo la Ec. (A.6) en la Ec. (A.9):

$$
\lim _{T \rightarrow 0} \frac{1}{V} \iiint_{\mathscr{V}(\mathbf{x})} f_{T}\left(\mathbf{x}^{\prime}, t\right) d V=\frac{1}{V} \iiint_{\mathscr{V}(\mathbf{x})} X_{k}\left(\mathbf{x}^{\prime}, t\right) \bullet_{k}\left(\mathbf{x}^{\prime}, t\right) d V .
$$

Utilizando la definición de la función indicadora de fase, dada por la Ec. (1.6):

$$
\lim _{T \rightarrow 0} \frac{1}{V} \iiint_{\mathscr{Y}(\mathbf{x})} f_{T}\left(\mathbf{x}^{\prime}, t\right) d V=\frac{1}{V} \iiint_{\mathscr{V} /(t)} \bullet_{k}\left(\mathbf{x}^{\prime}, t\right) d V
$$

o sustituyendo la Ec. (A.11) en la Ec. (A.1):

$$
\varepsilon_{k}\left\langle\bullet_{k}\right\rangle^{k}(\mathbf{x}, t)=\frac{1}{V} \iiint_{\sigma_{k}(t)} \bullet_{k}\left(\mathbf{x}^{\prime}, t\right) d V .
$$

Ahora, se aplica el límite de $\varepsilon_{k}$ cuando $T$ tiende a cero en la Ec. (1.19).

$$
\begin{aligned}
\lim _{T \rightarrow 0}\left\langle X_{k}\right\rangle(\mathbf{x}, t) & =\lim _{T \rightarrow 0} \varepsilon_{k}(\mathbf{x}, t) \\
& =\lim _{T \rightarrow 0} \frac{1}{V} \iiint_{\mathscr{Y}(\mathbf{x})}\left[\frac{1}{T} \int_{t-\frac{T}{2}}^{t+\frac{T}{2}} X_{k}\left(\mathbf{x}^{\prime}, t^{\prime}\right) d t^{\prime}\right] d V .
\end{aligned}
$$

Así como también en la Ec. (A.7), $\left|\frac{1}{T} \int_{t-\frac{T}{2}}^{t+\frac{T}{2}} X_{k}\left(\mathbf{x}^{\prime}, t^{\prime}\right) d t^{\prime}\right| \leq g_{2}\left(\mathbf{x}^{\prime}\right)$ y desarrollando la integral. 
El resultado es: $1 \leq g_{2}\left(\mathbf{x}^{\prime}\right)$ y puede aplicarse el Teorema de la convergencia dominada de Lebesgue en la Ec. (A.13) mediante los mismos argumentos acerca de la región $k$, para tener:

$$
\begin{aligned}
\lim _{T \rightarrow 0} \varepsilon_{k}(\mathbf{x}, t) & =\frac{1}{V} \iiint_{\mathscr{T}_{k}(\mathbf{x})} d V \\
& =\frac{V_{k}}{V}
\end{aligned}
$$

sustituyendo la Ec. (A.14) en el miembro izquierdo de la Ec. (A.12), obtenemos finalmente que:

$$
\left\langle\bullet_{k}\right\rangle^{k}(\mathbf{x}, t)=\frac{1}{V_{k}} \iiint_{\mathscr{\sigma}_{k}(\mathbf{x})} \bullet_{k}\left(\mathbf{x}^{\prime}, t\right) d V
$$

el cual es el operador promedio volumétrico intrínseco de fase aplicado a la función local $\bullet_{k}$.

\section{A.2. Promedio temporal}

La condición $\mathscr{V} \rightarrow \mathscr{D}$ nos da el promedio temporal como caso asintótico. Para probar esto, la región $\mathscr{D} \subset \mathbb{R}^{3}$ está definida con un volumen $\delta_{V}$ muy pequeño. Aplicando este límite a la Ec. (1.21).

$$
\lim _{\mathscr{Y} \rightarrow \mathscr{D}}\left\langle X_{k} \bullet_{k}\right\rangle(\mathbf{x}, t)=\lim _{\mathscr{Y} \rightarrow \mathscr{D}} \frac{1}{V T} \iiint_{\mathscr{\mathscr { V }}(\mathbf{x})} \int_{t-\frac{T}{2}}^{t+\frac{T}{2}} X_{k}\left(\mathbf{x}^{\prime}, t^{\prime}\right) \bullet_{k}\left(\mathbf{x}^{\prime}, t^{\prime}\right) d t^{\prime} d V
$$

cuando $\delta_{V} \rightarrow 0$.

Intercambiando el orden de integración en el miembro derecho.

$$
\varepsilon_{k}\left\langle\bullet_{k}\right\rangle^{k}(\mathbf{x}, t)=\lim _{\mathscr{V} \rightarrow \mathscr{O}} \frac{1}{T} \int_{t-\frac{T}{2}}^{t+\frac{T}{2}} f_{V}\left(\mathbf{x}, t^{\prime}\right) d t^{\prime}
$$

donde

$$
f_{V}\left(\mathbf{x}, t^{\prime}\right) \doteq \frac{1}{V} \iiint_{\mathscr{V}(\mathbf{x})} X_{k}\left(\mathbf{x}^{\prime}, t^{\prime}\right) \bullet_{k}\left(\mathbf{x}^{\prime}, t^{\prime}\right) d V
$$


Analizando la integral del límite de $f_{V}\left(\mathbf{x}, t^{\prime}\right)$ cuando la región de integración $\mathscr{Q}$ se colapsa a $\mathscr{D}$. Para hacer esto, supongamos que para una región fija, en un intervalo de tiempo $T$, la probabilidad de que un vector de posición esté en la superficie de una partícula es cero. Esto significa que, una fotografía instantánea al tiempo $t$ para una posición $\mathbf{x}^{\prime}$ dentro de la región $\mathscr{D}$ se colapsa a un punto $\mathbf{x}$, este vector de posición $\mathbf{x}^{\prime}$ permanece en $\mathscr{Y}$, de tal forma que $X_{k}\left(\mathbf{x}^{\prime}, t^{\prime}\right)=X_{k}\left(\mathbf{x}, t^{\prime}\right)$, excepto en un conjunto de medida cero de $t^{\prime}$.

$$
\lim _{\mathscr{V} \rightarrow \mathscr{D}} f_{V}\left(\mathbf{x}, t^{\prime}\right)=X_{k}\left(\mathbf{x}, t^{\prime}\right) \lim _{\mathscr{V} \rightarrow \mathscr{D}} \frac{1}{V} \iiint_{\mathscr{V}(\mathbf{x})} \bullet_{k}\left(\mathbf{x}^{\prime}, t^{\prime}\right) d V
$$

Si $\bullet{ }_{k}$ es continua, entonces es Riemann integrable, tal que puede aplicarse el Teorema del valor medio para integrales a la Ec. (A.19), para obtener:

$$
\lim _{\mathscr{V} \rightarrow \mathscr{D}} f_{V}\left(\mathbf{x}, t^{\prime}\right)=X_{k}\left(\mathbf{x}, t^{\prime}\right) \lim _{\mathscr{V} \rightarrow \mathscr{D}} \frac{1}{V} \bullet_{k}\left(\mathbf{x}^{\prime \prime}, t^{\prime}\right) \cdot V
$$

donde $\bullet_{k}\left(\mathbf{x}^{\prime \prime}, t^{\prime}\right)$ está evaluada en el punto $\mathbf{x}^{\prime \prime}$ el cual está en $\mathscr{W}$. Esto significa que $\mathbf{x}^{\prime \prime} \in \mathscr{V}$ y en el límite cuando la región $\mathscr{V}$ se colapsa a la región $\mathscr{D}, \mathbf{x}^{\prime \prime}$ tiende a $\mathbf{x}$ :

$$
\lim _{\mathscr{V} \rightarrow \mathscr{D}} f_{V}\left(\mathbf{x}, t^{\prime}\right)=X_{k}\left(\mathbf{x}, t^{\prime}\right) \bullet_{k}\left(\mathbf{x}, t^{\prime}\right)
$$

excepto en un conjunto de medida cero de $t^{\prime}$.

Note que el Teorema del valor medio para integrales no puede aplicarse directamente en la Ec. (A.18) ya que $X_{k}\left(\mathbf{x}^{\prime}, t^{\prime}\right)$ no es una función continua en $\mathscr{V}$.

El límite puede aplicarse bajo el signo integral si existe una función $g_{2}$ finita, que sea Lebesgue integrable y que acote el valor absoluto del valor promedio en el volumen de $\bullet_{k}\left(\mathbf{x}^{\prime}, t^{\prime}\right)$; es decir:

$$
\left|f_{V}\left(\mathbf{x}, t^{\prime}\right)\right| \leq g_{2}\left(t^{\prime}\right) \quad \text { y } \quad \frac{1}{T} \int_{t-\frac{T}{2}}^{t+\frac{T}{2}} g_{2}\left(t^{\prime}\right) d t^{\prime}<\infty .
$$


Si se satisfacen ambas condiciones, puede aplicarse nuevamente el Teorema de la convergencia dominada de Lebesgue, para tener:

$$
\lim _{\mathscr{V} \rightarrow \mathscr{D}} \frac{1}{T} \int_{t-\frac{T}{2}}^{t+\frac{T}{2}} f_{V}\left(\mathbf{x}, t^{\prime}\right) d t^{\prime}=\frac{1}{T} \int_{t-\frac{T}{2}}^{t+\frac{T}{2}} \lim _{\mathscr{V} \rightarrow \mathscr{D}} f_{V}\left(\mathbf{x}, t^{\prime}\right) d t^{\prime}
$$

Para el mismo caso de la densidad, $\bullet_{k}=\rho_{g}$ y $\rho_{g}=P_{g} / s^{2}$. Puede tomarse la presión de entrada como una cota superior de $g_{2}$, entonces:

$$
\begin{aligned}
\left|\frac{1}{V} \iiint_{\mathscr{\mathscr { V }}(\mathbf{x})} \rho_{g}\left(\mathbf{x}^{\prime}, t^{\prime}\right) d V\right| & \leq \frac{1}{V} \iiint_{\mathscr{V}(\mathbf{x})} 2.5981 d V \\
& \leq 2.5981 \mathrm{~kg} \cdot \mathrm{m}^{3}
\end{aligned}
$$

que tiene la misma interpretación que la Ec. (A.8).

Sustituyendo la Ec. (A.21) en la Ec. (A.23) y luego en la Ec. (A.17):

$$
\varepsilon_{k}\left\langle\bullet_{k}\right\rangle^{k}(\mathbf{x}, t)=\frac{1}{T} \int_{t-\frac{T}{2}}^{t+\frac{T}{2}} X_{k}\left(\mathbf{x}, t^{\prime}\right) \bullet_{k}\left(\mathbf{x}, t^{\prime}\right) d t^{\prime}
$$

Utilizando la definición de la función indicadora de fase dada en la Ec. (1.6):

$$
\varepsilon_{k}\left\langle\bullet_{k}\right\rangle^{k}(\mathbf{x}, t)=\frac{1}{T} \sum_{i} \int_{t-\frac{T_{k i}}{2}}^{t+\frac{T_{k i}}{2}} \bullet_{k}\left(\mathbf{x}, t^{\prime}\right) d t^{\prime}
$$

Aplicando el límite a $\varepsilon_{k}$ en la Ec. (1.19):

$$
\begin{aligned}
\lim _{\mathscr{Y} \rightarrow \mathscr{D}}\left\langle X_{k}\right\rangle(\mathbf{x}, t)= & \lim _{\mathscr{Y} \rightarrow \mathscr{D}} \varepsilon_{k}(\mathbf{x}, t) \\
& =\lim _{\mathscr{V} \rightarrow \mathscr{D}} \frac{1}{V T} \int_{t-\frac{T}{2}}^{t+\frac{T}{2}} \iiint_{\mathscr{Y}(\mathbf{x})} X_{k}\left(\mathbf{x}^{\prime}, t^{\prime}\right) d V d t^{\prime} .
\end{aligned}
$$

Así como también en la Ec. (A.22), $\left|\frac{1}{V} \iiint_{\mathscr{\mathscr { V }}(\mathbf{x})} X_{k}\left(\mathbf{x}^{\prime}, t^{\prime}\right) d V\right| \leq g_{2}\left(t^{\prime}\right)$ ó $1 \leq g_{2}\left(t^{\prime}\right)$.

Aplicando el Teorema de la convergencia dominada de Lebesgue en la Ec. (A.26) y por los mismos argumentos acerca de la región $k$ : 


$$
\begin{aligned}
\lim _{\mathscr{V} \rightarrow \mathscr{D}} \varepsilon_{k}(\mathbf{x}, t) & =\frac{1}{T} \int_{t-\frac{T}{2}}^{t+\frac{T}{2}} X_{k}\left(\mathbf{x}, t^{\prime}\right) d t^{\prime} \\
& =\frac{\sum_{i} T_{k_{i}}}{T} .
\end{aligned}
$$

La Ec. (A.27) es una fracción temporal y puede interpretarse como la fracción de $T$ en la cual $i$ elementos de la fase $k$, pasan por un determinado punto $\mathbf{x}$.

Sustituyendo la Ec. (A.27) en el miembro izquierdo de la Ec. (A.24) y simplificando, se obtiene el siguiente resultado:

$$
\left\langle\bullet_{k}\right\rangle^{k}(\mathbf{x}, t)=\frac{1}{\sum_{i} T_{k_{i}}} \sum_{i} \int_{t=\frac{T_{k_{i}}}{2}}^{t+\frac{T_{k_{i}}}{2}} \bullet_{k}\left(\mathbf{x}, t^{\prime}\right) d t^{\prime},
$$

donde la región temporal $T_{k_{i}}$ indica el tiempo en el cual la fase $k_{i}$ está presente. Esto significa, si $k$ representa partículas de catalizador, $T_{k_{i}}$ será el tiempo en el cual $i$ partículas pasan por un punto dado. La Ec. (A.28), corresponde al tiempo promedio en un medio multifásico. 
Casos asintóticos del campo de velocidad con promedio espacio-temporal ponderado en la masa

En este trabajo se considera el flujo de partículas en un fluido, el cual se considera compresible, por lo tanto es importante demostrar la ocurrencia de los casos asintóticos anteriores para el promedio ponderado en la masa del campo de velocidad definido en la Ec. (1.25). Para hacer esto, pueden extenderse los resultados encontrados en la Ec. (A.15) y en la Ec. (A.28) para los casos asintóticos del promedio espacio-temporal al definir la función $\bullet_{k}$ como $\rho_{k} \mathrm{y} \rho_{k} \mathbf{v}_{k}$, entonces en el límite cuando $T$ tiende a cero:

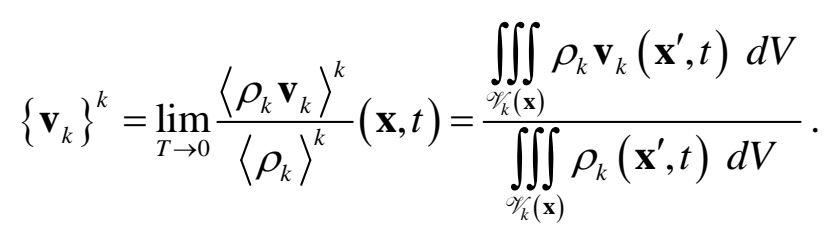

La Ec. (A.29) representa el operador promedio volumétrico intrínseco de fase ponderado en la masa del campo de velocidad.

En el límite cuando la región $\mathscr{Q}$ tiende a $\mathscr{D}$ y el volumen tiende a cero:

$$
\left\{\mathbf{v}_{k}\right\}^{k}=\lim _{\mathscr{D} \rightarrow 0} \frac{\left\langle\rho_{k} \mathbf{v}_{k}\right\rangle^{k}}{\left\langle\rho_{k}\right\rangle^{k}}(\mathbf{x}, t)=\frac{\sum_{i} \int_{t-\frac{T_{i}}{2}}^{t+\frac{T_{k_{i}}}{2}} \rho_{k}\left(\mathbf{x}, t^{\prime}\right) \mathbf{v}_{k}\left(\mathbf{x}, t^{\prime}\right) d t^{\prime}}{\sum_{i} \int_{t-\frac{T_{k_{i}}}{2}}^{t+\frac{T_{k_{i}}}{2}} \rho_{k}\left(\mathbf{x}, t^{\prime}\right) d t^{\prime}},
$$

que es, justamente un operador promedio temporal ponderado en la masa para el campo de velocidad de la fase $k$. 


\section{Apéndice $B$}

\section{Análisis de promedios ponderados de desviaciones}

En este Apéndice estudiaremos cómo se relacionan el promedio espacio-temporal de la desviación de una variable ponderada en la masa $\left\langle\tilde{\bullet}_{k}\right\rangle^{k}$ y el promedio ponderado en la masa de una desviación en el espacio-tiempo $\left\{\bullet_{k}^{\prime}\right\}^{k}$. Para lo cual tomaremos el promedio espacio-temporal de la Ec. (1.27):

$$
\left\langle\bullet_{k}\right\rangle^{k}=\left\langle\left\{\bullet_{k}\right\}^{k}\right\rangle^{k}+\left\langle\tilde{\bullet}_{k}\right\rangle^{k}
$$

utilizando la definición del promedio ponderado en la masa dada en la Ec. (1.25):

$$
\left\langle\bullet_{k}\right\rangle^{k}=\left\langle\frac{\left\langle\rho_{k} \bullet_{k}\right\rangle^{k}}{\left\langle\rho_{k}\right\rangle^{k}}\right\rangle^{k}+\left\langle\tilde{\bullet}_{k}\right\rangle^{k},
$$

podemos sacar del promedio las cantidades promediadas teniendo en cuenta las restricciones de escala siguientes (ver Sección 1.5):

$$
\frac{\left\langle\bullet_{k}^{\prime} \bullet_{k}^{\prime}\right\rangle^{k}}{\left\langle\bullet_{k}\right\rangle^{k}\left\langle\bullet_{k}\right\rangle^{k}} \ll 1, \quad \frac{r_{0}}{L_{\left\langle\bullet^{\prime} \cdot \bullet^{\prime}\right\rangle^{k}}} \ll 1, \quad \frac{T}{T_{\left\langle\bullet^{\prime} \cdot \bullet^{\prime}\right\rangle^{k}}} \ll 1 .
$$

Para tener:

$$
\left\langle\bullet_{k}\right\rangle^{k}=\frac{\left\langle\rho_{k} \bullet_{k}\right\rangle^{k}}{\left\langle\rho_{k}\right\rangle^{k}}+\left\langle\tilde{\bullet}_{k}\right\rangle^{k},
$$

o bien

$$
\left\langle\bullet_{k}\right\rangle^{k}=\left\{\bullet_{k}\right\}^{k}+\left\langle\tilde{\bullet}_{k}\right\rangle^{k}
$$

Por otro lado, tomaremos el promedio ponderado en la masa de la Ec. (1.26)

$$
\left\{\bullet_{k}\right\}^{k}=\left\{\left\langle\bullet_{k}\right\rangle^{k}\right\}^{k}+\left\{\bullet_{k}^{\prime}\right\}^{k}
$$


sustituyendo la definición del promedio ponderado en la masa dada en la Ec. (1.25):

$$
\left\{\bullet_{k}\right\}^{k}=\left\{\frac{\left\langle\rho_{k}\left\langle\bullet_{k}\right\rangle^{k}\right\rangle^{k}}{\left\langle\rho_{k}\right\rangle^{k}}\right\}^{k}+\left\{\bullet_{k}^{\prime}\right\}^{k},
$$

tomando la descomposición espacio-temporal de la densidad y considerando que $\frac{\rho_{k}^{\prime}}{\left\langle\rho_{k}\right\rangle^{k}} \ll 1$, se obtiene

$$
\left\{\bullet_{k}\right\}^{k}=\left\{\frac{\left\langle\rho_{k}\right\rangle^{k}\left\langle\bullet_{k}\right\rangle^{k}}{\left\langle\rho_{k}\right\rangle^{k}}\right\}^{k}+\left\{\bullet_{k}^{\prime}\right\}^{k} \quad \text { o bien } \quad\left\{\bullet_{k}\right\}^{k}=\left\{\left\langle\bullet_{k}\right\rangle^{k}\right\}^{k}+\left\{\bullet_{k}^{\prime}\right\}^{k}
$$

Podemos sacar del promedio las cantidades promediadas teniendo en cuenta las restricciones de escala siguientes (ver Sección 1.5):

$$
\frac{\left\langle\bullet_{k}^{\prime} \bullet_{k}^{\prime}\right\rangle^{k}}{\left\langle\bullet_{k}\right\rangle^{k}\left\langle\bullet_{k}\right\rangle^{k}} \ll 1, \quad \frac{r_{0}}{L_{\left\langle\bullet^{\prime} \bullet_{k^{\prime}}\right\rangle^{k}}} \ll 1, \quad \frac{T}{T_{\left\langle\bullet_{\bullet^{\prime} \cdot \boldsymbol{\bullet}^{\prime}}\right\rangle^{k}}} \ll 1 .
$$

Quedando finalmente:

$$
\begin{aligned}
& \left\{\bullet_{k}\right\}^{k}=\left\langle\bullet_{k}\right\rangle^{k}+\left\{\bullet_{k}^{\prime}\right\}^{k}, \\
& \left\langle\bullet_{k}\right\rangle^{k}=\left\{\bullet_{k}\right\}^{k}-\left\{\bullet_{k}^{\prime}\right\}^{k} .
\end{aligned}
$$

Restando la Ec. (B.8) de la Ec. (B.4), se obtiene la relación:

$$
\left\langle\tilde{\bullet}_{k}\right\rangle^{k}+\left\{\bullet_{k}^{\prime}\right\}^{k}=\mathbf{0}
$$

que implica que:

$$
\left\{\bullet_{k}^{\prime}\right\}^{k}=-\left\langle\tilde{\bullet}_{k}\right\rangle^{k} .
$$




\section{Apéndice $C$}

\section{Ecuaciones promediadas en el espacio-tiempo}

El procedimiento general para obtener las ecuaciones promediadas es el siguiente:

1. Multiplicar la ecuación de balance por $X_{k}$,

2. introducir la función indicadora de fase a las derivadas y

3. promediar usando el operador promedio espacio-temporal definido en la Ec. (1.17).

C.1 Ecuación de conservación de la masa

$$
\frac{\partial}{\partial t}\left\langle X_{k} \rho_{k}\right\rangle+\nabla \cdot\left\langle X_{k} \rho_{k} \mathbf{v}_{k}\right\rangle=\left\langle\rho_{k}\left(\frac{\partial}{\partial t^{\prime}} X_{k}+\mathbf{v}_{k} \cdot \nabla^{\prime} X_{k}\right)\right\rangle .
$$

Ahora, analizaremos el último término dentro del paréntesis:

$$
\frac{\partial}{\partial t^{\prime}} X_{k}+\mathbf{v}_{k} \cdot \nabla^{\prime} X_{k}
$$

para lo cual utilizaremos la Ec. (1.10) en la forma $\frac{\partial X_{k}}{\partial t^{\prime}}=-\mathbf{w}_{k \ell} \cdot \nabla^{\prime} X_{k}$, para obtener

$$
\begin{aligned}
\frac{\partial}{\partial t^{\prime}} X_{k}+\mathbf{v}_{k} \cdot \nabla^{\prime} X_{k} & =-\mathbf{w}_{k \ell} \cdot \nabla^{\prime} X_{k}+\mathbf{v}_{k} \cdot \nabla^{\prime} X_{k} \\
& =\left(\mathbf{v}_{k}-\mathbf{w}_{k \ell}\right) \cdot \nabla^{\prime} X_{k} .
\end{aligned}
$$

Introduciendo la Ec. (C.2) en la Ec. (C.1):

$$
\frac{\partial}{\partial t}\left(\varepsilon_{k}\left\langle\rho_{k}\right\rangle^{k}\right)+\nabla \cdot\left(\varepsilon_{k}\left\langle\rho_{k}\right\rangle^{k}\left\{\mathbf{v}_{k}\right\}^{k}\right)=\Gamma_{k}
$$

o bien, escrita en forma Lagrangiana como:

$$
\frac{D_{k}}{D t}\left(\varepsilon_{k}\left\langle\rho_{k}\right\rangle^{k}\right)+\varepsilon_{k}\left\langle\rho_{k}\right\rangle^{k} \nabla \cdot\left\{\mathbf{v}_{k}\right\}^{k}=\Gamma_{k} .
$$

Donde hemos hecho uso de las siguientes definiciones

$$
\begin{aligned}
& \left\langle\rho_{k}\right\rangle^{k} \doteq\left\langle X_{k} \rho_{k}\right\rangle / \varepsilon_{k}, \\
& \left\{\mathbf{v}_{k}\right\}^{k} \doteq\left\langle X_{k} \rho_{k} \mathbf{v}_{k}\right\rangle / \varepsilon_{k}\left\langle\rho_{k}\right\rangle^{k}, \\
& \Gamma_{k} \doteq\left\langle\rho_{k}\left(\mathbf{v}_{k}-\mathbf{w}_{k \ell}\right) \cdot \nabla^{\prime} X_{k}\right\rangle,
\end{aligned}
$$

siendo $\Gamma_{k}$, el flujo de masa en la interfaz. 
C.2 Ecuación de balance de cantidad de movimiento

$$
\begin{aligned}
\frac{\partial}{\partial t}\left\langle X_{k} \rho_{k} \mathbf{v}_{k}\right\rangle+\nabla \cdot\left\langle X_{k} \rho_{k} \mathbf{v}_{k} \mathbf{v}_{k}\right\rangle-\left\langle X_{k} \rho_{k} \mathbf{g}_{k}\right\rangle-\nabla \cdot\left\langle X_{k} \boldsymbol{\Pi}_{k}\right\rangle= & \\
& \left\langle\rho_{k} \mathbf{v}_{k}\left(\mathbf{v}_{k}-\mathbf{w}_{k \ell}\right) \cdot \nabla^{\prime} X_{k}\right\rangle-\left\langle\nabla^{\prime} X_{k} \cdot \boldsymbol{\Pi}_{k}\right\rangle
\end{aligned}
$$

o bien

$$
\frac{\partial}{\partial t}\left(\varepsilon_{k}\left\langle\rho_{k}\right\rangle^{k}\left\{\mathbf{v}_{k}\right\}^{k}\right)+\nabla \cdot\left\langle X_{k} \rho_{k} \mathbf{v}_{k} \mathbf{v}_{k}\right\rangle-\varepsilon_{k}\left\langle\rho_{k}\right\rangle^{k} \mathbf{g}_{k}-\nabla \cdot\left(\varepsilon_{k}\left\langle\boldsymbol{\Pi}_{k}\right\rangle^{k}\right)=\Gamma_{k} \mathbf{v}_{k i}+\mathbf{M}_{k}
$$

Donde hemos hecho uso de la Ec. (C.2) y de las siguientes definiciones

$$
\begin{aligned}
& \left\{\mathbf{v}_{k}\right\}^{k} \doteq\left\langle X_{k} \rho_{k} \mathbf{v}_{k}\right\rangle / \varepsilon_{k}\left\langle\rho_{k}\right\rangle^{k}, \\
& \left\langle\boldsymbol{\Pi}_{k}\right\rangle^{k} \doteq\left\langle X_{k} \boldsymbol{\Pi}_{k}\right\rangle / \varepsilon_{k}, \\
& \mathbf{g}_{k} \doteq\left\langle X_{k} \rho_{k} \mathbf{g}_{k}\right\rangle / \varepsilon_{k}\left\langle\rho_{k}\right\rangle^{k}, \\
& \mathbf{M}_{k} \doteq-\left\langle\boldsymbol{\Pi}_{k} \cdot \nabla^{\prime} X_{k}\right\rangle, \\
& \Gamma_{k} \mathbf{v}_{k i} \doteq\left\langle\rho_{k} \mathbf{v}_{k}\left(\mathbf{v}_{k}-\mathbf{w}_{k \ell}\right) \cdot \nabla^{\prime} X_{k}\right\rangle,
\end{aligned}
$$

$\mathbf{v}_{k i}$ es la velocidad de la fase $k$ en el límite cuando nos aproximamos a la interfaz desde el interior de la fase $k$. También utilizaremos la siguiente expansión:

$$
\begin{aligned}
\left\langle X_{k} \rho_{k} \mathbf{v}_{k} \mathbf{v}_{k}\right\rangle & =\nabla \cdot\left\langle X_{k} \rho_{k}\left(\tilde{\mathbf{v}}_{k}+\left\{\mathbf{v}_{k}\right\}^{k}\right)\left(\tilde{\mathbf{v}}_{k}+\left\{\mathbf{v}_{k}\right\}^{k}\right)\right\rangle \\
& =\left[\varepsilon_{k}\left(\left\langle\rho_{k}\right\rangle^{k}\left\{\mathbf{v}_{k}\right\}^{k}\left\{\mathbf{v}_{k}\right\}^{k}-\boldsymbol{\Pi}_{k}^{\mathrm{Re}}\right)\right],
\end{aligned}
$$

donde $\boldsymbol{\Pi}_{k}^{\mathrm{Re}} \doteq-\left\langle X_{k} \rho_{k} \tilde{\mathbf{v}}_{k} \tilde{\mathbf{v}}_{k}\right\rangle / \varepsilon_{k}$. Introduciendo la Ec. (C.6) en la Ec. (C.5), obtenemos:

$$
\begin{aligned}
\frac{\partial}{\partial t}\left(\varepsilon_{k}\left\langle\rho_{k}\right\rangle^{k}\left\{\mathbf{v}_{k}\right\}^{k}\right) & +\nabla \cdot\left(\varepsilon_{k}\left\langle\rho_{k}\right\rangle^{k}\left\{\mathbf{v}_{k}\right\}^{k}\left\{\mathbf{v}_{k}\right\}^{k}\right)-\varepsilon_{k}\left\langle\rho_{k}\right\rangle^{k} \mathbf{g}_{k} \\
& -\nabla \cdot\left[\varepsilon_{k}\left(\left\langle\boldsymbol{\Pi}_{k}\right\rangle^{k}+\boldsymbol{\Pi}_{k}^{\mathrm{Re}}\right)\right]=\Gamma_{k} \mathbf{v}_{k i}+\mathbf{M}_{k}
\end{aligned}
$$

o bien, con ayuda de la ecuación de continuidad Ec. (C.3), podemos reescribir la Ec. (C.7) en forma Lagrangiana como:

$$
\varepsilon_{k}\left\langle\rho_{k}\right\rangle^{k} \frac{D_{k}}{D t}\left\{\mathbf{v}_{k}\right\}^{k}-\varepsilon_{k}\left\langle\rho_{k}\right\rangle^{k} \mathbf{g}_{k}-\nabla \cdot\left[\varepsilon_{k}\left(\left\langle\boldsymbol{\Pi}_{k}\right\rangle^{k}+\boldsymbol{\Pi}_{k}^{\mathrm{Re}}\right)\right]=\Gamma_{k}\left(\mathbf{v}_{k i}-\left\{\mathbf{v}_{k}\right\}^{k}\right)+\mathbf{M}_{k}
$$




\section{C.3 Ecuación de balance de energía total}

$$
\begin{aligned}
& \frac{\partial}{\partial t}\left\langle X_{k} \rho_{k}\left(U_{k}+\frac{1}{2} \mathbf{v}_{k} \cdot \mathbf{v}_{k}\right)\right\rangle+\nabla \cdot\left\langle X_{k} \rho_{k} \mathbf{v}_{k}\left(U_{k}+\frac{1}{2} \mathbf{v}_{k} \cdot \mathbf{v}_{k}\right)\right\rangle-\left\langle X_{k} \rho_{k} \mathbf{g}_{k} \cdot \mathbf{v}_{k}\right\rangle \\
& -\nabla \cdot\left\langle X_{k}\left(\mathbf{\Pi}_{k} \cdot \mathbf{v}_{k}\right)\right\rangle+\nabla \cdot\left(\varepsilon_{k}\left\langle\mathbf{q}_{k}\right\rangle^{k}\right)-\varepsilon_{k}\left\langle Q_{k}\right\rangle^{k}=\Gamma_{k}\left[U_{k i}+\frac{1}{2}\left(\mathbf{v}_{k} \cdot \mathbf{v}_{k}\right)_{i}\right]+E_{k}+W_{k i} \cdot(C
\end{aligned}
$$

Donde hemos utilizado la Ec. (C.2) y las siguientes definiciones:

$$
\begin{aligned}
& \left\langle\mathbf{q}_{k}\right\rangle^{k} \doteq\left\langle X_{k} \mathbf{q}_{k}\right\rangle / \varepsilon_{k}, \\
& \left\langle Q_{k}\right\rangle^{k} \doteq\left\langle X_{k} Q_{k}\right\rangle / \varepsilon_{k}, \\
& \left\langle\rho_{k}\left(U_{k}+\frac{1}{2} \mathbf{v}_{k} \cdot \mathbf{v}_{k}\right)\left(\mathbf{v}_{k}-\mathbf{w}_{k \ell}\right) \cdot \nabla^{\prime} X_{k}\right\rangle \doteq \Gamma_{k}\left[U_{k i}+\frac{1}{2}\left(\mathbf{v}_{k} \cdot \mathbf{v}_{k}\right)_{i}\right], \\
& \left\langle\mathbf{q}_{k} \cdot \nabla^{\prime} X_{k}\right\rangle \doteq E_{k}, \\
& \left\langle\nabla^{\prime} X_{k} \cdot\left(\mathbf{\Pi}_{k} \cdot \mathbf{v}_{k}\right)\right\rangle \doteq-W_{k i} .
\end{aligned}
$$

Ahora analizaremos término por término de la Ec. (C.9).

Primer término:

$$
\frac{\partial}{\partial t}\left\langle X_{k} \rho_{k}\left(U_{k}+\frac{1}{2} \mathbf{v}_{k} \cdot \mathbf{v}_{k}\right)\right\rangle=\frac{\partial}{\partial t}\left\langle X_{k} \rho_{k}\left[\tilde{U}_{k}+\left\{U_{k}\right\}^{k}+\frac{1}{2}\left(\tilde{\mathbf{v}}_{k}+\left\{\mathbf{v}_{k}\right\}^{k}\right) \cdot\left(\tilde{\mathbf{v}}_{k}+\left\{\mathbf{v}_{k}\right\}^{k}\right)\right]\right\rangle
$$

o bien

$$
\frac{\partial}{\partial t}\left\langle X_{k} \rho_{k}\left(U_{k}+\frac{1}{2} \mathbf{v}_{k} \cdot \mathbf{v}_{k}\right)\right\rangle=\frac{\partial}{\partial t}\left[\varepsilon_{k}\left\langle\rho_{k}\right\rangle^{k}\left(\left\{U_{k}\right\}^{k}+\frac{1}{2}\left\{\mathbf{v}_{k}\right\}^{k} \cdot\left\{\mathbf{v}_{k}\right\}^{k}+K_{k}^{\mathrm{Re}}\right)\right]
$$

con las definiciones

$$
\begin{aligned}
& \left\langle\rho_{k}\right\rangle^{k} \doteq\left\langle X_{k} \rho_{k}\right\rangle / \varepsilon_{k}, \\
& K_{k}^{\mathrm{Re}} \doteq \frac{1}{2}\left\langle X_{k} \rho_{k} \tilde{\mathbf{v}}_{k} \cdot \tilde{\mathbf{v}}_{k}\right\rangle / \varepsilon_{k}\left\langle\rho_{k}\right\rangle^{k} .
\end{aligned}
$$

El segundo término en la Ec. (C.9) es:

$$
\begin{aligned}
& \nabla \cdot\left\langle X_{k} \rho_{k} \mathbf{v}_{k}\left(U_{k}+\frac{1}{2} \mathbf{v}_{k} \cdot \mathbf{v}_{k}\right)\right\rangle= \\
& \nabla \cdot\left\langle X_{k} \rho_{k}\left(\tilde{\mathbf{v}}_{k}+\left\{\mathbf{v}_{k}\right\}^{k}\right)\left[\tilde{U}_{k}+\left\{U_{k}\right\}^{k}+\frac{1}{2}\left(\tilde{\mathbf{v}}_{k}+\left\{\mathbf{v}_{k}\right\}^{k}\right) \cdot\left(\tilde{\mathbf{v}}_{k}+\left\{\mathbf{v}_{k}\right\}^{k}\right)\right]\right\rangle= \\
& \nabla \cdot\left[\varepsilon_{k}\left\langle\rho_{k}\right\rangle^{k}\left\{\mathbf{v}_{k}\right\}^{k}\left(\left\{U_{k}\right\}^{k}+\frac{1}{2}\left\{\mathbf{v}_{k}\right\}^{k} \cdot\left\{\mathbf{v}_{k}\right\}^{k}+K_{k}^{\mathrm{Re}}\right)+\varepsilon_{k} \mathbf{q}_{k}^{K}+\varepsilon_{k} \mathbf{q}_{k}^{r}-\varepsilon_{k} \boldsymbol{\Pi}_{k}^{\mathrm{Re}} \cdot\left\{\mathbf{v}_{k}\right\}^{k}\right] \cdot(\mathrm{C} .
\end{aligned}
$$


Con las siguientes definiciones:

$$
\begin{aligned}
& \mathbf{q}_{k}^{K} \doteq \frac{1}{2}\left\langle X_{k} \rho_{k} \tilde{\mathbf{v}}_{k} \tilde{\mathbf{v}}_{k} \cdot \tilde{\mathbf{v}}_{k}\right\rangle / \varepsilon_{k}, \\
& \mathbf{q}_{k}^{r} \doteq\left\langle X_{k} \rho_{k} \tilde{\mathbf{v}}_{k} \tilde{U}_{k}\right\rangle / \varepsilon_{k}, \\
& \boldsymbol{\Pi}_{k}^{\mathrm{Re}} \doteq-\left\langle X_{k} \rho_{k} \tilde{\mathbf{v}}_{k} \tilde{\mathbf{v}}_{k}\right\rangle / \varepsilon_{k} .
\end{aligned}
$$

El tercer término es:

$$
\left\langle X_{k} \rho_{k} \mathbf{g}_{k} \cdot\left(\tilde{\mathbf{v}}_{k}+\left\{\mathbf{v}_{k}\right\}^{k}\right)\right\rangle=\varepsilon_{k}\left\langle\rho_{k}\right\rangle^{k} \mathbf{g}_{k} \cdot\left\{\mathbf{v}_{k}\right\}^{k}
$$

El cuarto término es:

$$
\nabla \cdot\left\langle X_{k} \boldsymbol{\Pi}_{k} \cdot\left(\tilde{\mathbf{v}}_{k}+\left\{\mathbf{v}_{k}\right\}^{k}\right)\right\rangle=-\nabla \cdot\left[\varepsilon_{k}\left(\mathbf{q}_{k}^{p}+\mathbf{q}_{k}^{t}\right)\right]+\nabla \cdot\left(\varepsilon_{k}\left\langle\boldsymbol{\Pi}_{k}\right\rangle^{k} \cdot\left\{\mathbf{v}_{k}\right\}^{k}\right) .
$$

Donde

$$
\begin{aligned}
& \mathbf{q}_{k}^{p} \doteq\left\langle X_{k} P_{k} \tilde{\mathbf{v}}_{k}\right\rangle / \varepsilon_{k}, \\
& \mathbf{q}_{k}^{t} \doteq\left\langle X_{k} \boldsymbol{\tau}_{k} \cdot \tilde{\mathbf{v}}_{k}\right\rangle / \varepsilon_{k} .
\end{aligned}
$$

Finalmente, introduciendo las Ecs. (C.10)-(C.13) en la Ec. (C.9) obtenemos:

$$
\begin{aligned}
& \frac{\partial}{\partial t}\left[\varepsilon_{k}\left\langle\rho_{k}\right\rangle^{k}\left(\left\{U_{k}\right\}^{k}+\frac{1}{2}\left\{\mathbf{v}_{k}\right\}^{k} \cdot\left\{\mathbf{v}_{k}\right\}^{k}+K_{k}^{\mathrm{Re}}\right)\right] \\
& +\nabla \cdot\left[\varepsilon_{k}\left\langle\rho_{k}\right\rangle^{k}\left\{\mathbf{v}_{k}\right\}^{k}\left(\left\{U_{k}\right\}^{k}+\frac{1}{2}\left\{\mathbf{v}_{k}\right\}^{k} \cdot\left\{\mathbf{v}_{k}\right\}^{k}+K_{k}^{\mathrm{Re}}\right)\right]= \\
& \nabla \cdot\left[\varepsilon_{k}\left(\left\langle\boldsymbol{\Pi}_{k}\right\rangle^{k}+\boldsymbol{\Pi}_{k}^{\mathrm{Re}}\right) \cdot\left\{\mathbf{v}_{k}\right\}^{k}\right]-\nabla \cdot\left[\varepsilon_{k}\left(\left\langle\mathbf{q}_{k}\right\rangle^{k}+\mathbf{q}_{k}^{\mathrm{Re}}\right)\right] \\
& +\varepsilon_{k}\left\langle Q_{k}\right\rangle^{k}+\varepsilon_{k}\left\langle\rho_{k}\right\rangle^{k} \mathbf{g}_{k} \cdot\left\{\mathbf{v}_{k}\right\}^{k}+E_{k}+W_{k i}+\Gamma_{k}\left[U_{k i}+\frac{1}{2}\left(\mathbf{v}_{k} \cdot \mathbf{v}_{k}\right)_{i}\right] .
\end{aligned}
$$

Donde $\mathbf{q}_{k}^{\mathrm{Re}} \doteq \mathbf{q}_{k}^{p}+\mathbf{q}_{k}^{t}+\mathbf{q}_{k}^{K}+\mathbf{q}_{k}^{r}$.

Podemos reescribir la Ec. (C.14) en forma Lagrangiana con la ecuación de continuidad Ec. (C.3), como:

$$
\begin{aligned}
\varepsilon_{k}\left\langle\rho_{k}\right\rangle^{k} \frac{D_{k}}{D t}\left(\left\{U_{k}\right\}^{k}\right. & \left.+\frac{1}{2}\left\{\mathbf{v}_{k}\right\}^{k} \cdot\left\{\mathbf{v}_{k}\right\}^{k}+K_{k}^{\mathrm{Re}}\right)=\nabla \cdot\left[\varepsilon_{k}\left(\left\langle\boldsymbol{\Pi}_{k}\right\rangle^{k}+\boldsymbol{\Pi}_{k}^{\mathrm{Re}}\right) \cdot\left\{\mathbf{v}_{k}\right\}^{k}\right] \\
& -\nabla \cdot\left[\varepsilon_{k}\left(\left\langle\mathbf{q}_{k}\right\rangle^{k}+\mathbf{q}_{k}^{\mathrm{Re}}\right)\right]+\varepsilon_{k}\left\langle Q_{k}\right\rangle^{k}+\varepsilon_{k}\left\langle\rho_{k}\right\rangle^{k} \mathbf{g}_{k} \cdot\left\{\mathbf{v}_{k}\right\}^{k}+E_{k} \\
& +W_{k i}+\Gamma_{k}\left(U_{k i}-\left\{U_{k}\right\}^{k}+\frac{1}{2}\left[\left(\mathbf{v}_{k} \cdot \mathbf{v}_{k}\right)_{i}-\left\{\mathbf{v}_{k}\right\}^{k} \cdot\left\{\mathbf{v}_{k}\right\}^{k}\right]-K_{k}^{\operatorname{Re}}\right)
\end{aligned}
$$


C.4 Ecuación de balance para la energía interna

$$
\begin{array}{r}
\frac{\partial}{\partial t}\left\langle X_{k} \rho_{k} U_{k}\right\rangle+\nabla \cdot\left\langle X_{k} \rho_{k} U_{k} \mathbf{v}_{k}\right\rangle+\nabla \cdot\left(\varepsilon_{k}\left\langle\mathbf{q}_{k}\right\rangle^{k}\right)-\varepsilon_{k}\left\langle Q_{k}\right\rangle^{k}-\left\langle X_{k} \boldsymbol{\Pi}_{k}: \nabla^{\prime} \mathbf{v}_{k}\right\rangle= \\
\Gamma_{k} U_{k i}+E_{k},
\end{array}
$$

donde hemos utilizado la Ec. (C.2) y las siguientes definiciones:

$$
\begin{aligned}
& \left\langle\mathbf{q}_{k}\right\rangle^{k} \doteq\left\langle X_{k} \mathbf{q}_{k}\right\rangle / \varepsilon_{k}, \\
& \left\langle Q_{k}\right\rangle^{k} \doteq\left\langle X_{k} Q_{k}\right\rangle / \varepsilon_{k}, \\
& \left\langle\rho_{k} U_{k}\left(\mathbf{v}_{k}-\mathbf{w}_{k \ell}\right) \cdot \nabla^{\prime} X_{k}\right\rangle \doteq \Gamma_{k} U_{k i} \\
& \left\langle\mathbf{q}_{k} \cdot \nabla^{\prime} X_{k}\right\rangle \doteq E_{k} .
\end{aligned}
$$

Analizando término por término de la Ec. (C.16).

Primer término:

$$
\frac{\partial}{\partial t}\left\langle X_{k} \rho_{k}\left(\tilde{U}_{k}+\left\{U_{k}\right\}^{k}\right)\right\rangle=\frac{\partial}{\partial t}\left(\varepsilon_{k}\left\langle\rho_{k}\right\rangle^{k}\left\{U_{k}\right\}^{k}\right)
$$

El segundo es:

$$
\begin{aligned}
\nabla \cdot\left\langle X_{k} \rho_{k} U_{k} \mathbf{v}_{k}\right\rangle & =\nabla \cdot\left\langle X_{k} \rho_{k}\left(\tilde{U}_{k}+\left\{U_{k}\right\}^{k}\right)\left(\tilde{\mathbf{v}}_{k}+\left\{\mathbf{v}_{k}\right\}^{k}\right)\right\rangle \\
& =\nabla \cdot\left[\varepsilon_{k}\left(\left\langle\rho_{k}\right\rangle^{k}\left\{U_{k}\right\}^{k}\left\{\mathbf{v}_{k}\right\}^{k}+\mathbf{q}_{k}^{r}\right)\right] .
\end{aligned}
$$

Donde $\mathbf{q}_{k}^{r} \doteq\left\langle X_{k} \rho_{k} \tilde{U}_{k} \tilde{\mathbf{v}}_{k}\right\rangle / \varepsilon_{k}$. Finalmente, introduciendo la Ec. (C.17) y la Ec. (C.18) en la Ec. (C.16) obtenemos:

$$
\begin{aligned}
\frac{\partial}{\partial t}\left(\varepsilon_{k}\left\langle\rho_{k}\right\rangle^{k}\left\{U_{k}\right\}^{k}\right)+\nabla \cdot\left(\varepsilon_{k}\left\langle\rho_{k}\right\rangle^{k}\left\{U_{k}\right\}^{k}\left\{\mathbf{v}_{k}\right\}^{k}\right) & +\nabla \cdot\left[\varepsilon_{k}\left(\left\langle\mathbf{q}_{k}\right\rangle^{k}+\mathbf{q}_{k}^{r}\right)\right]-\varepsilon_{k}\left\langle Q_{k}\right\rangle^{k} \\
& -\left\langle X_{k} \boldsymbol{\Pi}_{k}: \nabla^{\prime} \mathbf{v}_{k}\right\rangle=\Gamma_{k} U_{k i}+E_{k} .
\end{aligned}
$$

Podemos reescribir la Ec. (C.19) en forma Lagrangiana con ayuda de la ecuación de continuidad Ec. (C.3), como:

$$
\begin{array}{r}
\varepsilon_{k}\left\langle\rho_{k}\right\rangle^{k} \frac{D_{k}}{D t}\left\{U_{k}\right\}^{k}+\nabla \cdot\left[\varepsilon_{k}\left(\left\langle\mathbf{q}_{k}\right\rangle^{k}+\mathbf{q}_{k}^{r}\right)\right]-\varepsilon_{k}\left\langle Q_{k}\right\rangle^{k}-\left\langle X_{k} \boldsymbol{\Pi}_{k}: \nabla^{\prime} \mathbf{v}_{k}\right\rangle= \\
\Gamma_{k}\left(U_{k i}-\left\{U_{k}\right\}^{k}\right)+E_{k} .
\end{array}
$$




\section{C.5 Ecuación de balance para la entalpía}

Partiendo de la ecuación de energía térmica local y utilizando la definición termodinámica de la entalpía obtenemos:

$$
\frac{\partial}{\partial t^{\prime}}\left[\rho_{k}\left(H_{k}-\frac{P_{k}}{\rho_{k}}\right)\right]+\nabla^{\prime} \cdot\left[\rho_{k}\left(H_{k}-\frac{P_{k}}{\rho_{k}}\right) \mathbf{v}_{k}\right]+\nabla^{\prime} \cdot \mathbf{q}_{k}-Q_{k}-\boldsymbol{\Pi}_{k}: \nabla^{\prime} \mathbf{v}_{k}=0,
$$

desarrollando términos, introduciendo la relación de comportamiento de Newton para los esfuerzos totales y simplificando (utilizando la notación de la derivada material de la presión),

$$
\frac{\partial}{\partial t^{\prime}}\left(\rho_{k} H_{k}\right)+\nabla^{\prime} \cdot\left(\rho_{k} H_{k} \mathbf{v}_{k}\right)-\frac{D_{k}}{D t^{\prime}} P_{k}+\nabla^{\prime} \cdot \mathbf{q}_{k}-Q_{k}+\boldsymbol{\tau}_{k}: \nabla^{\prime} \mathbf{v}_{k}=0
$$

Multiplicando esta ecuación por la función indicadora de fase e introduciéndola a las derivadas y aplicando el operador promedio espacio-temporal definido en la Ec. (1.17), se obtiene la ecuación de la energía térmica local expresada en términos de la entalpía:

$$
\begin{aligned}
& \frac{\partial}{\partial t}\left(\varepsilon_{k}\left\langle\rho_{k}\right\rangle^{k}\left\{H_{k}\right\}^{k}\right)+\nabla \cdot\left\langle X_{k} \rho_{k} H_{k} \mathbf{v}_{k}\right\rangle-\frac{D_{k}}{D t}\left(\varepsilon_{k}\left\langle P_{k}\right\rangle^{k}\right)+\nabla \cdot\left(\varepsilon_{k}\left\langle\mathbf{q}_{k}\right\rangle^{k}\right) \\
& -\left\langle\left(\rho_{k} H_{k}-P_{k}\right) \frac{D_{k}}{D t^{\prime}} X_{k}\right\rangle-\left\langle\mathbf{q}_{k} \cdot \nabla^{\prime} X_{k}\right\rangle-\varepsilon_{k}\left\langle Q_{k}\right\rangle^{k}+\left\langle X_{k} \boldsymbol{\tau}_{k}: \nabla^{\prime} \mathbf{v}_{k}\right\rangle=0 .
\end{aligned}
$$

Analizando el segundo término de la Ec. (C.21),

$$
\begin{aligned}
\nabla \cdot\left\langle X_{k} \rho_{k} H_{k} \mathbf{v}_{k}\right\rangle & =\nabla \cdot\left\langle X_{k} \rho_{k}\left(\tilde{H}_{k}+\left\{H_{k}\right\}^{k}\right)\left(\tilde{\mathbf{v}}_{k}+\left\{\mathbf{v}_{k}\right\}^{k}\right)\right\rangle \\
& =\nabla \cdot\left[\varepsilon_{k}\left(\left\langle\rho_{k}\right\rangle^{k}\left\{H_{k}\right\}^{k}\left\{\mathbf{v}_{k}\right\}^{k}+\hat{\mathbf{q}}_{k}^{r}\right)\right] .
\end{aligned}
$$

Analizando también el quinto término,

$$
-\left\langle\left(\rho_{k} H_{k}-P_{k}\right) \frac{D_{k}}{D t^{\prime}} X_{k}\right\rangle=-\left\langle\rho_{k} H_{k} \frac{D_{k}}{D t^{\prime}} X_{k}\right\rangle+\left\langle P_{k} \frac{D_{k}}{D t^{\prime}} X_{k}\right\rangle
$$


el último término es una integral de superficie en la cual podemos utilizar la condición de frontera:

$$
P_{k}=P_{k i} \quad \text { en } \quad \mathscr{O}_{k \ell}(\mathbf{x}, t)
$$

y considerando la aproximación:

$$
P_{k i} \sim\left\langle P_{k}\right\rangle_{i}
$$

podemos escribir al sustituir $\partial X_{k} / \partial t^{\prime}$ de la Ec. (1.8)

$$
-\left\langle\left(\rho_{k} H_{k}-P_{k}\right) \frac{D_{k}}{D t^{\prime}} X_{k}\right\rangle=-H_{k i} \Gamma_{k}+\left\langle P_{k}\right\rangle_{i} \frac{D_{k}}{D t} \varepsilon_{k}
$$

donde se ha sustituido la definición:

$$
\left\langle\rho_{k} H_{k}\left(\mathbf{v}_{k}-\mathbf{w}_{k \ell}\right) \cdot \nabla^{\prime} X_{k}\right\rangle \doteq H_{k i} \Gamma_{k}
$$

Sustituyendo la Ec. (C.22) y la Ec. (C.23) en la Ec. (C.21) y reagrupando, se tiene que

$$
\begin{aligned}
& \frac{\partial}{\partial t}\left(\varepsilon_{k}\left\langle\rho_{k}\right\rangle^{k}\left\{H_{k}\right\}^{k}\right)+\nabla \cdot\left[\varepsilon_{k}\left(\left\langle\rho_{k}\right\rangle^{k}\left\{H_{k}\right\}^{k}\left\{\mathbf{v}_{k}\right\}^{k}\right)\right]+\nabla \cdot\left[\varepsilon_{k}\left(\left\langle\mathbf{q}_{k}\right\rangle^{k}+\hat{\mathbf{q}}_{k}^{r}\right)\right] \\
& -\varepsilon_{k}\left\langle Q_{k}\right\rangle^{k}-\varepsilon_{k} \frac{D_{k}}{D t}\left\langle P_{k}\right\rangle^{k}-\left(\left\langle P_{k}\right\rangle^{k}-\left\langle P_{k}\right\rangle_{i}\right) \frac{D_{k}}{D t} \varepsilon_{k}-H_{k i} \Gamma_{k}-E_{k}+\left\langle X_{k} \boldsymbol{\tau}_{k}: \nabla^{\prime} \mathbf{v}_{k}\right\rangle=0,
\end{aligned}
$$

donde

$$
\begin{aligned}
& \hat{\mathbf{q}}_{k}^{r} \doteq\left\langle X_{k} \rho_{k} \tilde{H}_{k} \tilde{\mathbf{v}}_{k}\right\rangle / \varepsilon_{k}, \\
& \left\langle\mathbf{q}_{k} \cdot \nabla^{\prime} X_{k}\right\rangle \doteq E_{k} .
\end{aligned}
$$

Desarrollando las derivadas en los dos primeros términos de la Ec. (C.24) y sustituyendo la ecuación de continuidad de campo promedio, tenemos:

$$
\begin{aligned}
& \varepsilon_{k}\left\langle\rho_{k}\right\rangle^{k} \frac{D_{k}}{D t}\left\{H_{k}\right\}^{k}+\nabla \cdot\left[\varepsilon_{k}\left(\left\langle\mathbf{q}_{k}\right\rangle^{k}+\hat{\mathbf{q}}_{k}^{r}\right)\right]-\varepsilon_{k}\left\langle Q_{k}\right\rangle^{k}-\varepsilon_{k} \frac{D_{k}}{D t}\left\langle P_{k}\right\rangle^{k} \\
& -\left(\left\langle P_{k}\right\rangle^{k}-\left\langle P_{k}\right\rangle_{i}\right) \frac{D_{k}}{D t} \varepsilon_{k}+\Gamma_{k}\left(\left\{H_{k}\right\}^{k}-H_{k i}\right)-E_{k}+\left\langle X_{k} \boldsymbol{\tau}_{k}: \nabla^{\prime} \mathbf{v}_{k}\right\rangle=0 .
\end{aligned}
$$


C.6 Ecuación de balance para la entropía

$$
\begin{array}{r}
\frac{\partial}{\partial t}\left\langle X_{k} \rho_{k} S_{k}\right\rangle+\nabla \cdot\left\langle X_{k} \rho_{k} S_{k} \mathbf{v}_{k}\right\rangle+\nabla \cdot\left(\varepsilon_{k}\left\langle\frac{\mathbf{q}_{k}}{T_{k}}\right\rangle^{k}\right)-\varepsilon_{k}\left\langle\frac{Q_{k}}{T_{k}}\right\rangle^{k}= \\
\left\langle\nabla^{\prime} X_{k} \cdot\left(\frac{\mathbf{q}_{k}}{T_{k}}\right)\right\rangle+\Gamma_{k} S_{k i}+\left\langle X_{k} \Delta_{k}\right\rangle,
\end{array}
$$

donde hemos utilizado la Ec. (C.2) y las definiciones:

$$
\begin{aligned}
& \left\langle\frac{\mathbf{q}_{k}}{T_{k}}\right\rangle^{k} \doteq\left\langle X_{k} \frac{\mathbf{q}_{k}}{T_{k}}\right\rangle / \varepsilon_{k}, \\
& \left\langle\frac{Q_{k}}{T_{k}}\right\rangle^{k} \doteq\left\langle X_{k} \frac{Q_{k}}{T_{k}}\right\rangle / \varepsilon_{k}, \\
& \left\langle\rho_{k} S_{k}\left(\mathbf{v}_{k}-\mathbf{w}_{k \ell}\right) \cdot \nabla^{\prime} X_{k}\right\rangle \doteq \Gamma_{k} S_{k i} .
\end{aligned}
$$

Analizando término por término de la Ec. (C.26), el primer término es:

$$
\frac{\partial}{\partial t}\left\langle X_{k} \rho_{k} S_{k}\right\rangle=\frac{\partial}{\partial t}\left\langle X_{k} \rho_{k}\left(\tilde{S}_{k}+\left\{S_{k}\right\}^{k}\right)\right\rangle=\frac{\partial}{\partial t}\left(\varepsilon_{k}\left\langle\rho_{k}\right\rangle^{k}\left\{S_{k}\right\}^{k}\right)
$$

El argumento de la divergencia en el segundo término:

$$
\left\langle X_{k} \rho_{k}\left(\tilde{S}_{k}+\left\{S_{k}\right\}^{k}\right)\left(\tilde{\mathbf{v}}_{k}+\left\{\mathbf{v}_{k}\right\}^{k}\right)\right\rangle=\left(\varepsilon_{k}\left\langle\rho_{k}\right\rangle^{k}\left\{\tilde{S}_{k} \tilde{\mathbf{v}}_{k}\right\}^{k}\right)+\left(\varepsilon_{k}\left\langle\rho_{k}\right\rangle^{k}\left\{S_{k}\right\}^{k}\left\{\mathbf{v}_{k}\right\}^{k}\right) .
$$

Finalmente, introduciendo la Ec. (C.27) y la Ec. (C.28) en la Ec. (C.26) obtenemos:

$$
\begin{aligned}
& \frac{\partial}{\partial t}\left(\varepsilon_{k}\left\langle\rho_{k}\right\rangle^{k}\left\{S_{k}\right\}^{k}\right)+\nabla \cdot\left(\varepsilon_{k}\left\langle\rho_{k}\right\rangle^{k}\left\{\tilde{S}_{k} \tilde{\mathbf{v}}_{k}\right\}^{k}\right)+\nabla \cdot\left(\varepsilon_{k}\left\langle\rho_{k}\right\rangle^{k}\left\{S_{k}\right\}^{k}\left\{\mathbf{v}_{k}\right\}^{k}\right) \\
& +\nabla \cdot\left(\varepsilon_{k}\left\langle\frac{\mathbf{q}_{k}}{T_{k}}\right\rangle^{k}\right)-\varepsilon_{k}\left\langle\frac{Q_{k}}{T_{k}}\right\rangle^{k}=\left\langle\nabla^{\prime} X_{k} \cdot\left(\frac{\mathbf{q}_{k}}{T_{k}}\right)\right\rangle+\Gamma_{k} S_{k i}+\left\langle X_{k} \Delta_{k}\right\rangle .
\end{aligned}
$$

Donde $\left\{\tilde{S}_{k} \tilde{\mathbf{v}}_{k}\right\}^{k} \doteq\left\langle X_{k} \rho_{k} \tilde{S}_{k} \tilde{\mathbf{v}}_{k}\right\rangle / \varepsilon_{k}\left\langle\rho_{k}\right\rangle^{k}$. Podemos reescribir la Ec. (C.29) en forma Lagrangiana con ayuda de la ecuación de continuidad Ec. (C.3), como:

$$
\begin{array}{r}
\varepsilon_{k}\left\langle\rho_{k}\right\rangle^{k} \frac{D_{k}}{D t}\left\{S_{k}\right\}^{k}+\nabla \cdot\left(\varepsilon_{k}\left\langle\rho_{k}\right\rangle^{k}\left\{\tilde{S}_{k} \tilde{\mathbf{v}}_{k}\right\}^{k}\right)+\nabla \cdot\left(\varepsilon_{k}\left\langle\frac{\mathbf{q}_{k}}{T_{k}}\right\rangle^{k}\right)-\varepsilon_{k}\left\langle\frac{Q_{k}}{T_{k}}\right\rangle^{k}= \\
\left\langle\nabla^{\prime} X_{k} \cdot\left(\frac{\mathbf{q}_{k}}{T_{k}}\right)\right\rangle+\Gamma_{k}\left(S_{k i}-\left\{S_{k}\right\}^{k}\right)+\left\langle X_{k} \Delta_{k}\right\rangle .
\end{array}
$$




\section{Apéndice $D$}

\section{Derivación del conjunto de ecuaciones representado en la Ec. (2.40)}

En la desigualdad de la entropía hay dos tipos de presiones: $P_{k s} \mathrm{y}\left\langle P_{k}\right\rangle^{k}$. Estudiemos su diferencia:

$$
P_{k s}-\left\langle P_{k}\right\rangle^{k}=P_{k}\left(\left\{S_{k}\right\}^{k},\left\langle\rho_{k}\right\rangle^{k}\right)-\left\langle P_{k}\right\rangle^{k}
$$

Considere la siguiente obtención de la presión promedio, para lo cual expandiremos el primer sumando del miembro derecho de la Ec. (D.1) mediante una serie de Taylor alrededor del punto $\left(\left\{S_{k}\right\}^{k},\left\langle\rho_{k}\right\rangle^{k}\right)$,

$$
\begin{aligned}
P_{k}=P_{k}\left(S_{k}, \rho_{k}\right)=P_{k}\left(\left\{S_{k}\right\}^{k}+\tilde{S}_{k},\left\langle\rho_{k}\right\}^{k}+\rho_{k}^{\prime}\right) & \\
=P_{k}\left(\left\{S_{k}\right\}^{k},\left\langle\rho_{k}\right\rangle^{k}\right) & +\left[\left(\frac{\partial P_{k}}{\partial S_{k}}\right)_{\rho_{k}}\right]_{\left\{S_{k}\right\}^{k},\left\{\rho_{k}\right\rangle^{k}}\left(S_{k}-\left\{S_{k}\right\}^{k}\right) \\
& +\left[\left(\frac{\partial P_{k}}{\partial \rho_{k}}\right)_{S_{k}}\right]_{\left\{S_{k}\right\}^{k},\left\{\rho_{k}\right\rangle^{k}}\left(\rho_{k}-\left\langle\rho_{k}\right\rangle^{k}\right)+\cdots
\end{aligned}
$$

Truncando la serie a primer orden y promediando en el espacio-tiempo ambos lados:

$$
\begin{aligned}
\left\langle P_{k}\right\rangle^{k} & \approx P_{k s}+\left[\left(\frac{\partial P_{k}}{\partial S_{k}}\right)_{\rho_{k}}\right]_{\left\{S_{k}\right\}^{k},\left\{\rho_{k}\right\rangle^{k}}\left\langle\tilde{S}_{k}\right\rangle^{k}+\left[\left(\frac{\partial P_{k}}{\partial \rho_{k}}\right)_{S_{k}}\right]_{\left\{S_{k}\right\}^{k},\left\{\rho_{k}\right\}^{k}}\left\langle\rho_{k}^{\prime}\right\rangle^{k} \\
& \approx P_{k s}+\left[\left(\frac{\partial P_{k}}{\partial S_{k}}\right)_{\rho_{k}}\right]_{\left\{S_{k}\right\}^{k},\left\langle\rho_{k}\right\rangle^{k}}\left\langle\tilde{S}_{k}\right\rangle^{k} .
\end{aligned}
$$


Analizando ahora el término: $\left\langle\tilde{S}_{k}\right\rangle^{k}$

$$
\begin{aligned}
& \left\langle\tilde{S}_{k}\right\rangle^{k}=\left\langle S_{k}-\left\{S_{k}\right\}^{k}\right\rangle^{k} \\
& =\left\langle S_{k}-\frac{\left\langle X_{k} \rho_{k} S_{k}\right\rangle}{\left\langle X_{k} \rho_{k}\right\rangle}\right\rangle^{k} \\
& =\left\langle S_{k}-\frac{\left\langle X_{k}\left(\left\langle\rho_{k}\right\rangle^{k}+\rho_{k}^{\prime}\right) S_{k}\right\rangle}{\left\langle X_{k} \rho_{k}\right\rangle}\right\rangle^{k} \\
& =\left\langle S_{k}\right\rangle^{k}-\left\langle\frac{\left\langle\rho_{k}\right\rangle^{k} \varepsilon_{k}\left\langle S_{k}\right\rangle^{k}}{\varepsilon_{k}\left\langle\rho_{k}\right\rangle^{k}}\right\rangle^{k}-\left\langle\frac{\left\langle X_{k} \rho_{k}^{\prime} S_{k}\right\rangle}{\varepsilon_{k}\left\langle\rho_{k}\right\rangle^{k}}\right\rangle^{k} \\
& =\left\langle S_{k}\right\rangle^{k}-\left\langle S_{k}\right\rangle^{k}-\frac{\left\langle X_{k} \rho_{k}^{\prime} S_{k}\right\rangle}{\varepsilon_{k}\left\langle\rho_{k}\right\rangle^{k}} \\
& =-\frac{\left\langle X_{k} \rho_{k}^{\prime}\left(\left\{S_{k}\right\}^{k}+\tilde{S}_{k}\right)\right\rangle}{\varepsilon_{k}\left\langle\rho_{k}\right\rangle^{k}} \\
& =-\frac{\varepsilon_{k}\left\langle\rho_{k}^{\prime}\right\rangle^{k}\left\{S_{k}\right\}^{k}}{\varepsilon_{k}\left\langle\rho_{k}\right\rangle^{k}}-\frac{\left\langle X_{k} \rho_{k}^{\prime} \tilde{S}_{k}\right\rangle}{\varepsilon_{k}\left\langle\rho_{k}\right\rangle^{k}} \\
& \left\langle\tilde{S}_{k}\right\rangle^{k}=-\frac{\left\langle\rho_{k}^{\prime} \tilde{S}_{k}\right\rangle^{k}}{\left\langle\rho_{k}\right\rangle^{k}} .
\end{aligned}
$$

Esta ecuación nos permite representar la diferencia entre los dos tipos de presión en la Ec. (D.1) como:

$$
P_{k s}-\left\langle P_{k}\right\rangle^{k}=\left[\left(\frac{\partial P_{k}}{\partial S_{k}}\right)_{\rho_{k}}\right]_{\left\{S_{k}\right\}^{k},\left\langle\rho_{k}\right\rangle^{k}} \frac{\left\langle\rho_{k}^{\prime} \tilde{S}_{k}\right\rangle^{k}}{\left\langle\rho_{k}\right\rangle^{k}} .
$$

Por lo que la diferencia $P_{k s}-\left\langle P_{k}\right\rangle^{k}$ es cuadrática en las desviaciones y podemos aproximar su diferencia a primer orden por cero:

$$
P_{k s}-\left\langle P_{k}\right\rangle^{k} \approx 0 \text {. }
$$


La temperatura del fluido en la ecuación de la entropía promedio aparece en dos formas también: $\left\{T_{k}\right\}^{k}$ y $T_{k s}$. Estudiemos su diferencia, en forma similar a como lo hicimos con la presión:

$$
\begin{aligned}
& T_{k}=T_{k}\left(S_{k}, \rho_{k}\right)=T_{k}\left(\left\{S_{k}\right\}^{k}+\tilde{S}_{k},\left\langle\rho_{k}\right\rangle^{k}+\rho_{k}^{\prime}\right) \\
&=T_{k}\left(\left\{S_{k}\right\}^{k},\left\langle\rho_{k}\right\rangle^{k}\right)+ {\left[\left(\frac{\partial T_{k}}{\partial S_{k}}\right)_{\rho_{k}}\right]_{\left\{S_{k}\right\}^{k},\left\{\rho_{k}\right\rangle^{k}}\left(S_{k}-\left\{S_{k}\right\}^{k}\right) } \\
&+ {\left[\left(\frac{\partial T_{k}}{\partial \rho_{k}}\right)_{S_{k}}\right]_{\left\{S_{k}\right\}^{k},\left\langle\rho_{k}\right\rangle^{k}}\left(\rho_{k}-\left\langle\rho_{k}\right\rangle^{k}\right)+\cdots }
\end{aligned}
$$

Truncando la serie a primer orden y tomando el promediando ponderado en la masa de ambos lados:

$$
\begin{aligned}
\left\{T_{k}\right\}^{k} & \approx T_{k s}+\left[\left(\frac{\partial T_{k}}{\partial S_{k}}\right)_{\rho_{k}}\right]_{\left\{S_{k}\right\}^{k},\left\{\rho_{k}\right\rangle^{k}}\left\{\tilde{S}_{k}\right\}^{k}+\left[\left(\frac{\partial T_{k}}{\partial \rho_{k}}\right)_{S_{k}}\right]_{\left\{S_{k}\right\}^{k},\left\{\rho_{k}\right\rangle^{k}}\left\{\rho_{k}^{\prime}\right\}^{k} \\
& \approx T_{k s}+\left[\left(\frac{\partial T_{k}}{\partial \rho_{k}}\right)_{S_{k}}\right]_{\left\{S_{k}\right\}^{k},\left\langle\rho_{k}\right\rangle^{k}}\left\{\rho_{k}^{\prime}\right\}^{k} .
\end{aligned}
$$

Como ya se demostró anteriormente:

$$
\left\{\rho_{k}^{\prime}\right\}^{k}=O\left(\frac{\left\langle\rho_{k}^{\prime} \rho_{k}^{\prime}\right\rangle^{k}}{\left\langle\rho_{k}\right\rangle^{k}}\right)
$$

Por lo tanto, $T_{k s}$ puede remplazarse por $\left\{T_{k}\right\}^{k}$. 
La generación de calor en el volumen $Q_{k}$ aparece dos veces en la ecuación de la entropía: $\left\langle X_{k} Q_{k}\right\rangle$ y $\left\langle X_{k} Q_{k} / T_{k}\right\rangle$. Analicemos la diferencia (escrita aquí en forma adecuada para que sean dimensionalmente comparables) entre ambas:

$$
\begin{aligned}
\left\langle X_{k} Q_{k}\right\rangle-\left\{T_{k}\right\}^{k}\left\langle X_{k} Q_{k} / T_{k}\right\rangle & =\left\{T_{k}\right\}^{k}\left\langle X_{k} Q_{k}\left(\frac{1}{\left\{T_{k}\right\}^{k}}-\frac{1}{T_{k}}\right)\right\rangle \\
& =\varepsilon_{k}\left\{T_{k}\right\}^{k}\left\langle\left(\left\langle Q_{k}\right\rangle^{k}+Q_{k}^{\prime}\right) \frac{T_{k}-\left\{T_{k}\right\}^{k}}{T_{k}\left\{T_{k}\right\}^{k}}\right\rangle^{k} \\
& =\varepsilon_{k}\left[\left\langle Q_{k}\right\rangle^{k}\left\langle\frac{\tilde{T}_{k}}{T_{k}}\right\rangle^{k}+\left\langle\frac{Q_{k}^{\prime} \tilde{T_{k}}}{T_{k}}\right\rangle^{k}\right] .
\end{aligned}
$$

Por otro lado,

$$
\begin{aligned}
\left\langle\tilde{T}_{k}\right\rangle^{k} & =\left\langle T_{k}-\left\{T_{k}\right\}^{k}\right\rangle^{k} \\
& =\left\langle T_{k}-\frac{\left\langle X_{k} \rho_{k} T_{k}\right\rangle}{\left\langle X_{k} \rho_{k}\right\rangle}\right\rangle^{k} \\
& =\left\langle T_{k}-\frac{\left\langle X_{k}\left(\left\langle\rho_{k}\right\rangle^{k}+\rho_{k}^{\prime}\right) T_{k}\right\rangle}{\left\langle X_{k} \rho_{k}\right\rangle}\right\rangle \\
& =\left\langle T_{k}\right\rangle^{k}-\left\langle\frac{\left\langle\rho_{k}\right\rangle^{k} \varepsilon_{k}\left\langle T_{k}\right\rangle^{k}}{\varepsilon_{k}\left\langle\rho_{k}\right\rangle^{k}}\right\rangle^{k}-\left\langle\frac{\left\langle X_{k} \rho_{k}^{\prime} T_{k}\right\rangle}{\varepsilon_{k}\left\langle\rho_{k}\right\rangle^{k}}\right\rangle^{k} \\
& =\left\langle T_{k}\right\rangle^{k}-\left\langle T_{k}\right\rangle^{k}-\frac{\left\langle X_{k} \rho_{k}^{\prime} T_{k}\right\rangle}{\varepsilon_{k}\left\langle\rho_{k}\right\rangle^{k}} \\
& =-\frac{\left\langle X_{k} \rho_{k}^{\prime}\left(\left\{T_{k}\right\}^{k}+\tilde{T}_{k}\right)\right\rangle}{\varepsilon_{k}\left\langle\rho_{k}\right\rangle^{k}} \\
& =-\frac{\varepsilon_{k}\left\langle\rho_{k}^{\prime}\right\rangle^{k}\left\{T_{k}\right\}^{k}}{\varepsilon_{k}\left\langle\rho_{k}\right\rangle^{k}}-\frac{\left\langle X_{k} \rho_{k}^{\prime} \tilde{T}_{k}\right\rangle}{\varepsilon_{k}\left\langle\rho_{k}\right\rangle^{k}} \\
& =-\frac{\left\langle\rho_{k}^{\prime} \tilde{T}_{k}\right\rangle^{k}}{\left\langle\rho_{k}\right\rangle^{k}} .
\end{aligned}
$$


Entonces podemos reescribir la Ec. (D.9) como:

$$
\left\langle X_{k} Q_{k}\right\rangle-\left\{T_{k}\right\}^{k}\left\langle X_{k} Q_{k} / T_{k}\right\rangle=\varepsilon_{k}\left[-\left\langle Q_{k}\right\rangle^{k} \frac{\left\langle\rho_{k}^{\prime} \tilde{T}_{k}\right\rangle^{k}}{\left\langle\rho_{k}\right\rangle^{k}\left\langle T_{k}\right\rangle^{k}}+\left\langle\frac{Q_{k}^{\prime} \tilde{T}_{k}}{T_{k}}\right\rangle^{k}\right]
$$

y entonces $\left\langle X_{k} Q_{k}\right\rangle-\left\{T_{k}\right\}^{k}\left\langle X_{k} Q_{k} / T_{k}\right\rangle \approx 0$, a primer orden en las desviaciones.

Aunque no es inmediatamente obvio, debe haber alguna relación entre los flujos de energía interna turbulenta $\mathbf{q}_{k}^{r}$ y la ecuación de la entropía, pero en formas algo diferentes.

Es útil examinar la diferencia entre los términos donde esto ocurre.

$$
\begin{aligned}
\left\{T_{k}\right\}^{k} \nabla & \cdot\left(\varepsilon_{k}\left\langle\rho_{k}\right\rangle^{k}\left\{\tilde{S}_{k} \tilde{\mathbf{v}}_{k}\right\}^{k}\right)-\nabla \cdot\left(\varepsilon_{k} \mathbf{q}_{k}^{r}\right)= \\
& =\nabla \cdot\left[\varepsilon_{k}\left(\left\{T_{k}\right\}^{k}\left\langle\rho_{k}\right\rangle^{k}\left\{\tilde{S}_{k} \tilde{\mathbf{v}}_{k}\right\}^{k}-\mathbf{q}_{k}^{r}\right)\right]-\varepsilon_{k}\left\langle\rho_{k}\right\rangle^{k}\left\{\tilde{S}_{k} \tilde{\mathbf{v}}_{k}\right\}^{k} \cdot \nabla\left\{T_{k}\right\}^{k} \\
& =\nabla \cdot\left[\varepsilon_{k}\left\langle\rho_{k}\right\rangle^{k}\left(\left\{T_{k}\right\}^{k}\left\{\tilde{S}_{k} \tilde{\mathbf{v}}_{k}\right\}^{k}-\frac{\left\langle X_{k} \rho_{k} \tilde{\mathbf{v}}_{k} \tilde{U}_{k}\right\rangle}{\left\langle X_{k} \rho_{k}\right\rangle}\right)\right]-\varepsilon_{k}\left\langle\rho_{k}\right\rangle^{k}\left\{\tilde{S}_{k} \tilde{\mathbf{v}}_{k}\right\}^{k} \cdot \nabla\left\{T_{k}\right\}^{k} \\
& =\nabla \cdot\left[\varepsilon_{k}\left\langle\rho_{k}\right\rangle^{k}\left(\left\{T_{k}\right\}^{k}\left\{\tilde{S}_{k} \tilde{\mathbf{v}}_{k}\right\}^{k}-\left\{\tilde{\mathbf{v}}_{k} \tilde{U}_{k}\right\}^{k}\right)\right]-\varepsilon_{k}\left\langle\rho_{k}\right\rangle^{k}\left\{\tilde{S}_{k} \tilde{\mathbf{v}}_{k}\right\}^{k} \cdot \nabla\left\{T_{k}\right\}^{k} .
\end{aligned}
$$

Utilizando la relación de Gibbs:

$$
d U_{k}=T_{k} d S_{k}-\frac{P_{k}}{\rho_{k}^{2}} d \rho_{k},
$$

$\varepsilon_{k}\left\langle\rho_{k}\right\rangle^{k}\left\{\tilde{\mathbf{v}}_{k} \tilde{U}_{k}\right\}^{k}$ se puede representar como: 


$$
\begin{aligned}
\varepsilon_{k}\left\langle\rho_{k}\right\rangle^{k}\left\{\tilde{\mathbf{v}}_{k} \tilde{U}_{k}\right\}^{k} & =\varepsilon_{k}\left\langle\rho_{k}\right\rangle^{k}\left\{\left\{T_{k}\right\}^{k} \tilde{\mathbf{v}}_{k} \tilde{S}_{k}-\frac{P_{k s}}{\left\langle\rho_{k}\right\rangle^{k}\left\langle\rho_{k}\right\rangle^{k}} \tilde{\mathbf{v}}_{k} \rho_{k}^{\prime}\right\}^{k} \\
& =\varepsilon_{k}\left\langle\rho_{k}\right\rangle^{k}\left[\left\{T_{k}\right\}^{k}\left\{\tilde{\mathbf{v}}_{k} \tilde{S}_{k}\right\}^{k}-\frac{P_{k s}}{\left\langle\rho_{k}\right\rangle^{k}\left\langle\rho_{k}\right\rangle^{k}}\left\{\tilde{\mathbf{v}}_{k} \rho_{k}^{\prime}\right\}^{k}\right] \\
& =\varepsilon_{k}\left\langle\rho_{k}\right\rangle^{k}\left[\left\{T_{k}\right\}^{k}\left\{\tilde{\mathbf{v}}_{k} \tilde{S}_{k}\right\}^{k}-\frac{P_{k s}}{\left\langle\rho_{k}\right\rangle^{k}\left\langle\rho_{k}\right\rangle^{k}} \frac{\partial \rho_{k}}{\partial P_{k}}\left\{\tilde{\mathbf{v}}_{k} P_{k}^{\prime}\right\}^{k}\right]
\end{aligned}
$$

por lo que

$$
\begin{aligned}
\left\{T_{k}\right\}^{k} \nabla \cdot\left(\varepsilon_{k}\left\langle\rho_{k}\right\rangle^{k}\left\{\tilde{S}_{k} \tilde{\mathbf{v}}_{k}\right\}^{k}\right)-\nabla \cdot\left(\varepsilon_{k} \mathbf{q}_{k}^{r}\right)= \\
=\nabla \cdot\left[\varepsilon_{k}\left\langle\rho_{k}\right\rangle^{k}\left(\left\{T_{k}\right\}^{k}\left\{\tilde{S}_{k} \tilde{\mathbf{v}}_{k}\right\}^{k}-\left[\left\{T_{k}\right\}^{k}\left\{\tilde{\mathbf{v}}_{k} \tilde{S}_{k}\right\}^{k}-\frac{P_{k s}}{\left\langle\rho_{k}\right\rangle^{k}\left\langle\rho_{k}\right\rangle^{k}} \frac{\partial \rho_{k}}{\partial P_{k}}\left\{\tilde{\mathbf{v}}_{k} P_{k}^{\prime}\right\}^{k}\right]\right)\right] \\
\quad-\varepsilon_{k}\left\langle\rho_{k}\right\rangle^{k}\left\{\tilde{S}_{k} \tilde{\mathbf{v}}_{k}\right\}^{k} \cdot \nabla\left\{T_{k}\right\}^{k} \\
=\nabla \cdot\left[\varepsilon_{k} \frac{P_{k s}}{\left\langle\rho_{k}\right\rangle^{k}} \frac{\partial \rho_{k}}{\partial P_{k}}\left\{\tilde{\mathbf{v}}_{k} P_{k}^{\prime}\right\}^{k}\right]-\varepsilon_{k}\left\langle\rho_{k}\right\rangle^{k}\left\{\tilde{S}_{k} \tilde{\mathbf{v}}_{k}\right\}^{k} \cdot \nabla\left\{T_{k}\right\}^{k}
\end{aligned}
$$

que puede aproximarse por

$$
\left\{T_{k}\right\}^{k} \nabla \cdot\left(\varepsilon_{k}\left\langle\rho_{k}\right\rangle^{k}\left\{\tilde{S}_{k} \tilde{\mathbf{v}}_{k}\right\}^{k}\right)-\nabla \cdot\left(\varepsilon_{k} \mathbf{q}_{k}^{r}\right) \approx-\varepsilon_{k}\left\langle\rho_{k}\right\rangle^{k}\left\{\tilde{S}_{k} \tilde{\mathbf{v}}_{k}\right\}^{k} \cdot \nabla\left\{T_{k}\right\}^{k}
$$


El término de flujo de calor $\mathbf{q}_{k}$ aparece en dos formas en la ecuación de la entropía, $\left\langle X_{k} \mathbf{q}_{k}\right\rangle$ y $\left\langle X_{k} \mathbf{q}_{k} / T_{k}\right\rangle$. Puede demostrarse que en el orden de aproximación de este análisis, la diferencia entre estos dos términos (escrita en forma adecuada para que sean dimensionalmente comparables) puede relacionarse con el flujo de calor a través del gradiente de temperatura.

$$
\begin{gathered}
\left\{T_{k}\right\}^{k} \nabla \cdot\left[\varepsilon_{k}\left\langle\frac{\mathbf{q}_{k}}{T_{k}}\right\rangle^{k}\right]-\nabla \cdot\left[\varepsilon_{k}\left\langle\mathbf{q}_{k}\right\rangle^{k}\right]=\nabla \cdot\left[\varepsilon_{k}\left(\left\{T_{k}\right\}^{k}\left\langle\frac{\mathbf{q}_{k}}{T_{k}}\right\rangle^{k}-\left\langle\mathbf{q}_{k}\right\rangle^{k}\right]\right]-\varepsilon_{k}\left\langle\frac{\mathbf{q}_{k}}{T_{k}}\right\rangle^{k} \cdot \nabla\left\{T_{k}\right\}^{k} \\
=\nabla \cdot\left[\varepsilon_{k}\left[\left\langle\frac{\left\{T_{k}\right\}^{k}-T_{k}}{T_{k}} \mathbf{q}_{k}\right\rangle^{k}\right)\right]-\varepsilon_{k}\left\langle\frac{\mathbf{q}_{k}}{T_{k}}\right\rangle^{k} \cdot \nabla\left\{T_{k}\right\}^{k}+\varepsilon_{k} \frac{\left\langle\mathbf{q}_{k}\right\rangle^{k}}{\left\{T_{k}\right\}^{k}} \cdot \nabla\left\{T_{k}\right\}^{k}-\varepsilon_{k} \frac{\left\langle\mathbf{q}_{k}\right\rangle^{k}}{\left\{T_{k}\right\}^{k}} \cdot \nabla\left\{T_{k}\right\}^{k} \\
=\nabla \cdot\left[\varepsilon_{k}\left(\left\langle\frac{\left\{T_{k}\right\}^{k}-T_{k}}{T_{k}} \mathbf{q}_{k}\right\rangle^{k}\right)\right]+\varepsilon_{k}\left\langle\left(\frac{1}{\left\{T_{k}\right\}^{k}}-\frac{1}{T_{k}}\right) \mathbf{q}_{k}\right\rangle^{k} \cdot \nabla\left\{T_{k}\right\}^{k} \\
-\varepsilon_{k} \frac{\left\langle\mathbf{q}_{k}\right\rangle^{k}}{\left\{T_{k}\right\}^{k}} \cdot \nabla\left\{T_{k}\right\}^{k}
\end{gathered}
$$

quedando finalmente:

$$
\begin{aligned}
\left\{T_{k}\right\}^{k} \nabla \cdot\left[\varepsilon_{k}\left\langle\frac{\mathbf{q}_{k}}{T_{k}}\right\rangle^{k}\right]-\nabla \cdot\left[\varepsilon_{k}\left\langle\mathbf{q}_{k}\right\rangle^{k}\right]= \\
\nabla \cdot\left[-\varepsilon_{k}\left(\left\langle\frac{\tilde{T}_{k}}{T_{k}} \mathbf{q}_{k}\right\rangle^{k}\right)\right]+\varepsilon_{k} \frac{1}{\left\{T_{k}\right\}^{k}}\left\langle\frac{\tilde{T}_{k}}{T_{k}} \mathbf{q}_{k}\right\rangle^{k} \cdot \nabla\left\{T_{k}\right\}^{k}-\varepsilon_{k} \frac{\left\langle\mathbf{q}_{k}\right\rangle^{k}}{\left\{T_{k}\right\}^{k}} \cdot \nabla\left\{T_{k}\right\}^{k} \cdot(\mathrm{D}
\end{aligned}
$$

Los primeros dos términos del miembro derecho son promedios espacio-temporales de desviaciones ponderadas en la masa. De una forma similar a la desarrollada para el promedio espacio-temporal de la desviación de la entropía ponderada en la masa, estos promedios pueden expresarse como el promedio de productos de desviaciones. Para el orden de aproximación de este análisis tales términos se despreciarán. Por lo tanto la Ec. (D.16) se reduce a:

$$
\left\{T_{k}\right\}^{k} \nabla \cdot\left[\varepsilon_{k}\left\langle\frac{\mathbf{q}_{k}}{T_{k}}\right\rangle^{k}\right]-\nabla \cdot\left[\varepsilon_{k}\left\langle\mathbf{q}_{k}\right\rangle^{k}\right] \approx-\varepsilon_{k} \frac{\left\langle\mathbf{q}_{k}\right\rangle^{k}}{\left\{T_{k}\right\}^{k}} \cdot \nabla\left\{T_{k}\right\}^{k} .
$$


Similarmente la transferencia de calor interfacial aparece como transporte de calor interfacial y como transporte de entropía interfacial.

$$
\begin{aligned}
\left\langle\mathbf{q}_{k} \cdot \nabla X_{k}\right\rangle-\left\{T_{k}\right\}^{k}\left\langle\frac{\mathbf{q}_{k}}{T_{k}} \cdot \nabla X_{k}\right\rangle & =\left\langle\mathbf{q}_{k} \cdot \nabla X_{k}\left(1-\frac{\left\{T_{k}\right\}^{k}}{T_{k}}\right)\right\rangle \\
& =\left\langle\mathbf{q}_{k} \cdot \nabla X_{k} \frac{T_{k}-\left\{T_{k}\right\}^{k}}{T_{k}}\right\rangle \\
& =\left\langle\mathbf{q}_{k} \cdot \nabla X_{k} \frac{\tilde{T}_{k}}{T_{k}}\right\rangle=\left\langle\mathbf{q}_{k} \cdot \nabla X_{k}\right\rangle \frac{\left\langle\tilde{T}_{k}\right\rangle}{\left\{T_{k}\right\}^{k}}+\text { h.o.t. }
\end{aligned}
$$

y como anteriormente ya demostramos que $\left\langle\tilde{T}_{k}\right\rangle^{k}=-\left\langle\rho_{k}^{\prime} \tilde{T}_{k}\right\rangle^{k} /\left\langle\rho_{k}\right\rangle^{k}$. En el orden de aproximación que hemos estado utilizando, reducimos la Ec. (D.18) a:

$$
\left\langle\mathbf{q}_{k} \cdot \nabla X_{k}\right\rangle-\left\{T_{k}\right\}^{k}\left\langle\frac{\mathbf{q}_{k}}{T_{k}} \cdot \nabla X_{k}\right\rangle \approx 0 .
$$

En las ecuaciones promediadas tenemos dos tipos de temperaturas promedio, $\left\langle T_{k}\right\rangle^{k} \mathrm{y}$ $\left\{T_{k}\right\}^{k}$, analicemos como están relacionadas:

$$
\begin{aligned}
\left\langle T_{k}\right\rangle^{k}-\left\{T_{k}\right\}^{k} & =\frac{\left\langle X_{k} T_{k}\right\rangle}{\left\langle X_{k}\right\rangle}-\frac{\left\langle X_{k} \rho_{k} T_{k}\right\rangle}{\left\langle X_{k} \rho_{k}\right\rangle} \\
& =\frac{\left\langle\rho_{k}\right\rangle^{k}\left\langle X_{k} T_{k}\right\rangle-\left\langle X_{k} \rho_{k} T_{k}\right\rangle}{\varepsilon_{k}\left\langle\rho_{k}\right\rangle^{k}} \\
& =\frac{\left\langle\left\langle\rho_{k}\right\rangle^{k} X_{k} T_{k}-X_{k} \rho_{k} T_{k}\right\rangle}{\varepsilon_{k}\left\langle\rho_{k}\right\rangle^{k}} \\
& =\frac{\left\langle X_{k} T_{k}\left(\left\langle\rho_{k}\right\rangle^{k}-\rho_{k}\right)\right\rangle}{\varepsilon_{k}\left\langle\rho_{k}\right\rangle^{k}}=-\frac{\varepsilon_{k}\left\langle T_{k} \rho_{k}^{\prime}\right\rangle^{k}}{\varepsilon_{k}\left\langle\rho_{k}\right\rangle^{k}} \\
& =-\left\langle T_{k}\right\rangle^{k} \frac{\left\langle\rho_{k}^{\prime}\right\rangle^{k}}{\left\langle\rho_{k}\right\rangle^{k}}-\frac{\left\langle T_{k}^{\prime} \rho_{k}^{\prime}\right\rangle^{k}}{\left\langle\rho_{k}\right\rangle^{k}}=-\frac{\left\langle T_{k}^{\prime} \rho_{k}^{\prime}\right\rangle^{k}}{\left\langle\rho_{k}\right\rangle^{k}} .
\end{aligned}
$$

Por lo tanto:

$$
\left\langle T_{k}\right\rangle^{k}-\left\{T_{k}\right\}^{k} \approx 0 .
$$




\section{Apéndice $E$}

\section{Evaluación de la derivada del módulo de compresibilidad del sólido}

Una cerradura conveniente para la presión promedio de la fase sólida en modelos de dos campos de presión debe tomar en cuenta dos contribuciones. La primera es que el campo de presión del gas ocurriendo en la posición del sólido como si la partícula sólida no estuviera, conocida como la aproximación hidrostática; la segunda es un efecto colectivo debido a la aglomeración de las partículas sólidas, dando la fuerza tomada en cuenta por el módulo de compresibilidad del sólido. El resultado puede expresarse como $P_{s}=P_{g}+\Phi\left(\varepsilon_{s}\right)$. El primer efecto puede tomarse como una consecuencia directa de la consideración fundamental, conocida en hidrostática como la base para demostrar los teoremas de Arquímedes. El segundo término fue introducido para superar el mal planteamiento del modelo al ajustar el módulo de compresibilidad del sólido y después al proponer otras relaciones (Gidaspow, 1994).

Para encontrar la función $\Phi_{0}^{\prime}$ que garantice rapideces de propagación reales, en el discriminante de segundo orden del modelo incompresible, dado en la Ec. (4.21), debe ser mayor o igual a cero. Después de algunas sustituciones se llega a

$$
\Phi_{0}^{\prime} \geq \frac{\rho_{s 0} \rho_{g 0}\left(v_{g 0}-v_{s 0}\right)^{2}}{\varepsilon_{0} \rho_{s 0}+\varepsilon_{s 0} \rho_{g 0}} .
$$

Más aún, considerando que $\varepsilon_{s 0} \rho_{g 0}<<\varepsilon_{0} \rho_{s 0}$, la desigualdad también se satisface por

$$
\varepsilon_{0} \Phi_{0}^{\prime} \geq \rho_{g 0}\left(v_{g 0}-v_{s 0}\right)^{2}
$$

y por lo tanto, puede proponerse la siguiente igualdad

$$
\varepsilon_{0} \Phi_{0}^{\prime}=\xi \rho_{g 0}\left(v_{g 0}-v_{s 0}\right)^{2}, \text { donde } \xi \geq 1
$$

que tiene la misma estructura que la propuesta por (Stuhmiller, 1977) para columnas de burbujeo, desarrollada con la teoría de flujo potencial, con $\xi=1 / 4$. 


\section{Apéndice $F$}

\section{Expresiones explícitas de las matrices \\ B, C y D dadas en la Ec. (4.6)}

$$
\begin{aligned}
& \mathbf{B}=\left(\begin{array}{cccc}
b_{11} & b_{12} & 0 & 0 \\
b_{21} & 0 & 0 & 0 \\
0 & 0 & b_{33} & 0 \\
0 & 0 & 0 & b_{44}
\end{array}\right) \\
& \mathbf{C}=\left(\begin{array}{cccc}
c_{11} & c_{12} & c_{13} & 0 \\
c_{21} & 0 & 0 & c_{24} \\
0 & c_{32} & c_{33} & 0 \\
c_{41} & c_{42} & 0 & c_{44}
\end{array}\right) \\
& \mathbf{D}=\left(\begin{array}{cccc}
0 & 0 & 0 & 0 \\
0 & 0 & 0 & 0 \\
d_{31} & d_{32} & d_{33} & d_{34} \\
d_{41} & d_{42} & d_{43} & d_{44}
\end{array}\right) .
\end{aligned}
$$

\begin{tabular}{|c|c|c|}
\hline$b_{11}=\rho_{g 0}$ & $c_{11}=\rho_{g 0} v_{g 0}$ & $d_{31}=\beta\left(\varepsilon_{s 0}-\varepsilon_{0}\right)\left(v_{g 0}-v_{s 0}\right)$ \\
\hline$b_{12}=\frac{\varepsilon_{0}}{s^{2}}$ & $c_{12}=v_{g 0} \frac{\varepsilon_{0}}{s^{2}}$ & $d_{32}=g\left[\rho_{g 0}\left(p_{0}\right) / p_{0}\right]$ \\
\hline$b_{21}=-\rho_{s 0}$ & $c_{13}=\varepsilon_{0} \rho_{g 0}$ & $d_{33}=\beta \varepsilon_{s 0} \varepsilon_{0}$ \\
\hline$b_{33}=\varepsilon_{0} \rho_{g 0}$ & $c_{21}=-\rho_{s 0} v_{s 0}$ & $d_{34}=-\beta \varepsilon_{s 0} \varepsilon_{0}$ \\
\hline \multirow[t]{6}{*}{$b_{44}=\varepsilon_{s 0} \rho_{s 0}$} & $c_{24}=\varepsilon_{s 0} \rho_{s 0}$ & $d_{41}=-\left[\beta\left(\varepsilon_{s 0}-\varepsilon_{0}\right)\left(v_{g 0}-v_{s 0}\right)+\left(\rho_{s 0}-\rho_{g 0}\right) g\right]$, \\
\hline & $c_{32}=\varepsilon_{0}$ & $d_{42}=-\varepsilon_{s 0} g\left[\rho_{g 0}\left(p_{0}\right) / p_{0}\right]$, \\
\hline & $c_{33}=\varepsilon_{0} \rho_{g 0} v_{g 0}$ & $d_{43}=-d_{44}=-\beta \varepsilon_{s 0} \varepsilon_{0}$ \\
\hline & $c_{41}=-\varepsilon_{s 0} \Phi_{0}^{\prime}$ & \\
\hline & $c_{42}=\varepsilon_{s 0}$, & \\
\hline & $c_{44}=\varepsilon_{s 0} \rho_{s 0} v_{s 0}$, & \\
\hline
\end{tabular}

Donde los coeficientes están definidos en la Tabla F.1, con $\frac{d \rho_{g}}{d P_{g}}=s^{-2}$.

Tabla F.1. Coeficientes de las matrices B, C y D. 


\section{Apéndice $G$}

\section{Sistemas de ecuaciones cuyas raíces son las rapideces de propagación del Capítulo 4}

$\underline{\text { Sistema incompresible de segundo orden }}$

$$
\begin{aligned}
& c_{1}+c_{2}=2 \frac{\left(1-\varepsilon_{0}\right) \rho_{g o} v_{g 0}+\varepsilon_{0} \rho_{s o} v_{s 0}}{\left(1-\varepsilon_{0}\right) \rho_{g o}+\varepsilon_{0} \rho_{s o}} \doteq p_{1} \\
& c_{1} c_{2}=2 \frac{\left(1-\varepsilon_{0}\right) \rho_{g o} v_{g 0}^{2}+\varepsilon_{0} \rho_{s o} v_{s 0}^{2}-\varepsilon_{0}\left(1-\varepsilon_{0}\right) \Phi_{0}^{\prime}}{\left(1-\varepsilon_{0}\right) \rho_{g o}+\varepsilon_{0} \rho_{s o}} \doteq p_{2} .
\end{aligned}
$$

Combinando la Ec. (G.1) con la Ec. (G.2) se obtiene la ecuación: $c^{2}-p_{1} c+p_{2}=0$ cuyas raíces están dadas por la Ec. (4.21).

Sistema compresible de cuarto orden

$$
\begin{aligned}
& \eta_{44}+\eta_{43}+\eta_{42}+\eta_{41}=2\left(v_{g 0}+v_{s 0}\right) \\
& \eta_{44} \eta_{41}+\eta_{43} \eta_{41}+\eta_{44} \eta_{43}+\eta_{44} \eta_{42}+\eta_{43} \eta_{42}+\eta_{42} \eta_{41}=\frac{\rho_{s o}\left(v_{g 0}^{2}+4 v_{g 0} v_{s 0}+v_{s 0}^{2}\right)-\left(1-\varepsilon_{0}\right) \Phi_{0}^{\prime}}{\rho_{s o}} \\
& \eta_{44} \eta_{43} \eta_{42}+\eta_{44} \eta_{43} \eta_{41}+\eta_{44} \eta_{42} \eta_{41}+\eta_{43} \eta_{42} \eta_{41}=2 \frac{\rho_{s o} v_{s 0}\left(v_{g 0}+v_{s 0}\right)-\left(1-\varepsilon_{0}\right) \Phi_{0}^{\prime}}{\rho_{s o}} v_{g 0} \\
& \eta_{44} \eta_{43} \eta_{42} \eta_{41}=\frac{\rho_{s o} v_{s 0}^{2}-\left(1-\varepsilon_{0}\right) \Phi_{0}^{\prime}}{\rho_{s o}} v_{g 0}^{2} .
\end{aligned}
$$

Cuyas raíces están dadas en la Ec. (4.24) y en la Ec. (4.25). 


$$
\begin{aligned}
& \eta_{33}+\eta_{32}+\eta_{31}=P_{1} \\
& \eta_{33} \eta_{31}+\eta_{33} \eta_{32}+\eta_{32} \eta_{31}=P_{2} \\
& \eta_{33} \eta_{32} \eta_{31}=P_{3}
\end{aligned}
$$

donde los polinomios $P_{k}, k=1,2,3$ son respectivamente:

$$
P_{1} \doteq \frac{\varepsilon_{0}\left(4 \varepsilon_{0}-1\right) v_{g 0}+\varepsilon_{0}\left(1-\varepsilon_{0}\right) v_{s 0}+\varepsilon_{0}\left(1-\varepsilon_{0}\right) \frac{\rho_{s o}}{\rho_{g o}}\left(v_{g 0}+2 v_{s 0}\right)+\left[\rho_{g o}-\left(1+\varepsilon_{0}\right) \rho_{s o}\right] \frac{g}{\beta}}{\varepsilon_{0}^{2}+\varepsilon_{0}\left(1-\varepsilon_{0}\right) \frac{\rho_{s o}}{\rho_{g o}}}
$$

$$
P_{2} \doteq \frac{\left[\begin{array}{c}
\varepsilon_{0}\left(5 \varepsilon_{0}-2\right) v_{g 0}^{2}+2 \varepsilon_{0}\left(1-\varepsilon_{0}\right) v_{g 0} v_{s 0}+\varepsilon_{0}\left(1-\varepsilon_{0}\right) \frac{\rho_{s o}}{\rho_{g o}} v_{s 0}\left(2 v_{g 0}+v_{s 0}\right) \\
-2\left(\rho_{s o} v_{s 0}-\rho_{g o} v_{g 0}+\varepsilon_{0} \rho_{s o} v_{g 0}\right) \frac{g}{\beta}-\frac{\varepsilon_{0}\left(1-\varepsilon_{0}\right)^{2}}{\rho_{g o}} \Phi_{0}^{\prime}
\end{array}\right]}{\varepsilon_{0}^{2}+\varepsilon_{0}\left(1-\varepsilon_{0}\right) \frac{\rho_{s o}}{\rho_{g o}}}
$$

$$
P_{3} \doteq \frac{\left[\begin{array}{c}
\varepsilon_{0}\left(2 \varepsilon_{0}-1\right) v_{g 0}^{3}+\varepsilon_{0}\left(1-\varepsilon_{0}\right) v_{g 0}^{2} v_{s 0}+\varepsilon_{0}\left(1-\varepsilon_{0}\right) \frac{\rho_{s o}}{\rho_{g o}} v_{g 0} v_{s 0}^{2}-\frac{\varepsilon_{0}\left(1-\varepsilon_{0}\right)^{2}}{\rho_{g o}} v_{g 0} \Phi_{0}^{\prime} \\
+\left[\left(1-\varepsilon_{0}\right) \Phi_{0}^{\prime}-\rho_{s o} v_{s 0}^{2}-\left(\varepsilon_{0} \rho_{s o}-\rho_{g o}\right) v_{g 0}^{2}\right] \frac{g}{\beta}
\end{array}\right]}{\varepsilon_{0}^{2}+\varepsilon_{0}\left(1-\varepsilon_{0}\right) \frac{\rho_{s o}}{\rho_{g o}}}
$$

y cuyos valores son las raíces $\left(\eta_{31}, \eta_{32}, \eta_{33}\right)$ de la ecuación cúbica

$$
\eta^{3}-P_{1} \eta^{2}+P_{2} \eta-P_{3}=0
$$


Comportamiento completo (efectos compresible e incompresible) sistema de tercer orden

$\alpha_{1}+\alpha_{2}+\alpha_{3}=P_{1}$

$\alpha_{1} \alpha_{2}+\alpha_{2} \alpha_{3}+\alpha_{3} \alpha_{1}=P_{2}-\frac{s^{2}}{C}$

$\alpha_{1} \alpha_{2} \alpha_{3}=P_{3}-\frac{s^{2} a}{C}$

cuyos valores de las raíces $\left(\alpha_{1}, \alpha_{2}, \alpha_{3}\right)$ de la ecuación cúbica representada en la Ec. (4.30). 


\section{Apéndice $H$}

\section{Expresiones explícitas de los componentes de la matriz $D^{*}$ dada en la Ec. (5.11)}

$$
\mathbf{D}^{*}=\left(\begin{array}{cccc}
0 & 0 & 0 & 0 \\
0 & 0 & 0 & 0 \\
d_{31} & d_{32} & d_{33} & d_{34} \\
d_{41} & d_{42} & d_{43} & d_{44}
\end{array}\right) .
$$

Donde los coeficientes están definidos en la Tabla H.1.

Tabla H.1. Coeficientes de las matriz D*.

\begin{tabular}{ll}
\hline$d_{31}=\beta\left(\varepsilon_{s 0}-\varepsilon_{0}\right)\left(v_{g 0}-v_{s 0}\right)$, & $d_{41}=-\left[\beta\left(\varepsilon_{s 0}-\varepsilon_{0}\right)\left(v_{g 0}-v_{s 0}\right)+\left(\rho_{s 0}-\rho_{g 0}\right) g\right]$, \\
$d_{32}=\left(\beta_{g W} v_{g 0}{ }^{2}+g\right) \frac{\rho_{g 0}\left(p_{0}\right)}{p_{0}}$, & $d_{42}=-\varepsilon_{s 0} g \frac{\rho_{g 0}\left(p_{0}\right)}{p_{0}}$, \\
$d_{33}=\beta \varepsilon_{s 0} \varepsilon_{0}+2 \beta_{g W} \rho_{g 0} v_{g 0}$, & $d_{43}=-\beta \varepsilon_{s 0} \varepsilon_{0}$, \\
$d_{34}=-\beta \varepsilon_{s 0} \varepsilon_{0}$, & $d_{44}=\beta \varepsilon_{s 0} \varepsilon_{0}+2 \beta_{s W} \rho_{s 0} v_{s 0}$. \\
\hline
\end{tabular}




\section{Apéndice $I$}

\section{Coeficientes de la parte de segundo y tercer orden compresible del modelo del Capítulo 5}

$$
\begin{aligned}
M_{31}= & -\frac{2 \rho_{s 0}}{1-\varepsilon_{0}} \frac{1}{C_{w}}\left[\varepsilon_{0} \beta_{s W} v_{s 0}\left(2 v_{g 0}+v_{s 0}\right)+\left(1-\varepsilon_{0}\right) \beta_{g W} v_{g 0}\left(2 v_{s 0}+\frac{v_{g 0}}{2}\right)\right] \\
M_{32}= & -\frac{2 \rho_{s 0}}{1-\varepsilon_{0}} \frac{1}{C_{w}}\left[\varepsilon_{0} \beta_{s W} v_{s 0} v_{g 0}\left(v_{g 0}+2 v_{s 0}\right)+\left(1-\varepsilon_{0}\right) \beta_{g W} v_{s 0} v_{g 0}\left(v_{s 0}+v_{g 0}\right)-\left(1-\varepsilon_{0}\right)^{2} \frac{\beta_{g W} v_{g 0} \Phi_{0}^{\prime}}{\rho_{s 0}}\right]
\end{aligned}
$$




\section{Apéndice $J$}

\section{Ecuación de estado de campo promedio para la entalpía}

La entalpía es una función de estado de la temperatura y de la presión:

$$
H_{k}=H_{k}\left(T_{k}, P_{k}\right)
$$

o bien, en términos de cantidades promediadas:

$$
H_{k}=H_{k}\left(T_{k}+\left\{T_{k}\right\}^{k}, P_{k}+\left\langle P_{k}\right\rangle^{k}\right)
$$

Desarrollando en serie de Taylor alrededor del punto $\left(\left\{T_{k}\right\}^{k},\left\langle P_{k}\right\rangle^{k}\right)$ :

$$
\begin{aligned}
H_{k}=H_{k}\left(\left\{T_{k}\right\}^{k},\left\langle P_{k}\right\rangle^{k}\right) & +\left[\left(\frac{\partial H_{k}}{\partial T_{k}}\right)_{P_{k}}\right]_{\left\{T_{k}\right\}^{k},\left\langle P_{k}\right\rangle^{k}}\left(T_{k}-\left\{T_{k}\right\}^{k}\right) \\
& +\left[\left(\frac{\partial H_{k}}{\partial P_{k}}\right)_{T_{k}}\right]_{\left\{T_{k}\right\}^{k},\left\langle P_{k}\right\rangle^{k}}\left(P_{k}-\left\langle P_{k}\right\rangle^{k}\right)+\cdots
\end{aligned}
$$

La capacidad calorífica a presión constante de la fase $k, c_{p k}$ se define por:

$$
\left(\frac{\partial H_{k}}{\partial T_{k}}\right)_{P_{k}} \doteq c_{p k}
$$

Utilizando la siguiente ecuación básica de la termostática:

$$
d H_{k}=T_{k} d S_{k}+\frac{1}{\rho_{k}} d P_{k},
$$

dividiéndola por $d P_{k}$ a $T_{k}$ constante se obtiene:

$$
\left(\frac{\partial H_{k}}{\partial P_{k}}\right)_{T_{k}}=T_{k}\left(\frac{\partial S_{k}}{\partial P_{k}}\right)_{T_{k}}+\frac{1}{\rho_{k}}
$$


Utilizando también la relación de Maxwell siguiente:

$$
\begin{aligned}
\left(\frac{\partial S_{k}}{\partial P_{k}}\right)_{T_{k}} & =-\left(\frac{\partial V_{k}}{\partial T_{k}}\right)_{P_{k}} \\
& =\frac{1}{\rho_{k}^{2}}\left(\frac{\partial \rho_{k}}{\partial T_{k}}\right)_{P_{k}} .
\end{aligned}
$$

Sustituyendo las Ecs. (J.4), (J.3) y (J.2) en la Ec. (J.1) reducida a primer orden obtenemos:

$$
\begin{aligned}
H_{k} \approx H_{k}\left(\left\{T_{k}\right\}^{k},\left\langle P_{k}\right\rangle^{k}\right) & +\left[c_{p k}\right]_{\left\{T_{k}\right\}^{k},\left\{P_{k}\right\rangle^{k}}\left(T_{k}-\left\{T_{k}\right\}^{k}\right) \\
& +\left[\frac{1}{\rho_{k}}+\frac{T_{k}}{\rho_{k}{ }^{2}}\left(\frac{\partial \rho_{k}}{\partial T_{k}}\right)_{P_{k}}\right]_{\left\{T_{k}\right\}^{k},\left\langle P_{k}\right\rangle^{k}}\left(P_{k}-\left\langle P_{k}\right\rangle^{k}\right),
\end{aligned}
$$

o bien reagrupando

$$
H_{k}-H_{k S} \approx c_{p k S}\left(T_{k}-\left\{T_{k}\right\}^{k}\right)+\left[\frac{1}{\rho_{k}}\left\{1+\left(\frac{\partial \ln \rho_{k}}{\partial \ln T_{k}}\right)_{P_{k}}\right\}\right]_{\left\{T_{k}\right\}^{k},\left\langle P_{k}\right\rangle^{k}}\left(P_{k}-\left\langle P_{k}\right\rangle^{k}\right),
$$

donde $h_{k S}$ y $c_{p k s}$ están evaluadas a temperatura y presión promedios. Especializando esta ecuación para una fase gaseosa $g$ y para una fase sólida $s$ :

$$
H_{g}-H_{g S} \approx c_{p g S}\left(T_{g}-\left\{T_{g}\right\}^{g}\right)+\left[\frac{1}{\rho_{g}}\left\{1+\left(\frac{\partial \ln \rho_{g}}{\partial \ln T_{g}}\right)_{P_{g}}\right\}\right]_{\left\{T_{g}\right\}^{g},\left\langle P_{g}\right\rangle^{g}}\left(P_{g}-\left\langle P_{g}\right\rangle^{g}\right)
$$

$\mathrm{y}$

$$
H_{s}-H_{s S} \approx c_{p s S}\left(T_{s}-\left\{T_{s}\right\}^{s}\right)+\left[\frac{1}{\rho_{s}}\left\{1+\left(\frac{\partial \ln \rho_{s}}{\partial \ln T_{s}}\right)_{P_{s}}\right\}\right]_{\left\{T_{s}\right\}^{s},\left\langle P_{s}\right\rangle^{s}}\left(P_{s}-\left\langle P_{s}\right\rangle^{s}\right) .
$$

Tomando logaritmos naturales a ambos lados de la ecuación de estado del gas ideal:

$$
\ln \rho_{g}=\ln P_{g}-\ln T_{g}-\ln \left(\frac{R}{M_{g}}\right)
$$


por lo que para un gas ideal

$$
\left(\frac{\partial \ln \rho_{g}}{\partial \ln T_{g}}\right)_{P_{g}}=-1
$$

Entonces, sustituyendo la Ec. (J.7) en la Ec. (J.5):

$$
H_{g}-H_{g S} \approx c_{p g}\left(T_{g}-\left\{T_{g}\right\}^{g}\right)
$$

Con la finalidad de no aumentar la notación, $c_{p g}$ es la capacidad calorífica de gas ideal a presión constante de la fase gaseosa $g$.

Tomando el promedio ponderado en la masa de esta ecuación se obtiene:

$$
\left\{H_{g}\right\}^{g}-H_{g S} \approx 0 \text {. }
$$

Sustituyendo este resultado en la Ec. (J.8)

$$
H_{g}-\left\{H_{g}\right\}^{g} \approx c_{p g}\left(T_{g}-\left\{T_{g}\right\}^{g}\right)
$$

o bien

$$
\tilde{H}_{g} \approx c_{p g} \tilde{T}_{g}
$$

Por lo que obtenemos finalmente para el gas:

$$
\left\{H_{g}\right\}^{g} \approx c_{p g}\left\{T_{g}\right\}^{g} \text {. }
$$

Aplicando la derivada material siguiendo el movimiento del fluido de ambos lados:

$$
\frac{D_{g}}{D t}\left\{H_{g}\right\}^{g} \approx c_{p g} \frac{D_{g}}{D t}\left\{T_{g}\right\}^{g} \text {. }
$$

Por otro lado, para un sólido rígido esférico y de tamaño muy pequeño (del orden de micrómetros) podemos trabajar adicionalmente el término

$$
\left(\frac{\partial \ln \rho_{s}}{\partial \ln T_{s}}\right)_{P_{s}}=-\frac{T_{s}}{V_{s}}\left(\frac{\partial V_{s}}{\partial T_{s}}\right)_{P_{s}}=-3 \frac{T_{s}}{d_{p}}\left(\frac{\partial d_{p}}{\partial T_{s}}\right)_{P_{s}} .
$$


De Tablas para sólidos (Incropera y DeWitt, 1996), el término $\left(\frac{\partial d_{p}}{\partial T_{s}}\right)_{P_{s}}$ es del orden de $10^{-6}$, por lo que lo podemos despreciar y la Ec. (J.6) se reduce a

$$
H_{s}-H_{s S} \approx c_{p s}\left(T_{s}-\left\{T_{s}\right\}^{s}\right)+\left[\frac{1}{\rho_{s}}\right]_{\left\{T_{s}\right\}^{s},\left\langle P_{s}\right\rangle^{s}}\left(P_{s}-\left\langle P_{s}\right\rangle^{s}\right)
$$

o bien,

$$
\left\{H_{s}\right\}^{s} \approx c_{p s}\left\{T_{s}\right\}^{s}+\frac{1}{\rho_{s}}\left\langle P_{s}\right\rangle^{s}
$$

Por último, tomamos la derivada material siguiendo el movimiento del sólido en ambos lados para tener:

$$
\frac{D_{s}}{D t}\left\{H_{s}\right\}^{s} \approx c_{p s} \frac{D_{s}}{D t}\left\{T_{s}\right\}^{s}+\frac{D_{s}}{D t}\left(\frac{1}{\rho_{s}}\left\langle P_{s}\right\rangle^{s}\right)
$$




\section{Apéndice $K$}

\section{Evaluación de la derivada de la densidad del gas}

\section{K.1 Derivada de la densidad de la fase gas}

La densidad es una función de estado de la presión y de la temperatura, por lo que:

$$
\frac{\partial}{\partial \chi} \rho_{g}\left(P_{g}(z, t), T_{g}(z, t)\right)=\left(\frac{\partial \rho_{g}}{\partial P_{g}}\right)_{T_{g}} \frac{\partial P_{g}}{\partial \chi}+\left(\frac{\partial \rho_{g}}{\partial T_{g}}\right)_{P_{g}} \frac{\partial T_{g}}{\partial \chi}, \quad \chi=t, z
$$

Además, contamos con las siguientes definiciones de la termostática (Firoozabadi, 1999):

$$
\begin{gathered}
\frac{1}{\rho_{g}}\left(\frac{\partial \rho_{g}}{\partial P_{g}}\right)_{T_{g}} \equiv \kappa_{T} \quad \text { (compresibilidad isotérmica) } \\
\frac{1}{\rho_{g}}\left(\frac{\partial \rho_{g}}{\partial P_{g}}\right)_{S_{g}} \equiv \kappa_{S} \quad \text { (compresibilidad isentrópica) } \\
\left(\frac{\partial \rho_{g}}{\partial P_{g}}\right)_{S_{g}} \equiv s^{-2} \quad(s, \text { velocidad adiabática del sonido) } \\
-\frac{1}{\rho_{g}}\left(\frac{\partial \rho_{g}}{\partial T_{g}}\right)_{P_{g}} \equiv \beta_{V} \quad \text { (expansión volumétrica) }
\end{gathered}
$$

y algunas relaciones entre ellas:

$$
\begin{aligned}
& \kappa_{T}=\gamma \kappa_{S} \\
& c_{p g}=c_{v g}+\frac{T_{g}}{\rho_{g}} \frac{\beta_{V}{ }^{2}}{\kappa_{T}} \\
& c_{v g}(\gamma-1) \frac{\rho_{g}}{T_{g}} \kappa_{T}=\beta_{V}{ }^{2}
\end{aligned}
$$


o también

$$
\begin{aligned}
& \frac{c_{p g}(\gamma-1)}{s^{2} T_{g}}=\beta_{V}{ }^{2} \\
& \frac{1}{\rho_{g}}=s^{2} \kappa_{S}=s^{2} \frac{\kappa_{T}}{\gamma} \\
& \frac{\gamma}{s^{2} \rho_{g}}=\kappa_{T} .
\end{aligned}
$$

Por lo que la Ec. (K.1) se reduce a:

$$
\frac{\partial}{\partial \chi} \rho_{g}=\rho_{g}\left(\kappa_{T} \frac{\partial P_{g}}{\partial \chi}-\beta_{V} \frac{\partial T_{g}}{\partial \chi}\right), \quad \chi=t, z,
$$

o bien

$$
\frac{\partial}{\partial \chi} \rho_{g}=\frac{\gamma}{s^{2}} \frac{\partial P_{g}}{\partial \chi}-\rho_{g} \beta_{V} \frac{\partial T_{g}}{\partial \chi}, \quad \chi=t, z .
$$

K.2 Linealización de $\rho_{g}\left(P_{g}, T_{g}\right)$

$$
\begin{gathered}
\left.\rho_{g}\left(P_{g}, T_{g}\right)\right|_{P_{g 0}, T_{g 0}}=\rho_{g}\left(P_{g 0}, T_{g 0}\right)+\left(\frac{\partial \rho_{g}}{\partial P_{g}}\right)_{T_{g 0}}\left(P_{g}-P_{g 0}\right)+\left(\frac{\partial \rho_{g}}{\partial T_{g}}\right)_{P_{g 0}}\left(T_{g}-T_{g 0}\right)+\cdots \\
\left.\rho_{g}\left(P_{g}, T_{g}\right)\right|_{P_{g 0}, T_{g 0}} \approx \rho_{g 0}+\rho_{g 0} \kappa_{T}\left(P_{g}-P_{g 0}\right)-\rho_{g 0} \beta_{V}\left(T_{g}-T_{g 0}\right) \\
\left.\rho_{g}\left(P_{g}, T_{g}\right)\right|_{P_{g 0}, T_{g 0}} \approx \rho_{g 0}+\frac{\gamma}{s^{2}}\left(P_{g}-P_{g 0}\right)-\rho_{g 0} \beta_{V}\left(T_{g}-T_{g 0}\right) .
\end{gathered}
$$

Restando el estado base:

$$
\left.\rho_{g}\left(P_{g}, T_{g}\right)\right|_{P_{g 0}, T_{g 0}} \approx\left[\rho_{g 0}+\frac{\gamma}{s^{2}}\left(P_{g}-P_{g 0}\right)-\rho_{g 0} \beta_{V}\left(T_{g}-T_{g 0}\right)\right]-\rho_{g 0} .
$$

Finalmente, la linealización de $\rho_{g}\left(P_{g}, T_{g}\right)$ queda como:

$$
\left.\rho_{g}\left(P_{g}, T_{g}\right)\right|_{P_{g 0}, T_{g 0}} \approx \frac{\gamma}{s^{2}}\left(P_{g}-P_{g 0}\right)-\rho_{g 0} \beta_{V}\left(T_{g}-T_{g 0}\right) .
$$




\section{Apéndice $L$}

\section{Expresiones explícitas de las matrices}

\section{B, C y D dadas en la Ec. (7.8)}

$$
\begin{aligned}
\mathbf{B} & \doteq\left(\begin{array}{cccccc}
b_{11} & b_{12} & 0 & 0 & b_{15} & 0 \\
b_{21} & 0 & 0 & 0 & 0 & 0 \\
0 & 0 & b_{33} & 0 & 0 & 0 \\
0 & 0 & 0 & b_{44} & 0 & 0 \\
0 & b_{52} & 0 & 0 & b_{55} & 0 \\
0 & 0 & 0 & 0 & 0 & b_{66}
\end{array}\right) \\
\mathbf{C} & \doteq\left(\begin{array}{cccccc}
c_{11} & c_{12} & c_{13} & 0 & c_{15} & 0 \\
c_{21} & 0 & 0 & c_{24} & 0 & 0 \\
0 & c_{32} & c_{33} & 0 & 0 & 0 \\
c_{41} & c_{42} & 0 & c_{44} & 0 & 0 \\
0 & c_{52} & 0 & 0 & c_{55} & 0 \\
0 & 0 & 0 & 0 & 0 & c_{66}
\end{array}\right) \\
\mathbf{D} & \doteq\left(\begin{array}{cccccc}
0 & 0 & 0 & 0 & 0 & 0 \\
0 & 0 & 0 & 0 & 0 & 0 \\
d_{31} & d_{32} & d_{33} & d_{34} & d_{35} & 0 \\
d_{41} & d_{42} & d_{43} & d_{44} & d_{45} & 0 \\
d_{51} & 0 & 0 & 0 & d_{55} & 0 \\
d_{61} & 0 & 0 & 0 & d_{65} & 0
\end{array}\right) .
\end{aligned}
$$

Donde los coeficientes están definidos en la Tabla L.1, con $\left(\frac{\partial \rho_{g}}{\partial P_{g}}\right)_{S_{g}}=s^{-2}$ y $-\frac{1}{\rho_{g}}\left(\frac{\partial \rho_{g}}{\partial T_{g}}\right)_{P_{g}}=\beta_{V}$ 
Tabla L.1. Coeficientes de las matrices B, C y D.

\begin{tabular}{|c|c|c|}
\hline$b_{11}=\rho_{g 0}$, & $c_{11}=\rho_{g 0} v_{g 0}$ & $d_{31}=\beta\left(\varepsilon_{s 0}-\varepsilon_{0}\right)\left(v_{g 0}-v_{s 0}\right)$, \\
\hline \multirow[t]{2}{*}{$b_{12}=\varepsilon_{0} \frac{\gamma}{s^{2}}$} & $c_{12}=\varepsilon_{0} \frac{\gamma}{s^{2}} v_{g 0}$ & $d_{32}=g \frac{\gamma}{s^{2}}$ \\
\hline & $c_{13}=\varepsilon_{0} \rho_{g 0}$ & $d_{33}=\beta \varepsilon_{s 0} \varepsilon_{0}$ \\
\hline$b_{15}=-\varepsilon_{0} \rho_{g 0} \beta_{V}$ & $c_{15}=-\varepsilon_{0} \rho_{g 0} \beta_{V} v_{g 0}$, & $\begin{array}{l}d_{34}=-\beta \varepsilon_{s 0} \varepsilon_{0} \\
d_{35}=-g \rho_{g 0} \beta_{V}\end{array}$ \\
\hline \multirow{3}{*}{$b_{21}=-\rho_{s 0}$} & $c_{21}=-\rho_{s 0} v_{s 0}$ & $d_{41}=-\left[\beta\left(1-2 \varepsilon_{0}\right)\left(v_{g 0}-v_{s 0}\right)+\left(\rho_{s 0}-\rho_{g 0}\right) g\right]$ \\
\hline & $c_{24}=\varepsilon_{s 0} \rho_{s 0}$ & $d_{42}=-\left(1-\varepsilon_{0}\right) \frac{\gamma}{s^{2}} g$ \\
\hline & $c_{32}=\varepsilon_{0}$ & $d_{43}=-\beta\left(1-\varepsilon_{0}\right) \varepsilon_{0}$ \\
\hline$b_{33}=\varepsilon_{0} \rho_{g 0}$ & $c_{33}=\varepsilon_{0} \rho_{g 0} v_{g 0}$ & $\begin{array}{l}d_{44}=\beta\left(1-\varepsilon_{0}\right) \varepsilon_{0} \\
d_{45}=\left(1-\varepsilon_{0}\right) g \rho_{g 0} \beta_{V}\end{array}$ \\
\hline \multirow[t]{2}{*}{$b_{44}=\varepsilon_{s 0} \rho_{s 0}$} & $\begin{array}{l}c_{41}=-\left(1-\varepsilon_{0}\right) \Phi_{0}^{\prime}, \\
c_{42}=1-\varepsilon_{0}, \\
c_{44}=\left(1-\varepsilon_{0}\right) \rho_{s 0} v_{s 0},\end{array}$ & $d_{51}=-\frac{6}{d_{p}} \bar{h}_{i 0}\left(T_{i}-T_{g 0}\right)$ \\
\hline & & $d_{55}=-\frac{6}{d_{p}} \bar{h}_{i 0}\left(1-\varepsilon_{0}\right)$ \\
\hline$b_{52}=-\varepsilon_{0}$ & $c_{52}=-\varepsilon_{0} v_{g 0}$ & $d_{61}=\frac{6}{d_{p}} \bar{h}_{i 0}\left(T_{i}-T_{g 0}\right)$ \\
\hline$b_{55}=\varepsilon_{0} \rho_{g 0} c_{p g}$ & $c_{55}=\varepsilon_{0} \rho_{g 0} c_{p g} v_{g 0}$ & $d_{65}=\frac{6}{d_{p}} \bar{h}_{i 0}\left(1-\varepsilon_{0}\right)$ \\
\hline$b_{66}=\left(1-\varepsilon_{0}\right) \rho_{g s} c_{p s}$, & $c_{66}=\left(1-\varepsilon_{0}\right) \rho_{s 0} c_{p s} v_{s 0}$, & \\
\hline
\end{tabular}




\section{Apéndice $M$}

\section{Sistemas de ecuaciones cuyas raíces son las rapideces de propagación de la Ec. (7.34)}

Sistema no-isotérmico incompresible de pseudo-tercer orden

$$
\begin{aligned}
& c_{21 T}+c_{22 T}=M_{1 \gamma} \\
& c_{21 T} c_{22 T}=M_{2 \gamma}
\end{aligned}
$$

donde los polinomios $M_{k \gamma}, k=1,2$ son respectivamente:

$$
\begin{gathered}
M_{1 \gamma} \doteq \frac{1}{\Upsilon_{3}}\left[\begin{array}{c}
-g \varepsilon_{0} \beta\left(v_{s 0}+v_{g 0}\right)+g^{2} \frac{\rho_{s 0}-\rho_{g 0}}{1-\varepsilon_{0}}-2 g \varepsilon_{0} \beta v_{g 0} \frac{\rho_{s 0}}{\rho_{g 0}} \\
+2 g \varepsilon_{0} \beta v_{g 0} \frac{1-2 \varepsilon_{0}}{1-\varepsilon_{0}}-\frac{6 \beta \bar{h}_{i 0}\left(T_{g 0}-T_{i}\right)}{\rho_{g 0} d_{p}}
\end{array}\right] \\
M_{2 \gamma} \doteq \frac{1}{\Upsilon_{3}}\left[\begin{array}{c}
\frac{6 g \bar{h}_{i 0}\left(T_{g 0}-T_{i}\right)}{d_{p}} \frac{1+\varepsilon_{0}}{\varepsilon_{0}}-g \varepsilon_{0} \beta v_{s 0} v_{g 0}+g \varepsilon_{0} \beta v_{g 0}^{2} \frac{1-2 \varepsilon_{0}}{1-\varepsilon_{0}} \\
-\frac{6 \beta \bar{h}_{i 0}\left(T_{g 0}-T_{i}\right) v_{g 0}}{\rho_{g 0} d_{p}}+g^{2} v_{g 0} \frac{\rho_{s 0}-\rho_{g 0}}{1-\varepsilon_{0}}-g \varepsilon_{0} \beta v_{g 0}^{2} \frac{\rho_{s 0}}{\rho_{g 0}}
\end{array}\right]
\end{gathered}
$$

y cuyos valores son las raíces $\left(c_{21 T}, c_{22 T}\right)$ de la ecuación cuadrática

$$
c_{2 T}^{2}-M_{1 \gamma} c_{2 T}+M_{2 \gamma}=0 .
$$


Sistema no-isotérmico incompresible de cuarto orden

$$
\begin{aligned}
& c_{44 T}+c_{43 T}+c_{42 T}+c_{41 T}=N_{1 \gamma} \\
& c_{44 T} c_{41 T}+c_{43 T} c_{41 T}+c_{44 T} c_{43 T}+c_{44 T} c_{42 T}+c_{43 T} c_{42 T}+c_{42 T} c_{41 T}=N_{2 \gamma} \\
& c_{44 T} c_{43 T} c_{42 T}+c_{44 T} c_{43 T} c_{41 T}+c_{44 T} c_{42 T} c_{41 T}+c_{43 T} c_{42 T} c_{41 T}=N_{3 \gamma} \\
& c_{44 T} c_{43 T} c_{42 T} c_{41 T}=N_{4 \gamma}
\end{aligned}
$$

donde los polinomios $N_{k \gamma}, k=1,2,3,4$ son respectivamente:

$$
N_{1 \gamma} \doteq \frac{1}{\Upsilon_{4}}\left[\varepsilon_{0} \beta\left(3 v_{g 0}+v_{s 0}\right)-4 \frac{1-2 \varepsilon_{0}}{1-\varepsilon_{0}} \varepsilon_{0} \beta v_{g 0}+\frac{g\left[\rho_{g 0}-\left(1+\varepsilon_{0}\right) \rho_{s 0}\right]}{1-\varepsilon_{0}}+2 \varepsilon_{0} \beta\left(v_{g 0}+v_{s 0}\right) \frac{\rho_{s 0}}{\rho_{g 0}}\right]
$$

$$
N_{2 \gamma} \doteq \frac{1}{\Upsilon_{4}}\left[\begin{array}{l}
-\frac{6 \bar{h}_{i 0}}{d_{p}}\left(T_{g 0}-T_{i}\right)+3 \varepsilon_{0} v_{g 0} \beta\left(v_{g 0}+v_{s 0}\right)-6 v_{g 0}^{2} \beta \varepsilon_{0} \frac{1-2 \varepsilon_{0}}{1-\varepsilon_{0}} \\
-\frac{g}{1-\varepsilon_{0}}\left(-3 \rho_{g 0} v_{g 0}+\rho_{s 0} v_{g 0}+2 \rho_{s 0} v_{s 0}+3 \varepsilon_{0} \rho_{s 0} v_{g 0}\right) \\
+\varepsilon_{0} \beta \frac{\rho_{s 0}}{\rho_{g 0}}\left(v_{g 0}^{2}+4 v_{g 0} v_{s 0}+v_{s 0}^{2}\right)-\frac{\beta}{\rho_{g 0}} \varepsilon_{0}\left(1-\varepsilon_{0}\right) \Phi_{0}^{\prime}
\end{array}\right]
$$

$$
N_{3 \gamma} \doteq \frac{1}{\Upsilon_{4}}\left[\begin{array}{l}
-\frac{12 \bar{h}_{i 0}}{d_{p}}\left(T_{g 0}-T_{i}\right) v_{g 0}+\varepsilon_{0} \beta v_{g 0}^{2}\left(v_{g 0}+3 v_{s 0}\right)+\frac{v_{g 0}^{2}}{1-\varepsilon_{0}}\left(-4 \beta \varepsilon_{0} v_{g 0}+8 \beta \varepsilon_{0}^{2} v_{g 0}+3 \rho_{g 0} g\right) \\
-\frac{g \rho_{s 0}}{1-\varepsilon_{0}}\left(2 v_{s 0} v_{g 0}+v_{s 0}^{2}+3 \varepsilon_{0} v_{g 0}^{2}\right)+2 \varepsilon_{0} \beta v_{s 0} v_{g 0}\left(v_{s 0}+v_{g 0}\right) \frac{\rho_{s 0}}{\rho_{g 0}}+g \Phi_{0}^{\prime}-\frac{2 \varepsilon_{0}\left(1-\varepsilon_{0}\right) \beta v_{g 0} \Phi_{0}^{\prime}}{\rho_{g 0}}
\end{array}\right]
$$

$$
N_{4 \gamma} \doteq \frac{1}{\Upsilon_{4}}\left[\begin{array}{l}
-\frac{6 \bar{h}_{i 0}}{d_{p}}\left(T_{g 0}-T_{i}\right) v_{g 0}^{2}+\varepsilon_{0} \beta v_{s 0} v_{g 0}^{3}-\frac{\beta v_{g 0}^{4} \varepsilon_{0}\left(1-2 \varepsilon_{0}\right)}{1-\varepsilon_{0}}-\frac{g v_{g 0}^{3}\left(\varepsilon_{0} \rho_{s 0}-\rho_{g 0}\right)}{1-\varepsilon_{0}} \\
-\frac{g v_{g 0} v_{s 0}^{2} \rho_{s 0}}{1-\varepsilon_{0}}+\frac{\varepsilon_{0} \rho_{s 0} \beta v_{g 0}^{2} v_{s 0}^{2}}{\rho_{g 0}}+g v_{g 0} \Phi_{0}^{\prime}-\frac{\beta v_{g 0}^{2}\left(1-\varepsilon_{0}\right) \varepsilon_{0} \Phi_{0}^{\prime}}{\rho_{g 0}}
\end{array}\right]
$$

y cuyos valores son las raíces $\left(c_{41 T}, c_{42 T}, c_{43 T}, c_{44 T}\right)$ de la ecuación cuártica

$$
c_{4 T}^{4}-N_{1 \gamma} c_{4 T}^{3}+N_{2 \gamma} c_{4 T}^{2}-N_{3 \gamma} c_{4 T}+N_{4 \gamma}=0 .
$$


Sistema no-isotérmico incompresible de quinto orden

$$
\begin{aligned}
& c_{5,1 T}+c_{5,2 T}+c_{5,3 T}+c_{5,4 T}+c_{5,5 T}=O_{1 \gamma} \\
& c_{5,1 T} c_{5,2 T}+c_{5,3 T} c_{5,4 T}+c_{5,1 T} c_{5,3 T} \\
& +c_{5,1 T} c_{5,4 T}+c_{5,2 T} c_{5,3 T}+c_{5,2 T} c_{5,4 T} \\
& +c_{5,1 T} c_{5,5 T}+c_{5,2 T} c_{5,5 T}+c_{5,3 T} c_{5,5 T}+c_{5,4 T} c_{5,5 T}=O_{2 \gamma} \\
& c_{5,1 T} c_{5,2 T} c_{5,3 T}+c_{5,1 T} c_{5,2 T} c_{5,4 T}+c_{5,1 T} c_{5,3 T} c_{5,4 T}+c_{5,2 T} c_{5,3 T} c_{5,4 T} \\
& +c_{5,1 T} c_{5,2 T} c_{5,5 T}+c_{5,3 T} c_{5,4 T} c_{5,5 T}+c_{5,1 T} c_{5,3 T} c_{5,5 T} \\
& +c_{5,1 T} c_{5,4 T} c_{5,5 T}+c_{5,2 T} c_{5,3 T} c_{5,5 T}+c_{5,2 T} c_{5,4 T} c_{5,5 T}=O_{3 \gamma} \\
& c_{5,1 T} c_{5,2 T} c_{5,3 T} c_{5,4 T} \\
& +c_{5,1 T} c_{5,2 T} c_{5,3 T} c_{5,5 T}+c_{5,1 T} c_{5,2 T} c_{5,4 T} c_{5,5 T} \\
& +c_{5,1 T} c_{5,3 T} c_{5,4 T} c_{5,5 T}+c_{5,2 T} c_{5,3 T} c_{5,4 T} c_{5,5 T}=O_{4 \gamma} \\
& c_{5,1 T} c_{5,2 T} c_{5,3 T} c_{5,4 T} c_{5,5 T}=O_{5 \gamma}
\end{aligned}
$$

donde los polinomios $O_{k \gamma}, k=1,2,3,4,5$ son respectivamente:

$$
\begin{aligned}
O_{1 \gamma} \doteq \frac{1}{\Upsilon_{5}}\left[\frac{\left(3 v_{g 0}+2 v_{s 0}\right) \varepsilon_{0}}{1-\varepsilon_{0}} \rho_{s 0}\right] \\
O_{2 \gamma} \doteq \frac{1}{\Upsilon_{5}}\left[\frac{\left(3 v_{g 0}^{2}+6 v_{g 0} v_{s 0}+v_{s 0}^{2}\right) \varepsilon_{0}}{1-\varepsilon_{0}} \rho_{s 0}-\varepsilon_{0} \Phi_{0}^{\prime}\right] \\
O_{3 \gamma} \doteq \frac{1}{\Upsilon_{5}}\left[\frac{v_{g 0}\left(v_{g 0}^{2}+6 v_{g 0} v_{s 0}+3 v_{s 0}^{2}\right) \varepsilon_{0}}{1-\varepsilon_{0}} \rho_{s 0}-3 \varepsilon_{0} v_{g 0} \Phi_{0}^{\prime}\right] \\
O_{4 \gamma} \doteq \frac{1}{\Upsilon_{5}}\left[\frac{v_{g 0}^{2} v_{s 0}\left(2 v_{g 0}+3 v_{s 0}\right) \varepsilon_{0}}{1-\varepsilon_{0}} \rho_{s 0}-3 \varepsilon_{0} v_{g 0}^{2} \Phi_{0}^{\prime}\right] \\
O_{5 \gamma} \doteq \frac{1}{\Upsilon_{5}}\left[\frac{v_{g 0}^{3} v_{s 0}^{2} \varepsilon_{0}}{1-\varepsilon_{0}} \rho_{s 0}-\varepsilon_{0} v_{g 0}^{3} \Phi_{0}^{\prime}\right]
\end{aligned}
$$

y cuyos valores son las raíces $\left(c_{51 T}, c_{52 T}, c_{53 T}, c_{54 T}, c_{55 T}\right)$ de la ecuación quíntica

$$
c_{5 T}^{5}-O_{1 \gamma} c_{5 T}^{4}+O_{2 \gamma} c_{5 T}^{3}-O_{3 \gamma} c_{5 T}^{2}+O_{4 \gamma} c_{5 T}-O_{5 \gamma}=0 .
$$




\section{Apéndice $N$}

\section{Sistemas de ecuaciones cuyas raíces son las rapideces de propagación de la Ec. (7.43)}

Sistema no-isotérmico completo de quinto orden

$$
\begin{aligned}
& A_{5}\left(\frac{\partial}{\partial t}+\alpha_{5,1 T} \frac{\partial}{\partial z}\right)\left(\frac{\partial}{\partial t}+\alpha_{5,2 T} \frac{\partial}{\partial z}\right)\left(\frac{\partial}{\partial t}+\alpha_{5,3 T} \frac{\partial}{\partial z}\right)\left(\frac{\partial}{\partial t}+\alpha_{5,4 T} \frac{\partial}{\partial z}\right)\left(\frac{\partial}{\partial t}+\alpha_{5,5 T} \frac{\partial}{\partial z}\right) \\
& =A_{5}\left(\frac{\partial^{5}}{\partial t^{5}}+M_{5,4 C} \frac{\partial^{5}}{\partial t^{4} \partial z}+M_{5,3 C} \frac{\partial^{5}}{\partial t^{3} \partial z^{2}}+M_{5,2 C} \frac{\partial^{5}}{\partial t^{2} \partial z^{3}}+M_{5,1 C} \frac{\partial^{5}}{\partial t \partial z^{4}}+M_{5,0 C} \frac{\partial^{5}}{\partial z^{5}}\right)
\end{aligned}
$$

cuya amplitud es,

$$
A_{5} \doteq-\varepsilon_{0} \rho_{g 0}\left[c_{p g} \frac{\gamma}{s^{2}} T+\left(1-\varepsilon_{0}\right) \frac{\beta_{V}}{\beta} \Upsilon_{5}\right]
$$

y las rapideces de propagación se obtienen a partir del sistema de ecuaciones algebraicas:

$$
\begin{aligned}
& \alpha_{5,1 T}+\alpha_{5,2 T}+\alpha_{5,3 T}+\alpha_{5,4 T}+\alpha_{5,5 T}=M_{5,4 C} \\
& \alpha_{5,1 T} \alpha_{5,2 T}+\alpha_{5,3 T} \alpha_{5,4 T}+\alpha_{5,1 T} \alpha_{5,3 T} \\
& +\alpha_{5,1 T} \alpha_{5,4 T}+\alpha_{5,2 T} \alpha_{5,3 T}+\alpha_{5,2 T} \alpha_{5,4 T} \\
& +\alpha_{5,1 T} \alpha_{5,5 T}+\alpha_{5,2 T} \alpha_{5,5 T}+\alpha_{5,3 T} \alpha_{5,5 T}+\alpha_{5,4 T} \alpha_{5,5 T}=M_{5,3 C} \\
& \alpha_{5,1 T} \alpha_{5,2 T} \alpha_{5,3 T}+\alpha_{5,1 T} \alpha_{5,2 T} \alpha_{5,4 T}+\alpha_{5,1 T} \alpha_{5,3 T} \alpha_{5,4 T}+\alpha_{5,2 T} \alpha_{5,3 T} \alpha_{5,4 T} \\
& +\alpha_{5,1 T} \alpha_{5,2 T} \alpha_{5,5 T}+\alpha_{5,3 T} \alpha_{5,4 T} \alpha_{5,5 T}+\alpha_{5,1 T} \alpha_{5,3 T} \alpha_{5,5 T} \\
& +\alpha_{5,1 T} \alpha_{5,4 T} \alpha_{5,5 T}+\alpha_{5,2 T} \alpha_{5,3 T} \alpha_{5,5 T}+\alpha_{5,2 T} \alpha_{5,4 T} \alpha_{5,5 T}=M_{5,2 C} \\
& \alpha_{5,1 T} \alpha_{5,2 T} \alpha_{5,3 T} \alpha_{5,4 T} \\
& +\alpha_{5,1 T} \alpha_{5,2 T} \alpha_{5,3 T} \alpha_{5,5 T}+\alpha_{5,1 T} \alpha_{5,2 T} \alpha_{5,4 T} \alpha_{5,5 T} \\
& +\alpha_{5,1 T} \alpha_{5,3 T} \alpha_{5,4 T} \alpha_{5,5 T}+\alpha_{5,2 T} \alpha_{5,3 T} \alpha_{5,4 T} \alpha_{5,5 T}=M_{5,1 C} \\
& \alpha_{5,1 T} \alpha_{5,2 T} \alpha_{5,3 T} \alpha_{5,4 T} \alpha_{5,5 T}=M_{5,0 C}
\end{aligned}
$$


donde los polinomios $M_{5, i C}, i=0,1,2,3,4$ son respectivamente:

$$
\begin{aligned}
& M_{5,4 C}=-\frac{\varepsilon_{0} \rho_{g 0}}{A_{5}}\left[\begin{array}{l}
c_{p g} \frac{\gamma}{s^{2}} T\left(\eta_{41}+\eta_{42}+\eta_{43}+\eta_{44}+v_{g 0}\right) \\
+\left(1-\varepsilon_{0}\right) \frac{\beta_{V}}{\beta} \Upsilon_{5}\left(c_{51 T}+c_{52 T}+c_{53 T}+c_{54 T}+c_{55 T}\right)
\end{array}\right] \\
& M_{5,3 C}=-\frac{\varepsilon_{0} \rho_{g 0}}{A_{5}}\left[\begin{array}{l}
-\tau c_{p g} \\
+c_{p g} \frac{\gamma}{s^{2}} T\left(\begin{array}{l}
\eta_{41} \eta_{42}+\eta_{41} \eta_{43}+\eta_{42} \eta_{43}+\eta_{41} \eta_{44}+\eta_{42} \eta_{44} \\
+\eta_{43} \eta_{44}+\eta_{41} v_{g 0}+\eta_{42} v_{g 0}+\eta_{43} v_{g 0}+\eta_{44} v_{g 0}
\end{array}\right) \\
+\left(1-\varepsilon_{0}\right) \frac{\beta_{V}}{\beta} \Upsilon_{5}\left(\begin{array}{l}
c_{51 T} c_{52 T}+c_{51 T} c_{53 T}+c_{52 T} c_{53 T}+c_{51 T} c_{54 T}+c_{52 T} c_{54 T} \\
+c_{53 T} c_{54 T}+c_{51 T} c_{55 T}+c_{52 T} c_{55 T}+c_{53 T} c_{55 T}+c_{54 T} c_{55 T}
\end{array}\right)
\end{array}\right)
\end{aligned}
$$

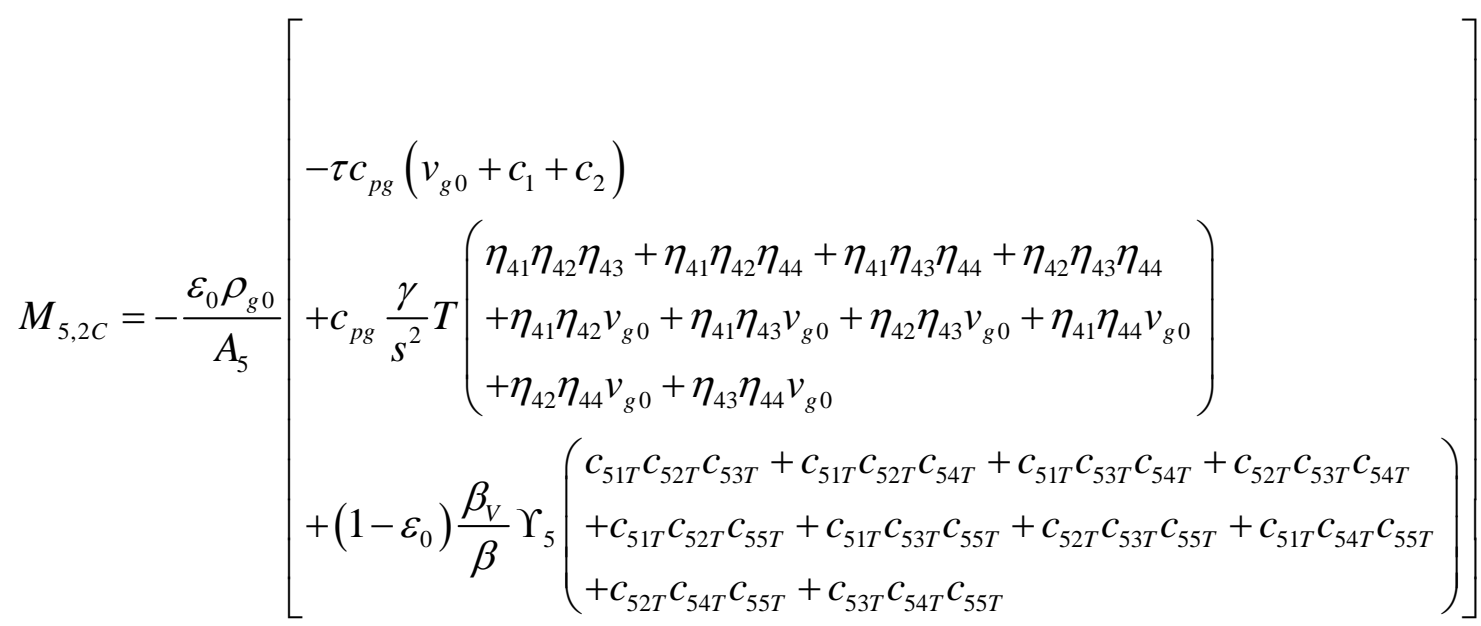

$$
M_{5,1 C}=-\frac{\varepsilon_{0} \rho_{g 0}}{A_{5}}\left[\begin{array}{l}
-\tau c_{p g}\left(v_{g 0} c_{1}+v_{g 0} c_{2}+c_{1} c_{2}\right) \\
+c_{p g} \frac{\gamma}{s^{2}} T\left(\begin{array}{l}
\eta_{41} \eta_{42} \eta_{43} \eta_{44}+\eta_{41} \eta_{42} \eta_{43} v_{g 0}+\eta_{41} \eta_{42} \eta_{44} v_{g 0} \\
+\eta_{41} \eta_{43} \eta_{44} v_{g 0}+\eta_{42} \eta_{43} \eta_{44} v_{g 0}
\end{array}\right) \\
+\left(1-\varepsilon_{0}\right) \frac{\beta_{V}}{\beta} \Upsilon_{5}\left(\begin{array}{l}
c_{51 T} c_{52 T} c_{53 T} c_{54 T}+c_{51 T} c_{52 T} c_{53 T} c_{55 T}+c_{51 T} c_{52 T} c_{54 T} c_{55 T} \\
+c_{51 T} c_{53 T} c_{54 T} c_{55 T}+c_{52 T} c_{53 T} c_{54 T} c_{55 T}
\end{array}\right)
\end{array}\right]
$$




$$
M_{5,0 C}=-\frac{\varepsilon_{0} \rho_{g 0}}{A_{5}}\left[\begin{array}{l}
-\tau c_{p g} v_{g 0} c_{1} c_{2} \\
+c_{p g} \frac{\gamma}{s^{2}} T \eta_{41} \eta_{42} \eta_{43} \eta_{44} v_{g 0} \\
+\left(1-\varepsilon_{0}\right) \frac{\beta_{V}}{\beta} \Upsilon_{5} c_{51 T} c_{52 T} c_{53 T} c_{54 T} c_{55 T}
\end{array}\right]
$$

y cuyos valores son las raíces $\left(\alpha_{5,1 T}, \alpha_{5,2 T}, \alpha_{5,3 T}, \alpha_{5,4 T}, \alpha_{5,5 T}\right)$ de la ecuación de quinto grado

$$
\alpha_{5 T}^{5}-M_{5,4 C} \alpha_{5 T}^{4}+M_{5,3 C} \alpha_{5 T}^{3}-M_{5,2 C} \alpha_{5 T}^{2}+M_{5,1 C} \alpha_{5 T}-M_{5,0 C}=0 .
$$

\section{$\underline{\text { Sistema no-isotérmico completo de cuarto orden }}$}

$$
\begin{aligned}
& A_{4}\left(\frac{\partial}{\partial t}+\alpha_{4,1 T} \frac{\partial}{\partial z}\right)\left(\frac{\partial}{\partial t}+\alpha_{4,2 T} \frac{\partial}{\partial z}\right)\left(\frac{\partial}{\partial t}+\alpha_{4,3 T} \frac{\partial}{\partial z}\right)\left(\frac{\partial}{\partial t}+\alpha_{4,4 T} \frac{\partial}{\partial z}\right) \\
& =A_{4}\left(\frac{\partial^{4}}{\partial t^{4}}+M_{4,3 C} \frac{\partial^{4}}{\partial t^{3} \partial z}+M_{4,2 C} \frac{\partial^{4}}{\partial t^{2} \partial z^{2}}+M_{4,1 C} \frac{\partial^{4}}{\partial t \partial z^{3}}+M_{4,0 C} \frac{\partial^{4}}{\partial z^{4}}\right)
\end{aligned}
$$

cuya amplitud es,

$$
A_{4} \doteq\left[-\frac{\gamma}{s^{2}} C \varepsilon_{0} \rho_{g 0} c_{p g}+\frac{6}{d_{p}} \bar{h}_{i 0}\left(1-\varepsilon_{0}\right) \frac{\gamma}{s^{2}} T-\varepsilon_{0}\left(1-\varepsilon_{0}\right) \rho_{g 0} \frac{\beta_{V}}{\beta} \Upsilon_{4}\right]
$$

y las rapideces de propagación se obtienen a partir del sistema de ecuaciones algebraicas:

$$
\begin{aligned}
& \alpha_{4,1 T}+\alpha_{4,2 T}+\alpha_{4,3 T}+\alpha_{4,4 T}=M_{4,3 C} \\
& \alpha_{4,1 T} \alpha_{4,2 T}+\alpha_{4,3 T} \alpha_{4,4 T}+\alpha_{4,1 T} \alpha_{4,3 T} \\
& +\alpha_{4,1 T} \alpha_{4,4 T}+\alpha_{4,2 T} \alpha_{4,3 T}+\alpha_{4,2 T} \alpha_{4,4 T}=M_{4,2 C} \\
& \alpha_{4,1 T} \alpha_{4,2 T} \alpha_{4,3 T}+\alpha_{4,1 T} \alpha_{4,2 T} \alpha_{4,4 T}+\alpha_{4,1 T} \alpha_{4,3 T} \alpha_{4,4 T}+\alpha_{4,2 T} \alpha_{4,3 T} \alpha_{4,4 T}=M_{4,1 C} \\
& \alpha_{4,1 T} \alpha_{4,2 T} \alpha_{4,3 T} \alpha_{4,4 T}=M_{4,0 C}
\end{aligned}
$$


donde los polinomios $M_{4, i C}, i=0,1,2,3$ son respectivamente:

$$
\begin{aligned}
& M_{4,3 C}=\frac{1}{A_{4}}\left[\begin{array}{l}
-\frac{\gamma}{s^{2}} C \varepsilon_{0} \rho_{g 0} c_{p g}\left(\eta_{31}+\eta_{32}+\eta_{33}+a_{4}\right) \\
+\frac{6}{d_{p}} \bar{h}_{i 0}\left(1-\varepsilon_{0}\right) \frac{\gamma}{s^{2}} T\left(\eta_{41}+\eta_{42}+a_{3}+a_{4}\right) \\
-\varepsilon_{0}\left(1-\varepsilon_{0}\right) \rho_{g 0} \frac{\beta_{V}}{\beta} \Upsilon_{4}\left(c_{41 T}+c_{42 T}+c_{43 T}+c_{44 T}\right)
\end{array}\right]
\end{aligned}
$$

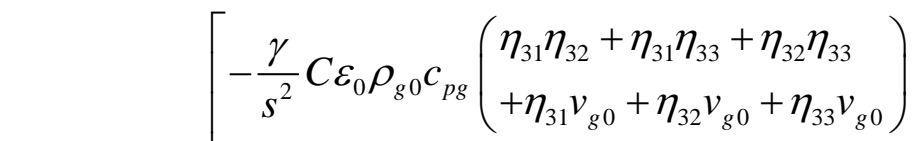

$$
\begin{aligned}
& M_{4,2 C}=\frac{1}{A_{4}}\left(\begin{array}{l}
+\frac{6}{d_{p}} \bar{h}_{i 0}\left(1-\varepsilon_{0}\right) \frac{\gamma}{s^{2}} T\left(\begin{array}{l}
\eta_{41} \eta_{42}+\eta_{41} \eta_{43}+\eta_{42} \eta_{43} \\
+\eta_{41} \eta_{44}+\eta_{42} \eta_{44}+\eta_{43} \eta_{44}
\end{array}\right) \\
-\varepsilon_{0}\left(1-\varepsilon_{0}\right) \rho_{g 0} \frac{\beta_{V}}{\beta} \Upsilon_{4}\left(\begin{array}{l}
c_{41 T} c_{42 T}+c_{41 T} c_{43 T}+c_{42 T} c_{43 T} \\
+c_{41 T} c_{44 T}+c_{42 T} c_{44 T}+c_{43 T} c_{44 T}
\end{array}\right)
\end{array}\right. \\
& +\varepsilon_{0} \rho_{g 0} c_{p g}-\frac{6}{d_{p}} \bar{h}_{i 0}\left(1-\varepsilon_{0}\right) \tau \\
& M_{4,1 C}=\frac{1}{A_{4}}\left[\begin{array}{c}
-\frac{\gamma}{s^{2}} C \varepsilon_{0} \rho_{g 0} c_{p g}\left(\begin{array}{l}
\eta_{31} \eta_{32} \eta_{33}+\eta_{31} \eta_{32} v_{g 0} \\
+\eta_{31} \eta_{33} v_{g 0}+\eta_{32} \eta_{33} v_{g 0}
\end{array}\right) \\
+\frac{6}{d_{p}} \bar{h}_{i 0}\left(1-\varepsilon_{0}\right) \frac{\gamma}{s^{2}} T\left(\begin{array}{l}
\eta_{41} \eta_{42} \eta_{43}+\eta_{41} \eta_{42} \eta_{44} \\
+\eta_{41} \eta_{43} \eta_{44}+\eta_{42} \eta_{43} \eta_{44}
\end{array}\right) \\
-\varepsilon_{0}\left(1-\varepsilon_{0}\right) \rho_{g 0} \frac{\beta_{V}}{\beta} \Upsilon_{4}\left(\begin{array}{l}
c_{41 T} c_{42 T} c_{43 T}+c_{41 T} c_{42 T} c_{44 T} \\
+c_{41 T} c_{43 T} c_{44 T}+c_{42 T} c_{43 T} c_{44 T}
\end{array}\right) \\
+\left(v_{g 0}+a\right) \varepsilon_{0} \rho_{g 0} c_{p g}-\frac{6}{d_{p}} \bar{h}_{i 0}\left(1-\varepsilon_{0}\right) \tau\left(c_{1}+c_{2}\right)
\end{array}\right] \\
& M_{4,0 C}=\frac{1}{A_{4}}\left[\begin{array}{l}
-\frac{\gamma}{s^{2}} C \varepsilon_{0} \rho_{g 0} c_{p g} \eta_{31} \eta_{32} \eta_{33} v_{g 0}+\frac{6}{d_{p}} \bar{h}_{i 0}\left(1-\varepsilon_{0}\right) \frac{\gamma}{s^{2}} T \eta_{41} \eta_{42} \eta_{43} \eta_{44} \\
-\varepsilon_{0}\left(1-\varepsilon_{0}\right) \rho_{g 0} \frac{\beta_{V}}{\beta} \Upsilon_{4} c_{41 T} c_{42 T} c_{43 T} c_{44 T} \\
+v_{g 0} a \varepsilon_{0} \rho_{g 0} c_{p g}-\frac{6}{d_{p}} \bar{h}_{i 0}\left(1-\varepsilon_{0}\right) \tau c_{1} c_{2}
\end{array}\right]
\end{aligned}
$$


y cuyos valores son las raíces $\left(\alpha_{4,1 T}, \alpha_{4,2 T}, \alpha_{4,3 T}, \alpha_{4,4 T}\right)$ de la ecuación de cuarto grado

$$
\alpha_{4 T}^{4}-M_{4,3 C} \alpha_{4 T}^{3}+M_{4,2 C} \alpha_{4 T}^{2}-M_{4,1 C} \alpha_{4 T}+M_{4,0 C}=0
$$

\section{Sistema no-isotérmico completo de tercer orden}

$$
\begin{aligned}
& A_{3}\left(\frac{\partial}{\partial t}+\alpha_{3,1 T} \frac{\partial}{\partial z}\right)\left(\frac{\partial}{\partial t}+\alpha_{3,2 T} \frac{\partial}{\partial z}\right)\left(\frac{\partial}{\partial t}+\alpha_{3,3 T} \frac{\partial}{\partial z}\right) \\
& =A_{3}\left(\frac{\partial^{3}}{\partial t^{3}}+M_{3,2 C} \frac{\partial^{3}}{\partial t^{2} \partial z}+M_{3,1 C} \frac{\partial^{3}}{\partial t \partial z^{2}}+M_{3,0 C} \frac{\partial^{3}}{\partial z^{3}}\right)
\end{aligned}
$$

cuya amplitud es,

$$
A_{3} \doteq \frac{6}{d_{p}} \bar{h}_{i 0}\left(1-\varepsilon_{0}\right) \frac{\gamma}{s^{2}} C
$$

y las rapideces de propagación se obtienen a partir del sistema de ecuaciones algebraicas:

$$
\begin{aligned}
& \alpha_{3,1 T}+\alpha_{3,2 T}+\alpha_{3,3 T}=M_{3,2 C} \\
& \alpha_{3,1 T} \alpha_{3,2 T}+\alpha_{3,1 T} \alpha_{3,3 T}+\alpha_{3,2 T} \alpha_{3,3 T}=M_{3,1 C} \\
& \alpha_{3,1 T} \alpha_{3,2 T} \alpha_{3,3 T}=M_{3,0 C}
\end{aligned}
$$

donde los polinomios $M_{3, i C}, i=0,1,2$ son respectivamente:

$$
M_{3,2 C}=\frac{1}{A_{3}}\left[\begin{array}{l}
\varepsilon_{0} \rho_{g 0} c_{p g} \frac{\gamma}{s^{2}} g C \\
-\varepsilon_{0}\left(1-\varepsilon_{0}\right) \rho_{g 0} \frac{\beta_{V}}{\beta} \Upsilon_{3} \\
+\frac{6}{d_{p}} \bar{h}_{i 0}\left(1-\varepsilon_{0}\right) \frac{\gamma}{s^{2}} C\left(\eta_{31}+\eta_{32}+\eta_{33}\right)
\end{array}\right]
$$




$$
\begin{aligned}
& M_{3,1 C}=\frac{1}{A_{3}}\left[\begin{array}{l}
\varepsilon_{0} \rho_{g 0} c_{p g} \frac{\gamma}{s^{2}} g C\left(\eta_{21}+v_{g 0}\right) \\
-\varepsilon_{0}\left(1-\varepsilon_{0}\right) \rho_{g 0} \frac{\beta_{V}}{\beta} \Upsilon_{3}\left(c_{21 T}+c_{22 T}\right) \\
+\frac{6}{d_{p}} \bar{h}_{i 0}\left(1-\varepsilon_{0}\right) \frac{\gamma}{s^{2}} C\left(\eta_{31} \eta_{32}+\eta_{31} \eta_{33}+\eta_{32} \eta_{33}\right) \\
-\frac{6}{d_{p}} \bar{h}_{i 0}\left(1-\varepsilon_{0}\right)
\end{array}\right] \\
& M_{3,0 C}=\frac{1}{A_{3}}\left[\begin{array}{l}
\varepsilon_{0} \rho_{g 0} c_{p g} \frac{\gamma}{s^{2}} g C \eta_{21} v_{g 0} \\
-\varepsilon_{0}\left(1-\varepsilon_{0}\right) \rho_{g 0} \frac{\beta_{V}}{\beta} \Upsilon_{3} c_{21 T} c_{22 T} \\
+\frac{6}{d_{p}} \bar{h}_{i 0}\left(1-\varepsilon_{0}\right) \frac{\gamma}{s^{2}} C \eta_{31} \eta_{32} \eta_{33} \\
-\frac{6}{d_{p}} \bar{h}_{i 0}\left(1-\varepsilon_{0}\right) a
\end{array}\right]
\end{aligned}
$$

y cuyos valores son las raíces $\left(\alpha_{3,1 T}, \alpha_{3,2 T}, \alpha_{3,3 T}\right)$ de la ecuación cúbica

$$
\alpha_{3 T}^{3}-M_{3,2 C} \alpha_{3 T}^{2}+M_{3,1 C} \alpha_{3 T}-M_{3,0 C}=0
$$




\section{Apéndice $O$}

\section{Expresiones explícitas de los componentes de la matriz $D^{* *}$ dada en la Ec. (8.16)}

$$
\mathbf{D}^{* *}=\left(\begin{array}{cccccc}
0 & 0 & 0 & 0 & 0 & 0 \\
0 & 0 & 0 & 0 & 0 & 0 \\
d_{31} & d_{32} & d_{33} & d_{34} & d_{35} & 0 \\
d_{41} & d_{42} & d_{43} & d_{44} & d_{45} & 0 \\
d_{51} & 0 & 0 & 0 & d_{55} & 0 \\
d_{61} & 0 & 0 & 0 & d_{65} & d_{66}
\end{array}\right) .
$$

Donde los coeficientes están definidos en la Tabla O.1.

Tabla O.1. Coeficientes de las matriz $\mathrm{D}^{* *}$.

\begin{tabular}{ll}
\hline$d_{31}=\beta\left(\varepsilon_{s 0}-\varepsilon_{0}\right)\left(v_{g 0}-v_{s 0}\right)$, & $d_{44}=\beta \varepsilon_{s 0} \varepsilon_{0}+2 \beta_{s W} \rho_{s 0} v_{s 0}$, \\
$d_{32}=\frac{\gamma}{s^{2}}\left(g+\beta_{g W} v_{g 0}{ }^{2}\right)$, & $d_{45}=\left(1-\varepsilon_{0}\right) g \rho_{g 0} \beta_{V}$, \\
$d_{33}=\beta \varepsilon_{s 0} \varepsilon_{0}+2 \beta_{g W} \rho_{g 0} v_{g 0}$, & $d_{51}=-\frac{6}{d_{p}} \bar{h}_{i 0}\left(T_{i}-T_{g 0}\right)+\frac{4}{D_{t}} \bar{h}_{g W 0}\left(T_{W 0}-T_{g 0}\right)$, \\
$d_{34}=-\beta \varepsilon_{s 0} \varepsilon_{0}$, & $d_{55}=-\frac{6}{d_{p}} \bar{h}_{i 0}\left(1-\varepsilon_{0}\right)-\frac{4}{D_{t}} \bar{h}_{g W 0} \varepsilon_{0}$, \\
$d_{35}=-\left(g+\beta_{g W} v_{g 0}{ }^{2}\right) \rho_{g 0} \beta_{V}$, & $d_{61}=\frac{6}{d_{p}} \bar{h}_{i 0}\left(T_{i}-T_{g 0}\right)-\frac{4}{D_{t}} \bar{h}_{s W 0}\left(T_{W 0}-T_{g 0}\right)$, \\
$d_{41}=-\left[\beta\left(\varepsilon_{s 0}-\varepsilon_{0}\right)\left(v_{g 0}-v_{s 0}\right)\right]$, & $d_{65}=\frac{6}{d_{p}} \bar{h}_{i 0}\left(1-\varepsilon_{0}\right)$, \\
$+\left(\rho_{s 0}-\rho_{g 0}\right) g$ & $\gamma$ \\
$d_{42}=-\varepsilon_{s 0} g \frac{\gamma}{s^{2}}$, & $d_{60}=-\frac{4}{D_{t}} \bar{h}_{s W 0} \varepsilon_{s 0}$. \\
$d_{43}=-\beta \varepsilon_{s 0} \varepsilon_{0}$, &
\end{tabular}


